Key Words:

Homogeneity, Coupled

Operations, DWPF

Retention:

Permanent

\title{
REDUCTION OF CONSTRAINTS FOR COUPLED OPERATIONS
}

\author{
F.C. Raszewski
}

T.B. Edwards

DECEMBER 2009

Savannah River National Laboratory

Savannah River Nuclear Solutions

Aiken. SC 29808

Prepared for the U.S. Department of Energy Under

Contract Number DE-AC09-08SR22470

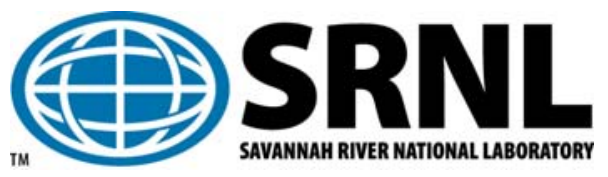




\section{DISCLAIMER}

This work was prepared under an agreement with and funded by the U.S. Government. Neither the U. S. Government or its employees, nor any of its contractors, subcontractors or their employees, makes any express or implied:

1. warranty or assumes any legal liability for the accuracy, completeness, or for the use or results of such use of any information, product, or process disclosed; or

2. representation that such use or results of such use would not infringe privately owned rights; or

3. endorsement or recommendation of any specifically identified commercial product, process, or service.

Any views and opinions of authors expressed in this work do not necessarily state or reflect those of the United States Government, or its contractors, or subcontractors.

Printed in the United States of America

Prepared for

U.S. Department of Energy 


\section{REVIEWS AND APPROVALS}

F.C. Raszewski, Co-author, Process Technology Programs

Date

T.B. Edwards, Co-author, Applied Computational Engineering and Statistics

Date

D.K. Peeler, Peer Reviewer, Process Technology Programs

Date

K.M. Fox, Peer Reviewer, Process Technology Programs

Date

C. C. Herman, Manager, Process Technology Programs

Date

S. L. Marra, Manager,

Date

Environmental \& Chemical Process Technology Research Programs

J. E. Occhipinti, Manager

Date

Waste Solidification Engineering

- ii - 


\section{TABLE OF CONTENTS}

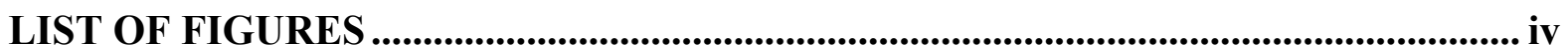

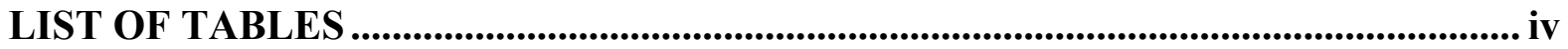

LIST OF ABBREVIATIONS ............................................................................................... V

1.0 EXECUTIVE SUMMARY ........................................................................................ vi

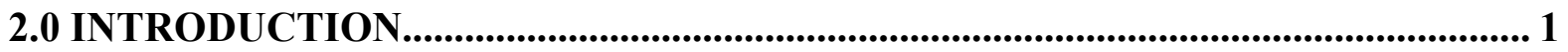

2.1 GLASS SELECTION STRATEGY ........................................................................ 2

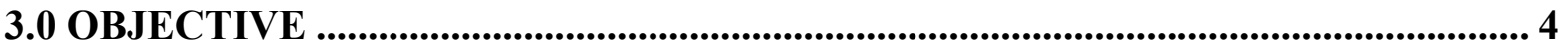

4.0 EXPERIMENTAL PROCEDURES ............................................................................. 4

4.1 TARGET GLASS COMPOSITIONS................................................................ 4

4.2 GLASS FABRICATION......................................................................................... 4

4.3 PROPERTY MEASUREMENTS .............................................................................. 5

4.3.1 Compositional Analysis.................................................................................................. 5

4.3.2 Product Consistency Test (PCT) .................................................................................. 5

5.0 RESULTS AND DISCUSSION .................................................................................... 7

5.1 CHEMICAL COMPOSITION MEASUREMENTS ....................................................... 7

5.1.1 Measurements in Analytical Sequence............................................................................. 7

5.1.2 Composition Measurements by Glass Identifier ......................................................... 7

5.1.3 Batch 1 and Uranium Standard Results ...................................................................... 8

5.1.4 Composition Measurements by Glass Identifier with Targeted Compositions. 9

5.1.5 Measured versus Target Compositions ........................................................................ 15

5.2 MAR ASSESSMENT .......................................................................................... 15

5.3 PCT ......................................................................................................................... 18

5.3.1 Measurements in Analytical Sequence..................................................................... 18

5.3.2 Results for the Samples of the Multi-Element Solution Standard...................... 18

5.3.3 Measurements by Glass Identifier .............................................................................. 19

5.3.4 Normalized PCT Results .............................................................................................. 19

5.3.5 Effects of Heat Treatment ..................................................................................... 24

5.3.6 Predicted versus Measured PCT Results .................................................................... 25

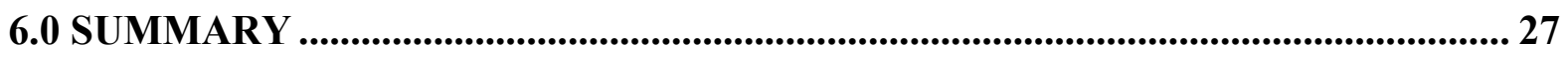

6.1 CHEMICAL COMPOSITION ASSESSMENT ........................................................ 27

6.2 PCT ASSESSMENT ...................................................................................................... 27

7.0 RECOMMENDATIONS............................................................................................. 28

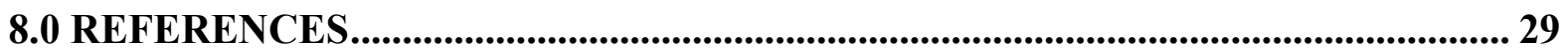




\section{LIST OF FIGURES}

Figure 1. XRD patterns of the ROC-09 quenched (a) and ccc (b) glasses. 24

Figure 2. $\log \mathrm{NL}[\mathrm{B}(\mathrm{g} / \mathrm{L})]$ versus del $\mathrm{Gp}\left(\Delta \mathrm{G}_{\mathrm{p}}\right)$ model with $95 \%$ confidence interval for individual PCT measurements. 26

\section{LIST OF TABLES}

Table 1. Projection Ranges Based on the HLW System Plan Revision 14 (a) with AlDissolution and (b) without Al-Dissolution................................................................ 3

Table 2. Oxide Intervals (wt\%) Used to Develop Glass Compositions.................................... 3

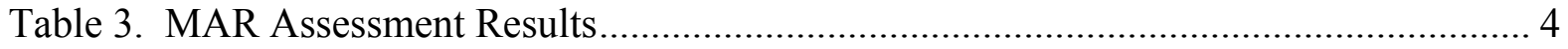

Table 4. Target Compositions .......................................................................................... 6

Table 5. ROC-01, ROC-07, and ROC-13 Peroxide Fusion Measurements............................ 10

Table 6. ROC-06 Lithium Metaborate Measurements …….............................................. 11

Table 7. ROC-08 Lithium Metaborate Measurements …………………............................. 12

Table 8. ROC-17 Lithium Metaborate Measurements ……………………………............. 13

Table 9. ROC-28 Lithium Metaborate Measurements ………….......................................... 14

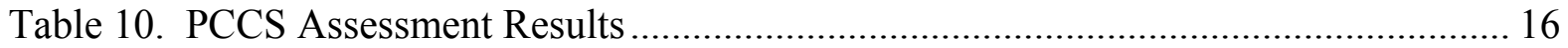

Table 11. Results from Samples of the Multi-Element Solution Standard........................... 18

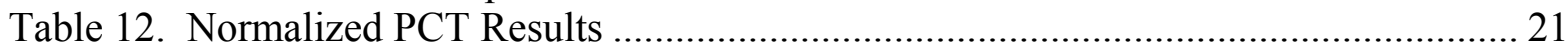

Table 13. Re-measured PCT Responses of ROC-09 ………............................................. 24

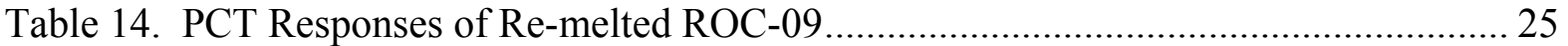




\section{LIST OF ABBREVIATIONS}

$\begin{array}{ll}\Delta \mathrm{G}_{\mathrm{p}} & \text { Free Energy of Hydration } \\ \text { AD } & \text { Analytical Development } \\ \text { ANOVA } & \text { Analysis of Variance } \\ \text { ARM } & \text { Approved Reference Material } \\ \text { B Del } \mathrm{G}_{\mathrm{p}} & \Delta \mathrm{G}_{\mathrm{p} \text { Value for Boron }} \\ \text { bc } & \text { Bias Correction } \\ \text { ccc } & \text { Centerline Canister Cooled } \\ \text { DWPF } & \text { Defense Waste Processing Facility } \\ \text { EA } & \text { Environmental Assessment } \\ \text { homg } & \text { Homogeneity } \\ \text { HLW } & \text { High Level Waste } \\ \text { ICP-AES } & \text { Inductively Coupled Plasma-Atomic Emission Spectroscopy } \\ \text { ID } & \text { Identification } \\ \text { lfrit } & \text { low frit } \\ \text { LM } & \text { Lithium Metaborate Fusion } \\ \text { lsum } & \text { Low Sum of Oxides } \\ \text { MAR } & \text { Measurement Acceptability Region } \\ \text { newhv } & \text { new high viscosity } \\ \text { newlv } & \text { new low viscosity } \\ \text { NL [B (g/L)] } & \text { Normalized Boron Release (g/L) } \\ \text { PAR } & \text { Property Acceptability Region } \\ \text { PCCS } & \text { Process Composition Control System } \\ \text { PCT } & \text { Product Consistency Test } \\ \text { PF } & \text { Sodium Peroxide Fusion } \\ \text { Pred } & \text { Prediction } \\ \text { PSAL } & \text { Process Science Analytical Laboratory } \\ \text { ROC } & \text { Reduction of Constraints } \\ \text { SB } & \text { Sludge Batch } \\ \text { SWPF } & \text { Salt Waste Processing Facility } \\ \text { Th } & \text { Liquidus Temperature } \\ \text { TT\&QAP } & \text { Task Technical and Quality Assurance Plan } \\ \text { TTR } & \text { Task Technical Request } \\ \mathrm{U}_{\text {std }} & \text { Uranium Standard } \\ \text { WL } & \text { Waste Loading } \\ \text { XRD } & \text { X-ray Diffraction } \\ & \end{array}$




\subsection{EXECUTIVE SUMMARY}

The homogeneity constraint was implemented in the Defense Waste Processing Facility (DWPF) Product Composition Control System (PCCS) to help ensure that the current durability models would be applicable to the glass compositions being processed during DWPF operations. While the homogeneity constraint is typically an issue at lower waste loadings (WLs), it may impact the operating windows for DWPF operations, where the glass forming systems may be limited to lower waste loadings based on fissile or heat load limits. In the sludge batch 1b (SB1b) variability study, application of the homogeneity constraint at the measurement acceptability region (MAR) limit eliminated much of the potential operating window for DWPF. As a result, Edwards and Brown developed criteria that allowed DWPF to relax the homogeneity constraint from the MAR to the property acceptance region (PAR) criterion, which opened up the operating window for DWPF operations. These criteria are defined as:

(1) use the alumina constraint as currently implemented in PCCS $\left(\mathrm{Al}_{2} \mathrm{O}_{3} \geq 3 \mathrm{wt} \%\right)$ and add a sum of alkali constraint with an upper limit of $19.3 \mathrm{wt} \%\left(\sum \mathrm{M}_{2} \mathrm{O}<19.3 \mathrm{wt} \%\right)$, or

(2) adjust the lower limit on the $\mathrm{Al}_{2} \mathrm{O}_{3}$ constraint to $4 \mathrm{wt} \%\left(\mathrm{Al}_{2} \mathrm{O}_{3} \geq 4 \mathrm{wt} \%\right)$.

Herman et al. previously demonstrated that these criteria could be used to replace the homogeneity constraint for future sludge-only batches. The compositional region encompassing coupled operations flowsheets could not be bounded as these flowsheets were unknown at the time. With the initiation of coupled operations at DWPF in 2008, the need to revisit the homogeneity constraint was realized. This constraint was specifically addressed through the variability study for SB5 where it was shown that the homogeneity constraint could be ignored if the alumina and alkali constraints were imposed. Additional benefit could be gained if the homogeneity constraint could be replaced by the $\mathrm{Al}_{2} \mathrm{O}_{3}$ and sum of alkali constraint for future coupled operations processing based on projections from Revision 14 of the High Level Waste (HLW) System Plan.

As with the first phase of testing for sludge-only operations, replacement of the homogeneity constraint with the alumina and sum of alkali constraints will ensure acceptable product durability over the compositional region evaluated. Although these study glasses only provide limited data in a large compositional region, the approach and results are consistent with previous studies that challenged the homogeneity constraint for sludge-only operations. That is, minimal benefit is gained by imposing the homogeneity constraint if the other PCCS constraints are satisfied. The normalized boron releases of all of the glasses are well below the Environmental Assessment (EA) glass results, regardless of thermal history. Although one of the glasses had a normalized boron release of approximately $10 \mathrm{~g} / \mathrm{L}$ and was not predictable, the glass is still considered acceptable. This particular glass has a low $\mathrm{Al}_{2} \mathrm{O}_{3}$ concentration, which may have attributed to the anomalous behavior. Given that poor durability has been previously observed in other glasses with low $\mathrm{Al}_{2} \mathrm{O}_{3}$ and $\mathrm{Fe}_{2} \mathrm{O}_{3}$ concentrations, including the sludge-only reduction of constraints study, further investigations appear to be warranted.

Based on the results of this study, it is recommended that the homogeneity constraint (in its entirety with the associated low frit/high frit constraints) be eliminated for coupled operations as 
defined by Revision 14 of the HLW System Plan with up to $2 \mathrm{wt} \% \mathrm{TiO}_{2}$. The use of the alumina and sum of alkali constraints should be continued along with the variability study to determine the predictability of the current durability models and/or that the glasses are acceptable with respect to durability. The use of a variability study for each batch is consistent with the glass product control program and it will help to assess new streams or compositional changes.

It is also recommended that the influence of alumina and alkali on durability be studied in greater detail. Limited data suggests that there may be a need to adjust the lower $\mathrm{Al}_{2} \mathrm{O}_{3}$ limit and/or the upper alkali limit in order to prevent the fabrication of unacceptable glasses. An in-depth evaluation of all previous data as well as any new data would help to better define an alumina and alkali combination that would avoid potential phase separation and ensure glass durability. 


\subsection{INTRODUCTION}

The homogeneity constraint was implemented in the Defense Waste Processing Facility (DWPF) Product Composition Control System (PCCS) to help ensure that the current durability models would be applicable to the glass compositions being processed during DWPF operations. The Product Consistency Test (PCT) response of phase separated glasses has been shown to be unpredictable $e^{1,2}$. Thus, a discriminator to avoid unpredictable behavior was developed so that the durability model could be limited to homogeneous glasses or glasses containing isotropic crystals, such as spinel. The homogeneity constraint was derived from a discriminant analysis ${ }^{\mathrm{A}}$ of 110 glasses ( 88 homogeneous and 22 phase separated) and is a linear discriminant function of terms representing sludge and frit, in which higher concentrations of sludge components appear to lessen the chances of phase separation. While the homogeneity constraint is typically an issue at lower waste loadings (WLs), it may impact the operating windows ${ }^{\mathrm{B}}$ for DWPF operations, where the glass forming systems may be limited to lower waste loadings based on fissile or heat load limits. In the sludge batch $1 \mathrm{~b}$ (SB1b) variability study, application of the homogeneity constraint at the measurement acceptability region (MAR) limit eliminated much of the potential operating window for DWPF. ${ }^{3}$ As a result, Edwards and Brown developed criteria that allowed DWPF to relax the homogeneity constraint from the MAR to the property acceptance region (PAR) criterion, which opened up the operating window for DWPF operations. ${ }^{3}$ These criteria are defined as:

(1) use the alumina constraint as currently implemented in PCCS $\left(\mathrm{Al}_{2} \mathrm{O}_{3} \geq 3 \mathrm{wt} \%\right)$ and add a sum of alkali constraint with an upper limit of $19.3 \mathrm{wt} \%\left(\sum \mathrm{M}_{2} \mathrm{O}<19.3 \mathrm{wt} \%\right)$, or

(2) adjust the lower limit on the $\mathrm{Al}_{2} \mathrm{O}_{3}$ constraint to $4 \mathrm{wt} \%\left(\mathrm{Al}_{2} \mathrm{O}_{3} \geq 4 \mathrm{wt} \%\right)$.

Historical glasses of interest to DWPF having these criteria were found to be acceptable using a normalized boron release (NL [B]) of $10 \mathrm{~g} / \mathrm{L}$ as a benchmark. ${ }^{3}$ This value was chosen so that the boron releases of the study glasses were well below that of the Environmental Assessment (EA) glass when accounting for measurement uncertainty. It should be emphasized that this limit was selected only as a guide to develop the $\mathrm{Al}_{2} \mathrm{O}_{3}$ and/or sum of alkali criteria.

Herman et al. later demonstrated that the $\mathrm{Al}_{2} \mathrm{O}_{3}$ and/or sum of alkali criteria could be used to replace the homogeneity constraint over a bounding glass composition region for sludge-only processing. ${ }^{4}$ The compositional region encompassing coupled operations flowsheets could not be bounded at that time as these flowsheets were unknown. In the sludge-only study, replacing the homogeneity constraint was considered defensible if all of the glasses were predictable by PCCS and/or acceptable compared to the $10 \mathrm{~g} / \mathrm{L}$ normalized boron release benchmark. Two of the glasses that failed the homogeneity constraint did not meet these criteria, but they did meet the alternative $\mathrm{Al}_{2} \mathrm{O}_{3}$ and/or sum of alkali criteria; however, replacing the homogeneity constraint with the $\mathrm{Al}_{2} \mathrm{O}_{3}$ and/or sum of alkali constraint was recommended along with the continued performance of variaibilty studies for each sludge batch.

\footnotetext{
A Linear discriminant analysis is a classification method used in statistics to determine a linear combination of features, which best separate two or more groups of objects or events. In this case, a discriminator based on frit and sludge terms was developed to separate homogenous glasses from phase-separated glasses.

B The WL interval over which a particular glass system is considered to be acceptable based on model predictions.
} 
With the initiation of coupled operations at DWPF in 2008, the need to revisit the homogeneity constraint was realized. This constraint was specifically addressed through the variability study for SB5. ${ }^{5}$ Additional benefit would be gained for future coupled operations processing if the homogeneity constraint could be replaced by the $\mathrm{Al}_{2} \mathrm{O}_{3}$ and/or sum of alkali constraint using a similar methodology as was done for sludge-only processing.

This study has been performed in order to address technical issues discussed in the Technical Task Request (TTR) and was performed in accordance with the Task Technical and Quality Assurance Plan (TT\&QAP).,

\subsection{GLASS SELECTION STRATEGY}

As stated in the TT\&QAP, the latest version of the High Level Waste (HLW) System Plan (Revision 14) was used as the compositional basis for future coupled operations flowsheets. ${ }^{\mathrm{C}}, 8$ The minimum and maximum ranges for each sludge oxide are included in Table 1 (with and without Al-dissolution). The minimum and maximum sludge oxide ranges were combined with the primary frit components $\left(\mathrm{B}_{2} \mathrm{O}_{3}, \mathrm{Li}_{2} \mathrm{O}, \mathrm{Na}_{2} \mathrm{O}\right.$ and $\left.\mathrm{SiO}_{2}\right)$ over waste loading ranges of interest to develop a glass compositional region that potentially bounds future DWPF processing for coupled operations. This range represents WLs from 30 to $50 \%$ with the exception of $\mathrm{Al}_{2} \mathrm{O}_{3}$ concentration, which was lowered to ensure constraint coverage. Table 2 summarizes the bounding glass compositional region used in this study as a result of these assumptions. JMP ${ }^{\mathrm{TM}}$ was used to D-optimally ${ }^{\mathrm{D}}$ select 29 glasses based on the oxide ranges ${ }^{\mathrm{E}}$ in Table 2 that challenged (or were close to challenging) the homogeneity constraint or its associated constraints.

A summary of the MAR assessment results is provided in Table 3. In addition to homogeneity (homg), some of the glasses also challenge the $\mathrm{TiO}_{2} \mathrm{~F}$, liquidus temperature $\left(\mathrm{T}_{\mathrm{L}}\right)$, low frit/high frit ${ }^{G}$ (lfrit/hfrit) and viscosity (newhv) constraints. ${ }^{9}{ }^{, H}$ It should be noted that all ${ }^{\mathrm{I}}$ of the glasses satisfy the $\mathrm{Al}_{2} \mathrm{O}_{3}$ and/or sum or alkali criteria while falling outside of the MAR for the homogeneity constraint, which is consistent with the objectives of this study. The nomenclature for the glass identification (ID) can be described as follows: "ROC" refers to Reduction of Constraints. Glasses ROC-01 through ROC-29 represent extreme vertices and ROC-30 represents the centroid of the glass region of interest for coupled operations.

\footnotetext{
${ }^{\mathrm{C}}$ While it is realized that some processing scenarios exist for elevated levels of $\mathrm{TiO}_{2}$ (i.e., $>2 \mathrm{wt} \%$ ) or the addition of other process streams, the DWPF flowsheets for these scenarios were not available and SRNL could only use what was defined in the system plan.

$\mathrm{D}$ D-optimality is an experimental design method that minimizes the variance of the estimates of the coefficients of the proposed model. In this study, the proposed model was taken to be a linear function of the oxides listed in Table 2.

$\mathrm{E}$ "Others" in Table 2 includes: $\mathrm{BaO}, \mathrm{CdO}, \mathrm{Ce}_{2} \mathrm{O}_{3}, \mathrm{La}_{2} \mathrm{O}_{3}, \mathrm{PbO}, \mathrm{SO}_{4}, \mathrm{ZnO}$ and $\mathrm{ZrO}_{2}$.

F While the PCCS limit for $\mathrm{TiO}_{2}$ in glass is $2 \mathrm{wt} \%$, select compositions in this study fail the $\mathrm{TiO}_{2} \mathrm{MAR}$ once measurement uncertainties are applied to the $2 \mathrm{wt} \%$ value.

$\mathrm{G}$ Both the low frit and high frit constraints are associated with homogeneity.

$\mathrm{H}$ Although not waste form affecting, a risk-based management decision regarding $\mathrm{T}_{\mathrm{L}}$ and viscosity predictions may be necessary with respect to melter feed acceptance; however, failure of these constraints will not impact the outcome of this study.

${ }^{I}$ ROC-08 failed the alkali constraint $\left(\mathrm{R}_{2} \mathrm{O}\right)$ for the given amount of $\mathrm{Al}_{2} \mathrm{O}_{3}$ due to the measurement uncertainty accounted for in PCCS.
} 
SRNL-STI-2009-00465, REVISION 0

Table 1. Projection Ranges Based on the HLW System Plan Revision 14 (a) with Al-Dissolution and (b) without Al-Dissolution

\begin{tabular}{|c|c|c|}
\hline Oxide & $\begin{array}{c}\text { Min } \\
(\mathrm{wt} \%)\end{array}$ & $\begin{array}{c}\text { Max } \\
(\mathrm{wt} \%)\end{array}$ \\
\hline $\mathrm{Al}_{2} \mathrm{O}_{3}$ & 11.95 & 30.60 \\
\hline $\mathrm{BaO}$ & 0.07 & 0.28 \\
\hline $\mathrm{CaO}$ & 1.91 & 3.66 \\
\hline $\mathrm{Ce}_{2} \mathrm{O}_{3}$ & 0.20 & 0.96 \\
\hline $\mathrm{Cr}_{2} \mathrm{O}_{3}$ & 0.19 & 0.41 \\
\hline $\mathrm{CuO}$ & 0.05 & 0.14 \\
\hline $\mathrm{Fe}_{2} \mathrm{O}_{3}$ & 24.32 & 41.22 \\
\hline $\mathrm{K}_{2} \mathrm{O}$ & 0.07 & 0.29 \\
\hline $\mathrm{La}_{2} \mathrm{O}_{3}$ & 0.03 & 0.31 \\
\hline $\mathrm{MgO}$ & 0.35 & 2.65 \\
\hline $\mathrm{MnO}$ & 2.09 & 10.66 \\
\hline $\mathrm{Na}_{2} \mathrm{O}$ & 19.91 & 27.21 \\
\hline $\mathrm{NiO}$ & 0.34 & 4.51 \\
\hline $\mathrm{PbO}$ & 0.09 & 0.39 \\
\hline $\mathrm{SO}_{4}$ & 0.09 & 1.88 \\
\hline $\mathrm{SiO}_{2}$ & 1.79 & 7.26 \\
\hline $\mathrm{ThO}_{2}$ & 0.01 & 3.12 \\
\hline $\mathrm{TiO}_{2}$ & 0.00 & 3.89 \\
\hline $\mathrm{U}_{3} \mathrm{O}_{8}$ & 0.63 & 17.66 \\
\hline $\mathrm{ZnO}$ & 0.05 & 0.24 \\
\hline $\mathrm{ZrO}_{2}$ & 0.10 & 0.67 \\
\hline
\end{tabular}

(a)

\begin{tabular}{|c|c|c|}
\hline Oxide & $\begin{array}{c}\text { Min } \\
(w t \%)\end{array}$ & $\begin{array}{c}\text { Max } \\
(\mathrm{wt} \%)\end{array}$ \\
\hline $\mathrm{Al}_{2} \mathrm{O}_{3}$ & 19.14 & 34.31 \\
\hline $\mathrm{BaO}$ & 0.12 & 0.27 \\
\hline $\mathrm{CaO}$ & 1.74 & 2.89 \\
\hline $\mathrm{Ce}_{2} \mathrm{O}_{3}$ & 0.08 & 0.81 \\
\hline $\mathrm{Cr}_{2} \mathrm{O}_{3}$ & 0.20 & 0.36 \\
\hline $\mathrm{CuO}$ & 0.04 & 0.14 \\
\hline $\mathrm{Fe}_{2} \mathrm{O}_{3}$ & 18.71 & 34.25 \\
\hline $\mathrm{K}_{2} \mathrm{O}$ & 0.13 & 0.41 \\
\hline $\mathrm{La}_{2} \mathrm{O}_{3}$ & 0.09 & 0.26 \\
\hline $\mathrm{MgO}$ & 0.27 & 2.26 \\
\hline $\mathrm{MnO}$ & 1.21 & 8.79 \\
\hline $\mathrm{Na}_{2} \mathrm{O}$ & 19.12 & 27.85 \\
\hline $\mathrm{NiO}$ & 0.17 & 3.94 \\
\hline $\mathrm{PbO}$ & 0.04 & 0.33 \\
\hline $\mathrm{SO}_{4}$ & 0.16 & 1.07 \\
\hline $\mathrm{SiO}_{2}$ & 2.54 & 8.27 \\
\hline $\mathrm{ThO}_{2}$ & 0.00 & 1.84 \\
\hline $\mathrm{TiO}_{2}$ & 0.46 & 3.35 \\
\hline $\mathrm{U}_{3} \mathrm{O}_{8}$ & 0.54 & 18.80 \\
\hline $\mathrm{ZnO}$ & 0.05 & 0.23 \\
\hline $\mathrm{ZrO}_{2}$ & 0.23 & 0.56 \\
\hline
\end{tabular}

(b)

Table 2. Oxide Intervals (wt\%) Used to Develop Glass Compositions

\begin{tabular}{|c|c|c|}
\hline Oxide & $\begin{array}{c}\text { Min } \\
(\mathrm{wt} \%)\end{array}$ & $\begin{array}{c}\text { Max } \\
(\mathrm{wt} \%)\end{array}$ \\
\hline $\mathrm{Al}_{2} \mathrm{O}_{3}$ & 3.25 & 18 \\
\hline $\mathrm{B}_{2} \mathrm{O}_{3}$ & 4.5 & 14 \\
\hline $\mathrm{CaO}$ & 0 & 4 \\
\hline $\mathrm{Cr}_{2} \mathrm{O}_{3}$ & 0 & 0.2 \\
\hline $\mathrm{Fe}_{2} \mathrm{O}_{3}$ & 5 & 21 \\
\hline $\mathrm{Li}_{2} \mathrm{O}$ & 4 & 7 \\
\hline $\mathrm{MgO}$ & 0 & 1.5 \\
\hline $\mathrm{MnO}$ & 0.3 & 5.5 \\
\hline $\mathrm{Na}_{2} \mathrm{O}$ & 10 & 18 \\
\hline $\mathrm{NiO}^{2}$ & 0 & 2.5 \\
\hline $\mathrm{SiO}_{2}$ & 30 & 55 \\
\hline $\mathrm{TiO}_{2}$ & 0.5 & 2 \\
\hline $\mathrm{U}_{3} \mathrm{O}_{8}$ & 0 & 9.5 \\
\hline $\mathrm{Others}_{2}$ & 0 & 2 \\
\hline \multicolumn{3}{|c}{}
\end{tabular}


Table 3. MAR Assessment Results

\begin{tabular}{|c|c|}
\hline Glass ID & MAR Results \\
\hline ROC-01 & $\mathrm{TiO}_{2}$ \\
\hline ROC-02 & $\mathrm{T}_{\mathrm{L}} \mathrm{TiO}_{2}$ Homg \\
\hline ROC-03 & $\mathrm{TiO}_{2}$ 1Frit \\
\hline ROC-04 & $\mathrm{TiO}_{2}$ \\
\hline ROC-05 & Homg lFrit \\
\hline ROC-06 & Homg \\
\hline ROC-07 & Homg hFrit \\
\hline ROC-08 & $\mathrm{TiO}_{2}$ Homg $\mathrm{R}_{2} \mathrm{O}$ lFrit \\
\hline \multicolumn{2}{|l|}{ ROC-09 } \\
\hline ROC-10 & 1Frit \\
\hline ROC-11 & Homg lFrit \\
\hline ROC-12 & Homg \\
\hline ROC-13 & Homg \\
\hline ROC-14 & $\mathrm{T}_{\mathrm{L}} \mathrm{TiO}_{2}$ \\
\hline ROC-15 & $\mathrm{T}_{\mathrm{L}}$ Homg \\
\hline
\end{tabular}

\begin{tabular}{|c|c|}
\hline Glass ID & MAR Results \\
\hline ROC-16 & $\mathrm{TiO}_{2}$ Homg \\
\hline ROC-17 & $\mathrm{TiO}_{2}$ Homg \\
\hline ROC-18 & $\mathrm{TiO}_{2}$ Homg lFrit \\
\hline ROC-19 & $\mathrm{TiO}_{2}$ Homg \\
\hline ROC-20 & $\mathrm{TiO}_{2}$ \\
\hline ROC-21 & $\mathrm{T}_{\mathrm{L}} \mathrm{TiO}_{2}$ Homg \\
\hline ROC-22 & $\mathrm{T}_{\mathrm{L}} \mathrm{Homg}$ lFrit \\
\hline ROC-23 & $\mathrm{T}_{\mathrm{L}}$ newhv TiO \\
\hline ROC-24 & $\mathrm{TiO}_{2}$ \\
\hline ROC-25 & $\mathrm{Homg}$ \\
\hline ROC-26 & $\mathrm{Homg}$ \\
\hline ROC-27 & $\mathrm{T}_{\mathrm{L}} \mathrm{TiO}_{2} 1$ Frit \\
\hline ROC-28 & $\mathrm{TiO}_{2} \mathrm{Homg}$ \\
\hline ROC-29 & $\mathrm{T}_{\mathrm{L}}$ \\
\hline ROC-30 & $\mathrm{Homg}$ \\
\hline
\end{tabular}

\subsection{OBJECTIVE}

The objective of this task is to develop a technical basis for replacing the homogeneity constraint (in its entirety with the associated low/high frit constraints) with the $\mathrm{Al}_{2} \mathrm{O}_{3}$ and/or sum or alkali criteria for future coupled operations flowsheets (as projected by Revision 14 of the HLW System Plan using the $2 \mathrm{wt} \% \mathrm{TiO}_{2}$ glass limit).

\subsection{EXPERIMENTAL PROCEDURES}

\subsection{TARGET GLASS COMPOSITIONS}

Target compositions of the thirty test glasses (expressed as wt $\%$ oxides) are presented in Table 4 .

\subsection{GLASS FABRICATION}

Each glass was prepared from the proper proportions of reagent-grade metal oxides, carbonates, $\mathrm{H}_{3} \mathrm{BO}_{3}$, and salts in $150 \mathrm{~g}$ batches. ${ }^{10}$ The raw materials were thoroughly mixed and placed into a platinum alloy, $250 \mathrm{~mL}$ crucible. Batched materials were placed into a high-temperature furnace at the target melt temperature of $1150^{\circ} \mathrm{C}$. The crucible was removed from the furnace after an isothermal hold at $1150^{\circ} \mathrm{C}$ for 1 hour. The molten glass was quenched by pouring the liquid onto a clean, stainless steel plate. The glass pour patty was used as a sampling stock for the various property measurements (i.e., chemical composition and durability testing). Approximately $25 \mathrm{~g}$ of each glass was heat-treated to simulate cooling along the centerline of a DWPF-type canister to gauge the effects of thermal history on the product performance. ${ }^{11}$ This cooling schedule is referred to as the centerline canister cooling (ccc) curve. 


\subsection{PROPERTY MEASUREMENTS}

\subsubsection{Compositional Analysis}

To confirm that the as-fabricated glasses met the target compositions, a representative sample from each glass was submitted to Analytical Development (AD) for chemical analysis under the auspices of two analytical plans. ${ }^{12,13}$ Two dissolution methods were performed by the Process Science Analytical Laboratory (PSAL) to allow measurement of these chemical compositions: lithium metaborate fusion (LM) and sodium peroxide fusion (PF). For each glass, measurements were obtained from samples prepared in duplicate by each of these dissolution methods. All of the prepared samples were analyzed twice for each element of interest by Inductively Coupled Plasma-Atomic Emission Spectroscopy (ICP-AES) with the instrumentation being re-calibrated between the duplicate analyses. Analytical plans were developed in such a way as to provide the opportunity to evaluate potential sources of bias and error. Glass standards were also intermittently measured to assess the performance of the ICP-AES instrument over the course of these analyses.

\subsubsection{Product Consistency Test (PCT)}

The PCT was performed in triplicate on each quenched and ccc glass to assess chemical durability using Method A of the procedure. ${ }^{14}$ Also included in the experimental test matrix were the EA glass ${ }^{15}$, the Approved Reference Material (ARM) glass ${ }^{15}$, and blanks from the sample cleaning batch. Samples were ground, washed, and prepared according to the standard procedure. The resulting solutions were sampled (filtered and acidified) and analyzed by AD under the auspices of three analytical plans. ${ }^{16-18}$ Samples of a multi-element, standard solution were also included in each analytical plan to verify the accuracy of the ICP-AES instrument. Normalized release rates were calculated based on the elemental concentrations of different compositional views using the average of the leachate concentrations. 
SRNL-STI-2009-00465, REVISION 0

Table 4. Target Compositions

\begin{tabular}{|c|c|c|c|c|c|c|c|c|c|c|c|c|c|c|c|c|c|c|c|c|c|c|}
\hline Glass ID & $\mathrm{Al}_{2} \mathrm{O}_{3}$ & $\mathbf{B}_{2} \mathbf{O}_{3}$ & $\mathrm{BaO}$ & $\mathrm{CaO}$ & $\mathrm{CdO}$ & $\mathrm{Ce}_{2} \mathrm{O}_{3}$ & $\mathrm{Cr}_{2} \mathrm{O}_{3}$ & $\mathrm{CuO}$ & $\mathrm{Fe}_{2} \mathrm{O}_{3}$ & $\mathrm{La}_{2} \mathrm{O}_{3}$ & $\mathbf{L i}_{2} \mathbf{O}$ & MgO & MnO & $\mathrm{Na}_{2} \mathrm{O}$ & $\mathrm{NiO}$ & $\mathrm{PbO}$ & $\mathrm{SO}_{4}$ & $\mathrm{SiO}_{2}$ & $\mathrm{TiO}_{2}$ & $\mathrm{U}_{3} \mathbf{O}_{8}$ & $\mathrm{ZnO}$ & $\mathrm{ZrO}_{2}$ \\
\hline ROC-01 & 10.86 & 6.34 & 0.03 & 0.60 & 0.10 & $\begin{array}{l}0.12 \\
\end{array}$ & 0.20 & 0.04 & 8.62 & 0.03 & 5.88 & 1.50 & 5.50 & 10.46 & 0.00 & 0.07 & 0.16 & 37.90 & 2.00 & 9.50 & 0.04 & 0.07 \\
\hline ROC-02 & 5.91 & 10.19 & 0.00 & 4.00 & 0.00 & 0.00 & 0.18 & 0.00 & 6.70 & 0.00 & 4.00 & 1.35 & 0.30 & 13.89 & 2.50 & 0.00 & 0.00 & 44.71 & 1.96 & 4.31 & 0.00 & 0.00 \\
\hline ROC-03 & 3.76 & 9.98 & 0.08 & 0.00 & 0.30 & 0.36 & 0.00 & 0.13 & 14.14 & 0.10 & 4.00 & 0.00 & 0.48 & 10.00 & 0.00 & 0.22 & 0.48 & 44.25 & 2.00 & 9.38 & 0.13 & 0.21 \\
\hline ROC-04 & 7.96 & 5.42 & 0.08 & 4.00 & 0.30 & 0.36 & 0.00 & 0.13 & 6.76 & 0.10 & 6.01 & 0.00 & 0.30 & 11.81 & 2.50 & 0.22 & 0.48 & 41.74 & 2.00 & 9.50 & 0.13 & 0.21 \\
\hline ROC-05 & 4.55 & 6.86 & 0.00 & 0.10 & 0.00 & 0.00 & 0.00 & 0.00 & 14.52 & 0.00 & 4.00 & 1.50 & 5.50 & 10.53 & 0.07 & 0.00 & 0.00 & 42.36 & 0.50 & 9.50 & 0.00 & 0.00 \\
\hline $\begin{array}{l}\text { ROC-06 } \\
\end{array}$ & 5.10 & 14.00 & 0.00 & 4.00 & 0.00 & 0.00 & 0.20 & 0.00 & 5.00 & 0.00 & 4.00 & 0.00 & 5.50 & 10.62 & 0.00 & 0.00 & 0.00 & 41.58 & 0.50 & 9.50 & 0.00 & 0.00 \\
\hline ROC-07 & 3.46 & 13.69 & 0.00 & 0.00 & 0.00 & 0.00 & 0.20 & 0.00 & 10.23 & 0.00 & 5.40 & 0.00 & 0.30 & 10.10 & 0.00 & 0.00 & 0.00 & 53.88 & 0.50 & 2.24 & 0.00 & 0.00 \\
\hline ROC-08 & 3.51 & 4.50 & 0.00 & 0.00 & 0.00 & 0.00 & 0.20 & 0.00 & 6.22 & 0.00 & 4.75 & 1.50 & 5.50 & 13.92 & 2.50 & 0.00 & 0.00 & 47.74 & 2.00 & 7.67 & 0.00 & 0.00 \\
\hline ROC-09 & 3.91 & 10.07 & 0.08 & 4.00 & 0.30 & 0.36 & 0.00 & 0.13 & 8.29 & 0.10 & 4.00 & 1.50 & 3.79 & 13.30 & 2.50 & 0.22 & 0.48 & 45.94 & 0.50 & 0.20 & 0.13 & 0.21 \\
\hline ROC-10 & 3.33 & 5.36 & 0.08 & 0.00 & 0.30 & 0.36 & 0.20 & 0.13 & 15.02 & 0.10 & 4.52 & 1.50 & 1.29 & 11.16 & 0.00 & 0.22 & 0.48 & 45.63 & 0.50 & 9.50 & 0.13 & 0.21 \\
\hline ROC-11 & 3.25 & 4.50 & 0.08 & 1.32 & 0.30 & 0.36 & 0.20 & 0.13 & 5.60 & 0.10 & 4.56 & 0.21 & 5.50 & 10.00 & 2.50 & 0.22 & 0.48 & 50.37 & 0.50 & 9.50 & 0.13 & 0.21 \\
\hline ROC-12 & 5.48 & 7.60 & 0.00 & 3.96 & 0.00 & 0.00 & 0.00 & 0.00 & $\begin{array}{l}5.91 \\
\end{array}$ & 0.00 & 6.45 & 1.50 & 0.30 & 13.43 & 2.50 & 0.00 & 0.00 & 49.74 & 0.50 & 2.63 & 0.00 & 0.00 \\
\hline ROC-13 & 10.63 & $\begin{array}{l}4.50 \\
\end{array}$ & 0.00 & 1.42 & 0.00 & 0.00 & 0.00 & 0.00 & 6.62 & 0.00 & 5.57 & 1.50 & 5.50 & 10.00 & 0.00 & 0.00 & 0.00 & 45.44 & 0.50 & 8.31 & 0.00 & 0.00 \\
\hline ROC-14 & 11.40 & 13.01 & 0.08 & 0.00 & 0.30 & 0.36 & 0.00 & 0.13 & 6.54 & 0.10 & 5.26 & 1.50 & 4.20 & 10.34 & 2.50 & 0.22 & 0.48 & 38.66 & 1.97 & 2.62 & 0.13 & 0.21 \\
\hline ROC-15 & 7.02 & 4.50 & 0.08 & 0.00 & 0.30 & 0.36 & 0.20 & 0.13 & 10.27 & 0.10 & 4.00 & 0.00 & 1.05 & 15.14 & 2.50 & 0.22 & 0.48 & 43.83 & 0.50 & 9.00 & 0.13 & 0.21 \\
\hline ROC-16 & 3.25 & 9.43 & 0.08 & 0.00 & 0.30 & 0.36 & 0.00 & 0.13 & 5.00 & 0.10 & 6.68 & 0.00 & 5.44 & 10.00 & 0.00 & 0.22 & 0.48 & 47.79 & 2.00 & 8.41 & 0.13 & 0.21 \\
\hline ROC-17 & 6.34 & 4.86 & 0.00 & 0.16 & 0.00 & 0.00 & 0.20 & 0.00 & 10.03 & 0.00 & 4.66 & 0.00 & 5.50 & 16.00 & 2.50 & 0.00 & 0.00 & 47.56 & 2.00 & 0.18 & 0.00 & 0.00 \\
\hline ROC-18 & 3.80 & 5.05 & 0.00 & 2.01 & 0.00 & 0.00 & 0.00 & 0.00 & 111.32 & 0.00 & 4.00 & 0.00 & 4.87 & 10.00 & 2.20 & 0.00 & 0.00 & 49.52 & 2.00 & 5.23 & 0.00 & 0.00 \\
\hline ROC-19 & 3.94 & 13.27 & 0.00 & 0.00 & 0.00 & 0.00 & 0.00 & 0.00 & 5.00 & 0.00 & 4.00 & 1.50 & 0.30 & 10.13 & 2.50 & 0.00 & 0.00 & 50.39 & 2.00 & 6.97 & 0.00 & 0.00 \\
\hline ROC-20 & 3.58 & 10.30 & 0.08 & 4.00 & 0.30 & 0.36 & 0.20 & 0.13 & 8.38 & 0.10 & 4.08 & 0.38 & 5.50 & 12.92 & 0.00 & 0.22 & 0.48 & 46.47 & 2.00 & 0.19 & 0.13 & 0.21 \\
\hline ROC-21 & 10.37 & 11.66 & 0.00 & 0.09 & 0.00 & 0.00 & 0.20 & 0.00 & 7.82 & 0.00 & 4.23 & 1.11 & 5.28 & 10.00 & 1.52 & 0.00 & 0.00 & 36.22 & 2.00 & 9.50 & 0.00 & 0.00 \\
\hline ROC-22 & 3.25 & 6.43 & 0.00 & 0.00 & 0.00 & 0.00 & 0.00 & 0.00 & 14.69 & 0.00 & 4.52 & 0.00 & 5.50 & 10.00 & 2.50 & 0.00 & 0.00 & 46.84 & 1.82 & 4.43 & 0.00 & 0.00 \\
\hline ROC-23 & 9.99 & 5.69 & 0.08 & 0.00 & 0.30 & 0.36 & 0.20 & 0.13 & 7.17 & 0.10 & 4.91 & 1.42 & 1.89 & 10.89 & 1.93 & 0.22 & 0.48 & 49.35 & 2.00 & 2.55 & 0.13 & 0.21 \\
\hline $\begin{array}{l}\text { ROC-24 } \\
\end{array}$ & 10.21 & 11.09 & 0.08 & 0.00 & 0.30 & 0.36 & 0.20 & 0.13 & 6.89 & 0.10 & 4.04 & 1.50 & 5.44 & 10.28 & 0.15 & 0.22 & 0.48 & 44.95 & 2.00 & 1.25 & 0.13 & 0.21 \\
\hline ROC-25 & 4.88 & 4.50 & 0.08 & 0.00 & 0.30 & 0.36 & 0.20 & 0.13 & 11.84 & 0.10 & 4.00 & 1.50 & 5.50 & 15.45 & 0.05 & 0.22 & 0.48 & 47.08 & 0.50 & 2.51 & 0.13 & 0.21 \\
\hline ROC-26 & 9.07 & 6.49 & 0.00 & 0.00 & 0.00 & 0.00 & 0.00 & 0.00 & 5.00 & 0.00 & 4.00 & 0.00 & 3.29 & 17.17 & 0.00 & 0.00 & 0.00 & 48.68 & 0.50 & 5.80 & 0.00 & 0.00 \\
\hline ROC-27 & 3.94 & 4.50 & 0.08 & 0.00 & 0.30 & 0.36 & 0.00 & 0.13 & 15.17 & 0.10 & 4.00 & 1.50 & 5.50 & 11.80 & 1.95 & 0.22 & 0.48 & 43.65 & 2.00 & 3.99 & 0.13 & 0.21 \\
\hline ROC-28 & 4.49 & 4.50 & 0.08 & 0.00 & 0.30 & 0.36 & 0.00 & 0.13 & 6.34 & 0.10 & 4.13 & 1.50 & 0.47 & $\begin{array}{l}17.67 \\
\end{array}$ & 0.00 & 0.22 & 0.48 & 55.00 & 2.00 & 1.89 & 0.13 & 0.21 \\
\hline ROC-29 & 10.76 & 12.48 & 0.08 & 0.06 & 0.30 & 0.36 & 0.00 & 0.13 & 7.76 & 0.10 & 4.60 & 0.00 & 4.91 & 10.67 & 2.50 & 0.22 & 0.48 & 38.10 & 0.50 & 5.66 & 0.13 & 0.21 \\
\hline ROC-30 & 6.14 & 7.96 & 0.04 & 1.03 & 0.16 & 0.19 & 0.10 & 0.07 & 8.72 & 0.05 & 4.63 & 0.83 & 3.60 & 11.99 & 1.31 & 0.11 & 0.25 & 45.70 & 1.37 & 5.58 & 0.07 & 0.11 \\
\hline
\end{tabular}




\subsection{RESULTS AND DISCUSSION}

\subsection{CHEMICAL COMPOSITION MEASUREMENTS}

Table A1 (in two parts) in Appendix A provides the elemental concentration measurements from the study glasses that were prepared using LM, and Table A2 in Appendix A provides the measurements from the samples of these glasses prepared using PF. Measurements for the Batch 1 and uranium standard $\left(\mathrm{U}_{\mathrm{std}}\right)$ glass are also provided in these two tables. Note that these two tables contain measurements for glasses from another glass study that were included in the analytical plans (with IDs FY09EM21-28, FY09EM21-29, and FY09EM21-30). These three glasses will not be used to support the objectives of this study.

Elemental concentrations were converted to oxide concentrations by multiplying the values for each element by the gravimetric factor for the corresponding oxide. During this process, an elemental concentration that was determined to be below the detection limit of the analytical procedures used was reduced to half ${ }^{\mathrm{J}}$ of that detection limit as the oxide concentration was determined. ${ }^{19}$

\subsubsection{Measurements in Analytical Sequence}

Exhibit A1 in Appendix A provides plots in analytical sequence of the sample measurements generated by $\mathrm{AD}$ for each oxide by preparation method (i.e., LM and PF) and analytical set. The plots are in analytical sequence over the two sets of measurements. These plots include all of the measurement data from Tables A1 and A2. K A close review of these plots suggests that there are consistency issues in the measurements for some oxides for some of the glasses. These issues are explored in more detail in the following sections.

\subsubsection{Composition Measurements by Glass Identifier}

Exhibit A2 in Appendix A provides plots of the oxide concentration measurements by Glass ID/Lab ID for each analytical set (including Batch 1 and $U_{\text {std }}$ ). These plots demonstrate the individual measurements across the duplicates of each preparation method and the two ICP-AES calibrations for each glass within each analytical set. While there appears to be good repeatability among the measurements for each of the oxides for most of the glasses, there are some issues that should be noted. The measured values for the oxides derived using the PF method for Set \#1 suggest a dissolution problem for ROC-01, ROC-07, and ROC-13, specifically the values of $\mathrm{Al}_{2} \mathrm{O}_{3}, \mathrm{~B}_{2} \mathrm{O}_{3}, \mathrm{Fe}_{2} \mathrm{O}_{3}, \mathrm{Li}_{2} \mathrm{O}$ and $\mathrm{SiO}_{2}$. In addition, the measured values for the oxides derived using the LM method suggest dissolution problems for ROC-06, ROC-08, ROC-17, and ROC-28 (for example, see the $\mathrm{SiO}_{2}$ plot for Set \#2). A more thorough discussion of these analytical issues is provided in the following section along with a path forward for resolving the problem to obtain representative chemical compositions of the study glasses.

\footnotetext{
J Historically, the United States Environmental Protection Agency (USEPA) has advocated that non-detected measurements be replaced by one-half of the detection limit. The data should then be analyzed as if all of the measurements were observed with equal precision.

$\mathrm{K}$ In the Set \#2 results, glasses from another study are included in these plots for completeness (i.e., glasses FY09EM21-28, FY09EM21-29 and FY09EM21-30).
} 


\subsubsection{Batch 1 and Uranium Standard Results}

Exhibit A3 in Appendix A provides statistical analyses of the Batch 1 and $\mathrm{U}_{\text {std }}$ results by analytical set/calibration block for each oxide of interest for the LM preparation method. The results also include analysis of variance (ANOVA) investigations, which determine statistically significant differences among the means of these groups for each of the oxides for each of the standards. The following components indicate a significant ICP-AES calibration effect on the block averages at the 5\% significance level:

- $\quad$ Batch 1: $\mathrm{BaO}, \mathrm{CaO}, \mathrm{CuO}, \mathrm{MgO}, \mathrm{MnO}, \mathrm{Na}_{2} \mathrm{O}, \mathrm{NiO}, \mathrm{TiO}_{2}$, and $\mathrm{ZrO}_{2}$

- $\mathrm{U}_{\text {std }}: \mathrm{CaO}, \mathrm{Cr}_{2} \mathrm{O}_{3}, \mathrm{CuO}, \mathrm{MgO}, \mathrm{MnO}, \mathrm{Na}_{2} \mathrm{O}, \mathrm{NiO}, \mathrm{TiO}_{2}$, and $\mathrm{U}_{3} \mathrm{O}_{8}$

Exhibit A4 in Appendix A provides statistical analyses of the Batch 1 and $U_{\text {std }}$ results by analytical set/calibration block and ANOVA investigations for each oxide of interest for the PF preparation method. The following components indicate a significant ICP-AES calibration effect on the block averages at the $5 \%$ significance level:

- Batch 1: $\mathrm{Al}_{2} \mathrm{O}_{3}, \mathrm{Fe}_{2} \mathrm{O}_{3}$, and $\mathrm{Li}_{2} \mathrm{O}$

- $\mathrm{U}_{\text {std }}: \mathrm{B}_{2} \mathrm{O}_{3}$ and $\mathrm{Li}_{2} \mathrm{O}$

Reference values for the oxide concentrations of the standards are given in the header for each set of measurements in Exhibits A3 and A4.

Results from these statistical analyses provide incentive for adjusting the measurements by the effects of the ICP-AES calibration. Thus, bias correction of these data was pursued in order to determine if the adjusted values impacted the conclusions of this study. ${ }^{\mathrm{L}}$ Batch 1 results were used to bias correct all of the oxides (except uranium) as long as the reference value for the oxide concentration in the Batch 1 glass was greater than or equal to $0.1 \mathrm{wt} \%$. $\mathrm{U}_{\text {std }}$ results were used to bias correct the uranium values. By applying this approach, the Batch 1 results were used to bias correct the $\mathrm{Al}_{2} \mathrm{O}_{3}, \mathrm{~B}_{2} \mathrm{O}_{3}, \mathrm{BaO}, \mathrm{CaO}, \mathrm{Cr}_{2} \mathrm{O}_{3}, \mathrm{CuO}, \mathrm{Fe}_{2} \mathrm{O}_{3}, \mathrm{~K}_{2} \mathrm{O}, \mathrm{Li}_{2} \mathrm{O}, \mathrm{MgO}, \mathrm{MnO}, \mathrm{Na}{ }_{2} \mathrm{O}, \mathrm{NiO}$, $\mathrm{SiO}_{2}$ and $\mathrm{TiO}_{2}$ measurements, and the $\mathrm{U}_{\text {std }}$ values were used to bias correct the $\mathrm{U}_{3} \mathrm{O}_{8}$. Bias correction was not conducted on $\mathrm{CdO}, \mathrm{Ce}_{2} \mathrm{O}_{3}, \mathrm{La}_{2} \mathrm{O}_{3}, \mathrm{PbO}, \mathrm{SO}_{4}, \mathrm{ThO}_{2}, \mathrm{ZnO}$, or $\mathrm{ZrO}_{2}$.

The bias-correction was calculated as follows. For each oxide, let $\bar{a}_{i j}$ be the average measurement for the $i^{\text {th }}$ oxide at analytical block $j$ for Batch 1 (or $\mathrm{U}_{\text {std }}$ for uranium), and let $t_{i}$ be the reference value for the $i^{\text {th }}$ oxide for Batch 1 (or for $U_{\text {std }}$ if uranium). The averages and reference values are provided in Exhibits A3. Let $\bar{c}_{i j k}$ be the average measurement for the $i^{\text {th }}$ oxide at analytical block $j$ for the $k^{\text {th }}$ glass. The bias adjustment is given by:

\footnotetext{
${ }^{\mathrm{L}}$ It should be emphasized that bias correction is considered in order to demonstrate that the results and conclusions from this study are not affected by the compositional view (target, measured, or measured-bc). Demonstrating that the compositional view does not alter or change the conclusions shows a degree of robustness for the objective of the study.
} 


$$
\bar{c}_{i j k} \bullet\left(1-\frac{\bar{a}_{i j}-t_{i}}{\bar{a}_{i j}}\right)=\bar{c}_{i j k} \bullet \frac{t_{i}}{\bar{a}_{i j}}
$$

Bias-corrected measurements are indicated by a "bc" suffix in the remainder of the document. Both measured and measured "bc" values are included in the following discussion. For completeness, the original values of $\mathrm{CdO}, \mathrm{Ce}_{2} \mathrm{O}_{3}, \mathrm{La}_{2} \mathrm{O}_{3}, \mathrm{PbO}, \mathrm{SO}_{4}, \mathrm{ThO}_{2}, \mathrm{ZnO}$, and $\mathrm{ZrO}_{2}$ were included in the bias-corrected results in order to calculate a "bc" sum of oxides.

\subsubsection{Composition Measurements by Glass Identifier with Targeted Compositions}

Exhibit A5 in Appendix provides plots of the oxide concentration measurements by Glass ID/ Lab ID (including Batch 1 and $\mathrm{U}_{\text {std }}$ ). The data are sorted by increasing target oxide content. A review of the plots reveals the repeatability of the individual oxide values for each glass. The measurements for the oxides derived using the PF method for Set \#1 suggest a dissolution problem for ROC-01, ROC-07, and ROC-13. Table 5 provides a listing of the measurements corresponding to these glasses. Notice that both the measured and measured bc values are well below the target values for the first dissolution. These entries have been shaded in Table 5. Due to these anomalies, the first dissolution for each of these glasses was not used to determine the measured or the measured bias-corrected compositions for these glasses. The measurements for the oxides derived using the LM method suggest a dissolution problem for the second dissolution of ROC-06 and the first dissolution of ROC-08 in Set \#1 and for the first dissolution of ROC-17 and the second dissolution of ROC-28 in Set \#2. Table 6 through Table 9 provides a listing of the measurements corresponding to these glasses. Once again, note the discrepancies between the measured (and measured bc) values and the targeted values for dissolutions being questioned. These entries have been shaded in Table 6 through Table 9. Due to these anomalies, the shaded entries were not used to determine the measured or the measured bias-corrected compositions for the ROC-06, ROC-08, ROC-17, and ROC-28 glasses. 
SRNL-STI-2009-00465, REVISION 0

Table 5. ROC-01, ROC-07, and ROC-13 Peroxide Fusion Measurements

\begin{tabular}{|c|c|c|c|c|c|c|c|c|c|c|c|}
\hline \multirow{2}{*}{ Glass ID } & \multirow{2}{*}{ Lab ID } & \multirow{2}{*}{ Analyte } & Measured & Measured bc & Target & \multirow{2}{*}{ Glass ID } & \multirow{2}{*}{ Lab ID } & \multirow{2}{*}{ Analyte } & \multirow{2}{*}{\multicolumn{3}{|c|}{\begin{tabular}{c|c|} 
Measured & Measured bc \\
$(\mathrm{wt} \%)$
\end{tabular}}} \\
\hline & & & \multicolumn{3}{|c|}{$(\mathrm{wt} \%)$} & & & & & & \\
\hline \multirow{20}{*}{ ROC-01 } & a05PF11 & \multirow{4}{*}{$\mathrm{Al}_{2} \mathrm{O}_{3}$} & 8.45 & 8.55 & 10.86 & \multirow{20}{*}{ ROC-07 } & \multirow{5}{*}{\begin{tabular}{|l|} 
a07PF11 \\
a07PF12 \\
a07PF21 \\
a07PF22 \\
a07PF11
\end{tabular}} & \multirow{4}{*}{$\mathrm{Al}_{2} \mathrm{O}_{3}$} & 1.64 & 1.66 & 3.46 \\
\hline & a05PF12 & & 8.47 & 8.53 & 10.86 & & & & 1.64 & 1.66 & 3.46 \\
\hline & a05PF21 & & 10.98 & 11.11 & 10.86 & & & & 3.70 & 3.74 & 3.46 \\
\hline & a05PF22 & & 11.00 & 11.09 & 10.86 & & & & 3.68 & 3.72 & 3.46 \\
\hline & a05PF11 & \multirow{4}{*}{$\mathrm{B}_{2} \mathrm{O}_{3}$} & 4.83 & 5.13 & 6.34 & & & \multirow{4}{*}{$\mathrm{B}_{2} \mathrm{O}_{3}$} & 5.83 & 6.22 & 13.69 \\
\hline & a05PF12 & & 4.80 & 5.10 & 6.34 & & a07PF12 & & 5.73 & 6.22 & 13.69 \\
\hline & a05PF21 & & 6.12 & 6.50 & 6.34 & & a07PF21 & & 13.07 & 13.95 & 13.69 \\
\hline & a05PF22 & & 6.09 & 6.47 & 6.34 & & a07PF22 & & 12.98 & 14.08 & 13.69 \\
\hline & a05PF11 & \multirow{4}{*}{$\mathrm{Fe}_{2} \mathrm{O}_{3}$} & 6.55 & 6.62 & 8.62 & & a07PF11 & \multirow{4}{*}{$\mathrm{Fe}_{2} \mathrm{O}_{3}$} & 4.30 & 4.34 & 10.23 \\
\hline & a05PF12 & & 6.65 & 6.65 & 8.62 & & a07PF12 & & 4.30 & 4.37 & 10.23 \\
\hline & a05PF21 & & 8.56 & 8.66 & 8.62 & & a07PF21 & & 9.75 & 9.83 & 10.23 \\
\hline & a05PF22 & & 8.66 & 8.66 & 8.62 & & a07PF22 & & 9.71 & 9.87 & 10.23 \\
\hline & a05PF11 & \multirow{4}{*}{$\mathrm{Li}_{2} \mathrm{O}$} & 4.50 & 4.70 & 5.88 & & a07PF11 & \multirow{4}{*}{$\mathrm{Li}_{2} \mathrm{O}$} & 2.30 & 2.41 & 5.40 \\
\hline & a05PF12 & & 4.39 & 4.67 & 5.88 & & a07PF12 & & 2.26 & 2.38 & 5.40 \\
\hline & a05PF21 & & 5.68 & 5.94 & 5.88 & & a07PF21 & & 5.12 & 5.36 & 5.40 \\
\hline & a05PF22 & & 5.55 & 5.90 & 5.88 & & a07PF22 & & 5.15 & 5.41 & 5.40 \\
\hline & a05PF11 & \multirow{4}{*}{$\mathrm{SiO}_{2}$} & 29.09 & 29.70 & 37.90 & & a07PF11 & \multirow{4}{*}{$\mathrm{SiO}_{2}$} & 23.10 & 23.62 & 53.88 \\
\hline & a05PF12 & & 29.52 & 29.96 & 37.90 & & a07PF12 & & 23.10 & 23.58 & 53.88 \\
\hline & a05PF21 & & 37.87 & 38.65 & 37.90 & & a07PF21 & & 52.20 & 53.35 & 53.88 \\
\hline & a05PF22 & & 38.08 & 38.64 & 37.90 & & a07PF22 & & 52.20 & 53.28 & 53.88 \\
\hline
\end{tabular}

\begin{tabular}{|c|c|c|c|c|c|}
\hline \multirow{2}{*}{ Glass ID } & \multirow{2}{*}{ Lab ID } & \multirow{2}{*}{ Analyte } & Measured & Measured bc & Target \\
\hline & & & \multicolumn{3}{|c|}{$(w t \%)$} \\
\hline \multirow{20}{*}{ ROC-13 } & a14PF11 & \multirow{4}{*}{$\mathrm{Al}_{2} \mathrm{O}_{3}$} & 8.90 & 8.98 & 10.63 \\
\hline & a14PF12 & & 8.86 & 8.95 & 10.63 \\
\hline & a14PF21 & & 10.83 & 10.93 & 10.63 \\
\hline & a14PF22 & & 10.81 & 10.91 & 10.63 \\
\hline & a14PF11 & \multirow{4}{*}{$\mathrm{B}_{2} \mathrm{O}_{3}$} & 3.57 & 3.81 & 4.50 \\
\hline & a14PF12 & & 3.41 & 3.70 & 4.50 \\
\hline & a14PF21 & & 4.31 & 4.60 & 4.50 \\
\hline & a14PF22 & & 4.25 & 4.61 & 4.50 \\
\hline & a14PF11 & \multirow{4}{*}{$\mathrm{Fe}_{2} \mathrm{O}_{3}$} & 5.48 & 5.52 & 6.62 \\
\hline & a14PF12 & & 5.45 & 5.54 & 6.62 \\
\hline & a14PF21 & & 6.62 & 6.67 & 6.62 \\
\hline & a14PF22 & & 6.56 & 6.67 & 6.62 \\
\hline & a14PF11 & \multirow{4}{*}{$\mathrm{Li}_{2} \mathrm{O}$} & 4.50 & 4.71 & 5.57 \\
\hline & a14PF12 & & 4.48 & 4.71 & 5.57 \\
\hline & a14PF21 & & 5.53 & 5.79 & 5.57 \\
\hline & a14PF22 & & 5.43 & 5.70 & 5.57 \\
\hline & a14PF11 & \multirow{4}{*}{$\mathrm{SiO}_{2}$} & 37.22 & 38.05 & 45.44 \\
\hline & a14PF12 & & 37.44 & 38.21 & 45.44 \\
\hline & a14PF21 & & 45.35 & 46.36 & 45.44 \\
\hline & a14PF22 & & 45.14 & 46.07 & 45.44 \\
\hline
\end{tabular}


SRNL-STI-2009-00465, REVISION 0

Table 6. ROC-06 Lithium Metaborate Measurements

\begin{tabular}{|c|c|c|c|c|c|c|c|c|c|c|c|}
\hline \multirow{2}{*}{ Glass ID } & \multirow{2}{*}{ Lab ID } & \multirow{2}{*}{ Analyte } & Measured & Measured bc & Target & \multirow{2}{*}{ Glass ID } & \multirow{2}{*}{ Lab ID } & \multirow{2}{*}{ Analyte } & \multirow{2}{*}{\multicolumn{3}{|c|}{\begin{tabular}{c|c} 
Measured & Measured bc \\
$(w t \%)$
\end{tabular}}} \\
\hline & & & \multicolumn{3}{|c|}{$(w t \%)$} & & & & & & \\
\hline \multirow{20}{*}{ ROC-06 } & a03LM12 & \multirow{2}{*}{$\mathrm{BaO}$} & 0.00 & 0.00 & 0.00 & \multirow{20}{*}{ ROC-06 } & a03LM12 & \multirow{2}{*}{$\mathrm{MnO}$} & 5.38 & 5.41 & 5.50 \\
\hline & a03LM22 & & 0.00 & 0.00 & 0.00 & & a03LM22 & & 4.34 & 4.36 & 5.50 \\
\hline & a03LM11 & \multirow{2}{*}{$\mathrm{CaO}$} & 3.88 & 3.78 & 4.00 & & a03LM11 & \multirow{2}{*}{$\mathrm{Na}_{2} \mathrm{O}$} & 10.73 & 10.47 & 10.62 \\
\hline & a03LM12 & & 3.86 & 3.78 & 4.00 & & a03LM12 & & 10.64 & 10.36 & 10.62 \\
\hline & a03LM11 & \multirow{4}{*}{$\mathrm{CdO}$} & 0.00 & 0.00 & 0.00 & & a03LM11 & \multirow{4}{*}{$\mathrm{NiO}$} & 0.03 & 0.03 & 0.00 \\
\hline & a03LM12 & & 0.00 & 0.00 & 0.00 & & a03LM12 & & 0.03 & 0.02 & 0.00 \\
\hline & a03LM21 & & 0.00 & 0.00 & 0.00 & & a03LM21 & & 0.02 & 0.02 & 0.00 \\
\hline & a03LM22 & & 0.00 & 0.00 & 0.00 & & a03LM22 & & 0.03 & 0.02 & 0.00 \\
\hline & a03LM11 & $\mathrm{Ce}_{2} \mathrm{O}_{3}$ & 0.02 & 0.02 & 0.00 & & a03LM11 & $\mathrm{PbO}$ & 0.01 & 0.01 & 0.00 \\
\hline & a03LM12 & \multirow{3}{*}{$\mathrm{Cr}_{2} \mathrm{O}_{3}$} & 0.19 & 0.18 & 0.20 & & a03LM12 & \multirow{3}{*}{$\mathrm{SO}_{4}$} & 0.22 & 0.22 & 0.00 \\
\hline & a03LM21 & & 0.15 & 0.15 & 0.20 & & a03LM21 & & 0.22 & 0.22 & 0.00 \\
\hline & a03LM22 & & 0.15 & 0.15 & 0.20 & & a03LM22 & & 0.22 & 0.22 & 0.00 \\
\hline & a03LM11 & \multirow{4}{*}{$\mathrm{CuO}$} & 0.02 & 0.02 & 0.00 & & a03LM11 & \multirow{4}{*}{$\mathrm{TiO}_{2}$} & 0.43 & 0.44 & 0.50 \\
\hline & a03LM12 & & 0.02 & 0.02 & 0.00 & & a03LM12 & & 0.43 & 0.44 & 0.50 \\
\hline & a03LM21 & & 0.02 & 0.02 & 0.00 & & a03LM21 & & 0.35 & 0.35 & 0.50 \\
\hline & a03LM22 & & 0.02 & 0.02 & 0.00 & & a03LM22 & & 0.35 & 0.35 & 0.50 \\
\hline & a03LM11 & \multirow{4}{*}{$\mathrm{K}_{2} \mathrm{O}$} & 0.03 & 0.03 & 0.00 & & a03LM11 & \multirow{4}{*}{$\mathrm{U}_{3} \mathrm{O}_{8}$} & 9.21 & 9.11 & 9.50 \\
\hline & a03LM12 & & 0.09 & 0.09 & 0.00 & & a03LM12 & & 9.20 & 8.94 & 9.50 \\
\hline & a03LM21 & & 0.06 & 0.06 & 0.00 & & a03LM21 & & 7.18 & 7.10 & 9.50 \\
\hline & a03LM22 & & 0.03 & 0.03 & 0.00 & & a03LM22 & & 7.19 & 6.99 & 9.50 \\
\hline
\end{tabular}


SRNL-STI-2009-00465, REVISION 0

Table 7. ROC-08 Lithium Metaborate Measurements

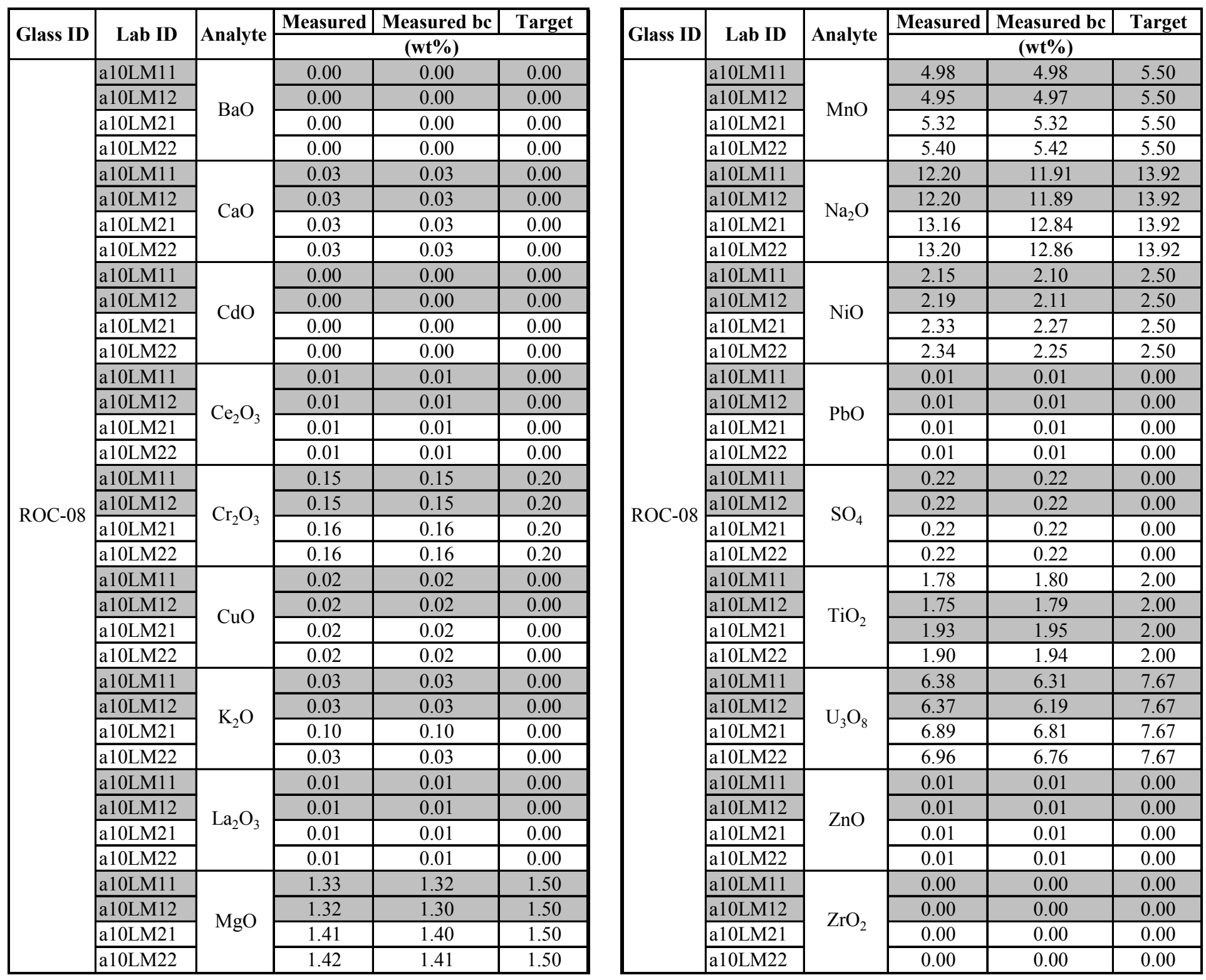

$-12-$ 
SRNL-STI-2009-00465, REVISION 0

Table 8. ROC-17 Lithium Metaborate Measurements

\begin{tabular}{|c|c|c|c|c|c|}
\hline \multirow[t]{2}{*}{ Glass ID } & \multirow[t]{2}{*}{ Lab ID } & \multirow[t]{2}{*}{ Analyte } & Measured & Measured bc & Target \\
\hline & & & \multicolumn{3}{|c|}{$(w t \%)$} \\
\hline \multirow{36}{*}{ ROC-17 } & b09LM11 & \multirow{4}{*}{$\mathrm{BaO}$} & 0.00 & 0.00 & 0.00 \\
\hline & b09LM12 & & 0.00 & 0.00 & 0.00 \\
\hline & b09LM21 & & 0.00 & 0.00 & 0.00 \\
\hline & b09LM22 & & 0.00 & 0.00 & 0.00 \\
\hline & b09LM11 & \multirow{4}{*}{$\mathrm{CaO}$} & 0.16 & 0.16 & 0.16 \\
\hline & b09LM12 & & 0.16 & 0.16 & 0.16 \\
\hline & b09LM21 & & 0.17 & 0.17 & 0.16 \\
\hline & b09LM22 & & 0.17 & 0.17 & 0.16 \\
\hline & b09LM11 & \multirow{4}{*}{$\mathrm{CdO}$} & 0.00 & 0.00 & 0.00 \\
\hline & b09LM12 & & 0.00 & 0.00 & 0.00 \\
\hline & b09LM21 & & 0.00 & 0.00 & 0.00 \\
\hline & b09LM22 & & 0.00 & 0.00 & 0.00 \\
\hline & b09LM11 & \multirow{4}{*}{$\mathrm{Ce}_{2} \mathrm{O}_{3}$} & 0.01 & 0.01 & 0.00 \\
\hline & b09LM12 & & 0.01 & 0.01 & 0.00 \\
\hline & b09LM21 & & 0.01 & 0.01 & 0.00 \\
\hline & b09LM22 & & 0.01 & 0.01 & 0.00 \\
\hline & b09LM11 & \multirow{4}{*}{$\mathrm{Cr}_{2} \mathrm{O}_{3}$} & 0.15 & 0.15 & 0.20 \\
\hline & b09LM12 & & 0.15 & 0.15 & 0.20 \\
\hline & b09LM21 & & 0.17 & 0.17 & 0.20 \\
\hline & b09LM22 & & 0.17 & 0.17 & 0.20 \\
\hline & b09LM11 & \multirow{4}{*}{$\mathrm{CuO}$} & 0.01 & 0.01 & 0.00 \\
\hline & b09LM12 & & 0.01 & 0.01 & 0.00 \\
\hline & b09LM21 & & 0.02 & 0.02 & 0.00 \\
\hline & b09LM22 & & 0.01 & 0.01 & 0.00 \\
\hline & b09LM11 & \multirow{4}{*}{$\mathrm{K}_{2} \mathrm{O}$} & 0.03 & 0.03 & 0.00 \\
\hline & b09LM12 & & 0.03 & 0.03 & 0.00 \\
\hline & b09LM21 & & 0.03 & 0.03 & 0.00 \\
\hline & b09LM22 & & 0.03 & 0.03 & 0.00 \\
\hline & b09LM11 & \multirow{4}{*}{$\mathrm{La}_{2} \mathrm{O}_{3}$} & 0.01 & 0.01 & 0.00 \\
\hline & b09LM12 & & 0.01 & 0.01 & 0.00 \\
\hline & b09LM21 & & 0.01 & 0.01 & 0.00 \\
\hline & b09LM22 & & 0.01 & 0.01 & 0.00 \\
\hline & b09LM11 & \multirow{4}{*}{$\mathrm{MgO}$} & 0.01 & 0.01 & 0.00 \\
\hline & b09LM12 & & 0.01 & 0.01 & 0.00 \\
\hline & b09LM21 & & 0.02 & 0.02 & 0.00 \\
\hline & b09LM22 & & 0.02 & 0.02 & 0.00 \\
\hline
\end{tabular}

\begin{tabular}{|c|c|c|c|c|c|}
\hline \multirow{2}{*}{ Glass ID } & \multirow{2}{*}{ Lab ID } & \multirow{2}{*}{ Analyte } & Measured & Measured bc & Target \\
\hline & & & \multicolumn{3}{|c|}{$(w t \%)$} \\
\hline \multirow{40}{*}{ ROC-17 } & b09LM11 & \multirow{4}{*}{$\mathrm{MnO}$} & 4.87 & 5.07 & 5.50 \\
\hline & b09LM12 & & 4.92 & 5.06 & 5.50 \\
\hline & b09LM21 & & 5.28 & 5.50 & 5.50 \\
\hline & b09LM22 & & 5.36 & 5.51 & 5.50 \\
\hline & b09LM11 & \multirow{4}{*}{$\mathrm{Na}_{2} \mathrm{O}$} & 15.37 & 14.96 & 16.00 \\
\hline & b09LM12 & & 14.83 & 15.15 & 16.00 \\
\hline & b09LM21 & & 16.85 & 16.40 & 16.00 \\
\hline & b09LM22 & & 15.64 & 15.98 & 16.00 \\
\hline & b09LM11 & \multirow{4}{*}{$\mathrm{NiO}$} & 2.07 & 2.04 & 2.50 \\
\hline & b09LM12 & & 2.09 & 2.04 & 2.50 \\
\hline & b09LM21 & & 2.29 & 2.25 & 2.50 \\
\hline & b09LM22 & & 2.28 & 2.23 & 2.50 \\
\hline & b09LM11 & \multirow{4}{*}{$\mathrm{PbO}$} & 0.01 & 0.01 & 0.00 \\
\hline & b09LM12 & & 0.01 & 0.01 & 0.00 \\
\hline & b09LM21 & & 0.01 & 0.01 & 0.00 \\
\hline & b09LM22 & & 0.01 & 0.01 & 0.00 \\
\hline & b09LM11 & \multirow{4}{*}{$\mathrm{SiO}_{2}$} & 42.57 & 43.08 & 47.56 \\
\hline & b09LM12 & & 42.36 & 43.11 & 47.56 \\
\hline & b09LM21 & & 46.85 & 47.41 & 47.56 \\
\hline & b09LM22 & & 46.42 & 47.24 & 47.56 \\
\hline & b09LM11 & \multirow{4}{*}{$\mathrm{SO}_{4}$} & 0.22 & 0.22 & 0.00 \\
\hline & b09LM12 & & 0.22 & 0.22 & 0.00 \\
\hline & b09LM21 & & 0.22 & 0.22 & 0.00 \\
\hline & b09LM22 & & 0.22 & 0.22 & 0.00 \\
\hline & b09LM11 & \multirow{4}{*}{$\mathrm{TiO}_{2}$} & 1.88 & 2.00 & 2.00 \\
\hline & b09LM12 & & 1.90 & 2.01 & 2.00 \\
\hline & b09LM21 & & 2.04 & 2.16 & 2.00 \\
\hline & b09LM22 & & 2.05 & 2.16 & 2.00 \\
\hline & b09LM11 & \multirow{4}{*}{$\mathrm{U}_{3} \mathrm{O}_{8}$} & 0.26 & 0.26 & 0.18 \\
\hline & b09LM12 & & 0.29 & 0.28 & 0.18 \\
\hline & b09LM21 & & 0.30 & 0.30 & 0.18 \\
\hline & b09LM22 & & 0.31 & 0.31 & 0.18 \\
\hline & b09LM11 & \multirow{4}{*}{$\mathrm{ZnO}$} & 0.01 & 0.01 & 0.00 \\
\hline & b09LM12 & & 0.01 & 0.01 & 0.00 \\
\hline & b09LM21 & & 0.01 & 0.01 & 0.00 \\
\hline & b09LM22 & & 0.01 & 0.01 & 0.00 \\
\hline & b09LM11 & \multirow{4}{*}{$\mathrm{ZrO}_{2}$} & 0.00 & 0.00 & 0.00 \\
\hline & b09LM12 & & 0.00 & 0.00 & 0.00 \\
\hline & b09LM21 & & 0.00 & 0.00 & 0.00 \\
\hline & b09LM22 & & 0.00 & 0.00 & 0.00 \\
\hline
\end{tabular}

$-13-$ 
SRNL-STI-2009-00465, REVISION 0

Table 9. ROC-28 Lithium Metaborate Measurements

\begin{tabular}{|c|c|c|c|c|c|}
\hline \multirow{2}{*}{ Glass ID } & \multirow{2}{*}{ Lab ID } & \multirow{2}{*}{ Analyte } & Measured & Measured bc & Target \\
\hline & & & \multicolumn{3}{|c|}{$($ wt\%) } \\
\hline \multirow{36}{*}{ ROC-28 } & b08LM11 & \multirow{4}{*}{$\mathrm{BaO}$} & 0.08 & 0.08 & 0.08 \\
\hline & b08LM12 & & 0.08 & 0.08 & 0.08 \\
\hline & b08LM21 & & 0.07 & 0.07 & 0.08 \\
\hline & b08LM22 & & 0.07 & 0.07 & 0.08 \\
\hline & b08LM11 & \multirow{4}{*}{$\mathrm{CaO}$} & 0.01 & 0.01 & 0.00 \\
\hline & b08LM12 & & 0.01 & 0.01 & 0.00 \\
\hline & b08LM21 & & 0.03 & 0.03 & 0.00 \\
\hline & b08LM22 & & 0.02 & 0.02 & 0.00 \\
\hline & b08LM11 & \multirow{4}{*}{$\mathrm{CdO}$} & 0.27 & 0.27 & 0.30 \\
\hline & b08LM12 & & 0.26 & 0.26 & 0.30 \\
\hline & b08LM21 & & 0.25 & 0.25 & 0.30 \\
\hline & b08LM22 & & 0.25 & 0.25 & 0.30 \\
\hline & b08LM11 & \multirow{4}{*}{$\mathrm{Ce}_{2} \mathrm{O}_{3}$} & 0.32 & 0.32 & 0.36 \\
\hline & b08LM12 & & 0.32 & 0.32 & 0.36 \\
\hline & b08LM21 & & 0.29 & 0.29 & 0.36 \\
\hline & b08LM22 & & 0.30 & 0.30 & 0.36 \\
\hline & b08LM11 & \multirow{4}{*}{$\mathrm{Cr}_{2} \mathrm{O}_{3}$} & 0.02 & 0.02 & 0.00 \\
\hline & b08LM12 & & 0.02 & 0.02 & 0.00 \\
\hline & b08LM21 & & 0.02 & 0.02 & 0.00 \\
\hline & b08LM22 & & 0.02 & 0.02 & 0.00 \\
\hline & b08LM11 & \multirow{4}{*}{$\mathrm{CuO}$} & 0.14 & 0.14 & 0.13 \\
\hline & b08LM12 & & 0.13 & 0.14 & 0.13 \\
\hline & b08LM21 & & 0.13 & 0.13 & 0.13 \\
\hline & b08LM22 & & 0.12 & 0.12 & 0.13 \\
\hline & b08LM11 & \multirow{4}{*}{$\mathrm{K}_{2} \mathrm{O}$} & 0.03 & 0.03 & 0.00 \\
\hline & b08LM12 & & 0.03 & 0.03 & 0.00 \\
\hline & b08LM21 & & 0.03 & 0.03 & 0.00 \\
\hline & b08LM22 & & 0.03 & 0.03 & 0.00 \\
\hline & b08LM11 & \multirow{4}{*}{$\mathrm{La}_{2} \mathrm{O}_{3}$} & 0.07 & 0.07 & 0.10 \\
\hline & b08LM12 & & 0.07 & 0.07 & 0.10 \\
\hline & b08LM21 & & 0.07 & 0.07 & 0.10 \\
\hline & b08LM22 & & 0.07 & 0.07 & 0.10 \\
\hline & b08LM11 & \multirow{4}{*}{$\mathrm{MgO}$} & 1.48 & 1.49 & 1.50 \\
\hline & b08LM12 & & 1.49 & 1.48 & 1.50 \\
\hline & b08LM21 & & 1.39 & 1.40 & 1.50 \\
\hline & b08LM22 & & 1.40 & 1.39 & 1.50 \\
\hline
\end{tabular}

\begin{tabular}{|c|c|c|c|c|c|}
\hline \multirow[t]{2}{*}{ Glass ID } & \multirow[t]{2}{*}{ Lab ID } & \multirow[t]{2}{*}{ Analyte } & \begin{tabular}{|l} 
Measured \\
\end{tabular} & Measured bc & Target \\
\hline & & & \multicolumn{3}{|c|}{$(w t \%)$} \\
\hline \multirow{40}{*}{ ROC-28 } & b08LM1 1 & \multirow{4}{*}{$\mathrm{MnO}$} & 0.48 & 0.49 & 0.47 \\
\hline & b08LM12 & & 0.48 & 0.49 & 0.47 \\
\hline & b08LM21 & & 0.45 & 0.47 & 0.47 \\
\hline & b08LM22 & & 0.45 & 0.46 & 0.47 \\
\hline & b08LM1 1 & \multirow{4}{*}{$\mathrm{Na}_{2} \mathrm{O}$} & 17.25 & 17.27 & 17.67 \\
\hline & b08LM12 & & 16.98 & 16.99 & 17.67 \\
\hline & b08LM21 & & 16.31 & 16.32 & 17.67 \\
\hline & b08LM22 & & 16.04 & 16.05 & 17.67 \\
\hline & b08LM11 & \multirow{4}{*}{$\mathrm{NiO}$} & 0.03 & 0.03 & 0.00 \\
\hline & b08LM12 & & 0.02 & 0.02 & 0.00 \\
\hline & b08LM21 & & 0.03 & 0.03 & 0.00 \\
\hline & b08LM22 & & 0.03 & 0.03 & 0.00 \\
\hline & b08LM11 & \multirow{4}{*}{$\mathrm{PbO}$} & 0.19 & 0.19 & 0.22 \\
\hline & b08LM12 & & 0.18 & 0.18 & 0.22 \\
\hline & b08LM21 & & 0.18 & 0.18 & 0.22 \\
\hline & b08LM22 & & 0.16 & 0.16 & 0.22 \\
\hline & b08LM11 & \multirow{4}{*}{$\mathrm{SiO}_{2}$} & 52.84 & 54.25 & 55.00 \\
\hline & b08LM12 & & 53.05 & 54.23 & 55.00 \\
\hline & b08LM21 & & 50.27 & 51.61 & 55.00 \\
\hline & b08LM22 & & 49.85 & 50.95 & 55.00 \\
\hline & b08LM11 & \multirow{4}{*}{$\mathrm{SO}_{4}$} & 0.22 & 0.22 & 0.48 \\
\hline & b08LM12 & & 0.54 & 0.54 & 0.48 \\
\hline & b08LM21 & & 0.22 & 0.22 & 0.48 \\
\hline & b08LM22 & & 0.22 & 0.22 & 0.48 \\
\hline & b08LM11 & \multirow{4}{*}{$\mathrm{TiO}_{2}$} & 1.90 & 1.96 & 2.00 \\
\hline & b08LM12 & & 1.92 & 1.97 & 2.00 \\
\hline & b08LM21 & & 1.80 & 1.85 & 2.00 \\
\hline & b08LM22 & & 1.80 & 1.85 & 2.00 \\
\hline & b08LM11 & \multirow{4}{*}{$\mathrm{U}_{3} \mathrm{O}_{8}$} & 1.83 & 1.85 & 1.89 \\
\hline & b08LM12 & & 1.82 & 1.82 & 1.89 \\
\hline & b08LM21 & & 1.67 & 1.70 & 1.89 \\
\hline & b08LM22 & & 1.73 & 1.73 & 1.89 \\
\hline & b08LM11 & \multirow{4}{*}{$\mathrm{ZnO}$} & 0.11 & 0.11 & 0.13 \\
\hline & b08LM12 & & 0.11 & 0.11 & 0.13 \\
\hline & b08LM21 & & 0.11 & 0.11 & 0.13 \\
\hline & b08LM22 & & 0.10 & 0.10 & 0.13 \\
\hline & b08LM11 & \multirow{4}{*}{$\mathrm{ZrO}_{2}$} & 0.18 & 0.18 & 0.21 \\
\hline & b08LM12 & & 0.18 & 0.18 & 0.21 \\
\hline & b08LM21 & & 0.17 & 0.17 & 0.21 \\
\hline & b08LM22 & & 0.17 & 0.17 & 0.21 \\
\hline
\end{tabular}

$-14-$ 


\subsubsection{Measured versus Target Compositions}

Table A4 in Appendix A provides a summary of the average compositions as well as the target compositions and some associated differences and relative differences. Exhibit A6 in Appendix A provides plots showing results for each glass for each oxide to help highlight the comparisons among the measured, bias-corrected, and targeted values. In general, the measured/measured bias-corrected values are consistent with the target oxide content in each of the study glasses. It should be noted that there is some scatter in the $\mathrm{SO}_{4}$ values around their intended targets. In addition, there also appears to be a detection limit issue for $\mathrm{SO}_{4}$ around $0.2 \mathrm{wt} \%$. The measured $\mathrm{NiO}, \mathrm{Na}_{2} \mathrm{O}$ and $\mathrm{ZrO}_{2}$ values for some of the study glasses are below the target values and the $\mathrm{Cr}_{2} \mathrm{O}_{3}$ values $^{\mathrm{M}}$ for the $\mathrm{U}_{\text {std }}$ are both high. None of these issues impact the outcome of this study.

The sums of oxides (measured and bias-corrected) for the study glasses fall within the interval of 95 to $105 \mathrm{wt} \%$ except for the measured values of ROC-20 ${ }^{\mathrm{N}}$; however, bias corrected measurements for this glass do have a sum of oxides above the lower limit of $95 \%$.

\subsection{MAR ASSESSMENT}

MAR assessment results are provided in Table $10 .^{\circ}$ The columns in the table list the glass identifier with compositional view, nepheline value, $\mathrm{TiO}_{2}$ concentration ( $\mathrm{wt} \%$ ), homogeneity constraint $(\mathrm{wt} \%)$ and the overall MAR assessment and the predicted values for: $\Delta \mathrm{G}_{\mathrm{p}}$ value for boron (B Del $G_{p}$ value), normalized leachate for boron in grams/Liter (NL[B (g/L)]), liquidus temperature in degrees Celsius $\left(\mathrm{T}_{\mathrm{L}}\left({ }^{\circ} \mathrm{C}\right)\right)$ and viscosity at $1150^{\circ} \mathrm{C}$ in Poise $(\operatorname{Visc}(\mathrm{P}))$.

Note that the PAR value of the homogeneity constraint is 210.92 (wt $\%)$. The MAR assessment conducted by current version of PCCS requires that the homogeneity constraint be satisfied at the PAR (i.e., the glass composition must yield a value for the homogeneity constraint that is greater than $210.92 \mathrm{wt} \%$ ). The MAR assessment for homogeneity may be satisfied by either (1) the homogeneity constraint being satisfied at the MAR, or (2) the homogeneity constraint being satisfied at the PAR and the alumina and/or sum of alkali constraint being satisfied at the MAR. The results from Herman et al. justified the use of the MAR assessment of the alumina and alkali constraints to provide an administrative override to satisfy the homogeneity requirement in its entirety (i.e., the homogeneity constraint being met at the PAR could be ignored) for sludge-only processing. ${ }^{4}$ For this study, the largest value of the homogeneity constraint over the target compositions is $213.11(\mathrm{wt} \%$ ). Note that while all of the target compositions of the study glasses fail or just satisfy the PAR, none of the target compositions satisfy the homogeneity constraint itself at the MAR value.

\footnotetext{
${ }^{M}$ The reference value of $\mathrm{Cr}_{2} \mathrm{O}_{3}$ was $0 \mathrm{wt} \%$; however, the measured value has been consistently around $0.25 \mathrm{wt} \%$, indicating that there is some $\mathrm{Cr}_{2} \mathrm{O}_{3}$ present in the standard.

$\mathrm{N}$ Note that the target sums of oxides for the standard glasses do not equal $100 \%$ due to an incomplete coverage of the oxides in the Batch 1 and $\mathrm{U}_{\text {std }}$ glasses.

$\mathrm{O}$ If the MAR status is blank, then the glass could be processed at DWPF.
} 
SRNL-STI-2009-00465, REVISION 0

Table 10. PCCS Assessment Results

\begin{tabular}{|c|c|c|c|c|c|c|c|c|c|}
\hline Glass ID & $\begin{array}{c}\text { Compositional } \\
\text { View }\end{array}$ & B Del $G_{p}$ & $\begin{array}{c}\text { NL } \\
{[B(g / L)]}\end{array}$ & $\mathbf{T}_{\mathbf{L}}\left({ }^{\circ} \mathbf{C}\right)$ & $\begin{array}{c}\text { Viscosity } \\
\text { (P) }\end{array}$ & \begin{tabular}{|c|}
$\mathrm{TiO}_{2}$ \\
$(\mathrm{wt} \%)$
\end{tabular} & $\begin{array}{c}\text { Homogeneity } \\
(w t \%)\end{array}$ & $\begin{array}{c}\text { Nepheline } \\
\text { Value }\end{array}$ & MAR Status \\
\hline \multirow{3}{*}{ ROC-01 } & target & -9.43 & 0.64 & 964 & 27 & 2.00 & 211.35 & 0.64 & $\mathrm{TiO}_{2}$ \\
\hline & measured & -8.89 & 0.51 & 967 & 33 & 1.91 & 211.07 & 0.64 & \\
\hline & measured bc & -8.87 & 0.51 & 967 & 32 & 1.93 & 213.67 & 0.65 & $\mathrm{TiO}_{2}$ \\
\hline \multirow{3}{*}{ ROC-02 } & target & -11.84 & 1.75 & 1023 & 29 & 1.96 & 210.52 & 0.69 & $\mathrm{~T}_{\mathrm{L}} \mathrm{TiO}_{2}$ Homg \\
\hline & measured & -11.15 & 1.32 & 1026 & 32 & 1.88 & 203.03 & 0.69 & $\mathrm{~T}_{\mathrm{L}}$ Homg \\
\hline & measured bc & -11.12 & 1.30 & 1020 & 32 & 1.90 & 205.79 & 0.70 & $\mathrm{~T}_{\mathrm{L}}$ Homg \\
\hline \multirow{3}{*}{ ROC-03 } & target & -7.68 & 0.31 & 890 & 27 & 2.00 & 213.11 & 0.76 & $\mathrm{TiO}_{2}$ IFrit \\
\hline & measured & -7.47 & 0.28 & 898 & 31 & 1.79 & 212.92 & 0.76 & 1Frit \\
\hline & measured bc & -7.43 & 0.28 & 901 & 31 & 1.82 & 215.75 & 0.77 & 1Frit \\
\hline \multirow{3}{*}{ ROC-04 } & target & -10.36 & 0.94 & 963 & 27 & 2.00 & 212.53 & 0.68 & $\mathrm{TiO}_{2}$ \\
\hline & measured & -9.80 & 0.75 & 955 & 32 & 1.80 & 211.89 & 0.68 & \\
\hline & measured bc & -9.84 & 0.76 & 948 & 31 & 1.83 & 213.88 & 0.69 & \\
\hline \multirow{3}{*}{ ROC-05 } & target & -9.74 & 0.73 & 924 & 27 & 0.50 & 210.52 & 0.74 & Homg lFrit \\
\hline & measured & -9.30 & 0.61 & 943 & 30 & 0.51 & 208.64 & 0.74 & Homg \\
\hline & measured bc & -9.31 & 0.61 & 945 & 30 & 0.52 & 212.16 & 0.74 & \\
\hline \multirow{3}{*}{ ROC-06 } & target & -12.30 & 2.12 & 724 & 29 & 0.50 & 192.21 & 0.73 & Homg \\
\hline & measured & -12.18 & 2.02 & 725 & 30 & 0.43 & 191.35 & 0.72 & Homg \\
\hline & measured bc & -12.16 & 2.00 & 728 & 30 & 0.44 & 194.44 & 0.73 & Homg \\
\hline \multirow{3}{*}{ ROC-07 } & target & -9.25 & 0.60 & 830 & 37 & 0.50 & 210.52 & 0.80 & Homg hFrit \\
\hline & measured & -8.74 & 0.48 & 832 & 41 & 0.47 & 204.52 & 0.80 & Homg \\
\hline & measured bc & -8.78 & 0.49 & 834 & 40 & 0.48 & 208.79 & 0.80 & Homg hFrit \\
\hline \multirow{3}{*}{ ROC-08 } & target & -13.25 & 3.16 & 896 & 37 & 2.00 & 168.64 & 0.73 & $\mathrm{TiO}_{2}$ Homg $\mathrm{R}_{2} \mathrm{O}$ lFrit \\
\hline & measured & -12.46 & 2.27 & 895 & 41 & 1.92 & 164.65 & 0.73 & Homg lFrit \\
\hline & measured bc & -12.34 & 2.16 & 891 & 42 & 1.94 & 166.66 & 0.74 & $\mathrm{TiO}_{2}$ Homg lFrit \\
\hline \multirow{3}{*}{ ROC-09 } & target & -13.28 & 3.20 & 939 & 27 & 0.50 & 211.65 & 0.73 & \\
\hline & measured & -11.69 & 1.65 & 974 & 35 & 0.55 & 204.97 & 0.74 & Homg lFrit \\
\hline & measured bc & -11.60 & 1.58 & 970 & 35 & 0.56 & 207.10 & 0.74 & Homg 1Frit \\
\hline \multirow{3}{*}{ ROC-10 } & target & -9.20 & 0.58 & 975 & 27 & 0.50 & 213.11 & 0.76 & 1Frit \\
\hline & measured & -8.28 & 0.40 & 975 & 36 & 0.47 & 209.80 & 0.77 & Homg lFrit \\
\hline & measured bc & -8.22 & 0.39 & 977 & 37 & 0.48 & 212.25 & 0.77 & 1Frit \\
\hline \multirow{3}{*}{ ROC-11 } & target & -10.23 & 0.89 & 916 & 88 & 0.50 & 171.33 & 0.79 & Homg lFrit \\
\hline & measured & -9.94 & 0.80 & 914 & 92 & 0.55 & 170.30 & 0.78 & Homg IFrit \\
\hline & measured bc & -9.87 & 0.77 & 907 & 93 & 0.56 & 172.33 & 0.79 & Homg IFrit \\
\hline \multirow{3}{*}{ ROC-12 } & target & -13.20 & 3.09 & 891 & 27 & 0.50 & 210.52 & 0.73 & Homg \\
\hline & measured & -12.48 & 2.29 & 912 & 29 & 0.50 & 207.20 & 0.72 & Homg \\
\hline & measured bc & -12.53 & 2.34 & 903 & 28 & 0.51 & 210.03 & 0.73 & Homg \\
\hline \multirow{3}{*}{ ROC-13 } & target & -8.75 & 0.48 & 843 & 86 & 0.50 & 210.52 & 0.69 & Homg \\
\hline & measured & -7.60 & 0.30 & 868 & 106 & 0.47 & 208.55 & 0.70 & newhv Homg \\
\hline & measured bc & -7.64 & 0.30 & 867 & 104 & 0.47 & 211.68 & 0.70 & newhy \\
\hline \multirow{3}{*}{ ROC-14 } & target & -9.01 & 0.54 & 1087 & 27 & 1.97 & 211.78 & 0.64 & $\mathrm{~T}_{\mathrm{L}} \mathrm{TiO}_{2}$ \\
\hline & measured & -8.45 & 0.43 & 1076 & 33 & 2.01 & 210.06 & 0.64 & $\mathrm{~T}_{\mathrm{L}} \mathrm{TiO}_{2}$ Homg \\
\hline & measured bc & -8.56 & 0.45 & 1068 & 31 & 2.04 & 214.13 & 0.65 & TL TiO2 \\
\hline \multirow{3}{*}{ ROC-15 } & target & -10.89 & 1.18 & 1041 & 30 & 0.50 & 208.42 & 0.66 & $\mathrm{~T}_{\mathrm{L}}$ Homg \\
\hline & measured & -9.53 & 0.67 & 1043 & 42 & 0.48 & 207.50 & 0.68 & $\mathrm{~T}_{\mathrm{L}}$ Homg \\
\hline & measured bc & -9.47 & 0.65 & 1036 & 43 & 0.49 & 209.93 & 0.68 & $\mathrm{~T}_{\mathrm{L}}$ Homg \\
\hline
\end{tabular}


SRNL-STI-2009-00465, REVISION 0

Table 10 cont. PCCS Assessment Results

\begin{tabular}{|c|c|c|c|c|c|c|c|c|c|}
\hline Glass ID & $\begin{array}{c}\text { Compositional } \\
\text { View }\end{array}$ & B Del $G_{p}$ & $\begin{array}{c}\text { NL } \\
{[B(g / L)]}\end{array}$ & $\mathbf{T}_{\mathbf{L}}\left({ }^{\circ} \mathbf{C}\right)$ & $\begin{array}{l}\text { Viscosity } \\
\text { (P) }\end{array}$ & \begin{tabular}{|c|}
$\mathrm{TiO}_{2}$ \\
$(\mathrm{wt} \%)$
\end{tabular} & $\begin{array}{c}\text { Homogeneity } \\
(w t \%)\end{array}$ & $\begin{array}{c}\text { Nepheline } \\
\text { Value }\end{array}$ & MAR Status \\
\hline \multirow{3}{*}{ ROC-16 } & target & -12.21 & 2.05 & 592 & 27 & 2.00 & 167.69 & 0.78 & $\mathrm{TiO}_{2}$ Homg \\
\hline & measured & -11.74 & 1.68 & 635 & 25 & 2.38 & 165.10 & 0.77 & $\mathrm{TiO}_{2}$ Homg lFrit \\
\hline & measured bc & -11.90 & 1.80 & 631 & 26 & 2.45 & 167.89 & 0.78 & $\mathrm{TiO}_{2}$ Homg lFrit \\
\hline \multirow{3}{*}{ ROC-17 } & target & -13.23 & 3.13 & 957 & 27 & 2.00 & 210.52 & 0.68 & $\mathrm{TiO}_{2}$ Homg \\
\hline & measured & -13.29 & 3.21 & 936 & 26 & 2.04 & 208.40 & 0.67 & $\mathrm{TiO}_{2}$ Homg \\
\hline & measured bc & -13.39 & 3.35 & 935 & 25 & 2.16 & 213.11 & 0.67 & $\mathrm{TiO}_{2}$ \\
\hline \multirow{3}{*}{ ROC-18 } & target & -8.67 & 0.47 & 986 & 72 & 2.00 & 206.70 & 0.78 & $\mathrm{TiO}_{2}$ Homg lFrit \\
\hline & measured & -8.98 & 0.53 & 978 & 60 & 1.90 & 204.60 & 0.77 & Homg 1Frit \\
\hline & measured bc & -9.07 & 0.55 & 970 & 62 & 1.95 & 207.01 & 0.77 & $\mathrm{TiO}_{2}$ Homg lFrit \\
\hline \multirow{3}{*}{ ROC-19 } & target & -8.70 & 0.47 & 980 & 63 & 2.00 & 175.24 & 0.78 & $\mathrm{TiO}_{2}$ Homg \\
\hline & measured & -8.80 & 0.49 & 979 & 61 & 1.83 & 171.27 & 0.77 & Homg \\
\hline & measured bc & -8.89 & 0.51 & 972 & 60 & 1.94 & 173.65 & 0.77 & $\mathrm{TiO}_{2} \mathrm{Homg}$ \\
\hline \multirow{3}{*}{ ROC-20 } & target & -13.23 & 3.13 & 762 & 28 & 2.00 & 211.03 & 0.74 & $\mathrm{TiO}_{2}$ \\
\hline & measured & -12.34 & 2.16 & 787 & 26 & 2.01 & 201.21 & 0.73 & lsum $\mathrm{TiO}_{2}$ Homg lFrit \\
\hline & measured bc & -12.52 & 2.33 & 790 & 24 & 2.12 & 205.67 & 0.73 & newlv $\mathrm{TiO}_{2}$ Homg lFrit \\
\hline \multirow{3}{*}{ ROC-21 } & target & -8.59 & 0.45 & 1098 & 30 & 2.00 & 202.87 & 0.64 & $\mathrm{~T}_{\mathrm{L}} \mathrm{TiO}_{2}$ Homg \\
\hline & measured & -8.65 & 0.46 & 1077 & 31 & 1.97 & 202.34 & 0.63 & $\mathrm{~T}_{\mathrm{L}} \mathrm{TiO}_{2}$ Homg \\
\hline & measured bc & -8.78 & 0.49 & 1075 & 30 & 2.03 & 208.52 & 0.64 & $\mathrm{~T}_{\mathrm{L}} \mathrm{TiO}_{2}$ Homg \\
\hline \multirow{3}{*}{ ROC-22 } & target & -9.11 & 0.56 & 1026 & 34 & 1.82 & 210.06 & 0.78 & $\mathrm{~T}_{\mathrm{L}}$ Homg lFrit \\
\hline & measured & -9.26 & 0.60 & 1013 & 33 & 1.86 & 209.94 & 0.77 & $\mathrm{~T}_{\mathrm{L}}$ Homg lFrit \\
\hline & measured bc & -9.42 & 0.64 & 1011 & 32 & 1.91 & 215.75 & 0.77 & $\mathrm{~T}_{\mathrm{L}}$ lFrit \\
\hline \multirow{3}{*}{ ROC-23 } & target & -7.01 & 0.23 & 1085 & 101 & 2.00 & 213.10 & 0.70 & $\mathrm{~T}_{\mathrm{L}}$ newhv $\mathrm{TiO}_{2}$ \\
\hline & measured & -6.73 & 0.21 & 1044 & 96 & 1.90 & 208.72 & 0.68 & $\mathrm{~T}_{\mathrm{L}}$ lsum Homg \\
\hline & measured bc & -6.80 & 0.21 & 1038 & 98 & 1.95 & 211.19 & 0.69 & $\mathrm{~T}_{\mathrm{L}} \mathrm{TiO}_{2}$ \\
\hline \multirow{3}{*}{ ROC-24 } & target & -8.05 & 0.36 & 967 & 78 & 2.00 & 211.98 & 0.69 & $\mathrm{TiO}_{2}$ \\
\hline & measured & -8.05 & 0.36 & 954 & 80 & 1.71 & 212.02 & 0.68 & \\
\hline & measured bc & -8.17 & 0.38 & 955 & 76 & 1.81 & 217.62 & 0.68 & \\
\hline \multirow{3}{*}{ ROC-25 } & target & -13.19 & 3.08 & 846 & 28 & 0.50 & 210.91 & 0.70 & Homg \\
\hline & measured & -12.44 & 2.26 & 876 & 24 & 0.49 & 208.87 & 0.69 & newlv Homg \\
\hline & measured bc & -12.54 & 2.35 & 871 & 26 & 0.50 & 210.93 & 0.69 & \\
\hline \multirow{3}{*}{ ROC-26 } & target & -13.00 & 2.85 & 669 & 48 & 0.50 & 201.89 & 0.65 & Homg \\
\hline & measured & -12.87 & 2.69 & 690 & 47 & 0.49 & 203.80 & 0.64 & Homg \\
\hline & measured bc & -12.93 & 2.77 & 689 & 47 & 0.51 & 205.59 & 0.64 & Homg \\
\hline \multirow{3}{*}{ ROC-27 } & target & -10.09 & 0.84 & 1034 & 27 & 2.00 & 213.11 & 0.74 & $\mathrm{~T}_{\mathrm{L}} \mathrm{TiO}_{2}$ lFrit \\
\hline & measured & -9.78 & 0.74 & 1027 & 28 & 1.88 & 211.13 & 0.73 & $\mathrm{~T}_{\mathrm{L}}$ lFrit \\
\hline & measured bc & -9.86 & 0.77 & 1019 & 29 & 1.93 & 213.02 & 0.73 & $\mathrm{~T}_{\mathrm{L}} \mathrm{TiO}_{2} 1$ Frit \\
\hline \multirow{3}{*}{ ROC-28 } & target & -13.28 & 3.20 & 665 & 48 & 2.00 & 194.13 & 0.71 & $\mathrm{TiO}_{2}$ Homg \\
\hline & measured & -12.82 & 2.64 & 681 & 48 & 1.91 & 189.62 & 0.71 & Homg \\
\hline & measured bc & -12.85 & 2.67 & 678 & 51 & 1.97 & 192.17 & 0.71 & $\mathrm{TiO}_{2}$ Homg \\
\hline \multirow{3}{*}{ ROC-29 } & target & -9.24 & 0.59 & 1052 & 28 & 0.50 & 213.11 & 0.64 & $\mathrm{~T}_{\mathrm{L}}$ \\
\hline & measured & -9.02 & 0.54 & 1046 & 30 & 0.48 & 213.58 & 0.63 & $\mathrm{~T}_{\mathrm{L}}$ \\
\hline & measured bc & -9.17 & 0.58 & 1043 & 28 & 0.51 & 219.47 & 0.63 & $\mathrm{~T}_{\mathrm{L}}$ \\
\hline \multirow{3}{*}{ ROC-30 } & target & -10.55 & 1.02 & 938 & 38 & 1.37 & 203.76 & 0.72 & Homg \\
\hline & measured & -10.60 & 1.05 & 940 & 37 & 1.36 & 207.36 & 0.71 & Homg \\
\hline & measured bc & -10.75 & 1.11 & 940 & 35 & 1.44 & 212.33 & 0.71 & \\
\hline
\end{tabular}




\subsection{PCT}

Table B1 in Appendix B provides the elemental leachate concentration measurements for the solution samples generated by the PCTs. Any measurement below the detection limit of the analytical procedure (indicated by a " $<$ ") was replaced by one half of the detection limit in subsequent analyses. In addition to adjustments for detection limits, the values were adjusted for the dilution factors: study glasses, blanks and the ARM glass were multiplied by 1.6667 to determine the values in parts per million ( $\mathrm{ppm}$ ) and the values for EA were multiplied by 16.6667. Table B2 in Appendix B provides the resulting values. There were no solution weight loss issues over the course of the seven day test.

\subsubsection{Measurements in Analytical Sequence}

Exhibit B1 in Appendix B provides plots of the leachate concentrations in analytical sequence for all of the data from all three sets of PCTs. No issues were observed in these plots.

\subsubsection{Results for the Samples of the Multi-Element Solution Standard}

Exhibit B2 in Appendix B provides analyses of measurements of the multi-element solution standard by analytical set/ICP-AES calibration block, and ANOVA investigations for each element of interest. A statistically significant difference (at a 5\% level) among the averages of these measurements was indicated for B, Li, and Si. No attempt was made to bias correct for these effects since averaging the ppm values for each set of triplicates minimizes the impact of any potential ICP-AES effects.

Table 11 summarizes the average measurements and the reference values for the four elements of interest. The results indicate consistent and accurate measurements throughout the measurement process.

Table 11. Results from Samples of the Multi-Element Solution Standard

\begin{tabular}{|c|c|c|c|c|}
\hline Analytical & Avg B & Avg Li & $\begin{array}{c}\text { Avg } \\
\text { Na }\end{array}$ & Avg Si \\
\hline Set/Block & $\mathbf{( p p m )}$ & $\mathbf{( p p m )}$ & $\mathbf{( p p m )}$ & $\mathbf{( p p m )}$ \\
\hline $1 / 1$ & 19.93 & 10.07 & 82.37 & 50.43 \\
\hline $1 / 2$ & 19.77 & 9.97 & 81.73 & 50.03 \\
\hline $1 / 3$ & 20.07 & 9.99 & 81.53 & 50.50 \\
\hline $2 / 1$ & 20.10 & 9.90 & 81.43 & 50.03 \\
\hline $2 / 2$ & 20.07 & 9.97 & 82.00 & 50.10 \\
\hline $2 / 3$ & 20.20 & 10.07 & 82.03 & 49.93 \\
\hline $3 / 1$ & 20.10 & 10.00 & 81.63 & 50.07 \\
\hline $3 / 2$ & 20.47 & 10.07 & 80.53 & 50.77 \\
\hline $3 / 3$ & 20.20 & 10.03 & 81.00 & 50.63 \\
\hline Grand Average & 20.10 & 10.01 & 81.59 & 50.28 \\
\hline Reference Value & 20 & 10 & 81 & 50 \\
\hline \% difference & $0.5 \%$ & $0.1 \%$ & $0.7 \%$ & $0.6 \%$ \\
\hline
\end{tabular}




\subsubsection{Measurements by Glass Identifier}

Exhibit B3 in Appendix B provides plots of the leachate concentrations for both the quenched and ccc version of each glass, as well as the reference samples (EA, ARM ${ }^{16}$, multielement solution standard and blanks). ${ }^{2}$ Two units of measure are used in these plots: ppm and the common logarithms of the ppm values. The common logarithm plots allow for the assessment of the repeatability of the measurements and any differences between the quenched and ccc version of a given glass.

For some of the glasses, scatter in the triplicate values of some analytes is observed; however, these results do not affect the outcome of this study.

\subsubsection{Normalized PCT Results}

PCT leachate concentrations were normalized using the target, measured and bias-corrected cation compositions $(\mathrm{wt} \%)$ in the glass to obtain a grams-per-liter $(\mathrm{g} / \mathrm{L})$ leachate concentration.

As is the usual convention, the common logarithm of the normalized PCT (normalized leachate, NL) for each element of interest was determined and used for comparison. To accomplish this computation, one must:

1. Determine the common logarithm of the elemental leachate concentration (ppm) for each of the triplicates and each of the elements of interest (these values are provided in Table B1 of Appendix B).

2. Average the common logarithms over the triplicates for each element of interest.

Normalizing Using Measured Composition

3. Subtract a quantity equal to 1 plus the common logarithm of the average cation measured concentration (expressed as a weight percent of the glass) from the average computed in step 2 .

Or Normalizing Using Target Composition

3. Subtract a quantity equal to 1 plus the common logarithm of the target cation concentration (expressed as a weight percent of the glass) from the average computed in step 2 .

Or Normalizing Using Measured Bias-Corrected Composition

3 . Subtract a quantity equal to 1 plus the common logarithm of the measured biascorrected cation concentration (expressed as a weight percent of the glass) from the average computed in step 2.

\footnotetext{
16 The concentrations of each element of interest for ARM are within the control limits in THERMO ${ }^{\mathrm{TM}}$.
} 
Exhibit B4 in Appendix B provides scatter plots that contain normalized released rates for both the quenched and ccc version of the glass based on the target, measured and biascorrected compositions. These plots offer an opportunity to investigate the consistency in the leaching across the elements for the glasses of this study. Consistency in the leaching across the elements is typically demonstrated by a high degree of linear correlation among the values for pairs of these elements. The smallest correlation in this plot is for $\mathrm{Li}$ and $\mathrm{Si}$, with a value of $\sim 91.5 \%$, which demonstrates the consistency of the results among the four analytes.

Table 12 summarizes the normalized PCT results for the glasses of this study, which are listed by heat treatment and compositional view for each glass. In general, glasses with higher $\mathrm{Al}_{2} \mathrm{O}_{3}$ concentrations are more durable, which is to be expected. The PCT results for all of the glasses are acceptable based upon comparisons to the EA results. The NL [B] value for ROC-09 based on the measured compositional view of ROC-09 is $10.14 \mathrm{~g} / \mathrm{L}$, which is slightly above the $10 \mathrm{~g} / \mathrm{L}$ benchmark ${ }^{17}$ used by Edwards and Brown, but it is still well below that of the EA glass when measurement uncertainty is applied and is thus considered to be acceptable. $^{3}$

\footnotetext{
${ }^{17}$ It should be emphasized that this limit was selected only as a guide to develop the $\mathrm{Al}_{2} \mathrm{O}_{3}$ and/or sum or alkali criteria.
} 
SRNL-STI-2009-00465, REVISION 0

Table 12. Normalized PCT Results

\begin{tabular}{|c|c|c|c|c|c|c|c|c|c|c|c|}
\hline Set & Glass ID & \begin{tabular}{|c|} 
Heat \\
Treatment
\end{tabular} & Comp View & $\begin{array}{c}\log N \mathrm{~L} \\
{[\mathrm{~B}(\mathrm{~g} / \mathrm{L})]}\end{array}$ & $\begin{array}{c}\log \mathrm{NL} \\
{[\mathrm{Li}(\mathrm{g} / \mathrm{L})]}\end{array}$ & $\begin{array}{c}\log \mathrm{NL} \\
{[\mathrm{Na}(\mathrm{g} / \mathrm{L})]}\end{array}$ & $\begin{array}{c}\log \mathrm{NL} \\
{[\mathrm{Si}(\mathrm{g} / \mathrm{L})]}\end{array}$ & $\begin{array}{l}\text { NL B } \\
(\mathrm{g} / \mathrm{L})\end{array}$ & $\begin{array}{c}\text { NL Li } \\
(\mathrm{g} / \mathrm{L})\end{array}$ & $\begin{array}{c}\text { NL Na } \\
(\mathrm{g} / \mathbf{L})\end{array}$ & $\begin{array}{c}\text { NL Si } \\
(\mathrm{g} / \mathrm{L})\end{array}$ \\
\hline 1 & ARM & reference & reference & -0.31 & -0.23 & -0.29 & -0.55 & 0.49 & 0.59 & 0.51 & 0.28 \\
\hline 2 & ARM & reference & reference & -0.26 & -0.21 & -0.28 & -0.54 & 0.54 & 0.61 & 0.53 & 0.29 \\
\hline 3 & ARM & reference & reference & -0.28 & -0.22 & -0.29 & -0.54 & 0.52 & 0.60 & 0.52 & 0.29 \\
\hline 1 & EA & reference & reference & 1.23 & 0.98 & 1.12 & 0.61 & 17.00 & 9.52 & 13.24 & 4.02 \\
\hline 2 & EA & reference & reference & 1.24 & 0.98 & 1.13 & 0.60 & 17.43 & 9.64 & 13.56 & 3.99 \\
\hline 3 & $\overline{\mathbf{E A}}$ & reference & reference & 1.24 & 0.99 & 1.13 & 0.61 & 17.55 & 9.77 & 13.41 & 4.04 \\
\hline 1 & \multirow{6}{*}{ ROC-01 } & $\mathrm{ccc}$ & target & 0.05 & 0.12 & -0.03 & -0.15 & 1.11 & 1.30 & 0.94 & 0.71 \\
\hline 1 & & $\mathrm{ccc}$ & measured & 0.06 & 0.14 & -0.02 & -0.15 & 1.15 & 1.36 & 0.96 & 0.71 \\
\hline 1 & & $\mathrm{ccc}$ & measured bc & 0.04 & 0.11 & -0.01 & -0.16 & 1.09 & 1.30 & 0.99 & 0.70 \\
\hline 1 & & quenched & target & 0.02 & 0.02 & -0.04 & -0.23 & 1.05 & 1.04 & 0.91 & 0.59 \\
\hline 1 & & quenched & measured & 0.04 & 0.04 & -0.03 & -0.23 & 1.10 & 1.09 & 0.93 & 0.58 \\
\hline 1 & & quenched & measured bc & 0.01 & 0.01 & -0.02 & -0.24 & 1.03 & 1.03 & 0.96 & 0.57 \\
\hline 1 & \multirow{6}{*}{ ROC-02 } & $\mathrm{ccc}$ & target & -0.11 & -0.10 & -0.07 & -0.39 & 0.77 & 0.79 & 0.85 & 0.41 \\
\hline 1 & & $\mathrm{ccc}$ & measured & -0.08 & -0.08 & -0.05 & -0.37 & 0.83 & 0.84 & 0.89 & 0.43 \\
\hline 1 & & $\mathrm{ccc}$ & measured bc & -0.11 & -0.10 & -0.05 & -0.38 & 0.78 & 0.80 & 0.90 & 0.42 \\
\hline 1 & & quenched & target & -0.04 & -0.03 & 0.02 & -0.32 & 0.92 & 0.93 & 1.04 & 0.48 \\
\hline 1 & & quenched & measured & 0.00 & -0.01 & 0.04 & -0.30 & 0.99 & 0.98 & 1.09 & 0.50 \\
\hline 1 & & quenched & measured bc & -0.04 & -0.03 & 0.04 & -0.31 & 0.92 & 0.94 & 1.11 & 0.49 \\
\hline 1 & \multirow{6}{*}{ ROC-03 } & $\mathrm{ccc}$ & \begin{tabular}{|l|} 
target \\
\end{tabular} & -0.09 & -0.04 & -0.24 & -0.34 & 0.81 & 0.91 & 0.57 & 0.46 \\
\hline 1 & & $\mathrm{ccc}$ & measured & -0.08 & -0.03 & -0.24 & -0.34 & 0.83 & 0.94 & 0.58 & 0.45 \\
\hline 1 & & $\mathrm{ccc}$ & measured bc & -0.11 & -0.05 & -0.23 & -0.35 & 0.78 & 0.89 & 0.59 & 0.45 \\
\hline 1 & & quenched & target & -0.10 & -0.03 & -0.26 & -0.35 & 0.80 & 0.93 & 0.55 & 0.45 \\
\hline 1 & & quenched & measured & -0.09 & -0.02 & -0.26 & -0.35 & 0.81 & 0.96 & 0.55 & 0.44 \\
\hline 1 & & quenched & measured bc & -0.12 & -0.04 & -0.25 & -0.36 & 0.76 & 0.91 & 0.57 & 0.44 \\
\hline 1 & \multirow{6}{*}{ ROC-04 } & $\mathrm{ccc}$ & target & -0.24 & 0.05 & -0.08 & -0.33 & 0.57 & 1.12 & 0.83 & 0.47 \\
\hline 1 & & $\mathrm{ccc}$ & measured & -0.22 & 0.06 & -0.07 & -0.33 & 0.60 & 1.15 & 0.85 & 0.47 \\
\hline 1 & & $\mathrm{ccc}$ & measured bc & -0.25 & 0.04 & -0.06 & -0.33 & 0.56 & 1.09 & 0.87 & 0.46 \\
\hline 1 & & quenched & target & -0.14 & -0.08 & -0.05 & -0.36 & 0.73 & 0.84 & 0.89 & 0.44 \\
\hline 1 & & quenched & measured & -0.12 & -0.06 & -0.04 & -0.36 & 0.76 & 0.86 & 0.92 & 0.44 \\
\hline 1 & & quenched & measured bc & -0.14 & -0.09 & -0.03 & -0.37 & 0.72 & 0.82 & 0.93 & 0.43 \\
\hline 1 & \multirow{6}{*}{ ROC-05 } & $\mathrm{ccc}$ & target & 0.09 & 0.18 & 0.00 & -0.16 & 1.24 & 1.51 & 1.00 & 0.70 \\
\hline 1 & & $\mathrm{ccc}$ & measured & 0.12 & 0.20 & 0.01 & -0.15 & 1.32 & 1.58 & 1.03 & 0.71 \\
\hline 1 & & $\mathrm{ccc}$ & measured bc & 0.09 & 0.18 & 0.02 & -0.16 & 1.22 & 1.50 & 1.05 & 0.69 \\
\hline 1 & & quenched & target & 0.10 & 0.07 & -0.01 & -0.23 & 1.24 & 1.18 & 0.98 & 0.59 \\
\hline 1 & & quenched & measured & 0.12 & 0.09 & 0.00 & -0.22 & 1.32 & 1.23 & 1.01 & 0.60 \\
\hline 1 & & quenched & measured bc & 0.09 & 0.07 & 0.01 & -0.23 & 1.23 & 1.18 & 1.02 & 0.59 \\
\hline 1 & \multirow{6}{*}{ ROC-06 } & $\mathrm{ccc}$ & target & 0.31 & 0.30 & 0.21 & -0.29 & 2.02 & 1.97 & 1.62 & 0.51 \\
\hline 1 & & $\mathrm{ccc}$ & measured & 0.32 & 0.30 & 0.21 & -0.29 & 2.09 & 1.99 & 1.61 & 0.52 \\
\hline 1 & & $\mathrm{ccc}$ & measured bc & 0.29 & 0.28 & 0.22 & -0.30 & 1.94 & 1.90 & 1.65 & 0.50 \\
\hline 1 & & quenched & target & 0.35 & 0.33 & 0.24 & -0.31 & 2.23 & 2.13 & 1.73 & 0.50 \\
\hline 1 & & quenched & measured & 0.36 & 0.33 & 0.24 & -0.30 & 2.30 & 2.16 & 1.72 & 0.50 \\
\hline 1 & & quenched & measured bc & 0.33 & 0.31 & 0.25 & -0.31 & 2.14 & 2.06 & 1.77 & 0.49 \\
\hline 1 & \multirow{6}{*}{ ROC-07 } & $\mathrm{ccc}$ & target & 0.24 & 0.22 & 0.07 & -0.16 & 1.73 & 1.67 & 1.17 & 0.70 \\
\hline 1 & & $\mathrm{ccc}$ & measured & 0.26 & 0.25 & 0.09 & -0.14 & 1.82 & 1.76 & 1.22 & 0.72 \\
\hline 1 & & $\mathrm{ccc}$ & measured bc & 0.23 & 0.22 & 0.10 & -0.15 & 1.69 & 1.67 & 1.25 & 0.70 \\
\hline 1 & & quenched & target & 0.32 & 0.29 & 0.12 & -0.15 & 2.07 & 1.96 & 1.33 & 0.70 \\
\hline 1 & & quenched & measured & 0.34 & 0.31 & 0.14 & -0.14 & 2.17 & 2.06 & 1.38 & 0.73 \\
\hline 1 & & quenched & measured bc & 0.31 & 0.29 & 0.15 & -0.15 & 2.02 & 1.96 & 1.42 & 0.71 \\
\hline 1 & \multirow{6}{*}{ ROC-08 } & $\mathrm{ccc}$ & target & 0.49 & 0.45 & 0.41 & 0.22 & 3.11 & 2.82 & 2.58 & 1.67 \\
\hline 1 & & ccc & measured & 0.51 & 0.47 & 0.44 & 0.24 & 3.24 & 2.94 & 2.73 & 1.73 \\
\hline 1 & & $\mathrm{ccc}$ & measured bc & 0.48 & 0.45 & 0.45 & 0.23 & 3.04 & 2.79 & 2.80 & 1.70 \\
\hline 1 & & quenched & \begin{tabular}{|l|} 
target \\
\end{tabular} & 0.52 & 0.44 & 0.44 & 0.21 & 3.33 & 2.73 & 2.74 & 1.63 \\
\hline 1 & & quenched & measured & 0.54 & 0.46 & 0.46 & 0.23 & 3.47 & 2.85 & 2.89 & 1.68 \\
\hline 1 & & quenched & measured bc & 0.51 & 0.43 & 0.47 & 0.22 & 3.27 & 2.71 & 2.97 & 1.66 \\
\hline 1 & \multirow{6}{*}{ ROC-09 } & $\mathrm{ccc}$ & target & 0.55 & 0.46 & 0.48 & 0.05 & 3.57 & 2.89 & 2.98 & 1.12 \\
\hline 1 & & $\mathrm{ccc}$ & measured & 0.57 & 0.49 & 0.52 & 0.06 & 3.74 & 3.07 & 3.31 & 1.15 \\
\hline 1 & & $\mathrm{ccc}$ & measured bc & 0.55 & 0.47 & 0.53 & 0.05 & 3.52 & 2.92 & 3.39 & 1.13 \\
\hline 1 & & quenched & \begin{tabular}{|l|} 
target \\
\end{tabular} & 0.99 & 0.86 & 0.89 & 0.35 & 9.68 & 7.25 & 7.83 & 2.21 \\
\hline 1 & & quenched & measured & 1.01 & 0.89 & 0.94 & 0.35 & 10.14 & 7.72 & 8.68 & 2.26 \\
\hline 1 & & quenched & measured bc & 0.98 & 0.87 & 0.95 & 0.35 & 9.55 & 7.33 & 8.90 & 2.22 \\
\hline
\end{tabular}


SRNL-STI-2009-00465, REVISION 0

Table 12 cont. Normalized PCT Results

\begin{tabular}{|c|c|c|c|c|c|c|c|c|c|c|c|}
\hline Set & Glass ID & \begin{tabular}{c|} 
Heat \\
Treatment
\end{tabular} & Comp View & $\begin{array}{c}\log N \mathbf{L} \\
{[B(g / L)]}\end{array}$ & $\begin{array}{c}\log \mathrm{NL} \\
{[\mathrm{Li}(\mathrm{g} / \mathrm{L})]}\end{array}$ & $\begin{array}{c}\log \mathrm{NL} \\
{[\mathrm{Na}(\mathrm{g} / \mathrm{L})]} \\
\end{array}$ & $\begin{array}{c}\log \mathrm{NL} \\
{[\mathrm{Si}(\mathrm{g} / \mathrm{L})]}\end{array}$ & $\begin{array}{l}\text { NL B } \\
(g / L)\end{array}$ & $\begin{array}{c}\text { NL Li } \\
(\mathrm{g} / \mathrm{L})\end{array}$ & $\begin{array}{c}\text { NL Na } \\
(\mathrm{g} / \mathrm{L})\end{array}$ & $\begin{array}{c}\text { NL Si } \\
(\mathrm{g} / \mathrm{L})\end{array}$ \\
\hline 1 & \multirow{6}{*}{ ROC-10 } & $\mathrm{ccc}$ & target & 0.07 & 0.06 & -0.02 & -0.18 & 1.18 & 1.15 & 0.97 & 0.67 \\
\hline 1 & & $\operatorname{ccc}$ & measured & 0.08 & 0.08 & 0.02 & -0.18 & 1.20 & 1.19 & 1.04 & 0.66 \\
\hline 1 & & $\mathrm{ccc}$ & measured bc & 0.05 & 0.05 & 0.03 & -0.19 & 1.13 & 1.13 & 1.06 & 0.65 \\
\hline 1 & & quenched & target & 0.11 & 0.09 & 0.03 & -0.14 & 1.29 & 1.24 & 1.06 & 0.72 \\
\hline 1 & & quenched & measured & 0.12 & 0.11 & 0.06 & -0.15 & 1.32 & 1.27 & 1.14 & 0.72 \\
\hline 1 & & quenched & measured bc & 0.09 & 0.08 & 0.07 & -0.15 & 1.24 & 1.21 & 1.17 & 0.70 \\
\hline 2 & \multirow{6}{*}{ ROC-11 } & $\operatorname{ccc}$ & target & -0.01 & 0.02 & -0.06 & -0.21 & 0.98 & 1.05 & 0.86 & 0.62 \\
\hline 2 & & $\mathrm{ccc}$ & measured & 0.01 & 0.05 & -0.06 & -0.20 & 1.02 & 1.11 & 0.86 & 0.63 \\
\hline 2 & & $\mathrm{ccc}$ & measured bc & -0.02 & 0.02 & -0.05 & -0.21 & 0.96 & 1.05 & 0.88 & 0.62 \\
\hline 2 & & quenched & target & 0.05 & 0.03 & -0.01 & -0.19 & 1.11 & 1.07 & 0.97 & 0.64 \\
\hline 2 & & quenched & measured & 0.06 & 0.05 & -0.01 & -0.18 & 1.15 & 1.13 & 0.97 & 0.66 \\
\hline 2 & & quenched & measured bc & 0.04 & 0.03 & 0.00 & -0.19 & 1.08 & 1.08 & 0.99 & 0.64 \\
\hline 2 & \multirow{6}{*}{ ROC-12 } & $\mathrm{ccc}$ & target & -0.07 & 0.04 & 0.03 & -0.28 & 0.85 & 1.10 & 1.08 & 0.53 \\
\hline 2 & & $\mathrm{ccc}$ & measured & -0.05 & 0.05 & 0.05 & -0.27 & 0.90 & 1.12 & 1.13 & 0.54 \\
\hline 2 & & $\mathrm{ccc}$ & measured bc & -0.08 & 0.03 & 0.06 & -0.28 & 0.83 & 1.07 & 1.14 & 0.53 \\
\hline 2 & & quenched & target & -0.05 & 0.05 & 0.08 & -0.25 & 0.90 & 1.12 & 1.20 & 0.56 \\
\hline 2 & & quenched & measured & -0.03 & 0.06 & 0.10 & -0.24 & 0.94 & 1.15 & 1.26 & 0.58 \\
\hline 2 & & quenched & measured bc & -0.06 & 0.04 & 0.11 & -0.25 & 0.88 & 1.10 & 1.27 & 0.56 \\
\hline 2 & \multirow{6}{*}{ ROC-13 } & $\operatorname{ccc}$ & target & -0.10 & -0.05 & -0.16 & -0.30 & 0.79 & 0.89 & 0.69 & 0.50 \\
\hline 2 & & $\mathrm{ccc}$ & measured & -0.08 & -0.04 & -0.12 & -0.30 & 0.83 & 0.91 & 0.77 & 0.50 \\
\hline 2 & & $\operatorname{ccc}$ & measured bc & -0.11 & -0.06 & -0.11 & -0.31 & 0.77 & 0.87 & 0.78 & 0.49 \\
\hline 2 & & quenched & target & -0.11 & -0.07 & -0.15 & -0.30 & 0.78 & 0.85 & 0.71 & 0.50 \\
\hline 2 & & quenched & measured & -0.09 & -0.06 & -0.10 & -0.30 & 0.82 & 0.87 & 0.79 & 0.50 \\
\hline 2 & & quenched & measured bc & -0.12 & -0.08 & -0.10 & -0.31 & 0.76 & 0.83 & 0.80 & 0.49 \\
\hline 2 & \multirow{6}{*}{ ROC-14 } & $\mathrm{ccc}$ & target & 0.18 & 0.14 & -0.02 & -0.37 & 1.52 & 1.38 & 0.96 & 0.43 \\
\hline 2 & & $\mathrm{ccc}$ & measured & 0.20 & 0.16 & 0.00 & -0.37 & 1.59 & 1.44 & 0.99 & 0.43 \\
\hline 2 & & $\mathrm{ccc}$ & measured bc & 0.17 & 0.14 & 0.00 & -0.38 & 1.48 & 1.37 & 1.01 & 0.42 \\
\hline 2 & & quenched & target & 0.01 & -0.02 & -0.13 & -0.39 & 1.01 & 0.95 & 0.73 & 0.40 \\
\hline 2 & & quenched & measured & 0.02 & -0.01 & -0.12 & -0.39 & 1.06 & 0.99 & 0.76 & 0.40 \\
\hline 2 & & quenched & measured bc & -0.01 & -0.03 & -0.11 & -0.40 & 0.98 & 0.94 & 0.77 & 0.40 \\
\hline 2 & \multirow{6}{*}{ ROC-15 } & $\mathrm{ccc}$ & target & -0.10 & -0.03 & 0.04 & -0.19 & 0.80 & 0.94 & 1.08 & 0.65 \\
\hline 2 & & $\mathrm{ccc}$ & measured & -0.08 & -0.01 & 0.07 & -0.19 & 0.83 & 0.99 & 1.18 & 0.65 \\
\hline 2 & & $\mathrm{ccc}$ & measured bc & -0.11 & -0.03 & 0.08 & -0.20 & 0.78 & 0.94 & 1.20 & 0.64 \\
\hline 2 & & quenched & target & -0.08 & -0.11 & 0.00 & -0.24 & 0.83 & 0.77 & 1.01 & 0.58 \\
\hline 2 & & quenched & measured & -0.06 & -0.09 & 0.04 & -0.24 & 0.86 & 0.81 & 1.10 & 0.57 \\
\hline 2 & & quenched & measured bc & -0.09 & -0.12 & 0.05 & -0.25 & 0.81 & 0.77 & 1.11 & 0.56 \\
\hline 2 & \multirow{6}{*}{ ROC-16 } & $\mathrm{ccc}$ & target & 0.68 & 0.63 & 0.49 & 0.27 & 4.80 & 4.23 & 3.11 & 1.87 \\
\hline 2 & & $\mathrm{ccc}$ & measured & 0.71 & 0.64 & 0.50 & 0.29 & 5.11 & 4.39 & 3.15 & 1.96 \\
\hline 2 & & $\mathrm{ccc}$ & measured bc & 0.68 & 0.63 & 0.50 & 0.28 & 4.82 & 4.29 & 3.14 & 1.92 \\
\hline 2 & & quenched & target & 0.70 & 0.63 & 0.51 & 0.28 & 4.96 & 4.29 & 3.23 & 1.89 \\
\hline 2 & & quenched & measured & 0.72 & 0.65 & 0.52 & 0.30 & 5.29 & 4.45 & 3.27 & 1.98 \\
\hline 2 & & quenched & measured bc & 0.70 & 0.64 & 0.52 & 0.29 & 4.98 & 4.35 & 3.27 & 1.93 \\
\hline 2 & \multirow{6}{*}{ ROC-17 } & $\mathrm{ccc}$ & target & 0.01 & 0.11 & 0.13 & -0.12 & 1.01 & 1.28 & 1.36 & 0.76 \\
\hline 2 & & $\mathrm{ccc}$ & measured & 0.01 & 0.13 & 0.13 & -0.11 & 1.02 & 1.33 & 1.34 & 0.78 \\
\hline 2 & & $\operatorname{ccc}$ & measured bc & -0.02 & 0.10 & 0.13 & -0.11 & 0.96 & 1.26 & 1.34 & 0.77 \\
\hline 2 & & quenched & target & 0.07 & 0.05 & 0.14 & -0.13 & 1.18 & 1.13 & 1.37 & 0.75 \\
\hline 2 & & quenched & measured & 0.08 & 0.07 & 0.13 & -0.12 & 1.19 & 1.17 & 1.35 & 0.76 \\
\hline 2 & & quenched & measured bc & 0.05 & 0.04 & 0.13 & -0.12 & 1.12 & 1.10 & 1.35 & 0.75 \\
\hline 2 & \multirow{6}{*}{ ROC-18 } & $\mathrm{ccc}$ & target & -0.11 & -0.02 & -0.12 & -0.29 & 0.79 & 0.96 & 0.75 & 0.52 \\
\hline 2 & & $\mathrm{ccc}$ & measured & -0.09 & -0.02 & -0.13 & -0.26 & 0.81 & 0.95 & 0.74 & 0.54 \\
\hline 2 & & $\mathrm{ccc}$ & measured bc & -0.12 & -0.03 & -0.13 & -0.27 & 0.77 & 0.93 & 0.74 & 0.53 \\
\hline 2 & & quenched & target & 0.00 & 0.02 & -0.04 & -0.24 & 0.99 & 1.05 & 0.91 & 0.57 \\
\hline 2 & & quenched & measured & 0.01 & 0.02 & -0.05 & -0.22 & 1.03 & 1.04 & 0.89 & 0.60 \\
\hline 2 & & quenched & measured bc & -0.01 & 0.01 & -0.05 & -0.23 & 0.97 & 1.02 & 0.89 & 0.58 \\
\hline 2 & \multirow{6}{*}{ ROC-19 } & $\mathrm{ccc}$ & target & 0.31 & 0.28 & 0.15 & -0.14 & 2.02 & 1.90 & 1.41 & 0.73 \\
\hline 2 & & $\mathrm{ccc}$ & measured & 0.33 & 0.30 & 0.14 & -0.12 & 2.15 & 1.98 & 1.38 & 0.76 \\
\hline 2 & & $\mathrm{ccc}$ & measured bc & 0.31 & 0.29 & 0.14 & -0.13 & 2.03 & 1.93 & 1.38 & 0.75 \\
\hline 2 & & quenched & target & 0.31 & 0.29 & 0.15 & -0.14 & 2.06 & 1.94 & 1.41 & 0.72 \\
\hline 2 & & quenched & measured & 0.34 & 0.31 & 0.14 & -0.13 & 2.19 & 2.02 & 1.38 & 0.75 \\
\hline 2 & & quenched & measured bc & 0.32 & 0.30 & 0.14 & -0.13 & 2.06 & 1.98 & 1.39 & 0.74 \\
\hline
\end{tabular}


SRNL-STI-2009-00465, REVISION 0

Table 12 cont. Normalized PCT Results

\begin{tabular}{|c|c|c|c|c|c|c|c|c|c|c|c|}
\hline Set & Glass ID & \begin{tabular}{|c|} 
Heat \\
Treatment
\end{tabular} & Comp View & $\begin{array}{c}\log \mathrm{NL} \\
{[\mathrm{B}(\mathrm{g} / \mathrm{L})]}\end{array}$ & $\begin{array}{c}\log \mathrm{NL} \\
{[\mathrm{Li}(\mathrm{g} / \mathrm{L})]}\end{array}$ & $\begin{array}{c}\log \mathrm{NL} \\
{[\mathrm{Na}(\mathrm{g} / \mathrm{L})]}\end{array}$ & $\begin{array}{c}\log \mathbf{N L} \\
{[\mathrm{Si}(\mathrm{g} / \mathrm{L})]}\end{array}$ & $\begin{array}{l}\text { NL B } \\
(\mathrm{g} / \mathrm{L})\end{array}$ & $\begin{array}{c}\text { NL Li } \\
(\mathrm{g} / \mathrm{L})\end{array}$ & $\begin{array}{c}\text { NL Na } \\
(\mathrm{g} / \mathrm{L})\end{array}$ & $\begin{array}{l}\text { NL } S i \\
(g / L)\end{array}$ \\
\hline 2 & \multirow{6}{*}{ ROC-20 } & \begin{tabular}{|c|}
$\mathrm{ccc}$ \\
\end{tabular} & target & 0.80 & 0.71 & \begin{tabular}{|l|}
0.70 \\
\end{tabular} & 0.23 & 6.34 & 5.18 & 5.02 & 1.70 \\
\hline 2 & & $\mathrm{ccc}$ & measured & 0.83 & 0.74 & 0.73 & 0.26 & 6.74 & 5.53 & 5.32 & 1.83 \\
\hline 2 & & $\mathrm{ccc}$ & measured bc & 0.80 & 0.72 & 0.73 & 0.26 & 6.35 & 5.21 & 5.33 & 1.81 \\
\hline 2 & & quenched & \begin{tabular}{|c|} 
target \\
\end{tabular} & 0.92 & 0.80 & 0.81 & 0.27 & 8.23 & 6.28 & 6.39 & 1.87 \\
\hline 2 & & quenched & measured & 0.94 & 0.83 & 0.83 & 0.31 & 8.75 & 6.70 & 6.77 & 2.02 \\
\hline 2 & & quenched & measured bc & 0.92 & 0.80 & 0.83 & 0.30 & 8.24 & 6.31 & 6.79 & 1.99 \\
\hline 2 & \multirow{6}{*}{ ROC-21 } & $\mathrm{ccc}$ & \begin{tabular}{|l|} 
target \\
\end{tabular} & -0.03 & -0.02 & -0.13 & -0.30 & 0.94 & 0.96 & 0.74 & 0.51 \\
\hline 2 & & $\mathrm{ccc}$ & measured & -0.01 & 0.00 & -0.15 & -0.29 & 0.99 & 1.01 & 0.71 & 0.51 \\
\hline 3 & & $\mathrm{ccc}$ & measured bc & -0.03 & -0.02 & -0.15 & -0.30 & 0.93 & 0.95 & 0.71 & 0.50 \\
\hline 2 & & quenched & \begin{tabular}{|c|} 
target \\
\end{tabular} & 0.00 & 0.00 & -0.11 & -0.32 & 1.00 & 0.99 & 0.77 & 0.48 \\
\hline 2 & & quenched & measured & 0.02 & 0.02 & -0.13 & -0.31 & 1.04 & 1.04 & 0.74 & 0.49 \\
\hline 3 & & quenched & measured bc & -0.01 & -0.01 & -0.13 & -0.32 & 0.98 & 0.98 & 0.74 & 0.48 \\
\hline 3 & \multirow{6}{*}{ ROC-22 } & $\mathrm{ccc}$ & \begin{tabular}{|l|} 
target \\
\end{tabular} & 0.06 & 0.10 & -0.02 & -0.16 & 1.14 & 1.25 & 0.95 & 0.69 \\
\hline 3 & & $\mathrm{ccc}$ & measured & 0.05 & 0.10 & -0.03 & -0.15 & 1.13 & 1.27 & 0.93 & 0.70 \\
\hline 3 & & $\mathrm{ccc}$ & measured bc & 0.03 & 0.08 & -0.03 & -0.16 & 1.06 & 1.20 & 0.93 & 0.69 \\
\hline 3 & & quenched & \begin{tabular}{|c|} 
target \\
\end{tabular} & 0.20 & 0.18 & 0.10 & -0.11 & 1.57 & 1.52 & 1.25 & 0.78 \\
\hline 3 & & quenched & measured & 0.19 & 0.19 & 0.09 & -0.10 & 1.55 & 1.54 & 1.23 & 0.79 \\
\hline 3 & & quenched & measured bc & 0.16 & 0.16 & 0.09 & -0.11 & 1.46 & 1.46 & 1.23 & 0.77 \\
\hline 3 & \multirow{6}{*}{ ROC-23 } & $\mathrm{ccc}$ & target & -0.26 & -0.16 & -0.28 & -0.36 & 0.55 & 0.69 & 0.53 & 0.44 \\
\hline 3 & & $\mathrm{ccc}$ & measured & -0.24 & -0.15 & -0.27 & -0.33 & 0.58 & 0.71 & 0.54 & 0.47 \\
\hline 3 & & $\mathrm{ccc}$ & measured bc & -0.26 & -0.16 & -0.27 & -0.34 & 0.55 & 0.69 & 0.54 & 0.46 \\
\hline 3 & & quenched & \begin{tabular}{|c|} 
target \\
\end{tabular} & -0.17 & -0.12 & -0.24 & -0.34 & 0.68 & 0.76 & 0.58 & 0.45 \\
\hline 3 & & quenched & measured & -0.15 & -0.11 & -0.23 & -0.31 & 0.71 & 0.78 & 0.59 & 0.49 \\
\hline 3 & & quenched & measured bc & -0.18 & -0.12 & -0.23 & -0.32 & 0.67 & 0.76 & 0.59 & 0.47 \\
\hline 3 & \multirow{6}{*}{ ROC-24 } & $\mathrm{ccc}$ & \begin{tabular}{|c|} 
target \\
\end{tabular} & -0.23 & -0.18 & -0.29 & -0.42 & 0.59 & 0.67 & 0.52 & 0.38 \\
\hline 3 & & $\mathrm{ccc}$ & \begin{tabular}{|l|} 
measured \\
\end{tabular} & -0.22 & -0.16 & -0.30 & -0.41 & 0.60 & 0.69 & 0.50 & 0.39 \\
\hline 3 & & ccc & measured bc & -0.25 & -0.19 & -0.30 & -0.42 & 0.57 & 0.65 & 0.51 & 0.38 \\
\hline 3 & & quenched & \begin{tabular}{|c|} 
target \\
\end{tabular} & -0.22 & -0.15 & -0.30 & -0.42 & 0.61 & 0.71 & 0.51 & 0.38 \\
\hline 3 & & quenched & measured & -0.21 & -0.14 & -0.31 & -0.42 & 0.62 & 0.73 & 0.50 & 0.38 \\
\hline 3 & & quenched & \begin{tabular}{|l|} 
measured bc \\
\end{tabular} & -0.24 & -0.16 & -0.30 & -0.43 & 0.58 & 0.69 & 0.50 & 0.38 \\
\hline 3 & \multirow{6}{*}{ ROC-25 } & $\mathrm{ccc}$ & \begin{tabular}{|l|} 
target \\
\end{tabular} & 0.09 & 0.07 & 0.09 & -0.13 & 1.23 & 1.17 & 1.23 & 0.74 \\
\hline 3 & & $\mathrm{ccc}$ & measured & 0.10 & 0.07 & 0.11 & -0.10 & 1.25 & 1.17 & 1.29 & 0.79 \\
\hline 3 & & $\mathrm{ccc}$ & measured bc & 0.07 & 0.06 & 0.11 & -0.11 & 1.17 & 1.14 & 1.29 & 0.78 \\
\hline 3 & & quenched & \begin{tabular}{|c|} 
target \\
\end{tabular} & 0.14 & 0.08 & 0.16 & -0.11 & 1.37 & 1.20 & 1.44 & 0.77 \\
\hline 3 & & quenched & measured & 0.14 & 0.08 & 0.18 & -0.08 & 1.38 & 1.19 & 1.51 & 0.83 \\
\hline 3 & & quenched & measured bc & 0.12 & 0.07 & 0.18 & -0.09 & 1.30 & 1.17 & 1.51 & 0.81 \\
\hline 3 & \multirow{6}{*}{ ROC-26 } & $\mathrm{ccc}$ & \begin{tabular}{|c|} 
target \\
\end{tabular} & -0.14 & -0.09 & -0.02 & -0.26 & 0.72 & 0.81 & 0.96 & 0.55 \\
\hline 3 & & $\mathrm{ccc}$ & measured & -0.13 & -0.09 & -0.02 & -0.25 & 0.75 & 0.81 & 0.96 & 0.56 \\
\hline 3 & & $\mathrm{ccc}$ & measured bc & -0.15 & -0.10 & -0.02 & -0.26 & 0.71 & 0.79 & 0.96 & 0.55 \\
\hline 3 & & quenched & \begin{tabular}{|c|} 
target \\
\end{tabular} & -0.08 & -0.08 & 0.05 & -0.24 & 0.83 & 0.84 & 1.12 & 0.57 \\
\hline 3 & & quenched & \begin{tabular}{|c|} 
measured \\
\end{tabular} & -0.07 & -0.08 & 0.05 & -0.23 & 0.86 & 0.83 & 1.12 & 0.58 \\
\hline 3 & & quenched & measured bc & -0.09 & -0.09 & 0.05 & -0.24 & 0.81 & 0.81 & 1.13 & 0.57 \\
\hline 3 & \multirow{6}{*}{ ROC-27 } & ccc & \begin{tabular}{|c|} 
target \\
\end{tabular} & 0.09 & 0.12 & 0.06 & -0.11 & 1.22 & 1.33 & 1.14 & 0.77 \\
\hline 3 & & $\mathrm{ccc}$ & measured & 0.10 & 0.13 & 0.06 & -0.10 & 1.26 & 1.34 & 1.16 & 0.79 \\
\hline 3 & & $\mathrm{ccc}$ & measured bc & 0.07 & 0.12 & 0.06 & -0.11 & 1.19 & 1.31 & 1.16 & 0.77 \\
\hline 3 & & quenched & \begin{tabular}{|c|} 
target \\
\end{tabular} & 0.18 & 0.14 & 0.12 & -0.10 & 1.51 & 1.37 & 1.33 & 0.80 \\
\hline 3 & & quenched & \begin{tabular}{|c|} 
measured \\
\end{tabular} & 0.19 & 0.14 & 0.13 & -0.09 & 1.56 & 1.38 & 1.35 & 0.81 \\
\hline 3 & & quenched & \begin{tabular}{|l|} 
measured bc \\
\end{tabular} & 0.17 & 0.13 & 0.13 & -0.10 & 1.48 & 1.34 & 1.35 & 0.79 \\
\hline 3 & \multirow{6}{*}{ ROC-28 } & $\mathrm{ccc}$ & \begin{tabular}{|c|} 
target \\
\end{tabular} & 0.29 & 0.22 & 0.28 & 0.06 & 1.93 & 1.67 & 1.91 & 1.15 \\
\hline 3 & & $\mathrm{ccc}$ & \begin{tabular}{|l|} 
measured \\
\end{tabular} & 0.31 & 0.23 & 0.30 & 0.08 & 2.02 & 1.69 & 1.98 & 1.20 \\
\hline 3 & & $\mathrm{ccc}$ & measured bc & 0.28 & 0.22 & 0.30 & 0.07 & 1.91 & 1.65 & 1.98 & 1.17 \\
\hline 3 & & quenched & \begin{tabular}{|c|} 
target \\
\end{tabular} & 0.41 & 0.32 & 0.39 & 0.13 & 2.56 & 2.06 & 2.44 & 1.33 \\
\hline 3 & & quenched & \begin{tabular}{|l|} 
measured \\
\end{tabular} & 0.43 & 0.32 & 0.40 & 0.14 & 2.68 & 2.09 & 2.51 & 1.38 \\
\hline 3 & & quenched & measured bc & 0.40 & 0.31 & 0.40 & 0.13 & 2.53 & 2.04 & 2.51 & 1.35 \\
\hline 3 & \multirow{6}{*}{ ROC-29 } & $\mathrm{ccc}$ & \begin{tabular}{|c|} 
target \\
\end{tabular} & 0.21 & 0.18 & 0.00 & -0.33 & 1.63 & 1.49 & 0.99 & 0.47 \\
\hline 3 & & $\mathrm{ccc}$ & measured & 0.22 & 0.19 & -0.01 & -0.32 & 1.66 & 1.54 & 0.98 & 0.48 \\
\hline 3 & & $\mathrm{ccc}$ & measured bc & 0.19 & 0.16 & -0.01 & -0.33 & 1.56 & 1.45 & 0.99 & 0.47 \\
\hline 3 & & quenched & \begin{tabular}{|c|} 
target \\
\end{tabular} & 0.07 & 0.06 & -0.06 & -0.34 & 1.19 & 1.14 & 0.86 & 0.46 \\
\hline 3 & & quenched & \begin{tabular}{|l|} 
measured \\
\end{tabular} & 0.08 & 0.07 & -0.07 & -0.33 & 1.21 & 1.18 & 0.85 & 0.47 \\
\hline 3 & & quenched & measured bc & 0.06 & 0.05 & -0.07 & -0.34 & 1.14 & 1.11 & 0.86 & 0.46 \\
\hline 3 & & $\mathrm{ccc}$ & \begin{tabular}{|c|} 
target \\
\end{tabular} & -0.03 & -0.01 & -0.06 & -0.25 & 0.93 & 0.97 & 0.88 & 0.56 \\
\hline 3 & & ccc & measured & -0.03 & 0.00 & -0.06 & -0.25 & 0.93 & 0.99 & 0.86 & 0.56 \\
\hline 3 & ROC-30 & $\mathrm{ccc}$ & measured bc & -0.06 & -0.03 & -0.06 & -0.25 & 0.88 & 0.93 & 0.87 & 0.56 \\
\hline 3 & RUC-30 & quenched & \begin{tabular}{|c|} 
target \\
\end{tabular} & -0.04 & -0.03 & -0.05 & -0.26 & 0.91 & 0.93 & 0.90 & 0.55 \\
\hline 3 & & quenched & measured & -0.04 & -0.03 & -0.06 & -0.26 & 0.91 & 0.94 & 0.88 & 0.55 \\
\hline 3 & & quenched & measured bc & -0.07 & -0.05 & -0.06 & -0.26 & 0.86 & 0.89 & 0.88 & 0.55 \\
\hline
\end{tabular}




\subsubsection{Effects of Heat Treatment}

Exhibit B5 in Appendix B provides plots of the normalized PCT responses between the two heat treatments. These plots provide a basis for judging the practical impact of differences in the PCT response due to the heat treatment of the glass. In general, the PCT responses of the ccc versions are relatively consistent with those of the quenched versions (within experimental error), except for ROC-09, in which the normalized release rates of the quenched glass are considerably higher than those of the ccc glass. ${ }^{18}$ Due to these anomalous results, the durability of ROC-09 was re-measured (both heat treatments) and the new data is designated as ROC-09R (Table 13). All of the re-measured values are comparable to those determined in the initial measurement. For the quenched ROC-09R glass, all three compositional views have a NL $[\mathrm{B}] \geq 10 \mathrm{~g} / \mathrm{L}$. Despite these results, the boron releases of each compositional view are still well below the EA benchmark, acceptable and consistent with the results of the initial measurement. In order to confirm that the quenched and ccc glasses were not inadvertently switched, both were submitted for X-ray Diffraction (XRD) analysis. Each of the glasses was determined to be amorphous (within the $0.5 \mathrm{vol} \%$ detection limit of the instrument) as shown in Figure 1. Since crystalline peaks were not detected in either the quenched or ccc glass, no insight was gained on crystallization impacts that could have lead to the significant difference in PCT response.

Table 13. Re-measured PCT Responses of ROC-09

\begin{tabular}{|c|c|c|c|c|c|c|c|c|c|c|c|}
\hline Set & Glass ID & \begin{tabular}{|c|} 
Heat \\
Treatment
\end{tabular} & Comp View & $\begin{array}{c}\log \mathrm{NL} \\
{[\mathrm{B}(\mathrm{g} / \mathrm{L})]}\end{array}$ & $\begin{array}{c}\log \mathrm{NL} \\
{[\mathrm{Li}(\mathrm{g} / \mathrm{L})]}\end{array}$ & $\begin{array}{c}\log \mathrm{NL} \\
{[\mathrm{Na}(\mathrm{g} / \mathrm{L})]}\end{array}$ & $\begin{array}{c}\log \mathbf{N L} \\
{[\mathrm{Si}(\mathrm{g} / \mathrm{L})]}\end{array}$ & $\begin{array}{l}\text { NL B } \\
(\mathrm{g} / \mathrm{L})\end{array}$ & $\begin{array}{c}\text { NL Li } \\
(\mathrm{g} / \mathrm{L})\end{array}$ & $\begin{array}{c}\text { NL Na } \\
(\mathrm{g} / \mathrm{L})\end{array}$ & $\begin{array}{l}\text { NL Si } \\
(\mathrm{g} / \mathrm{L})\end{array}$ \\
\hline 3 & \multirow{6}{*}{ ROC-09M } & \begin{tabular}{|c|}
$\mathrm{ccc}$ \\
\end{tabular} & target & 0.57 & 0.48 & 0.50 & 0.06 & 3.68 & 3.05 & 3.13 & 1.15 \\
\hline 3 & & ccc & measured & 0.59 & 0.51 & 0.54 & 0.07 & 3.86 & 3.25 & 3.47 & 1.17 \\
\hline 3 & & ccc & measured bc & 0.56 & 0.49 & 0.55 & 0.06 & 3.63 & 3.08 & 3.56 & 1.15 \\
\hline 3 & & quenched & target & 1.01 & 0.88 & 0.90 & 0.36 & 10.17 & 7.66 & 8.01 & 2.27 \\
\hline 3 & & quenched & measured & 1.03 & 0.91 & 0.95 & 0.37 & 10.66 & 8.15 & 8.88 & 2.32 \\
\hline 3 & & quenched & measured bc & 1.00 & 0.89 & 0.96 & 0.36 & 10.03 & 7.74 & 9.10 & 2.28 \\
\hline
\end{tabular}
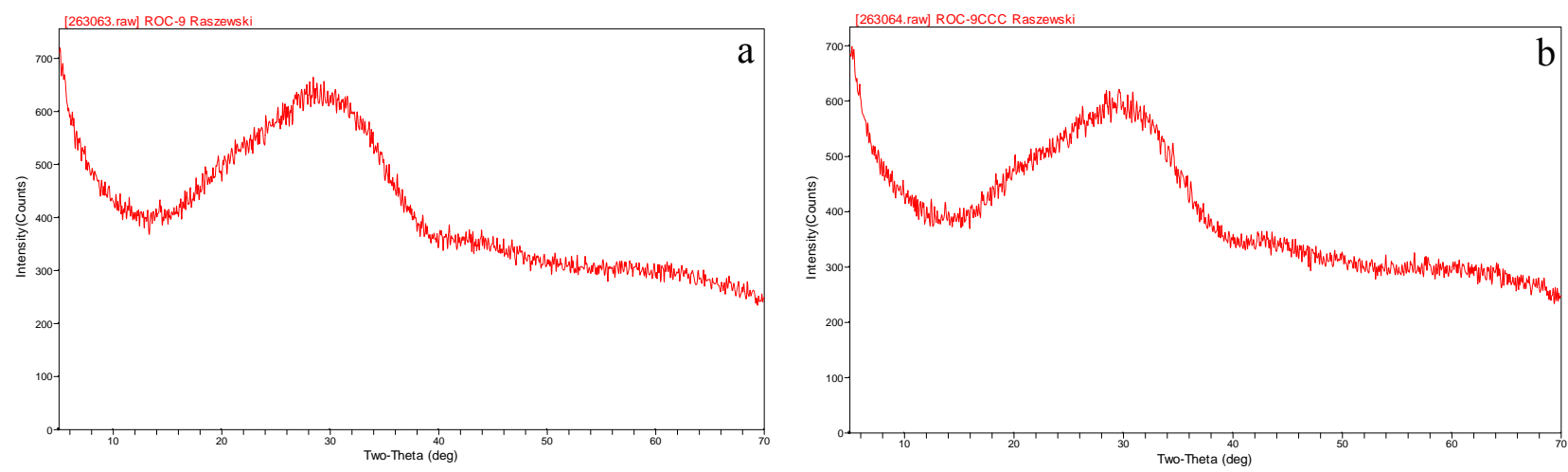

Figure 1. XRD patterns of the ROC-09 quenched (a) and cec (b) glasses.

18 It should be noted that boron releases of the quenched ROC-04, -18, -20, -22 and -28 are slightly higher than those of the ccc versions. Recognizing that there is measurement uncertainty, there is not enough variation between the quenched and ccc glasses to be of concern. 
Due to these results, ROC-09 was completely re-batched, re-melted and re-measured via XRD and PCT. The XRD results are similar to those shown in Figure 1 and the PCT results are consistent with those previously determined for ROC-09 (Table 14). ${ }^{19}$ Based on consistent results of the ROC-09 glasses (original and re-fabricated), this particular glass composition is prone to low durability (although still acceptable based on the use of the 10 $\mathrm{g} / \mathrm{L}$ guideline) even though no crystallization was detected.

Table 14. PCT Responses of Re-melted ROC-09

\begin{tabular}{|c|c|c|c|c|c|c|c|c|c|c|c|}
\hline Set & Glass ID & $\begin{array}{c}\text { Heat } \\
\text { Treatment }\end{array}$ & Comp View & $\begin{array}{c}\log \mathbf{N L} \\
{[\mathbf{B}(\mathbf{g} / \mathbf{L})]}\end{array}$ & $\begin{array}{c}\log \mathbf{N L} \\
{[\mathbf{L i}(\mathbf{g} / \mathbf{L})]}\end{array}$ & $\begin{array}{c}\log \mathbf{N L} \\
{[\mathbf{N a}(\mathbf{g} / \mathbf{L})]}\end{array}$ & $\begin{array}{c}\log \mathbf{N L} \\
{[\mathbf{S i}(\mathbf{g} / \mathbf{L})]}\end{array}$ & $\begin{array}{c}\mathbf{N L} \mathbf{B} \\
(\mathbf{g} / \mathbf{L})\end{array}$ & $\begin{array}{c}\mathbf{N L} \mathbf{L i} \\
(\mathbf{g} / \mathbf{L})\end{array}$ & $\begin{array}{c}\mathbf{N L} \mathbf{~ N a} \\
(\mathbf{g} / \mathbf{L})\end{array}$ & $\begin{array}{c}\mathbf{N L} \mathbf{S i} \\
(\mathbf{g} / \mathbf{L})\end{array}$ \\
\hline 4 & ROC-09RM & ccc & target & 0.56 & 0.47 & 0.48 & 0.02 & 3.65 & 2.94 & 3.01 & 1.04 \\
\hline 4 & ROC-09RM & quenched & target & 0.98 & 0.86 & 0.91 & 0.33 & 9.62 & 7.24 & 8.05 & 2.14 \\
\hline
\end{tabular}

\subsubsection{Predicted versus Measured PCT Results}

Exhibits B7 through B10 in Appendix B provide plots of the DWPF models for B, Li, Na, and Si that relate the logarithm of the normalized PCT result (for each element of interest) to a linear function of a free energy of hydration term $\left(\Delta \mathrm{G}_{\mathrm{p}}, \mathrm{kcal} / 100 \mathrm{~g}\right.$ glass $)$ derived for each of the glass compositional views and heat treatments. ${ }^{2}$ Prediction limits at a $95 \%$ confidence level for an individual PCT result are plotted along with the linear fit. The EA and ARM results are also indicated on these plots.

Note that there are some points that fall above the confidence limits for NL $[\mathrm{B}(\mathrm{g} / \mathrm{L})]$ as shown in Figure 2, specifically ROC-09, ROC-16 and ROC-20. Each of these glasses is unpredictable with respect to the $\Delta \mathrm{G}_{\mathrm{p}}$ model except for their target views; however, these glasses are acceptable, which demonstrates that the alumina/sum of alkali constraint prevents the production of unacceptable glasses based on coupled operations flowsheets. It is worth noting that these glasses have relatively low $\mathrm{Al}_{2} \mathrm{O}_{3}$ and $\mathrm{Fe}_{2} \mathrm{O}_{3}$ concentrations, and their poor durability behavior is consistent with similar glasses from the reduction of constraints study for sludge-only operations. Glasses at higher $\Delta \mathrm{G}_{\mathrm{p}}$ values (less negative) are also unpredictable; however, none of these glasses have a normalized boron releases greater than $2.5 \mathrm{~g} / \mathrm{L}$. There are also unpredictable glasses that fall below the confidence band around a $\Delta \mathrm{G}_{\mathrm{p}}$ value of -13 . In this region, the durability model is somewhat conservative in that it over-predicted the release rates for boron. These glasses are of no concern as the release rates of boron are very low.

\footnotetext{
${ }^{19}$ PCT responses were only normalized with respect to the target compositions as the glasses were not submitted for compositional analysis.
} 
SRNL-STI-2009-00465, REVISION 0

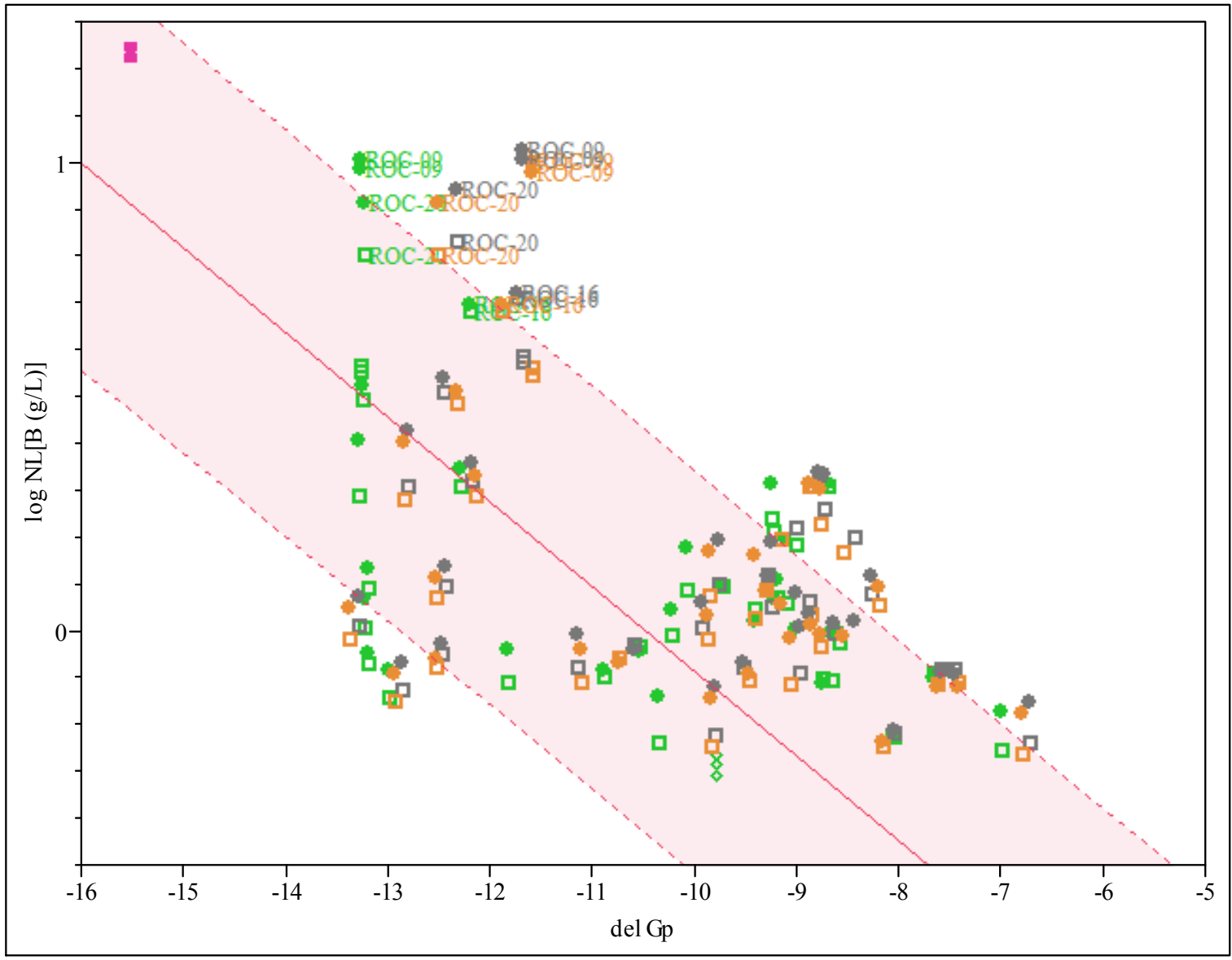

Legend

\begin{tabular}{|c|c|}
\hline Symbol & $\begin{array}{c}\text { Standard/ } \\
\text { Comp View-Heat Treatment }\end{array}$ \\
\hline $\mathrm{Z}$ & EA \\
\hline$\diamond$ & ARM \\
\hline$\square$ & Measured-ccc \\
\hline$\square$ & Measured bc -ccc \\
\hline$\square$ & Targeted-ccc \\
\hline$\bullet$ & Measured-quenched \\
\hline$\bullet$ & Measured bc - quenched \\
\hline$\bullet$ & Targeted- quenched \\
\hline
\end{tabular}

Figure 2. $\log$ NL $[B(g / L)]$ versus del $G p\left(\Delta G_{p}\right)$ model with $95 \%$ confidence interval for individual PCT measurements. 


\subsection{SUMMARY}

\subsection{CHEMICAL COMPOSITION ASSESSMENT}

Due to a significant ICP-AES calibration effect (at the 5\% significance value), biascorrection of the glass composition data was pursued. Batch 1 results were used to bias correct the $\mathrm{Al}_{2} \mathrm{O}_{3}, \mathrm{~B}_{2} \mathrm{O}_{3}, \mathrm{BaO}, \mathrm{CaO}, \mathrm{Cr}_{2} \mathrm{O}_{3}, \mathrm{CuO}, \mathrm{Fe}_{2} \mathrm{O}_{3}, \mathrm{~K}_{2} \mathrm{O}, \mathrm{Li}_{2} \mathrm{O}, \mathrm{MgO}, \mathrm{MnO}, \mathrm{Na}_{2} \mathrm{O}, \mathrm{NiO}$, $\mathrm{SiO}_{2}$, and $\mathrm{TiO}_{2}$ measurements, and the $\mathrm{U}_{\text {std }}$ values were used to bias correct the $\mathrm{U}_{3} \mathrm{O}_{8}$ measurements. Bias correction was not conducted on the $\mathrm{CdO}, \mathrm{Ce}_{2} \mathrm{O}_{3}, \mathrm{La}_{2} \mathrm{O}_{3}, \mathrm{PbO}, \mathrm{SO}_{4}$, $\mathrm{ThO}_{2}, \mathrm{ZnO}$, or $\mathrm{ZrO}_{2}$ measurements. While a bias-corrected view was included as part of these evaluations, the conclusions of the study were not sensitive to compositional views used to represent the study glasses.

A PF dissolution issue was observed for glasses ROC-01, ROC-07 and ROC-13, and a LM dissolution issue was seen in the results for glasses ROC-06, ROC-08, ROC-17, and ROC28. The measurements from the questionable dissolutions for these glasses were not used to determine the measured or measured bias-corrected values for the chemical compositions for these glasses.

In general, the measured/measured bias-corrected values, once screened for the issues identified in the previous paragraph, were consistent with the target oxide content in each of the study glasses. It should be noted that there was some scatter in the $\mathrm{SO}_{4}$ values around their intended targets. In addition, the measured $\mathrm{NiO}$ values for some of the study glasses were below the target values.

The sums of oxides (measured and bias-corrected) for the study glasses fall within the interval of 95 to $105 \mathrm{wt} \%$ except for the measured value of ROC-20; however, bias corrected measurements for this glass did have a sum of oxides above the low limit of $95 \%$. Although compositional issues were identified, none of the issues had an impact on the results or conclusions drawn from this study.

\subsection{PCT ASSESSMENT}

In general, PCT results for the ccc versions of the study glasses are relatively consistent with those of the quenched versions (within experimental error), except for ROC-09 in which the release rates of the quenched glass are considerably higher than those of the ccc glass. Due to these anomalous results, ROC-09 was re-measured. All of the re-measured values were comparable to those determined in the primary measurement. In order to confirm that the quenched and ccc glasses were not switched, both were submitted for XRD analysis. Each of the glasses was determined to be amorphous (within the $0.5 \mathrm{vol} \%$ detection limit of the instrument). As a final step, ROC-09 was completely re-batched, re-melted and re-measured via XRD and PCT. Both the XRD and PCT results are consistent with those of the original glass. Thus, there is some other factor influencing the durability of this glass and it is recommended that the influence of alumina and alkali on durability be studied in greater detail. Similar results have been observed in glasses fabricated for the sludge-only reduction of constraints task and in support of a current EM-31 task. ${ }^{20}$ The anomalous behaviors of all 
of these glasses warrant further study using advanced characterization methods in order to determine the mechanism(s) for the poor durability.

The PCT results for all of the glasses are acceptable based upon comparisons to the EA results and use of the normalized boron $10 \mathrm{~g} / \mathrm{L}$ benchmark established by Brown and Edwards in support of implementation of the homogeneity constraint. ${ }^{3}$ While the boron releases for some compositional views of ROC-09 (initial and re-measured) are slightly above the $10 \mathrm{~g} / \mathrm{L}$, the values are still well below the EA results. Even though ROC-09 had a boron release of approximately $10 \mathrm{~g} / \mathrm{L}$ and the measured composition failed the homogeneity constraint, SRNL recommends the elimination of the homogeneity constraint, which is consistent with the recommendation that was made for sludge-only processing. The use of the alumina and sum of alkali constraints should be continued along with the variability study to determine the applicability of the current durability models and/or that the glasses are acceptable with respect to durability. As with the first phase of testing for sludge-only operations, replacement of the homogeneity constraint with the alumina and sum of alkali constraints will ensure acceptable product durability over the compositional region evaluated. Although these study glasses only provide limited data in a large compositional region, the approach and results are consistent with previous studies that challenged the homogeneity constraint for sludge-only operations. That is, no added benefit is gained by implementation of the homogeneity constraint if the other PCCS constraints are protected and variability studies are continued.

Of the thirty glasses tested in this study, three glasses (ROC-09, ROC-16 and ROC-20) were determined to be unpredictable with respect to the $\Delta \mathrm{G}_{\mathrm{p}}$ model for durability; however, these glasses are acceptable, which demonstrates that the alumina/sum of alkali constraint prevents the production of unacceptable glasses based on coupled operations flowsheets. Glasses at higher $\Delta \mathrm{G}_{\mathrm{p}}$ values (less negative) are also unpredictable; however, none of these glasses have a normalized boron releases greater than $2.5 \mathrm{~g} / \mathrm{L}$. There are also unpredictable glasses that fall below the confidence band around a $\Delta G_{p}$ value of -13 . In this region, the durability model is somewhat conservative in that it over-predicted the release rates for boron. These glasses are of no concern as the release rates of boron are very low.

\subsection{RECOMMENDATIONS}

Based on the results of this study and conclusions from other SRNL glass studies, it is recommended that the homogeneity constraint (in its entirety with the associated low frit/high frit constraints) be eliminated for coupled operations (up to $2 \mathrm{wt} \% \mathrm{TiO}_{2}$ ). The variability study should be used to verify that the current durability models are applicable and/or that the glasses are acceptable with respect to durability.

Based on the anomalous results of ROC-09, it is also recommended that the influence of alumina and alkali on durability be studied in greater detail. Limited data suggests that there may be a need to adjust the lower $\mathrm{Al}_{2} \mathrm{O}_{3}$ limit and/or the upper alkali limit in order to prevent the fabrication of unacceptable glasses. An in-depth evaluation of all previous data as well as any new data would help to better define an alumina and alkali combination that would avoid potential phase separation and ensure glass durability. 


\subsection{REFERENCES}

1. C.M. Jantzen and K.G. Brown, "Predicting Phase Separation in Nuclear Watste Glasses," Ceram. Trans., 107 289-300 (2000).

2. C.M. Jantzen, J.B. Pickett, K.G. Brown, T.B. Edwards, and D.C. Beam, "Process/Product Models for the Defense Waste Processing Facility (DWPF): Part I. Predicting Glass Durability from Composition Using a Thermodynamic Hydration Energy Reaction Model (THERMO)," Westinghouse Savannah River Company, Aiken, SC, WSRC-TR-93-672, Rev. 1, 1995.

3. T.B. Edwards and K.G. Brown, "Evaluating the Glasses Batched for the Tank 42 Variability Study," Westinghouse Savannah River Company, Aiken, SC, SRT-SCS98-017, Rev. 0, 1998.

4. C.C. Herman, T.B. Edwards, D.R. Best, D.M. Marsh, and R.J. Workman, "Reduction of Constraints: Phase 2 Experimental Assessment for Sludge-Only Processing," Savannah River National Laboratory, Aiken, SC, WSRC-TR-2002-00482 Rev. 0, 2002.

5. F.C. Raszewski, T.B. Edwards, and D.K. Peeler, "Sludge Batch 5 Variability Study with Frit 418," Savannah River National Laboratory, Aiken, SC, SRNS-STI-2008$00065,2008$.

6. F.C. Raszewski, "Reduction of Constraints for Coupled Operations," Savannah River National Laboratory, Aiken, SC, SRNL-STI-2008-00529, 2008.

7. J.W. Ray, "Replacing the Homogeneity Constraint for Coupled DWPF Operations," Washington Savannah River Company, Aiken, SC, HLW-DWPF-TTR-2008-0044, 2008 .

8. D.P. Chew, M.J. Mahoney, and J.R. Vitali, "Life-Cycle Liquid Waste Disposition System Plan - Revision 14," Washington Savannah River Company, Aiken, SC, LWO-PIT-2007-00062, 2007.

9. K.G. Brown, R.L. Postles, and T.B. Edwards, "SME Acceptability Determination for DWPF Process Control," Westinghouse Savannah River Company, Aiken, SC, WSRC-TR-95-00364, Rev. 5, 2006.

10. F.C. Raszewski and M.E. Stone, "Experimental Testing to Support Additional SB4 Tank 40 Decants (TT\&QAP)," Savannah River National Laboratory, Aiken, SC, WSRC-STI-2008-00254, Rev. 0, 2008. 
11. S.L. Marra and C.M. Jantzen, "Characterization of Projected DWPF Glass Heat Treated to Simulate Canister Centerline Cooling," Westinghouse Savannah River Company, Aiken, SC, WSRC-TR-92-142, Rev. 1, 1993.

12. T.B. Edwards, "An Analytical Plan for Measuring the Chemical Compositions of the First Set of Glasses Fabricated in Support of the Reduction of Constraints Study for Coupled Operations," Savannah River National Laboratory, Aiken, SC, SRNLL5100-2009-00002, 2009.

13. T.B. Edwards, "An Analytical Plan for Measuring the Chemical Compositions of the Second Set of ROC Glasses and Three Glasses from the EM-21 FY09 Task," Savannah River National Laboratory, Aiken, SC, SRNL-L5100-2009-00003, 2009.

14. "Standard Test Methods for Determining Chemical Durability of Nuclear, Hazardous, and Mixed Waste Glasses and Multiphase Glass Ceramics: The Product Consistency Test (PCT)," ASTM International, West Conshohocken, PA, ASTM C 128502(2008), 2008.

15. C.M. Jantzen, N.E. Bibler, D.C. Beam, C.L. Crawford, and M.A. Pickett, "Characterization of the Defense Waste Processing Facility (DWPF) Environmental Assessment (EA) Glass Standard Reference Material," Westinghouse Savannah River Company, Aiken, SC, WSRC-TR-92-346, Rev. 1, 1993.

16. T.B. Edwards, "An Analytical Plan for Measuring the First Set of PCTs for Glasses Fabricated in Support of the Reduction of Constraints Study for Coupled Operations," Savannah River National Laboratory, Aiken, SC, SRNL-L5100-2009-00005, 2009.

17. T.B. Edwards, "An Analytical Plan for Measuring the Second Set of PCTs for Glasses Fabricated in Support of the Reduction of Constraints Study for Coupled Operations," Savannah River National Laboratory, Aiken, SC, SRNL-L5200-200900004, 2009.

18. T.B. Edwards, "Analytical Plans for Measuring the Chemical Compositions of the Matrix 1 Glasses Supporting the EM-21 FY09 Task," Savannah River National Laboratory, Aiken, SC, SRNL-L5200-2009-00026, 2009.

19. "Toxicity Characteristic Leaching Procedure," U.S. Environmental Protection Agency (EPA), Washington, D.C., SW-846 Method 1311, 1992.

20. F.C. Raszewski and T.B. Edwards, "Results of the FY09 Enhanced DOE High-Level Waste Melter Throughput Studies at SRNL," Savannah River National Laboratory, Aiken, SC, SRNL-STI-2009-00465, Draft. 


\section{Appendix A:}

\section{Tables and Exhibits Supporting the Analysis of the Chemical Composition Measurements of the Study Glasses}


SRNL-STI-2009-00465, Revision 0

This page intentionally left blank. 
SRNL-STI-2009-00465, Revision 0

\section{Table A1. Measured Elemental Concentrations (wt\%) for the Study Glasses Prepared Using Lithium Metaborate (part 1)}

\begin{tabular}{|c|c|c|c|c|c|c|c|c|c|c|c|c|c|c|c|}
\hline Set & Glass ID & Block & $\begin{array}{l}\text { Sub- } \\
\text { Block }\end{array}$ & Sequence & Lab ID & $\begin{array}{l}\mathrm{Ba} \\
\text { (wt\%) }\end{array}$ & $\begin{array}{l}\mathrm{Ca} \\
\text { (wt\%) }\end{array}$ & $\begin{array}{l}\mathrm{Cd} \\
\text { (wt\%) }\end{array}$ & $\begin{array}{l}\mathrm{Ce} \\
\text { (wt\%) } \\
\end{array}$ & $\begin{array}{l}\mathrm{Cr} \\
\text { (wt\%) }\end{array}$ & $\begin{array}{l}\mathrm{Cu} \\
\text { (wt\%) }\end{array}$ & $\begin{array}{l}\mathrm{K} \\
\text { (wt\%) }\end{array}$ & $\begin{array}{l}\mathrm{La} \\
\text { (wt\%) }\end{array}$ & $\begin{array}{l}\mathrm{Mg} \\
\text { (wt\%) }\end{array}$ & $\begin{array}{l}\text { Mn } \\
\text { (wt\%) }\end{array}$ \\
\hline 1 & Batch 1 & 1 & 1 & 1 & BCHLM111 & 0.133 & 0.903 & $<0.007$ & $<0.010$ & 0.072 & 0.301 & 2.68 & $<0.009$ & 0.86 & 1.32 \\
\hline 1 & Ustd & 1 & 1 & 2 & UstdLM111 & $<0.002$ & 0.95 & $<0.007$ & $<0.010$ & 0.157 & 0.011 & 2.41 & $<0.009$ & 0.73 & 2.03 \\
\hline 1 & ROC-01 & 1 & 1 & 3 & a05LM11 & 0.0234 & 0.436 & 0.075 & 0.106 & 0.118 & 0.044 & 0.065 & 0.01 & 0.86 & 4.06 \\
\hline 1 & ROC-08 & 1 & 1 & 4 & a10LM21 & $<0.002$ & 0.0227 & $<0.007$ & $<0.010$ & 0.11 & 0.015 & 0.085 & $<0.009$ & 0.85 & 4.12 \\
\hline 1 & ROC-07 & 1 & 1 & 5 & a07LM21 & $<0.002$ & 0.0199 & $<0.007$ & $<0.010$ & 0.114 & 0.008 & 0.088 & $<0.009$ & 0.01 & 0.232 \\
\hline 1 & ROC-09 & 1 & 1 & 6 & a12LM11 & 0.066 & 2.53 & 0.226 & 0.238 & $<0.010$ & 0.089 & 0.092 & 0.068 & 0.82 & 2.63 \\
\hline 1 & ROC-06 & 1 & 1 & 7 & a03LM21 & $<0.002$ & 2.21 & $<0.007$ & 0.012 & 0.102 & 0.013 & 0.05 & $<0.009$ & 0 & 3.35 \\
\hline 1 & ROC-11 & 1 & 1 & 8 & a08LM11 & 0.0724 & 0.913 & 0.238 & 0.32 & 0.112 & 0.104 & 0.052 & 0.062 & 0.14 & 4.21 \\
\hline 1 & ROC-01 & 1 & 1 & 9 & a05LM21 & 0.0234 & 0.433 & 0.072 & 0.104 & 0.121 & 0.046 & 0.06 & 0.01 & 0.86 & 4.07 \\
\hline 1 & ROC-03 & 1 & 1 & 10 & a01LM11 & 0.0722 & 0.123 & 0.236 & 0.314 & $<0.010$ & 0.104 & 0.05 & 0.063 & 0.01 & 0.383 \\
\hline 1 & Batch 1 & 1 & 1 & 11 & BCHLM121 & 0.134 & 0.893 & $<0.007$ & $<0.010$ & 0.074 & 0.299 & 2.75 & $<0.009$ & 0.86 & 1.34 \\
\hline 1 & Ustd & 1 & 1 & 12 & UstdLM121 & $<0.002$ & 0.942 & $<0.007$ & $<0.010$ & 0.159 & 0.011 & 2.47 & $<0.009$ & 0.722 & 2.05 \\
\hline 1 & ROC-07 & 1 & 1 & 13 & a07LM11 & $<0.002$ & 0.021 & $<0.007$ & $<0.010$ & 0.115 & 0.007 & $<0.050$ & $<0.009$ & 0.01 & 0.232 \\
\hline 1 & ROC-09 & 1 & 1 & 14 & a12LM21 & 0.0673 & 2.57 & 0.23 & 0.248 & $<0.010$ & 0.09 & 0.053 & 0.069 & 0.83 & 2.69 \\
\hline 1 & ROC-08 & 1 & 1 & 15 & a10LM11 & $<0.002$ & 0.0192 & $<0.007$ & $<0.010$ & 0.102 & 0.014 & $<0.050$ & $<0.009$ & 0.8 & 3.86 \\
\hline 1 & ROC-10 & 1 & 1 & 16 & a13LM11 & 0.0683 & 0.0179 & 0.224 & 0.297 & 0.104 & 0.095 & $<0.050$ & 0.058 & 0.84 & 0.936 \\
\hline 1 & ROC-10 & 1 & 1 & 17 & a13LM21 & 0.0682 & 0.0175 & 0.224 & 0.298 & 0.104 & 0.095 & $<0.050$ & 0.058 & 0.84 & 0.935 \\
\hline 1 & ROC-03 & 1 & 1 & 18 & a01LM21 & 0.0704 & 0.0292 & 0.232 & 0.306 & $<0.010$ & 0.104 & $<0.050$ & 0.075 & 0.01 & 0.359 \\
\hline 1 & ROC-06 & 1 & 1 & 19 & a03LM11 & $<0.002$ & 2.77 & $<0.007$ & 0.019 & 0.128 & 0.015 & $<0.050$ & $<0.009$ & 0 & 4.25 \\
\hline 1 & ROC-11 & 1 & 1 & 20 & a08LM21 & 0.0711 & 0.89 & 0.233 & 0.308 & 0.113 & 0.103 & $<0.050$ & 0.06 & 0.14 & 4.18 \\
\hline 1 & Batch 1 & 1 & 1 & 21 & BCHLM131 & 0.134 & 0.887 & $<0.007$ & $<0.010$ & 0.074 & 0.298 & 2.78 & $<0.009$ & 0.86 & 1.35 \\
\hline 1 & Ustd & 1 & 1 & 22 & UstdLM131 & $<0.002$ & 0.934 & $<0.007$ & $<0.010$ & 0.162 & 0.011 & 2.49 & $<0.009$ & 0.72 & 2.08 \\
\hline 1 & Batch 1 & 1 & 2 & 1 & BCHLM112 & 0.133 & 0.905 & $<0.007$ & $<0.010$ & 0.073 & 0.298 & 2.71 & $<0.009$ & 0.858 & 1.31 \\
\hline 1 & Ustd & 1 & 2 & 2 & UstdLM112 & $<0.002$ & 0.94 & $<0.007$ & $<0.010$ & 0.16 & 0.011 & 2.38 & $<0.009$ & 0.722 & 2.04 \\
\hline 1 & ROC-06 & 1 & 2 & 3 & a03LM12 & $<0.002$ & 2.76 & $<0.007$ & 0.029 & 0.127 & 0.016 & 0.078 & $<0.009$ & 0.005 & 4.17 \\
\hline 1 & ROC-01 & 1 & 2 & 4 & a05LM22 & 0.0234 & 0.433 & 0.074 & 0.115 & 0.118 & 0.047 & 0.068 & 0.01 & 0.853 & 4.04 \\
\hline 1 & ROC-10 & 1 & 2 & 5 & a13LM22 & 0.0682 & 0.0186 & 0.226 & 0.308 & 0.104 & 0.095 & 0.056 & 0.06 & 0.833 & 0.927 \\
\hline 1 & ROC-07 & 1 & 2 & 6 & a07LM22 & $<0.002$ & 0.0209 & $<0.007$ & $<0.010$ & 0.115 & 0.009 & 0.06 & $<0.009$ & 0.006 & 0.232 \\
\hline 1 & ROC-11 & 1 & 2 & 7 & a08LM22 & 0.0715 & 0.896 & 0.235 & 0.322 & 0.112 & 0.103 & $<0.050$ & 0.06 & 0.144 & 4.13 \\
\hline 1 & ROC-07 & 1 & 2 & 8 & a07LM12 & $<0.002$ & 0.022 & $<0.007$ & $<0.010$ & 0.117 & 0.008 & $<0.050$ & $<0.009$ & 0.007 & 0.231 \\
\hline 1 & ROC-03 & 1 & 2 & 9 & a01LM22 & 0.0709 & 0.0306 & 0.233 & 0.317 & $<0.010$ & 0.103 & $<0.050$ & 0.08 & 0.007 & 0.357 \\
\hline 1 & ROC-09 & 1 & 2 & 10 & a12LM12 & 0.0667 & 2.53 & 0.228 & 0.269 & $<0.010$ & 0.089 & $<0.050$ & 0.07 & 0.82 & 2.63 \\
\hline 1 & Batch 1 & 1 & 2 & 11 & BCHLM122 & 0.134 & 0.885 & $<0.007$ & $<0.010$ & 0.074 & 0.297 & 2.79 & $<0.009$ & 0.866 & 1.34 \\
\hline 1 & Ustd & 1 & 2 & 12 & UstdLM122 & $<0.002$ & 0.93 & $<0.007$ & $<0.010$ & 0.162 & 0.012 & 2.53 & $<0.009$ & 0.727 & 2.07 \\
\hline 1 & ROC-10 & 1 & 2 & 13 & a13LM12 & 0.0676 & 0.019 & 0.226 & 0.306 & 0.102 & 0.095 & $<0.050$ & 0.06 & 0.827 & 0.93 \\
\hline 1 & ROC-09 & 1 & 2 & 14 & $\begin{array}{l}\text { a12LM22 } \\
\end{array}$ & 0.0671 & 2.56 & 0.23 & 0.272 & $<0.010$ & 0.089 & $<0.050$ & 0.07 & 0.823 & 2.67 \\
\hline 1 & ROC-08 & 1 & 2 & 15 & a10LM12 & $<0.002$ & 0.0203 & $<0.007$ & $<0.010$ & 0.1 & 0.015 & $<0.050$ & $<0.009$ & 0.793 & 3.83 \\
\hline 1 & ROC-03 & 1 & 2 & 16 & a01LM12 & 0.0725 & 0.125 & 0.237 & 0.326 & $<0.010$ & 0.105 & $<0.050$ & 0.06 & 0.009 & 0.384 \\
\hline 1 & ROC-11 & 1 & 2 & 17 & a08LM12 & 0.0729 & 0.913 & 0.239 & 0.33 & 0.112 & 0.105 & $<0.050$ & 0.06 & 0.141 & 4.2 \\
\hline 1 & ROC-06 & 1 & 2 & 18 & a03LM22 & $<0.002$ & 2.21 & $<0.007$ & 0.022 & 0.102 & 0.014 & $<0.050$ & $<0.009$ & 0.005 & 3.36 \\
\hline 1 & ROC-01 & 1 & 2 & 19 & a05LM12 & 0.0236 & 0.434 & 0.076 & 0.117 & 0.119 & 0.045 & $<0.050$ & 0.01 & 0.872 & 4.16 \\
\hline 1 & ROC-08 & 1 & 2 & 20 & a10LM22 & $<0.002$ & 0.0246 & $<0.007$ & $<0.010$ & 0.11 & 0.017 & $<0.050$ & $<0.009$ & 0.857 & 4.18 \\
\hline 1 & Batch 1 & 1 & 2 & 21 & BCHLM132 & 0.133 & 0.879 & $<0.007$ & $<0.010$ & 0.0737 & 0.296 & 2.83 & $<0.009$ & 0.866 & 1.34 \\
\hline 1 & Ustd & 1 & 2 & 22 & UstdLM132 & $<0.002$ & 0.929 & $<0.007$ & $<0.010$ & 0.161 & 0.0115 & 2.5 & $<0.009$ & 0.724 & 2.07 \\
\hline 1 & Batch 1 & 2 & 1 & 1 & BCHLM211 & 0.133 & 0.903 & $<0.007$ & $<0.010$ & 0.072 & 0.297 & 2.66 & $<0.009$ & 0.859 & 1.31 \\
\hline 1 & Ustd & 2 & 1 & 2 & UstdLM211 & $<0.002$ & 0.951 & $<0.007$ & $<0.010$ & 0.16 & 0.011 & 2.37 & $<0.009$ & 0.713 & 2.04 \\
\hline 1 & ROC-13 & 2 & 1 & 3 & a14LM21 & $<0.002$ & 0.91 & $<0.007$ & 0.022 & $<0.010$ & 0.012 & $<0.050$ & $<0.009$ & 0.817 & 3.91 \\
\hline 1 & ROC-12 & 2 & 1 & 4 & a06LM21 & $<0.002$ & 2.69 & $<0.007$ & $<0.010$ & $<0.010$ & 0.01 & $<0.050$ & $<0.009$ & 0.876 & 0.235 \\
\hline 1 & ROC-05 & 2 & 1 & 5 & a11LM21 & $<0.002$ & 0.102 & $<0.007$ & 0.028 & $<0.010$ & 0.018 & $<0.050$ & $<0.009$ & 0.897 & 4.24 \\
\hline 1 & ROC-02 & 2 & 1 & 6 & a09LM11 & $<0.002$ & 2.73 & $<0.007$ & $<0.010$ & 0.107 & 0.013 & $<0.050$ & $<0.009$ & 0.792 & 0.234 \\
\hline 1 & ROC-13 & 2 & 1 & 7 & a14LM11 & $<0.002$ & 0.902 & $<0.007$ & 0.021 & $<0.010$ & 0.013 & $<0.050$ & $<0.009$ & 0.825 & 3.96 \\
\hline 1 & ROC-05 & 2 & 1 & 8 & a11LM11 & $<0.002$ & 0.1 & $<0.007$ & 0.027 & $<0.010$ & 0.019 & $<0.050$ & $<0.009$ & 0.874 & 4.11 \\
\hline 1 & ROC-14 & 2 & 1 & 9 & a04LM21 & 0.0713 & 0.0433 & 0.236 & 0.284 & $<0.010$ & 0.1 & $<0.050$ & 0.06 & 0.867 & 3.15 \\
\hline 1 & Batch 1 & 2 & 1 & 10 & BCHLM221 & 0.134 & 0.881 & $<0.007$ & $<0.010$ & 0.074 & 0.294 & 2.75 & $<0.009$ & 0.852 & 1.33 \\
\hline 1 & Ustd & 2 & 1 & 11 & UstdLM221 & $<0.002$ & 0.932 & $<0.007$ & $<0.010$ & 0.163 & 0.012 & 2.44 & $<0.009$ & 0.718 & 2.05 \\
\hline 1 & ROC-04 & 2 & 1 & 12 & a02LM21 & 0.0679 & 2.71 & 0.226 & 0.32 & $<0.010$ & 0.101 & $<0.050$ & 0.05 & 0.006 & 0.271 \\
\hline 1 & ROC-14 & 2 & 1 & 13 & $\begin{array}{l}\text { a04LM11 } \\
\end{array}$ & 0.0703 & 0.0357 & 0.234 & 0.285 & $<0.010$ & 0.102 & $<0.050$ & 0.06 & 0.851 & 3.07 \\
\hline 1 & ROC-12 & 2 & 1 & 14 & a06LM11 & $<0.002$ & 2.66 & $<0.007$ & $<0.010$ & $<0.010$ & 0.01 & $<0.050$ & $<0.009$ & 0.865 & 0.233 \\
\hline 1 & ROC-15 & 2 & 1 & 15 & a15LM21 & 0.0683 & 0.0208 & 0.225 & 0.309 & 0.113 & 0.098 & $<0.050$ & 0.05 & 0.008 & 0.768 \\
\hline 1 & ROC-04 & 2 & 1 & 16 & a02LM11 & 0.0694 & 2.7 & 0.228 & 0.326 & $<0.010$ & 0.102 & $<0.050$ & 0.06 & 0.006 & 0.224 \\
\hline 1 & ROC-15 & 2 & 1 & 17 & a15LM11 & 0.0686 & 0.0224 & 0.226 & 0.308 & 0.11 & 0.097 & $<0.050$ & 0.05 & 0.008 & 0.767 \\
\hline 1 & ROC-02 & 2 & 1 & 18 & a09LM21 & $<0.002$ & 2.67 & $<0.007$ & $<0.010$ & 0.106 & 0.012 & $<0.050$ & $<0.009$ & 0.781 & 0.23 \\
\hline 1 & Batch 1 & 2 & 1 & 19 & BCHLM231 & 0.134 & 0.882 & $<0.007$ & $<0.010$ & 0.074 & 0.295 & 2.74 & $<0.009$ & 0.855 & 1.33 \\
\hline 1 & Ustd & 2 & 1 & 20 & UstdLM231 & $<0.002$ & 0.926 & $<0.007$ & $<0.010$ & 0.162 & 0.011 & 2.46 & $<0.009$ & 0.718 & 2.04 \\
\hline 1 & Batch 1 & 2 & 2 & 1 & BCHLM212 & 0.138 & 0.92 & $<0.007$ & $<0.010$ & 0.075 & 0.301 & 2.69 & $<0.009$ & 0.852 & 1.33 \\
\hline 1 & Ustd & 2 & 2 & 2 & UstdLM212 & $<0.002$ & 0.968 & $<0.007$ & $<0.010$ & 0.165 & 0.013 & 2.39 & $<0.009$ & 0.715 & 2.07 \\
\hline 1 & ROC-04 & 2 & 2 & 3 & a02LM12 & 0.0708 & 2.69 & 0.225 & 0.335 & $<0.010$ & 0.105 & 0.058 & 0.06 & 0.006 & 0.223 \\
\hline 1 & ROC-14 & 2 & 2 & 4 & a04LM12 & 0.0724 & 0.0366 & 0.231 & 0.288 & $<0.010$ & 0.105 & 0.062 & 0.06 & 0.877 & 3.1 \\
\hline 1 & ROC-02 & 2 & 2 & 5 & a09LM12 & $<0.002$ & 2.73 & $<0.007$ & $<0.010$ & 0.109 & 0.014 & 0.067 & $<0.009$ & 0.816 & 0.237 \\
\hline 1 & ROC-13 & 2 & 2 & 6 & a14LM12 & $<0.002$ & 0.935 & $<0.007$ & 0.025 & $<0.010$ & 0.014 & 0.068 & $<0.009$ & 0.85 & 3.98 \\
\hline
\end{tabular}


SRNL-STI-2009-00465, Revision 0

\section{Table A1. Measured Elemental Concentrations (wt\%) for the Study Glasses Prepared Using Lithium Metaborate (part 1)}

\begin{tabular}{|c|c|c|c|c|c|c|c|c|c|c|c|c|c|c|c|}
\hline Set & Glass ID & Block & $\begin{array}{l}\text { Sub- } \\
\text { Block }\end{array}$ & Sequence & Lab ID & $\begin{array}{l}\mathrm{Ba} \\
\text { (wt\%) }\end{array}$ & $\begin{array}{l}\mathrm{Ca} \\
\text { (wt\%) }\end{array}$ & $\begin{array}{l}\mathrm{Cd} \\
\text { (wt\%) }\end{array}$ & $\begin{array}{l}\mathrm{Ce} \\
\text { (wt\%) } \\
\end{array}$ & $\begin{array}{l}\mathrm{Cr} \\
\text { (wt\%) }\end{array}$ & $\begin{array}{l}\mathrm{Cu} \\
\text { (wt\%) }\end{array}$ & $\begin{array}{l}\mathrm{K} \\
\text { (wt\%) }\end{array}$ & $\begin{array}{l}\mathrm{La} \\
\text { (wt\%) }\end{array}$ & $\begin{array}{l}\mathrm{Mg} \\
\text { (wt\%) }\end{array}$ & $\begin{array}{l}\text { Mn } \\
\text { (wt\%) }\end{array}$ \\
\hline 1 & ROC-05 & 2 & 2 & 7 & a11LM22 & $<0.002$ & 0.104 & $<0.007$ & 0.033 & $<0.010$ & 0.019 & 0.052 & $<0.009$ & 0.922 & 4.29 \\
\hline 1 & ROC-14 & 2 & 2 & 8 & a04LM22 & 0.0734 & 0.0449 & 0.235 & 0.291 & $<0.010$ & 0.103 & $<0.050$ & 0.06 & 0.893 & 3.17 \\
\hline 1 & ROC-12 & 2 & 2 & 9 & a06LM22 & $<0.002$ & 2.7 & $<0.007$ & $<0.010$ & $<0.010$ & 0.011 & 0.055 & $<0.009$ & 0.933 & 0.246 \\
\hline 1 & Batch 1 & 2 & 2 & 10 & BCHLM222 & 0.138 & 0.907 & $<0.007$ & $<0.010$ & 0.076 & 0.299 & 2.68 & $<0.009$ & 0.853 & 1.34 \\
\hline 1 & Ustd & 2 & 2 & 11 & UstdLM222 & $<0.002$ & 0.954 & $<0.007$ & $<0.010$ & 0.165 & 0.013 & 2.47 & $<0.009$ & 0.721 & 2.07 \\
\hline 1 & ROC-13 & 2 & 2 & 12 & a14LM22 & $<0.002$ & 0.935 & $<0.007$ & 0.025 & $<0.010$ & 0.013 & $<0.050$ & $<0.009$ & 0.848 & 3.97 \\
\hline 1 & ROC-04 & 2 & 2 & 13 & a02LM22 & 0.0685 & 2.71 & 0.224 & 0.321 & $<0.010$ & 0.102 & $<0.050$ & 0.05 & 0.006 & 0.268 \\
\hline 1 & ROC-02 & 2 & 2 & 14 & a09LM22 & $<0.002$ & 2.66 & $<0.007$ & $<0.010$ & 0.109 & 0.013 & $<0.050$ & $<0.009$ & 0.807 & 0.234 \\
\hline 1 & ROC-15 & 2 & 2 & 15 & a15LM22 & 0.0703 & 0.0214 & 0.223 & 0.315 & 0.117 & 0.102 & $<0.050$ & 0.05 & 0.008 & 0.78 \\
\hline 1 & ROC-12 & 2 & 2 & 16 & a06LM12 & $<0.002$ & 2.68 & $<0.007$ & $<0.010$ & $<0.010$ & 0.011 & $<0.050$ & $<0.009$ & 0.903 & 0.238 \\
\hline 1 & ROC-15 & 2 & 2 & 17 & a15LM12 & 0.0706 & 0.0229 & 0.226 & 0.318 & 0.114 & 0.1 & $<0.050$ & 0.06 & 0.009 & 0.782 \\
\hline 1 & ROC-05 & 2 & 2 & 18 & a11LM12 & $<0.002$ & 0.104 & $<0.007$ & 0.031 & $<0.010$ & 0.02 & $<0.050$ & $<0.009$ & 0.912 & 4.2 \\
\hline 1 & Batch 1 & 2 & 2 & 19 & BCHLM232 & 0.138 & 0.903 & $<0.007$ & $<0.010$ & 0.076 & 0.3 & 2.71 & $<0.009$ & 0.858 & 1.35 \\
\hline 1 & Ustd & 2 & 2 & 20 & UstdLM232 & $<0.002$ & 0.95 & $<0.007$ & $<0.010$ & 0.166 & 0.013 & 2.4 & $<0.009$ & 0.722 & 2.09 \\
\hline 2 & Batch 1 & 1 & 1 & 1 & BCHLM111 & 0.133 & 0.887 & $<0.007$ & $<0.020$ & 0.072 & 0.307 & 2.61 & $<0.009$ & 0.851 & 1.29 \\
\hline 2 & Ustd 1 & 1 & 1 & 2 & UstdLM111 & $<0.002$ & 0.922 & $<0.007$ & $<0.020$ & 0.164 & 0.008 & 2.31 & $<0.009$ & 0.718 & 2.08 \\
\hline 2 & ROC-16 & 1 & 1 & 3 & b05LM11 & 0.07 & 0.014 & 0.277 & 0.28 & 0.02 & 0.112 & $<0.050$ & 0.0518 & 0.009 & 3.98 \\
\hline 2 & ROC-23 & 1 & 1 & 4 & b10LM21 & 0.077 & $<0.010$ & 0.227 & 0.26 & 0.093 & 0.11 & $<0.050$ & 0.0545 & 0.813 & 1.35 \\
\hline 2 & ROC-22 & 1 & 1 & 5 & b07LM21 & $<0.002$ & 0.012 & $<0.007$ & $<0.020$ & 0.023 & 0.009 & $<0.050$ & $<0.009$ & 0.005 & 4.2 \\
\hline 2 & ROC-25 & 1 & 1 & 6 & b12LM11 & 0.071 & 0.019 & 0.233 & 0.266 & 0.123 & 0.107 & $<0.050$ & 0.0634 & 0.857 & 3.95 \\
\hline 2 & ROC-21 & 1 & 1 & 7 & b03LM21 & $<0.002$ & 0.068 & $<0.007$ & 0.026 & 0.108 & 0.016 & $<0.050$ & $<0.009$ & 0.652 & 4.03 \\
\hline 2 & ROC-28 & 1 & 1 & 8 & b08LM11 & 0.069 & $<0.010$ & 0.233 & 0.269 & 0.011 & 0.11 & $<0.050$ & 0.0599 & 0.89 & 0.37 \\
\hline 2 & ROC-16 & 1 & 1 & 9 & b05LM21 & 0.07 & 0.014 & 0.279 & 0.281 & 0.018 & 0.108 & $<0.050$ & 0.0514 & 0.011 & 4 \\
\hline 2 & ROC-18 & 1 & 1 & 10 & b01LM11 & $<0.002$ & 1.44 & $<0.007$ & $<0.020$ & 0.024 & 0.012 & $<0.050$ & $<0.009$ & 0.011 & 3.64 \\
\hline 2 & FY09EM21-30 & 1 & 1 & 11 & b18LM11 & 0.038 & 1.17 & 0.12 & 0.147 & 0.058 & 0.068 & $<0.050$ & 0.0233 & 0.381 & 1.78 \\
\hline 2 & Batch 1 & 1 & 1 & 12 & BCHLM121 & 0.133 & 0.865 & $<0.007$ & $<0.020$ & 0.073 & 0.311 & 2.73 & $<0.009$ & 0.85 & 1.29 \\
\hline 2 & Ustd 1 & 1 & 1 & 13 & UstdLM121 & $<0.002$ & 0.919 & $<0.007$ & $<0.020$ & 0.16 & 0.009 & 2.4 & $<0.009$ & 0.712 & 2.09 \\
\hline 2 & ROC-22 & 1 & 1 & 14 & b07LM11 & $<0.002$ & 0.013 & $<0.007$ & $<0.020$ & 0.024 & 0.01 & $<0.050$ & $<0.009$ & 0.006 & 4.29 \\
\hline 2 & ROC-25 & 1 & 1 & 15 & b12LM21 & 0.071 & 0.019 & 0.234 & 0.262 & 0.122 & 0.109 & $<0.050$ & 0.0627 & 0.858 & 3.94 \\
\hline 2 & ROC-23 & 1 & 1 & 16 & b10LM11 & 0.08 & 0.011 & 0.235 & 0.262 & 0.094 & 0.111 & $<0.050$ & 0.0556 & 0.829 & 1.39 \\
\hline 2 & ROC-27 & 1 & 1 & 17 & b13LM11 & 0.072 & 0.01 & 0.24 & 0.273 & 0.025 & 0.112 & $<0.050$ & 0.0625 & 0.881 & 3.96 \\
\hline 2 & ROC-27 & 1 & 1 & 18 & b13LM21 & 0.073 & 0.01 & 0.242 & 0.276 & 0.026 & 0.116 & $<0.050$ & 0.0641 & 0.889 & 4.05 \\
\hline 2 & ROC-18 & 1 & 1 & 19 & b01LM21 & $<0.002$ & 1.51 & $<0.007$ & $<0.020$ & 0.022 & 0.012 & $<0.050$ & $<0.009$ & 0.008 & 3.74 \\
\hline 2 & ROC-21 & 1 & 1 & 20 & b03LM11 & $<0.002$ & 0.067 & $<0.007$ & 0.025 & 0.109 & 0.016 & $<0.050$ & $<0.009$ & 0.638 & 3.98 \\
\hline 2 & ROC-28 & 1 & 1 & 21 & b08LM21 & 0.064 & 0.018 & 0.221 & 0.251 & 0.012 & 0.104 & $<0.050$ & 0.0561 & 0.837 & 0.35 \\
\hline 2 & FY09EM21-30 & 1 & 1 & 22 & b18LM21 & 0.039 & 1.2 & 0.127 & 0.149 & 0.061 & 0.067 & $<0.050$ & 0.0306 & 0.393 & 1.84 \\
\hline 2 & Batch 1 & 1 & 1 & 23 & BCHLM131 & 0.134 & 0.863 & $<0.007$ & $<0.020$ & 0.075 & 0.315 & 2.88 & $<0.009$ & 0.85 & 1.31 \\
\hline 2 & Ustd 1 & 1 & 1 & 24 & UstdLM131 & $<0.002$ & 0.918 & $<0.007$ & $<0.020$ & 0.163 & 0.01 & 2.42 & $<0.009$ & 0.713 & 2.1 \\
\hline 2 & Batch 1 & 1 & 2 & 1 & BCHLM112 & 0.133 & 0.874 & $<0.007$ & $<0.020$ & 0.072 & 0.305 & 2.69 & $<0.009$ & 0.86 & 1.31 \\
\hline 2 & Ustd 1 & 1 & 2 & 2 & UstdLM112 & $<0.002$ & 0.891 & $<0.007$ & $<0.020$ & 0.162 & 0.008 & 2.36 & $<0.009$ & 0.724 & 2.12 \\
\hline 2 & ROC-21 & 1 & 2 & 3 & b03LM12 & $<0.002$ & 0.065 & $<0.007$ & 0.03 & 0.105 & 0.015 & $<0.050$ & $<0.009$ & 0.648 & 4.02 \\
\hline 2 & ROC-16 & 1 & 2 & 4 & b05LM22 & 0.069 & 0.012 & 0.275 & 0.286 & 0.018 & 0.105 & 0.061 & 0.0517 & 0.011 & 4.06 \\
\hline 2 & ROC-27 & 1 & 2 & 5 & b13LM22 & 0.073 & $<0.010$ & 0.239 & 0.285 & 0.025 & 0.109 & 0.062 & 0.0642 & 0.902 & 4.09 \\
\hline 2 & ROC-22 & 1 & 2 & 6 & b07LM22 & $<0.002$ & $<0.010$ & $<0.007$ & $<0.020$ & 0.022 & 0.009 & 0.067 & $<0.009$ & 0.005 & 4.27 \\
\hline 2 & ROC-28 & 1 & 2 & 7 & b08LM22 & 0.065 & 0.015 & 0.217 & 0.258 & 0.011 & 0.094 & $<0.050$ & 0.057 & 0.846 & 0.35 \\
\hline 2 & ROC-22 & 1 & 2 & 8 & b07LM12 & $<0.002$ & 0.011 & $<0.007$ & $<0.020$ & 0.023 & 0.01 & $<0.050$ & $<0.009$ & 0.006 & 4.33 \\
\hline 2 & ROC-18 & 1 & 2 & 9 & b01LM22 & $<0.002$ & 1.51 & $<0.007$ & $<0.020$ & 0.021 & 0.011 & $<0.050$ & $<0.009$ & 0.007 & 3.8 \\
\hline 2 & ROC-25 & 1 & 2 & 10 & b12LM12 & 0.071 & 0.017 & 0.234 & 0.274 & 0.121 & 0.103 & $<0.050$ & 0.0641 & 0.863 & 4.02 \\
\hline 2 & FY09EM21-30 & 1 & 2 & 11 & b18LM22 & 0.038 & 1.18 & 0.124 & 0.157 & 0.059 & 0.063 & $<0.050$ & 0.0313 & 0.395 & 1.86 \\
\hline 2 & Batch 1 & 1 & 2 & 12 & BCHLM122 & 0.133 & 0.832 & $<0.007$ & $<0.020$ & 0.072 & 0.306 & 2.83 & $<0.009$ & 0.862 & 1.32 \\
\hline 2 & Ustd 1 & 1 & 2 & 13 & UstdLM122 & $<0.002$ & 0.884 & $<0.007$ & $<0.020$ & 0.164 & 0.008 & 2.58 & $<0.009$ & 0.723 & 2.13 \\
\hline 2 & ROC-27 & 1 & 2 & 14 & b13LM12 & 0.071 & $<0.010$ & 0.236 & 0.282 & 0.024 & 0.103 & $<0.050$ & 0.0636 & 0.889 & 4.02 \\
\hline 2 & ROC-25 & 1 & 2 & 15 & b12LM22 & 0.07 & 0.017 & 0.231 & 0.269 & 0.12 & 0.104 & $<0.050$ & 0.0634 & 0.871 & 4.02 \\
\hline 2 & ROC-23 & 1 & 2 & 16 & b10LM12 & 0.078 & $<0.010$ & 0.231 & 0.273 & 0.093 & 0.108 & $<0.050$ & 0.0568 & 0.841 & 1.41 \\
\hline 2 & ROC-18 & 1 & 2 & 17 & b01LM12 & $<0.002$ & 1.43 & $<0.007$ & $<0.020$ & 0.023 & 0.011 & $<0.050$ & $<0.009$ & 0.011 & 3.72 \\
\hline 2 & ROC-28 & 1 & 2 & 18 & b08LM12 & 0.069 & $<0.010$ & 0.231 & 0.274 & 0.011 & 0.105 & $<0.050$ & 0.0603 & 0.901 & 0.37 \\
\hline 2 & ROC-21 & 1 & 2 & 19 & b03LM22 & $<0.002$ & 0.065 & $<0.007$ & 0.029 & 0.107 & 0.016 & $<0.050$ & $<0.009$ & 0.658 & 4.1 \\
\hline 2 & ROC-16 & 1 & 2 & 20 & b05LM12 & 0.069 & 0.011 & 0.275 & 0.287 & 0.02 & 0.104 & $<0.050$ & 0.0523 & 0.009 & 4.06 \\
\hline 2 & ROC-23 & 1 & 2 & 21 & b10LM22 & 0.077 & $<0.010$ & 0.227 & 0.265 & 0.0931 & 0.103 & $<0.050$ & 0.0554 & 0.823 & 1.37 \\
\hline 2 & FY09EM21-30 & 1 & 2 & 22 & b18LM12 & 0.038 & 1.15 & 0.119 & 0.148 & 0.0573 & 0.059 & $<0.050$ & 0.0235 & 0.385 & 1.8 \\
\hline 2 & Batch 1 & 1 & 2 & 23 & BCHLM132 & 0.133 & 0.828 & $<0.007$ & $<0.020$ & 0.0727 & 0.303 & 2.68 & $<0.009$ & 0.863 & 1.31 \\
\hline 2 & Ustd 1 & 1 & 2 & 24 & UstdLM132 & $<0.002$ & 0.886 & $<0.007$ & $<0.020$ & 0.161 & 0.009 & 2.42 & $<0.009$ & 0.724 & 2.12 \\
\hline 2 & Batch 1 & 2 & 1 & 1 & BCHLM211 & 0.132 & 0.88 & $<0.007$ & $<0.020$ & 0.071 & 0.313 & 2.76 & $<0.009$ & 0.853 & 1.29 \\
\hline 2 & Ustd 1 & 2 & 1 & 2 & UstdLM211 & $<0.002$ & 0.94 & $<0.007$ & $<0.020$ & 0.167 & 0.011 & 2.5 & $<0.009$ & 0.721 & 2.09 \\
\hline 2 & ROC-30 & 2 & 1 & 3 & b14LM21 & 0.038 & 0.755 & 0.126 & 0.176 & 0.064 & 0.063 & 0.064 & 0.03 & 0.503 & 2.7 \\
\hline 2 & ROC-29 & 2 & 1 & 4 & b06LM21 & 0.069 & 0.056 & 0.219 & 0.313 & 0.02 & 0.109 & 0.059 & 0.061 & 0.006 & 3.63 \\
\hline 2 & ROC-20 & 2 & 1 & 5 & b11LM21 & 0.07 & 2.7 & 0.228 & 0.283 & 0.13 & 0.095 & $<0.050$ & 0.063 & 0.219 & 3.88 \\
\hline 2 & ROC-17 & 2 & 1 & 6 & b09LM11 & $<0.002$ & 0.111 & $<0.007$ & $<0.020$ & 0.103 & 0.008 & $<0.050$ & $<0.009$ & 0.006 & 3.77 \\
\hline 2 & ROC-30 & 2 & 1 & 7 & b14LM11 & 0.039 & 0.762 & 0.129 & 0.175 & 0.065 & 0.064 & $<0.050$ & 0.03 & 0.512 & 2.74 \\
\hline 2 & ROC-20 & 2 & 1 & 8 & b11LM11 & 0.07 & 2.66 & 0.227 & 0.273 & 0.128 & 0.097 & $<0.050$ & 0.061 & 0.215 & 3.84 \\
\hline
\end{tabular}


SRNL-STI-2009-00465, Revision 0

Table A1. Measured Elemental Concentrations (wt\%) for the Study Glasses Prepared Using Lithium Metaborate (part 1)

\begin{tabular}{|c|c|c|c|c|c|c|c|c|c|c|c|c|c|c|c|}
\hline Set & Glass ID & Block & $\begin{array}{l}\text { Sub- } \\
\text { Block }\end{array}$ & Sequence & Lab ID & $\begin{array}{l}\mathrm{Ba} \\
(\mathrm{wt} \%)\end{array}$ & $\begin{array}{l}\mathrm{Ca} \\
\text { (wt\%) }\end{array}$ & $\begin{array}{l}\text { Cd } \\
\text { (wt\%) }\end{array}$ & $\begin{array}{l}\mathrm{Ce} \\
\text { (wt\%) }\end{array}$ & $\begin{array}{l}\mathrm{Cr} \\
(\mathrm{wt} \%)\end{array}$ & $\begin{array}{l}\mathrm{Cu} \\
\text { (wt\%) }\end{array}$ & $\begin{array}{l}\text { K } \\
\text { (wt\%) }\end{array}$ & $\begin{array}{l}\mathrm{La} \\
(\mathrm{wt} \%)\end{array}$ & $\begin{array}{l}\mathrm{Mg} \\
(\mathrm{wt} \%)\end{array}$ & $\begin{array}{l}\text { Mn } \\
\text { (wt\%) }\end{array}$ \\
\hline 2 & FY09EM21-28 & 2 & 1 & 9 & b04LM21 & 0.039 & 1.29 & 0.121 & 0.157 & 0.068 & 0.065 & $<0.050$ & 0.035 & 0.412 & 1.91 \\
\hline 2 & ROC-26 & 2 & 1 & 10 & b17LM21 & $<0.002$ & 0.01 & $<0.007$ & $<0.020$ & 0.015 & 0.022 & $<0.050$ & $<0.009$ & 0.005 & 2.52 \\
\hline 2 & ROC-24 & 2 & 1 & 11 & b16LM11 & 0.073 & $<0.010$ & 0.237 & 0.303 & 0.107 & 0.111 & $<0.050$ & 0.066 & 0.886 & 4.07 \\
\hline 2 & Batch 1 & 2 & 1 & 12 & BCHLM221 & 0.131 & 0.862 & $<0.007$ & $<0.020$ & 0.073 & 0.314 & 2.72 & $<0.009$ & 0.849 & 1.28 \\
\hline 2 & Ustd 1 & 2 & 1 & 13 & UstdLM221 & $<0.002$ & 0.916 & $<0.007$ & $<0.020$ & 0.168 & 0.014 & 2.41 & $<0.009$ & 0.716 & 2.07 \\
\hline 2 & ROC-19 & 2 & 1 & 14 & b02LM21 & $<0.002$ & 0.014 & $<0.007$ & $<0.020$ & 0.016 & 0.017 & $<0.050$ & $<0.009$ & 0.886 & 0.239 \\
\hline 2 & FY09EM21-28 & 2 & 1 & 15 & b04LM11 & 0.035 & 1.16 & 0.11 & 0.147 & 0.064 & 0.055 & $<0.050$ & 0.033 & 0.374 & 1.72 \\
\hline 2 & ROC-29 & 2 & 1 & 16 & b06LM11 & 0.069 & 0.053 & 0.221 & 0.314 & 0.02 & 0.108 & $<0.050$ & 0.061 & 0.006 & 3.61 \\
\hline 2 & FY09EM21-29 & 2 & 1 & 17 & b15LM21 & 0.039 & 1.24 & 0.129 & 0.159 & 0.062 & 0.062 & $<0.050$ & 0.037 & 0.397 & 1.83 \\
\hline 2 & ROC-19 & 2 & 1 & 18 & b02LM11 & $<0.002$ & 0.013 & $<0.007$ & $<0.020$ & 0.018 & 0.018 & $<0.050$ & $\begin{array}{l}<0.009 \\
\end{array}$ & 0.915 & 0.234 \\
\hline 2 & FY09EM21-29 & 2 & 1 & 19 & b15LM11 & 0.04 & 1.25 & 0.131 & 0.162 & 0.064 & 0.069 & $<0.050$ & 0.038 & 0.4 & 1.84 \\
\hline 2 & ROC-17 & 2 & 1 & 20 & b09LM21 & $<0.002$ & 0.12 & $<0.007$ & $<0.020$ & 0.116 & 0.012 & $<0.050$ & $<0.009$ & 0.012 & 4.09 \\
\hline 2 & ROC-24 & 2 & 1 & 21 & b16LM21 & 0.073 & $<0.010$ & 0.239 & 0.293 & 0.109 & 0.114 & $<0.050$ & 0.067 & 0.884 & 4.06 \\
\hline 2 & ROC-26 & 2 & 1 & 22 & b17LM11 & $<0.002$ & 0.012 & $<0.007$ & $<0.020$ & 0.016 & 0.021 & $<0.050$ & $<0.009$ & 0.005 & 2.47 \\
\hline 2 & Batch 1 & 2 & 1 & 23 & BCHLM231 & 0.132 & 0.855 & $<0.007$ & $<0.020$ & 0.074 & 0.323 & 2.6 & $<0.009$ & 0.857 & 1.28 \\
\hline 2 & Ustd 1 & 2 & 1 & 24 & UstdLM231 & $<0.002$ & 0.911 & $<0.007$ & $<0.020$ & 0.168 & 0.016 & 2.27 & $<0.009$ & 0.721 & 2.08 \\
\hline 2 & Batch 1 & 2 & 2 & 1 & BCHLM212 & 0.132 & 0.875 & $<0.007$ & $<0.020$ & 0.072 & 0.307 & 2.79 & $<0.009$ & 0.852 & 1.3 \\
\hline 2 & Ustd 1 & 2 & 2 & 2 & UstdLM212 & $<0.002$ & 0.936 & $<0.007$ & $<0.020$ & 0.166 & 0.012 & 2.47 & $<0.009$ & 0.717 & 2.11 \\
\hline 2 & ROC-19 & 2 & 2 & 3 & b02LM12 & $<0.002$ & 0.014 & $<0.007$ & $<0.020$ & 0.018 & 0.016 & 0.081 & $<0.009$ & 0.898 & 0.234 \\
\hline 2 & FY09EM21-28 & 2 & 2 & 4 & b04LM12 & 0.035 & 1.16 & 0.106 & 0.141 & 0.065 & 0.062 & 0.057 & 0.033 & 0.369 & 1.73 \\
\hline 2 & ROC-17 & 2 & 2 & 5 & b09LM12 & $<0.002$ & 0.112 & $<0.007$ & $<0.020$ & 0.106 & 0.008 & $<0.050$ & $<0.009$ & 0.007 & 3.81 \\
\hline 2 & ROC-30 & 2 & 2 & 6 & b14LM12 & 0.038 & 0.76 & 0.126 & 0.17 & 0.067 & 0.062 & $<0.050$ & 0.031 & 0.509 & 2.76 \\
\hline 2 & ROC-20 & 2 & 2 & 7 & b11LM22 & 0.07 & 2.71 & 0.225 & 0.279 & 0.135 & 0.097 & $<0.050$ & 0.063 & 0.218 & 3.92 \\
\hline 2 & FY09EM21-28 & 2 & 2 & 8 & b04LM22 & 0.038 & 1.29 & 0.118 & 0.155 & 0.071 & 0.07 & $<0.050$ & 0.036 & 0.411 & 1.93 \\
\hline 2 & ROC-29 & 2 & 2 & 9 & b06LM22 & 0.069 & 0.056 & 0.216 & 0.304 & 0.021 & 0.111 & $<0.050$ & 0.061 & 0.007 & 3.68 \\
\hline 2 & ROC-24 & 2 & 2 & 10 & b16LM22 & 0.073 & 0.01 & 0.229 & 0.296 & 0.11 & 0.111 & $<0.050$ & 0.066 & 0.87 & 4.08 \\
\hline 2 & ROC-26 & 2 & 2 & 11 & b17LM12 & $<0.002$ & 0.013 & $<0.007$ & $<0.020$ & 0.016 & 0.022 & $<0.050$ & $<0.009$ & 0.005 & 2.47 \\
\hline 2 & Batch 1 & 2 & 2 & 12 & BCHLM222 & 0.131 & 0.861 & $<0.007$ & $<0.020$ & 0.076 & 0.31 & 2.6 & $<0.009$ & 0.842 & 1.29 \\
\hline 2 & Ustd 1 & 2 & 2 & 13 & UstdLM222 & $<0.002$ & 0.907 & $<0.007$ & $<0.020$ & 0.162 & 0.017 & 2.52 & $<0.009$ & 0.701 & 2.06 \\
\hline 2 & ROC-30 & 2 & 2 & 14 & b14LM22 & 0.038 & 0.749 & 0.126 & 0.17 & 0.068 & 0.063 & $<0.050$ & 0.031 & 0.504 & 2.77 \\
\hline 2 & ROC-19 & 2 & 2 & 15 & b02LM22 & $<0.002$ & 0.015 & $<0.007$ & $<0.020$ & 0.017 & 0.019 & $<0.050$ & $<0.009$ & 0.888 & 0.244 \\
\hline 2 & ROC-17 & 2 & 2 & 16 & b09LM22 & $<0.002$ & 0.121 & $<0.007$ & $<0.020$ & 0.118 & 0.01 & $<0.050$ & $<0.009$ & 0.012 & 4.15 \\
\hline 2 & FY09EM21-29 & 2 & 2 & 17 & b15LM22 & 0.04 & 1.25 & 0.127 & 0.154 & 0.065 & 0.069 & $<0.050$ & 0.038 & 0.394 & 1.86 \\
\hline 2 & ROC-29 & 2 & 2 & 18 & b06LM12 & 0.069 & 0.054 & 0.219 & 0.305 & 0.021 & 0.11 & $<0.050$ & 0.061 & 0.007 & 3.68 \\
\hline 2 & FY09EM21-29 & 2 & 2 & 19 & b15LM12 & 0.04 & 1.26 & 0.128 & 0.155 & 0.067 & 0.067 & $<0.050$ & 0.038 & 0.397 & 1.87 \\
\hline 2 & ROC-20 & 2 & 2 & 20 & b11LM12 & 0.071 & 2.66 & 0.227 & 0.264 & 0.133 & 0.1 & $<0.050$ & 0.062 & 0.216 & 3.92 \\
\hline 2 & ROC-26 & 2 & 2 & 21 & b17LM22 & $<0.002$ & 0.011 & $<0.007$ & $<0.020$ & 0.016 & 0.025 & $<0.050$ & $<0.009$ & 0.005 & 2.57 \\
\hline 2 & ROC-24 & 2 & 2 & 22 & b16LM12 & 0.074 & $<0.010$ & 0.237 & 0.296 & 0.111 & 0.114 & $<0.050$ & 0.067 & 0.887 & 4.17 \\
\hline 2 & Batch 1 & 2 & 2 & 23 & BCHLM232 & 0.134 & 0.856 & $<0.007$ & $<0.020$ & 0.077 & 0.319 & 2.6 & $<0.009$ & 0.851 & 1.31 \\
\hline 2 & Ustd 1 & 2 & 2 & 24 & UstdLM232 & $<0.002$ & 0.912 & $<0.007$ & $<0.020$ & 0.166 & 0.015 & 2.39 & $<0.009$ & 0.716 & 2.11 \\
\hline
\end{tabular}


SRNL-STI-2009-00465, Revision 0

\section{Table A1. Measured Elemental Concentrations (wt\%) for the Study Glasses Prepared Using Lithium Metaborate (part 2)}

\begin{tabular}{|c|c|c|c|c|c|c|c|c|c|c|c|c|c|c|c|}
\hline Set & Glass ID & Block & $\begin{array}{l}\text { Sub- } \\
\text { Block }\end{array}$ & Sequence & Lab ID & $\begin{array}{l}\mathrm{Na} \\
\text { (wt\%) }\end{array}$ & $\begin{array}{l}\mathrm{Ni} \\
\text { (wt\%) }\end{array}$ & $\begin{array}{l}\mathrm{P} \\
(\mathrm{wt} \%) \\
\end{array}$ & $\begin{array}{l}\mathrm{Pb} \\
\text { (wt\%) }\end{array}$ & $\begin{array}{l}\mathrm{S} \\
\text { (wt\%) }\end{array}$ & $\begin{array}{l}\mathrm{Si} \\
\text { (wt\%) }\end{array}$ & $\begin{array}{l}\mathrm{Ti} \\
\text { (wt\%) }\end{array}$ & $\begin{array}{l}\mathrm{U} \\
(\mathrm{wt} \%)\end{array}$ & $\begin{array}{l}\mathrm{Zn} \\
(\mathrm{wt} \%)\end{array}$ & $\begin{array}{l}\mathrm{Zr} \\
\text { (wt\%) }\end{array}$ \\
\hline 1 & Batch 1 & 1 & 1 & 1 & BCHLM111 & 6.78 & 0.607 & $<0.015$ & 0.0101 & $<0.150$ & & 0.4 & $<0.267$ & $<0.020$ & 0.065 \\
\hline 1 & Ustd & 1 & 1 & 2 & UstdLM111 & 8.83 & 0.77 & $<0.015$ & $<0.010$ & $<0.150$ & & 0.56 & 2.07 & $<0.020$ & $<0.005$ \\
\hline 1 & ROC-01 & 1 & 1 & 3 & a05LM11 & 7.63 & 0.0208 & 0.0173 & $<0.010$ & $<0.150$ & & 1.16 & 7.52 & 0.038 & $<0.005$ \\
\hline 1 & ROC-08 & 1 & 1 & 4 & a10LM21 & 9.76 & 1.83 & 0.0177 & $<0.010$ & $<0.150$ & & 1.16 & 5.84 & $<0.020$ & $<0.005$ \\
\hline 1 & ROC-07 & 1 & 1 & 5 & a07LM21 & 7.24 & 0.0285 & $<0.015$ & $<0.010$ & $<0.150$ & & 0.28 & 1.8 & $<0.020$ & $<0.005$ \\
\hline 1 & ROC-09 & 1 & 1 & 6 & a12LM11 & 8.84 & 1.81 & $<0.015$ & 0.177 & 0.232 & & 0.33 & 0.47 & 0.11 & 0.133 \\
\hline 1 & ROC-06 & 1 & 1 & 7 & a03LM21 & 6.33 & 0.0196 & $<0.015$ & $<0.010$ & $<0.150$ & & 0.21 & 6.09 & $<0.020$ & $<0.005$ \\
\hline 1 & ROC-11 & 1 & 1 & 8 & a08LM11 & 7.47 & 1.9 & 0.0227 & 0.226 & 0.208 & & 0.3 & 7.84 & 0.121 & 0.139 \\
\hline 1 & ROC-01 & 1 & 1 & 9 & a05LM21 & 7.48 & 0.0234 & 0.0215 & $<0.010$ & $<0.150$ & & 1.14 & 7.44 & 0.039 & $<0.005$ \\
\hline 1 & ROC-03 & 1 & 1 & 10 & a01LM11 & 7.39 & 0.0221 & $<0.015$ & 0.2 & 0.201 & & 1.11 & 7.53 & 0.113 & 0.146 \\
\hline 1 & Batch 1 & 1 & 1 & 11 & BCHLM121 & 6.86 & 0.606 & $<0.015$ & 0.0105 & $<0.150$ & & 0.4 & $<0.267$ & $<0.020$ & 0.065 \\
\hline 1 & Ustd & 1 & 1 & 12 & UstdLM121 & 8.85 & 0.757 & $<0.015$ & $<0.010$ & $<0.150$ & & 0.6 & 2.13 & $<0.020$ & $<0.005$ \\
\hline 1 & ROC-07 & 1 & 1 & 13 & a07LM11 & 7.13 & 0.0356 & $<0.015$ & 0.0102 & $<0.150$ & & 0.3 & 1.76 & $<0.020$ & $<0.005$ \\
\hline 1 & ROC-09 & 1 & 1 & 14 & a12LM21 & 8.95 & 1.83 & $<0.015$ & 0.185 & 0.232 & & 0.34 & 0.484 & 0.113 & 0.135 \\
\hline 1 & ROC-08 & 1 & 1 & 15 & a10LM11 & 9.05 & 1.69 & 0.015 & $<0.010$ & $<0.150$ & & 1.07 & 5.41 & $<0.020$ & $<0.005$ \\
\hline 1 & ROC-10 & 1 & 1 & 16 & a13LM11 & 7.71 & 0.0244 & $<0.015$ & 0.192 & 0.223 & & 0.28 & 7.2 & 0.102 & 0.132 \\
\hline 1 & ROC-10 & 1 & 1 & 17 & a13LM21 & 7.68 & 0.0224 & $<0.015$ & 0.191 & 0.21 & & 0.3 & 7.23 & 0.102 & 0.132 \\
\hline 1 & ROC-03 & 1 & 1 & 18 & a01LM21 & 7.29 & 0.0209 & $<0.015$ & 0.198 & 0.204 & & 1.06 & 7.4 & 0.109 & 0.138 \\
\hline 1 & ROC-06 & 1 & 1 & 19 & a03LM11 & 7.96 & 0.0267 & 0.0219 & 0.01 & $<0.150$ & & 0.26 & 7.81 & $<0.020$ & $<0.005$ \\
\hline 1 & ROC-11 & 1 & 1 & 20 & a08LM21 & 7.43 & 1.85 & 0.0227 & 0.227 & 0.223 & & 0.34 & 7.61 & 0.12 & 0.137 \\
\hline 1 & Batch 1 & 1 & 1 & 21 & BCHLM131 & 6.89 & 0.602 & $<0.015$ & $<0.010$ & $<0.150$ & & 0.41 & $<0.267$ & $<0.020$ & 0.066 \\
\hline 1 & Ustd & 1 & 1 & 22 & UstdLM131 & 8.88 & 0.754 & $<0.015$ & $<0.010$ & $<0.150$ & & 0.57 & 1.99 & $<0.020$ & $<0.005$ \\
\hline 1 & Batch 1 & 1 & 2 & 1 & BCHLM112 & 6.75 & 0.61 & $<0.015$ & $<0.010$ & $<0.150$ & & 0.399 & $<0.267$ & $<0.020$ & 0.066 \\
\hline 1 & Ustd & 1 & 2 & 2 & UstdLM112 & 8.77 & 0.77 & $<0.015$ & $<0.010$ & $<0.150$ & & 0.558 & 2.1 & $<0.020$ & $<0.005$ \\
\hline 1 & ROC-06 & 1 & 2 & 3 & a03LM12 & 7.89 & 0.02 & 0.033 & $<0.010$ & $<0.150$ & & 0.259 & 7.8 & $<0.020$ & $<0.005$ \\
\hline 1 & ROC-01 & 1 & 2 & 4 & a05LM22 & 7.47 & 0.02 & 0.031 & $<0.010$ & 0.17 & & 1.12 & 7.4 & 0.041 & $<0.005$ \\
\hline 1 & ROC-10 & 1 & 2 & 5 & a13LM22 & 7.71 & 0.02 & 0.015 & 0.19 & 0.244 & & 0.277 & 7.2 & 0.104 & 0.13 \\
\hline 1 & ROC-07 & 1 & 2 & 6 & a07LM22 & 7.21 & 0.03 & $<0.015$ & $<0.010$ & $<0.150$ & & 0.273 & 1.8 & $<0.020$ & $<0.005$ \\
\hline 1 & ROC-11 & 1 & 2 & 7 & a08LM22 & 7.37 & 1.89 & 0.036 & 0.23 & 0.271 & & 0.337 & 7.6 & 0.123 & 0.135 \\
\hline 1 & ROC-07 & 1 & 2 & 8 & a07LM12 & 7.22 & 0.03 & $<0.015$ & $<0.010$ & $<0.150$ & & 0.271 & 1.8 & $<0.020$ & $<0.005$ \\
\hline 1 & ROC-03 & 1 & 2 & 9 & a01LM22 & 7.32 & 0.02 & 0.02 & 0.2 & 0.244 & & 1.04 & 7.5 & 0.112 & 0.135 \\
\hline 1 & ROC-09 & 1 & 2 & 10 & a12LM12 & 8.85 & 1.84 & $<0.015$ & 0.19 & 0.293 & & 0.327 & 0.495 & 0.114 & 0.132 \\
\hline 1 & Batch 1 & 1 & 2 & 11 & BCHLM122 & 6.87 & 0.62 & $<0.015$ & 0.01 & $<0.150$ & & 0.399 & $<0.267$ & $<0.020$ & 0.065 \\
\hline 1 & Ustd & 1 & 2 & 12 & UstdLM122 & 8.91 & 0.78 & $<0.015$ & $<0.010$ & $<0.150$ & & 0.557 & 2.1 & $<0.020$ & $<0.005$ \\
\hline 1 & ROC-10 & 1 & 2 & 13 & a13LM12 & 7.69 & 0.02 & $<0.015$ & 0.19 & 0.206 & & 0.276 & 7.3 & 0.102 & 0.129 \\
\hline 1 & ROC-09 & 1 & 2 & 14 & a12LM22 & 8.97 & 1.85 & $<0.015$ & 0.19 & 0.259 & & 0.331 & 0.501 & 0.114 & 0.134 \\
\hline 1 & ROC-08 & 1 & 2 & 15 & a10LM12 & 9.05 & 1.72 & 0.022 & $<0.010$ & $<0.150$ & & 1.05 & 5.4 & $<0.020$ & $<0.005$ \\
\hline 1 & ROC-03 & 1 & 2 & 16 & a01LM12 & 7.47 & 0.02 & 0.017 & 0.21 & 0.241 & & 1.09 & 7.6 & 0.115 & 0.143 \\
\hline 1 & ROC-11 & 1 & 2 & 17 & a08LM12 & 7.5 & 1.93 & 0.03 & 0.23 & 0.262 & & 0.34 & 7.8 & 0.125 & 0.137 \\
\hline 1 & ROC-06 & 1 & 2 & 18 & a03LM22 & 6.39 & 0.02 & 0.025 & $<0.010$ & $<0.150$ & & 0.207 & 6.1 & $<0.020$ & 0.006 \\
\hline 1 & ROC-01 & 1 & 2 & 19 & a05LM12 & 7.69 & 0.02 & 0.027 & $<0.010$ & $<0.150$ & & 1.15 & 7.6 & 0.04 & $<0.005$ \\
\hline 1 & ROC-08 & 1 & 2 & 20 & a10LM22 & 9.79 & 1.84 & 0.023 & $<0.010$ & $<0.150$ & & 1.14 & 5.9 & $<0.020$ & $<0.005$ \\
\hline 1 & Batch 1 & 1 & 2 & 21 & BCHLM132 & 6.94 & 0.61 & $<0.015$ & $<0.010$ & $<0.150$ & & 0.395 & $<0.267$ & $<0.020$ & 0.065 \\
\hline 1 & Ustd & 1 & 2 & 22 & UstdLM132 & 8.94 & 0.778 & $<0.015$ & $<0.010$ & $<0.150$ & & 0.557 & 2.1 & $<0.020$ & $<0.005$ \\
\hline 1 & Batch 1 & 2 & 1 & 1 & BCHLM211 & 6.82 & 0.63 & $<0.015$ & $<0.010$ & $<0.150$ & & 0.397 & $<0.267$ & $<0.020$ & 0.065 \\
\hline 1 & Ustd & 2 & 1 & 2 & UstdLM211 & 8.77 & 0.78 & $<0.015$ & $<0.010$ & $<0.150$ & & 0.562 & 2.1 & $<0.020$ & $<0.005$ \\
\hline 1 & ROC-13 & 2 & 1 & 3 & a14LM21 & 6.7 & 0.03 & 0.02 & $<0.010$ & $<0.150$ & & 0.276 & 6.1 & $<0.020$ & $<0.005$ \\
\hline 1 & ROC-12 & 2 & 1 & 4 & a06LM21 & 9.55 & 1.95 & $<0.015$ & $<0.010$ & $<0.150$ & & 0.296 & 2.2 & $<0.020$ & 0.008 \\
\hline 1 & ROC-05 & 2 & 1 & 5 & a11LM21 & 7.74 & 0.1 & $<0.015$ & 0.01 & $<0.150$ & & 0.308 & 7.7 & $<0.020$ & $<0.005$ \\
\hline 1 & ROC-02 & 2 & 1 & 6 & a09LM11 & 10 & 1.95 & $<0.015$ & $<0.010$ & $<0.150$ & & 1.12 & 3.5 & $<0.020$ & 0.008 \\
\hline 1 & ROC-13 & 2 & 1 & 7 & a14LM11 & 6.78 & 0.03 & 0.021 & $<0.010$ & $<0.150$ & & 0.277 & 6.2 & $<0.020$ & $<0.005$ \\
\hline 1 & ROC-05 & 2 & 1 & 8 & a11LM11 & 7.54 & 0.09 & $<0.015$ & $<0.010$ & $<0.150$ & & 0.295 & 7.6 & $<0.020$ & $<0.005$ \\
\hline 1 & ROC-14 & 2 & 1 & 9 & a04LM21 & 7.5 & 1.81 & $<0.015$ & 0.21 & 0.234 & & 1.2 & 2.1 & 0.119 & 0.017 \\
\hline 1 & Batch 1 & 2 & 1 & 10 & BCHLM221 & 6.8 & 0.61 & $<0.015$ & 0.01 & $<0.150$ & & 0.395 & $<0.267$ & $<0.020$ & 0.065 \\
\hline 1 & Ustd & 2 & 1 & 11 & UstdLM221 & 8.88 & 0.79 & $<0.015$ & $<0.010$ & $<0.150$ & & 0.555 & 2.1 & $<0.020$ & $<0.005$ \\
\hline 1 & ROC-04 & 2 & 1 & 12 & a02LM21 & 8.57 & 1.83 & 0.031 & 0.17 & 0.236 & & 1.09 & 7.7 & 0.117 & 0.14 \\
\hline 1 & ROC-14 & 2 & 1 & 13 & a04LM11 & 7.39 & 1.73 & $<0.015$ & 0.2 & 0.222 & & 1.18 & 2.1 & 0.116 & 0.018 \\
\hline 1 & ROC-12 & 2 & 1 & 14 & a06LM11 & 9.49 & 1.95 & $<0.015$ & $<0.010$ & $<0.150$ & & 0.292 & 2.2 & $<0.020$ & 0.008 \\
\hline 1 & ROC-15 & 2 & 1 & 15 & a15LM21 & 10.3 & 1.8 & 0.021 & 0.2 & 0.277 & & 0.285 & 6.8 & 0.116 & 0.129 \\
\hline 1 & ROC-04 & 2 & 1 & 16 & a02LM11 & 8.56 & 1.73 & 0.035 & 0.18 & 0.235 & & 1.06 & 7.7 & 0.119 & 0.14 \\
\hline 1 & ROC-15 & 2 & 1 & 17 & a15LM11 & 10.4 & 1.79 & 0.021 & 0.2 & 0.255 & & 0.285 & 6.8 & 0.116 & 0.129 \\
\hline 1 & ROC-02 & 2 & 1 & 18 & a09LM21 & 9.83 & 1.91 & $<0.015$ & $<0.010$ & $<0.150$ & & 1.11 & 3.5 & $<0.020$ & 0.007 \\
\hline 1 & Batch 1 & 2 & 1 & 19 & BCHLM231 & 6.82 & 0.62 & $<0.015$ & 0.01 & $<0.150$ & & 0.395 & $<0.267$ & $<0.020$ & 0.065 \\
\hline 1 & Ustd & 2 & 1 & 20 & UstdLM231 & 8.82 & 0.78 & $<0.015$ & $<0.010$ & $<0.150$ & & 0.552 & 2.1 & $<0.020$ & $<0.005$ \\
\hline 1 & Batch 1 & 2 & 2 & 1 & BCHLM212 & 6.7 & 0.6 & $<0.015$ & 0.01 & $<0.150$ & & 0.408 & $<0.267$ & $<0.020$ & 0.066 \\
\hline 1 & Ustd & 2 & 2 & 2 & UstdLM212 & 8.69 & 0.76 & $<0.015$ & $<0.010$ & $<0.150$ & & 0.573 & 2.1 & $<0.020$ & $<0.005$ \\
\hline 1 & ROC-04 & 2 & 2 & 3 & a02LM12 & 8.42 & 1.69 & 0.038 & 0.19 & 0.275 & & 1.08 & 7.6 & 0.125 & 0.142 \\
\hline 1 & ROC-14 & 2 & 2 & 4 & a04LM12 & 7.32 & 1.68 & $<0.015$ & 0.22 & 0.239 & & 1.21 & 2.1 & 0.122 & 0.018 \\
\hline 1 & ROC-02 & 2 & 2 & 5 & a09LM12 & 9.92 & 1.9 & $<0.015$ & $<0.010$ & $<0.150$ & & 1.14 & 3.5 & $<0.020$ & 0.009 \\
\hline 1 & ROC-13 & 2 & 2 & 6 & a14LM12 & 6.74 & 0.02 & 0.032 & $<0.010$ & $<0.150$ & & 0.283 & 6.1 & $<0.020$ & $<0.005$ \\
\hline
\end{tabular}


SRNL-STI-2009-00465, Revision 0

\section{Table A1. Measured Elemental Concentrations (wt\%) for the Study Glasses Prepared Using Lithium Metaborate (part 2)}

\begin{tabular}{|c|c|c|c|c|c|c|c|c|c|c|c|c|c|c|c|}
\hline Set & Glass ID & Block & $\begin{array}{l}\text { Sub- } \\
\text { Block }\end{array}$ & Sequence & Lab ID & $\begin{array}{l}\mathrm{Na} \\
\text { (wt\%) }\end{array}$ & $\begin{array}{l}\mathrm{Ni} \\
\text { (wt\%) }\end{array}$ & $\begin{array}{l}\mathrm{P} \\
\text { (wt\%) }\end{array}$ & $\begin{array}{l}\mathrm{Pb} \\
\text { (wt\%) }\end{array}$ & $\begin{array}{l}\text { S } \\
\text { (wt\%) }\end{array}$ & $\begin{array}{l}\mathrm{Si} \\
\text { (wt\%) }\end{array}$ & $\begin{array}{l}\mathrm{Ti} \\
\text { (wt\%) }\end{array}$ & $\begin{array}{l}\mathrm{U} \\
\text { (wt\%) }\end{array}$ & $\begin{array}{l}\mathrm{Zn} \\
\text { (wt\%) }\end{array}$ & $\begin{array}{l}\mathrm{Zr} \\
\text { (wt\%) }\end{array}$ \\
\hline 1 & ROC-05 & 2 & 2 & 7 & a11LM22 & 7.69 & 0.09 & 0.025 & 0.01 & $<0.150$ & & 0.314 & 7.7 & $<0.020$ & $<0.005$ \\
\hline 1 & ROC-14 & 2 & 2 & 8 & a04LM22 & 7.43 & 1.76 & $<0.015$ & 0.22 & 0.21 & & 1.23 & 2.1 & 0.125 & 0.017 \\
\hline 1 & ROC-12 & 2 & 2 & 9 & a06LM22 & 9.53 & 1.92 & $<0.015$ & $<0.010$ & $<0.150$ & & 0.31 & 2.2 & $<0.020$ & 0.009 \\
\hline 1 & Batch 1 & 2 & 2 & 10 & BCHLM222 & 6.75 & 0.6 & $<0.015$ & $<0.010$ & $<0.150$ & & 0.404 & $<0.267$ & $<0.020$ & 0.066 \\
\hline 1 & Ustd & 2 & 2 & 11 & UstdLM222 & 8.79 & 0.76 & $<0.015$ & $<0.010$ & $<0.150$ & & 0.567 & 2.1 & $<0.020$ & $<0.005$ \\
\hline 1 & ROC-13 & 2 & 2 & 12 & a14LM22 & 6.63 & 0.03 & 0.028 & $<0.010$ & $<0.150$ & & 0.282 & 6 & $<0.020$ & $<0.005$ \\
\hline 1 & ROC-04 & 2 & 2 & 13 & a02LM22 & 8.45 & 1.77 & 0.036 & 0.18 & 0.273 & & 1.09 & 7.8 & 0.122 & 0.138 \\
\hline 1 & ROC-02 & 2 & 2 & 14 & a09LM22 & 9.68 & 1.85 & 0.016 & $<0.010$ & $<0.150$ & & 1.13 & 3.4 & $<0.020$ & 0.008 \\
\hline 1 & ROC-15 & 2 & 2 & 15 & a15LM22 & 10.2 & 1.75 & 0.025 & 0.21 & 0.247 & & 0.291 & 6.8 & 0.123 & 0.131 \\
\hline 1 & ROC-12 & 2 & 2 & 16 & a06LM12 & 9.44 & 1.9 & $<0.015$ & $<0.010$ & $<0.150$ & & 0.299 & 2.1 & $<0.020$ & 0.009 \\
\hline 1 & ROC-15 & 2 & 2 & 17 & a15LM12 & 10.3 & 1.75 & 0.03 & 0.22 & 0.253 & & 0.292 & 6.9 & 0.122 & 0.131 \\
\hline 1 & ROC-05 & 2 & 2 & 18 & a11LM12 & 7.47 & 0.08 & 0.022 & 0.01 & $<0.150$ & & 0.306 & 7.5 & $<0.020$ & $<0.005$ \\
\hline 1 & Batch 1 & 2 & 2 & 19 & BCHLM232 & 6.76 & 0.6 & $<0.015$ & 0.01 & $<0.150$ & & 0.405 & $<0.267$ & $<0.020$ & 0.066 \\
\hline 1 & Ustd & 2 & 2 & 20 & UstdLM232 & 8.77 & 0.76 & $<0.015$ & $<0.010$ & $<0.150$ & & 0.569 & 2.1 & $<0.020$ & $<0.005$ \\
\hline 2 & Batch 1 & 1 & 1 & 1 & BCHLM111 & 6.59 & 0.593 & $<0.0150$ & $<0.010$ & $<0.150$ & 22.7 & 0.387 & $<0.200$ & $<0.020$ & 0.065 \\
\hline 2 & Ustd 1 & 1 & 1 & 2 & UstdLM111 & 8.64 & 0.841 & $<0.0150$ & $<0.010$ & $<0.150$ & 20.6 & 0.564 & 2 & $<0.020$ & $<0.005$ \\
\hline 2 & ROC-16 & 1 & 1 & 3 & b05LM11 & 7.34 & 0.02 & 0.026 & 0.189 & $<0.150$ & 21.2 & 1.42 & 6.64 & 0.092 & 0.125 \\
\hline 2 & ROC-23 & 1 & 1 & 4 & b10LM21 & 7.86 & 1.21 & $<0.0150$ & 0.174 & $<0.150$ & 21.2 & 1.12 & 1.94 & 0.096 & 0.124 \\
\hline 2 & ROC-22 & 1 & 1 & 5 & b07LM21 & 7.54 & 1.67 & $<0.0150$ & $<0.010$ & $<0.150$ & 21.3 & 1.1 & 3.53 & $<0.020$ & $<0.005$ \\
\hline 2 & ROC-25 & 1 & 1 & 6 & b12LM11 & 11 & 0.066 & $<0.0150$ & 0.169 & 0.188 & 20.5 & 0.29 & 2.02 & 0.092 & 0.128 \\
\hline 2 & ROC-21 & 1 & 1 & 7 & b03LM21 & 7.68 & 1.16 & 0.028 & $<0.010$ & $<0.150$ & 16.9 & 1.19 & 7.68 & $<0.020$ & $<0.005$ \\
\hline 2 & ROC-28 & 1 & 1 & 8 & b08LM11 & 12.8 & 0.02 & $<0.0150$ & 0.173 & $<0.150$ & 24.7 & 1.14 & 1.55 & 0.091 & 0.134 \\
\hline 2 & ROC-16 & 1 & 1 & 9 & b05LM21 & 7.38 & 0.021 & 0.028 & 0.192 & $<0.150$ & 21.3 & 1.43 & 6.64 & 0.093 & 0.13 \\
\hline 2 & ROC-18 & 1 & 1 & 10 & b01LM11 & 7.52 & 1.48 & $<0.0150$ & $<0.010$ & $<0.150$ & 21.4 & 1.11 & 4.11 & $<0.020$ & 0.007 \\
\hline 2 & FY09EM21-30 & 1 & 1 & 11 & b18LM11 & 8.55 & 0.806 & $<0.0150$ & 0.078 & $<0.150$ & 17.9 & 2.14 & 4.67 & 0.05 & 0.071 \\
\hline 2 & Batch 1 & 1 & 1 & 12 & BCHLM121 & 6.69 & 0.601 & $<0.0150$ & $<0.010$ & $<0.150$ & 22.8 & 0.397 & $<0.200$ & $<0.020$ & 0.064 \\
\hline 2 & $\begin{array}{l}\text { Ustd } 1 \\
\end{array}$ & 1 & 1 & 13 & UstdLM121 & 8.67 & 0.853 & $<0.0150$ & $<0.010$ & $<0.150$ & 20.7 & 0.576 & 2.01 & $<0.020$ & $<0.005$ \\
\hline 2 & ROC-22 & 1 & 1 & 14 & b07LM11 & 7.72 & 1.71 & $<0.0150$ & $<0.010$ & $<0.150$ & 21.8 & 1.12 & 3.61 & $<0.020$ & $<0.005$ \\
\hline 2 & ROC-25 & 1 & 1 & 15 & b12LM21 & 11 & 0.065 & $<0.0150$ & 0.173 & 0.161 & 20.6 & 0.289 & 1.99 & 0.091 & 0.127 \\
\hline 2 & ROC-23 & 1 & 1 & 16 & b10LM11 & 8.1 & 1.25 & $<0.0150$ & 0.181 & $<0.150$ & 21.8 & 1.15 & 2.05 & 0.1 & 0.128 \\
\hline 2 & ROC-27 & 1 & 1 & 17 & b13LM11 & 8.7 & 1.15 & $<0.0150$ & 0.182 & $<0.150$ & 19.9 & 1.12 & 3.2 & 0.098 & 0.131 \\
\hline 2 & ROC-27 & 1 & 1 & 18 & b13LM21 & 8.73 & 1.18 & $<0.0150$ & 0.185 & $<0.150$ & 20.1 & 1.13 & 3.22 & 0.1 & 0.132 \\
\hline 2 & ROC-18 & 1 & 1 & 19 & b01LM21 & 7.75 & 1.56 & $<0.0150$ & $<0.010$ & $<0.150$ & 22.6 & 1.16 & 4.29 & $<0.020$ & 0.008 \\
\hline 2 & ROC-21 & 1 & 1 & 20 & b03LM11 & 7.74 & 1.15 & 0.028 & $<0.010$ & $<0.150$ & 16.8 & 1.17 & 7.54 & $<0.020$ & $<0.005$ \\
\hline 2 & ROC-28 & 1 & 1 & 21 & b08LM21 & 12.1 & 0.027 & $<0.0150$ & 0.169 & $<0.150$ & 23.5 & 1.08 & 1.42 & 0.09 & 0.124 \\
\hline 2 & FY09EM21-30 & 1 & 1 & 22 & b18LM21 & 8.82 & 0.846 & $<0.0150$ & 0.083 & $<0.150$ & 18.6 & 2.19 & 4.8 & 0.052 & 0.072 \\
\hline 2 & Batch 1 & 1 & 1 & 23 & BCHLM131 & 6.74 & 0.612 & $<0.0150$ & $<0.010$ & $<0.150$ & 23.1 & 0.399 & $<0.200$ & $<0.020$ & 0.065 \\
\hline 2 & Ustd 1 & 1 & 1 & 24 & UstdLM131 & 8.73 & 0.864 & $<0.0150$ & $<0.010$ & $<0.150$ & 20.9 & 0.572 & 2.03 & $<0.020$ & $<0.005$ \\
\hline 2 & Batch 1 & 1 & 2 & 1 & BCHLM112 & 6.62 & 0.586 & $<0.0150$ & $<0.010$ & $<0.150$ & 23 & 0.395 & $<0.200$ & $<0.020$ & 0.065 \\
\hline 2 & Ustd 1 & 1 & 2 & 2 & UstdLM112 & 8.56 & 0.846 & $<0.0150$ & $<0.010$ & $<0.150$ & 20.7 & 0.572 & 2.05 & $<0.020$ & $<0.005$ \\
\hline 2 & ROC-21 & 1 & 2 & 3 & b03LM12 & 7.56 & 1.14 & 0.032 & $<0.010$ & $<0.150$ & 16.7 & 1.17 & 7.62 & $<0.020$ & $<0.005$ \\
\hline 2 & ROC-16 & 1 & 2 & 4 & b05LM22 & 7.3 & 0.019 & 0.034 & 0.177 & $<0.150$ & 21.4 & 1.43 & 6.7 & 0.085 & 0.128 \\
\hline 2 & ROC-27 & 1 & 2 & 5 & b13LM22 & 8.63 & 1.17 & $<0.0150$ & 0.166 & $<0.150$ & 20 & 1.13 & 3.28 & 0.09 & 0.131 \\
\hline 2 & ROC-22 & 1 & 2 & 6 & b07LM22 & 7.49 & 1.68 & $<0.0150$ & $<0.010$ & $<0.150$ & 21.4 & 1.11 & 3.61 & $<0.020$ & $<0.005$ \\
\hline 2 & ROC-28 & 1 & 2 & 7 & b08LM22 & 11.9 & 0.023 & $<0.0150$ & 0.152 & $<0.150$ & 23.3 & 1.08 & 1.47 & 0.081 & 0.124 \\
\hline 2 & ROC-22 & 1 & 2 & 8 & b07LM12 & 7.6 & 1.69 & $<0.0150$ & $<0.010$ & $<0.150$ & 21.8 & 1.12 & 3.65 & $<0.020$ & $<0.005$ \\
\hline 2 & ROC-18 & 1 & 2 & 9 & b01LM22 & 7.67 & 1.54 & $<0.0150$ & $<0.010$ & $<0.150$ & 22.6 & 1.16 & 4.38 & $<0.020$ & 0.008 \\
\hline 2 & ROC-25 & 1 & 2 & 10 & b12LM12 & 10.8 & 0.06 & $<0.0150$ & 0.166 & 0.156 & 20.7 & 0.293 & 1.95 & 0.087 & 0.126 \\
\hline 2 & FY09EM21-30 & 1 & 2 & 11 & b18LM22 & 8.7 & 0.83 & $<0.0150$ & 0.077 & $<0.150$ & 18.5 & 2.2 & 4.91 & 0.048 & 0.072 \\
\hline 2 & Batch 1 & 1 & 2 & 12 & BCHLM122 & 6.64 & 0.589 & $<0.0150$ & $<0.010$ & $<0.150$ & 23 & 0.395 & $<0.200$ & $<0.020$ & 0.064 \\
\hline 2 & Ustd 1 & 1 & 2 & 13 & UstdLM122 & 8.6 & 0.847 & $<0.0150$ & $<0.010$ & $<0.150$ & 20.8 & 0.572 & 2.03 & $<0.020$ & $<0.005$ \\
\hline 2 & ROC-27 & 1 & 2 & 14 & b13LM12 & 8.42 & 1.13 & $<0.0150$ & 0.169 & 0.169 & 19.9 & 1.12 & 3.26 & 0.092 & 0.131 \\
\hline 2 & ROC-25 & 1 & 2 & 15 & b12LM22 & 10.8 & 0.061 & $<0.0150$ & 0.164 & $<0.150$ & 20.6 & 0.291 & 2.03 & 0.087 & 0.127 \\
\hline 2 & ROC-23 & 1 & 2 & 16 & b10LM12 & 7.92 & 1.25 & $<0.0150$ & 0.175 & $<0.150$ & 21.8 & 1.15 & 2.02 & 0.095 & 0.126 \\
\hline 2 & ROC-18 & 1 & 2 & 17 & b01LM12 & 7.48 & 1.47 & $<0.0150$ & $<0.010$ & $<0.150$ & 21.6 & 1.12 & 4.18 & $<0.020$ & 0.008 \\
\hline 2 & ROC-28 & 1 & 2 & 18 & b08LM12 & 12.6 & 0.019 & $<0.0150$ & 0.169 & 0.18 & 24.8 & 1.15 & 1.54 & 0.088 & 0.132 \\
\hline 2 & ROC-21 & 1 & 2 & 19 & b03LM22 & 7.7 & 1.15 & 0.029 & $<0.010$ & $<0.150$ & 16.9 & 1.19 & 7.77 & $<0.020$ & $<0.005$ \\
\hline 2 & ROC-16 & 1 & 2 & 20 & b05LM12 & 7.29 & 0.02 & 0.032 & 0.188 & $<0.150$ & 21.3 & 1.43 & 6.69 & 0.09 & 0.125 \\
\hline 2 & ROC-23 & 1 & 2 & 21 & b10LM22 & 7.93 & 1.21 & $<0.0150$ & 0.173 & $<0.150$ & 21.4 & 1.13 & 1.98 & 0.0945 & 0.124 \\
\hline 2 & FY09EM21-30 & 1 & 2 & 22 & b18LM12 & 8.58 & 0.803 & $<0.0150$ & 0.075 & $<0.150$ & 17.9 & 2.13 & 4.7 & 0.0473 & 0.071 \\
\hline 2 & Batch 1 & 1 & 2 & 23 & BCHLM132 & 6.77 & 0.593 & $<0.0150$ & $<0.010$ & $<0.150$ & 22.9 & 0.393 & $<0.200$ & $<0.020$ & 0.065 \\
\hline 2 & Ustd 1 & 1 & 2 & 24 & UstdLM132 & 8.71 & 0.84 & $<0.0150$ & $<0.010$ & $<0.150$ & 20.7 & 0.573 & 2.04 & $<0.020$ & $<0.005$ \\
\hline 2 & Batch 1 & 2 & 1 & 1 & BCHLM211 & 6.7 & 0.595 & $<0.0150$ & $<0.010$ & $<0.150$ & 23.1 & 0.39 & $<0.200$ & $<0.020$ & 0.063 \\
\hline 2 & Ustd 1 & 2 & 1 & 2 & UstdLM211 & 8.87 & 0.848 & $<0.0150$ & $<0.010$ & $<0.150$ & 21 & 0.566 & 1.99 & $<0.020$ & $<0.005$ \\
\hline 2 & ROC-30 & 2 & 1 & 3 & b14LM21 & 9.12 & 0.956 & $<0.0150$ & 0.103 & $<0.150$ & 21.1 & 0.805 & 4.64 & 0.063 & 0.07 \\
\hline 2 & ROC-29 & 2 & 1 & 4 & b06LM21 & 8.14 & 1.76 & $<0.0150$ & 0.192 & 0.191 & 17.6 & 0.288 & 4.53 & 0.114 & 0.129 \\
\hline 2 & ROC-20 & 2 & 1 & 5 & b11LM21 & 9.24 & 0.02 & $<0.0150$ & 0.185 & $<0.150$ & 20.2 & 1.21 & 0.563 & 0.101 & 0.136 \\
\hline 2 & ROC-17 & 2 & 1 & 6 & b09LM11 & 11.4 & 1.63 & $<0.0150$ & $<0.010$ & $<0.150$ & 19.9 & 1.13 & 0.221 & $<0.020$ & $<0.005$ \\
\hline 2 & ROC-30 & 2 & 1 & 7 & b14LM11 & 9.43 & 0.963 & $<0.0150$ & 0.104 & $<0.150$ & 21.6 & 0.815 & 4.63 & 0.064 & 0.072 \\
\hline 2 & ROC-20 & 2 & 1 & 8 & b11LM11 & 9.2 & 0.017 & $<0.0150$ & 0.185 & $<0.150$ & 19.9 & 1.19 & 0.532 & 0.101 & 0.13 \\
\hline
\end{tabular}


SRNL-STI-2009-00465, Revision 0

Table A1. Measured Elemental Concentrations (wt\%) for the Study Glasses Prepared Using Lithium Metaborate (part 2)

\begin{tabular}{|c|c|c|c|c|c|c|c|c|c|c|c|c|c|c|c|}
\hline Set & Glass ID & Block & $\begin{array}{l}\text { Sub- } \\
\text { Block }\end{array}$ & Sequence & Lab ID & $\begin{array}{l}\mathrm{Na} \\
(\mathrm{wt} \%)\end{array}$ & $\begin{array}{l}\mathrm{Ni} \\
\text { (wt\%) }\end{array}$ & $\begin{array}{l}\text { P } \\
\text { (wt\%) }\end{array}$ & $\begin{array}{l}\mathrm{Pb} \\
\text { (wt\%) }\end{array}$ & $\begin{array}{l}\text { S } \\
\text { (wt\%) }\end{array}$ & $\begin{array}{l}\text { Si } \\
\text { (wt\%) }\end{array}$ & $\begin{array}{l}\mathrm{Ti} \\
\text { (wt\%) }\end{array}$ & $\begin{array}{l}\mathrm{U} \\
\text { (wt\%) }\end{array}$ & $\begin{array}{l}\mathrm{Zn} \\
\text { (wt\%) }\end{array}$ & $\begin{array}{l}\mathrm{Zr} \\
\text { (wt\%) }\end{array}$ \\
\hline 2 & FY09EM21-28 & 2 & 1 & 9 & b04LM21 & 9.64 & 0.911 & $<0.0150$ & 0.098 & $<0.150$ & 20 & 2.14 & 1.87 & 0.065 & 0.077 \\
\hline 2 & ROC-26 & 2 & 1 & 10 & b17LM21 & 13.1 & 0.018 & $<0.0150$ & $<0.010$ & $<0.150$ & 22.5 & 0.294 & 4.62 & $<0.020$ & $<0.005$ \\
\hline 2 & ROC-24 & 2 & 1 & 11 & b16LM11 & 7.99 & 0.151 & $<0.0150$ & 0.199 & $<0.150$ & 20.8 & 1.03 & 1.1 & 0.107 & 0.128 \\
\hline 2 & Batch 1 & 2 & 1 & 12 & BCHLM221 & 6.84 & 0.605 & $<0.0150$ & $<0.010$ & $<0.150$ & 23.1 & 0.381 & $<0.200$ & $<0.020$ & 0.063 \\
\hline 2 & Ustd 1 & 2 & 1 & 13 & UstdLM221 & 8.97 & 0.861 & $<0.0150$ & $<0.010$ & $<0.150$ & 21 & 0.553 & 2.03 & $<0.020$ & $<0.005$ \\
\hline 2 & ROC-19 & 2 & 1 & 14 & b02LM21 & 7.74 & 1.85 & $<0.0150$ & $<0.010$ & $<0.150$ & 22.5 & 1.08 & 5.38 & $<0.020$ & $<0.005$ \\
\hline 2 & FY09EM21-28 & 2 & 1 & 15 & b04LM11 & 8.81 & 0.837 & $<0.0150$ & 0.093 & $<0.150$ & 18.1 & 1.93 & 1.58 & 0.06 & 0.07 \\
\hline 2 & ROC-29 & 2 & 1 & 16 & b06LM11 & 8.24 & 1.76 & $<0.0150$ & 0.203 & 0.234 & 17.7 & 0.282 & 4.43 & 0.119 & 0.129 \\
\hline 2 & FY09EM21-29 & 2 & 1 & 17 & b15LM21 & 9.41 & 0.85 & $<0.0150$ & 0.117 & $<0.150$ & 19 & 2.12 & 3.37 & 0.059 & 0.062 \\
\hline 2 & ROC-19 & 2 & 1 & 18 & b02LM11 & 8.1 & 1.93 & $<0.0150$ & $<0.010$ & $<0.150$ & 23.2 & 1.11 & 5.57 & $<0.020$ & $<0.005$ \\
\hline 2 & FY09EM21-29 & 2 & 1 & 19 & b15LM11 & 9.56 & 0.845 & $<0.0150$ & 0.119 & $<0.150$ & 19.5 & 2.13 & 3.39 & 0.06 & 0.077 \\
\hline 2 & ROC-17 & 2 & 1 & 20 & b09LM21 & 12.5 & 1.8 & $<0.0150$ & $<0.010$ & $<0.150$ & 21.9 & 1.22 & 0.251 & $<0.020$ & $<0.005$ \\
\hline 2 & ROC-24 & 2 & 1 & 21 & b16LM21 & 8.05 & 0.148 & $<0.0150$ & 0.206 & $<0.150$ & 20.9 & 1.01 & 1.18 & 0.111 & 0.129 \\
\hline 2 & ROC-26 & 2 & 1 & 22 & b17LM11 & 13.2 & 0.02 & $<0.0150$ & $<0.010$ & $<0.150$ & 22.3 & 0.281 & 4.52 & $<0.020$ & $<0.005$ \\
\hline 2 & Batch 1 & 2 & 1 & 23 & BCHLM231 & 7.04 & 0.601 & $<0.0150$ & $<0.010$ & $<0.150$ & 23.4 & 0.378 & $<0.200$ & $<0.020$ & 0.063 \\
\hline 2 & Ustd 1 & 2 & 1 & 24 & UstdLM231 & 9.2 & 0.872 & $<0.0150$ & $<0.010$ & $<0.150$ & 21.2 & 0.548 & 2.07 & $<0.020$ & $<0.005$ \\
\hline 2 & Batch 1 & 2 & 2 & 1 & BCHLM212 & 6.55 & 0.595 & $<0.0150$ & $<0.010$ & $<0.150$ & 22.9 & 0.385 & $<0.200$ & $<0.020$ & 0.062 \\
\hline 2 & Ustd 1 & 2 & 2 & 2 & UstdLM212 & 8.64 & 0.848 & $<0.0150$ & $<0.010$ & $<0.150$ & 20.9 & 0.565 & 2.15 & $<0.020$ & $<0.005$ \\
\hline 2 & ROC-19 & 2 & 2 & 3 & b02LM12 & 7.5 & 1.87 & $<0.0150$ & $<0.010$ & $<0.150$ & 22.6 & 1.11 & 5.64 & $<0.020$ & $<0.005$ \\
\hline 2 & FY09EM21-28 & 2 & 2 & 4 & b04LM12 & 8.32 & 0.811 & $<0.0150$ & 0.09 & $<0.150$ & 17.7 & 1.94 & 1.68 & 0.061 & 0.067 \\
\hline 2 & ROC-17 & 2 & 2 & 5 & b09LM12 & 11 & 1.64 & $<0.0150$ & $<0.010$ & $<0.150$ & 19.8 & 1.14 & 0.246 & $<0.020$ & $<0.005$ \\
\hline 2 & ROC-30 & 2 & 2 & 6 & b14LM12 & 9.02 & 0.956 & $<0.0150$ & 0.108 & $<0.150$ & 21.3 & 0.822 & 4.8 & 0.067 & 0.072 \\
\hline 2 & ROC-20 & 2 & 2 & 7 & b11LM22 & 8.92 & 0.022 & $<0.0150$ & 0.199 & $<0.150$ & 20.2 & 1.21 & 0.584 & 0.108 & 0.135 \\
\hline 2 & FY09EM21-28 & 2 & 2 & 8 & b04LM22 & 9.17 & 0.909 & $<0.0150$ & 0.101 & $<0.150$ & 19.7 & 2.15 & 1.87 & 0.068 & 0.078 \\
\hline 2 & ROC-29 & 2 & 2 & 9 & b06LM22 & 7.81 & 1.77 & $<0.0150$ & 0.208 & 0.188 & 17.6 & 0.292 & 4.46 & 0.122 & 0.131 \\
\hline 2 & ROC-24 & 2 & 2 & 10 & b16LM22 & 7.54 & 0.141 & $<0.0150$ & 0.207 & $<0.150$ & 20.4 & 1.02 & 1.18 & 0.112 & 0.13 \\
\hline 2 & ROC-26 & 2 & 2 & 11 & b17LM12 & 12.2 & 0.021 & $<0.0150$ & $<0.010$ & $<0.150$ & 21.7 & 0.288 & 4.53 & $<0.020$ & $<0.005$ \\
\hline 2 & Batch 1 & 2 & 2 & 12 & BCHLM222 & 6.51 & 0.6 & $<0.0150$ & $<0.010$ & $<0.150$ & 22.9 & 0.383 & $<0.200$ & $<0.020$ & 0.063 \\
\hline 2 & Ustd 1 & 2 & 2 & 13 & UstdLM222 & 8.38 & 0.839 & $<0.0150$ & $<0.010$ & $<0.150$ & 20.4 & 0.551 & 2.07 & $<0.020$ & $<0.005$ \\
\hline 2 & ROC-30 & 2 & 2 & 14 & b14LM22 & 8.8 & 0.975 & $<0.0150$ & 0.112 & $<0.150$ & 21.3 & 0.812 & 4.64 & 0.068 & 0.07 \\
\hline 2 & ROC-19 & 2 & 2 & 15 & b02LM22 & 7.42 & 1.87 & $<0.0150$ & $<0.010$ & $<0.150$ & 22.6 & 1.09 & 5.44 & $<0.020$ & $<0.005$ \\
\hline 2 & ROC-17 & 2 & 2 & 16 & b09LM22 & 11.6 & 1.79 & $<0.0150$ & $<0.010$ & $<0.150$ & 21.7 & 1.23 & 0.267 & $<0.020$ & $<0.005$ \\
\hline 2 & FY09EM21-29 & 2 & 2 & 17 & b15LM22 & 8.83 & 0.855 & $<0.0150$ & 0.123 & $<0.150$ & 19 & 2.15 & 3.42 & 0.062 & 0.065 \\
\hline 2 & ROC-29 & 2 & 2 & 18 & b06LM12 & 7.79 & 1.78 & $<0.0150$ & 0.213 & 0.211 & 17.7 & 0.288 & 4.55 & 0.125 & 0.132 \\
\hline 2 & FY09EM21-29 & 2 & 2 & 19 & b15LM12 & 8.85 & 0.839 & $<0.0150$ & 0.123 & $<0.150$ & 19.4 & 2.16 & 3.44 & 0.062 & 0.074 \\
\hline 2 & ROC-20 & 2 & 2 & 20 & b11LM12 & 8.82 & 0.02 & $<0.0146$ & 0.202 & $<0.150$ & 20.1 & 1.2 & 0.57 & 0.108 & 0.129 \\
\hline 2 & ROC-26 & 2 & 2 & 21 & b17LM22 & 12.5 & 0.02 & $<0.0146$ & $<0.010$ & $<0.150$ & 22.7 & 0.3 & 4.77 & $<0.020$ & $<0.005$ \\
\hline 2 & ROC-24 & 2 & 2 & 22 & b16LM12 & 7.61 & 0.144 & $<0.0149$ & 0.215 & $<0.150$ & 21 & 1.04 & 1.21 & 0.114 & 0.131 \\
\hline 2 & Batch 1 & 2 & 2 & 23 & BCHLM232 & 6.55 & 0.614 & $<0.0150$ & $<0.010$ & $<0.150$ & 23.4 & 0.386 & $<0.200$ & $<0.020$ & 0.063 \\
\hline 2 & Ustd 1 & 2 & 2 & 24 & UstdLM232 & 8.56 & 0.871 & $<0.0150$ & $<0.010$ & $<0.150$ & 21.1 & 0.556 & 2.08 & $<0.020$ & $<0.005$ \\
\hline
\end{tabular}


SRNL-STI-2009-00465, Revision 0

Table A2. Measured Elemental Concentrations (wt\%) for the Study Glasses Prepared Using Peroxide Fusion

\begin{tabular}{|c|c|c|c|c|c|c|c|c|c|}
\hline Set & Glass ID & Block & Sub-Block & Sequence & Al (wt\%) & B (wt\%) & Fe (wt\%) & $\mathrm{Li}(w \mathrm{t} \%)$ & $\mathrm{Si}(\mathrm{wt} \%)$ \\
\hline 1 & Batch 1 & 1 & 1 & 1 & 2.54 & 2.27 & 8.83 & 1.98 & 22.9 \\
\hline 1 & Ustd & 1 & 1 & 2 & 2.13 & 2.75 & 9.31 & 1.36 & 21.1 \\
\hline 1 & ROC-10 & 1 & 1 & 3 & 1.94 & 1.62 & 9.91 & 2.05 & 21.3 \\
\hline 1 & ROC-10 & 1 & 1 & 4 & 1.98 & 1.64 & 9.94 & 2.08 & 21.5 \\
\hline 1 & ROC-01 & 1 & 1 & 5 & 4.47 & 1.5 & 4.58 & 2.09 & 13.6 \\
\hline 1 & ROC-08 & 1 & 1 & 6 & 1.98 & 1.35 & 4.03 & 2.12 & 21.3 \\
\hline 1 & ROC-03 & 1 & 1 & 7 & 2.16 & 3.04 & 9.47 & 1.81 & 20.5 \\
\hline 1 & ROC-09 & 1 & 1 & 8 & 2.16 & 2.99 & 5.76 & 1.77 & 21 \\
\hline 1 & ROC-04 & 1 & 1 & 9 & 4.32 & 1.6 & 4.7 & 2.74 & 19.5 \\
\hline 1 & ROC-03 & 1 & 1 & 10 & 2.19 & 3.05 & 9.56 & 1.83 & 20.7 \\
\hline 1 & Batch 1 & 1 & 1 & 11 & 2.55 & 2.28 & 8.91 & 1.97 & 23.1 \\
\hline 1 & Ustd & 1 & 1 & 12 & 2.15 & 2.75 & 9.33 & 1.37 & 21.1 \\
\hline 1 & ROC-04 & 1 & 1 & 13 & 4.35 & 1.62 & 4.77 & 2.74 & 19.6 \\
\hline 1 & ROC-15 & 1 & 1 & 14 & 3.84 & 1.33 & 7.13 & 1.78 & 20.6 \\
\hline 1 & ROC-09 & 1 & 1 & 15 & 2.16 & 2.97 & 5.76 & 1.76 & 20.9 \\
\hline 1 & ROC-15 & 1 & 1 & 16 & 3.85 & 1.34 & 7.13 & 1.8 & 20.6 \\
\hline 1 & ROC-11 & 1 & 1 & 17 & 1.9 & 1.34 & 3.87 & 2.02 & 23 \\
\hline 1 & ROC-01 & 1 & 1 & 18 & 5.81 & 1.9 & 5.99 & 2.64 & 17.7 \\
\hline 1 & ROC-11 & 1 & 1 & 19 & 1.88 & 1.33 & 3.84 & 2.02 & 23.1 \\
\hline 1 & ROC-08 & 1 & 1 & 20 & 2.02 & 1.34 & 4.19 & 2.15 & 21.8 \\
\hline 1 & Batch 1 & 1 & 1 & 21 & 2.56 & 2.27 & 8.9 & 1.96 & 23.0 \\
\hline 1 & Ustd & 1 & 1 & 22 & 2.16 & 2.73 & 9.31 & 1.35 & 21.1 \\
\hline 1 & Batch 1 & 1 & 2 & 1 & 2.57 & 2.27 & 8.94 & 1.94 & 23.1 \\
\hline 1 & Ustd & 1 & 2 & 2 & 2.15 & 2.76 & 9.42 & 1.34 & 21.2 \\
\hline 1 & ROC-01 & 1 & 2 & 3 & 4.48 & 1.49 & 4.65 & 2.04 & 13.8 \\
\hline 1 & ROC-09 & 1 & 2 & 4 & 2.16 & 2.99 & 5.84 & 1.73 & 21.1 \\
\hline 1 & ROC-03 & 1 & 2 & 5 & 2.21 & 3.06 & 9.71 & 1.8 & 21 \\
\hline 1 & ROC-01 & 1 & 2 & 6 & 5.82 & 1.89 & 6.06 & 2.58 & 17.8 \\
\hline 1 & ROC-15 & 1 & 2 & 7 & 3.85 & 1.34 & 7.19 & 1.75 & 20.6 \\
\hline 1 & ROC-09 & 1 & 2 & 8 & 2.17 & 2.99 & 5.82 & 1.72 & 21 \\
\hline 1 & ROC-08 & 1 & 2 & 9 & 1.99 & 1.33 & 4.11 & 2.07 & 21.5 \\
\hline 1 & ROC-10 & 1 & 2 & 10 & 1.95 & 1.61 & 10 & 2 & 21.4 \\
\hline 1 & Batch 1 & 1 & 2 & 11 & 2.56 & 2.28 & 9.02 & 1.94 & 23.2 \\
\hline 1 & Ustd & 1 & 2 & 12 & 2.14 & 2.75 & 9.4 & 1.33 & 21.2 \\
\hline 1 & ROC-11 & 1 & 2 & 13 & 1.91 & 1.37 & 3.91 & 1.98 & 23.1 \\
\hline 1 & ROC-11 & 1 & 2 & 14 & 1.88 & 1.35 & 3.88 & 1.98 & 23.1 \\
\hline 1 & ROC-04 & 1 & 2 & 15 & 4.35 & 1.61 & 4.76 & 2.68 & 19.6 \\
\hline 1 & ROC-04 & 1 & 2 & 16 & 4.36 & 1.61 & 4.78 & 2.7 & 19.6 \\
\hline 1 & ROC-15 & 1 & 2 & 17 & 3.85 & 1.34 & 7.21 & 1.75 & 20.6 \\
\hline 1 & ROC-08 & 1 & 2 & 18 & 2.03 & 1.35 & 4.24 & 2.11 & 21.8 \\
\hline 1 & ROC-03 & 1 & 2 & 19 & 2.18 & 3.02 & 9.62 & 1.76 & 20.7 \\
\hline 1 & ROC-10 & 1 & 2 & 20 & 1.97 & 1.63 & 10.1 & 2.02 & 21.6 \\
\hline 1 & Batch 1 & 1 & 2 & 21 & 2.55 & 2.27 & 8.98 & 1.93 & 23.1 \\
\hline 1 & Ustd & 1 & 2 & 22 & 2.14 & 2.75 & 9.41 & 1.33 & 21.1 \\
\hline 1 & Batch 1 & 2 & 1 & 1 & 2.57 & 2.27 & 8.94 & 1.99 & 23 \\
\hline 1 & Ustd & 2 & 1 & 2 & 2.16 & 2.73 & 9.3 & 1.36 & 21.1 \\
\hline 1 & ROC-13 & 2 & 1 & 3 & 4.71 & 1.11 & 3.83 & 2.09 & 17.4 \\
\hline 1 & ROC-05 & 2 & 1 & 4 & 2.5 & 2.01 & 9.99 & 1.77 & 19.4 \\
\hline 1 & ROC-06 & 2 & 1 & 5 & 2.85 & 4.2 & 3.28 & 1.85 & 19.1 \\
\hline 1 & ROC-07 & 2 & 1 & 6 & 0.869 & 1.81 & 3.01 & 1.07 & 10.8 \\
\hline 1 & ROC-06 & 2 & 1 & 7 & 2.88 & 4.23 & 3.49 & 1.87 & 19.4 \\
\hline 1 & ROC-14 & 2 & 1 & 8 & 6.05 & 3.90 & 4.59 & 2.37 & 18 \\
\hline 1 & ROC-05 & 2 & 1 & 9 & 2.51 & 2.05 & 10.1 & 1.79 & 19.5 \\
\hline 1 & Batch 1 & 2 & 1 & 10 & 2.55 & 2.26 & 8.87 & 1.96 & 22.9 \\
\hline 1 & Ustd & 2 & 1 & 11 & 2.17 & 2.76 & 9.43 & 1.36 & 21.3 \\
\hline 1 & ROC-02 & 2 & 1 & 12 & 3.09 & 2.92 & 4.56 & 1.76 & 20 \\
\hline 1 & ROC-12 & 2 & 1 & 13 & 3.02 & 2.28 & 4.23 & 2.96 & 22.8 \\
\hline 1 & ROC-14 & 2 & 1 & 14 & 6.03 & 3.89 & 4.58 & 2.35 & 18.1 \\
\hline 1 & ROC-12 & 2 & 1 & 15 & 2.97 & 2.25 & 4.17 & 2.95 & 22.5 \\
\hline 1 & ROC-13 & 2 & 1 & 16 & 5.73 & 1.34 & 4.63 & 2.57 & 21.2 \\
\hline 1 & ROC-07 & 2 & 1 & 17 & 1.96 & 4.06 & 6.82 & 2.38 & 24.4 \\
\hline 1 & ROC-02 & 2 & 1 & 18 & 3.14 & 2.96 & 4.6 & 1.78 & 20.2 \\
\hline 1 & Batch 1 & 2 & 1 & 19 & 2.55 & 2.26 & 8.91 & 1.95 & 23.0 \\
\hline 1 & Ustd & 2 & 1 & 20 & 2.15 & 2.73 & 9.29 & 1.35 & 21.0 \\
\hline 1 & Batch 1 & 2 & 2 & 1 & 2.55 & 2.24 & 8.85 & 1.96 & 23 \\
\hline 1 & Ustd & 2 & 2 & 2 & 2.15 & 2.69 & 9.28 & 1.35 & 21.2 \\
\hline 1 & ROC-12 & 2 & 2 & 3 & 3.02 & 2.24 & 4.19 & 2.91 & 22.9 \\
\hline 1 & ROC-05 & 2 & 2 & 4 & 2.53 & 2.00 & 10.1 & 1.78 & 19.6 \\
\hline 1 & ROC-13 & 2 & 2 & 5 & 4.69 & 1.06 & 3.81 & 2.08 & 17.5 \\
\hline 1 & ROC-14 & 2 & 2 & 6 & 6.07 & 3.84 & 4.55 & 2.34 & 18 \\
\hline
\end{tabular}


SRNL-STI-2009-00465, Revision 0

Table A2. Measured Elemental Concentrations (wt\%) for the Study Glasses Prepared Using Peroxide Fusion

\begin{tabular}{|c|c|c|c|c|c|c|c|c|c|}
\hline Set & Glass ID & Block & Sub-Block & Sequence & Al (wt\%) & B (wt\%) & Fe (wt\%) & $\mathrm{Li}(w \mathrm{w} \%)$ & $\mathrm{Si}(\mathrm{wt} \%)$ \\
\hline 1 & ROC-07 & 2 & 2 & 7 & 0.87 & 1.78 & 3.01 & 1.05 & 10.8 \\
\hline 1 & ROC-14 & 2 & 2 & 8 & 6.04 & 3.85 & 4.56 & 2.34 & 18 \\
\hline 1 & ROC-07 & 2 & 2 & 9 & 1.95 & 4.03 & 6.79 & 2.39 & 24.4 \\
\hline 1 & Batch 1 & 2 & 2 & 10 & 2.56 & 2.21 & 8.81 & 1.96 & 23 \\
\hline 1 & Ustd & 2 & 2 & 11 & 2.17 & 2.71 & 9.31 & 1.36 & 21.3 \\
\hline 1 & ROC-12 & 2 & 2 & 12 & 2.99 & 2.24 & 4.15 & 2.9 & 22.5 \\
\hline 1 & ROC-02 & 2 & 2 & 13 & 3.16 & 2.95 & 4.58 & 1.74 & 20.3 \\
\hline 1 & ROC-02 & 2 & 2 & 14 & 3.09 & 2.91 & 4.51 & 1.72 & 19.9 \\
\hline 1 & ROC-06 & 2 & 2 & 15 & 2.85 & 4.18 & 3.27 & 1.81 & 19.1 \\
\hline 1 & ROC-13 & 2 & 2 & 16 & 5.72 & 1.32 & 4.59 & 2.52 & 21.1 \\
\hline 1 & ROC-06 & 2 & 2 & 17 & 2.88 & 4.23 & 3.49 & 1.82 & 19.5 \\
\hline 1 & ROC-05 & 2 & 2 & 18 & 2.49 & 1.98 & 9.95 & 1.76 & 19.4 \\
\hline 1 & Batch 1 & 2 & 2 & 19 & 2.56 & 2.23 & 8.84 & 1.95 & 23 \\
\hline 1 & Ustd & 2 & 2 & 20 & 2.15 & 2.69 & 9.28 & 1.34 & 21.1 \\
\hline 2 & Batch 1 & 1 & 1 & 1 & 2.550 & 2.230 & 8.910 & 1.960 & \\
\hline 2 & Ustd 1 & 1 & 1 & 2 & 2.140 & 2.710 & 9.380 & 1.360 & \\
\hline 2 & ROC-27 & 1 & 1 & 3 & 2.320 & 1.330 & 10.200 & 1.820 & \\
\hline 2 & ROC-27 & 1 & 1 & 4 & 2.280 & 1.310 & 10.100 & 1.830 & \\
\hline 2 & ROC-16 & 1 & 1 & 5 & 1.830 & 2.720 & 3.700 & 2.990 & \\
\hline 2 & ROC-23 & 1 & 1 & 6 & 5.610 & 1.640 & 4.840 & 2.190 & \\
\hline 2 & ROC-18 & 1 & 1 & 7 & 2.140 & 1.470 & 7.760 & 1.860 & \\
\hline 2 & ROC-25 & 1 & 1 & 8 & 2.900 & 1.380 & 8.640 & 1.910 & \\
\hline 2 & ROC-19 & 1 & 1 & 9 & 2.150 & 3.760 & 3.380 & 1.760 & \\
\hline 2 & ROC-18 & 1 & 1 & 10 & 2.160 & 1.490 & 7.880 & 1.870 & \\
\hline 2 & ROC-26 & 1 & 1 & 11 & 5.060 & 1.870 & 3.580 & 1.850 & \\
\hline 2 & Batch 1 & 1 & 1 & 12 & 2.580 & 2.210 & 8.960 & 2.030 & \\
\hline 2 & Ustd 1 & 1 & 1 & 13 & 2.170 & 2.640 & 9.330 & 1.390 & \\
\hline 2 & ROC-19 & 1 & 1 & 14 & 2.210 & 3.870 & 3.410 & 1.810 & \\
\hline 2 & FY09EM21-29 & 1 & 1 & 15 & 3.820 & 1.980 & 8.550 & 2.260 & \\
\hline 2 & ROC-25 & 1 & 1 & 16 & 2.810 & 1.360 & 8.150 & 1.820 & \\
\hline 2 & FY09EM21-29 & 1 & 1 & 17 & 3.860 & 2.020 & 8.720 & 2.290 & \\
\hline 2 & ROC-28 & 1 & 1 & 18 & 2.540 & 1.310 & 4.350 & 1.880 & \\
\hline 2 & ROC-16 & 1 & 1 & 19 & 1.850 & 2.730 & 3.630 & 3.020 & \\
\hline 2 & ROC-28 & 1 & 1 & 20 & 2.540 & 1.320 & 4.390 & 1.900 & \\
\hline 2 & ROC-23 & 1 & 1 & 21 & 5.640 & 1.680 & 4.850 & 2.230 & \\
\hline 2 & ROC-26 & 1 & 1 & 22 & 5.080 & 1.930 & 3.560 & 1.870 & \\
\hline 2 & Batch 1 & 1 & 1 & 23 & 2.580 & 2.260 & 8.950 & 2.030 & \\
\hline 2 & Ustd 1 & 1 & 1 & 24 & 2.190 & 2.720 & 9.420 & 1.400 & \\
\hline 2 & Batch 1 & 1 & 2 & 1 & 2.570 & 2.260 & 8.900 & 1.970 & \\
\hline 2 & Ustd 1 & 1 & 2 & 2 & 2.170 & 2.740 & 9.400 & 1.370 & \\
\hline 2 & ROC-16 & 1 & 2 & 3 & 1.810 & 2.790 & 3.710 & 2.980 & \\
\hline 2 & ROC-25 & 1 & 2 & 4 & 2.880 & 1.430 & 8.710 & 1.900 & \\
\hline 2 & ROC-18 & 1 & 2 & 5 & 2.150 & 1.530 & 7.930 & 1.870 & \\
\hline 2 & ROC-16 & 1 & 2 & 6 & 1.830 & 2.760 & 3.700 & 2.980 & \\
\hline 2 & FY09EM21-29 & 1 & 2 & 7 & 3.810 & 2.050 & 8.960 & 2.280 & \\
\hline 2 & ROC-25 & 1 & 2 & 8 & 2.780 & 1.360 & 8.360 & 1.830 & \\
\hline 2 & ROC-23 & 1 & 2 & 9 & 5.620 & 1.700 & 4.930 & 2.220 & \\
\hline 2 & ROC-27 & 1 & 2 & 10 & 2.380 & 1.350 & 10.600 & 1.880 & \\
\hline 2 & ROC-26 & 1 & 2 & 11 & 5.060 & 1.960 & 3.650 & 1.870 & \\
\hline 2 & Batch 1 & 1 & 2 & 12 & 2.570 & 2.300 & 9.230 & 2.030 & \\
\hline 2 & Ustd 1 & 1 & 2 & 13 & 2.160 & 2.790 & 9.670 & 1.400 & \\
\hline 2 & ROC-28 & 1 & 2 & 14 & 2.530 & 1.350 & 4.130 & 1.900 & \\
\hline 2 & ROC-28 & 1 & 2 & 15 & 2.570 & 1.360 & 4.160 & 1.910 & \\
\hline 2 & ROC-19 & 1 & 2 & 16 & 2.170 & 3.880 & 3.200 & 1.760 & \\
\hline 2 & ROC-19 & 1 & 2 & 17 & 2.220 & 4.010 & 3.320 & 1.810 & \\
\hline 2 & FY09EM21-29 & 1 & 2 & 18 & 3.840 & 2.070 & 8.650 & 2.250 & \\
\hline 2 & ROC-23 & 1 & 2 & 19 & 5.650 & 1.740 & 4.660 & 2.240 & \\
\hline 2 & ROC-18 & 1 & 2 & 20 & 2.170 & 1.580 & 7.860 & 1.890 & \\
\hline 2 & ROC-27 & 1 & 2 & 21 & 2.330 & 1.420 & 10.400 & 1.870 & \\
\hline 2 & ROC-26 & 1 & 2 & 22 & 5.110 & 2.010 & 3.500 & 1.880 & \\
\hline 2 & Batch 1 & 1 & 2 & 23 & 2.600 & 2.410 & 9.200 & 2.040 & \\
\hline 2 & Ustd 1 & 1 & 2 & 24 & 2.180 & 2.900 & 9.680 & 1.430 & \\
\hline 2 & Batch 1 & 2 & 1 & 1 & 2.450 & 2.270 & 8.670 & 1.930 & \\
\hline 2 & Ustd 1 & 2 & 1 & 2 & 2.060 & 2.730 & 9.180 & 1.350 & \\
\hline 2 & ROC-30 & 2 & 1 & 3 & 3.310 & 2.390 & 6.100 & 2.050 & \\
\hline 2 & ROC-20 & 2 & 1 & 4 & 1.850 & 2.910 & 5.680 & 1.710 & \\
\hline 2 & ROC-21 & 2 & 1 & 5 & 5.400 & 3.410 & 5.380 & 1.810 & \\
\hline 2 & ROC-22 & 2 & 1 & 6 & 1.790 & 1.990 & 10.000 & 2.030 & \\
\hline 2 & ROC-21 & 2 & 1 & 7 & 5.430 & 3.420 & 5.400 & 1.820 & \\
\hline 2 & FY09EM21-28 & 2 & 1 & 8 & 3.750 & 2.070 & 8.680 & 2.210 & \\
\hline
\end{tabular}


SRNL-STI-2009-00465, Revision 0

Table A2. Measured Elemental Concentrations (wt\%) for the Study Glasses Prepared Using Peroxide Fusion

\begin{tabular}{|c|c|c|c|c|c|c|c|c|c|}
\hline Set & Glass ID & Block & Sub-Block & Sequence & $\mathrm{Al}(\mathrm{wt} \%)$ & B (wt\%) & $\mathrm{Fe}(w t \%)$ & Li (wt\%) & $\mathrm{Si}(w \mathrm{t} \%)$ \\
\hline 2 & ROC-20 & 2 & 1 & 9 & 1.900 & 3.010 & 5.890 & 1.770 & \\
\hline 2 & FY09EM21-30 & 2 & 1 & 10 & 3.600 & 1.980 & 8.590 & 2.090 & \\
\hline 2 & ROC-24 & 2 & 1 & 11 & 5.470 & 3.280 & 4.580 & 1.760 & \\
\hline 2 & Batch 1 & 2 & 1 & 12 & 2.510 & 2.310 & 8.930 & 1.960 & \\
\hline 2 & Ustd 1 & 2 & 1 & 13 & 2.100 & 2.770 & 9.340 & 1.340 & \\
\hline 2 & ROC-17 & 2 & 1 & 14 & 3.430 & 1.490 & 6.780 & 2.030 & \\
\hline 2 & ROC-29 & 2 & 1 & 15 & 5.920 & 3.810 & 5.320 & 2.060 & \\
\hline 2 & FY09EM21-28 & 2 & 1 & 16 & 3.820 & 2.110 & 8.830 & 2.190 & \\
\hline 2 & ROC-29 & 2 & 1 & 17 & 5.910 & 3.790 & 5.320 & 2.030 & \\
\hline 2 & ROC-30 & 2 & 1 & 18 & 3.580 & 2.560 & 6.550 & 2.170 & \\
\hline 2 & ROC-22 & 2 & 1 & 19 & 1.910 & 2.050 & 10.400 & 2.090 & \\
\hline 2 & ROC-17 & 2 & 1 & 20 & 3.400 & 1.480 & 6.790 & 2.020 & \\
\hline 2 & FY09EM21-30 & 2 & 1 & 21 & 3.680 & 2.030 & 8.770 & 2.110 & \\
\hline 2 & ROC-24 & 2 & 1 & 22 & 5.640 & 3.400 & 4.710 & 1.810 & \\
\hline 2 & Batch 1 & 2 & 1 & 23 & 2.420 & 2.210 & 8.530 & 1.820 & \\
\hline 2 & Ustd 1 & 2 & 1 & 24 & 2.170 & 2.820 & 9.550 & 1.390 & \\
\hline 2 & Batch 1 & 2 & 2 & 1 & 2.530 & 2.260 & 8.630 & 1.960 & \\
\hline 2 & Ustd 1 & 2 & 2 & 2 & 2.140 & 2.780 & 9.240 & 1.370 & \\
\hline 2 & ROC-29 & 2 & 2 & 3 & 5.930 & 3.800 & 5.220 & 2.060 & \\
\hline 2 & ROC-20 & 2 & 2 & 4 & 1.960 & 3.100 & 5.890 & 1.810 & \\
\hline 2 & ROC-30 & 2 & 2 & 5 & 3.410 & 2.470 & 6.180 & 2.100 & \\
\hline 2 & FY09EM21-28 & 2 & 2 & 6 & 3.780 & 2.090 & 8.640 & 2.210 & \\
\hline 2 & ROC-22 & 2 & 2 & 7 & 1.870 & 2.020 & 10.100 & 2.070 & \\
\hline 2 & FY09EM21-28 & 2 & 2 & 8 & 3.830 & 2.110 & 8.730 & 2.240 & \\
\hline 2 & ROC-22 & 2 & 2 & 9 & 1.840 & 2.020 & 10.100 & 2.080 & \\
\hline 2 & FY09EM21-30 & 2 & 2 & 10 & 3.710 & 2.040 & 8.700 & 2.170 & \\
\hline 2 & ROC-24 & 2 & 2 & 11 & 5.630 & 3.410 & 4.610 & 1.790 & \\
\hline 2 & Batch 1 & 2 & 2 & 12 & 2.590 & 2.360 & 9.040 & 2.020 & \\
\hline 2 & Ustd 1 & 2 & 2 & 13 & 2.150 & 2.810 & 9.450 & 1.410 & \\
\hline 2 & ROC-29 & 2 & 2 & 14 & 5.980 & 3.770 & 5.310 & 2.130 & \\
\hline 2 & ROC-17 & 2 & 2 & 15 & 3.420 & 1.490 & 6.730 & 2.130 & \\
\hline 2 & ROC-17 & 2 & 2 & 16 & 3.500 & 1.510 & 6.890 & 2.160 & \\
\hline 2 & ROC-21 & 2 & 2 & 17 & 5.590 & 3.520 & 5.540 & 1.950 & \\
\hline 2 & ROC-30 & 2 & 2 & 18 & 3.380 & 2.440 & 6.260 & 2.130 & \\
\hline 2 & ROC-21 & 2 & 2 & 19 & 5.610 & 3.520 & 5.530 & 1.930 & \\
\hline 2 & ROC-20 & 2 & 2 & 20 & 1.970 & 3.010 & 5.860 & 1.820 & \\
\hline 2 & FY09EM21-30 & 2 & 2 & 21 & 3.690 & 2.030 & 8.840 & 2.240 & \\
\hline 2 & ROC-24 & 2 & 2 & 22 & 5.740 & 3.450 & 4.760 & 1.910 & \\
\hline 2 & Batch 1 & 2 & 2 & 23 & 2.460 & 2.230 & 8.670 & 1.950 & \\
\hline 2 & Ustd 1 & 2 & 2 & 24 & 2.190 & 2.860 & 9.630 & 1.460 & \\
\hline
\end{tabular}


SRNL-STI-2009-00465, Revision 0

Table A3. Average Measured and Bias-Corrected Chemical Compositions Versus Targeted Compositions by Oxide by Glass ID

\begin{tabular}{|c|c|c|c|c|c|c|c|c|c|}
\hline & & & & Measured & & & & & \\
\hline & & & Measured & Bias-Corrected & Targeted & Diff of & Diff of & $\%$ Diff of & \% Diff of \\
\hline Set & Glass \# & Oxide & (wt\%) & (wt\%) & (wt\%) & Measured & Meas BC & Measured & Meas BC \\
\hline 1 & Batch 1 & $\mathrm{Al} 2 \mathrm{O} 3$ (wt\%) & 4.8292 & 4.8770 & 4.8770 & -0.0478 & 0.0000 & $-1.0 \%$ & $0.0 \%$ \\
\hline 1 & Batch 1 & B2O3 (wt\%) & 7.2743 & 7.7770 & 7.7770 & -0.5027 & 0.0000 & $-6.5 \%$ & $0.0 \%$ \\
\hline 1 & Batch 1 & $\mathrm{BaO}$ (wt\%) & 0.1504 & 0.1510 & 0.1510 & -0.0006 & 0.0000 & $-0.4 \%$ & $0.0 \%$ \\
\hline 1 & Batch 1 & $\mathrm{CaO}(\mathrm{wt} \%)$ & 1.2532 & 1.2200 & 1.2200 & 0.0332 & 0.0000 & $2.7 \%$ & $0.0 \%$ \\
\hline 1 & Batch 1 & CdO (wt\%) & 0.0040 & 0.0040 & 0.0000 & 0.0040 & 0.0040 & & \\
\hline 1 & Batch 1 & Ce2O3 (wt\%) & 0.0059 & 0.0059 & 0.0000 & 0.0059 & 0.0059 & & \\
\hline 1 & Batch 1 & Cr2O3 (wt\%) & 0.1081 & 0.1070 & 0.1070 & 0.0011 & 0.0000 & $1.0 \%$ & $0.0 \%$ \\
\hline 1 & Batch 1 & $\mathrm{CuO}$ (wt\%) & 0.3729 & 0.3990 & 0.3990 & -0.0261 & 0.0000 & $-6.5 \%$ & $0.0 \%$ \\
\hline 1 & Batch 1 & $\mathrm{Fe} 2 \mathrm{O} 3$ (wt\%) & 12.7243 & 12.8390 & 12.8390 & -0.1147 & 0.0000 & $-0.9 \%$ & $0.0 \%$ \\
\hline 1 & Batch 1 & K2O (wt\%) & 3.2896 & 3.3270 & 3.3270 & -0.0374 & 0.0000 & $-1.1 \%$ & $0.0 \%$ \\
\hline 1 & Batch 1 & La2O3 (wt\%) & 0.0053 & 0.0053 & 0.0000 & 0.0053 & 0.0053 & & \\
\hline 1 & Batch 1 & Li2O (wt\%) & 4.2143 & 4.4290 & 4.4290 & -0.2147 & 0.0000 & $-4.8 \%$ & $0.0 \%$ \\
\hline 1 & Batch 1 & $\mathrm{MgO}(\mathrm{wt} \%)$ & 1.4232 & 1.4190 & 1.4190 & 0.0042 & 0.0000 & $0.3 \%$ & $0.0 \%$ \\
\hline 1 & Batch 1 & $\mathrm{MnO}(\mathrm{wt} \%)$ & 1.7205 & 1.7260 & 1.7260 & -0.0055 & 0.0000 & $-0.3 \%$ & $0.0 \%$ \\
\hline 1 & Batch 1 & $\mathrm{Na} 2 \mathrm{O}$ (wt\%) & 9.1821 & 9.0030 & 9.0030 & 0.1791 & 0.0000 & $2.0 \%$ & $0.0 \%$ \\
\hline 1 & Batch 1 & $\mathrm{NiO}(\mathrm{wt} \%)$ & 0.7757 & 0.7510 & 0.7510 & 0.0247 & 0.0000 & $3.3 \%$ & $0.0 \%$ \\
\hline 1 & Batch 1 & $\mathrm{PbO}(\mathrm{wt} \%)$ & 0.0086 & 0.0086 & 0.0000 & 0.0086 & 0.0086 & & \\
\hline 1 & Batch 1 & $\mathrm{SiO} 2$ (wt\%) & 49.2574 & 50.2200 & 50.2200 & -0.9626 & 0.0000 & $-1.9 \%$ & $0.0 \%$ \\
\hline 1 & Batch 1 & SO4 (wt\%) & 0.2247 & 0.2247 & 0.0000 & 0.2247 & 0.2247 & & \\
\hline 1 & Batch 1 & TiO2 (wt\%) & 0.6682 & 0.6770 & 0.6770 & -0.0088 & 0.0000 & $-1.3 \%$ & $0.0 \%$ \\
\hline 1 & Batch 1 & U3O8 (wt\%) & 0.1574 & 0.1536 & 0.0000 & 0.1574 & 0.1536 & & \\
\hline 1 & Batch 1 & $\mathrm{ZnO}$ (wt\%) & 0.0124 & 0.0124 & 0.0000 & 0.0124 & 0.0124 & & \\
\hline 1 & Batch 1 & $\mathrm{ZrO} 2$ (wt\%) & 0.0884 & 0.0884 & 0.0980 & -0.0096 & -0.0096 & $-9.8 \%$ & $-9.8 \%$ \\
\hline 1 & Batch 1 & Sum & 97.7501 & 99.4249 & 99.0200 & -1.2699 & 0.4049 & $-1.3 \%$ & $0.4 \%$ \\
\hline 2 & Batch 1 & $\mathrm{Al} 2 \mathrm{O} 3$ (wt\%) & 4.7883 & 4.8770 & 4.8770 & -0.0887 & 0.0000 & $-1.8 \%$ & $0.0 \%$ \\
\hline 2 & Batch 1 & B2O3 (wt\%) & 7.3280 & 7.7770 & 7.7770 & -0.4490 & 0.0000 & $-5.8 \%$ & $0.0 \%$ \\
\hline 2 & Batch 1 & $\mathrm{BaO}(\mathrm{wt} \%)$ & 0.1480 & 0.1510 & 0.1510 & -0.0030 & 0.0000 & $-2.0 \%$ & $0.0 \%$ \\
\hline 2 & Batch 1 & $\mathrm{CaO}(\mathrm{wt} \%)$ & 1.2054 & 1.2200 & 1.2200 & -0.0146 & 0.0000 & $-1.2 \%$ & $0.0 \%$ \\
\hline 2 & Batch 1 & $\mathrm{CdO}$ (wt\%) & 0.0040 & 0.0040 & 0.0000 & 0.0040 & 0.0040 & & \\
\hline 2 & Batch 1 & Ce2O3 (wt\%) & 0.0117 & 0.0117 & 0.0000 & 0.0117 & 0.0117 & & \\
\hline 2 & Batch 1 & Cr2O3 (wt\%) & 0.1071 & 0.1070 & 0.1070 & 0.0001 & 0.0000 & $0.1 \%$ & $0.0 \%$ \\
\hline 2 & Batch 1 & $\mathrm{CuO}$ (wt\%) & 0.3894 & 0.3990 & 0.3990 & -0.0096 & 0.0000 & $-2.4 \%$ & $0.0 \%$ \\
\hline 2 & Batch 1 & Fe2O3 (wt\%) & 12.7029 & 12.8390 & 12.8390 & -0.1361 & 0.0000 & $-1.1 \%$ & $0.0 \%$ \\
\hline 2 & Batch 1 & K2O (wt\%) & 3.2615 & 3.3270 & 3.3270 & -0.0655 & 0.0000 & $-2.0 \%$ & $0.0 \%$ \\
\hline 2 & Batch 1 & La2O3 (wt\%) & 0.0053 & 0.0053 & 0.0000 & 0.0053 & 0.0053 & & \\
\hline 2 & Batch 1 & Li2O (wt\%) & 4.2520 & 4.4290 & 4.4290 & -0.1770 & 0.0000 & $-4.0 \%$ & $0.0 \%$ \\
\hline 2 & Batch 1 & $\mathrm{MgO}(\mathrm{wt} \%)$ & 1.4151 & 1.4190 & 1.4190 & -0.0039 & 0.0000 & $-0.3 \%$ & $0.0 \%$ \\
\hline 2 & Batch 1 & $\mathrm{MnO}(\mathrm{wt} \%)$ & 1.6764 & 1.7260 & 1.7260 & -0.0496 & 0.0000 & $-2.9 \%$ & $0.0 \%$ \\
\hline 2 & Batch 1 & $\mathrm{Na} 2 \mathrm{O}$ (wt\%) & 9.0136 & 9.0030 & 9.0030 & 0.0106 & 0.0000 & $0.1 \%$ & $0.0 \%$ \\
\hline 2 & Batch 1 & $\mathrm{NiO}(\mathrm{wt} \%)$ & 0.7618 & 0.7510 & 0.7510 & 0.0108 & 0.0000 & $1.4 \%$ & $0.0 \%$ \\
\hline 2 & Batch 1 & $\mathrm{PbO}(\mathrm{wt} \%)$ & 0.0054 & 0.0054 & 0.0000 & 0.0054 & 0.0054 & & \\
\hline 2 & Batch 1 & $\mathrm{SiO} 2$ (wt\%) & 49.2574 & 50.2200 & 50.2200 & -0.9626 & 0.0000 & $-1.9 \%$ & $0.0 \%$ \\
\hline 2 & Batch 1 & SO4 (wt \%) & 0.2247 & 0.2247 & 0.0000 & 0.2247 & 0.2247 & & \\
\hline 2 & Batch 1 & TiO2 (wt\%) & 0.6490 & 0.6770 & 0.6770 & -0.0280 & 0.0000 & $-4.1 \%$ & $0.0 \%$ \\
\hline 2 & Batch 1 & U3O8 (wt\%) & 0.1179 & 0.1176 & 0.0000 & 0.1179 & 0.1176 & & \\
\hline 2 & Batch 1 & $\mathrm{ZnO}(\mathrm{wt} \%)$ & 0.0124 & 0.0124 & 0.0000 & 0.0124 & 0.0124 & & \\
\hline 2 & Batch 1 & $\mathrm{ZrO} 2$ (wt\%) & 0.0861 & 0.0861 & 0.0980 & -0.0119 & -0.0119 & $-12.1 \%$ & $-12.1 \%$ \\
\hline 2 & Batch 1 & Sum & 97.4234 & 99.3893 & 99.0200 & -1.5966 & 0.3693 & $-1.6 \%$ & $0.4 \%$ \\
\hline 1 & ROC-01 & $\mathrm{Al} 2 \mathrm{O} 3$ (wt\%) & 10.9874 & 11.0997 & 10.8584 & 0.1291 & 0.2414 & $1.2 \%$ & $2.2 \%$ \\
\hline 1 & ROC-01 & B2O3 (wt\%) & 6.1017 & 6.4827 & 6.3394 & -0.2377 & 0.1433 & $-3.7 \%$ & $2.3 \%$ \\
\hline 1 & ROC-01 & $\mathrm{BaO}(\mathrm{wt} \%)$ & 0.0262 & 0.0265 & 0.0259 & 0.0003 & 0.0007 & $1.2 \%$ & $2.6 \%$ \\
\hline 1 & ROC-01 & $\mathrm{CaO}(\mathrm{wt} \%)$ & 0.6073 & 0.5936 & 0.5962 & 0.0110 & -0.0026 & $1.9 \%$ & $-0.4 \%$ \\
\hline 1 & ROC-01 & $\mathrm{CdO}(\mathrm{wt} \%)$ & 0.0848 & 0.0848 & 0.0957 & -0.0109 & -0.0109 & $-11.4 \%$ & $-11.4 \%$ \\
\hline 1 & ROC-01 & Ce2O3 (wt\%) & 0.1294 & 0.1294 & 0.1164 & 0.0131 & 0.0131 & $11.2 \%$ & $11.2 \%$ \\
\hline 1 & ROC-01 & Cr2O3 (wt\%) & 0.1739 & 0.1734 & 0.2000 & -0.0261 & -0.0266 & $-13.0 \%$ & $-13.3 \%$ \\
\hline 1 & ROC-01 & $\mathrm{CuO}$ (wt\%) & 0.0570 & 0.0609 & 0.0414 & 0.0156 & 0.0195 & $37.7 \%$ & $47.2 \%$ \\
\hline 1 & ROC-01 & Fe2O3 (wt\%) & 8.6139 & 8.6624 & 8.6212 & -0.0073 & 0.0411 & $-0.1 \%$ & $0.5 \%$ \\
\hline 1 & ROC-01 & K2O (wt\%) & 0.0657 & 0.0658 & 0.0000 & 0.0657 & 0.0658 & & \\
\hline 1 & ROC-01 & La2O3 (wt\%) & 0.0117 & 0.0117 & 0.0316 & -0.0198 & -0.0198 & $-62.8 \%$ & $-62.8 \%$ \\
\hline 1 & ROC-01 & Li2O (wt\%) & 5.6191 & 5.9178 & 5.8767 & -0.2576 & 0.0411 & $-4.4 \%$ & $0.7 \%$ \\
\hline 1 & ROC-01 & MgO (wt\%) & 1.4282 & 1.4183 & 1.5000 & -0.0718 & -0.0817 & $-4.8 \%$ & $-5.4 \%$ \\
\hline 1 & ROC-01 & $\mathrm{MnO}(\mathrm{wt} \%)$ & 5.2713 & 5.2849 & 5.5000 & -0.2287 & -0.2151 & $-4.2 \%$ & $-3.9 \%$ \\
\hline 1 & ROC-01 & $\mathrm{Na} 2 \mathrm{O}$ (wt\%) & 10.2010 & 9.9484 & 10.4607 & -0.2598 & -0.5123 & $-2.5 \%$ & $-4.9 \%$ \\
\hline 1 & ROC-01 & $\mathrm{NiO}(\mathrm{wt} \%)$ & 0.0268 & 0.0260 & 0.0000 & 0.0268 & 0.0260 & & \\
\hline 1 & ROC-01 & $\mathrm{PbO}(\mathrm{wt} \%)$ & 0.0054 & 0.0054 & 0.0698 & -0.0644 & -0.0644 & $-92.3 \%$ & $-92.3 \%$ \\
\hline 1 & ROC-01 & $\mathrm{SiO} 2$ (wt\%) & 37.9726 & 38.6447 & 37.9018 & 0.0707 & 0.7429 & $0.2 \%$ & $2.0 \%$ \\
\hline 1 & ROC-01 & SO4 (wt\%) & 0.2958 & 0.2958 & 0.1552 & 0.1407 & 0.1407 & $90.7 \%$ & $90.7 \%$ \\
\hline 1 & ROC-01 & TiO2 (wt\%) & 1.9057 & 1.9313 & 2.0000 & -0.0943 & -0.0687 & $-4.7 \%$ & $-3.4 \%$ \\
\hline 1 & ROC-01 & U3O8 (wt\%) & 8.8322 & 8.6575 & 9.5000 & -0.6678 & -0.8425 & $-7.0 \%$ & $-8.9 \%$ \\
\hline
\end{tabular}


SRNL-STI-2009-00465, Revision 0

Table A3. Average Measured and Bias-Corrected Chemical Compositions Versus Targeted Compositions by Oxide by Glass ID

\begin{tabular}{|c|c|c|c|c|c|c|c|c|c|}
\hline & & & & Measured & & & & & \\
\hline & & & Measured & Bias-Corrected & Targeted & Diff of & Diff of & \% Diff of & $\%$ Diff of \\
\hline Set & Glass \# & Oxide & (wt\%) & (wt\%) & (wt\%) & Measured & Meas BC & Measured & Meas BC \\
\hline 1 & ROC-01 & $\mathrm{ZnO}$ (wt\%) & 0.0492 & 0.0492 & 0.0434 & 0.0057 & 0.0057 & $13.2 \%$ & $13.2 \%$ \\
\hline 1 & ROC-01 & $\mathrm{ZrO} 2$ (wt\%) & 0.0034 & 0.0034 & 0.0662 & -0.0628 & -0.0628 & $-94.9 \%$ & $-94.9 \%$ \\
\hline 1 & ROC-01 & Sum & 98.4697 & 99.5737 & 100.0000 & -1.5303 & -0.4263 & $-1.5 \%$ & $-0.4 \%$ \\
\hline 1 & ROC-02 & $\mathrm{Al} 2 \mathrm{O} 3$ (wt\%) & 5.8952 & 5.9516 & 5.9109 & -0.0156 & 0.0407 & $-0.3 \%$ & $0.7 \%$ \\
\hline 1 & ROC-02 & B2O3 (wt\%) & 9.4504 & 10.1678 & 10.1906 & -0.7402 & -0.0228 & $-7.3 \%$ & $-0.2 \%$ \\
\hline 1 & ROC-02 & $\mathrm{BaO}(\mathrm{wt} \%)$ & 0.0011 & 0.0011 & 0.0000 & 0.0011 & 0.0011 & & \\
\hline 1 & ROC-02 & $\mathrm{CaO}$ (wt\%) & 3.7743 & 3.6599 & 4.0000 & -0.2257 & -0.3401 & $-5.6 \%$ & $-8.5 \%$ \\
\hline 1 & ROC-02 & $\mathrm{CdO}(\mathrm{wt} \%)$ & 0.0040 & 0.0040 & 0.0000 & 0.0040 & 0.0040 & & \\
\hline 1 & ROC-02 & Ce2O3 (wt\%) & 0.0059 & 0.0059 & 0.0000 & 0.0059 & 0.0059 & & \\
\hline 1 & ROC-02 & Cr2O3 (wt\%) & 0.1575 & 0.1548 & 0.1800 & -0.0225 & -0.0252 & $-12.5 \%$ & $-14.0 \%$ \\
\hline 1 & ROC-02 & $\mathrm{CuO}$ (wt\%) & 0.0163 & 0.0174 & 0.0000 & 0.0163 & 0.0174 & & \\
\hline 1 & ROC-02 & Fe2O3 (wt\%) & 6.5230 & 6.6041 & 6.6982 & -0.1752 & -0.0942 & $-2.6 \%$ & $-1.4 \%$ \\
\hline 1 & ROC-02 & K2O (wt\%) & 0.0428 & 0.0437 & 0.0000 & 0.0428 & 0.0437 & & \\
\hline 1 & ROC-02 & La2O3 (wt\%) & 0.0053 & 0.0053 & 0.0000 & 0.0053 & 0.0053 & & \\
\hline 1 & ROC-02 & Li2O (wt\%) & 3.7676 & 3.9510 & 4.0000 & -0.2324 & -0.0490 & $-5.8 \%$ & $-1.2 \%$ \\
\hline 1 & ROC-02 & MgO (wt\%) & 1.3250 & 1.3263 & 1.3500 & -0.0250 & -0.0237 & $-1.9 \%$ & $-1.8 \%$ \\
\hline 1 & ROC-02 & $\mathrm{MnO}(\mathrm{wt} \%)$ & 0.3018 & 0.3030 & 0.3000 & 0.0018 & 0.0030 & $0.6 \%$ & $1.0 \%$ \\
\hline 1 & ROC-02 & $\mathrm{Na} 2 \mathrm{O}(\mathrm{wt} \%)$ & 13.2879 & 13.0992 & 13.8866 & -0.5987 & -0.7874 & $-4.3 \%$ & $-5.7 \%$ \\
\hline 1 & ROC-02 & $\mathrm{NiO}(\mathrm{wt} \%)$ & 2.4209 & 2.3423 & 2.5000 & -0.0791 & -0.1577 & $-3.2 \%$ & $-6.3 \%$ \\
\hline 1 & ROC-02 & $\mathrm{PbO}$ (wt\%) & 0.0054 & 0.0054 & 0.0000 & 0.0054 & 0.0054 & & \\
\hline 1 & ROC-02 & SiO2 (wt\%) & 42.9999 & 43.9198 & 44.7108 & -1.7109 & -0.7911 & $-3.8 \%$ & $-1.8 \%$ \\
\hline 1 & ROC-02 & SO4 (wt\%) & 0.2247 & 0.2247 & 0.0000 & 0.2247 & 0.2247 & & \\
\hline 1 & ROC-02 & $\mathrm{TiO} 2$ (wt\%) & 1.8765 & 1.9010 & 1.9601 & -0.0836 & -0.0591 & $-4.3 \%$ & $-3.0 \%$ \\
\hline 1 & ROC-02 & U3O8 (wt\%) & 4.0977 & 3.9814 & 4.3129 & -0.2151 & -0.3315 & $-5.0 \%$ & $-7.7 \%$ \\
\hline 1 & ROC-02 & $\mathrm{ZnO}(\mathrm{wt} \%)$ & 0.0124 & 0.0124 & 0.0000 & 0.0124 & 0.0124 & & \\
\hline 1 & ROC-02 & $\mathrm{ZrO} 2$ (wt\%) & 0.0108 & 0.0108 & 0.0000 & 0.0108 & 0.0108 & & \\
\hline 1 & ROC-02 & Sum & 96.2065 & 97.6927 & 100.0000 & -3.7935 & -2.3073 & $-3.8 \%$ & $-2.3 \%$ \\
\hline 1 & ROC-03 & $\mathrm{Al} 2 \mathrm{O} 3$ (wt\%) & 4.1286 & 4.1707 & 3.7616 & 0.3670 & 0.4091 & $9.8 \%$ & $10.9 \%$ \\
\hline 1 & ROC-03 & B2O3 (wt\%) & 9.7965 & 10.4083 & 9.9798 & -0.1833 & 0.4285 & $-1.8 \%$ & $4.3 \%$ \\
\hline 1 & ROC-03 & $\mathrm{BaO}(\mathrm{wt} \%)$ & 0.0798 & 0.0809 & 0.0801 & -0.0003 & 0.0007 & $-0.4 \%$ & $0.9 \%$ \\
\hline 1 & ROC-03 & $\mathrm{CaO}$ (wt\%) & 0.1077 & 0.1052 & 0.0009 & 0.1067 & 0.1043 & $11240.2 \%$ & $10985.4 \%$ \\
\hline 1 & ROC-03 & $\mathrm{CdO}(\mathrm{wt} \%)$ & 0.2679 & 0.2679 & 0.2965 & -0.0286 & -0.0286 & $-9.6 \%$ & $-9.6 \%$ \\
\hline 1 & ROC-03 & Ce2O3 (wt\%) & 0.3698 & 0.3698 & 0.3606 & 0.0093 & 0.0093 & $2.6 \%$ & $2.6 \%$ \\
\hline 1 & ROC-03 & Cr2O3 (wt\%) & 0.0073 & 0.0073 & 0.0000 & 0.0073 & 0.0073 & & \\
\hline 1 & ROC-03 & $\mathrm{CuO}(\mathrm{wt} \%)$ & 0.1302 & 0.1392 & 0.1282 & 0.0020 & 0.0110 & $1.5 \%$ & $8.6 \%$ \\
\hline 1 & ROC-03 & Fe2O3 (wt\%) & 13.7108 & 13.7877 & 14.1391 & -0.4283 & -0.3514 & $-3.0 \%$ & $-2.5 \%$ \\
\hline 1 & ROC-03 & K2O (wt\%) & 0.0376 & 0.0378 & 0.0000 & 0.0376 & 0.0378 & & \\
\hline 1 & ROC-03 & La2O3 (wt\%) & 0.0815 & 0.0815 & 0.0978 & -0.0162 & -0.0162 & $-16.6 \%$ & $-16.6 \%$ \\
\hline 1 & ROC-03 & Li2O (wt\%) & 3.8752 & 4.0812 & 4.0000 & -0.1248 & 0.0812 & $-3.1 \%$ & $2.0 \%$ \\
\hline 1 & ROC-03 & $\mathrm{MgO}(\mathrm{wt} \%)$ & 0.0149 & 0.0148 & 0.0000 & 0.0149 & 0.0148 & & \\
\hline 1 & ROC-03 & $\mathrm{MnO}(\mathrm{wt} \%)$ & 0.4787 & 0.4799 & 0.4843 & -0.0055 & -0.0043 & $-1.1 \%$ & $-0.9 \%$ \\
\hline 1 & ROC-03 & Na2O (wt\%) & 9.9314 & 9.6855 & 10.0010 & -0.0696 & -0.3155 & $-0.7 \%$ & $-3.2 \%$ \\
\hline 1 & ROC-03 & $\mathrm{NiO}(\mathrm{wt} \%)$ & 0.0264 & 0.0256 & 0.0000 & 0.0264 & 0.0256 & & \\
\hline 1 & ROC-03 & $\mathrm{PbO}(\mathrm{wt} \%)$ & 0.2176 & 0.2176 & 0.2163 & 0.0012 & 0.0012 & $0.6 \%$ & $0.6 \%$ \\
\hline 1 & ROC-03 & SiO2 (wt\%) & 44.3370 & 45.1214 & 44.2513 & 0.0857 & 0.8701 & $0.2 \%$ & $2.0 \%$ \\
\hline 1 & ROC-03 & SO4 (wt\%) & 0.6666 & 0.6666 & 0.4808 & 0.1858 & 0.1858 & $38.7 \%$ & $38.7 \%$ \\
\hline 1 & ROC-03 & TiO2 (wt\%) & 1.7931 & 1.8171 & 2.0000 & -0.2069 & -0.1829 & $-10.3 \%$ & $-9.1 \%$ \\
\hline 1 & ROC-03 & U3O8 (wt\%) & 8.8528 & 8.6774 & 9.3820 & -0.5291 & -0.7045 & $-5.6 \%$ & $-7.5 \%$ \\
\hline 1 & ROC-03 & $\mathrm{ZnO}$ (wt\%) & 0.1397 & 0.1397 & 0.1346 & 0.0051 & 0.0051 & $3.8 \%$ & $3.8 \%$ \\
\hline 1 & ROC-03 & $\mathrm{ZrO} 2$ (wt\%) & 0.1898 & 0.1898 & 0.2051 & -0.0153 & -0.0153 & $-7.5 \%$ & $-7.5 \%$ \\
\hline 1 & ROC-03 & Sum & 99.2411 & 100.5731 & 100.0000 & -0.7589 & 0.5731 & $-0.8 \%$ & $0.6 \%$ \\
\hline 1 & ROC-04 & $\mathrm{Al} 2 \mathrm{O} 3$ (wt\%) & 8.2099 & 8.2938 & 7.9615 & 0.2484 & 0.3323 & $3.1 \%$ & $4.2 \%$ \\
\hline 1 & ROC-04 & B2O3 (wt $\%)$ & 5.1840 & 5.5078 & 5.4163 & -0.2323 & 0.0914 & $-4.3 \%$ & $1.7 \%$ \\
\hline 1 & ROC-04 & $\mathrm{BaO}(\mathrm{wt} \%)$ & 0.0772 & 0.0769 & 0.0801 & -0.0029 & -0.0032 & $-3.6 \%$ & $-4.1 \%$ \\
\hline 1 & ROC-04 & $\mathrm{CaO}$ (wt\%) & 3.7813 & 3.6667 & 4.0000 & -0.2187 & -0.3333 & $-5.5 \%$ & $-8.3 \%$ \\
\hline 1 & ROC-04 & CdO (wt\%) & 0.2579 & 0.2579 & 0.2965 & -0.0386 & -0.0386 & $-13.0 \%$ & $-13.0 \%$ \\
\hline 1 & ROC-04 & Ce2O3 (wt\%) & 0.3813 & 0.3813 & 0.3606 & 0.0207 & 0.0207 & $5.7 \%$ & $5.7 \%$ \\
\hline 1 & ROC-04 & Cr2O3 (wt\%) & 0.0073 & 0.0072 & 0.0000 & 0.0073 & 0.0072 & & \\
\hline 1 & ROC-04 & $\mathrm{CuO}(\mathrm{wt} \%)$ & 0.1283 & 0.1374 & 0.1282 & 0.0001 & 0.0092 & $0.1 \%$ & $7.2 \%$ \\
\hline 1 & ROC-04 & Fe2O3 (wt\%) & 6.7946 & 6.8329 & 6.7642 & 0.0305 & 0.0688 & $0.5 \%$ & $1.0 \%$ \\
\hline 1 & ROC-04 & K2O (wt\%) & 0.0401 & 0.0409 & 0.0000 & 0.0401 & 0.0409 & & \\
\hline 1 & ROC-04 & La2O3 (wt\%) & 0.0645 & 0.0645 & 0.0978 & -0.0333 & -0.0333 & $-34.0 \%$ & $-34.0 \%$ \\
\hline 1 & ROC-04 & Li2O (wt\%) & 5.8451 & 6.1560 & 6.0107 & -0.1655 & 0.1453 & $-2.8 \%$ & $2.4 \%$ \\
\hline 1 & ROC-04 & $\mathrm{MgO}(\mathrm{wt} \%)$ & 0.0099 & 0.0100 & 0.0000 & 0.0099 & 0.0100 & & \\
\hline 1 & ROC-04 & $\mathrm{MnO}(\mathrm{wt} \%)$ & 0.3183 & 0.3195 & 0.3000 & 0.0183 & 0.0195 & $6.1 \%$ & $6.5 \%$ \\
\hline 1 & ROC-04 & Na2O (wt\%) & 11.4580 & 11.2951 & 11.8055 & -0.3475 & -0.5103 & $-2.9 \%$ & $-4.3 \%$ \\
\hline 1 & ROC-04 & $\mathrm{NiO}$ (wt\%) & 2.2332 & 2.1607 & 2.5000 & -0.2668 & -0.3393 & $-10.7 \%$ & $-13.6 \%$ \\
\hline 1 & ROC-04 & $\mathrm{PbO}$ (wt\%) & 0.1939 & 0.1939 & 0.2163 & -0.0225 & -0.0225 & $-10.4 \%$ & $-10.4 \%$ \\
\hline 1 & ROC-04 & $\mathrm{SiO} 2$ (wt\%) & 41.8768 & 42.6183 & 41.7419 & 0.1349 & 0.8764 & $0.3 \%$ & $2.1 \%$ \\
\hline
\end{tabular}


SRNL-STI-2009-00465, Revision 0

Table A3. Average Measured and Bias-Corrected Chemical Compositions Versus Targeted Compositions by Oxide by Glass ID

\begin{tabular}{|c|c|c|c|c|c|c|c|c|c|}
\hline & & & & "Measured & & & & & \\
\hline & & & Measured & Bias-Corrected & Targeted & Diff of & Diff of & \% Diff of & \% Diff of \\
\hline Set & Glass \# & Oxide & (wt\%) & (wt\%) & (wt\%) & Measured & Meas BC & Measured & Meas BC \\
\hline 1 & ROC-04 & SO4 (wt\%) & 0.7632 & 0.7632 & 0.4808 & 0.2824 & 0.2824 & $58.7 \%$ & $58.7 \%$ \\
\hline 1 & ROC-04 & TiO2 (wt\%) & 1.8014 & 1.8250 & 2.0000 & -0.1986 & -0.1750 & $-9.9 \%$ & $-8.7 \%$ \\
\hline$\frac{1}{1}$ & ROC-04 & U3O8 (wt\%) & 9.0798 & 8.8220 & 9.5000 & -0.4202 & -0.6780 & $-4.4 \%$ & $-7.1 \%$ \\
\hline 1 & ROC-04 & $\mathrm{ZnO}(\mathrm{wt} \%)$ & 0.1503 & 0.1503 & 0.1346 & 0.0157 & 0.0157 & $11.7 \%$ & $11.7 \%$ \\
\hline 1 & ROC-04 & $\mathrm{ZrO} 2$ (wt\%) & 0.1891 & 0.1891 & 0.2051 & -0.0160 & -0.0160 & $-7.8 \%$ & $-7.8 \%$ \\
\hline 1 & ROC-04 & Sum & 98.8456 & 99.7703 & 100.0000 & -1.1544 & -0.2297 & $-1.2 \%$ & $-0.2 \%$ \\
\hline 1 & ROC-05 & $\mathrm{Al} 2 \mathrm{O} 3$ (wt\%) & 4.7379 & 4.7832 & 4.5531 & 0.1848 & 0.2301 & $4.1 \%$ & $5.1 \%$ \\
\hline 1 & ROC-05 & B2O3 (wt\%) & 6.4720 & 6.9628 & 6.8633 & -0.3913 & 0.0995 & $-5.7 \%$ & $1.4 \%$ \\
\hline 1 & ROC-05 & $\mathrm{BaO}$ (wt\%) & 0.0011 & 0.0011 & 0.0000 & 0.0011 & 0.0011 & & \\
\hline 1 & ROC-05 & $\mathrm{CaO}(\mathrm{wt} \%)$ & 0.1434 & 0.1390 & 0.1048 & 0.0386 & 0.0342 & $36.8 \%$ & $32.6 \%$ \\
\hline 1 & ROC-05 & CdO (wt\%) & 0.0040 & 0.0040 & 0.0000 & 0.0040 & 0.0040 & & \\
\hline 1 & ROC-05 & Ce2O3 (wt\%) & 0.0348 & 0.0348 & 0.0000 & 0.0348 & 0.0348 & & \\
\hline 1 & ROC-05 & Cr2O3 (wt\%) & 0.0073 & 0.0072 & 0.0000 & 0.0073 & 0.0072 & & \\
\hline 1 & ROC-05 & $\mathrm{CuO}(\mathrm{wt} \%)$ & 0.0238 & 0.0255 & 0.0000 & 0.0238 & 0.0255 & & \\
\hline 1 & ROC-05 & $\mathrm{Fe} 2 \mathrm{O} 3(\mathrm{wt} \%)$ & 14.3470 & 14.5255 & 14.5163 & -0.1692 & 0.0092 & $-1.2 \%$ & $0.1 \%$ \\
\hline 1 & ROC-05 & K2O (wt\%) & 0.0382 & 0.0391 & 0.0000 & 0.0382 & 0.0391 & & \\
\hline 1 & ROC-05 & La2O3 (wt\%) & 0.0053 & 0.0053 & 0.0000 & 0.0053 & 0.0053 & & \\
\hline 1 & ROC-05 & Li2O (wt\%) & 3.8214 & 4.0075 & 4.0000 & -0.1786 & 0.0075 & $-4.5 \%$ & $0.2 \%$ \\
\hline 1 & ROC-05 & MgO (wt\%) & 1.4945 & 1.4961 & 1.5000 & -0.0055 & -0.0039 & $-0.4 \%$ & $-0.3 \%$ \\
\hline 1 & ROC-05 & $\mathrm{MnO}(\mathrm{wt} \%)$ & 5.4360 & 5.4566 & 5.5000 & -0.0640 & -0.0434 & $-1.2 \%$ & $-0.8 \%$ \\
\hline 1 & ROC-05 & $\mathrm{Na} 2 \mathrm{O}$ (wt\%) & 10.2583 & 10.1127 & 10.5327 & -0.2744 & -0.4200 & $-2.6 \%$ & $-4.0 \%$ \\
\hline$\frac{1}{1}$ & ROC-05 & $\mathrm{NiO}(\mathrm{wt} \%)$ & 0.1145 & 0.1107 & 0.0723 & 0.0422 & 0.0384 & $58.4 \%$ & $53.2 \%$ \\
\hline 1 & ROC-05 & $\mathrm{PbO}(\mathrm{wt} \%)$ & 0.0094 & 0.0094 & 0.0000 & 0.0094 & 0.0094 & & \\
\hline$\frac{1}{1}$ & ROC-05 & $\mathrm{SiO} 2$ (wt\%) & 41.6629 & 42.5541 & 42.3575 & -0.6946 & 0.1966 & $-1.6 \%$ & $0.5 \%$ \\
\hline 1 & ROC-05 & SO4 (wt\%) & 0.2247 & 0.2247 & 0.0000 & 0.2247 & 0.2247 & & \\
\hline 1 & ROC-05 & TiO2 (wt\%) & 0.5100 & 0.5166 & 0.5000 & 0.0100 & 0.0166 & $2.0 \%$ & $3.3 \%$ \\
\hline 1 & ROC-05 & U3O8 (wt\%) & 8.9914 & 8.7361 & 9.5000 & -0.5086 & -0.7639 & $-5.4 \%$ & $-8.0 \%$ \\
\hline 1 & ROC-05 & $\mathrm{ZnO}(\mathrm{wt} \%)$ & 0.0124 & 0.0124 & 0.0000 & 0.0124 & 0.0124 & & \\
\hline 1 & ROC-05 & $\mathrm{ZrO} 2$ (wt\%) & 0.0034 & 0.0034 & 0.0000 & 0.0034 & 0.0034 & & \\
\hline 1 & ROC-05 & Sum & 98.3539 & 99.7678 & 100.0000 & -1.6461 & -0.2322 & $-1.6 \%$ & $-0.2 \%$ \\
\hline 1 & ROC-06 & $\mathrm{Al} 2 \mathrm{O} 3$ (wt\%) & 5.4134 & 5.4652 & 5.1016 & 0.3119 & 0.3636 & $6.1 \%$ & $7.1 \%$ \\
\hline 1 & ROC-06 & B2O3 (wt\%) & 13.5558 & 14.5849 & 14.0000 & -0.4442 & 0.5849 & $-3.2 \%$ & $4.2 \%$ \\
\hline 1 & ROC-06 & $\mathrm{BaO}(\mathrm{wt} \%)$ & 0.0011 & 0.0011 & 0.0000 & 0.0011 & 0.0011 & & \\
\hline 1 & ROC-06 & $\mathrm{CaO}(\mathrm{wt} \%)$ & 3.8688 & 3.7817 & 4.0000 & -0.1312 & -0.2183 & $-3.3 \%$ & $-5.5 \%$ \\
\hline 1 & ROC-06 & $\mathrm{CdO}(\mathrm{wt} \%)$ & 0.0040 & 0.0040 & 0.0000 & 0.0040 & 0.0040 & & \\
\hline 1 & ROC-06 & Ce2O3 (wt\%) & 0.0281 & 0.0281 & 0.0000 & 0.0281 & 0.0281 & & \\
\hline 1 & ROC-06 & Cr2O3 (wt\%) & 0.1864 & 0.1857 & 0.2000 & -0.0136 & -0.0143 & $-6.8 \%$ & $-7.1 \%$ \\
\hline 1 & ROC-06 & $\mathrm{CuO}(\mathrm{wt} \%)$ & 0.0194 & 0.0207 & 0.0000 & 0.0194 & 0.0207 & & \\
\hline 1 & ROC-06 & Fe2O3 (wt\%) & 4.8360 & 4.8961 & 5.0000 & -0.1640 & -0.1039 & $-3.3 \%$ & $-2.1 \%$ \\
\hline 1 & ROC-06 & K2O (wt\%) & 0.0620 & 0.0619 & 0.0000 & 0.0620 & 0.0619 & & \\
\hline 1 & ROC-06 & La2O3 (wt\%) & 0.0053 & 0.0053 & 0.0000 & 0.0053 & 0.0053 & & \\
\hline 1 & ROC-06 & Li2O (wt\%) & 3.9560 & 4.1486 & 4.0000 & -0.0440 & 0.1486 & $-1.1 \%$ & $3.7 \%$ \\
\hline 1 & ROC-06 & $\mathrm{MgO}(\mathrm{wt} \%)$ & 0.0041 & 0.0041 & 0.0000 & 0.0041 & 0.0041 & & \\
\hline 1 & ROC-06 & $\mathrm{MnO}(\mathrm{wt} \%)$ & 5.4360 & 5.4497 & 5.5000 & -0.0640 & -0.0503 & $-1.2 \%$ & $-0.9 \%$ \\
\hline 1 & ROC-06 & $\mathrm{Na} 2 \mathrm{O}$ (wt\%) & 10.6829 & 10.4185 & 10.6188 & 0.0641 & -0.2003 & $0.6 \%$ & $-1.9 \%$ \\
\hline 1 & ROC-06 & $\mathrm{NiO}(\mathrm{wt} \%)$ & 0.0297 & 0.0288 & 0.0000 & 0.0297 & 0.0288 & & \\
\hline 1 & ROC-06 & $\mathrm{PbO}(\mathrm{wt} \%)$ & 0.0081 & 0.0081 & 0.0000 & 0.0081 & 0.0081 & & \\
\hline 1 & ROC-06 & $\mathrm{SiO} 2$ (wt\%) & 41.2350 & 42.1170 & 41.5797 & -0.3447 & 0.5374 & $-0.8 \%$ & $1.3 \%$ \\
\hline 1 & ROC-06 & SO4 (wt\%) & 0.2247 & 0.2247 & 0.0000 & 0.2247 & 0.2247 & & \\
\hline 1 & ROC-06 & $\mathrm{TiO} 2(\mathrm{wt} \%)$ & 0.4328 & 0.4387 & 0.5000 & -0.0672 & -0.0613 & $-13.4 \%$ & $-12.3 \%$ \\
\hline 1 & ROC-06 & U3O8 (wt\%) & 9.2037 & 9.0218 & 9.5000 & -0.2963 & -0.4782 & $-3.1 \%$ & $-5.0 \%$ \\
\hline 1 & ROC-06 & $\mathrm{ZnO}(\mathrm{wt} \%)$ & 0.0124 & 0.0124 & 0.0000 & 0.0124 & 0.0124 & & \\
\hline 1 & ROC-06 & $\mathrm{ZrO} 2$ (wt\%) & 0.0034 & 0.0034 & 0.0000 & 0.0034 & 0.0034 & & \\
\hline 1 & ROC-06 & Sum & 99.2090 & 100.9106 & 100.0000 & -0.7910 & 0.9106 & $-0.8 \%$ & $0.9 \%$ \\
\hline 1 & ROC-07 & $\mathrm{Al2O}$ (wt\%) & 3.6940 & 3.7293 & 3.4635 & 0.2305 & 0.2658 & $6.7 \%$ & $7.7 \%$ \\
\hline 1 & ROC-07 & $\mathrm{B} 2 \mathrm{O} 3(\mathrm{wt} \%)$ & 13.0245 & 14.0130 & 13.6919 & -0.6674 & 0.3211 & $-4.9 \%$ & $2.3 \%$ \\
\hline$\frac{1}{1}$ & ROC-07 & $\mathrm{BaO}(\mathrm{wt} \%)$ & 0.0011 & 0.0011 & 0.0000 & 0.0011 & 0.0011 & & \\
\hline 1 & ROC-07 & $\mathrm{CaO}(\mathrm{wt} \%)$ & 0.0293 & 0.0287 & 0.0000 & 0.0293 & 0.0287 & & \\
\hline 1 & ROC-07 & $\mathrm{CdO}(\mathrm{wt} \%)$ & 0.0040 & 0.0040 & 0.0000 & 0.0040 & 0.0040 & & \\
\hline 1 & ROC-07 & Ce2O3 (wt\%) & 0.0059 & 0.0059 & 0.0000 & 0.0059 & 0.0059 & & \\
\hline 1 & ROC-07 & Cr2O3 (wt\%) & 0.1684 & 0.1679 & 0.2000 & -0.0316 & -0.0321 & $-15.8 \%$ & $-16.1 \%$ \\
\hline 1 & ROC-07 & $\mathrm{CuO}(\mathrm{wt} \%)$ & 0.0100 & 0.0107 & 0.0000 & 0.0100 & 0.0107 & & \\
\hline 1 & ROC-07 & $\mathrm{Fe} 2 \mathrm{O} 3(\mathrm{wt} \%)$ & 9.7291 & 9.8501 & 10.2256 & -0.4965 & -0.3756 & $-4.9 \%$ & $-3.7 \%$ \\
\hline 1 & ROC-07 & K2O (wt\%) & 0.0596 & 0.0598 & 0.0000 & 0.0596 & 0.0598 & & \\
\hline 1 & ROC-07 & La2O3 (wt\%) & 0.0053 & 0.0053 & 0.0000 & 0.0053 & 0.0053 & & \\
\hline 1 & ROC-07 & Li2O (wt\%) & 5.1347 & 5.3849 & 5.4049 & -0.2703 & -0.0201 & $-5.0 \%$ & $-0.4 \%$ \\
\hline 1 & ROC-07 & $\mathrm{MgO}(\mathrm{wt} \%)$ & 0.0137 & 0.0136 & 0.0000 & 0.0137 & 0.0136 & & \\
\hline 1 & ROC-07 & $\mathrm{MnO}(\mathrm{wt} \%)$ & 0.2992 & 0.3000 & 0.3000 & -0.0008 & 0.0000 & $-0.3 \%$ & $0.0 \%$ \\
\hline 1 & ROC-07 & $\mathrm{Na} 2 \mathrm{O}$ (wt\%) & 9.7056 & 9.4653 & 10.0968 & -0.3912 & -0.6315 & $-3.9 \%$ & $-6.3 \%$ \\
\hline
\end{tabular}


SRNL-STI-2009-00465, Revision 0

Table A3. Average Measured and Bias-Corrected Chemical Compositions Versus Targeted Compositions by Oxide by Glass ID

\begin{tabular}{|c|c|c|c|c|c|c|c|c|c|}
\hline & & & & Measured & & & & & \\
\hline & & & Measured & Bias-Corrected & Targeted & Diff of & Diff of & \% Diff of & $\%$ Diff of \\
\hline Set & Glass \# & Oxide & (wt\%) & $(w t \%)$ & (wt\%) & Measured & Meas BC & Measured & Meas BC \\
\hline 1 & ROC-07 & $\mathrm{NiO}(\mathrm{wt} \%)$ & 0.0395 & 0.0383 & 0.0000 & 0.0395 & 0.0383 & & \\
\hline 1 & ROC-07 & $\mathrm{PbO}$ (wt\%) & 0.0068 & 0.0068 & 0.0000 & 0.0068 & 0.0068 & & \\
\hline 1 & ROC-07 & $\mathrm{SiO} 2$ (wt\%) & 52.1989 & 53.3155 & 53.8793 & -1.6803 & -0.5637 & $-3.1 \%$ & $-1.0 \%$ \\
\hline 1 & ROC-07 & SO4 (wt\%) & 0.2247 & 0.2247 & 0.0000 & 0.2247 & 0.2247 & & \\
\hline 1 & ROC-07 & $\mathrm{TiO} 2$ (wt\%) & 0.4687 & 0.4749 & 0.5000 & -0.0313 & -0.0251 & $-6.3 \%$ & $-5.0 \%$ \\
\hline 1 & ROC-07 & U3O8 (wt\%) & 2.1108 & 2.0689 & 2.2381 & -0.1273 & -0.1691 & $-5.7 \%$ & $-7.6 \%$ \\
\hline 1 & ROC-07 & $\mathrm{ZnO}(\mathrm{wt} \%)$ & 0.0124 & 0.0124 & 0.0000 & 0.0124 & 0.0124 & & \\
\hline 1 & ROC-07 & $\mathrm{ZrO} 2$ (wt\%) & 0.0034 & 0.0034 & 0.0000 & 0.0034 & 0.0034 & & \\
\hline 1 & ROC-07 & Sum & 96.9496 & 99.1843 & 100.0000 & -3.0504 & -0.8157 & $-3.1 \%$ & $-0.8 \%$ \\
\hline 1 & ROC-08 & $\mathrm{Al} 2 \mathrm{O} 3$ (wt\%) & 3.7884 & 3.8272 & 3.5110 & 0.2774 & 0.3161 & $7.9 \%$ & $9.0 \%$ \\
\hline 1 & ROC-08 & B2O3 (wt\%) & 4.3227 & 4.5926 & 4.5000 & -0.1773 & 0.0926 & $-3.9 \%$ & $2.1 \%$ \\
\hline 1 & ROC-08 & $\mathrm{BaO}$ (wt\%) & 0.0011 & 0.0011 & 0.0000 & 0.0011 & 0.0011 & & \\
\hline 1 & ROC-08 & $\mathrm{CaO}$ (wt\%) & 0.0331 & 0.0324 & 0.0000 & 0.0331 & 0.0324 & & \\
\hline 1 & ROC-08 & CdO (wt\%) & 0.0040 & 0.0040 & 0.0000 & 0.0040 & 0.0040 & & \\
\hline 1 & ROC-08 & Ce2O3 (wt\%) & 0.0059 & 0.0059 & 0.0000 & 0.0059 & 0.0059 & & \\
\hline 1 & ROC-08 & Cr2O3 (wt\%) & 0.1608 & 0.1602 & 0.2000 & -0.0392 & -0.0398 & $-19.6 \%$ & $-19.9 \%$ \\
\hline 1 & ROC-08 & $\mathrm{CuO}(\mathrm{wt} \%)$ & 0.0200 & 0.0214 & 0.0000 & 0.0200 & 0.0214 & & \\
\hline 1 & ROC-08 & Fe2O3 (wt\%) & 5.9225 & 5.9558 & 6.2185 & -0.2960 & -0.2627 & $-4.8 \%$ & $-4.2 \%$ \\
\hline 1 & ROC-08 & K2O (wt\%) & 0.0663 & 0.0666 & 0.0000 & 0.0663 & 0.0666 & & \\
\hline 1 & ROC-08 & La2O3 (wt\%) & 0.0053 & 0.0053 & 0.0000 & 0.0053 & 0.0053 & & \\
\hline 1 & ROC-08 & Li2O (wt\%) & 4.5480 & 4.7898 & 4.7458 & -0.1978 & 0.0440 & $-4.2 \%$ & $0.9 \%$ \\
\hline 1 & ROC-08 & $\mathrm{MgO}(\mathrm{wt} \%)$ & 1.4154 & 1.4055 & 1.5000 & -0.0846 & -0.0945 & $-5.6 \%$ & $-6.3 \%$ \\
\hline 1 & ROC-08 & $\mathrm{MnO}(\mathrm{wt} \%)$ & 5.3585 & 5.3723 & 5.5000 & -0.1415 & -0.1277 & $-2.6 \%$ & $-2.3 \%$ \\
\hline 1 & ROC-08 & Na2O (wt\%) & 13.1767 & 12.8505 & 13.9166 & -0.7399 & -1.0662 & $-5.3 \%$ & $-7.7 \%$ \\
\hline 1 & ROC-08 & $\mathrm{NiO}(\mathrm{wt} \%)$ & 2.3350 & 2.2623 & 2.5000 & -0.1650 & -0.2377 & $-6.6 \%$ & $-9.5 \%$ \\
\hline 1 & ROC-08 & $\mathrm{PbO}$ (wt\%) & 0.0054 & 0.0054 & 0.0000 & 0.0054 & 0.0054 & & \\
\hline 1 & ROC-08 & $\mathrm{SiO} 2(\mathrm{wt} \%)$ & 46.2089 & 47.0269 & 47.7401 & -1.5312 & -0.7132 & $-3.2 \%$ & $-1.5 \%$ \\
\hline 1 & ROC-08 & SO4 (wt\%) & 0.2247 & 0.2247 & 0.0000 & 0.2247 & 0.2247 & & \\
\hline 1 & ROC-08 & $\mathrm{TiO} 2(\mathrm{wt} \%)$ & 1.9182 & 1.9439 & 2.0000 & -0.0818 & -0.0561 & $-4.1 \%$ & $-2.8 \%$ \\
\hline 1 & ROC-08 & U3O8 (wt\%) & 6.9219 & 6.7848 & 7.6679 & -0.7460 & -0.8831 & $-9.7 \%$ & $-11.5 \%$ \\
\hline 1 & ROC-08 & $\mathrm{ZnO}(\mathrm{wt} \%)$ & 0.0124 & 0.0124 & 0.0000 & 0.0124 & 0.0124 & & \\
\hline 1 & ROC-08 & ZrO2 (wt\%) & 0.0034 & 0.0034 & 0.0000 & 0.0034 & 0.0034 & & \\
\hline 1 & ROC-08 & Sum & 96.4586 & 97.3544 & 100.0000 & -3.5414 & -2.6456 & $-3.5 \%$ & $-2.6 \%$ \\
\hline 1 & ROC-09 & $\mathrm{Al} 2 \mathrm{O} 3$ (wt\%) & 4.0860 & 4.1278 & 3.9112 & 0.1749 & 0.2166 & $4.5 \%$ & $5.5 \%$ \\
\hline 1 & ROC-09 & B2O3 (wt\%) & 9.6114 & 10.2116 & 10.0718 & -0.4604 & 0.1398 & $-4.6 \%$ & $1.4 \%$ \\
\hline 1 & ROC-09 & $\mathrm{BaO}(\mathrm{wt} \%)$ & 0.0746 & 0.0755 & 0.0801 & -0.0056 & -0.0046 & $-7.0 \%$ & $-5.7 \%$ \\
\hline 1 & ROC-09 & $\mathrm{CaO}$ (wt\%) & 3.5645 & 3.4843 & 4.0000 & -0.4355 & -0.5157 & $-10.9 \%$ & $-12.9 \%$ \\
\hline 1 & ROC-09 & CdO (wt\%) & 0.2610 & 0.2610 & 0.2965 & -0.0355 & -0.0355 & $-12.0 \%$ & $-12.0 \%$ \\
\hline 1 & ROC-09 & Ce2O3 (wt\%) & 0.3007 & 0.3007 & 0.3606 & -0.0598 & -0.0598 & $-16.6 \%$ & $-16.6 \%$ \\
\hline 1 & ROC-09 & Cr2O3 (wt\%) & 0.0073 & 0.0073 & 0.0000 & 0.0073 & 0.0073 & & \\
\hline 1 & ROC-09 & $\mathrm{CuO}(\mathrm{wt} \%)$ & 0.1117 & 0.1194 & 0.1282 & -0.0165 & -0.0088 & $-12.9 \%$ & $-6.8 \%$ \\
\hline 1 & ROC-09 & Fe2O3 (wt\%) & 8.2851 & 8.3317 & 8.2899 & -0.0048 & 0.0418 & $-0.1 \%$ & $0.5 \%$ \\
\hline 1 & ROC-09 & K2O (wt\%) & 0.0587 & 0.0590 & 0.0000 & 0.0587 & 0.0590 & & \\
\hline 1 & ROC-09 & La2O3 (wt\%) & 0.0812 & 0.0812 & 0.0978 & -0.0165 & -0.0165 & $-16.9 \%$ & $-16.9 \%$ \\
\hline 1 & ROC-09 & Li2O (wt\%) & 3.7568 & 3.9565 & 4.0010 & -0.2442 & -0.0445 & $-6.1 \%$ & $-1.1 \%$ \\
\hline 1 & ROC-09 & $\mathrm{MgO}(\mathrm{wt} \%)$ & 1.3652 & 1.3557 & 1.5000 & -0.1348 & -0.1443 & $-9.0 \%$ & $-9.6 \%$ \\
\hline 1 & ROC-09 & $\mathrm{MnO}(\mathrm{wt} \%)$ & 3.4281 & 3.4369 & 3.7862 & -0.3580 & -0.3493 & $-9.5 \%$ & $-9.2 \%$ \\
\hline 1 & ROC-09 & $\mathrm{Na} 2 \mathrm{O}$ (wt\%) & 12.0006 & 11.7035 & 13.2996 & -1.2990 & -1.5961 & $-9.8 \%$ & $-12.0 \%$ \\
\hline 1 & ROC-09 & $\mathrm{NiO}$ (wt\%) & 2.3319 & 2.2592 & 2.5000 & -0.1681 & -0.2408 & $-6.7 \%$ & $-9.6 \%$ \\
\hline 1 & ROC-09 & $\mathrm{PbO}(\mathrm{wt} \%)$ & 0.1998 & 0.1998 & 0.2163 & -0.0165 & -0.0165 & $-7.6 \%$ & $-7.6 \%$ \\
\hline 1 & ROC-09 & $\mathrm{SiO} 2$ (wt\%) & 44.9253 & 45.7206 & 45.9404 & -1.0151 & -0.2198 & $-2.2 \%$ & $-0.5 \%$ \\
\hline 1 & ROC-09 & SO4 (wt\%) & 0.7610 & 0.7610 & 0.4808 & 0.2802 & 0.2802 & $58.3 \%$ & $58.3 \%$ \\
\hline 1 & ROC-09 & $\mathrm{TiO} 2$ (wt\%) & 0.5538 & 0.5612 & 0.5000 & 0.0538 & 0.0612 & $10.8 \%$ & $12.2 \%$ \\
\hline 1 & ROC-09 & U3O8 (wt\%) & 0.5749 & 0.5634 & 0.2000 & 0.3749 & 0.3634 & $187.4 \%$ & $181.7 \%$ \\
\hline 1 & ROC-09 & $\mathrm{ZnO}(\mathrm{wt} \%)$ & 0.1404 & 0.1404 & 0.1346 & 0.0057 & 0.0057 & $4.3 \%$ & $4.3 \%$ \\
\hline 1 & ROC-09 & ZrO2 (wt\%) & 0.1803 & 0.1803 & 0.2051 & -0.0248 & -0.0248 & $-12.1 \%$ & $-12.1 \%$ \\
\hline 1 & ROC-09 & Sum & 96.6603 & 97.8980 & 100.0000 & -3.3397 & -2.1020 & $-3.3 \%$ & $-2.1 \%$ \\
\hline 1 & ROC-10 & $\mathrm{Al} 2 \mathrm{O} 3$ (wt\%) & 3.7034 & 3.7413 & 3.3256 & 0.3779 & 0.4157 & $11.4 \%$ & $12.5 \%$ \\
\hline 1 & ROC-10 & B2O3 (wt\%) & 5.2323 & 5.5591 & 5.3579 & -0.1255 & 0.2012 & $-2.3 \%$ & $3.8 \%$ \\
\hline 1 & ROC-10 & $\mathrm{BaO}(\mathrm{wt} \%)$ & 0.0760 & 0.0770 & 0.0801 & -0.0041 & -0.0031 & $-5.1 \%$ & $-3.9 \%$ \\
\hline 1 & ROC-10 & $\mathrm{CaO}$ (wt\%) & 0.0255 & 0.0250 & 0.0010 & 0.0245 & 0.0240 & $2453.5 \%$ & $2396.3 \%$ \\
\hline 1 & ROC-10 & $\mathrm{CdO}(\mathrm{wt} \%)$ & 0.2570 & 0.2570 & 0.2965 & -0.0395 & -0.0395 & $-13.3 \%$ & $-13.3 \%$ \\
\hline 1 & ROC-10 & Ce2O3 (wt\%) & 0.3540 & 0.3540 & 0.3606 & -0.0066 & -0.0066 & $-1.8 \%$ & $-1.8 \%$ \\
\hline 1 & ROC-10 & Cr2O3 (wt\%) & 0.1513 & 0.1508 & 0.2000 & -0.0487 & -0.0492 & $-24.4 \%$ & $-24.6 \%$ \\
\hline 1 & ROC-10 & $\mathrm{CuO}(\mathrm{wt} \%)$ & 0.1189 & 0.1271 & 0.1282 & -0.0093 & -0.0011 & $-7.2 \%$ & $-0.8 \%$ \\
\hline 1 & ROC-10 & Fe2O3 (wt\%) & 14.2791 & 14.3594 & 15.0212 & -0.7421 & -0.6619 & $-4.9 \%$ & $-4.4 \%$ \\
\hline 1 & ROC-10 & K2O (wt\%) & 0.0395 & 0.0395 & 0.0000 & 0.0395 & 0.0395 & & \\
\hline 1 & ROC-10 & La2O3 (wt\%) & 0.0692 & 0.0692 & 0.0978 & -0.0286 & -0.0286 & $-29.2 \%$ & $-29.2 \%$ \\
\hline 1 & ROC-10 & $\mathrm{Li} 2 \mathrm{O}$ (wt\%) & 4.3865 & 4.6196 & 4.5212 & -0.1346 & 0.0985 & $-3.0 \%$ & $2.2 \%$ \\
\hline
\end{tabular}


SRNL-STI-2009-00465, Revision 0

Table A3. Average Measured and Bias-Corrected Chemical Compositions Versus Targeted Compositions by Oxide by Glass ID

\begin{tabular}{|c|c|c|c|c|c|c|c|c|c|}
\hline & & & & Measured & & & & & \\
\hline & & & Measured & Bias-Corrected & Targeted & Diff of & Diff of & \% Diff of & $\%$ Diff of \\
\hline Set & Glass \# & Oxide & (wt\%) & $(w t \%)$ & (wt\%) & Measured & Meas BC & Measured & Meas BC \\
\hline 1 & ROC-10 & $\mathrm{MgO}(\mathrm{wt} \%)$ & 1.3847 & 1.3751 & 1.5000 & -0.1153 & -0.1249 & $-7.7 \%$ & $-8.3 \%$ \\
\hline 1 & ROC-10 & $\mathrm{MnO}(\mathrm{wt} \%)$ & 1.2034 & 1.2065 & 1.2887 & -0.0853 & -0.0822 & $-6.6 \%$ & $-6.4 \%$ \\
\hline 1 & ROC-10 & Na2O (wt\%) & 10.3762 & 10.1193 & 11.1585 & -0.7823 & -1.0392 & $-7.0 \%$ & $-9.3 \%$ \\
\hline 1 & ROC-10 & $\mathrm{NiO}(\mathrm{wt} \%)$ & 0.0276 & 0.0268 & 0.0000 & 0.0276 & 0.0268 & & \\
\hline 1 & ROC-10 & $\mathrm{PbO}$ (wt\%) & 0.2055 & 0.2055 & 0.2163 & -0.0109 & -0.0109 & $-5.0 \%$ & $-5.0 \%$ \\
\hline 1 & ROC-10 & $\mathrm{SiO} 2(\mathrm{wt} \%)$ & 45.8880 & 46.7003 & 45.6260 & 0.2620 & 1.0743 & $0.6 \%$ & $2.4 \%$ \\
\hline 1 & ROC-10 & SO4 (wt\%) & 0.6613 & 0.6613 & 0.4808 & 0.1806 & 0.1806 & $37.6 \%$ & $37.6 \%$ \\
\hline 1 & ROC-10 & $\mathrm{TiO} 2$ (wt $\%)$ & 0.4725 & 0.4787 & 0.5000 & -0.0275 & -0.0213 & $-5.5 \%$ & $-4.3 \%$ \\
\hline 1 & ROC-10 & U3O8 (wt\%) & 8.5286 & 8.3598 & 9.5000 & -0.9714 & -1.1402 & $-10.2 \%$ & $-12.0 \%$ \\
\hline 1 & ROC-10 & $\mathrm{ZnO}$ (wt\%) & 0.1276 & 0.1276 & 0.1346 & -0.0070 & -0.0070 & $-5.2 \%$ & $-5.2 \%$ \\
\hline 1 & ROC-10 & $\mathrm{ZrO} 2$ (wt\%) & 0.1766 & 0.1766 & 0.2051 & -0.0285 & -0.0285 & $-13.9 \%$ & $-13.9 \%$ \\
\hline 1 & ROC-10 & Sum & 97.7448 & 98.8165 & 100.0000 & -2.2552 & -1.1835 & $-2.3 \%$ & $-1.2 \%$ \\
\hline 1 & ROC-11 & $\mathrm{Al} 2 \mathrm{O} 3$ (wt\%) & 3.5759 & 3.6124 & 3.2500 & 0.3259 & 0.3624 & $10.0 \%$ & $11.2 \%$ \\
\hline 1 & ROC-11 & B2O3 (wt\%) & 4.3388 & 4.6098 & 4.5000 & -0.1612 & 0.1098 & $-3.6 \%$ & $2.4 \%$ \\
\hline 1 & ROC-11 & $\mathrm{BaO}(\mathrm{wt} \%)$ & 0.0804 & 0.0814 & 0.0801 & 0.0002 & 0.0013 & $0.3 \%$ & $1.6 \%$ \\
\hline 1 & ROC-11 & $\mathrm{CaO}$ (wt\%) & 1.2635 & 1.2351 & 1.3177 & -0.0542 & -0.0826 & $-4.1 \%$ & $-6.3 \%$ \\
\hline 1 & ROC-11 & $\mathrm{CdO}(\mathrm{wt} \%)$ & 0.2699 & 0.2699 & 0.2965 & -0.0266 & -0.0266 & $-9.0 \%$ & $-9.0 \%$ \\
\hline 1 & ROC-11 & Ce2O3 (wt\%) & 0.3748 & 0.3748 & 0.3606 & 0.0142 & 0.0142 & $3.9 \%$ & $3.9 \%$ \\
\hline 1 & ROC-11 & Cr2O3 (wt\%) & 0.1641 & 0.1635 & 0.2000 & -0.0359 & -0.0365 & $-18.0 \%$ & $-18.2 \%$ \\
\hline 1 & ROC-11 & $\mathrm{CuO}(\mathrm{wt} \%)$ & 0.1299 & 0.1388 & 0.1282 & 0.0017 & 0.0106 & $1.3 \%$ & $8.3 \%$ \\
\hline 1 & ROC-11 & Fe2O3 (wt $\%)$ & 5.5401 & 5.5712 & 5.5974 & -0.0573 & -0.0262 & $-1.0 \%$ & $-0.5 \%$ \\
\hline 1 & ROC-11 & K2O (wt\%) & 0.0382 & 0.0384 & 0.0000 & 0.0382 & 0.0384 & & \\
\hline 1 & ROC-11 & La2O3 (wt\%) & 0.0710 & 0.0710 & 0.0978 & -0.0268 & -0.0268 & $-27.4 \%$ & $-27.4 \%$ \\
\hline 1 & ROC-11 & Li2O (wt\%) & 4.3058 & 4.5348 & 4.5575 & -0.2517 & -0.0228 & $-5.5 \%$ & $-0.5 \%$ \\
\hline 1 & ROC-11 & $\mathrm{MgO}$ (wt\%) & 0.2342 & 0.2326 & 0.2094 & 0.0248 & 0.0232 & $11.9 \%$ & $11.1 \%$ \\
\hline 1 & ROC-11 & $\mathrm{MnO}(\mathrm{wt} \%)$ & 5.3972 & 5.4110 & 5.5000 & -0.1028 & -0.0890 & $-1.9 \%$ & $-1.6 \%$ \\
\hline 1 & ROC-11 & $\mathrm{Na} 2 \mathrm{O}$ (wt\%) & 10.0325 & 9.7841 & 10.0000 & 0.0325 & -0.2159 & $0.3 \%$ & $-2.2 \%$ \\
\hline 1 & ROC-11 & $\mathrm{NiO}(\mathrm{wt} \%)$ & 2.4082 & 2.3331 & 2.5000 & -0.0918 & -0.1669 & $-3.7 \%$ & $-6.7 \%$ \\
\hline 1 & ROC-11 & $\mathrm{PbO}$ (wt\%) & 0.2459 & 0.2459 & 0.2163 & 0.0295 & 0.0295 & $13.6 \%$ & $13.6 \%$ \\
\hline 1 & ROC-11 & $\mathrm{SiO} 2$ (wt\%) & 49.3643 & 50.2384 & 50.3680 & -1.0037 & -0.1296 & $-2.0 \%$ & $-0.3 \%$ \\
\hline 1 & ROC-11 & SO4 (wt\%) & 0.7220 & 0.7220 & 0.4808 & 0.2412 & 0.2412 & $50.2 \%$ & $50.2 \%$ \\
\hline 1 & ROC-11 & $\mathrm{TiO} 2$ (wt\%) & 0.5492 & 0.5567 & 0.5000 & 0.0492 & 0.0567 & $9.8 \%$ & $11.3 \%$ \\
\hline 1 & ROC-11 & U3O8 (wt\%) & 9.0946 & 8.9150 & 9.5000 & -0.4054 & -0.5850 & $-4.3 \%$ & $-6.2 \%$ \\
\hline 1 & ROC-11 & $\mathrm{ZnO}(\mathrm{wt} \%)$ & 0.1522 & 0.1522 & 0.1346 & 0.0176 & 0.0176 & $13.0 \%$ & $13.0 \%$ \\
\hline 1 & ROC-11 & ZrO2 (wt\%) & 0.1851 & 0.1851 & 0.2051 & -0.0201 & -0.0201 & $-9.8 \%$ & $-9.8 \%$ \\
\hline 1 & ROC-11 & Sum & 98.5376 & 99.4770 & 100.0000 & -1.4624 & -0.5230 & $-1.5 \%$ & $-0.5 \%$ \\
\hline 1 & ROC-12 & $\mathrm{Al} 2 \mathrm{O} 3$ (wt\%) & 5.6685 & 5.7227 & 5.4812 & 0.1873 & 0.2415 & $3.4 \%$ & $4.4 \%$ \\
\hline 1 & ROC-12 & B2O3 (wt\%) & 7.2528 & 7.8031 & 7.5976 & -0.3448 & 0.2055 & $-4.5 \%$ & $2.7 \%$ \\
\hline 1 & ROC-12 & $\mathrm{BaO}(\mathrm{wt} \%)$ & 0.0011 & 0.0011 & 0.0000 & 0.0011 & 0.0011 & & \\
\hline 1 & ROC-12 & $\mathrm{CaO}$ (wt\%) & 3.7534 & 3.6394 & 3.9570 & -0.2036 & -0.3176 & $-5.1 \%$ & $-8.0 \%$ \\
\hline 1 & ROC-12 & CdO (wt\%) & 0.0040 & 0.0040 & 0.0000 & 0.0040 & 0.0040 & & \\
\hline 1 & ROC-12 & Ce2O3 (wt\%) & 0.0059 & 0.0059 & 0.0000 & 0.0059 & 0.0059 & & \\
\hline 1 & ROC-12 & Cr2O3 (wt\%) & 0.0073 & 0.0072 & 0.0000 & 0.0073 & 0.0072 & & \\
\hline 1 & ROC-12 & $\mathrm{CuO}(\mathrm{wt} \%)$ & 0.0131 & 0.0141 & 0.0000 & 0.0131 & 0.0141 & & \\
\hline 1 & ROC-12 & Fe2O3 (wt\%) & 5.9833 & 6.0576 & 5.9144 & 0.0689 & 0.1432 & $1.2 \%$ & $2.4 \%$ \\
\hline 1 & ROC-12 & K2O (wt\%) & 0.0391 & 0.0400 & 0.0000 & 0.0391 & 0.0400 & & \\
\hline 1 & ROC-12 & La2O3 (wt\%) & 0.0053 & 0.0053 & 0.0000 & 0.0053 & 0.0053 & & \\
\hline 1 & ROC-12 & Li2O (wt\%) & 6.3080 & 6.6152 & 6.4512 & -0.1432 & 0.1639 & $-2.2 \%$ & $2.5 \%$ \\
\hline 1 & ROC-12 & $\mathrm{MgO}(\mathrm{wt} \%)$ & 1.4829 & 1.4845 & 1.5000 & -0.0171 & -0.0155 & $-1.1 \%$ & $-1.0 \%$ \\
\hline 1 & ROC-12 & $\mathrm{MnO}(\mathrm{wt} \%)$ & 0.3073 & 0.3085 & 0.3000 & 0.0073 & 0.0085 & $2.4 \%$ & $2.8 \%$ \\
\hline 1 & ROC-12 & $\mathrm{Na} 2 \mathrm{O}$ (wt\%) & 12.8094 & 12.6277 & 13.4279 & -0.6186 & -0.8002 & $-4.6 \%$ & $-6.0 \%$ \\
\hline 1 & ROC-12 & $\mathrm{NiO}(\mathrm{wt} \%)$ & 2.4559 & 2.3763 & 2.5000 & -0.0441 & -0.1237 & $-1.8 \%$ & $-4.9 \%$ \\
\hline 1 & ROC-12 & $\mathrm{PbO}(\mathrm{wt} \%)$ & 0.0054 & 0.0054 & 0.0000 & 0.0054 & 0.0054 & & \\
\hline 1 & ROC-12 & $\mathrm{SiO} 2$ (wt\%) & 48.5086 & 49.5463 & 49.7360 & -1.2274 & -0.1898 & $-2.5 \%$ & $-0.4 \%$ \\
\hline 1 & ROC-12 & SO4 (wt\%) & 0.2247 & 0.2247 & 0.0000 & 0.2247 & 0.2247 & & \\
\hline 1 & ROC-12 & TiO2 (wt\%) & 0.4991 & 0.5056 & 0.5000 & -0.0009 & 0.0056 & $-0.2 \%$ & $1.1 \%$ \\
\hline 1 & ROC-12 & U3O8 (wt\%) & 2.5648 & 2.4919 & 2.6345 & -0.0698 & -0.1426 & $-2.6 \%$ & $-5.4 \%$ \\
\hline 1 & ROC-12 & $\mathrm{ZnO}(\mathrm{wt} \%)$ & 0.0124 & 0.0124 & 0.0000 & 0.0124 & 0.0124 & & \\
\hline 1 & ROC-12 & $\mathrm{ZrO} 2$ (wt\%) & 0.0115 & 0.0115 & 0.0000 & 0.0115 & 0.0115 & & \\
\hline 1 & ROC-12 & Sum & 97.9239 & 99.5103 & 100.0000 & -2.0761 & -0.4897 & $-2.1 \%$ & $-0.5 \%$ \\
\hline 1 & ROC-13 & $\mathrm{Al} 2 \mathrm{O} 3$ (wt\%) & 10.8174 & 10.9208 & 10.6333 & 0.1841 & 0.2875 & $1.7 \%$ & $2.7 \%$ \\
\hline 1 & ROC-13 & B2O3 (wt\%) & 4.2825 & 4.6073 & 4.5000 & -0.2175 & 0.1073 & $-4.8 \%$ & $2.4 \%$ \\
\hline 1 & ROC-13 & $\mathrm{BaO}(\mathrm{wt} \%)$ & 0.0011 & 0.0011 & 0.0000 & 0.0011 & 0.0011 & & \\
\hline 1 & ROC-13 & $\mathrm{CaO}$ (wt\%) & 1.2880 & 1.2487 & 1.4250 & -0.1370 & -0.1763 & $-9.6 \%$ & $-12.4 \%$ \\
\hline 1 & ROC-13 & CdO (wt\%) & 0.0040 & 0.0040 & 0.0000 & 0.0040 & 0.0040 & & \\
\hline 1 & ROC-13 & Ce2O3 (wt\%) & 0.0272 & 0.0272 & 0.0000 & 0.0272 & 0.0272 & & \\
\hline 1 & ROC-13 & Cr2O3 (wt\%) & 0.0073 & 0.0072 & 0.0000 & 0.0073 & 0.0072 & & \\
\hline 1 & ROC-13 & $\mathrm{CuO}(\mathrm{wt} \%)$ & 0.0163 & 0.0174 & 0.0000 & 0.0163 & 0.0174 & & \\
\hline 1 & ROC-13 & Fe2O3 (wt\%) & 6.5909 & 6.6728 & 6.6168 & -0.0258 & 0.0561 & $-0.4 \%$ & $0.8 \%$ \\
\hline
\end{tabular}


SRNL-STI-2009-00465, Revision 0

Table A3. Average Measured and Bias-Corrected Chemical Compositions Versus Targeted Compositions by Oxide by Glass ID

\begin{tabular}{|c|c|c|c|c|c|c|c|c|c|}
\hline & & & & "Measured & & & & & \\
\hline & & & Measured & Bias-Corrected & Targeted & Diff of & Diff of & \% Diff of & \% Diff of \\
\hline Set & Glass \# & Oxide & (wt\%) & (wt\%) & (wt\%) & Measured & Meas BC & Measured & Meas BC \\
\hline 1 & ROC-13 & $\mathrm{K} 2 \mathrm{O}$ (wt\%) & 0.0431 & 0.0440 & 0.0000 & 0.0431 & 0.0440 & & \\
\hline 1 & ROC-13 & La2O3 (wt\%) & 0.0053 & 0.0053 & 0.0000 & 0.0053 & 0.0053 & & \\
\hline$\frac{1}{1}$ & ROC-13 & Li2O (wt\%) & 5.4791 & 5.7459 & 5.5719 & -0.0927 & 0.1741 & $-1.7 \%$ & $3.1 \%$ \\
\hline 1 & ROC-13 & $\mathrm{MgO}(\mathrm{wt} \%)$ & 1.3847 & 1.3861 & 1.5000 & -0.1153 & -0.1139 & $-7.7 \%$ & $-7.6 \%$ \\
\hline 1 & ROC-13 & $\mathrm{MnO}(\mathrm{wt} \%)$ & 5.1067 & 5.1262 & 5.5000 & -0.3933 & -0.3738 & $-7.2 \%$ & $-6.8 \%$ \\
\hline 1 & ROC-13 & $\mathrm{Na} 2 \mathrm{O}(\mathrm{wt} \%)$ & 9.0485 & 8.9200 & 10.0000 & -0.9515 & -1.0800 & $-9.5 \%$ & $-10.8 \%$ \\
\hline 1 & ROC-13 & $\mathrm{NiO}(\mathrm{wt} \%)$ & 0.0350 & 0.0338 & 0.0000 & 0.0350 & 0.0338 & & \\
\hline 1 & ROC-13 & $\mathrm{PbO}(\mathrm{wt} \%)$ & 0.0054 & 0.0054 & 0.0000 & 0.0054 & 0.0054 & & \\
\hline 1 & ROC-13 & $\mathrm{SiO} 2$ (wt\%) & 45.2462 & 46.2142 & 45.4395 & -0.1933 & 0.7746 & $-0.4 \%$ & $1.7 \%$ \\
\hline 1 & ROC-13 & SO4 (wt \%) & 0.2247 & 0.2247 & 0.0000 & 0.2247 & 0.2247 & & \\
\hline 1 & ROC-13 & $\mathrm{TiO} 2$ (wt\%) & 0.4662 & 0.4723 & 0.5000 & -0.0338 & -0.0277 & $-6.8 \%$ & $-5.5 \%$ \\
\hline 1 & ROC-13 & U3O8 (wt\%) & 7.1931 & 6.9889 & 8.3136 & -1.1205 & -1.3248 & $-13.5 \%$ & $-15.9 \%$ \\
\hline 1 & ROC-13 & $\mathrm{ZnO}(\mathrm{wt} \%)$ & 0.0124 & 0.0124 & 0.0000 & 0.0124 & 0.0124 & & \\
\hline 1 & ROC-13 & ZrO2 (wt\%) & 0.0034 & 0.0034 & 0.0000 & 0.0034 & 0.0034 & & \\
\hline 1 & ROC-13 & Sum & 97.2884 & 98.6891 & 100.0000 & -2.7116 & -1.3109 & $-2.7 \%$ & $-1.3 \%$ \\
\hline 1 & ROC-14 & $\mathrm{Al} 2 \mathrm{O} 3$ (wt\%) & 11.4268 & 11.5360 & 11.3993 & 0.0274 & 0.1366 & $0.2 \%$ & $1.2 \%$ \\
\hline 1 & ROC-14 & B2O3 (wt\%) & 12.4610 & 13.4064 & 13.0085 & -0.5475 & 0.3979 & $-4.2 \%$ & $3.1 \%$ \\
\hline 1 & ROC-14 & $\mathrm{BaO}$ (wt\%) & 0.0802 & 0.0799 & 0.0801 & 0.0001 & -0.0003 & $0.1 \%$ & $-0.3 \%$ \\
\hline 1 & ROC-14 & $\mathrm{CaO}(\mathrm{wt} \%)$ & 0.0561 & 0.0544 & 0.0000 & 0.0561 & 0.0544 & & \\
\hline 1 & ROC-14 & $\mathrm{CdO}(\mathrm{wt} \%)$ & 0.2673 & 0.2673 & 0.2965 & -0.0292 & -0.0292 & $-9.8 \%$ & $-9.8 \%$ \\
\hline 1 & ROC-14 & Ce2O3 (wt\%) & 0.3362 & 0.3362 & 0.3606 & -0.0244 & -0.0244 & $-6.8 \%$ & $-6.8 \%$ \\
\hline$\frac{1}{1}$ & ROC-14 & Cr2O3 (wt\%) & 0.0073 & 0.0072 & 0.0000 & 0.0073 & 0.0072 & & \\
\hline 1 & ROC-14 & $\mathrm{CuO}(\mathrm{wt} \%)$ & 0.1283 & 0.1374 & 0.1282 & 0.0001 & 0.0092 & $0.1 \%$ & $7.2 \%$ \\
\hline$\frac{1}{1}$ & ROC-14 & $\mathrm{Fe} 2 \mathrm{O} 3$ (wt\%) & 6.5337 & 6.6149 & 6.5397 & -0.0060 & 0.0752 & $-0.1 \%$ & $1.2 \%$ \\
\hline 1 & ROC-14 & K2O (wt\%) & 0.0413 & 0.0422 & 0.0000 & 0.0413 & 0.0422 & & \\
\hline 1 & ROC-14 & La2O3 (wt\%) & 0.0704 & 0.0704 & 0.0978 & -0.0274 & -0.0274 & $-28.0 \%$ & $-28.0 \%$ \\
\hline 1 & ROC-14 & Li2O (wt\%) & 5.0593 & 5.3057 & 5.2589 & -0.1996 & 0.0469 & $-3.8 \%$ & $0.9 \%$ \\
\hline 1 & ROC-14 & $\mathrm{MgO}(\mathrm{wt} \%)$ & 1.4460 & 1.4475 & 1.5000 & -0.0540 & -0.0525 & $-3.6 \%$ & $-3.5 \%$ \\
\hline 1 & ROC-14 & $\mathrm{MnO}(\mathrm{wt} \%)$ & 4.0318 & 4.0472 & 4.1973 & -0.1656 & -0.1501 & $-3.9 \%$ & $-3.6 \%$ \\
\hline 1 & ROC-14 & $\mathrm{Na} 2 \mathrm{O}$ (wt $\%)$ & 9.9887 & 9.8469 & 10.3446 & -0.3559 & -0.4977 & $-3.4 \%$ & $-4.8 \%$ \\
\hline 1 & ROC-14 & $\mathrm{NiO}(\mathrm{wt} \%)$ & 2.2205 & 2.1484 & 2.5000 & -0.2795 & -0.3516 & $-11.2 \%$ & $-14.1 \%$ \\
\hline 1 & ROC-14 & $\mathrm{PbO}(\mathrm{wt} \%)$ & 0.2289 & 0.2289 & 0.2163 & 0.0126 & 0.0126 & $5.8 \%$ & $5.8 \%$ \\
\hline 1 & ROC-14 & $\mathrm{SiO} 2$ (wt\%) & 38.5609 & 39.3858 & 38.6590 & -0.0981 & 0.7268 & $-0.3 \%$ & $1.9 \%$ \\
\hline 1 & ROC-14 & SO4 (wt\%) & 0.6778 & 0.6778 & 0.4808 & 0.1971 & 0.1971 & $41.0 \%$ & $41.0 \%$ \\
\hline 1 & ROC-14 & TiO2 (wt\%) & 2.0099 & 2.0361 & 1.9686 & 0.0414 & 0.0675 & $2.1 \%$ & $3.4 \%$ \\
\hline 1 & ROC-14 & U3O8 (wt\%) & 2.4763 & 2.4060 & 2.6241 & -0.1478 & -0.2181 & $-5.6 \%$ & $-8.3 \%$ \\
\hline 1 & ROC-14 & $\mathrm{ZnO}(\mathrm{wt} \%)$ & 0.1500 & 0.1500 & 0.1346 & 0.0154 & 0.0154 & $11.4 \%$ & $11.4 \%$ \\
\hline 1 & ROC-14 & $\mathrm{ZrO} 2$ (wt\%) & 0.0236 & 0.0236 & 0.2051 & -0.1815 & -0.1815 & $-88.5 \%$ & $-88.5 \%$ \\
\hline 1 & ROC-14 & Sum & 98.2824 & 100.2562 & 100.0000 & -1.7176 & 0.2562 & $-1.7 \%$ & $0.3 \%$ \\
\hline 1 & ROC-15 & $\mathrm{Al} 2 \mathrm{O} 3$ (wt\%) & 7.2699 & 7.3442 & 7.0171 & 0.2528 & 0.3271 & $3.6 \%$ & $4.7 \%$ \\
\hline 1 & ROC-15 & $\mathrm{B} 2 \mathrm{O} 3(\mathrm{wt} \%)$ & 4.3066 & 4.5755 & 4.5000 & -0.1934 & 0.0755 & $-4.3 \%$ & $1.7 \%$ \\
\hline 1 & ROC-15 & $\mathrm{BaO}(\mathrm{wt} \%)$ & 0.0775 & 0.0772 & 0.0801 & -0.0026 & -0.0029 & $-3.2 \%$ & $-3.6 \%$ \\
\hline 1 & ROC-15 & $\mathrm{CaO}(\mathrm{wt} \%)$ & 0.0306 & 0.0297 & 0.0011 & 0.0295 & 0.0286 & $2638.2 \%$ & $2554.7 \%$ \\
\hline 1 & ROC-15 & $\mathrm{CdO}(\mathrm{wt} \%)$ & 0.2570 & 0.2570 & 0.2965 & -0.0395 & -0.0395 & $-13.3 \%$ & $-13.3 \%$ \\
\hline 1 & ROC-15 & Ce2O3 (wt\%) & 0.3660 & 0.3660 & 0.3606 & 0.0055 & 0.0055 & $1.5 \%$ & $1.5 \%$ \\
\hline 1 & ROC-15 & Cr2O3 (wt\%) & 0.1659 & 0.1630 & 0.2000 & -0.0341 & -0.0370 & $-17.1 \%$ & $-18.5 \%$ \\
\hline 1 & ROC-15 & $\mathrm{CuO}(\mathrm{wt} \%)$ & 0.1242 & 0.1330 & 0.1282 & -0.0040 & 0.0048 & $-3.1 \%$ & $3.8 \%$ \\
\hline 1 & ROC-15 & Fe2O3 (wt\%) & 10.2438 & 10.3014 & 10.2719 & -0.0281 & 0.0295 & $-0.3 \%$ & $0.3 \%$ \\
\hline 1 & ROC-15 & K2O (wt\%) & 0.0301 & 0.0307 & 0.0000 & 0.0301 & 0.0307 & & \\
\hline 1 & ROC-15 & La2O3 (wt\%) & 0.0616 & 0.0616 & 0.0978 & -0.0362 & -0.0362 & $-37.0 \%$ & $-37.0 \%$ \\
\hline 1 & ROC-15 & Li2O (wt\%) & 3.8106 & 4.0132 & 4.0000 & -0.1894 & 0.0132 & $-4.7 \%$ & $0.3 \%$ \\
\hline 1 & ROC-15 & $\mathrm{MgO}(\mathrm{wt} \%)$ & 0.0137 & 0.0137 & 0.0000 & 0.0137 & 0.0137 & & \\
\hline 1 & ROC-15 & $\mathrm{MnO}(\mathrm{wt} \%)$ & 0.9997 & 1.0035 & 1.0459 & -0.0462 & -0.0424 & $-4.4 \%$ & $-4.1 \%$ \\
\hline 1 & ROC-15 & $\mathrm{Na} 2 \mathrm{O}$ (wt\%) & 13.8844 & 13.6873 & 15.1379 & -1.2535 & -1.4506 & $-8.3 \%$ & $-9.6 \%$ \\
\hline 1 & ROC-15 & $\mathrm{NiO}(\mathrm{wt} \%)$ & 2.2555 & 2.1823 & 2.5000 & -0.2445 & -0.3177 & $-9.8 \%$ & $-12.7 \%$ \\
\hline 1 & ROC-15 & $\mathrm{PbO}(\mathrm{wt} \%)$ & 0.2235 & 0.2235 & 0.2163 & 0.0072 & 0.0072 & $3.3 \%$ & $3.3 \%$ \\
\hline$\frac{1}{1}$ & ROC-15 & $\mathrm{SiO} 2(\mathrm{wt} \%)$ & 44.0696 & 44.8500 & 43.8261 & 0.2435 & 1.0239 & $0.6 \%$ & $2.3 \%$ \\
\hline 1 & ROC-15 & SO4 (wt \%) & 0.7729 & 0.7729 & 0.4808 & 0.2922 & 0.2922 & $60.8 \%$ & $60.8 \%$ \\
\hline 1 & ROC-15 & $\mathrm{TiO} 2(\mathrm{wt} \%)$ & 0.4808 & 0.4871 & 0.5000 & -0.0192 & -0.0129 & $-3.8 \%$ & $-2.6 \%$ \\
\hline 1 & ROC-15 & U3O8 (wt\%) & 8.0480 & 7.8195 & 9.0000 & -0.9520 & -1.1805 & $-10.6 \%$ & $-13.1 \%$ \\
\hline 1 & ROC-15 & $\mathrm{ZnO}(\mathrm{wt} \%)$ & 0.1484 & 0.1484 & 0.1346 & 0.0138 & 0.0138 & $10.3 \%$ & $10.3 \%$ \\
\hline 1 & ROC-15 & $\mathrm{ZrO} 2$ (wt\%) & 0.1756 & 0.1756 & 0.2051 & -0.0295 & -0.0295 & $-14.4 \%$ & $-14.4 \%$ \\
\hline 1 & ROC-15 & Sum & 97.8161 & 98.7165 & 100.0000 & -2.1839 & -1.2835 & $-2.2 \%$ & $-1.3 \%$ \\
\hline 2 & ROC-16 & $\mathrm{Al} 2 \mathrm{O} 3$ (wt\%) & 3.4578 & 3.4660 & 3.2500 & 0.2078 & 0.2160 & $6.4 \%$ & $6.6 \%$ \\
\hline 2 & ROC-16 & $\mathrm{B} 2 \mathrm{O} 3(\mathrm{wt} \%)$ & 8.8547 & 9.3890 & 9.4333 & -0.5786 & -0.0444 & $-6.1 \%$ & $-0.5 \%$ \\
\hline 2 & ROC-16 & $\mathrm{BaO}$ (wt\%) & 0.0776 & 0.0788 & 0.0801 & -0.0025 & -0.0013 & $-3.2 \%$ & $-1.6 \%$ \\
\hline 2 & ROC-16 & $\mathrm{CaO}(\mathrm{wt} \%)$ & 0.0178 & 0.0181 & 0.0000 & 0.0178 & 0.0181 & & \\
\hline 2 & ROC-16 & $\mathrm{CdO}$ (wt\%) & 0.3158 & 0.3158 & 0.2965 & 0.0194 & 0.0194 & $6.5 \%$ & $6.5 \%$ \\
\hline 2 & ROC-16 & Ce2O3 (wt\%) & 0.3321 & 0.3321 & 0.3606 & -0.0285 & -0.0285 & $-7.9 \%$ & $-7.9 \%$ \\
\hline
\end{tabular}


SRNL-STI-2009-00465, Revision 0

Table A3. Average Measured and Bias-Corrected Chemical Compositions Versus Targeted Compositions by Oxide by Glass ID

\begin{tabular}{|c|c|c|c|c|c|c|c|c|c|}
\hline & & & & Measured & & & & & \\
\hline & & & Measured & Bias-Corrected & Targeted & Diff of & Diff of & $\%$ Diff of & \% Diff of \\
\hline Set & Glass \# & Oxide & (wt\%) & $(w t \%)$ & $(w t \%)$ & Measured & Meas BC & Measured & Meas BC \\
\hline 2 & ROC-16 & Cr2O3 (wt\%) & 0.0278 & 0.0279 & 0.0000 & 0.0278 & 0.0279 & & \\
\hline 2 & ROC-16 & $\mathrm{CuO}$ (wt\%) & 0.1343 & 0.1390 & 0.1282 & 0.0060 & 0.0108 & $4.7 \%$ & $8.4 \%$ \\
\hline 2 & ROC-16 & Fe2O3 (wt\%) & 5.2684 & 5.2425 & 5.0000 & 0.2684 & 0.2425 & $5.4 \%$ & $4.8 \%$ \\
\hline 2 & ROC-16 & K2O (wt\%) & 0.0410 & 0.0413 & 0.0000 & 0.0410 & 0.0413 & & \\
\hline 2 & ROC-16 & La2O3 (wt\%) & 0.0608 & 0.0608 & 0.0978 & -0.0370 & -0.0370 & $-37.9 \%$ & $-37.9 \%$ \\
\hline 2 & ROC-16 & Li2O (wt\%) & 6.4426 & 6.5940 & 6.6785 & -0.2359 & -0.0845 & $-3.5 \%$ & $-1.3 \%$ \\
\hline 2 & ROC-16 & MgO (wt\%) & 0.0166 & 0.0166 & 0.0000 & 0.0166 & 0.0166 & & \\
\hline 2 & ROC-16 & $\mathrm{MnO}(\mathrm{wt} \%)$ & 5.1971 & 5.3234 & 5.4398 & -0.2427 & -0.1164 & $-4.5 \%$ & $-2.1 \%$ \\
\hline 2 & ROC-16 & $\mathrm{Na} 2 \mathrm{O}$ (wt\%) & 9.8775 & 9.8831 & 10.0000 & -0.1225 & -0.1169 & $-1.2 \%$ & $-1.2 \%$ \\
\hline 2 & ROC-16 & $\mathrm{NiO}$ (wt\%) & 0.0255 & 0.0252 & 0.0000 & 0.0255 & 0.0252 & & \\
\hline 2 & ROC-16 & $\mathrm{PbO}$ (wt\%) & 0.2009 & 0.2009 & 0.2163 & -0.0154 & -0.0154 & $-7.1 \%$ & $-7.1 \%$ \\
\hline 2 & ROC-16 & SiO2 (wt\%) & 45.5671 & 46.6772 & 47.7923 & -2.2252 & -1.1151 & $-4.7 \%$ & $-2.3 \%$ \\
\hline 2 & ROC-16 & SO4 (wt\%) & 0.2247 & 0.2247 & 0.4808 & -0.2561 & -0.2561 & $-53.3 \%$ & $-53.3 \%$ \\
\hline 2 & ROC-16 & TiO2 (wt\%) & 2.3811 & 2.4508 & 2.0000 & 0.3811 & 0.4508 & $19.1 \%$ & $22.5 \%$ \\
\hline 2 & ROC-16 & U3O8 (wt\%) & 7.8623 & 7.9156 & 8.4061 & -0.5437 & -0.4905 & $-6.5 \%$ & $-5.8 \%$ \\
\hline 2 & ROC-16 & $\mathrm{ZnO}(\mathrm{wt} \%)$ & 0.1120 & 0.1120 & 0.1346 & -0.0226 & -0.0226 & $-16.8 \%$ & $-16.8 \%$ \\
\hline 2 & ROC-16 & $\mathrm{ZrO} 2$ (wt\%) & 0.1716 & 0.1716 & 0.2051 & -0.0336 & -0.0336 & $-16.4 \%$ & $-16.4 \%$ \\
\hline 2 & ROC-16 & Sum & 96.6668 & 98.7063 & 100.0000 & -3.3332 & -1.2937 & $-3.3 \%$ & $-1.3 \%$ \\
\hline 2 & ROC-17 & Al2O3 (wt\%) & 6.4952 & 6.7244 & 6.3367 & 0.1585 & 0.3877 & $2.5 \%$ & $6.1 \%$ \\
\hline 2 & ROC-17 & B2O3 (wt\%) & 4.8057 & 5.1058 & 4.8628 & -0.0571 & 0.2430 & $-1.2 \%$ & $5.0 \%$ \\
\hline 2 & ROC-17 & $\mathrm{BaO}(\mathrm{wt} \%)$ & 0.0011 & 0.0011 & 0.0000 & 0.0011 & 0.0011 & & \\
\hline 2 & ROC-17 & $\mathrm{CaO}(\mathrm{wt} \%)$ & 0.1686 & 0.1700 & 0.1558 & 0.0128 & 0.0142 & $8.2 \%$ & $9.1 \%$ \\
\hline 2 & ROC-17 & $\mathrm{CdO}(\mathrm{wt} \%)$ & 0.0040 & 0.0040 & 0.0000 & 0.0040 & 0.0040 & & \\
\hline 2 & ROC-17 & Ce2O3 (wt\%) & 0.0117 & 0.0117 & 0.0000 & 0.0117 & 0.0117 & & \\
\hline 2 & ROC-17 & Cr2O3 (wt\%) & 0.1710 & 0.1696 & 0.2000 & -0.0290 & -0.0304 & $-14.5 \%$ & $-15.2 \%$ \\
\hline 2 & ROC-17 & $\mathrm{CuO}$ (wt\%) & 0.0138 & 0.0140 & 0.0000 & 0.0138 & 0.0140 & & \\
\hline 2 & ROC-17 & Fe2O3 (wt\%) & 9.7184 & 9.9799 & 10.0294 & -0.3110 & -0.0495 & $-3.1 \%$ & $-0.5 \%$ \\
\hline 2 & ROC-17 & K2O (wt\%) & 0.0301 & 0.0311 & 0.0000 & 0.0301 & 0.0311 & & \\
\hline 2 & ROC-17 & La2O3 (wt\%) & 0.0053 & 0.0053 & 0.0000 & 0.0053 & 0.0053 & & \\
\hline 2 & ROC-17 & Li2O (wt\%) & 4.4888 & 4.7591 & 4.6645 & -0.1757 & 0.0947 & $-3.8 \%$ & $2.0 \%$ \\
\hline 2 & ROC-17 & MgO (wt\%) & 0.0199 & 0.0200 & 0.0000 & 0.0199 & 0.0200 & & \\
\hline 2 & ROC-17 & $\mathrm{MnO}(\mathrm{wt} \%)$ & 5.3197 & 5.5054 & 5.5000 & -0.1803 & 0.0054 & $-3.3 \%$ & $0.1 \%$ \\
\hline 2 & ROC-17 & $\mathrm{Na} 2 \mathrm{O}$ (wt\%) & 16.2434 & 16.1908 & 16.0018 & 0.2416 & 0.1891 & $1.5 \%$ & $1.2 \%$ \\
\hline 2 & ROC-17 & $\mathrm{NiO}(\mathrm{wt} \%)$ & 2.2841 & 2.2405 & 2.5000 & -0.2159 & -0.2595 & $-8.6 \%$ & $-10.4 \%$ \\
\hline 2 & ROC-17 & $\mathrm{PbO}$ (wt\%) & 0.0054 & 0.0054 & 0.0000 & 0.0054 & 0.0054 & & \\
\hline 2 & ROC-17 & $\mathrm{SiO} 2(\mathrm{wt} \%)$ & 46.6367 & 47.3252 & 47.5645 & -0.9277 & -0.2392 & $-2.0 \%$ & $-0.5 \%$ \\
\hline 2 & ROC-17 & SO4 (wt\%) & 0.2247 & 0.2247 & 0.0000 & 0.2247 & 0.2247 & & \\
\hline 2 & ROC-17 & TiO2 (wt\%) & 2.0433 & 2.1606 & 2.0000 & 0.0433 & 0.1606 & $2.2 \%$ & $8.0 \%$ \\
\hline 2 & ROC-17 & U3O8 (wt\%) & 0.3054 & 0.3017 & 0.1845 & 0.1209 & 0.1172 & $65.5 \%$ & $63.5 \%$ \\
\hline 2 & ROC-17 & $\mathrm{ZnO}(\mathrm{wt} \%)$ & 0.0124 & 0.0124 & 0.0000 & 0.0124 & 0.0124 & & \\
\hline 2 & ROC-17 & ZrO2 (wt\%) & 0.0034 & 0.0034 & 0.0000 & 0.0034 & 0.0034 & & \\
\hline 2 & ROC-17 & Sum & 99.0122 & 100.9661 & 100.0000 & -0.9878 & 0.9661 & $-1.0 \%$ & $1.0 \%$ \\
\hline 2 & ROC-18 & $\mathrm{Al} 2 \mathrm{O} 3$ (wt\%) & 4.0719 & 4.0815 & 3.7950 & 0.2769 & 0.2865 & $7.3 \%$ & $7.6 \%$ \\
\hline 2 & ROC-18 & B2O3 (wt\%) & 4.8862 & 5.1794 & 5.0465 & -0.1603 & 0.1329 & $-3.2 \%$ & $2.6 \%$ \\
\hline 2 & ROC-18 & $\mathrm{BaO}(\mathrm{wt} \%)$ & 0.0011 & 0.0011 & 0.0000 & 0.0011 & 0.0011 & & \\
\hline 2 & ROC-18 & $\mathrm{CaO}$ (wt\%) & 2.0603 & 2.0938 & 2.0149 & 0.0455 & 0.0790 & $2.3 \%$ & $3.9 \%$ \\
\hline 2 & ROC-18 & $\mathrm{CdO}(\mathrm{wt} \%)$ & 0.0040 & 0.0040 & 0.0000 & 0.0040 & 0.0040 & & \\
\hline 2 & ROC-18 & Ce2O3 (wt\%) & 0.0117 & 0.0117 & 0.0000 & 0.0117 & 0.0117 & & \\
\hline 2 & ROC-18 & Cr2O3 (wt $\%)$ & 0.0329 & 0.0331 & 0.0000 & 0.0329 & 0.0331 & & \\
\hline 2 & ROC-18 & $\mathrm{CuO}(\mathrm{wt} \%)$ & 0.0144 & 0.0149 & 0.0000 & 0.0144 & 0.0149 & & \\
\hline 2 & ROC-18 & $\mathrm{Fe} 2 \mathrm{O} 3(\mathrm{wt} \%)$ & 11.2339 & 11.1786 & 11.3221 & -0.0882 & -0.1435 & $-0.8 \%$ & $-1.3 \%$ \\
\hline 2 & ROC-18 & K2O (wt\%) & 0.0301 & 0.0304 & 0.0000 & 0.0301 & 0.0304 & & \\
\hline 2 & ROC-18 & La2O3 (wt\%) & 0.0053 & 0.0053 & 0.0000 & 0.0053 & 0.0053 & & \\
\hline 2 & ROC-18 & Li2O (wt\%) & 4.0313 & 4.1260 & 4.0000 & 0.0313 & 0.1260 & $0.8 \%$ & $3.2 \%$ \\
\hline 2 & ROC-18 & MgO (wt\%) & 0.0153 & 0.0153 & 0.0000 & 0.0153 & 0.0153 & & \\
\hline 2 & ROC-18 & $\mathrm{MnO}(\mathrm{wt} \%)$ & 4.8097 & 4.9266 & 4.8704 & -0.0606 & 0.0563 & $-1.2 \%$ & $1.2 \%$ \\
\hline 2 & ROC-18 & $\mathrm{Na} 2 \mathrm{O}(\mathrm{wt} \%)$ & 10.2515 & 10.2574 & 10.0000 & 0.2515 & 0.2574 & $2.5 \%$ & $2.6 \%$ \\
\hline 2 & ROC-18 & $\mathrm{NiO}$ (wt\%) & 1.9247 & 1.9070 & 2.2033 & -0.2786 & -0.2963 & $-12.6 \%$ & $-13.4 \%$ \\
\hline 2 & ROC-18 & $\mathrm{PbO}$ (wt\%) & 0.0054 & 0.0054 & 0.0000 & 0.0054 & 0.0054 & & \\
\hline 2 & ROC-18 & $\mathrm{SiO} 2$ (wt\%) & 47.1716 & 48.3208 & 49.5168 & -2.3452 & -1.1960 & $-4.7 \%$ & $-2.4 \%$ \\
\hline 2 & ROC-18 & SO4 (wt\%) & 0.2247 & 0.2247 & 0.0000 & 0.2247 & 0.2247 & & \\
\hline 2 & ROC-18 & $\mathrm{TiO} 2$ (wt\%) & 1.8974 & 1.9529 & 2.0000 & -0.1027 & -0.0471 & $-5.1 \%$ & $-2.4 \%$ \\
\hline 2 & ROC-18 & U3O8 (wt\%) & 4.9998 & 5.0335 & 5.2311 & -0.2313 & -0.1976 & $-4.4 \%$ & $-3.8 \%$ \\
\hline 2 & ROC-18 & $\mathrm{ZnO}$ (wt\%) & 0.0124 & 0.0124 & 0.0000 & 0.0124 & 0.0124 & & \\
\hline 2 & ROC-18 & $\mathrm{ZrO} 2$ (wt\%) & 0.0105 & 0.0105 & 0.0000 & 0.0105 & 0.0105 & & \\
\hline 2 & ROC-18 & Sum & 97.7060 & 99.4264 & 100.0000 & -2.2940 & -0.5736 & $-2.3 \%$ & $-0.6 \%$ \\
\hline 2 & ROC-19 & $\mathrm{Al} 2 \mathrm{O} 3$ (wt\%) & 4.1333 & 4.1431 & 3.9421 & 0.1911 & 0.2009 & $4.8 \%$ & $5.1 \%$ \\
\hline 2 & ROC-19 & B2O3 (wt\%) & 12.4932 & 13.2450 & 13.2672 & -0.7740 & -0.0222 & $-5.8 \%$ & $-0.2 \%$ \\
\hline 2 & ROC-19 & $\mathrm{BaO}(\mathrm{wt} \%)$ & 0.0011 & 0.0011 & 0.0000 & 0.0011 & 0.0011 & & \\
\hline
\end{tabular}


SRNL-STI-2009-00465, Revision 0

Table A3. Average Measured and Bias-Corrected Chemical Compositions Versus Targeted Compositions by Oxide by Glass ID

\begin{tabular}{|c|c|c|c|c|c|c|c|c|c|}
\hline & & & & Measured & & & & & \\
\hline & & & Measured & Bias-Corrected & Targeted & Diff of & Diff of & $\%$ Diff of & \% Diff of \\
\hline Set & Glass \# & Oxide & (wt\%) & (wt\%) & (wt\%) & Measured & Meas BC & Measured & Meas BC \\
\hline 2 & ROC-19 & $\mathrm{CaO}(\mathrm{wt} \%)$ & 0.0196 & 0.0198 & 0.0000 & 0.0196 & 0.0198 & & \\
\hline 2 & ROC-19 & CdO (wt\%) & 0.0040 & 0.0040 & 0.0000 & 0.0040 & 0.0040 & & \\
\hline 2 & ROC-19 & Ce2O3 (wt\%) & 0.0117 & 0.0117 & 0.0000 & 0.0117 & 0.0117 & & \\
\hline 2 & ROC-19 & Cr2O3 (wt $\%)$ & 0.0252 & 0.0250 & 0.0000 & 0.0252 & 0.0250 & & \\
\hline 2 & ROC-19 & $\mathrm{CuO}(\mathrm{wt} \%)$ & 0.0219 & 0.0222 & 0.0000 & 0.0219 & 0.0222 & & \\
\hline 2 & ROC-19 & Fe2O3 (wt\%) & 4.7573 & 4.7350 & 5.0000 & -0.2427 & -0.2650 & $-4.9 \%$ & $-5.3 \%$ \\
\hline 2 & ROC-19 & K2O (wt\%) & 0.0470 & 0.0485 & 0.0000 & 0.0470 & 0.0485 & & \\
\hline 2 & ROC-19 & La2O3 (wt\%) & 0.0053 & 0.0053 & 0.0000 & 0.0053 & 0.0053 & & \\
\hline 2 & ROC-19 & Li2O (wt\%) & 3.8429 & 3.9332 & 4.0000 & -0.1571 & -0.0668 & $-3.9 \%$ & $-1.7 \%$ \\
\hline 2 & ROC-19 & $\mathrm{MgO}(\mathrm{wt} \%)$ & 1.4871 & 1.4959 & 1.5000 & -0.0129 & -0.0041 & $-0.9 \%$ & $-0.3 \%$ \\
\hline 2 & ROC-19 & $\mathrm{MnO}(\mathrm{wt} \%)$ & 0.3070 & 0.3177 & 0.3000 & 0.0070 & 0.0177 & $2.3 \%$ & $5.9 \%$ \\
\hline 2 & ROC-19 & $\mathrm{Na} 2 \mathrm{O}(\mathrm{wt} \%)$ & 10.3661 & 10.3344 & 10.1324 & 0.2337 & 0.2020 & $2.3 \%$ & $2.0 \%$ \\
\hline 2 & ROC-19 & $\mathrm{NiO}$ (wt\%) & 2.3923 & 2.3467 & 2.5000 & -0.1077 & -0.1533 & $-4.3 \%$ & $-6.1 \%$ \\
\hline 2 & ROC-19 & $\mathrm{PbO}(\mathrm{wt} \%)$ & 0.0054 & 0.0054 & 0.0000 & 0.0054 & 0.0054 & & \\
\hline 2 & ROC-19 & $\mathrm{SiO} 2(\mathrm{wt} \%)$ & 48.6156 & 49.3332 & 50.3904 & -1.7748 & -1.0572 & $-3.5 \%$ & $-2.1 \%$ \\
\hline 2 & ROC-19 & SO4 (wt\%) & 0.2247 & 0.2247 & 0.0000 & 0.2247 & 0.2247 & & \\
\hline 2 & ROC-19 & TiO2 (wt\%) & 1.8306 & 1.9358 & 2.0000 & -0.1694 & -0.0642 & $-8.5 \%$ & $-3.2 \%$ \\
\hline 2 & ROC-19 & U3O8 (wt\%) & 6.4944 & 6.4182 & 6.9679 & -0.4734 & -0.5497 & $-6.8 \%$ & $-7.9 \%$ \\
\hline 2 & ROC-19 & $\mathrm{ZnO}(\mathrm{wt} \%)$ & 0.0124 & 0.0124 & 0.0000 & 0.0124 & 0.0124 & & \\
\hline 2 & ROC-19 & $\mathrm{ZrO} 2$ (wt\%) & 0.0034 & 0.0034 & 0.0000 & 0.0034 & 0.0034 & & \\
\hline 2 & ROC-19 & Sum & 97.1016 & 98.6216 & 100.0000 & -2.8984 & -1.3784 & $-2.9 \%$ & $-1.4 \%$ \\
\hline 2 & ROC-20 & $\mathrm{Al} 2 \mathrm{O} 3$ (wt\%) & 3.6278 & 3.7550 & 3.5811 & 0.0467 & 0.1739 & $1.3 \%$ & $4.9 \%$ \\
\hline 2 & ROC-20 & B2O3 (wt\%) & 9.6838 & 10.2880 & 10.3037 & -0.6199 & -0.0157 & $-6.0 \%$ & $-0.2 \%$ \\
\hline 2 & ROC-20 & $\mathrm{BaO}$ (wt\%) & 0.0784 & 0.0804 & 0.0801 & -0.0017 & 0.0002 & $-2.1 \%$ & $0.3 \%$ \\
\hline 2 & ROC-20 & $\mathrm{CaO}(\mathrm{wt} \%)$ & 3.7534 & 3.7841 & 4.0000 & -0.2466 & -0.2159 & $-6.2 \%$ & $-5.4 \%$ \\
\hline 2 & ROC-20 & CdO (wt\%) & 0.2590 & 0.2590 & 0.2965 & -0.0375 & -0.0375 & $-12.6 \%$ & $-12.6 \%$ \\
\hline 2 & ROC-20 & Ce2O3 (wt\%) & 0.3218 & 0.3218 & 0.3606 & -0.0388 & -0.0388 & $-10.7 \%$ & $-10.7 \%$ \\
\hline 2 & ROC-20 & Cr2O3 (wt\%) & 0.1922 & 0.1906 & 0.2000 & -0.0078 & -0.0094 & $-3.9 \%$ & $-4.7 \%$ \\
\hline 2 & ROC-20 & $\mathrm{CuO}(\mathrm{wt} \%)$ & 0.1217 & 0.1235 & 0.1282 & -0.0065 & -0.0047 & $-5.0 \%$ & $-3.7 \%$ \\
\hline 2 & ROC-20 & Fe2O3 (wt\%) & 8.3352 & 8.5592 & 8.3788 & -0.0436 & 0.1804 & $-0.5 \%$ & $2.2 \%$ \\
\hline 2 & ROC-20 & K2O (wt\%) & 0.0301 & 0.0311 & 0.0000 & 0.0301 & 0.0311 & & \\
\hline 2 & ROC-20 & La2O3 (wt\%) & 0.0730 & 0.0730 & 0.0978 & -0.0247 & -0.0247 & $-25.3 \%$ & $-25.3 \%$ \\
\hline 2 & ROC-20 & Li2O (wt\%) & 3.8268 & 4.0578 & 4.0820 & -0.2553 & -0.0242 & $-6.3 \%$ & $-0.6 \%$ \\
\hline 2 & ROC-20 & $\mathrm{MgO}(\mathrm{wt} \%)$ & 0.3599 & 0.3620 & 0.3769 & -0.0170 & -0.0149 & $-4.5 \%$ & $-4.0 \%$ \\
\hline 2 & ROC-20 & $\mathrm{MnO}(\mathrm{wt} \%)$ & 5.0228 & 5.1980 & 5.5000 & -0.4772 & -0.3020 & $-8.7 \%$ & $-5.5 \%$ \\
\hline 2 & ROC-20 & $\mathrm{Na} 2 \mathrm{O}$ (wt\%) & 12.1927 & 12.1585 & 12.9201 & -0.7275 & -0.7616 & $-5.6 \%$ & $-5.9 \%$ \\
\hline 2 & ROC-20 & $\mathrm{NiO}(\mathrm{wt} \%)$ & 0.0251 & 0.0246 & 0.0000 & 0.0251 & 0.0246 & & \\
\hline 2 & ROC-20 & $\mathrm{PbO}$ (wt\%) & 0.2076 & 0.2076 & 0.2163 & -0.0087 & -0.0087 & $-4.0 \%$ & $-4.0 \%$ \\
\hline 2 & ROC-20 & $\mathrm{SiO} 2$ (wt\%) & 42.9999 & 43.6356 & 46.4681 & -3.4682 & -2.8325 & $-7.5 \%$ & $-6.1 \%$ \\
\hline 2 & ROC-20 & SO4 (wt\%) & 0.2247 & 0.2247 & 0.4808 & -0.2561 & -0.2561 & $-53.3 \%$ & $-53.3 \%$ \\
\hline 2 & ROC-20 & TiO2 (wt\%) & 2.0058 & 2.1210 & 2.0000 & 0.0058 & 0.1210 & $0.3 \%$ & $6.0 \%$ \\
\hline 2 & ROC-20 & U3O8 (wt\%) & 0.6630 & 0.6550 & 0.1892 & 0.4738 & 0.4658 & $250.4 \%$ & $246.2 \%$ \\
\hline 2 & ROC-20 & $\mathrm{ZnO}(\mathrm{wt} \%)$ & 0.1301 & 0.1301 & 0.1346 & -0.0045 & -0.0045 & $-3.4 \%$ & $-3.4 \%$ \\
\hline 2 & ROC-20 & $\mathrm{ZrO} 2$ (wt\%) & 0.1790 & 0.1790 & 0.2051 & -0.0261 & -0.0261 & $-12.7 \%$ & $-12.7 \%$ \\
\hline 2 & ROC-20 & Sum & 94.3138 & 96.4196 & 100.0000 & -5.6862 & -3.5804 & $-5.7 \%$ & $-3.6 \%$ \\
\hline 2 & ROC-21 & $\mathrm{Al} 2 \mathrm{O} 3$ (wt\%) & 10.4064 & 10.7723 & 10.3731 & 0.0333 & 0.3991 & $0.3 \%$ & $3.8 \%$ \\
\hline 2 & ROC-21 & $\mathrm{B} 2 \mathrm{O} 3(\mathrm{wt} \%)$ & 11.1650 & 11.8616 & 11.6624 & -0.4974 & 0.1992 & $-4.3 \%$ & $1.7 \%$ \\
\hline 2 & ROC-21 & $\mathrm{BaO}(\mathrm{wt} \%)$ & 0.0011 & 0.0011 & 0.0000 & 0.0011 & 0.0011 & & \\
\hline 2 & ROC-21 & $\mathrm{CaO}(\mathrm{wt} \%)$ & 0.0927 & 0.0942 & 0.0905 & 0.0022 & 0.0036 & $2.4 \%$ & $4.0 \%$ \\
\hline 2 & ROC-21 & $\mathrm{CdO}(\mathrm{wt} \%)$ & 0.0040 & 0.0040 & 0.0000 & 0.0040 & 0.0040 & & \\
\hline 2 & ROC-21 & Ce2O3 (wt\%) & 0.0322 & 0.0322 & 0.0000 & 0.0322 & 0.0322 & & \\
\hline 2 & ROC-21 & Cr2O3 (wt\%) & 0.1568 & 0.1577 & 0.2000 & -0.0432 & -0.0423 & $-21.6 \%$ & $-21.2 \%$ \\
\hline 2 & ROC-21 & $\mathrm{CuO}(\mathrm{wt} \%)$ & 0.0197 & 0.0204 & 0.0000 & 0.0197 & 0.0204 & & \\
\hline 2 & ROC-21 & Fe2O3 (wt\%) & 7.8097 & 8.0195 & 7.8215 & -0.0117 & 0.1980 & $-0.1 \%$ & $2.5 \%$ \\
\hline 2 & ROC-21 & K2O (wt\%) & 0.0301 & 0.0304 & 0.0000 & 0.0301 & 0.0304 & & \\
\hline 2 & ROC-21 & La2O3 (wt\%) & 0.0053 & 0.0053 & 0.0000 & 0.0053 & 0.0053 & & \\
\hline 2 & ROC-21 & Li2O (wt\%) & 4.0421 & 4.2851 & 4.2309 & -0.1889 & 0.0542 & $-4.5 \%$ & $1.3 \%$ \\
\hline 2 & ROC-21 & $\mathrm{MgO}(\mathrm{wt} \%)$ & 1.0762 & 1.0759 & 1.1057 & -0.0295 & -0.0299 & $-2.7 \%$ & $-2.7 \%$ \\
\hline 2 & ROC-21 & $\mathrm{MnO}(\mathrm{wt} \%)$ & 5.2068 & 5.3334 & 5.2781 & -0.0713 & 0.0553 & $-1.4 \%$ & $1.0 \%$ \\
\hline 2 & ROC-21 & $\mathrm{Na} 2 \mathrm{O}$ (wt\%) & 10.3392 & 10.3450 & 10.0010 & 0.3382 & 0.3440 & $3.4 \%$ & $3.4 \%$ \\
\hline 2 & ROC-21 & $\mathrm{NiO}(w \mathrm{t} \%)$ & 1.4634 & 1.4500 & 1.5154 & -0.0520 & -0.0654 & $-3.4 \%$ & $-4.3 \%$ \\
\hline 2 & ROC-21 & $\mathrm{PbO}(\mathrm{wt} \%)$ & 0.0054 & 0.0054 & 0.0000 & 0.0054 & 0.0054 & & \\
\hline 2 & ROC-21 & SiO2 (wt\%) & 35.9937 & 36.8709 & 36.2213 & -0.2276 & 0.6496 & $-0.6 \%$ & $1.8 \%$ \\
\hline 2 & ROC-21 & SO4 (wt\%) & 0.2247 & 0.2247 & 0.0000 & 0.2247 & 0.2247 & & \\
\hline 2 & ROC-21 & $\mathrm{TiO} 2(\mathrm{wt} \%)$ & 1.9682 & 2.0258 & 2.0000 & -0.0318 & 0.0258 & $-1.6 \%$ & $1.3 \%$ \\
\hline 2 & ROC-21 & U3O8 (wt\%) & 9.0238 & 9.0849 & 9.5000 & -0.4762 & -0.4151 & $-5.0 \%$ & $-4.4 \%$ \\
\hline 2 & ROC-21 & $\mathrm{ZnO}(\mathrm{wt} \%)$ & 0.0124 & 0.0124 & 0.0000 & 0.0124 & 0.0124 & & \\
\hline 2 & ROC-21 & ZrO2 (wt\%) & 0.0034 & 0.0034 & 0.0000 & 0.0034 & 0.0034 & & \\
\hline 2 & ROC-21 & Sum & 99.0823 & 101.7156 & 100.0000 & -0.9177 & 1.7156 & $-0.9 \%$ & $1.7 \%$ \\
\hline
\end{tabular}


SRNL-STI-2009-00465, Revision 0

Table A3. Average Measured and Bias-Corrected Chemical Compositions Versus Targeted Compositions by Oxide by Glass ID

\begin{tabular}{|c|c|c|c|c|c|c|c|c|c|}
\hline & & & & Measured & & & & & \\
\hline & & & Measured & Bias-Corrected & Targeted & Diff of & Diff of & \% Diff of & \% Diff of \\
\hline Set & Glass \# & Oxide & (wt\%) & $(\mathrm{wt} \%)$ & $(\mathrm{wt} \%)$ & Measured & Meas BC & Measured & Meas BC \\
\hline 2 & ROC-22 & $\mathrm{Al} 2 \mathrm{O} 3$ (wt\%) & 3.5003 & 3.6241 & 3.2510 & 0.2493 & 0.3731 & $7.7 \%$ & $11.5 \%$ \\
\hline 2 & ROC-22 & B2O3 (wt\%) & 6.5042 & 6.9105 & 6.4325 & 0.0717 & 0.4780 & $1.1 \%$ & $7.4 \%$ \\
\hline 2 & ROC-22 & $\mathrm{BaO}(\mathrm{wt} \%)$ & 0.0011 & 0.0011 & 0.0000 & 0.0011 & 0.0011 & & \\
\hline 2 & ROC-22 & $\mathrm{CaO}(\mathrm{wt} \%)$ & 0.0143 & 0.0145 & 0.0010 & 0.0133 & 0.0135 & $1334.2 \%$ & $1352.5 \%$ \\
\hline 2 & ROC-22 & $\mathrm{CdO}(\mathrm{wt} \%)$ & 0.0040 & 0.0040 & 0.0000 & 0.0040 & 0.0040 & & \\
\hline 2 & ROC-22 & Ce2O3 (wt\%) & 0.0117 & 0.0117 & 0.0000 & 0.0117 & 0.0117 & & \\
\hline 2 & ROC-22 & Cr2O3 (wt\%) & 0.0336 & 0.0338 & 0.0000 & 0.0336 & 0.0338 & & \\
\hline 2 & ROC-22 & $\mathrm{CuO}(\mathrm{wt} \%)$ & 0.0119 & 0.0123 & 0.0000 & 0.0119 & 0.0123 & & \\
\hline 2 & ROC-22 & Fe2O3 (wt\%) & 14.5115 & 14.9023 & 14.6920 & -0.1805 & 0.2103 & $-1.2 \%$ & $1.4 \%$ \\
\hline 2 & ROC-22 & K2O (wt\%) & 0.0428 & 0.0432 & 0.0000 & 0.0428 & 0.0432 & & \\
\hline 2 & ROC-22 & La2O3 (wt\%) & 0.0053 & 0.0053 & 0.0000 & 0.0053 & 0.0053 & & \\
\hline 2 & ROC-22 & Li2O (wt\%) & 4.4511 & 4.7214 & 4.5235 & -0.0724 & 0.1979 & $-1.6 \%$ & $4.4 \%$ \\
\hline 2 & ROC-22 & MgO (wt\%) & 0.0091 & 0.0091 & 0.0000 & 0.0091 & 0.0091 & & \\
\hline 2 & ROC-22 & $\mathrm{MnO}(\mathrm{wt} \%)$ & 5.5167 & 5.6508 & 5.5000 & 0.0167 & 0.1508 & $0.3 \%$ & $2.7 \%$ \\
\hline 2 & ROC-22 & $\mathrm{Na} 2 \mathrm{O}(\mathrm{wt} \%)$ & 10.2280 & 10.2338 & 10.0000 & 0.2280 & 0.2338 & $2.3 \%$ & $2.3 \%$ \\
\hline 2 & ROC-22 & $\mathrm{NiO}(\mathrm{wt} \%)$ & 2.1473 & 2.1278 & 2.5000 & -0.3527 & -0.3722 & $-14.1 \%$ & $-14.9 \%$ \\
\hline 2 & ROC-22 & $\mathrm{PbO}(\mathrm{wt} \%)$ & 0.0054 & 0.0054 & 0.0000 & 0.0054 & 0.0054 & & \\
\hline 2 & ROC-22 & $\mathrm{SiO} 2(\mathrm{wt} \%)$ & 46.1554 & 47.2800 & 46.8442 & -0.6888 & 0.4358 & $-1.5 \%$ & $0.9 \%$ \\
\hline 2 & ROC-22 & SO4 (wt\%) & 0.2247 & 0.2247 & 0.0000 & 0.2247 & 0.2247 & & \\
\hline 2 & ROC-22 & $\mathrm{TiO} 2(\mathrm{wt} \%)$ & 1.8557 & 1.9100 & 1.8222 & 0.0334 & 0.0877 & $1.8 \%$ & $4.8 \%$ \\
\hline 2 & ROC-22 & U3O8 (wt\%) & 4.2451 & 4.2738 & 4.4336 & -0.1885 & -0.1598 & $-4.3 \%$ & $-3.6 \%$ \\
\hline 2 & ROC-22 & $\mathrm{ZnO}(\mathrm{wt} \%)$ & 0.0124 & 0.0124 & 0.0000 & 0.0124 & 0.0124 & & \\
\hline 2 & ROC-22 & $\mathrm{ZrO} 2(\mathrm{wt} \%)$ & 0.0034 & 0.0034 & 0.0000 & 0.0034 & 0.0034 & & \\
\hline 2 & ROC-22 & Sum & 99.4949 & 102.0153 & 100.0000 & -0.5051 & 2.0153 & $-0.5 \%$ & $2.0 \%$ \\
\hline 2 & ROC-23 & $\mathrm{Al} 2 \mathrm{O} 3$ (wt\%) & 10.6379 & 10.6631 & 9.9919 & 0.6459 & 0.6712 & $6.5 \%$ & $6.7 \%$ \\
\hline 2 & ROC-23 & B2O3 (wt\%) & 5.4416 & 5.7690 & 5.6859 & -0.2443 & 0.0830 & $-4.3 \%$ & $1.5 \%$ \\
\hline 2 & ROC-23 & $\mathrm{BaO}$ (wt\%) & 0.0871 & 0.0884 & 0.0801 & 0.0070 & 0.0083 & $8.7 \%$ & $10.4 \%$ \\
\hline 2 & ROC-23 & $\mathrm{CaO}(\mathrm{wt} \%)$ & 0.0091 & 0.0092 & 0.0000 & 0.0091 & 0.0092 & & \\
\hline 2 & ROC-23 & $\mathrm{CdO}(\mathrm{wt} \%)$ & 0.2627 & 0.2627 & 0.2965 & -0.0337 & -0.0337 & $-11.4 \%$ & $-11.4 \%$ \\
\hline 2 & ROC-23 & Ce2O3 (wt\%) & 0.3104 & 0.3104 & 0.3606 & -0.0502 & -0.0502 & $-13.9 \%$ & $-13.9 \%$ \\
\hline 2 & ROC-23 & Cr2O3 (wt\%) & 0.1363 & 0.1371 & 0.1991 & -0.0628 & -0.0620 & $-31.5 \%$ & $-31.1 \%$ \\
\hline 2 & ROC-23 & $\mathrm{CuO}$ (wt\%) & 0.1352 & 0.1400 & 0.1282 & 0.0070 & 0.0118 & $5.5 \%$ & $9.2 \%$ \\
\hline 2 & ROC-23 & Fe2O3 (wt\%) & 6.8912 & 6.8579 & 7.1702 & -0.2791 & -0.3123 & $-3.9 \%$ & $-4.4 \%$ \\
\hline 2 & ROC-23 & K2O (wt\%) & 0.0301 & 0.0304 & 0.0000 & 0.0301 & 0.0304 & & \\
\hline 2 & ROC-23 & La2O3 (wt\%) & 0.0652 & 0.0652 & 0.0978 & -0.0326 & -0.0326 & $-33.3 \%$ & $-33.3 \%$ \\
\hline 2 & ROC-23 & Li2O (wt\%) & 4.7794 & 4.8917 & 4.9053 & -0.1259 & -0.0136 & $-2.6 \%$ & $-0.3 \%$ \\
\hline 2 & ROC-23 & $\mathrm{MgO}(\mathrm{wt} \%)$ & 1.3706 & 1.3701 & 1.4206 & -0.0500 & -0.0505 & $-3.5 \%$ & $-3.6 \%$ \\
\hline 2 & ROC-23 & $\mathrm{MnO}(\mathrm{wt} \%)$ & 1.7819 & 1.8252 & 1.8947 & -0.1128 & -0.0695 & $-6.0 \%$ & $-3.7 \%$ \\
\hline 2 & ROC-23 & $\mathrm{Na} 2 \mathrm{O}$ (wt\%) & 10.7200 & 10.7261 & 10.8896 & -0.1697 & -0.1636 & $-1.6 \%$ & $-1.5 \%$ \\
\hline 2 & ROC-23 & $\mathrm{NiO}(\mathrm{wt} \%)$ & 1.5652 & 1.5509 & 1.9340 & -0.3688 & -0.3830 & $-19.1 \%$ & $-19.8 \%$ \\
\hline 2 & ROC-23 & $\mathrm{PbO}(\mathrm{wt} \%)$ & 0.1893 & 0.1893 & 0.2163 & -0.0270 & -0.0270 & $-12.5 \%$ & $-12.5 \%$ \\
\hline 2 & ROC-23 & $\mathrm{SiO} 2$ (wt\%) & 46.1019 & 47.2250 & 49.3543 & -3.2524 & -2.1293 & $-6.6 \%$ & $-4.3 \%$ \\
\hline 2 & ROC-23 & SO4 (wt\%) & 0.2247 & 0.2247 & 0.4808 & -0.2561 & -0.2561 & $-53.3 \%$ & $-53.3 \%$ \\
\hline 2 & ROC-23 & TiO2 (wt\%) & 1.8974 & 1.9529 & 2.0000 & -0.1027 & -0.0471 & $-5.1 \%$ & $-2.4 \%$ \\
\hline 2 & ROC-23 & U3O8 (wt\%) & 2.3555 & 2.3715 & 2.5543 & -0.1989 & -0.1829 & $-7.8 \%$ & $-7.2 \%$ \\
\hline 2 & ROC-23 & $\mathrm{ZnO}(\mathrm{wt} \%)$ & 0.1200 & 0.1200 & 0.1346 & -0.0146 & -0.0146 & $-10.9 \%$ & $-10.9 \%$ \\
\hline 2 & ROC-23 & $\mathrm{ZrO} 2$ (wt\%) & 0.1695 & 0.1695 & 0.2051 & -0.0356 & -0.0356 & $-17.4 \%$ & $-17.4 \%$ \\
\hline 2 & ROC-23 & Sum & 95.2820 & 96.9503 & 100.0000 & -4.7180 & -3.0497 & $-4.7 \%$ & $-3.0 \%$ \\
\hline 2 & ROC-24 & $\mathrm{Al} 2 \mathrm{O} 3$ (wt\%) & 10.6190 & 10.9931 & 10.2084 & 0.4106 & 0.7846 & $4.0 \%$ & $7.7 \%$ \\
\hline 2 & ROC-24 & B2O3 (wt\%) & 10.8994 & 11.5795 & 11.0905 & -0.1911 & 0.4891 & $-1.7 \%$ & $4.4 \%$ \\
\hline 2 & ROC-24 & $\mathrm{BaO}(\mathrm{wt} \%)$ & 0.0818 & 0.0838 & 0.0801 & 0.0017 & 0.0037 & $2.1 \%$ & $4.6 \%$ \\
\hline 2 & ROC-24 & $\mathrm{CaO}(\mathrm{wt} \%)$ & 0.0087 & 0.0088 & 0.0005 & 0.0083 & 0.0084 & $1804.5 \%$ & $1820.5 \%$ \\
\hline 2 & ROC-24 & $\mathrm{CdO}$ (wt\%) & 0.2690 & 0.2690 & 0.2965 & -0.0275 & -0.0275 & $-9.3 \%$ & $-9.3 \%$ \\
\hline 2 & ROC-24 & Ce2O3 (wt\%) & 0.3479 & 0.3479 & 0.3606 & -0.0127 & -0.0127 & $-3.5 \%$ & $-3.5 \%$ \\
\hline 2 & ROC-24 & Cr2O3 (wt\%) & 0.1597 & 0.1583 & 0.2000 & -0.0403 & -0.0417 & $-20.2 \%$ & $-20.8 \%$ \\
\hline 2 & ROC-24 & $\mathrm{CuO}$ (wt\%) & 0.1408 & 0.1428 & 0.1282 & 0.0126 & 0.0146 & $9.8 \%$ & $11.4 \%$ \\
\hline 2 & ROC-24 & Fe2O3 (wt\%) & 6.6696 & 6.8489 & 6.8900 & -0.2204 & -0.0410 & $-3.2 \%$ & $-0.6 \%$ \\
\hline 2 & ROC-24 & K2O (wt\%) & 0.0301 & 0.0311 & 0.0000 & 0.0301 & 0.0311 & & \\
\hline 2 & ROC-24 & La2O3 (wt\%) & 0.0780 & 0.0780 & 0.0978 & -0.0198 & -0.0198 & $-20.2 \%$ & $-20.2 \%$ \\
\hline 2 & ROC-24 & Li2O (wt\%) & 3.9129 & 4.1494 & 4.0384 & -0.1255 & 0.1111 & $-3.1 \%$ & $2.8 \%$ \\
\hline 2 & ROC-24 & MgO (wt\%) & 1.4622 & 1.4708 & 1.5000 & -0.0378 & -0.0292 & $-2.5 \%$ & $-1.9 \%$ \\
\hline 2 & ROC-24 & $\mathrm{MnO}(\mathrm{wt} \%)$ & 5.2875 & 5.4719 & 5.4448 & -0.1573 & 0.0271 & $-2.9 \%$ & $0.5 \%$ \\
\hline 2 & ROC-24 & $\mathrm{Na} 2 \mathrm{O}$ (wt\%) & 10.5110 & 10.4792 & 10.2812 & 0.2299 & 0.1981 & $2.2 \%$ & $1.9 \%$ \\
\hline 2 & ROC-24 & $\mathrm{NiO}(\mathrm{wt} \%)$ & 0.1858 & 0.1822 & 0.1463 & 0.0395 & 0.0360 & $27.0 \%$ & $24.6 \%$ \\
\hline 2 & ROC-24 & $\mathrm{PbO}(\mathrm{wt} \%)$ & 0.2227 & 0.2227 & 0.2163 & 0.0064 & 0.0064 & $2.9 \%$ & $2.9 \%$ \\
\hline 2 & ROC-24 & $\mathrm{SiO} 2(\mathrm{wt} \%)$ & 44.4440 & 45.1002 & 44.9492 & -0.5052 & 0.1510 & $-1.1 \%$ & $0.3 \%$ \\
\hline 2 & ROC-24 & SO4 (wt\%) & 0.2247 & 0.2247 & 0.4808 & -0.2561 & -0.2561 & $-53.3 \%$ & $-53.3 \%$ \\
\hline 2 & ROC-24 & $\mathrm{TiO} 2$ (wt\%) & 1.7097 & 1.8079 & 2.0000 & -0.2903 & -0.1921 & $-14.5 \%$ & $-9.6 \%$ \\
\hline 2 & ROC-24 & U3O8 (wt\%) & 1.3767 & 1.3601 & 1.2509 & 0.1258 & 0.1092 & $10.1 \%$ & $8.7 \%$ \\
\hline
\end{tabular}


SRNL-STI-2009-00465, Revision 0

Table A3. Average Measured and Bias-Corrected Chemical Compositions Versus Targeted Compositions by Oxide by Glass ID

\begin{tabular}{|c|c|c|c|c|c|c|c|c|c|}
\hline & & & & "Measured & & & & & \\
\hline & & & Measured & Bias-Corrected & Targeted & Diff of & Diff of & \% Diff of & \% Diff of \\
\hline Set & Glass \# & Oxide & (wt\%) & (wt\%) & (wt\%) & Measured & Meas BC & Measured & Meas BC \\
\hline 2 & ROC-24 & $\mathrm{ZnO}(\mathrm{wt} \%)$ & 0.1382 & 0.1382 & 0.1346 & 0.0036 & 0.0036 & $2.6 \%$ & $2.6 \%$ \\
\hline 2 & ROC-24 & ZrO2 (wt\%) & 0.1749 & 0.1749 & 0.2051 & -0.0302 & -0.0302 & $-14.7 \%$ & $-14.7 \%$ \\
\hline 2 & ROC-24 & Sum & 98.9542 & 101.3236 & 100.0000 & -1.0458 & 1.3236 & $-1.0 \%$ & $1.3 \%$ \\
\hline 2 & ROC-25 & $\mathrm{Al} 2 \mathrm{O} 3$ (wt\%) & 5.3709 & 5.3837 & 4.8802 & 0.4907 & 0.5035 & $10.1 \%$ & $10.3 \%$ \\
\hline 2 & ROC-25 & B2O3 (wt\%) & 4.4515 & 4.7201 & 4.5000 & -0.0485 & 0.2201 & $-1.1 \%$ & $4.9 \%$ \\
\hline 2 & ROC-25 & $\mathrm{BaO}$ (wt\%) & 0.0790 & 0.0802 & 0.0801 & -0.0011 & 0.0001 & $-1.4 \%$ & $0.1 \%$ \\
\hline 2 & ROC-25 & $\mathrm{CaO}(\mathrm{wt} \%)$ & 0.0252 & 0.0256 & 0.0000 & 0.0252 & 0.0256 & & \\
\hline 2 & ROC-25 & $\mathrm{CdO}(\mathrm{wt} \%)$ & 0.2662 & 0.2662 & 0.2965 & -0.0303 & -0.0303 & $-10.2 \%$ & $-10.2 \%$ \\
\hline 2 & ROC-25 & Ce2O3 (wt\%) & 0.3136 & 0.3136 & 0.3606 & -0.0470 & -0.0470 & $-13.0 \%$ & $-13.0 \%$ \\
\hline 2 & ROC-25 & Cr2O3 (wt\%) & 0.1776 & 0.1786 & 0.2000 & -0.0224 & -0.0214 & $-11.2 \%$ & $-10.7 \%$ \\
\hline 2 & ROC-25 & $\mathrm{CuO}(\mathrm{wt} \%)$ & 0.1324 & 0.1371 & 0.1282 & 0.0042 & 0.0088 & $3.3 \%$ & $6.9 \%$ \\
\hline 2 & ROC-25 & Fe2O3 (wt\%) & 12.1024 & 12.0425 & 11.8414 & 0.2610 & 0.2011 & $2.2 \%$ & $1.7 \%$ \\
\hline 2 & ROC-25 & K2O (wt\%) & 0.0301 & 0.0304 & 0.0000 & 0.0301 & 0.0304 & & \\
\hline 2 & ROC-25 & La2O3 (wt\%) & 0.0744 & 0.0744 & 0.0978 & -0.0234 & -0.0234 & $-23.9 \%$ & $-23.9 \%$ \\
\hline 2 & ROC-25 & Li2O (wt\%) & 4.0152 & 4.1095 & 4.0000 & 0.0152 & 0.1095 & $0.4 \%$ & $2.7 \%$ \\
\hline 2 & ROC-25 & $\mathrm{MgO}(\mathrm{wt} \%)$ & 1.4299 & 1.4294 & 1.5000 & -0.0701 & -0.0706 & $-4.7 \%$ & $-4.7 \%$ \\
\hline 2 & ROC-25 & $\mathrm{MnO}(\mathrm{wt} \%)$ & 5.1422 & 5.2672 & 5.5000 & -0.3578 & -0.2328 & $-6.5 \%$ & $-4.2 \%$ \\
\hline 2 & ROC-25 & $\mathrm{Na} 2 \mathrm{O}(\mathrm{wt} \%)$ & 14.6932 & 14.7016 & 15.4453 & -0.7521 & -0.7438 & $-4.9 \%$ & $-4.8 \%$ \\
\hline 2 & ROC-25 & $\mathrm{NiO}(\mathrm{wt} \%)$ & 0.0802 & 0.0794 & 0.0493 & 0.0308 & 0.0301 & $62.5 \%$ & $61.0 \%$ \\
\hline 2 & ROC-25 & $\mathrm{PbO}(\mathrm{wt} \%)$ & 0.1810 & 0.1810 & 0.2163 & -0.0354 & -0.0354 & $-16.4 \%$ & $-16.4 \%$ \\
\hline 2 & ROC-25 & $\mathrm{SiO} 2$ (wt\%) & 44.0696 & 45.1432 & 47.0780 & -3.0084 & -1.9348 & $-6.4 \%$ & $-4.1 \%$ \\
\hline 2 & ROC-25 & SO4 (wt\%) & 0.4344 & 0.4344 & 0.4808 & -0.0464 & -0.0464 & $-9.6 \%$ & $-9.6 \%$ \\
\hline 2 & ROC-25 & $\mathrm{TiO} 2(\mathrm{wt} \%)$ & 0.4850 & 0.4992 & 0.5000 & -0.0150 & -0.0008 & $-3.0 \%$ & $-0.2 \%$ \\
\hline 2 & ROC-25 & U3O8 (wt\%) & 2.3555 & 2.3715 & 2.5058 & -0.1504 & -0.1343 & $-6.0 \%$ & $-5.4 \%$ \\
\hline 2 & ROC-25 & $\mathrm{ZnO}(\mathrm{wt} \%)$ & 0.1111 & 0.1111 & 0.1346 & -0.0235 & -0.0235 & $-17.5 \%$ & $-17.5 \%$ \\
\hline 2 & ROC-25 & $\mathrm{ZrO} 2$ (wt\%) & 0.1716 & 0.1716 & 0.2051 & -0.0336 & -0.0336 & $-16.4 \%$ & $-16.4 \%$ \\
\hline 2 & ROC-25 & Sum & 96.1918 & 97.7512 & 100.0000 & -3.8082 & -2.2488 & $-3.8 \%$ & $-2.2 \%$ \\
\hline 2 & ROC-26 & $\mathrm{Al} 2 \mathrm{O} 3$ (wt\%) & 9.5939 & 9.6167 & 9.0736 & 0.5203 & 0.5431 & $5.7 \%$ & $6.0 \%$ \\
\hline 2 & ROC-26 & B2O3 (wt\%) & 6.2547 & 6.6304 & 6.4909 & -0.2362 & 0.1395 & $-3.6 \%$ & $2.1 \%$ \\
\hline 2 & ROC-26 & $\mathrm{BaO}$ (wt\%) & 0.0011 & 0.0011 & 0.0000 & 0.0011 & 0.0011 & & \\
\hline 2 & ROC-26 & $\mathrm{CaO}(\mathrm{wt} \%)$ & 0.0161 & 0.0162 & 0.0000 & 0.0161 & 0.0162 & & \\
\hline 2 & ROC-26 & CdO (wt\%) & 0.0040 & 0.0040 & 0.0000 & 0.0040 & 0.0040 & & \\
\hline 2 & ROC-26 & Ce2O3 (wt\%) & 0.0117 & 0.0117 & 0.0000 & 0.0117 & 0.0117 & & \\
\hline 2 & ROC-26 & Cr2O3 (wt\%) & 0.0230 & 0.0228 & 0.0000 & 0.0230 & 0.0228 & & \\
\hline 2 & ROC-26 & $\mathrm{CuO}(\mathrm{wt} \%)$ & 0.0282 & 0.0286 & 0.0000 & 0.0282 & 0.0286 & & \\
\hline 2 & ROC-26 & Fe2O3 (wt\%) & 5.1076 & 5.0827 & 5.0000 & 0.1076 & 0.0827 & $2.2 \%$ & $1.7 \%$ \\
\hline 2 & ROC-26 & K2O (wt\%) & 0.0301 & 0.0311 & 0.0000 & 0.0301 & 0.0311 & & \\
\hline 2 & ROC-26 & La2O3 (wt\%) & 0.0053 & 0.0053 & 0.0000 & 0.0053 & 0.0053 & & \\
\hline 2 & ROC-26 & Li2O (wt\%) & 4.0205 & 4.1150 & 4.0000 & 0.0205 & 0.1150 & $0.5 \%$ & $2.9 \%$ \\
\hline 2 & ROC-26 & $\mathrm{MgO}(\mathrm{wt} \%)$ & 0.0083 & 0.0083 & 0.0000 & 0.0083 & 0.0083 & & \\
\hline 2 & ROC-26 & $\mathrm{MnO}(\mathrm{wt} \%)$ & 3.2377 & 3.3507 & 3.2934 & -0.0557 & 0.0573 & $-1.7 \%$ & $1.7 \%$ \\
\hline 2 & ROC-26 & $\mathrm{Na} 2 \mathrm{O}(\mathrm{wt} \%)$ & 17.1870 & 17.1338 & 17.1686 & 0.0184 & -0.0348 & $0.1 \%$ & $-0.2 \%$ \\
\hline 2 & ROC-26 & $\mathrm{NiO}(\mathrm{wt} \%)$ & 0.0251 & 0.0246 & 0.0000 & 0.0251 & 0.0246 & & \\
\hline 2 & ROC-26 & $\mathrm{PbO}(\mathrm{wt} \%)$ & 0.0054 & 0.0054 & 0.0000 & 0.0054 & 0.0054 & & \\
\hline 2 & ROC-26 & $\mathrm{SiO} 2$ (wt\%) & 47.7064 & 48.4107 & 48.6758 & -0.9694 & -0.2651 & $-2.0 \%$ & $-0.5 \%$ \\
\hline 2 & ROC-26 & SO4 (wt\%) & 0.2247 & 0.2247 & 0.0000 & 0.2247 & 0.2247 & & \\
\hline 2 & ROC-26 & TiO2 (wt\%) & 0.4850 & 0.5128 & 0.5000 & -0.0150 & 0.0128 & $-3.0 \%$ & $2.6 \%$ \\
\hline 2 & ROC-26 & U3O8 (wt\%) & 5.4361 & 5.3720 & 5.7977 & -0.3616 & -0.4257 & $-6.2 \%$ & $-7.3 \%$ \\
\hline 2 & ROC-26 & $\mathrm{ZnO}(\mathrm{wt} \%)$ & 0.0124 & 0.0124 & 0.0000 & 0.0124 & 0.0124 & & \\
\hline 2 & ROC-26 & $\mathrm{ZrO} 2$ (wt\%) & 0.0034 & 0.0034 & 0.0000 & 0.0034 & 0.0034 & & \\
\hline 2 & ROC-26 & Sum & 99.4277 & 100.6245 & 100.0000 & -0.5723 & 0.6245 & $-0.6 \%$ & $0.6 \%$ \\
\hline 2 & ROC-27 & $\mathrm{Al} 2 \mathrm{O} 3$ (wt\%) & 4.3978 & 4.4082 & 3.9423 & 0.4555 & 0.4659 & $11.6 \%$ & $11.8 \%$ \\
\hline 2 & ROC-27 & $\mathrm{B} 2 \mathrm{O} 3(\mathrm{wt} \%)$ & 4.3549 & 4.6163 & 4.5006 & -0.1456 & 0.1158 & $-3.2 \%$ & $2.6 \%$ \\
\hline 2 & ROC-27 & $\mathrm{BaO}(\mathrm{wt} \%)$ & 0.0807 & 0.0819 & 0.0801 & 0.0005 & 0.0018 & $0.7 \%$ & $2.2 \%$ \\
\hline 2 & ROC-27 & $\mathrm{CaO}(\mathrm{wt} \%)$ & 0.0105 & 0.0106 & 0.0010 & 0.0095 & 0.0097 & $1003.4 \%$ & $1015.5 \%$ \\
\hline 2 & ROC-27 & CdO (wt\%) & 0.2733 & 0.2733 & 0.2965 & -0.0232 & -0.0232 & $-7.8 \%$ & $-7.8 \%$ \\
\hline 2 & ROC-27 & Ce2O3 (wt\%) & 0.3268 & 0.3268 & 0.3606 & -0.0338 & -0.0338 & $-9.4 \%$ & $-9.4 \%$ \\
\hline 2 & ROC-27 & Cr2O3 (wt\%) & 0.0365 & 0.0367 & 0.0010 & 0.0355 & 0.0357 & $3546.7 \%$ & $3567.6 \%$ \\
\hline 2 & ROC-27 & $\mathrm{CuO}(\mathrm{wt} \%)$ & 0.1377 & 0.1425 & 0.1282 & 0.0095 & 0.0143 & $7.4 \%$ & $11.2 \%$ \\
\hline 2 & ROC-27 & Fe2O3 (wt\%) & 14.7617 & 14.6873 & 15.1747 & -0.4130 & -0.4873 & $-2.7 \%$ & $-3.2 \%$ \\
\hline 2 & ROC-27 & K2O (wt\%) & 0.0413 & 0.0417 & 0.0000 & 0.0413 & 0.0417 & & \\
\hline 2 & ROC-27 & La2O3 (wt\%) & 0.0746 & 0.0746 & 0.0978 & -0.0232 & -0.0232 & $-23.7 \%$ & $-23.7 \%$ \\
\hline 2 & ROC-27 & Li2O (wt\%) & 3.9829 & 4.0764 & 4.0010 & -0.0181 & 0.0754 & $-0.5 \%$ & $1.9 \%$ \\
\hline 2 & ROC-27 & $\mathrm{MgO}(\mathrm{wt} \%)$ & 1.4763 & 1.4758 & 1.5000 & -0.0237 & -0.0242 & $-1.6 \%$ & $-1.6 \%$ \\
\hline 2 & ROC-27 & $\mathrm{MnO}(\mathrm{wt} \%)$ & 5.2035 & 5.3301 & 5.4981 & -0.2946 & -0.1680 & $-5.4 \%$ & $-3.1 \%$ \\
\hline 2 & ROC-27 & $\mathrm{Na} 2 \mathrm{O}(\mathrm{wt} \%)$ & 11.6198 & 11.6264 & 11.7989 & -0.1791 & -0.1725 & $-1.5 \%$ & $-1.5 \%$ \\
\hline 2 & ROC-27 & $\mathrm{NiO}(\mathrm{wt} \%)$ & 1.4729 & 1.4594 & 1.9462 & -0.4732 & -0.4868 & $-24.3 \%$ & $-25.0 \%$ \\
\hline 2 & ROC-27 & $\mathrm{PbO}(\mathrm{wt} \%)$ & 0.1890 & 0.1890 & 0.2163 & -0.0273 & -0.0273 & $-12.6 \%$ & $-12.6 \%$ \\
\hline 2 & ROC-27 & $\mathrm{SiO} 2$ (wt\%) & 42.7325 & 43.7739 & 43.6480 & -0.9155 & 0.1259 & $-2.1 \%$ & $0.3 \%$ \\
\hline
\end{tabular}


SRNL-STI-2009-00465, Revision 0

Table A3. Average Measured and Bias-Corrected Chemical Compositions Versus Targeted Compositions by Oxide by Glass ID

\begin{tabular}{|c|c|c|c|c|c|c|c|c|c|}
\hline & & & & Measured & & & & & \\
\hline & & & Measured & Bias-Corrected & Targeted & Diff of & Diff of & $\%$ Diff of & \% Diff of \\
\hline Set & Glass \# & Oxide & (wt\%) & (wt\%) & (wt\%) & Measured & Meas BC & Measured & Meas BC \\
\hline 2 & ROC-27 & SO4 (wt \%) & 0.2951 & 0.2951 & 0.4808 & -0.1857 & -0.1857 & $-38.6 \%$ & $-38.6 \%$ \\
\hline 2 & ROC-27 & $\mathrm{TiO} 2$ (wt\%) & 1.8765 & 1.9314 & 2.0000 & -0.1235 & -0.0686 & $-6.2 \%$ & $-3.4 \%$ \\
\hline 2 & ROC-27 & U3O8 (wt\%) & 3.8206 & 3.8464 & 3.9884 & -0.1678 & -0.1420 & $-4.2 \%$ & $-3.6 \%$ \\
\hline 2 & ROC-27 & $\mathrm{ZnO}(\mathrm{wt} \%)$ & 0.1183 & 0.1183 & 0.1346 & -0.0164 & -0.0164 & $-12.2 \%$ & $-12.2 \%$ \\
\hline 2 & ROC-27 & $\mathrm{ZrO} 2$ (wt\%) & 0.1773 & 0.1773 & 0.2051 & -0.0278 & -0.0278 & $-13.6 \%$ & $-13.6 \%$ \\
\hline 2 & ROC-27 & Sum & 97.4604 & 98.9994 & 100.0000 & -2.5396 & -1.0006 & $-2.5 \%$ & $-1.0 \%$ \\
\hline 2 & ROC-28 & $\mathrm{Al} 2 \mathrm{O} 3$ (wt\%) & 4.8088 & 4.8202 & 4.4852 & 0.3236 & 0.3350 & $7.2 \%$ & $7.5 \%$ \\
\hline 2 & ROC-28 & B2O3 (wt\%) & 4.2986 & 4.5574 & 4.5000 & -0.2014 & 0.0574 & $-4.5 \%$ & $1.3 \%$ \\
\hline 2 & ROC-28 & $\mathrm{BaO}$ (wt\%) & 0.0770 & 0.0782 & 0.0801 & -0.0031 & -0.0019 & $-3.9 \%$ & $-2.4 \%$ \\
\hline 2 & ROC-28 & $\mathrm{CaO}(\mathrm{wt} \%)$ & 0.0070 & 0.0071 & 0.0000 & 0.0070 & 0.0071 & & \\
\hline 2 & ROC-28 & $\mathrm{CdO}$ (wt\%) & 0.2650 & 0.2650 & 0.2965 & -0.0315 & -0.0315 & $-10.6 \%$ & $-10.6 \%$ \\
\hline 2 & ROC-28 & Ce2O3 (wt\%) & 0.3180 & 0.3180 & 0.3606 & -0.0426 & -0.0426 & $-11.8 \%$ & $-11.8 \%$ \\
\hline 2 & ROC-28 & Cr2O3 (wt\%) & 0.0161 & 0.0162 & 0.0000 & 0.0161 & 0.0162 & & \\
\hline 2 & ROC-28 & $\mathrm{CuO}$ (wt\%) & 0.1346 & 0.1393 & 0.1282 & 0.0064 & 0.0111 & $5.0 \%$ & $8.7 \%$ \\
\hline 2 & ROC-28 & Fe2O3 (wt\%) & 6.0869 & 6.0588 & 6.3446 & -0.2577 & -0.2859 & $-4.1 \%$ & $-4.5 \%$ \\
\hline 2 & ROC-28 & K2O (wt\%) & 0.0301 & 0.0304 & 0.0000 & 0.0301 & 0.0304 & & \\
\hline 2 & ROC-28 & La2O3 (wt\%) & 0.0705 & 0.0705 & 0.0978 & -0.0273 & -0.0273 & $-27.9 \%$ & $-27.9 \%$ \\
\hline 2 & ROC-28 & Li2O (wt\%) & 4.0851 & 4.1811 & 4.1343 & -0.0492 & 0.0468 & $-1.2 \%$ & $1.1 \%$ \\
\hline 2 & ROC-28 & MgO (wt\%) & 1.4850 & 1.4845 & 1.5000 & -0.0150 & -0.0155 & $-1.0 \%$ & $-1.0 \%$ \\
\hline 2 & ROC-28 & $\mathrm{MnO}(\mathrm{wt} \%)$ & 0.4777 & 0.4894 & 0.4745 & 0.0033 & 0.0149 & $0.7 \%$ & $3.1 \%$ \\
\hline 2 & ROC-28 & $\mathrm{Na} 2 \mathrm{O}$ (wt\%) & 17.1196 & 17.1293 & 17.6732 & -0.5536 & -0.5439 & $-3.1 \%$ & $-3.1 \%$ \\
\hline 2 & ROC-28 & $\mathrm{NiO}(\mathrm{wt} \%)$ & 0.0248 & 0.0246 & 0.0000 & 0.0248 & 0.0246 & & \\
\hline 2 & ROC-28 & $\mathrm{PbO}(\mathrm{wt} \%)$ & 0.1842 & 0.1842 & 0.2163 & -0.0321 & -0.0321 & $-14.9 \%$ & $-14.9 \%$ \\
\hline 2 & ROC-28 & $\mathrm{SiO} 2(\mathrm{wt} \%)$ & 52.9477 & 54.2376 & 55.0000 & -2.0523 & -0.7624 & $-3.7 \%$ & $-1.4 \%$ \\
\hline 2 & ROC-28 & SO4 (wt\%) & 0.3820 & 0.3820 & 0.4808 & -0.0988 & -0.0988 & $-20.5 \%$ & $-20.5 \%$ \\
\hline 2 & ROC-28 & TiO2 (wt\%) & 1.9099 & 1.9658 & 2.0000 & -0.0901 & -0.0342 & $-4.5 \%$ & $-1.7 \%$ \\
\hline 2 & ROC-28 & U3O8 (wt\%) & 1.8219 & 1.8343 & 1.8882 & -0.0663 & -0.0539 & $-3.5 \%$ & $-2.9 \%$ \\
\hline 2 & ROC-28 & $\mathrm{ZnO}(\mathrm{wt} \%)$ & 0.1114 & 0.1114 & 0.1346 & -0.0232 & -0.0232 & $-17.2 \%$ & $-17.2 \%$ \\
\hline 2 & ROC-28 & $\mathrm{ZrO} 2$ (wt\%) & 0.1797 & 0.1797 & 0.2051 & -0.0255 & -0.0255 & $-12.4 \%$ & $-12.4 \%$ \\
\hline 2 & ROC-28 & Sum & 96.8415 & 98.5649 & 100.0000 & -3.1585 & -1.4351 & $-3.2 \%$ & $-1.4 \%$ \\
\hline 2 & ROC-29 & $\mathrm{Al} 2 \mathrm{O} 3$ (wt\%) & 11.2142 & 11.6105 & 10.7599 & 0.4543 & 0.8506 & $4.2 \%$ & $7.9 \%$ \\
\hline 2 & ROC-29 & B2O3 (wt\%) & 12.2115 & 12.9744 & 12.4790 & -0.2675 & 0.4954 & $-2.1 \%$ & $4.0 \%$ \\
\hline 2 & ROC-29 & $\mathrm{BaO}(\mathrm{wt} \%)$ & 0.0770 & 0.0789 & 0.0801 & -0.0031 & -0.0012 & $-3.9 \%$ & $-1.5 \%$ \\
\hline 2 & ROC-29 & $\mathrm{CaO}(\mathrm{wt} \%)$ & 0.0766 & 0.0772 & 0.0584 & 0.0182 & 0.0188 & $31.1 \%$ & $32.2 \%$ \\
\hline 2 & ROC-29 & CdO (wt\%) & 0.2499 & 0.2499 & 0.2965 & -0.0466 & -0.0466 & $-15.7 \%$ & $-15.7 \%$ \\
\hline 2 & ROC-29 & Ce2O3 (wt\%) & 0.3619 & 0.3619 & 0.3606 & 0.0014 & 0.0014 & $0.4 \%$ & $0.4 \%$ \\
\hline 2 & ROC-29 & Cr2O3 (wt\%) & 0.0300 & 0.0297 & 0.0000 & 0.0300 & 0.0297 & & \\
\hline 2 & ROC-29 & $\mathrm{CuO}$ (wt\%) & 0.1371 & 0.1390 & 0.1282 & 0.0089 & 0.0108 & $6.9 \%$ & $8.4 \%$ \\
\hline 2 & ROC-29 & $\mathrm{Fe} 2 \mathrm{O} 3$ (wt\%) & 7.5667 & 7.7705 & 7.7595 & -0.1928 & 0.0110 & $-2.5 \%$ & $0.1 \%$ \\
\hline 2 & ROC-29 & $\mathrm{K} 2 \mathrm{O}(\mathrm{wt} \%)$ & 0.0404 & 0.0416 & 0.0000 & 0.0404 & 0.0416 & & \\
\hline 2 & ROC-29 & La2O3 (wt\%) & 0.0715 & 0.0715 & 0.0978 & -0.0262 & -0.0262 & $-26.8 \%$ & $-26.8 \%$ \\
\hline 2 & ROC-29 & Li2O (wt\%) & 4.4565 & 4.7264 & 4.5994 & -0.1429 & 0.1270 & $-3.1 \%$ & $2.8 \%$ \\
\hline 2 & ROC-29 & $\mathrm{MgO}(\mathrm{wt} \%)$ & 0.0108 & 0.0108 & 0.0000 & 0.0108 & 0.0108 & & \\
\hline 2 & ROC-29 & $\mathrm{MnO}(\mathrm{wt} \%)$ & 4.7129 & 4.8773 & 4.9056 & -0.1927 & -0.0283 & $-3.9 \%$ & $-0.6 \%$ \\
\hline 2 & ROC-29 & Na2O (wt\%) & 10.7773 & 10.7457 & 10.6726 & 0.1047 & 0.0731 & $1.0 \%$ & $0.7 \%$ \\
\hline 2 & ROC-29 & $\mathrm{NiO}$ (wt\%) & 2.2491 & 2.2062 & 2.5000 & -0.2509 & -0.2938 & $-10.0 \%$ & $-11.8 \%$ \\
\hline 2 & ROC-29 & $\mathrm{PbO}(\mathrm{wt} \%)$ & 0.2197 & 0.2197 & 0.2163 & 0.0034 & 0.0034 & $1.6 \%$ & $1.6 \%$ \\
\hline 2 & ROC-29 & $\mathrm{SiO} 2$ (wt\%) & 37.7586 & 38.3166 & 38.1045 & -0.3459 & 0.2120 & $-0.9 \%$ & $0.6 \%$ \\
\hline 2 & ROC-29 & SO4 (wt\%) & 0.6172 & 0.6172 & 0.4808 & 0.1364 & 0.1364 & $28.4 \%$ & $28.4 \%$ \\
\hline 2 & ROC-29 & TiO2 (wt\%) & 0.4796 & 0.5071 & 0.5000 & -0.0205 & 0.0071 & $-4.1 \%$ & $1.4 \%$ \\
\hline 2 & ROC-29 & U3O8 (wt\%) & 5.2976 & 5.2356 & 5.6611 & -0.3636 & -0.4255 & $-6.4 \%$ & $-7.5 \%$ \\
\hline 2 & ROC-29 & $\mathrm{ZnO}(\mathrm{wt} \%)$ & 0.1494 & 0.1494 & 0.1346 & 0.0148 & 0.0148 & $11.0 \%$ & $11.0 \%$ \\
\hline 2 & ROC-29 & $\mathrm{ZrO} 2$ (wt\%) & 0.1759 & 0.1759 & 0.2051 & -0.0292 & -0.0292 & $-14.2 \%$ & $-14.2 \%$ \\
\hline 2 & ROC-29 & Sum & 98.9413 & 101.1931 & 100.0000 & -1.0587 & 1.1931 & $-1.1 \%$ & $1.2 \%$ \\
\hline 2 & ROC-30 & $\mathrm{Al} 2 \mathrm{O} 3$ (wt\%) & 6.4621 & 6.6914 & 6.1383 & 0.3238 & 0.5532 & $5.3 \%$ & $9.0 \%$ \\
\hline 2 & ROC-30 & $\mathrm{B} 2 \mathrm{O} 3(\mathrm{wt} \%)$ & 7.9371 & 8.4330 & 7.9577 & -0.0206 & 0.4753 & $-0.3 \%$ & $6.0 \%$ \\
\hline 2 & ROC-30 & $\mathrm{BaO}(\mathrm{wt} \%)$ & 0.0427 & 0.0438 & 0.0423 & 0.0004 & 0.0014 & $0.9 \%$ & $3.4 \%$ \\
\hline 2 & ROC-30 & $\mathrm{CaO}(\mathrm{wt} \%)$ & 1.0585 & 1.0672 & 1.0250 & 0.0335 & 0.0421 & $3.3 \%$ & $4.1 \%$ \\
\hline 2 & ROC-30 & $\mathrm{CdO}(\mathrm{wt} \%)$ & 0.1448 & 0.1448 & 0.1566 & -0.0119 & -0.0119 & $-7.6 \%$ & $-7.6 \%$ \\
\hline 2 & ROC-30 & Ce2O3 (wt\%) & 0.2023 & 0.2023 & 0.1905 & 0.0118 & 0.0118 & $6.2 \%$ & $6.2 \%$ \\
\hline 2 & ROC-30 & Cr2O3 (wt\%) & 0.0965 & 0.0956 & 0.0959 & 0.0006 & -0.0002 & $0.6 \%$ & $-0.2 \%$ \\
\hline 2 & ROC-30 & $\mathrm{CuO}$ (wt\%) & 0.0789 & 0.0800 & 0.0677 & 0.0111 & 0.0122 & $16.4 \%$ & $18.1 \%$ \\
\hline 2 & ROC-30 & Fe2O3 (wt\%) & 8.9678 & 9.2094 & 8.7193 & 0.2485 & 0.4902 & $2.9 \%$ & $5.6 \%$ \\
\hline 2 & ROC-30 & K2O (wt\%) & 0.0419 & 0.0431 & 0.0000 & 0.0419 & 0.0431 & & \\
\hline 2 & ROC-30 & La2O3 (wt\%) & 0.0358 & 0.0358 & 0.0517 & -0.0159 & -0.0159 & $-30.7 \%$ & $-30.7 \%$ \\
\hline 2 & ROC-30 & Li2O (wt\%) & 4.5480 & 4.8244 & 4.6296 & -0.0816 & 0.1949 & $-1.8 \%$ & $4.2 \%$ \\
\hline 2 & ROC-30 & $\mathrm{MgO}(\mathrm{wt} \%)$ & 0.8408 & 0.8457 & 0.8263 & 0.0145 & 0.0194 & $1.8 \%$ & $2.4 \%$ \\
\hline 2 & ROC-30 & $\mathrm{MnO}(\mathrm{wt} \%)$ & 3.5411 & 3.6646 & 3.6001 & -0.0589 & 0.0646 & $-1.6 \%$ & $1.8 \%$ \\
\hline 2 & ROC-30 & $\mathrm{Na} 2 \mathrm{O}(\mathrm{wt} \%)$ & 12.2567 & 12.2221 & 11.9887 & 0.2680 & 0.2334 & $2.2 \%$ & $1.9 \%$ \\
\hline
\end{tabular}


SRNL-STI-2009-00465, Revision 0

Table A3. Average Measured and Bias-Corrected Chemical Compositions Versus Targeted Compositions by Oxide by Glass ID

\begin{tabular}{|c|c|c|c|c|c|c|c|c|c|}
\hline & & & & Measured & & & & & \\
\hline & & & Measured & Bias-Corrected & Targeted & Diff of & Diff of & $\%$ Diff of & \% Diff of \\
\hline Set & Glass \# & Oxide & $(\mathrm{wt} \%)$ & $(w t \%)$ & $(\mathrm{wt} \%)$ & Measured & Meas BC & Measured & Meas BC \\
\hline 2 & ROC-30 & $\mathrm{NiO}$ (wt\%) & 1.2248 & 1.2014 & 1.3057 & -0.0810 & -0.1044 & $-6.2 \%$ & $-8.0 \%$ \\
\hline 2 & ROC-30 & $\mathrm{PbO}$ (wt\%) & 0.1150 & 0.1150 & 0.1143 & 0.0007 & 0.0007 & $0.6 \%$ & $0.6 \%$ \\
\hline 2 & ROC-30 & $\mathrm{SiO} 2(\mathrm{wt} \%)$ & 45.6206 & 46.2945 & 45.7022 & -0.0817 & 0.5923 & $-0.2 \%$ & $1.3 \%$ \\
\hline 2 & ROC-30 & SO4 (wt\%) & 0.2247 & 0.2247 & 0.2540 & -0.0293 & -0.0293 & $-11.5 \%$ & $-11.5 \%$ \\
\hline 2 & ROC-30 & $\mathrm{TiO} 2$ (wt\%) & 1.3569 & 1.4348 & 1.3707 & -0.0138 & 0.0641 & $-1.0 \%$ & $4.7 \%$ \\
\hline 2 & ROC-30 & U3O8 (wt\%) & 5.5157 & 5.4506 & 5.5839 & -0.0682 & -0.1332 & $-1.2 \%$ & $-2.4 \%$ \\
\hline 2 & ROC-30 & $\mathrm{ZnO}$ (wt\%) & 0.0815 & 0.0815 & 0.0711 & 0.0104 & 0.0104 & $14.6 \%$ & $14.6 \%$ \\
\hline 2 & ROC-30 & $\mathrm{ZrO} 2$ (wt\%) & 0.0959 & 0.0959 & 0.1084 & -0.0125 & -0.0125 & $-11.5 \%$ & $-11.5 \%$ \\
\hline 2 & ROC-30 & Sum & 100.4899 & 102.5018 & 100.0000 & 0.4899 & 2.5018 & $0.5 \%$ & $2.5 \%$ \\
\hline 1 & Ustd & $\mathrm{Al} 2 \mathrm{O} 3$ (wt\%) & 4.0656 & 4.1058 & 4.1000 & -0.0344 & 0.0058 & $-0.8 \%$ & $0.1 \%$ \\
\hline 1 & Ustd & B2O3 (wt\%) & 8.8011 & 9.4093 & 9.2090 & -0.4079 & 0.2003 & $-4.4 \%$ & $2.2 \%$ \\
\hline 1 & Ustd & $\mathrm{BaO}(\mathrm{wt} \%)$ & 0.0011 & 0.0011 & 0.0000 & 0.0011 & 0.0011 & & \\
\hline 1 & Ustd & $\mathrm{CaO}(\mathrm{wt} \%)$ & 1.3183 & 1.2833 & 1.3010 & 0.0173 & -0.0177 & $1.3 \%$ & $-1.4 \%$ \\
\hline 1 & Ustd & $\mathrm{CdO}(\mathrm{wt} \%)$ & 0.0040 & 0.0040 & 0.0000 & 0.0040 & 0.0040 & & \\
\hline 1 & Ustd & Ce2O3 (wt\%) & 0.0059 & 0.0059 & 0.0000 & 0.0059 & 0.0059 & & \\
\hline 1 & Ustd & Cr2O3 (wt\%) & 0.2365 & 0.2341 & 0.0000 & 0.2365 & 0.2341 & & \\
\hline 1 & Ustd & $\mathrm{CuO}$ (wt\%) & 0.0147 & 0.0157 & 0.0000 & 0.0147 & 0.0157 & & \\
\hline 1 & Ustd & Fe2O3 (wt\%) & 13.3522 & 13.4726 & 13.1960 & 0.1562 & 0.2766 & $1.2 \%$ & $2.1 \%$ \\
\hline 1 & Ustd & K2O (wt\%) & 2.9422 & 2.9758 & 2.9990 & -0.0568 & -0.0232 & $-1.9 \%$ & $-0.8 \%$ \\
\hline 1 & Ustd & La2O3 (wt\%) & 0.0053 & 0.0053 & 0.0000 & 0.0053 & 0.0053 & & \\
\hline 1 & Ustd & Li2O (wt\%) & 2.9064 & 3.0545 & 3.0570 & -0.1506 & -0.0025 & $-4.9 \%$ & $-0.1 \%$ \\
\hline 1 & Ustd & $\mathrm{MgO}(\mathrm{wt} \%)$ & 1.1956 & 1.1921 & 1.2100 & -0.0144 & -0.0179 & $-1.2 \%$ & $-1.5 \%$ \\
\hline 1 & Ustd & $\mathrm{MnO}(\mathrm{wt} \%)$ & 2.6577 & 2.6662 & 2.8920 & -0.2343 & -0.2258 & $-8.1 \%$ & $-7.8 \%$ \\
\hline 1 & Ustd & Na2O (wt\%) & 11.8961 & 11.6641 & 11.7950 & 0.1011 & -0.1309 & $0.9 \%$ & $-1.1 \%$ \\
\hline 1 & Ustd & $\mathrm{NiO}(w \mathrm{t} \%)$ & 0.9797 & 0.9485 & 1.1200 & -0.1403 & -0.1715 & $-12.5 \%$ & $-15.3 \%$ \\
\hline 1 & Ustd & $\mathrm{PbO}(\mathrm{wt} \%)$ & 0.0054 & 0.0054 & 0.0000 & 0.0054 & 0.0054 & & \\
\hline 1 & Ustd & SiO2 (wt\%) & 45.2462 & 46.1307 & 45.3530 & -0.1068 & 0.7777 & $-0.2 \%$ & $1.7 \%$ \\
\hline 1 & Ustd & SO4 (wt\%) & 0.2247 & 0.2247 & 0.0000 & 0.2247 & 0.2247 & & \\
\hline 1 & Ustd & $\mathrm{TiO} 2$ (wt\%) & 0.9424 & 0.9548 & 1.0490 & -0.1066 & -0.0942 & $-10.2 \%$ & $-9.0 \%$ \\
\hline 1 & Ustd & U3O8 (wt\%) & 2.4655 & 2.4060 & 2.4060 & 0.0595 & 0.0000 & $2.5 \%$ & $0.0 \%$ \\
\hline 1 & Ustd & $\mathrm{ZnO}(\mathrm{wt} \%)$ & 0.0124 & 0.0124 & 0.0000 & 0.0124 & 0.0124 & & \\
\hline 1 & Ustd & $\mathrm{ZrO} 2$ (wt\%) & 0.0034 & 0.0034 & 0.0000 & 0.0034 & 0.0034 & & \\
\hline 1 & Ustd & Sum & 99.2824 & 100.7758 & 99.6870 & -0.4046 & 1.0888 & $-0.4 \%$ & $1.1 \%$ \\
\hline 2 & Ustd & $\mathrm{Al} 2 \mathrm{O} 3(\mathrm{wt} \%)$ & 4.0656 & 4.1415 & 4.1000 & -0.0344 & 0.0415 & $-0.8 \%$ & $1.0 \%$ \\
\hline 2 & Ustd & B2O3 (wt\%) & 8.9272 & 9.4740 & 9.2090 & -0.2818 & 0.2650 & $-3.1 \%$ & $2.9 \%$ \\
\hline 2 & Ustd & $\mathrm{BaO}(\mathrm{wt} \%)$ & 0.0011 & 0.0011 & 0.0000 & 0.0011 & 0.0011 & & \\
\hline 2 & Ustd & $\mathrm{CaO}$ (wt\%) & 1.2758 & 1.2912 & 1.3010 & -0.0252 & -0.0098 & $-1.9 \%$ & $-0.8 \%$ \\
\hline 2 & Ustd & $\mathrm{CdO}$ (wt\%) & 0.0040 & 0.0040 & 0.0000 & 0.0040 & 0.0040 & & \\
\hline 2 & Ustd & Ce2O3 (wt\%) & 0.0117 & 0.0117 & 0.0000 & 0.0117 & 0.0117 & & \\
\hline 2 & Ustd & Cr2O3 (wt\%) & 0.2401 & 0.2398 & 0.0000 & 0.2401 & 0.2398 & & \\
\hline 2 & Ustd & $\mathrm{CuO}(\mathrm{wt} \%)$ & 0.0143 & 0.0146 & 0.0000 & 0.0143 & 0.0146 & & \\
\hline 2 & Ustd & Fe2O3 (wt\%) & 13.4952 & 13.6421 & 13.1960 & 0.2992 & 0.4461 & $2.3 \%$ & $3.4 \%$ \\
\hline 2 & Ustd & K2O (wt\%) & 2.9161 & 2.9754 & 2.9990 & -0.0829 & -0.0236 & $-2.8 \%$ & $-0.8 \%$ \\
\hline 2 & Ustd & La2O3 (wt\%) & 0.0053 & 0.0053 & 0.0000 & 0.0053 & 0.0053 & & \\
\hline 2 & Ustd & Li2O (wt\%) & 2.9907 & 3.1161 & 3.0570 & -0.0663 & 0.0591 & $-2.2 \%$ & $1.9 \%$ \\
\hline 2 & Ustd & $\mathrm{MgO}(\mathrm{wt} \%)$ & 1.1893 & 1.1926 & 1.2100 & -0.0207 & -0.0174 & $-1.7 \%$ & $-1.4 \%$ \\
\hline 2 & Ustd & $\mathrm{MnO}(\mathrm{wt} \%)$ & 2.7072 & 2.7873 & 2.8920 & -0.1848 & -0.1047 & $-6.4 \%$ & $-3.6 \%$ \\
\hline 2 & Ustd & Na2O (wt\%) & 11.7422 & 11.7277 & 11.7950 & -0.0528 & -0.0673 & $-0.4 \%$ & $-0.6 \%$ \\
\hline 2 & Ustd & $\mathrm{NiO}(\mathrm{wt} \%)$ & 1.0848 & 1.0695 & 1.1200 & -0.0352 & -0.0505 & $-3.1 \%$ & $-4.5 \%$ \\
\hline 2 & Ustd & $\mathrm{PbO}$ (wt\%) & 0.0054 & 0.0054 & 0.0000 & 0.0054 & 0.0054 & & \\
\hline 2 & Ustd & $\mathrm{SiO} 2$ (wt\%) & 44.5688 & 45.4396 & 45.3530 & -0.7843 & 0.0866 & $-1.7 \%$ & $0.2 \%$ \\
\hline 2 & Ustd & SO4 (wt\%) & 0.2247 & 0.2247 & 0.0000 & 0.2247 & 0.2247 & & \\
\hline 2 & Ustd & TiO2 (wt\%) & 0.9408 & 0.9814 & 1.0490 & -0.1082 & -0.0676 & $-10.3 \%$ & $-6.4 \%$ \\
\hline 2 & Ustd & U3O8 (wt\%) & 2.4124 & 2.4060 & 2.4060 & 0.0064 & 0.0000 & $0.3 \%$ & $0.0 \%$ \\
\hline 2 & Ustd & $\mathrm{ZnO}(\mathrm{wt} \%)$ & 0.0124 & 0.0124 & 0.0000 & 0.0124 & 0.0124 & & \\
\hline 2 & Ustd & $\mathrm{ZrO} 2$ (wt\%) & 0.0034 & 0.0034 & 0.0000 & 0.0034 & 0.0034 & & \\
\hline 2 & Ustd & Sum & 98.8385 & 100.7668 & 99.6870 & -0.8485 & 1.0798 & $-0.9 \%$ & $1.1 \%$ \\
\hline
\end{tabular}




\section{Exhibit A1. Measurements in Analytical Sequence for Samples by Oxide by Prep Method and by Analytical Set}

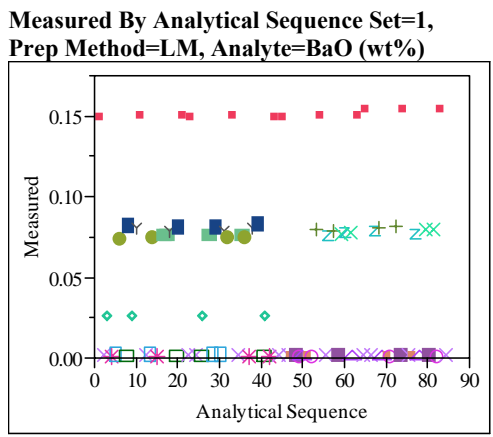

Measured By Analytical Sequence Set $=1$, Prep Method $=$ LM, Analyte $=\mathrm{CaO}(\mathbf{w t} \%)$

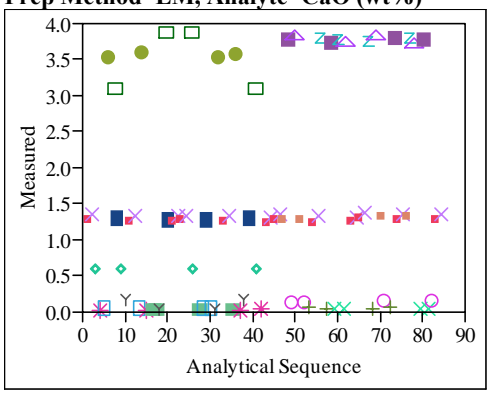

Measured By Analytical Sequence Set=1, Prep Method $=$ LM, Analyte $=\mathbf{C d O}(\mathbf{w t} \%)$

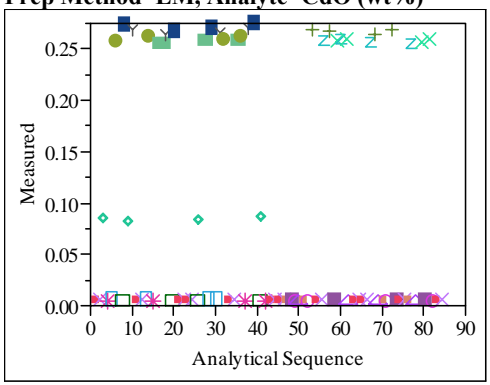

Measured By Analytical Sequence Set $=1$, Prep Method $=$ LM, Analyte $=\mathrm{Ce} 2 \mathrm{O3}(\mathrm{wt} \%)$

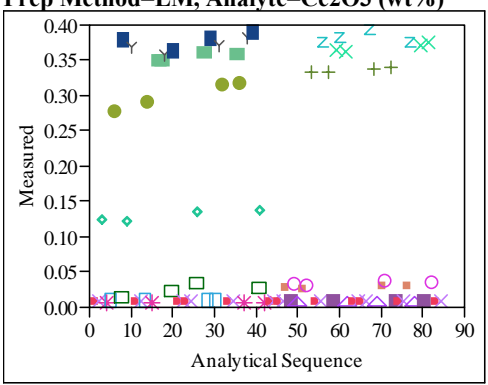

Measured By Analytical Sequence Set $=1$, Prep Method=LM, Analyte $=\mathbf{C r 2 O 3}\left(w_{t} \%\right)$

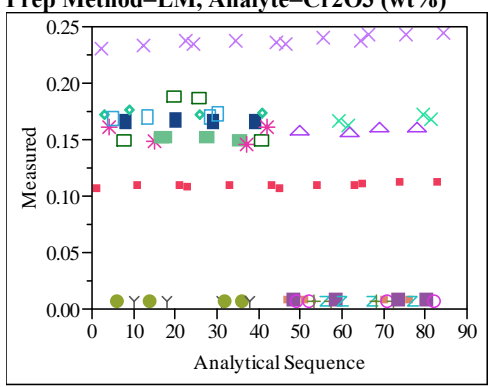

Measured By Analytical Sequence Set=1, Prep Method $=$ LM, Analyte $=\mathrm{CuO}\left(\mathrm{wt}^{\mathrm{O}} \mathrm{o}\right)$

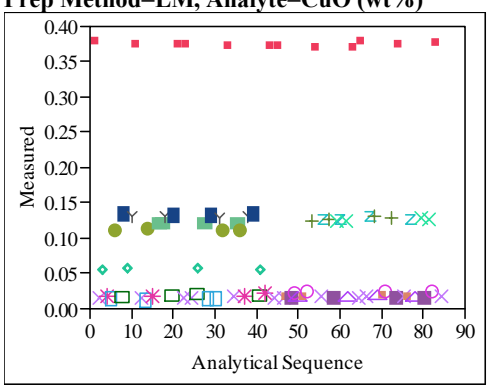

Measured By Analytical Sequence Set $=1$, Prep Method $=$ LM, Analyte $=$ K2O (wt $\%)$

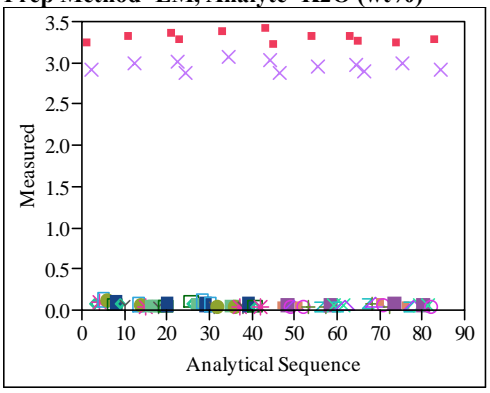

Measured By Analytical Sequence Set=1, Prep Method $=$ LM, Analyte $=\mathbf{L a 2 O 3}(\mathbf{w t} \%)$

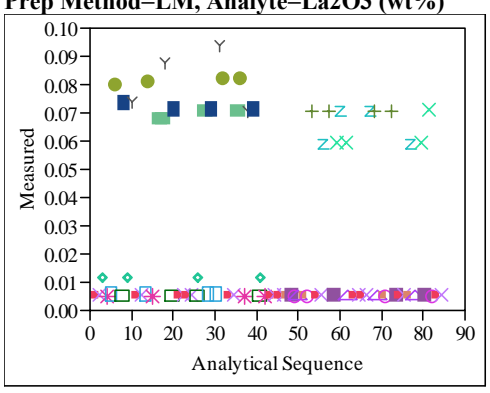

Measured By Analytical Sequence Set=1, Prep Method $=$ LM, Analyte $=$ MgO $(w t \%)$

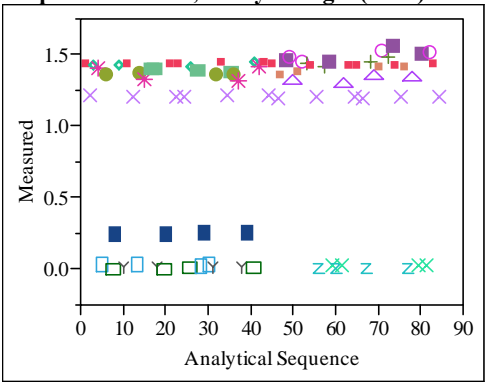

Measured By Analytical Sequence Set $=1$, Prep Method=LM, Analyte $=\mathrm{MnO}(\mathrm{wt} \%)$

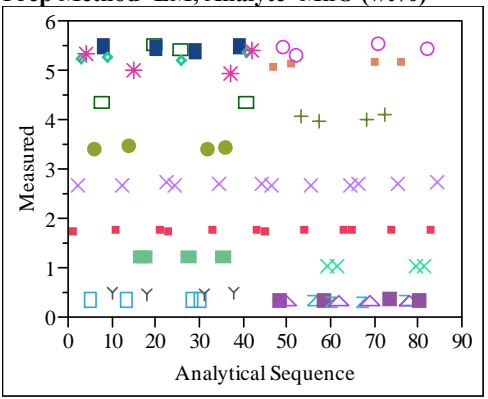

Measured By Analytical Sequence Set=1, Prep Method $=$ LM, Analyte $=\mathbf{N a 2 O}($ wt $\%)$

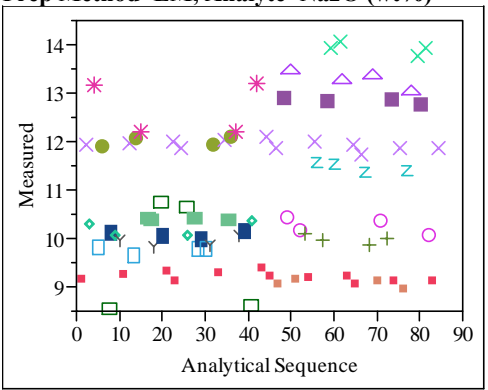

Measured By Analytical Sequence Set=1, Prep Method=LM, Analyte $=\mathrm{NiO}(\mathrm{wt} \%)$

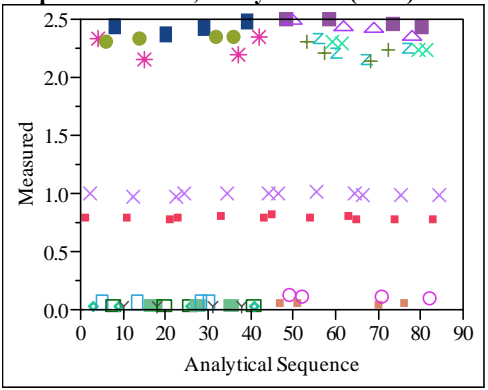




\section{Exhibit A1. Measurements in Analytical Sequence for Samples by Oxide by Prep Method and by Analytical Set}

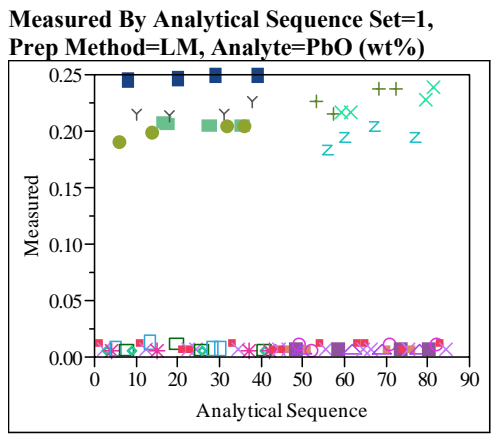

Measured By Analytical Sequence Set $=1$, Prep Method $=$ LM, Analyte $=\mathrm{SO} 4(\mathrm{wt} \%)$

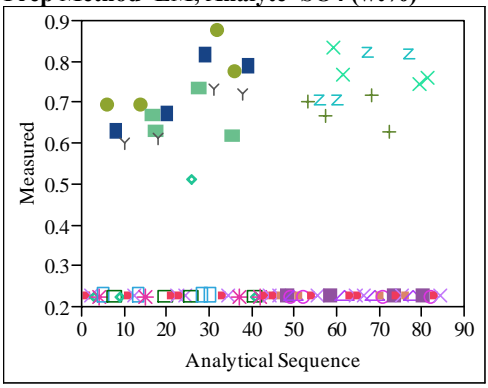

Measured By Analytical Sequence Set $=1$, Prep Method=LM, Analyte=TiO2 (wt \%)

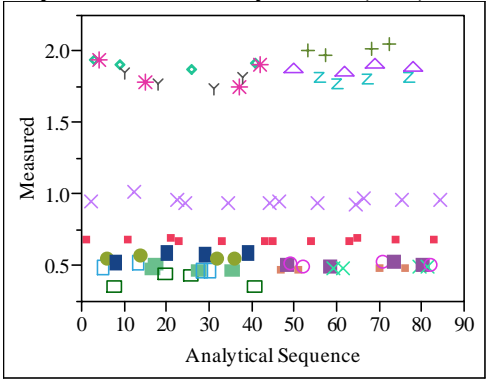

Measured By Analytical Sequence Set=1, Prep Method $=$ LM, Analyte $=$ U3O8 (wt $\%)$

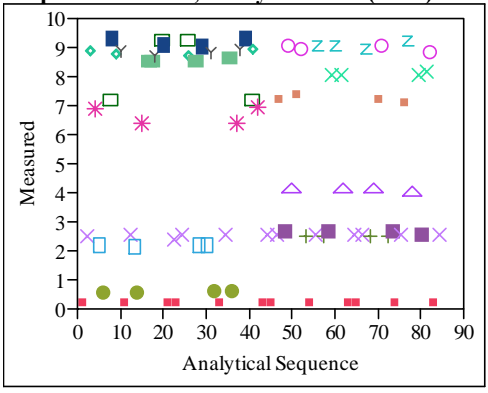

Measured By Analytical Sequence Set=1, Prep Method $=$ LM, Analyte $=\mathrm{ZnO}(\mathrm{wt} \%)$

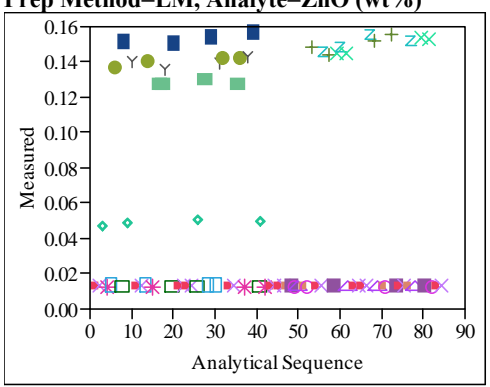

Measured By Analytical Sequence Set=1, Prep Method=LM, Analyte $=\mathrm{ZrO2}($ wt $\%)$

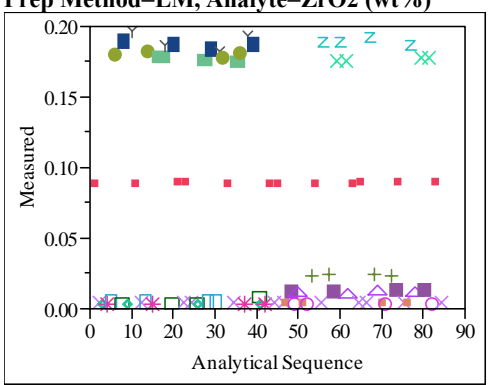

Measured By Analytical Sequence Set=1, Prep Method=PF, Analyte $=\mathbf{A l 2 O 3}(\mathbf{w t} \%)$

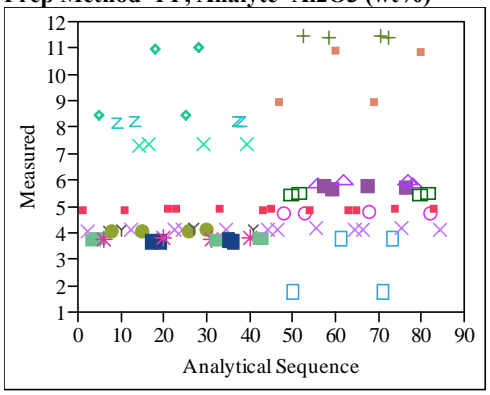

Measured By Analytical Sequence Set=1, Prep Method=PF, Analyte=B2O3 (wt\%)

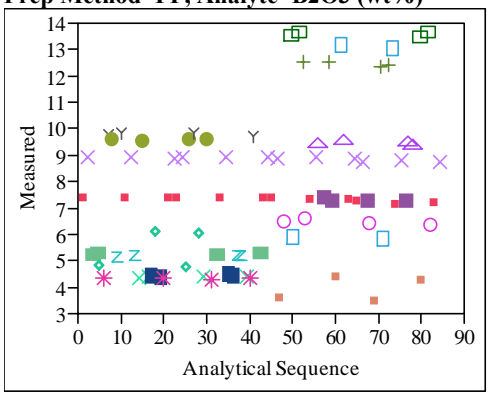

Measured By Analytical Sequence Set $=1$, Prep Method=PF, Analyte=Fe2O3 (wt \%)

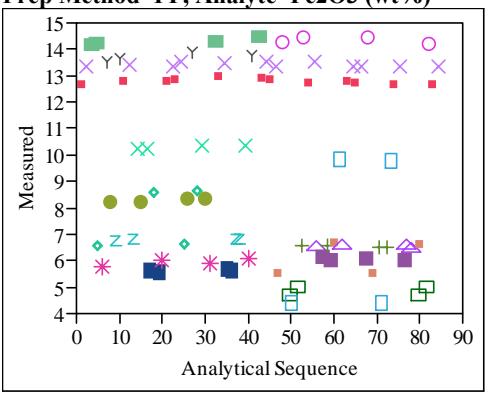

Measured By Analytical Sequence Set $=1$, Prep Method=PF, Analyte $=$ Li2O (wt\%)

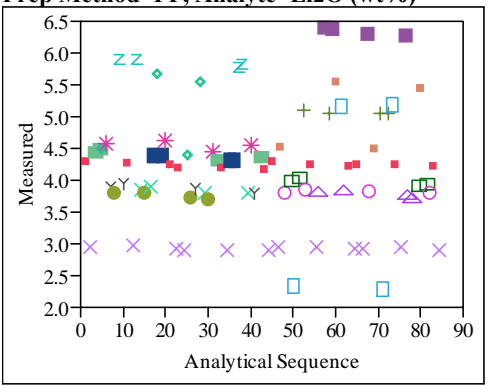

Measured By Analytical Sequence Set=1, Prep Method=PF, Analyte=SiO2 (wt\%)

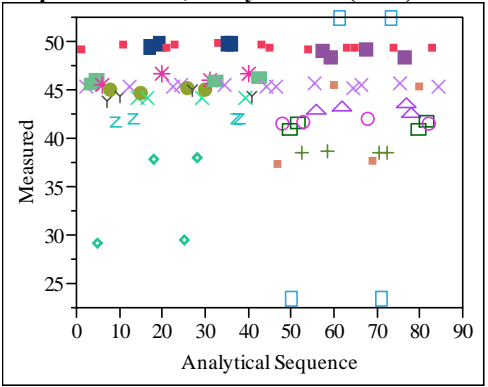

Measured By Analytical Sequence Set $=2$, Prep Method $=$ LM, Analyte $=\mathbf{B a O}(w t \%)$

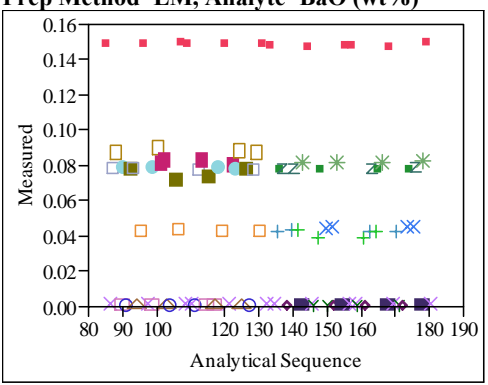




\section{Exhibit A1. Measurements in Analytical Sequence for Samples by Oxide by Prep Method and by Analytical Set}

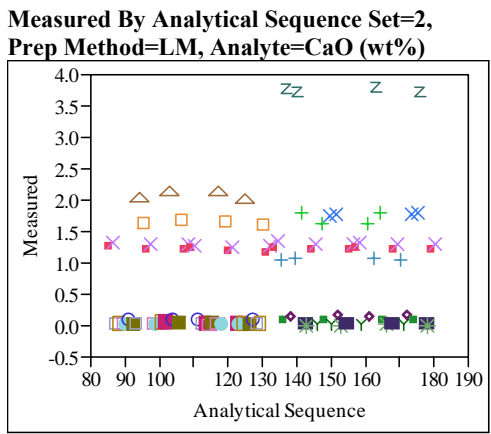

Measured By Analytical Sequence Set=2, Prep Method $=$ LM, Analyte $=\mathbf{C d O}(w t \%)$

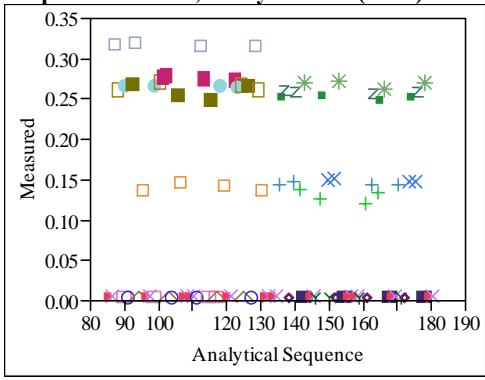

Measured By Analytical Sequence Set $=2$, Prep Method=LM, Analyte $=\mathrm{Ce} 2 \mathrm{O3}(\mathrm{wt} \%)$

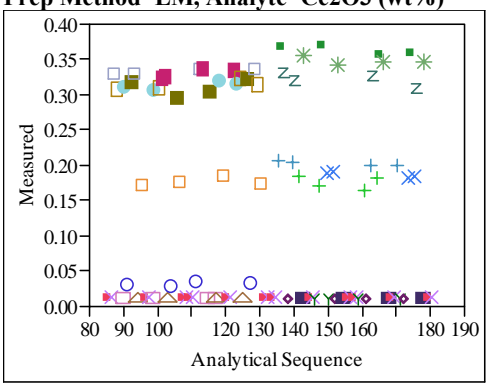

Measured By Analytical Sequence Set $=2$, Prep Method $=$ LM, Analyte $=\mathbf{C r 2 O 3}(\mathbf{w t} \%)$

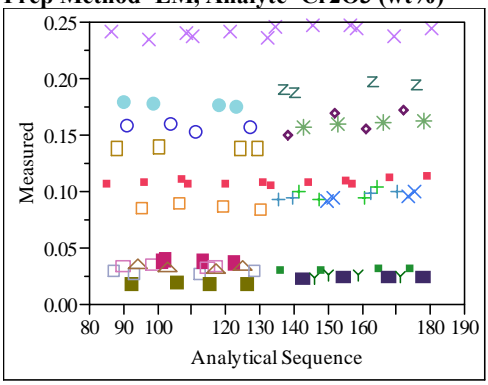

Measured By Analytical Sequence Set=2, Prep Method $=$ LM, Analyte $=\mathrm{CuO}(\mathrm{wt} \%)$

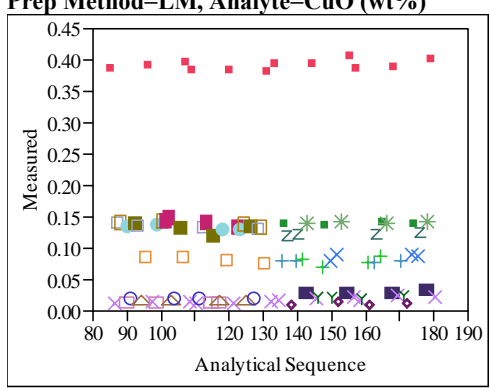

Measured By Analytical Sequence Set=2, Prep Method $=$ LM, Analyte $=$ K2O $\left(w^{2} \%\right)$

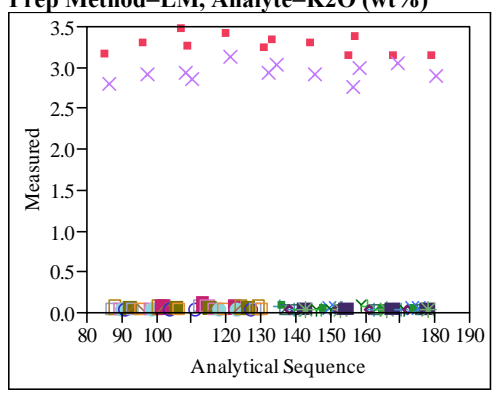

Measured By Analytical Sequence Set $=2$,

Prep Method $=$ LM, Analyte $=\mathbf{L a 2 O 3}\left(\mathrm{wt}^{\circ}\right)$

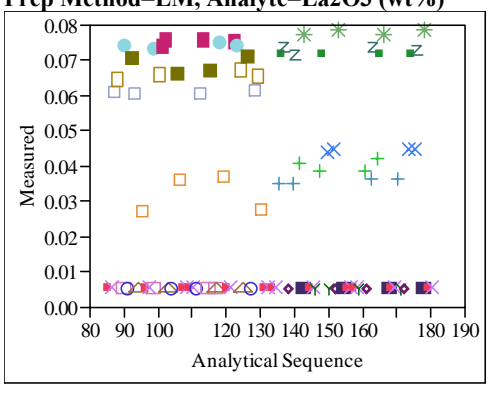

Measured By Analytical Sequence Set=2, Prep Method=LM, Analyte $=\mathbf{M g O}(\mathrm{wt} \%)$

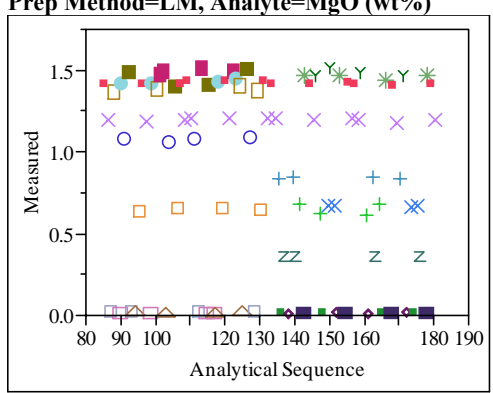

Measured By Analytical Sequence Set $=2$, Prep Method $=$ LM, Analyte $=$ MnO (wt\%)

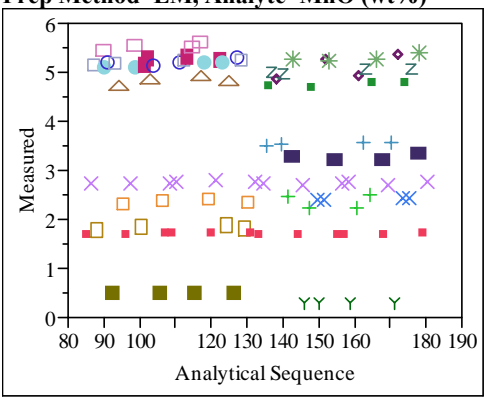

Measured By Analytical Sequence Set $=2$, Prep Method=LM, Analyte=Na2O (wt\%)

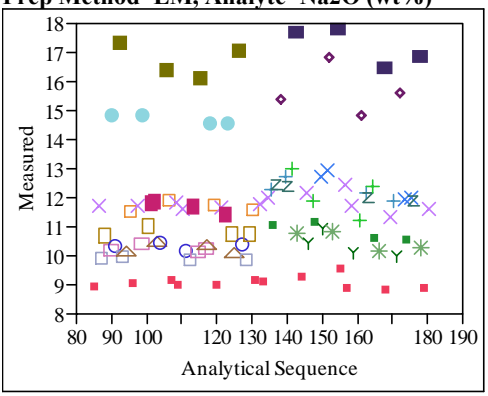

Measured By Analytical Sequence Set $=2$,

Prep Method=LM, Analyte $=\mathrm{NiO}(\mathbf{w t} \%)$

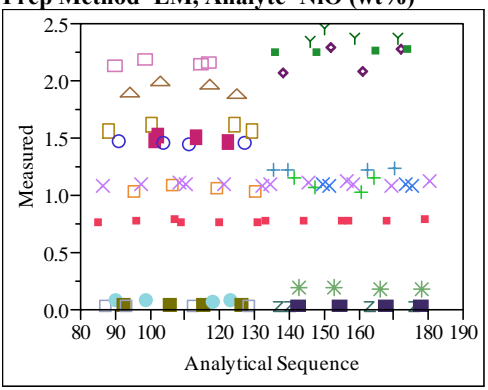

Measured By Analytical Sequence Set $=2$, Prep Method=LM, Analyte $=$ PbO (wt\%)

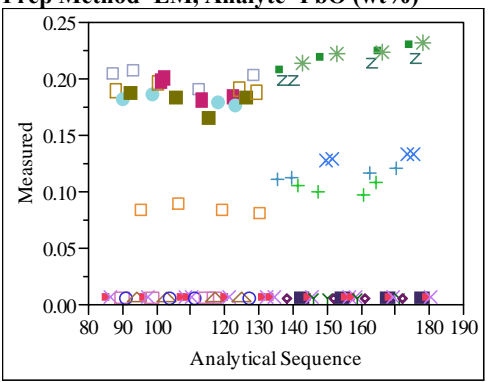




\section{Exhibit A1. Measurements in Analytical Sequence for Samples by Oxide by Prep Method and by Analytical Set}

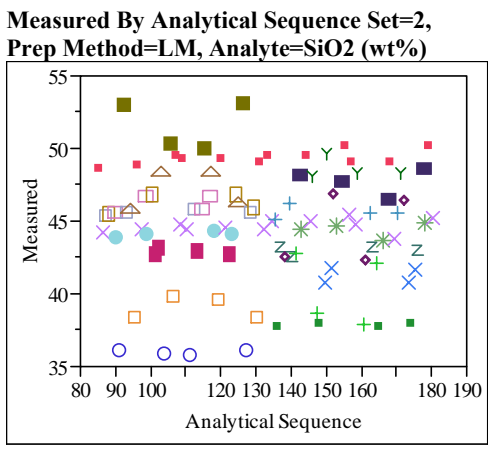

Measured By Analytical Sequence Set $=2$, Prep Method=LM, Analyte $=\mathrm{SO} 4(\mathrm{wt} \%)$

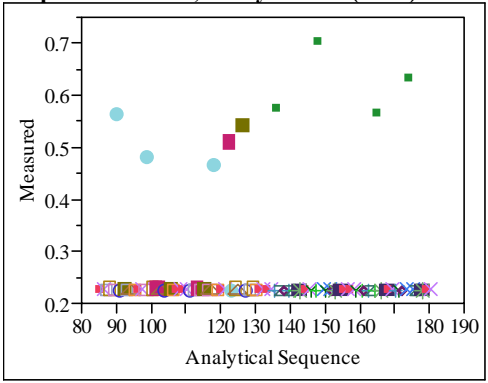

Measured By Analytical Sequence Set $=2$, Prep Method=LM, Analyte=TiO2 (wt\%)

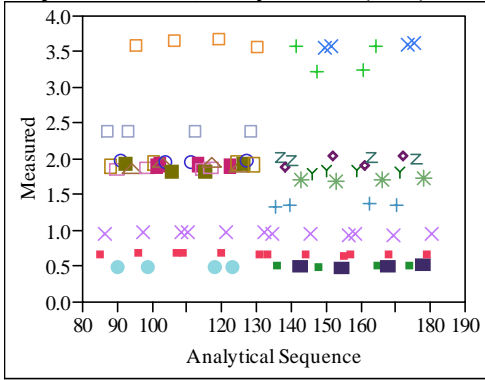

Measured By Analytical Sequence Set $=2$, Prep Method $=$ LM, Analyte $=\mathrm{U} 308(\mathrm{wt} \%)$

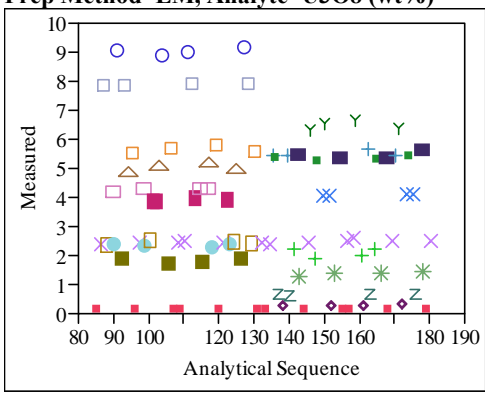

Measured By Analytical Sequence Set=2, Prep Method $=$ LM, Analyte $=\mathrm{ZnO}($ wt $\%)$

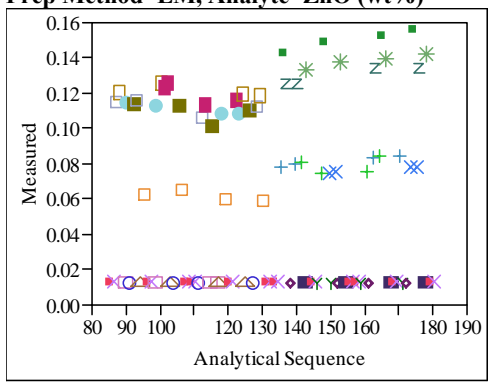

Measured By Analytical Sequence Set=2, Prep Method=LM, Analyte $=\mathrm{ZrO2}($ wt $\%)$

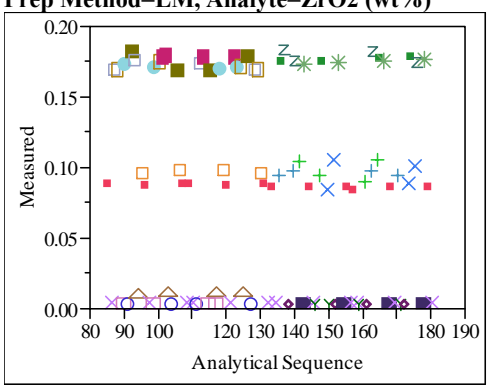

Measured By Analytical Sequence Set=2, Prep Method $=$ PF, Analyte $=A 1203$ (wt $\%)$

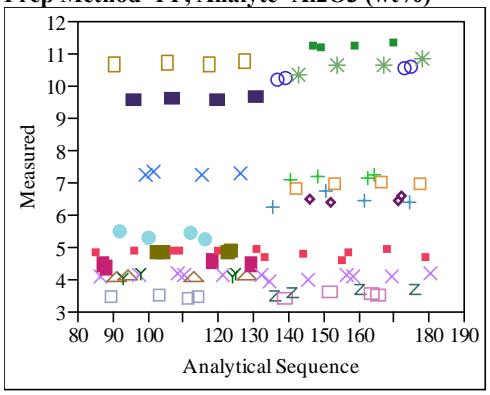

Measured By Analytical Sequence Set $=2$, Prep Method $=$ PF, Analyte $=\mathrm{B} 2 \mathrm{O} 3(\mathrm{wt} \%)$

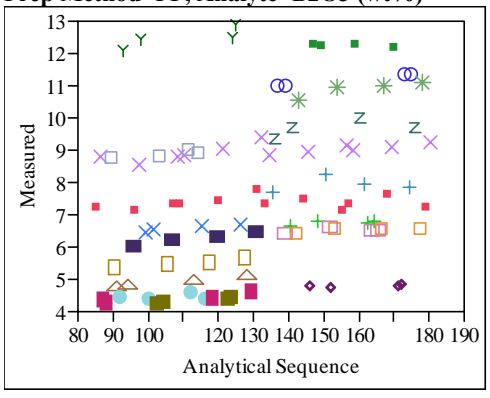

Measured By Analytical Sequence Set $=2$, Prep Method=PF, Analyte $=$ Fe2O3 (wt \%)

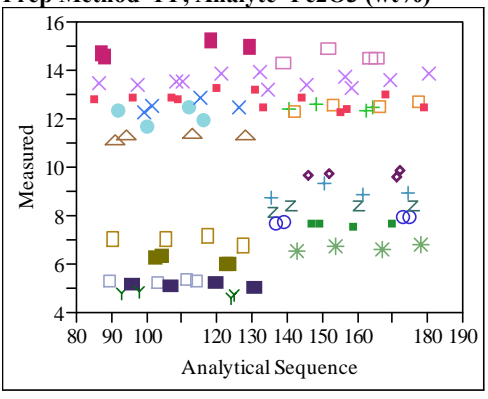

Measured By Analytical Sequence Set=2, Prep Method=PF, Analyte $=$ Li2O (wt\%)

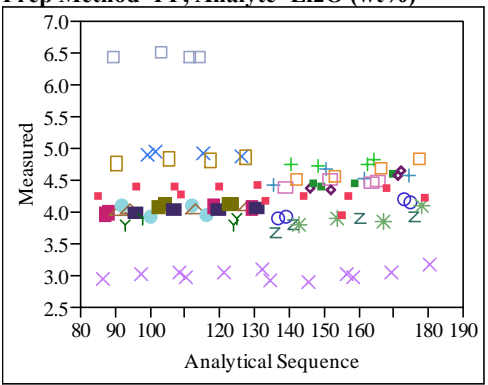


SRNL-STI-2009-00465, Revision 0

Exhibit A2. Measurements by Lab ID within Glass ID for Samples by Oxide and by Set

Set $=1$, Analyte $=\mathrm{Al2O3}(\mathrm{wt} \%)$ Variability Chart for Measured

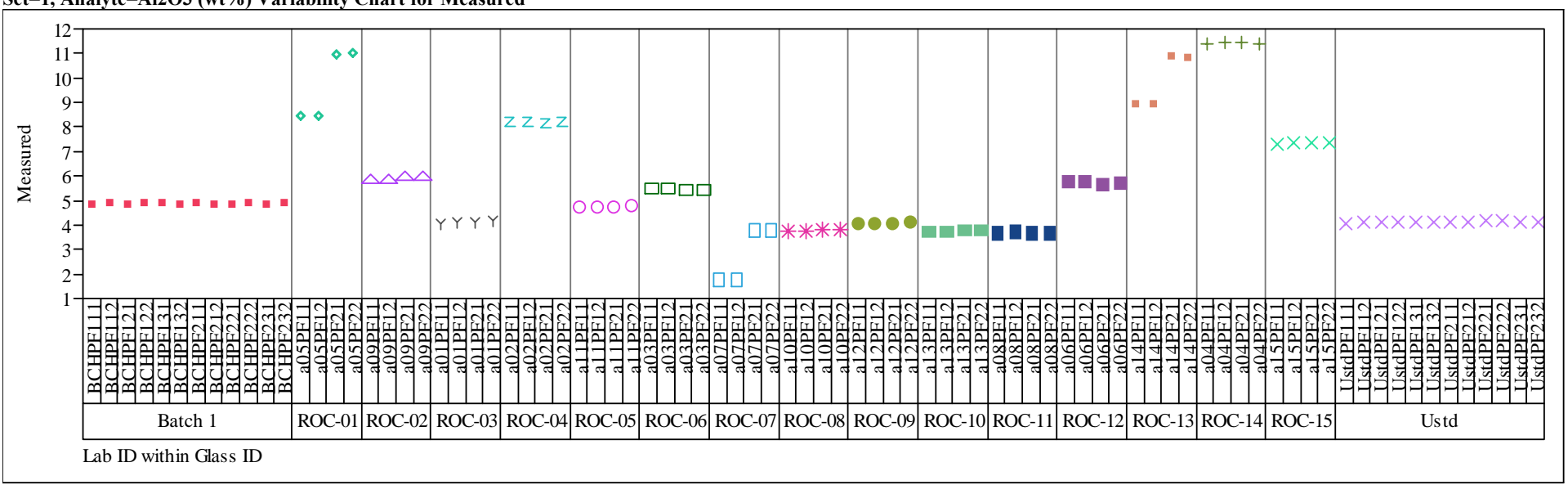

Set=1, Analyte $=\mathrm{B} 2 \mathrm{O} 3(\mathrm{wt} \%)$ Variability Chart for Measured

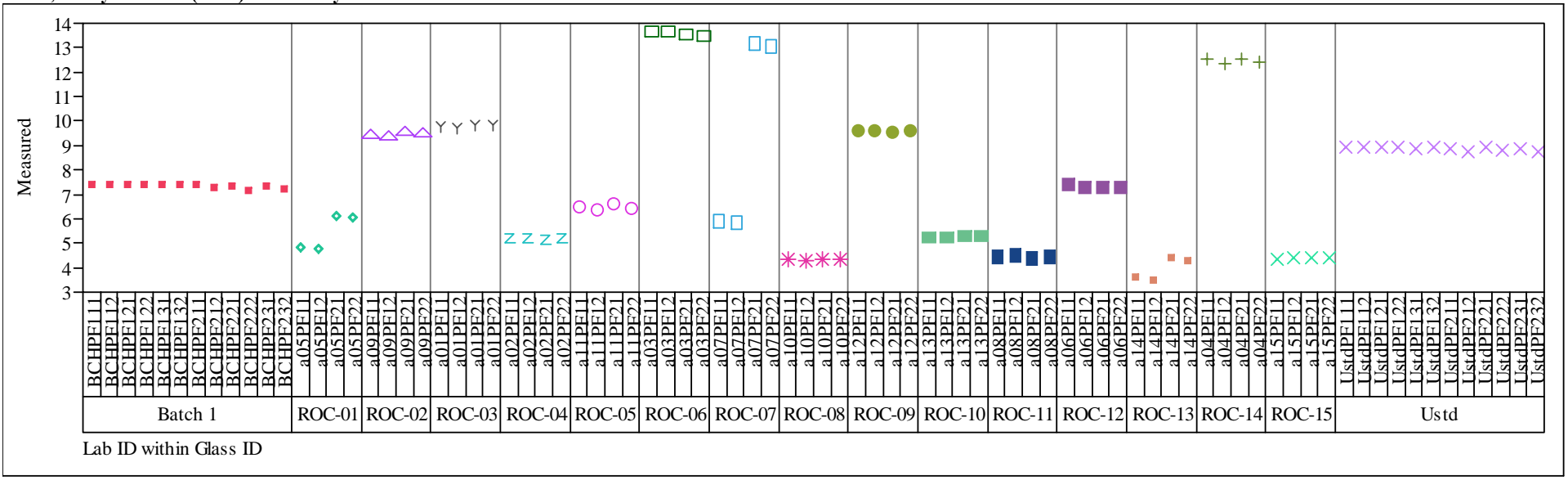


SRNL-STI-2009-00465, Revision 0

Exhibit A2. Measurements by Lab ID within Glass ID for Samples by Oxide and by Set

Set=1, Analyte=BaO (wt\%) Variability Chart for Measured

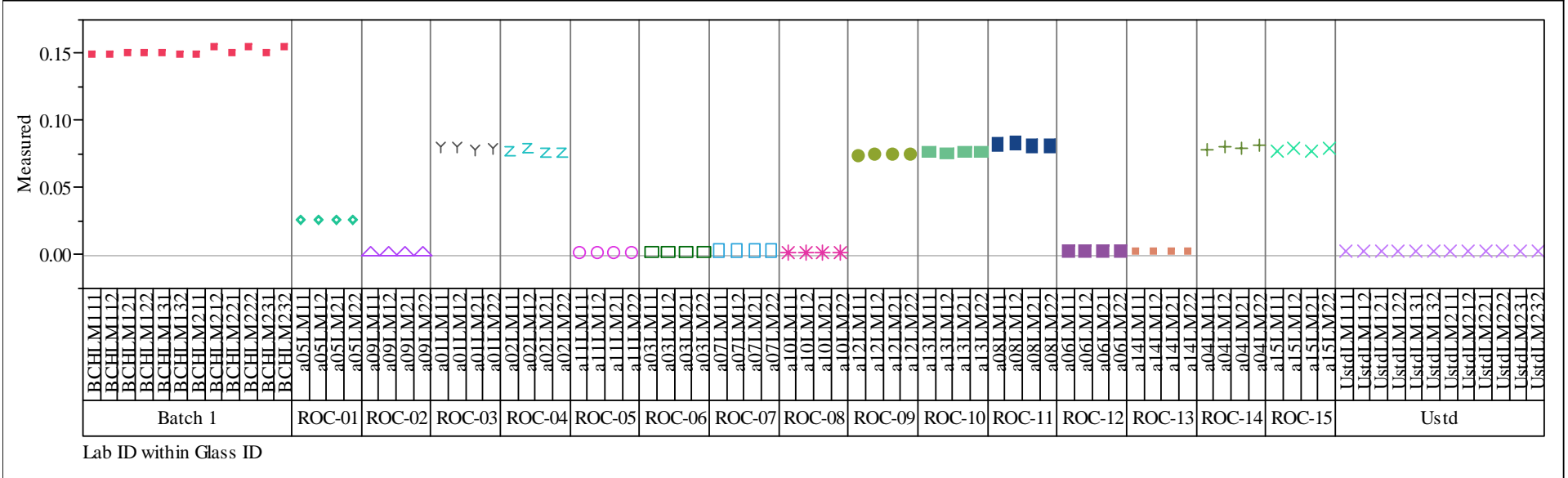

Set $=1$, Analyte $=\mathrm{CaO}($ wt $\%)$ Variability Chart for Measured

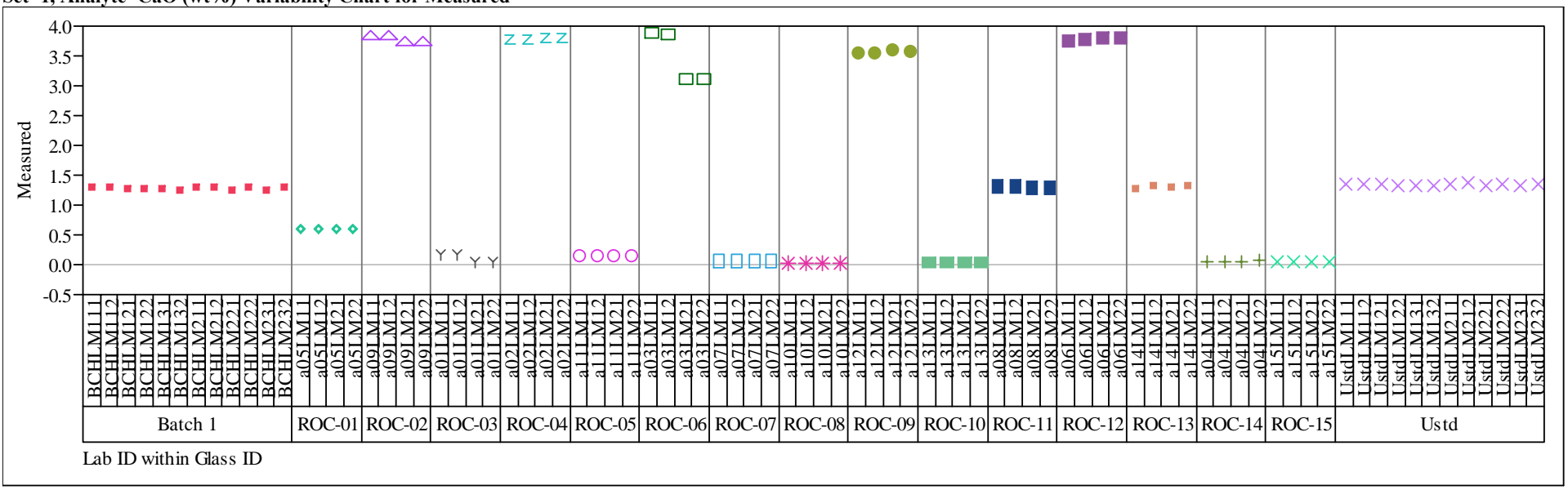


SRNL-STI-2009-00465, Revision 0

Exhibit A2. Measurements by Lab ID within Glass ID for Samples by Oxide and by Set

Set=1, Analyte=CdO (wt\%) Variability Chart for Measured

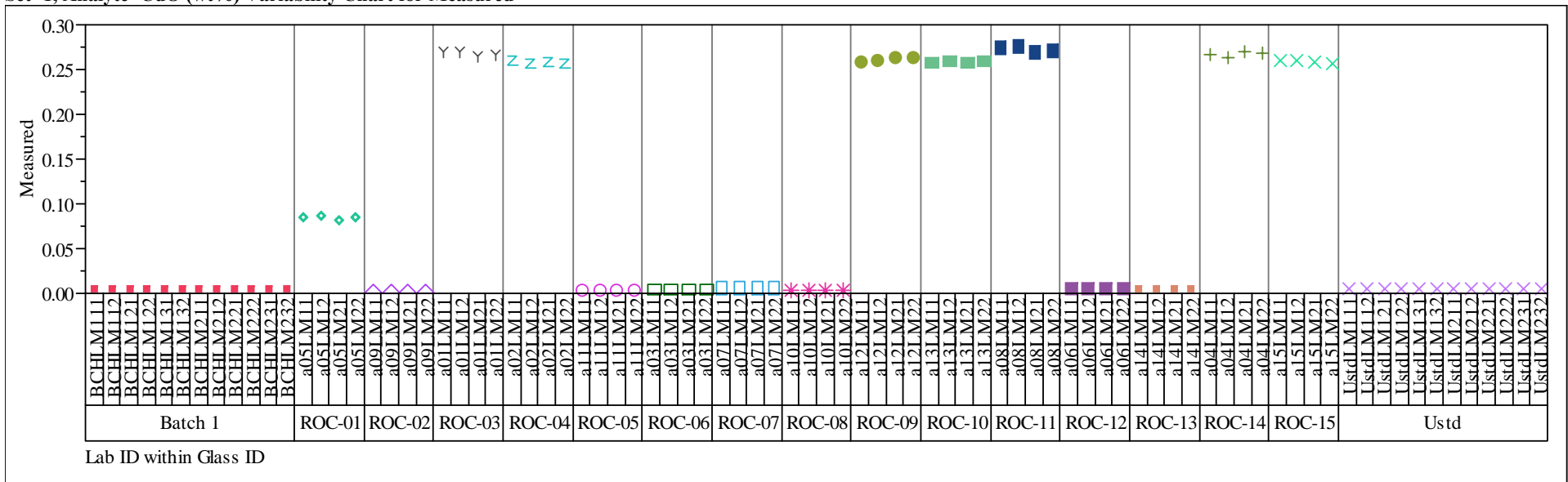

Set $=1$, Analyte $=\mathrm{Ce} 2 \mathrm{O} 3(\mathrm{wt} \%)$ Variability Chart for Measured

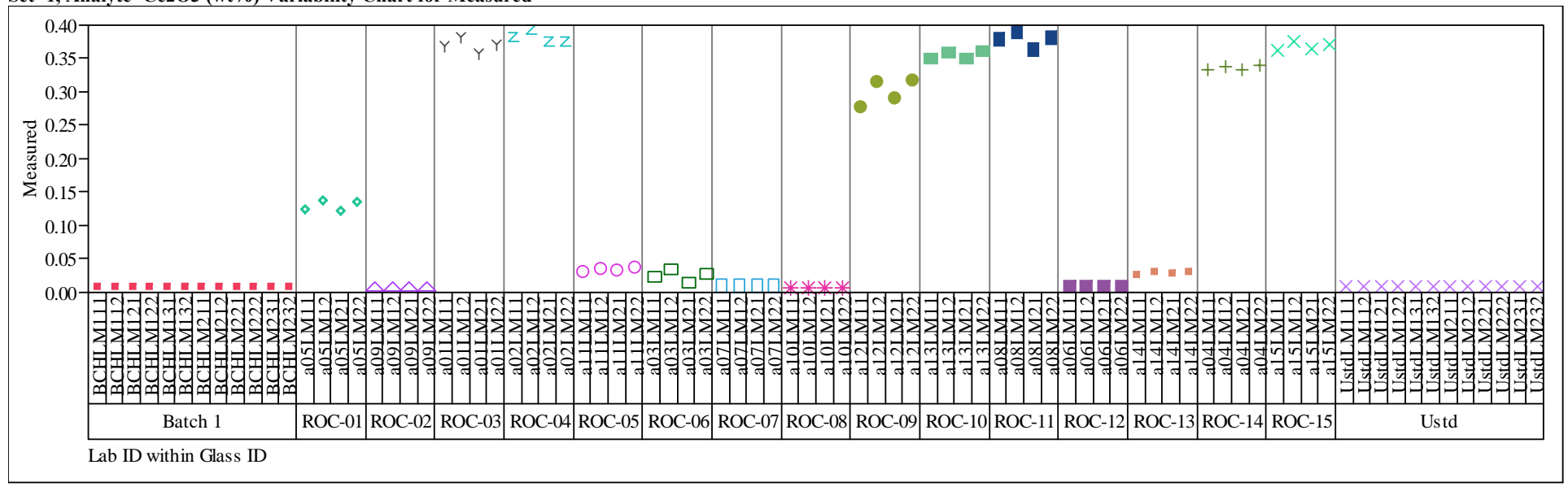


SRNL-STI-2009-00465, Revision 0

Exhibit A2. Measurements by Lab ID within Glass ID for Samples by Oxide and by Set

Set $=1$, Analyte $=\mathrm{Cr2O3}(\mathrm{wt} \%)$ Variability Chart for Measured

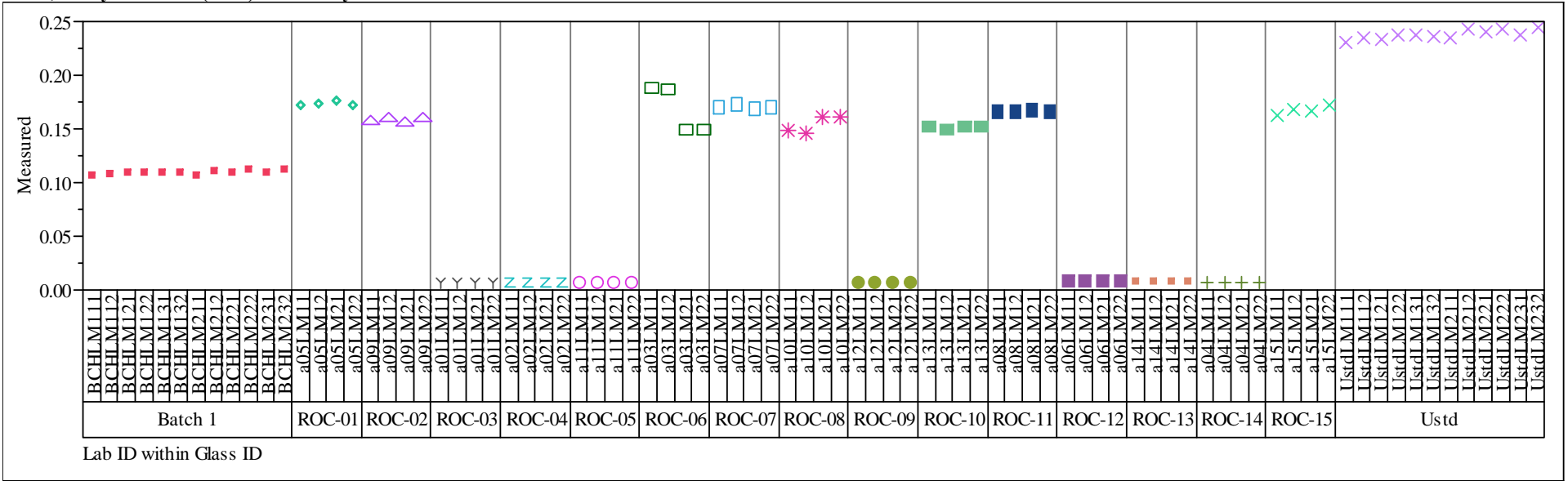

Set $=1$, Analyte $=\mathrm{CuO}(\mathrm{wt} \%)$ Variability Chart for Measured

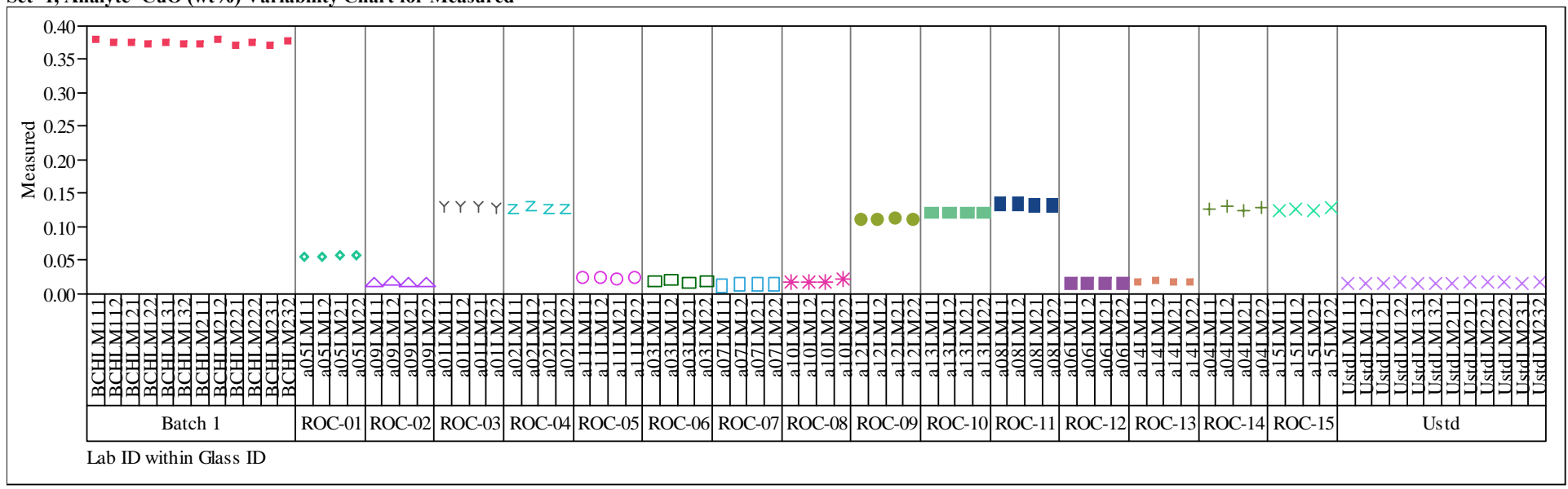


SRNL-STI-2009-00465, Revision 0

Exhibit A2. Measurements by Lab ID within Glass ID for Samples by Oxide and by Set

Set=1, Analyte=Fe2O3 (wt\%) Variability Chart for Measured

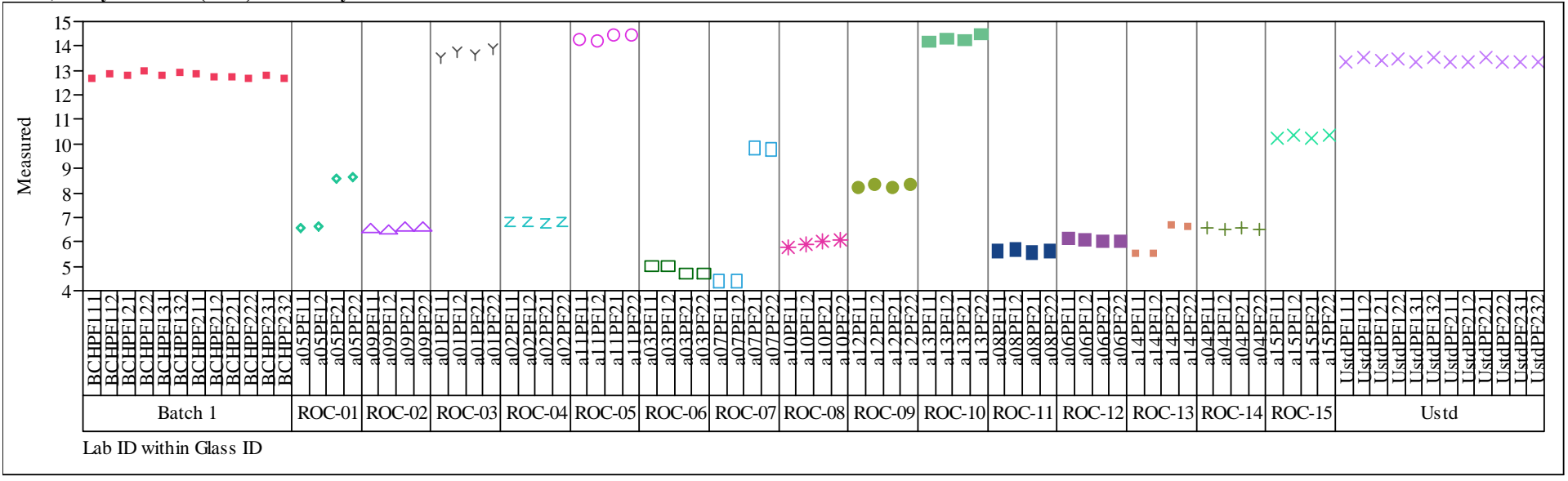

Set=1, Analyte=K2O (wt\%) Variability Chart for Measured

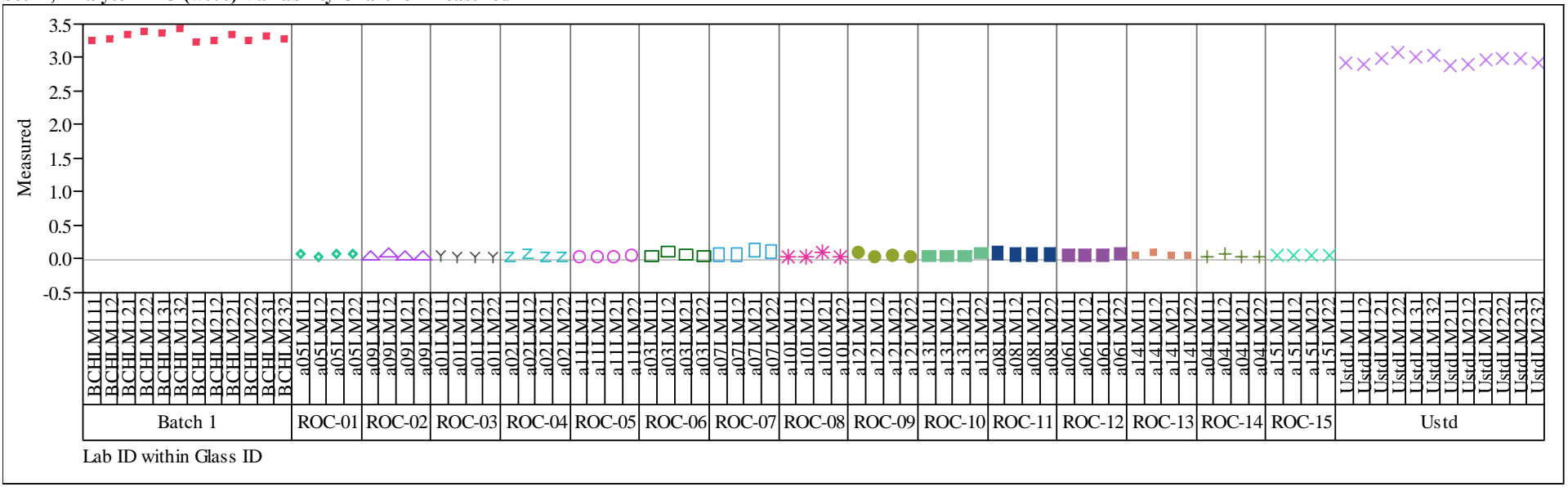


SRNL-STI-2009-00465, Revision 0

Exhibit A2. Measurements by Lab ID within Glass ID for Samples by Oxide and by Set

Set $=1$, Analyte $=$ La2O3 (wt\%) Variability Chart for Measured

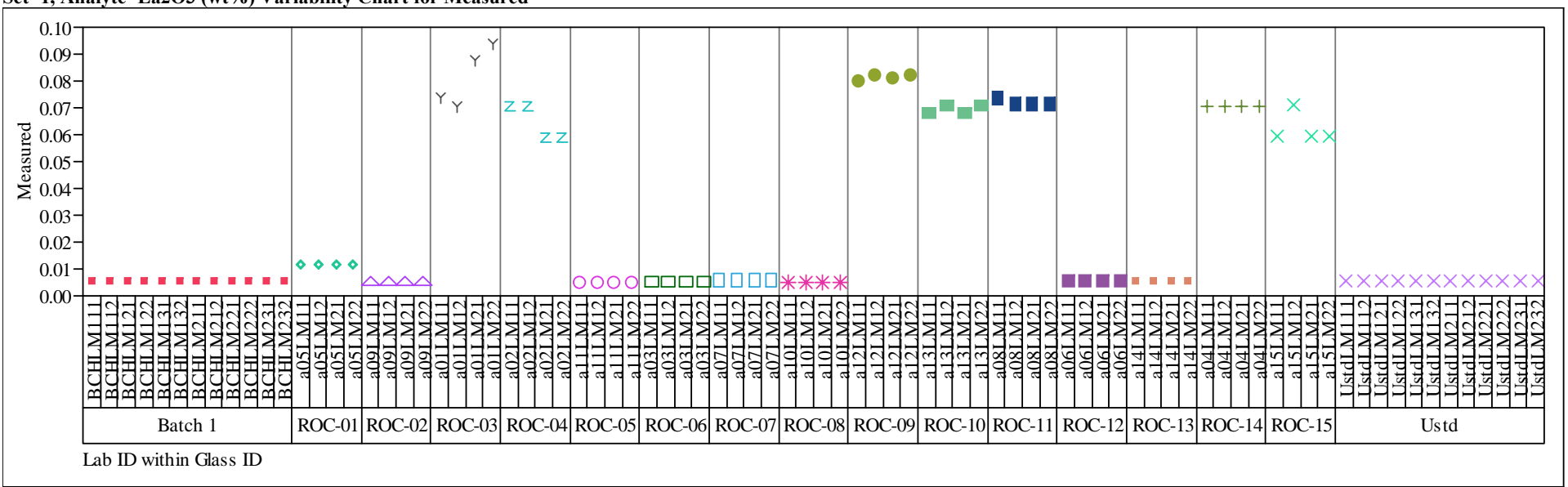

Set=1, Analyte=Li2O (wt\%) Variability Chart for Measured

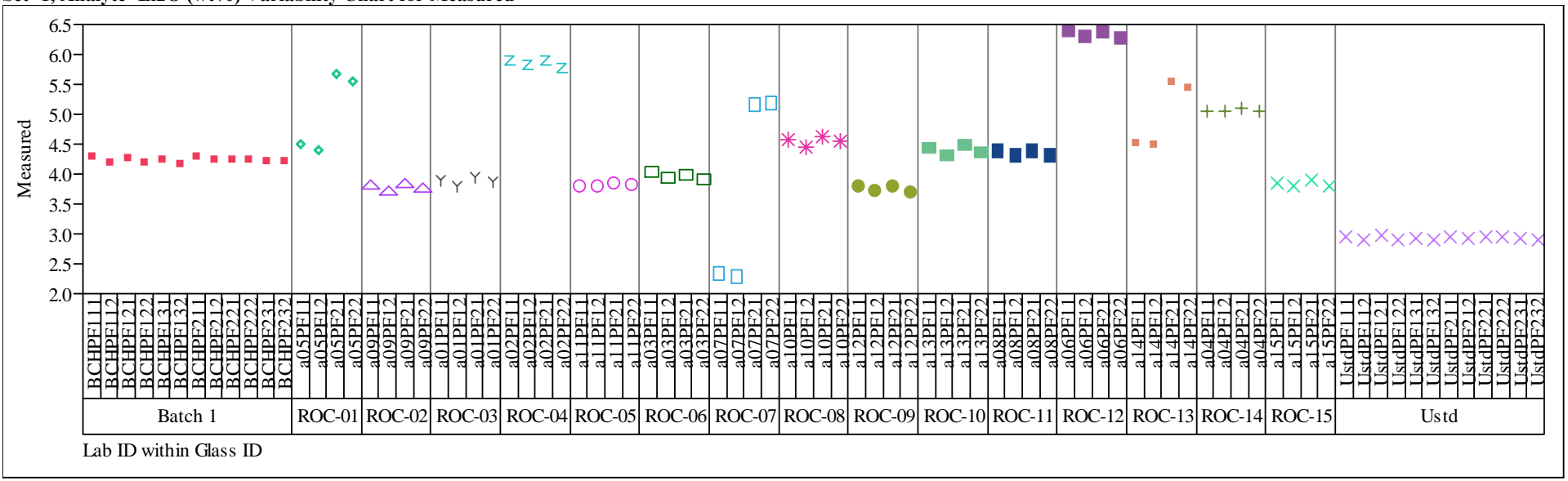


SRNL-STI-2009-00465, Revision 0

Exhibit A2. Measurements by Lab ID within Glass ID for Samples by Oxide and by Set

Set=1, Analyte $=\mathrm{MgO}(\mathrm{wt} \%)$ Variability Chart for Measured

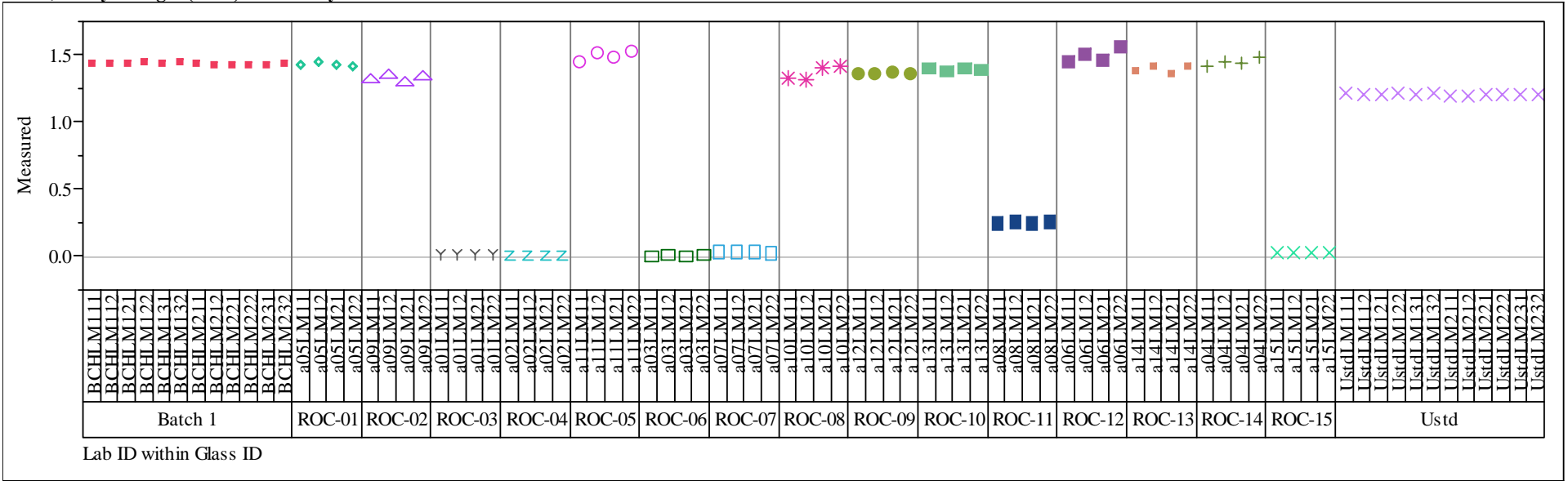

Set $=1$, Analyte $=\mathrm{MnO}(\mathrm{wt} \%)$ Variability Chart for Measured

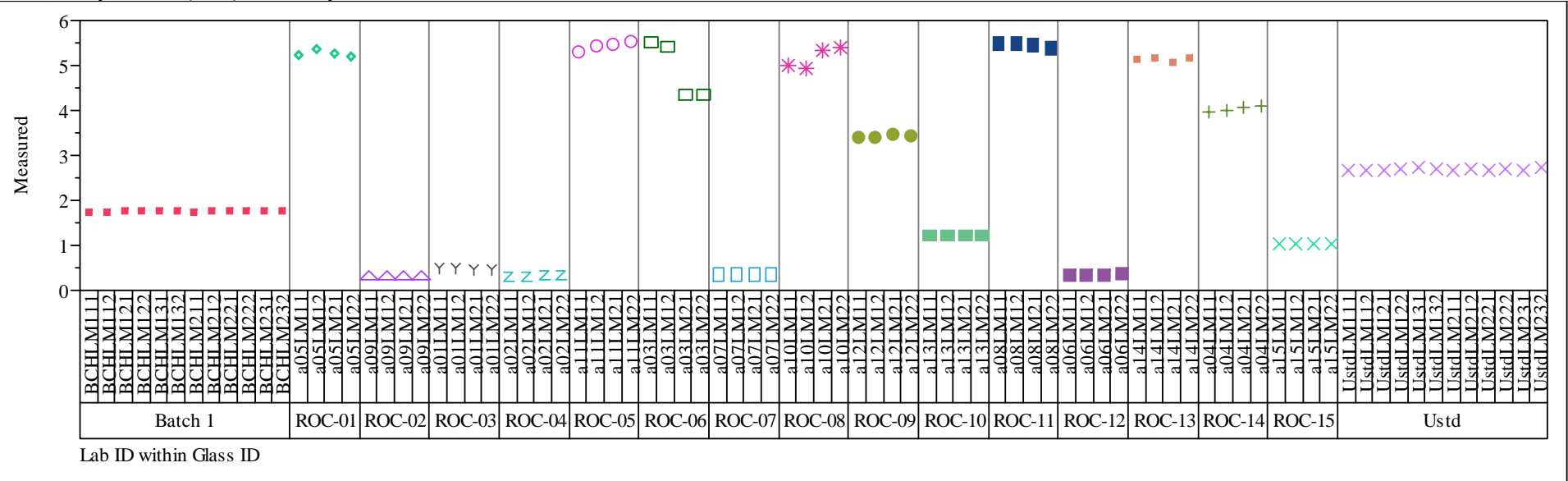


SRNL-STI-2009-00465, Revision 0

Exhibit A2. Measurements by Lab ID within Glass ID for Samples by Oxide and by Set

Set=1, Analyte $=\mathrm{Na} 2 \mathrm{O}(\mathrm{wt} \%)$ Variability Chart for Measured

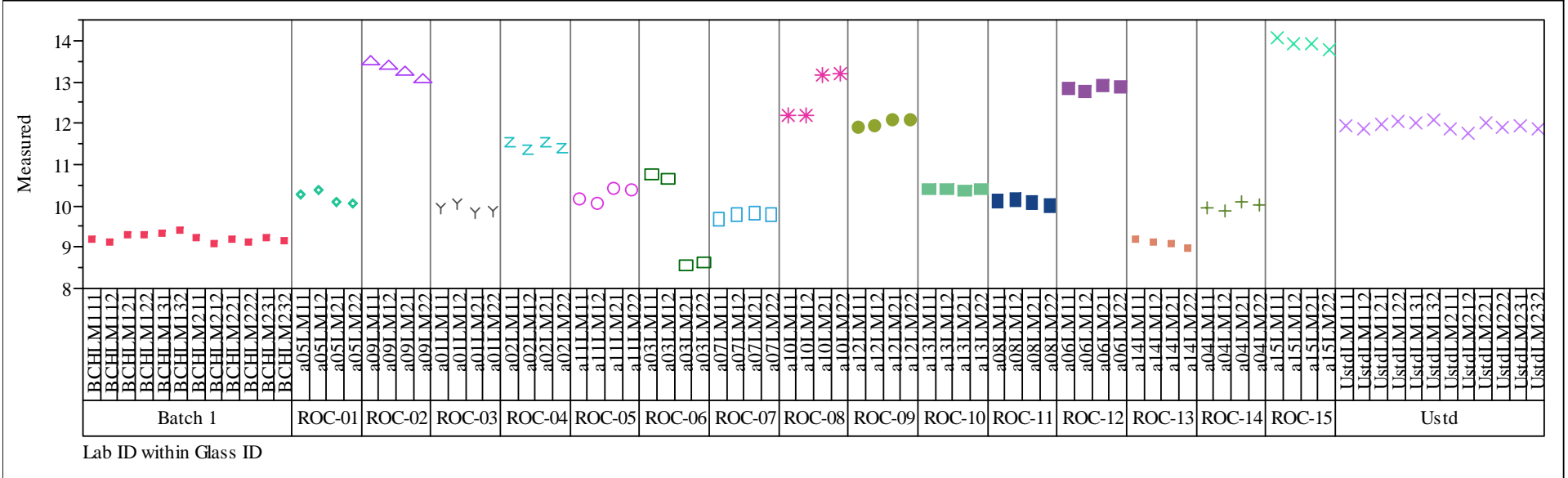

Set=1, Analyte $=\mathrm{NiO}(\mathrm{wt} \%)$ Variability Chart for Measured

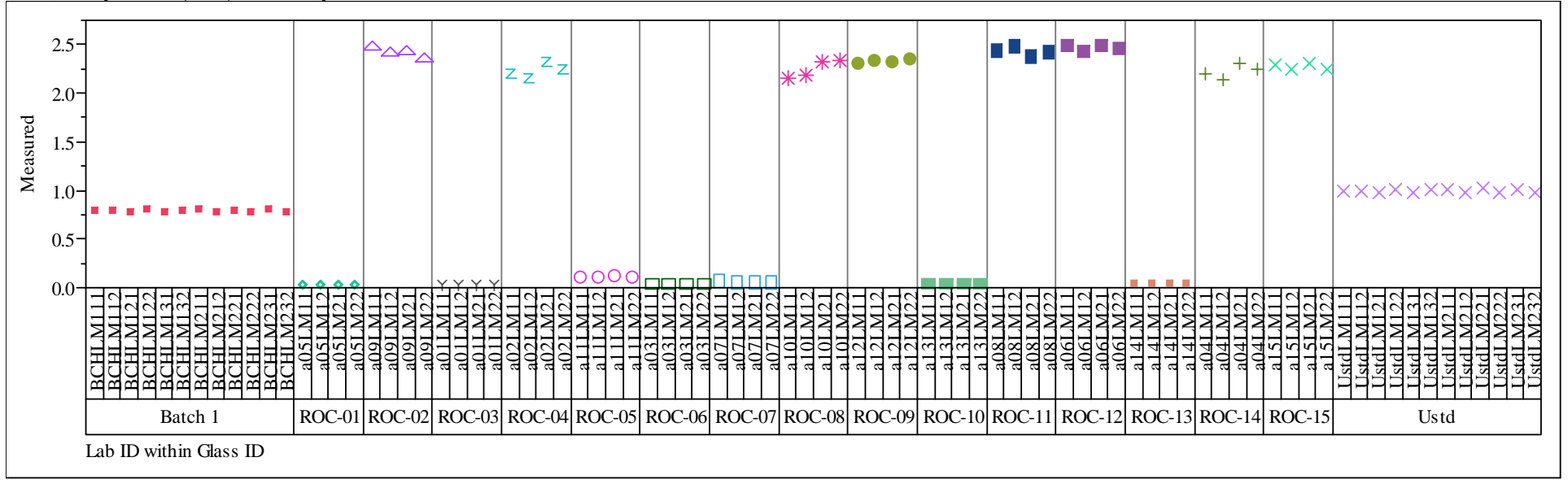


SRNL-STI-2009-00465, Revision 0

Exhibit A2. Measurements by Lab ID within Glass ID for Samples by Oxide and by Set

Set=1, Analyte=PbO (wt\%) Variability Chart for Measured

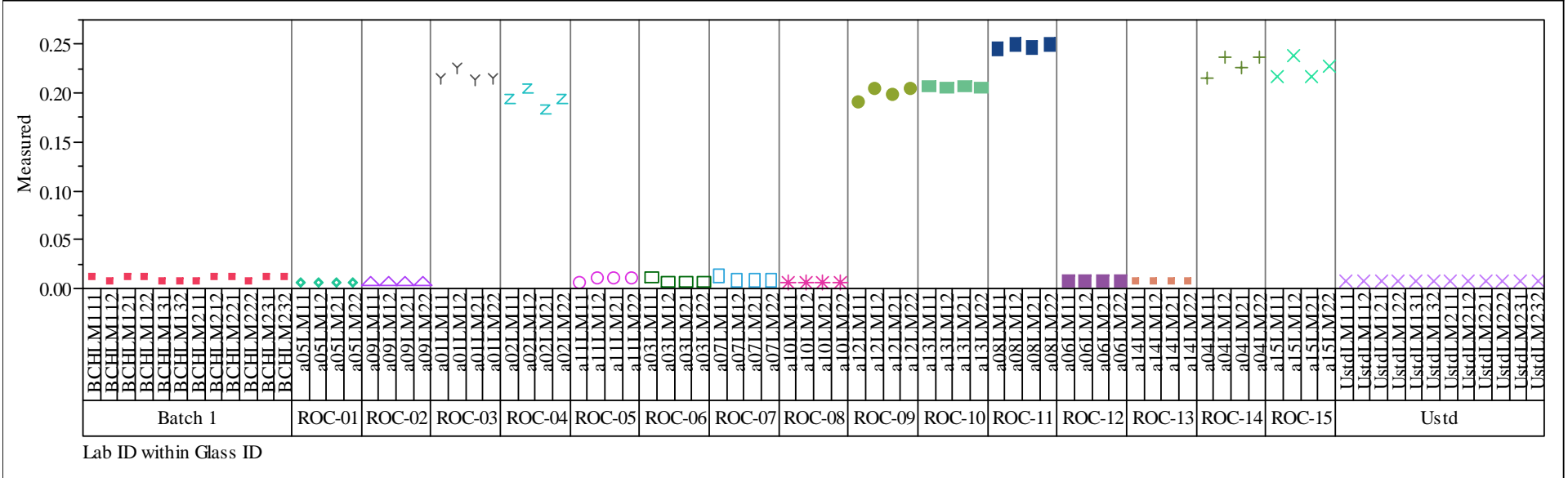

Set=1, Analyte=SiO2 (wt\%) Variability Chart for Measured

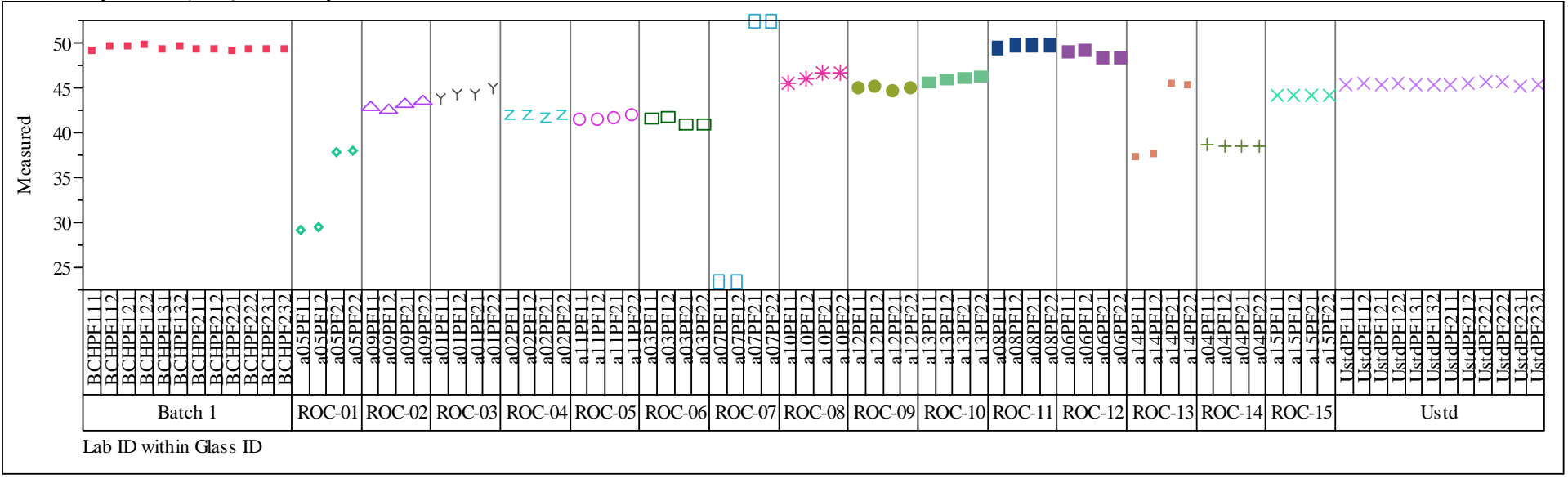


SRNL-STI-2009-00465, Revision 0

Exhibit A2. Measurements by Lab ID within Glass ID for Samples by Oxide and by Set

Set=1, Analyte=SO4 (wt\%) Variability Chart for Measured

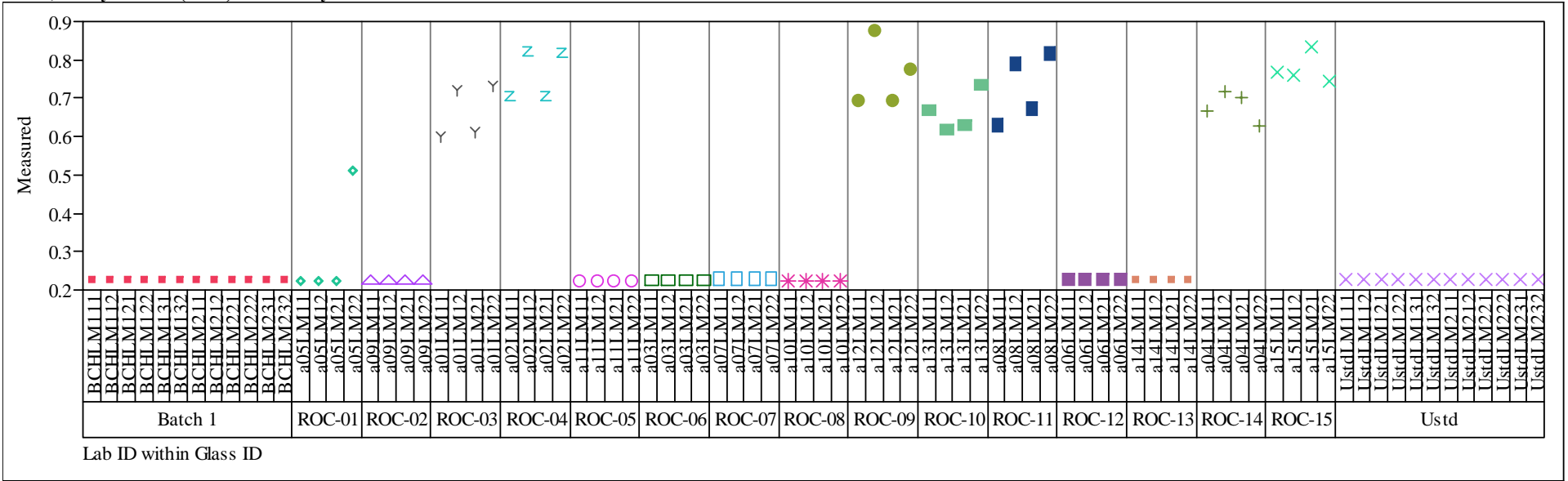

Set $=1$, Analyte=TiO2 $($ wt\%) Variability Chart for Measured

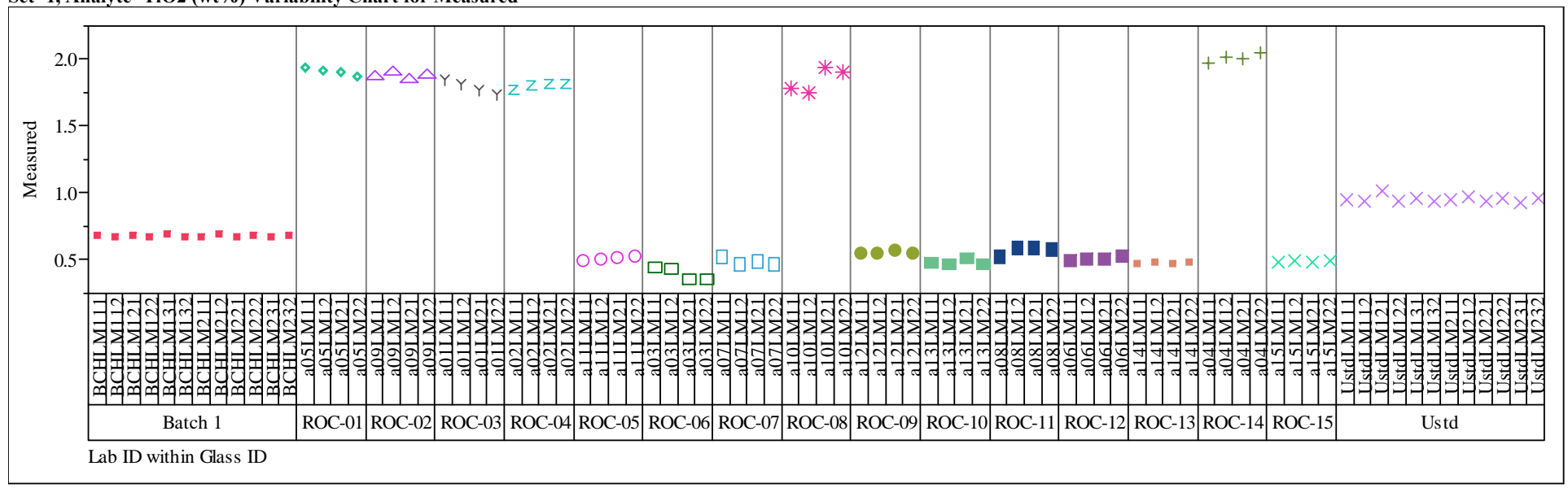


SRNL-STI-2009-00465, Revision 0

Exhibit A2. Measurements by Lab ID within Glass ID for Samples by Oxide and by Set

Set=1, Analyte=U3O8 (wt\%) Variability Chart for Measured

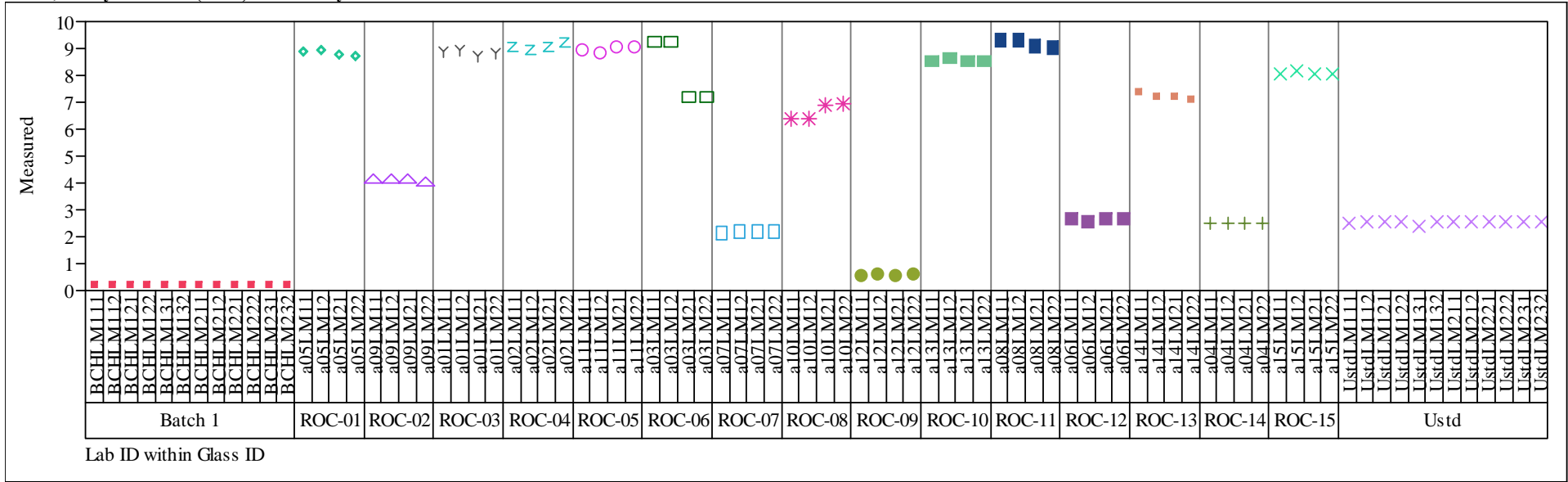

Set=1, Analyte= $\mathrm{ZnO}($ wt $\%)$ Variability Chart for Measured

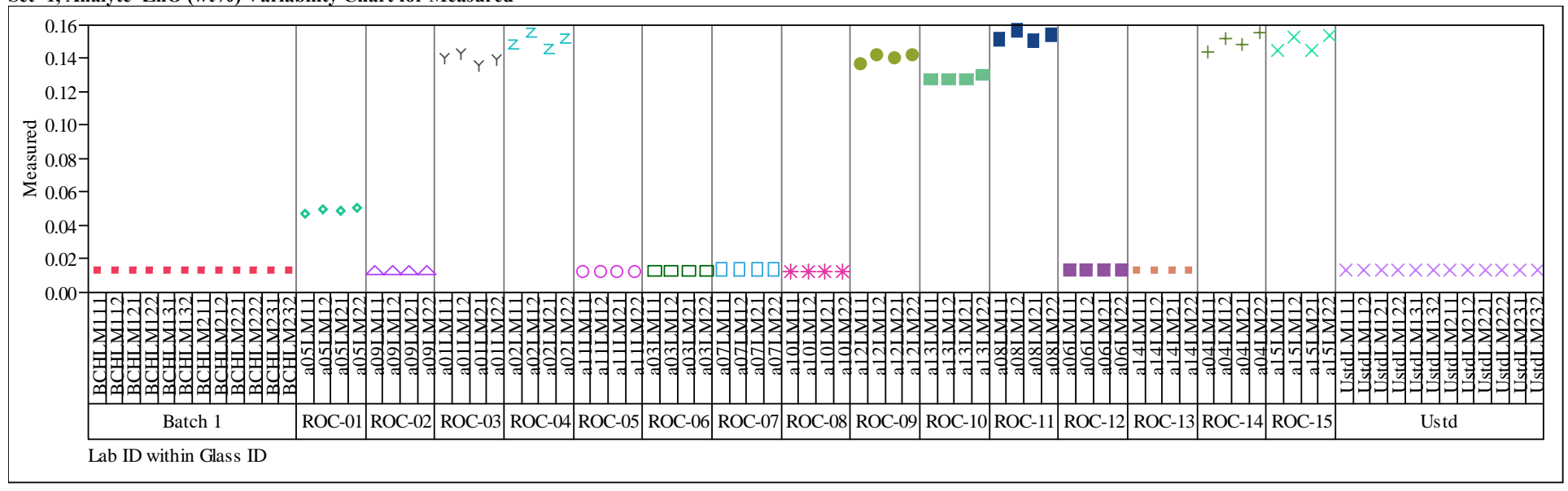


SRNL-STI-2009-00465, Revision 0

Exhibit A2. Measurements by Lab ID within Glass ID for Samples by Oxide and by Set

Set $=1$, Analyte $=\mathrm{ZrO} 2(\mathrm{wt} \%)$ Variability Chart for Measured

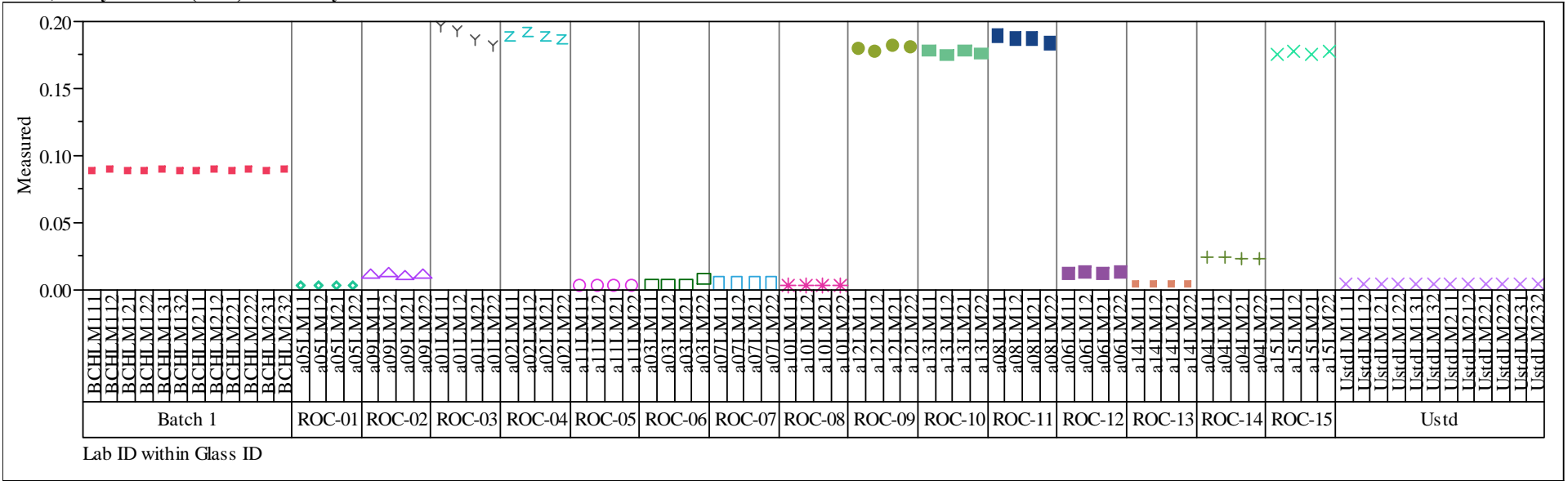

Set $=2$, Analyte $=\mathrm{Al2O3}(\mathrm{wt} \%)$ Variability Chart for Measured

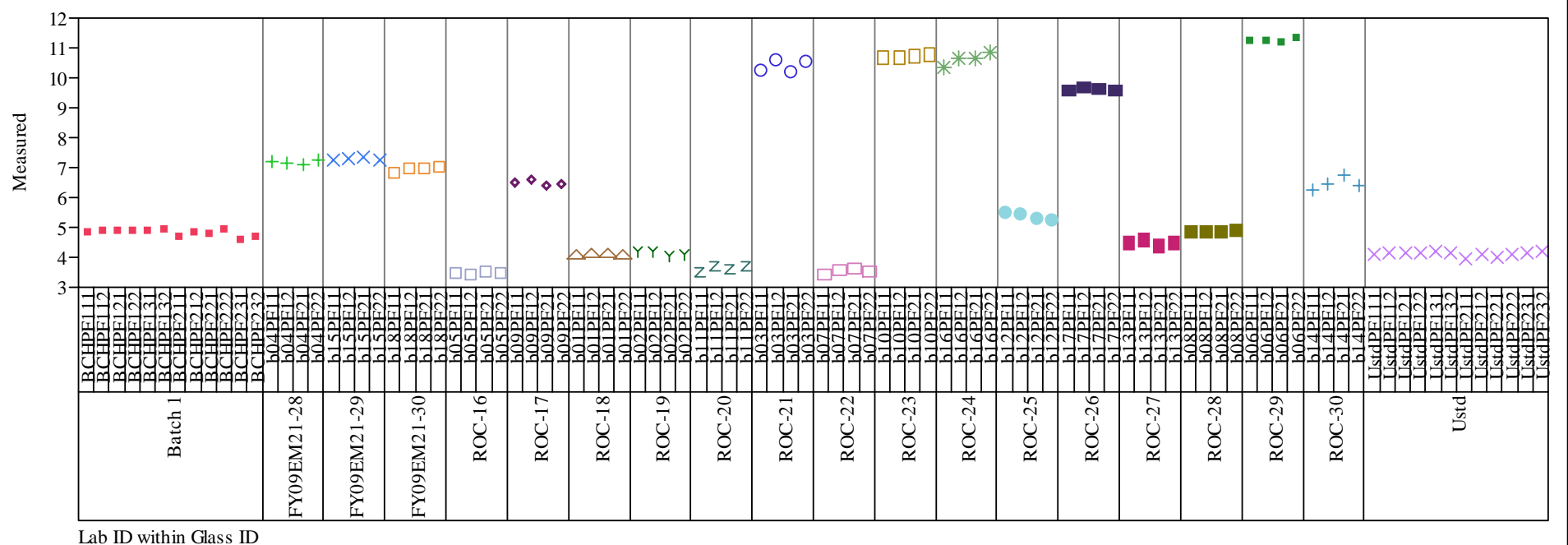


SRNL-STI-2009-00465, Revision 0

Exhibit A2. Measurements by Lab ID within Glass ID for Samples by Oxide and by Set

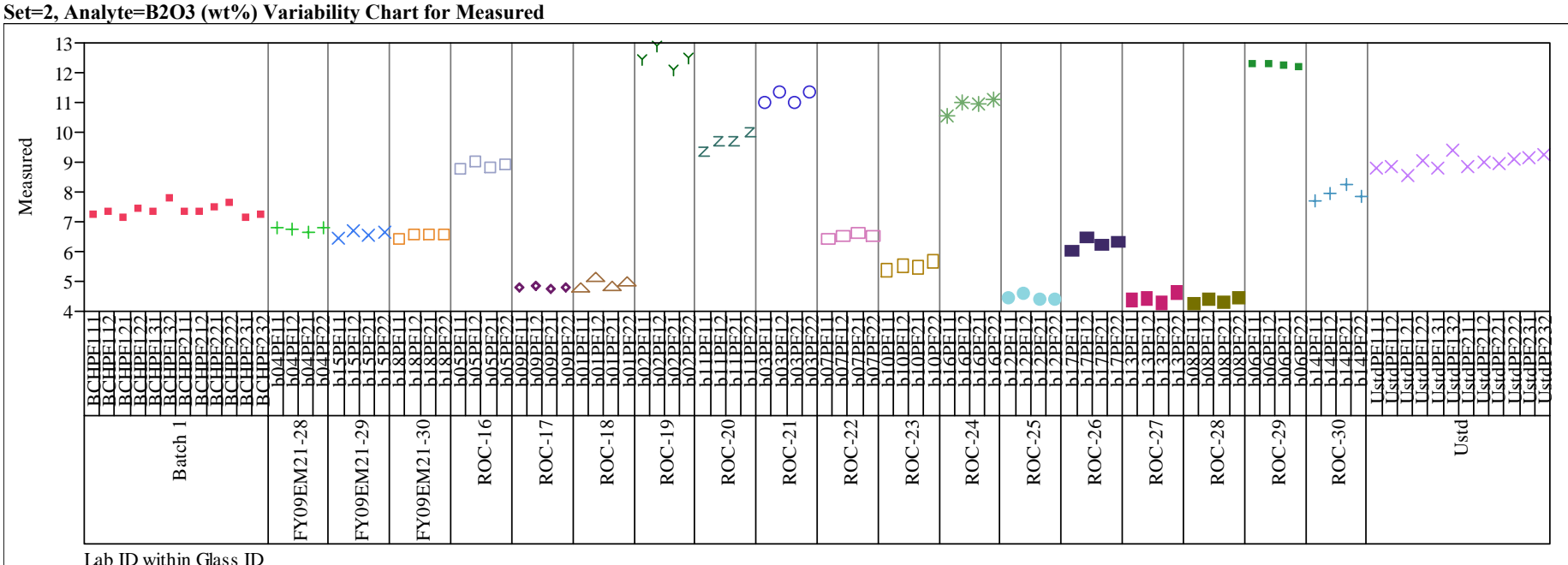

Set $=2$, Analyte $=\mathrm{BaO}(\mathrm{wt} \%)$ Variability Chart for Measured

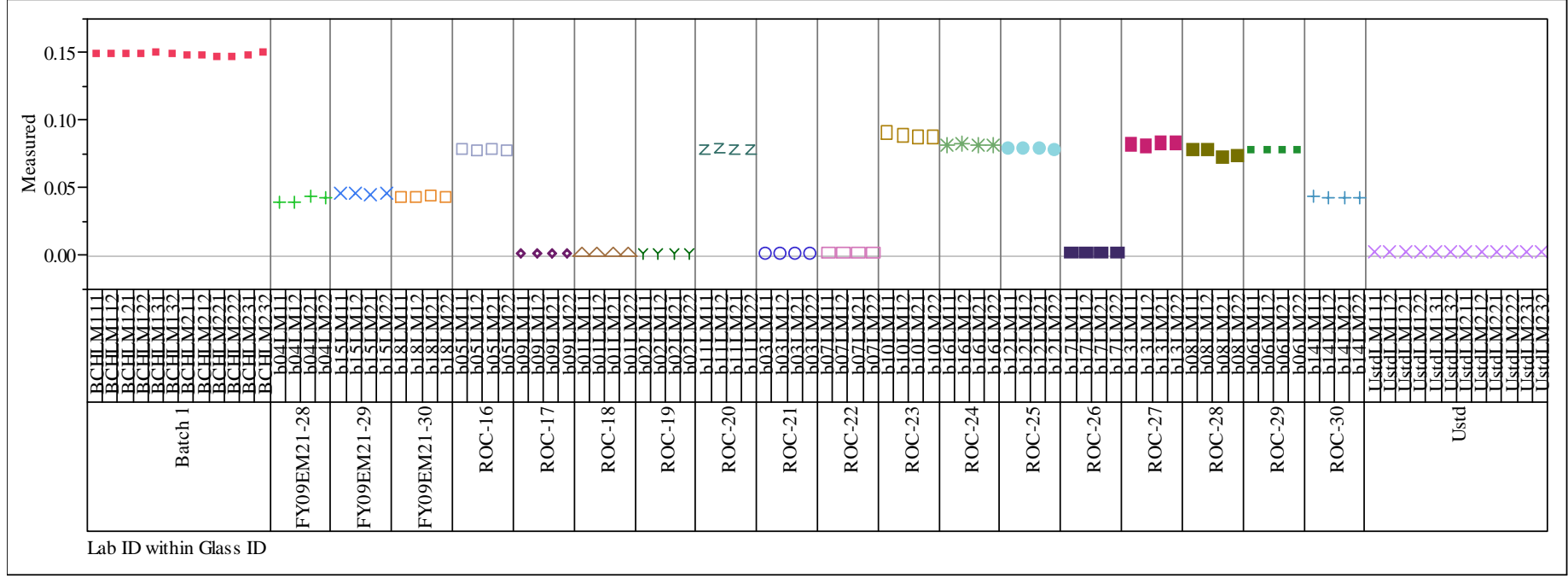


SRNL-STI-2009-00465, Revision 0

Exhibit A2. Measurements by Lab ID within Glass ID for Samples by Oxide and by Set

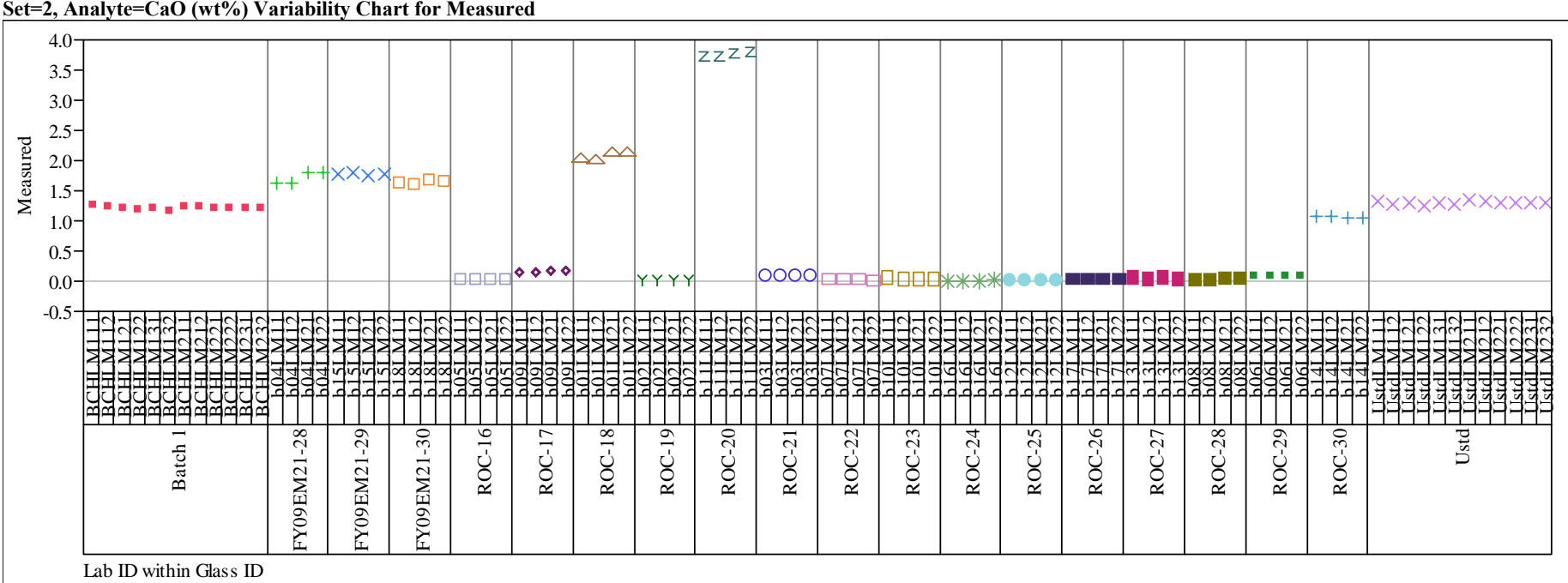

Set $=2$, Analyte $=\mathrm{CdO}(\mathrm{wt} \%)$ Variability Chart for Measured

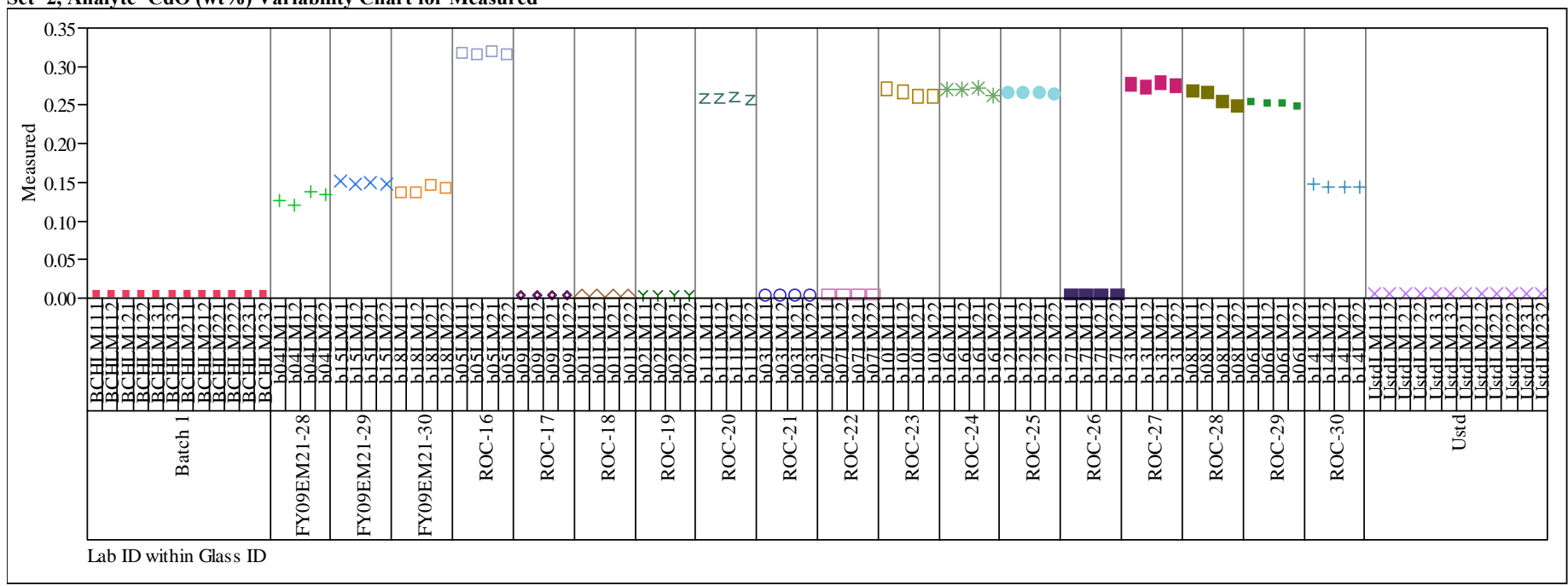


SRNL-STI-2009-00465, Revision 0

Exhibit A2. Measurements by Lab ID within Glass ID for Samples by Oxide and by Set

Set $=2$, Analyte $=\mathrm{Ce} 2 \mathrm{O3}(\mathrm{wt} \%)$ Variability Chart for Measured

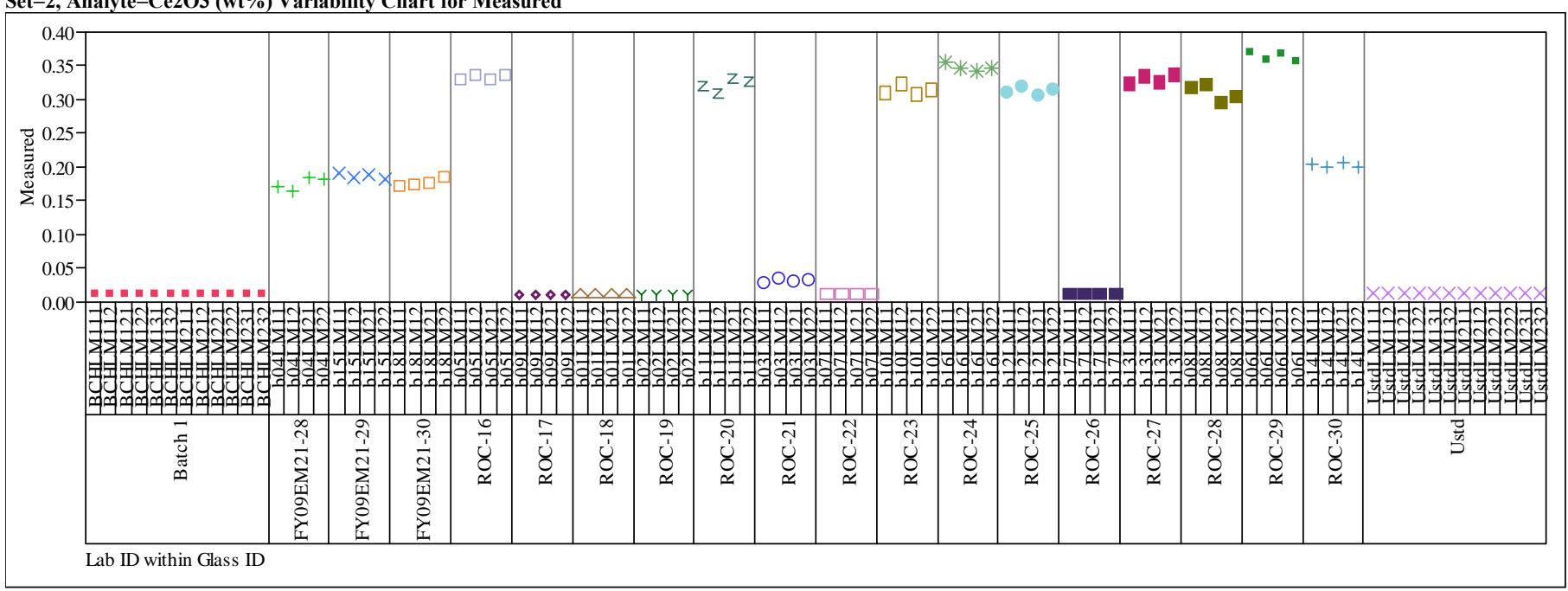

Set $=2$, Analyte $=\mathbf{C r 2 O 3}($ wt $\%)$ Variability Chart for Measured

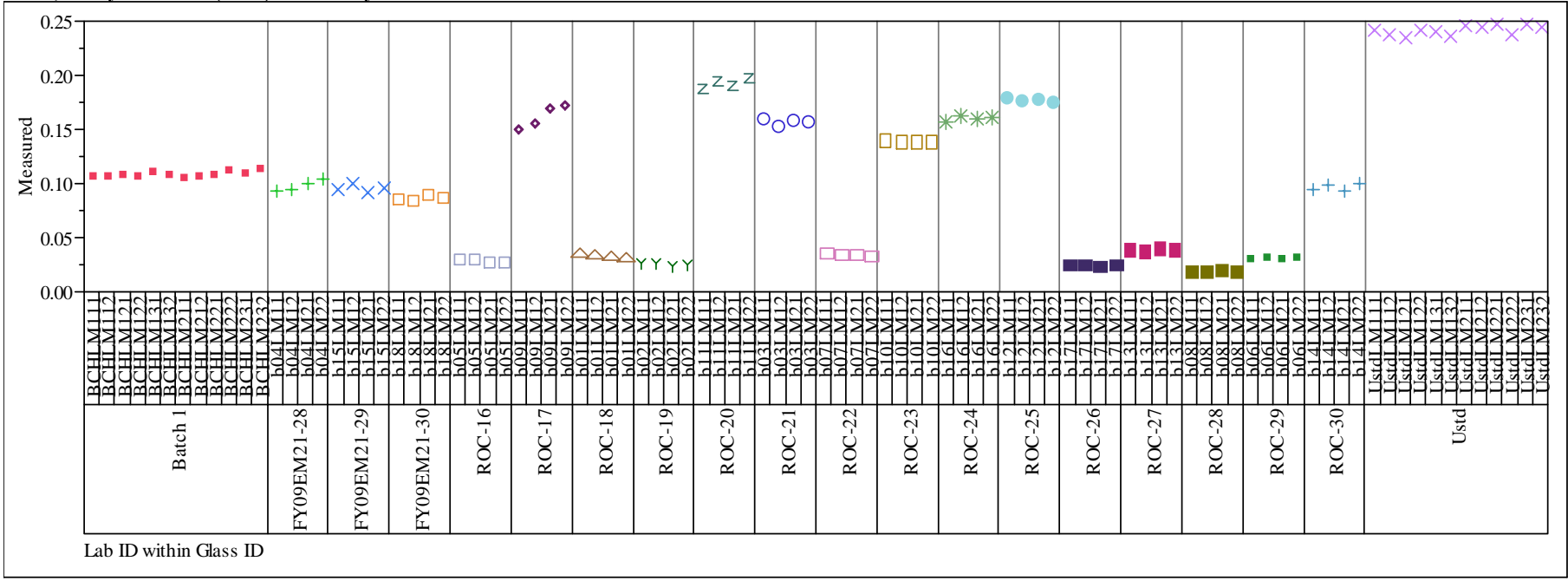


SRNL-STI-2009-00465, Revision 0

Exhibit A2. Measurements by Lab ID within Glass ID for Samples by Oxide and by Set

Set $=2$, Analyte $=\mathrm{CuO}(\mathrm{wt} \%)$ Variability Chart for Measured

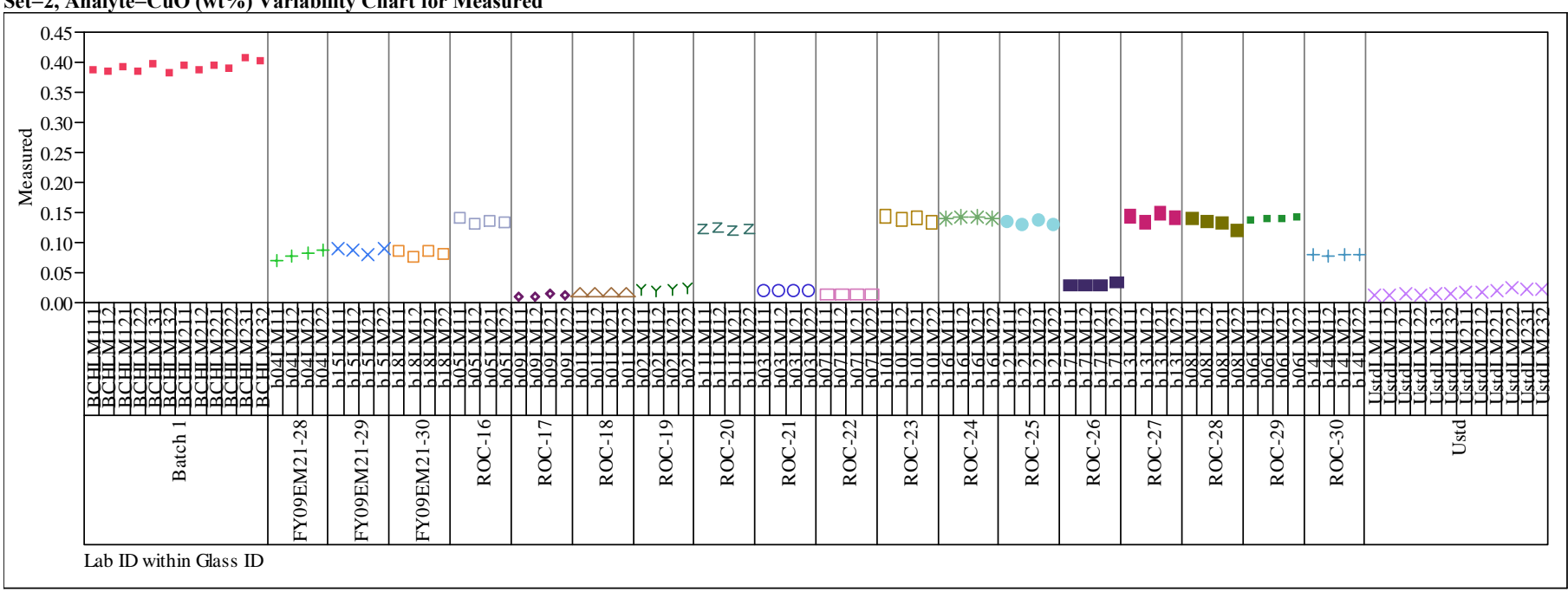

Set=2, Analyte=Fe2O3 (wt\%) Variability Chart for Measured

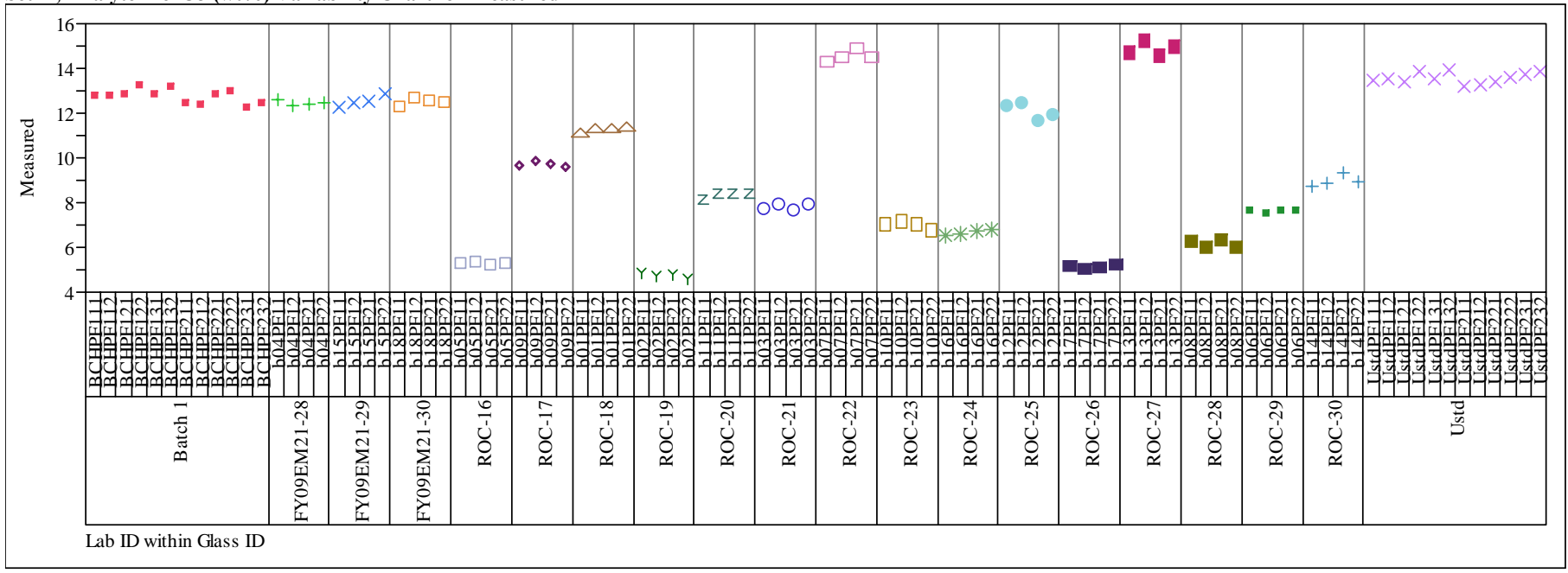


SRNL-STI-2009-00465, Revision 0

Exhibit A2. Measurements by Lab ID within Glass ID for Samples by Oxide and by Set

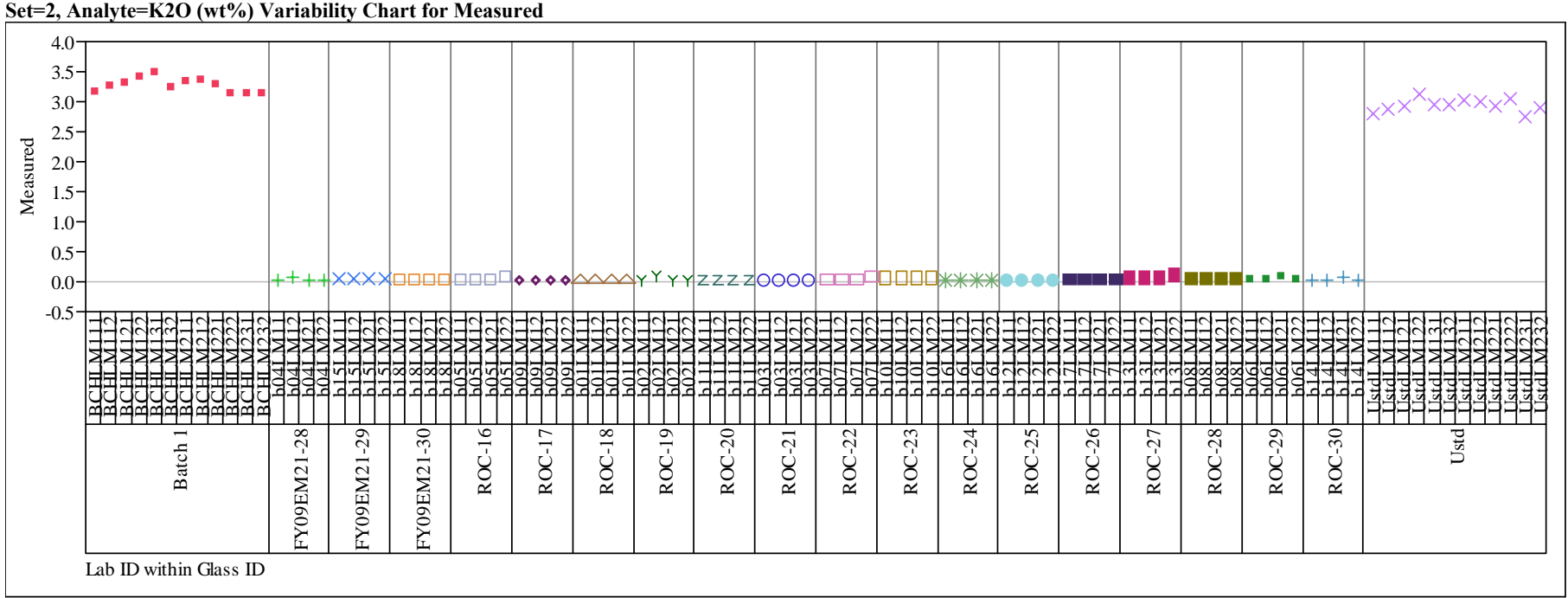

Set=2, Analyte=La2O3 (wt \%) Variability Chart for Measured

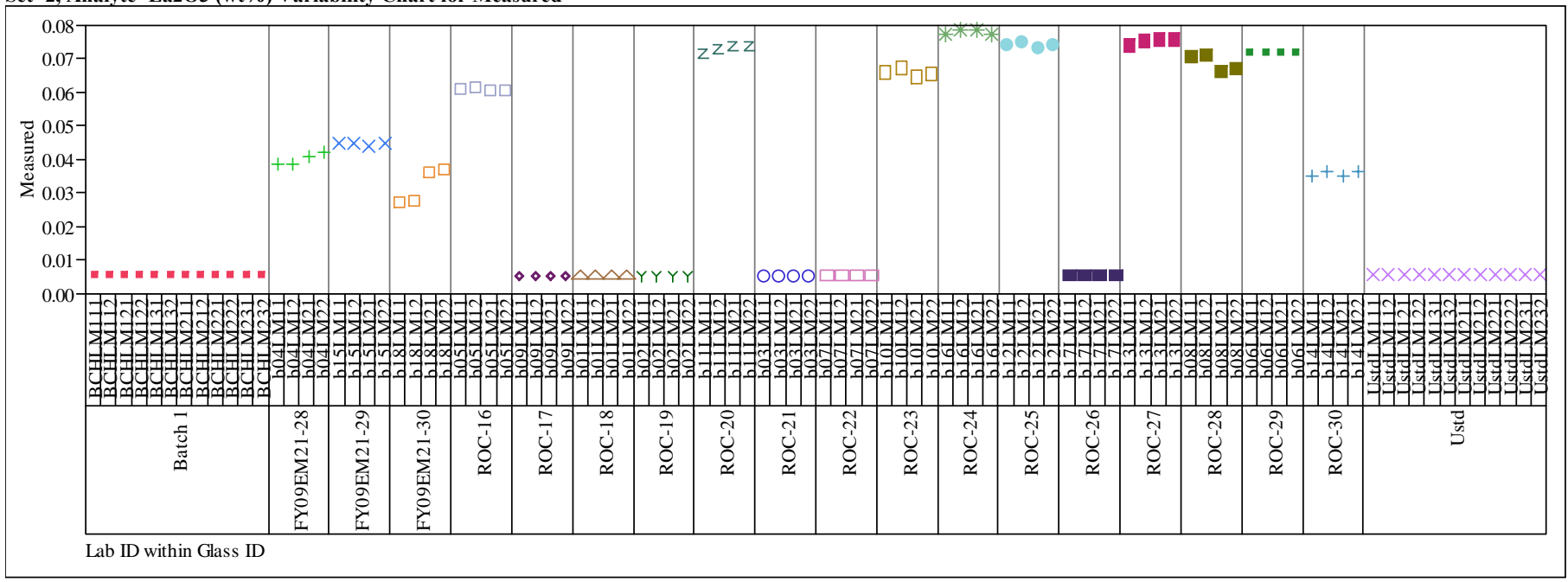


SRNL-STI-2009-00465, Revision 0

Exhibit A2. Measurements by Lab ID within Glass ID for Samples by Oxide and by Set

Set=2, Analyte $=$ Li2O (wt\%) Variability Chart for Measured

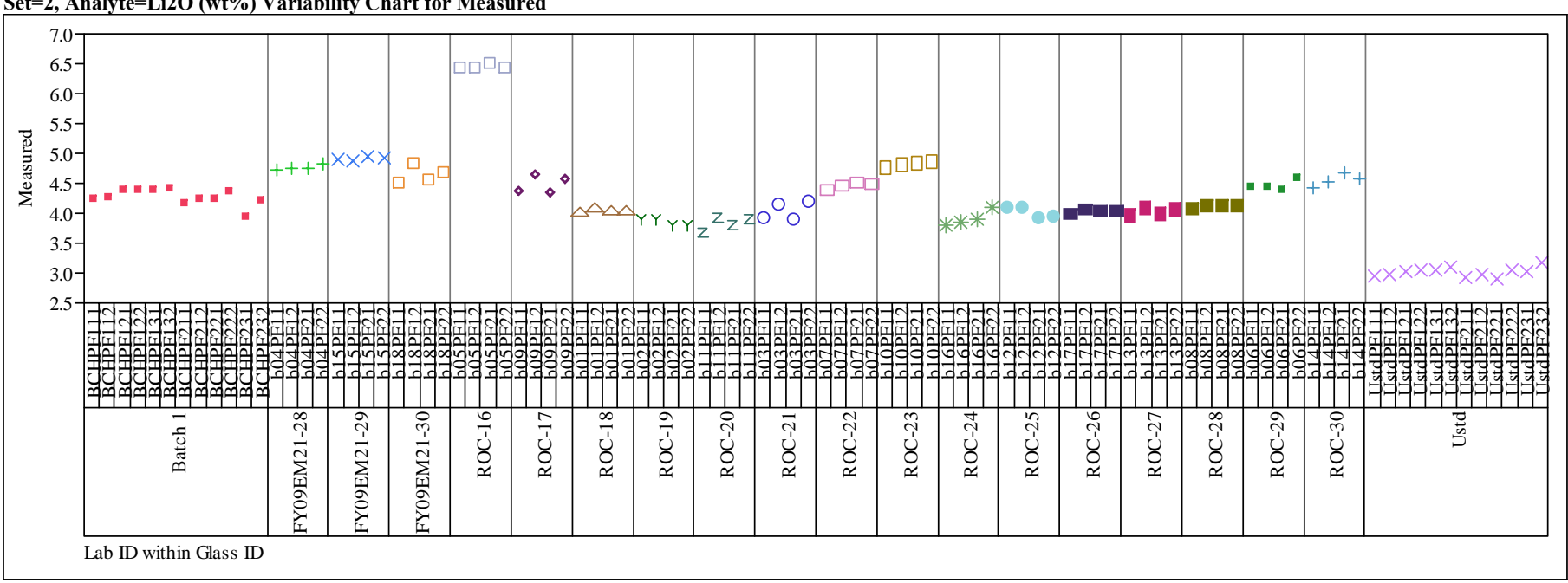

Set $=2$, Analyte $=\mathrm{MgO}(\mathbf{w t} \%)$ Variability Chart for Measured

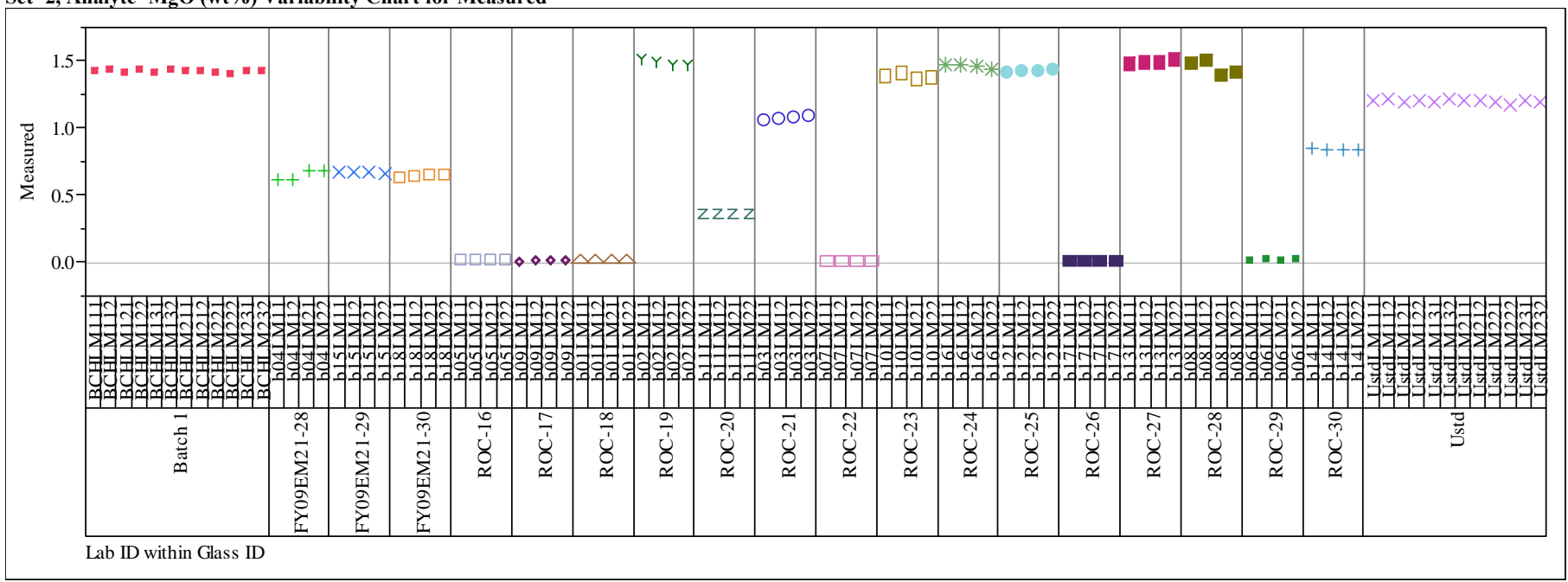


SRNL-STI-2009-00465, Revision 0

Exhibit A2. Measurements by Lab ID within Glass ID for Samples by Oxide and by Set

Set=2, Analyte $=\mathrm{MnO}(\mathrm{wt} \%)$ Variability Chart for Measured

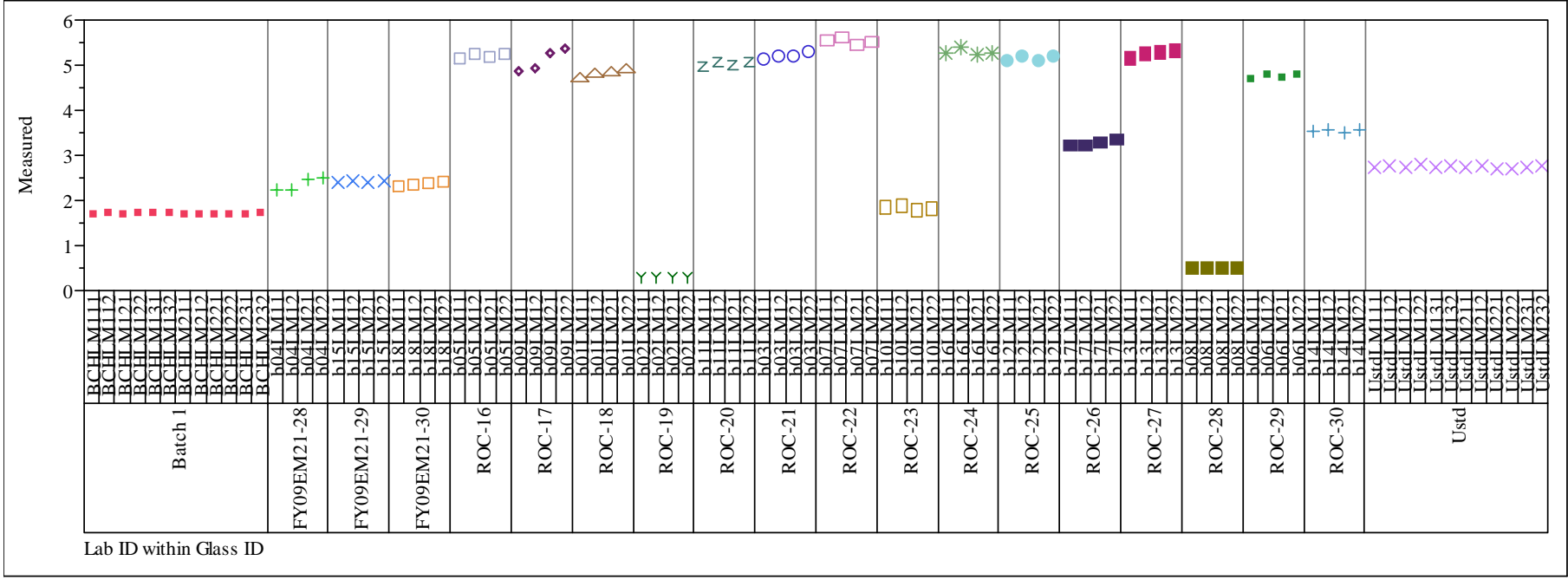

Set $=2$, Analyte $=\mathrm{Na2O}(\mathrm{wt} \%)$ Variability Chart for Measured

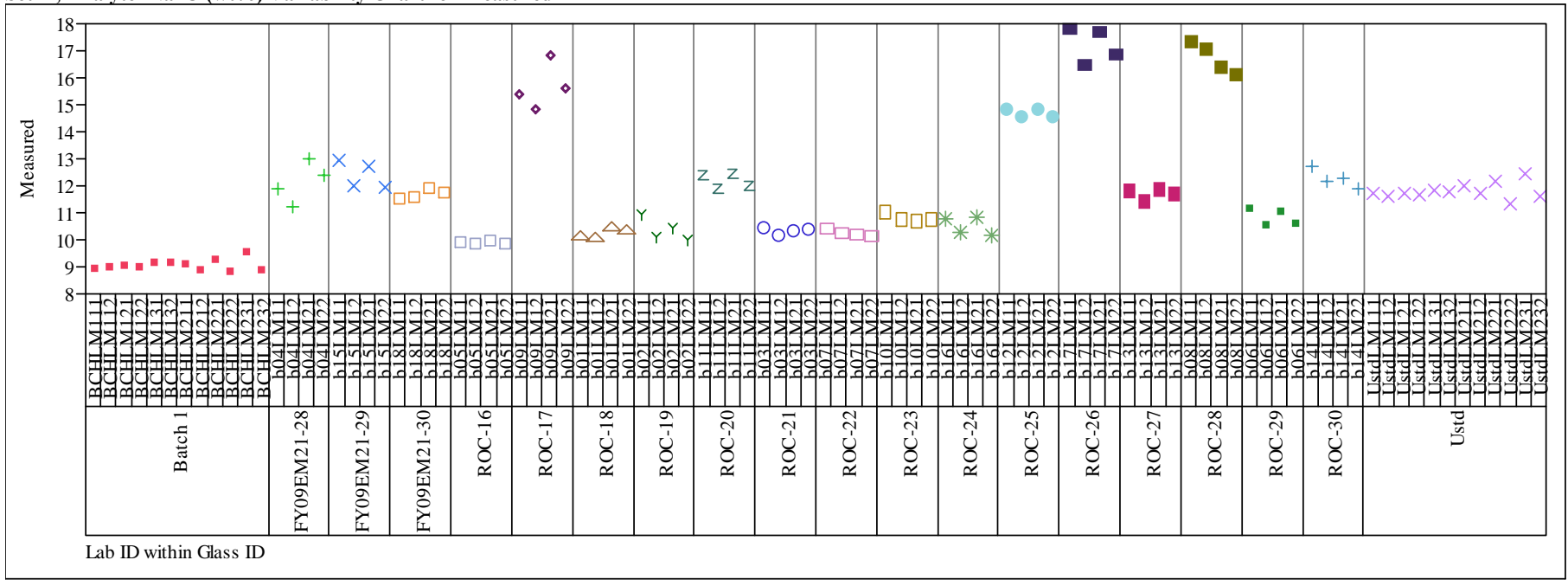


SRNL-STI-2009-00465, Revision 0

Exhibit A2. Measurements by Lab ID within Glass ID for Samples by Oxide and by Set

Set $=2$, Analyte $=\mathrm{NiO}(\mathrm{wt} \%)$ Variability Chart for Measured

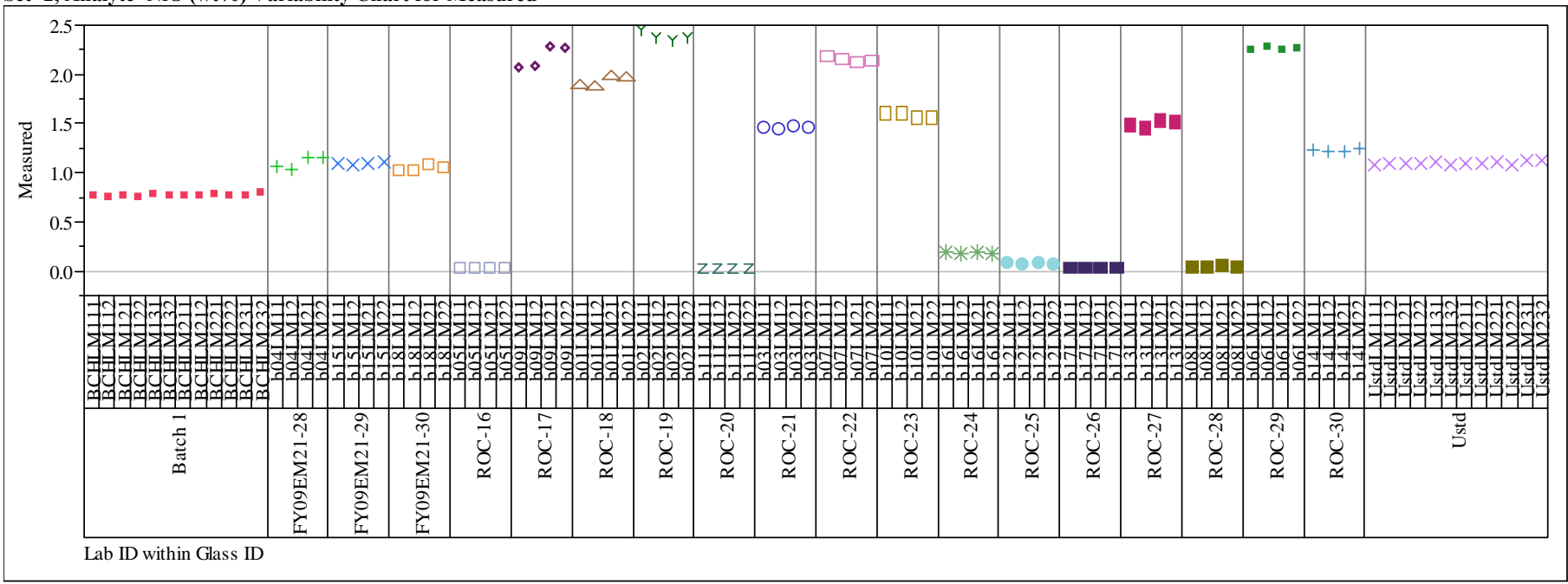

Set $=2$, Analyte $=\mathbf{P b O}($ wt $\%)$ Variability Chart for Measured

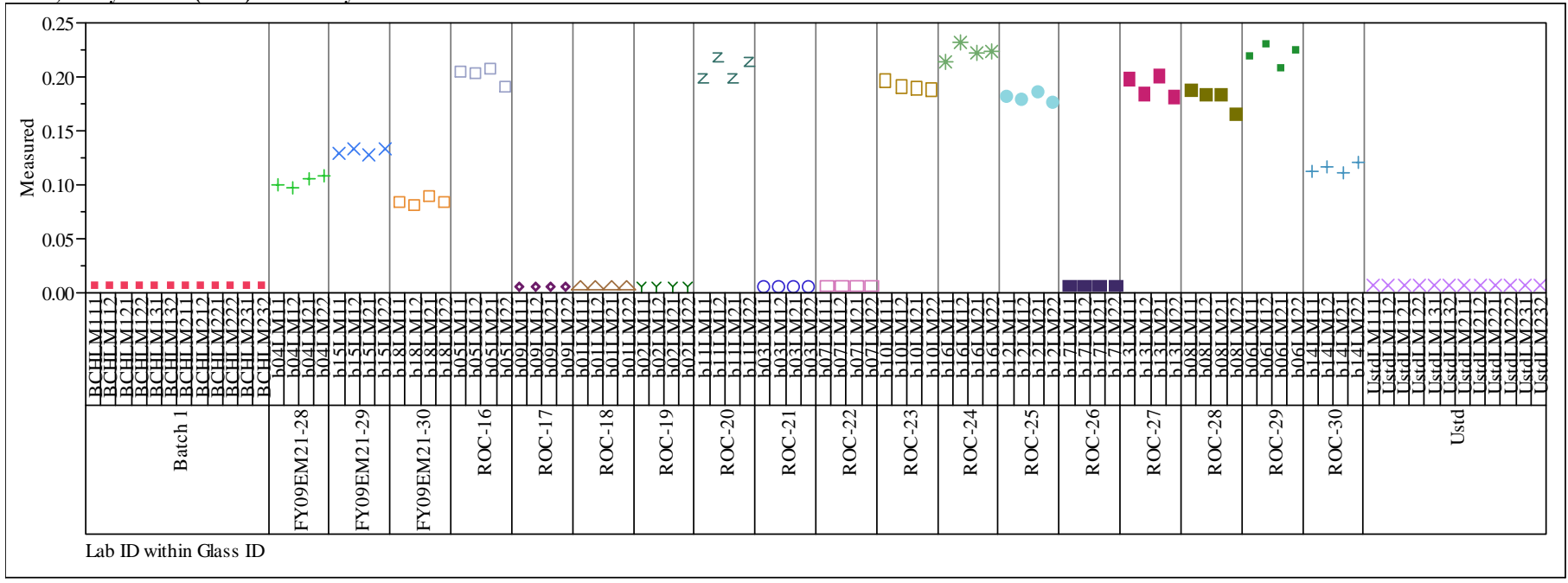


SRNL-STI-2009-00465, Revision 0

Exhibit A2. Measurements by Lab ID within Glass ID for Samples by Oxide and by Set

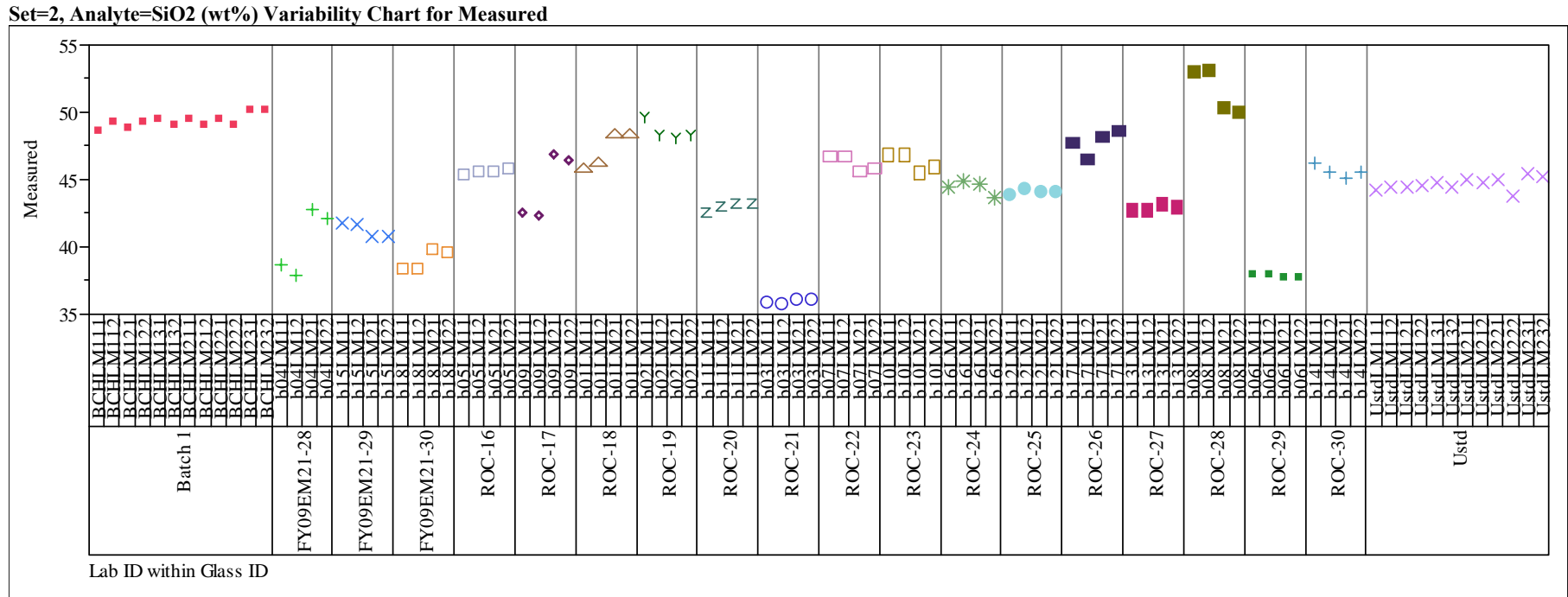

Set=2, Analyte=SO4 (wt\%) Variability Chart for Measured

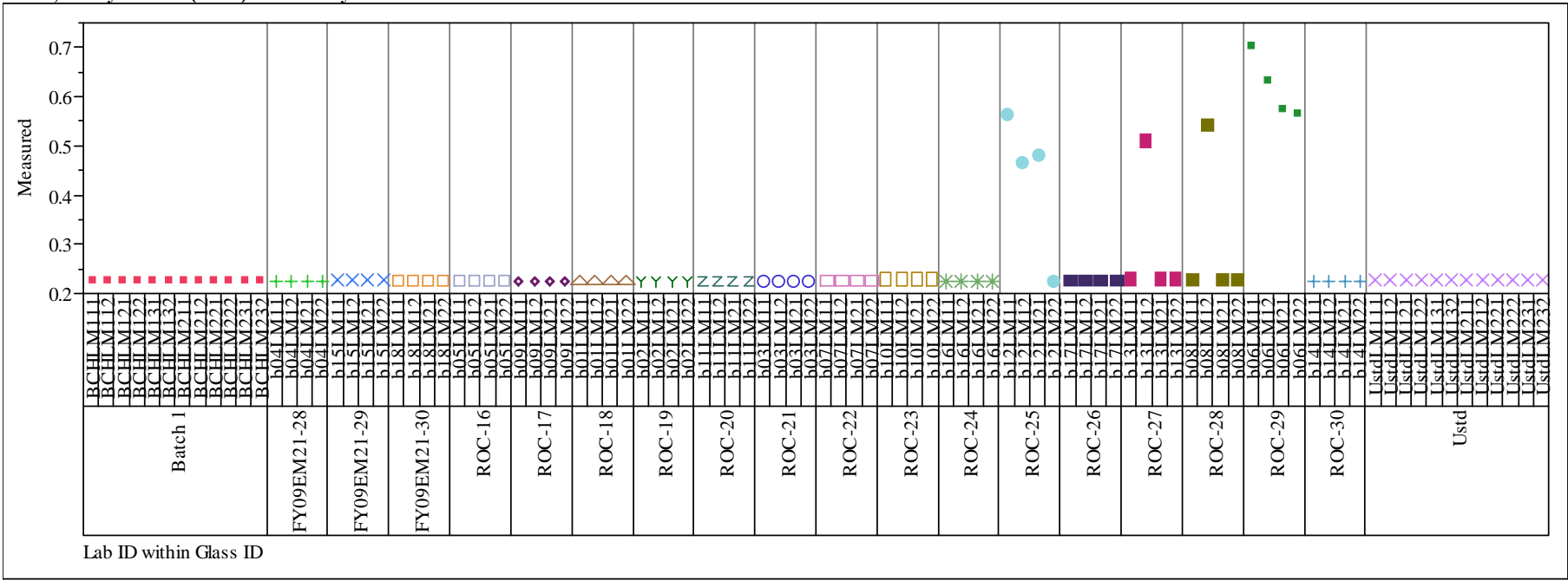


SRNL-STI-2009-00465, Revision 0

Exhibit A2. Measurements by Lab ID within Glass ID for Samples by Oxide and by Set

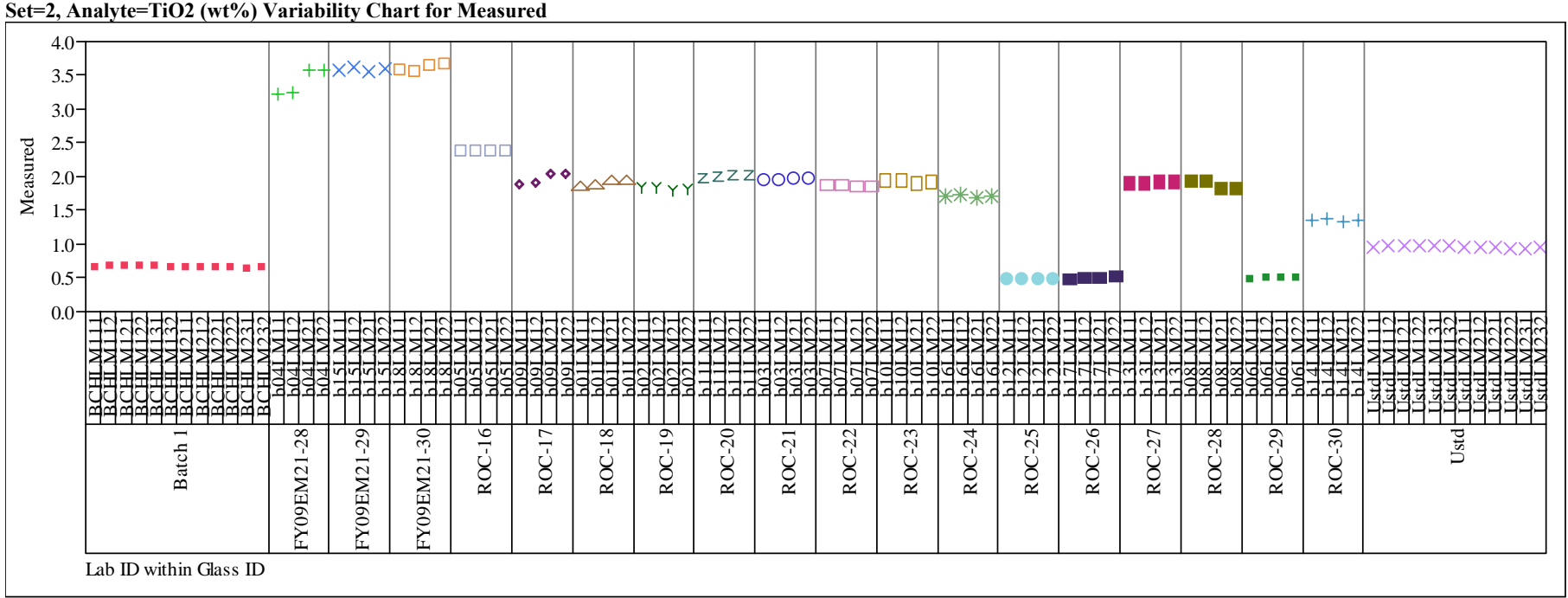

Set $=2$, Analyte $=\mathbf{U} 308$ (wt $\%$ ) Variability Chart for Measured

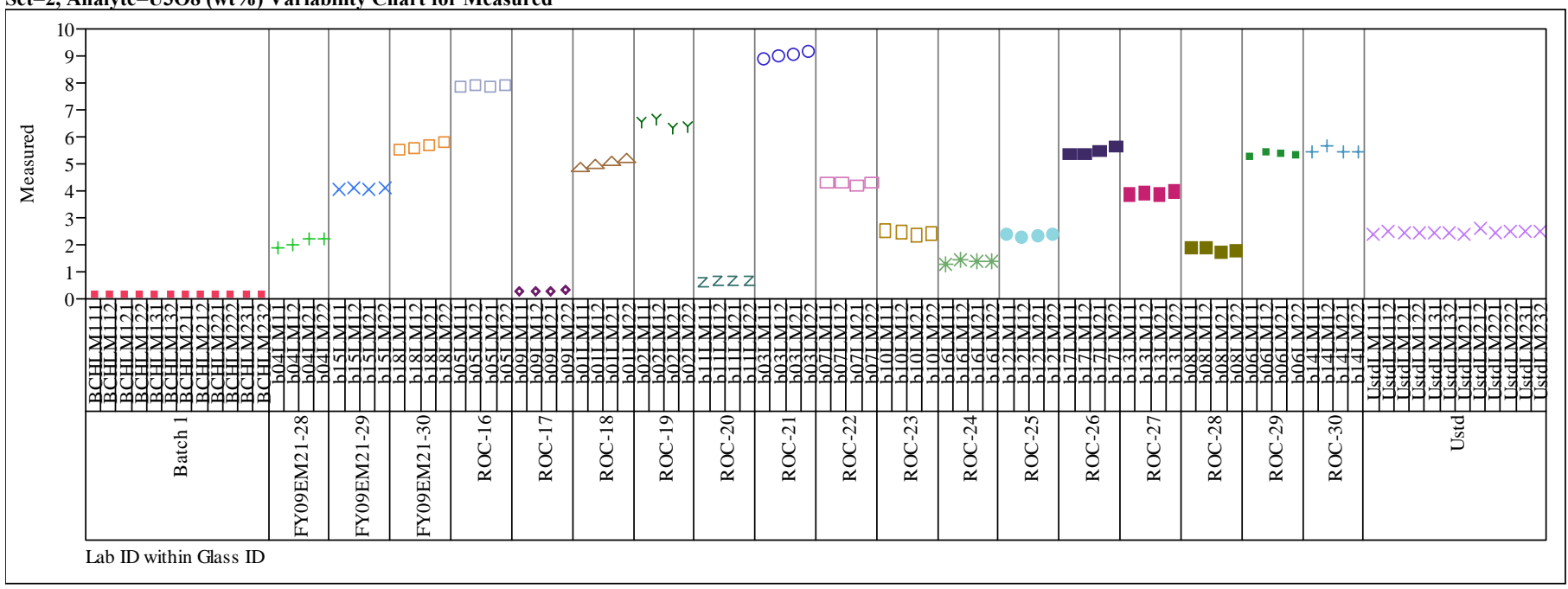


SRNL-STI-2009-00465, Revision 0

Exhibit A2. Measurements by Lab ID within Glass ID for Samples by Oxide and by Set

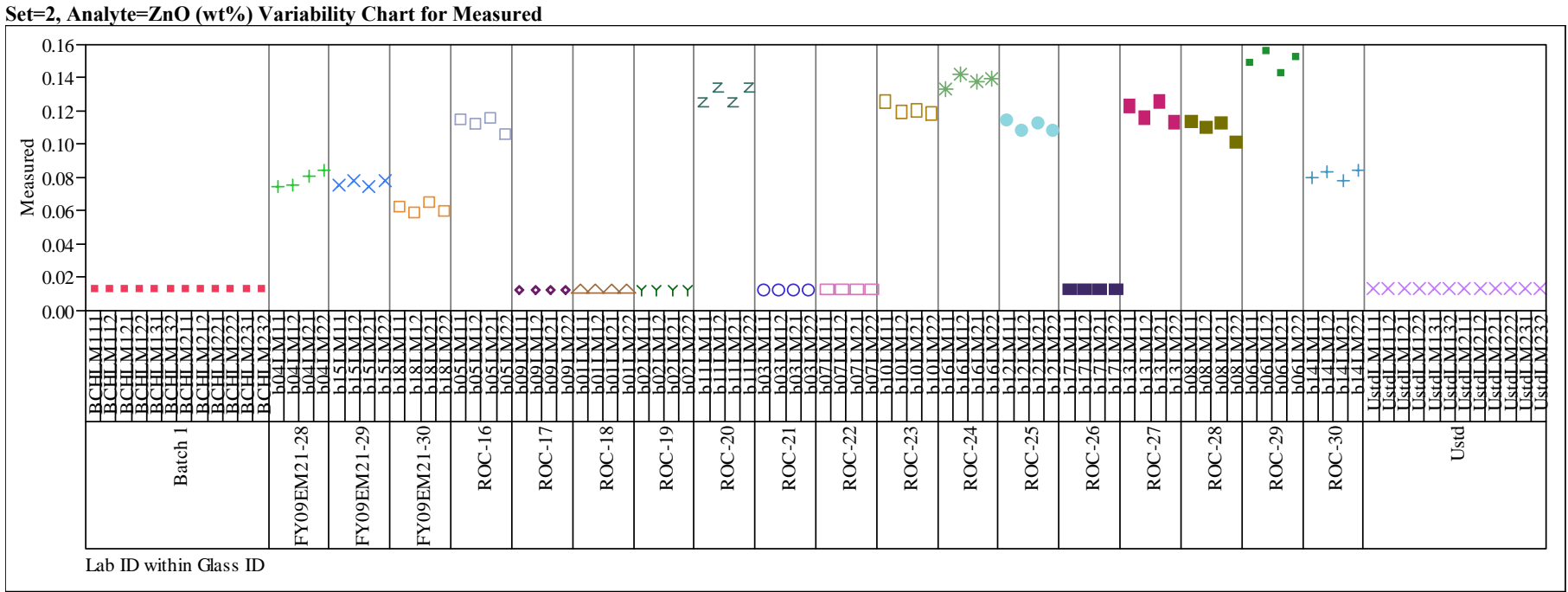

Set $=2$, Analyte $=\mathrm{ZrO} 2($ wt $\%)$ Variability Chart for Measured

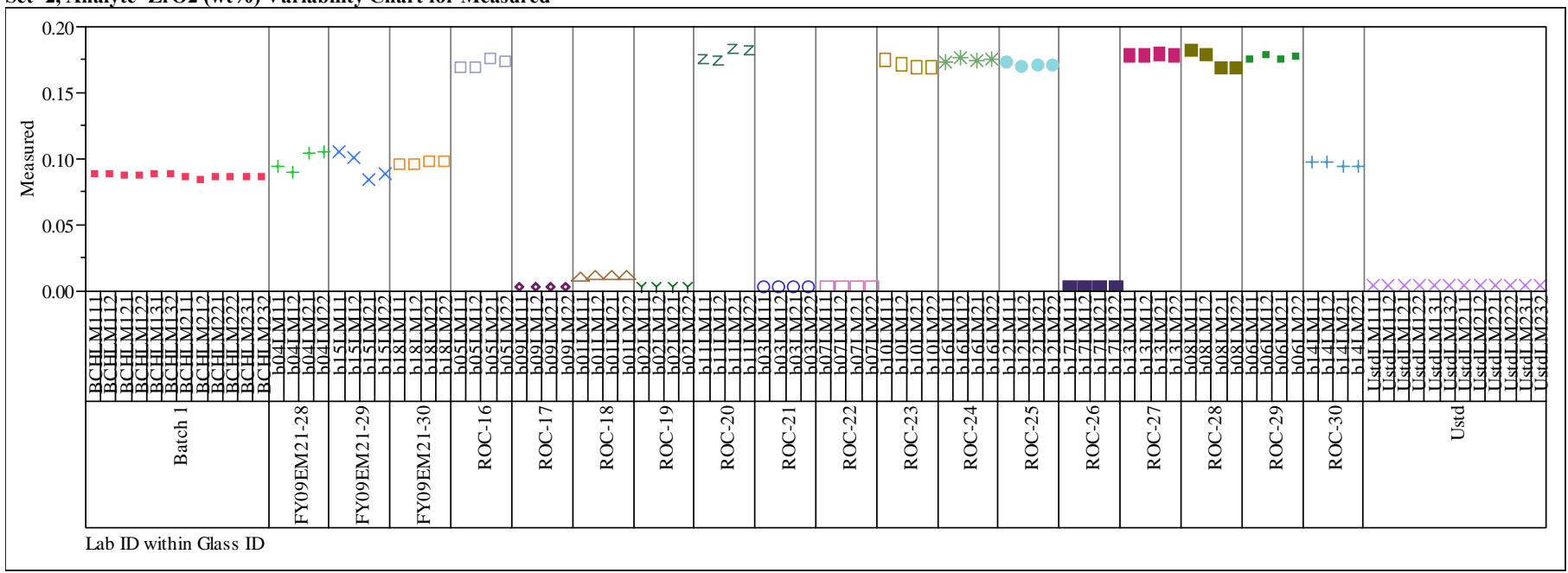


SRNL-STI-2009-00465, Revision 0

Exhibit A3. LM Measurements by Analytical Set, Block, and Sub-Block for Samples of the Batch 1 and Ustd Standards by Oxide

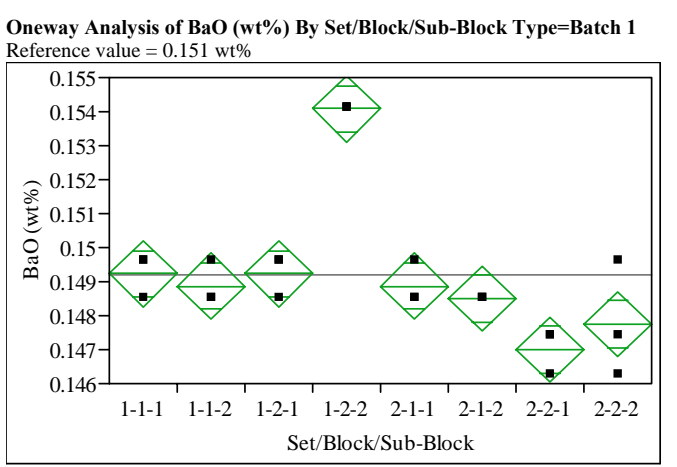

Oneway Anova
Summary of Fit

$\begin{array}{ll}\text { Rsquare } & 0.904335 \\ \text { Adj Rsquare } & 0.862481 \\ \text { Root Mean Square Error } & 0.000789 \\ \text { Mean of Response } & 0.149192\end{array}$

\begin{tabular}{ll} 
Adj Rsquare & 0.862481 \\
Root Mean Square Error & 0.000789 \\
\hline Mean of Response & 0.149192
\end{tabular}

$\begin{array}{lr}\text { Mean of Response } & 0.149192 \\ \text { Observations (or Sum Wgts) } & 24\end{array}$

Analysis of Variance
Source

$\begin{array}{lrrrrr}\text { Source } & \text { DF } & \text { Sum of Squares } & \text { Mean Square } & \text { F Ratio Prob }>\text { F } \\ \text { Set/Block/Sub-Block } & 7 & 0.00009427 & 0.000013 & 21.6071 & <.0001\end{array}$

$\begin{array}{llll}\text { Error } & 16 & 0.00000997 & 6.233 \mathrm{e}-7 \\ \text { C. Total } & 23 & 0.00010424 & \end{array}$

Means for Oneway Anova

\begin{tabular}{|c|c|c|c|c|c|}
\hline & Number & & Std Er & wer $95 \%$ & \\
\hline $1-1-1$ & & 0.149239 & 0.00046 & 0.14827 & \\
\hline $1-1-2$ & & 0.148867 & & & \\
\hline $2-$ & & & & & \\
\hline $2-$ & & & & & \\
\hline & & & & & \\
\hline & & & & & \\
\hline $2-1$ & & & 0.00046 & $\begin{array}{l}0.14604 \\
0.1467\end{array}$ & 0.147 \\
\hline
\end{tabular}

Std Error uses a pooled estimate of error variance
Oneway Analysis of CaO (wt\%) By Set/Block/Sub-Block Type=Batch 1

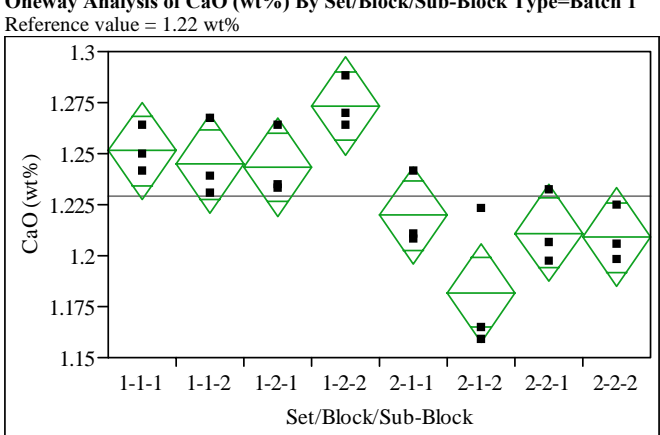

Oneway Anova
Summary of Fi

Rsquare
Adj Rsquar

0.630888
Root Mean Square Error $\quad 0.019626$

Mean of Response $\quad 1.229314$

Observations (or Sum Wgts) $\quad 24$

Analysis of Variance

Source
Set/Block/Sub-Block DF

$\begin{array}{lrr}\text { Set/Block/Sub-Block } & 7 & 0.01783879 \\ \text { rror } & 16 & 0.00616303\end{array}$ $\begin{array}{rrr}0.002548 & 6.6160 & 0.0009\end{array}$

C. Total

$\begin{array}{ll}16 & 0.00616303 \\ 23 & 0.02400182\end{array}$

Means for Oneway Anova

\begin{tabular}{|c|c|c|c|c|c|}
\hline Level & Number & Mean & Std Error & Lower 95\% & Upper 95\% \\
\hline 1-1-1 & & 1.25135 & 0.01133 & 1.2273 & 1.2754 \\
\hline $1-1-2$ & & 1.24482 & 0.01133 & & \\
\hline 1-2-1 & & 1.24342 & 0.011 & 1.2194 & 1.2674 \\
\hline $1-2-$ & & 1.27327 & 0.01133 & 1.2493 & 1.2973 \\
\hline 2-1-1 & & 1.21964 & 0.011 & 1.1956 & 1.2437 \\
\hline & & 1.18186 & 0.01133 & 1.1578 & 1.2059 \\
\hline $2-2-1$ & & 1.21124 & 0.01133 & 1.1872 & 1.2353 \\
\hline & & 1.20891 & 0.01133 & & \\
\hline
\end{tabular}

Std Error uses a pooled estimate of error variance
Oneway Analysis of CdO (wt\%) By Set/Block/Sub-Block Type=Batch 1 Reference value $=0.0 \mathrm{wt} \%$

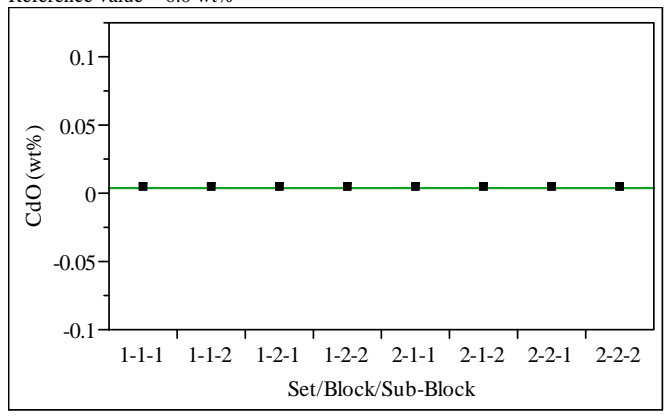

Oneway Anova

ummary of Fit

Rsquare

Root Mean Square Erro

Mean of Response $\quad 0.003998$

Observations (or Sum Wgts) 24

Analysis of Variance

Source DF Sum of Squares Mean Square F Ratio Prob $>$ F

$\begin{array}{lrrr}\text { Set/Block/Sub-Block } & 7 & 0 & 0 \\ \text { Error } & 16 & 0 & 0 \\ \text { C. Total } & 23 & 0 & \end{array}$

nova

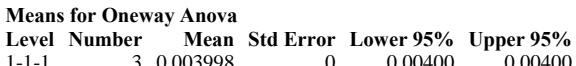

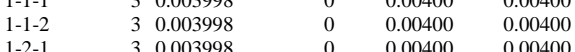

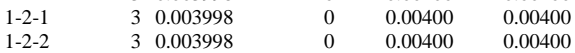

$\begin{array}{llllll}2-1-1 & 3 & 0.003998 & 0 & 0.00400 & 0.00400 \\ 2-1-2 & 3 & 0.003998 & 0 & 0.0400 & 0.0400\end{array}$

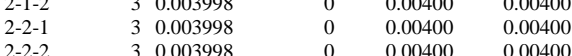

Std Error uses a pooled estimate of error variance 
SRNL-STI-2009-00465, Revision 0

Exhibit A3. LM Measurements by Analytical Set, Block, and Sub-Block for Samples of the Batch 1 and Ustd Standards by Oxide

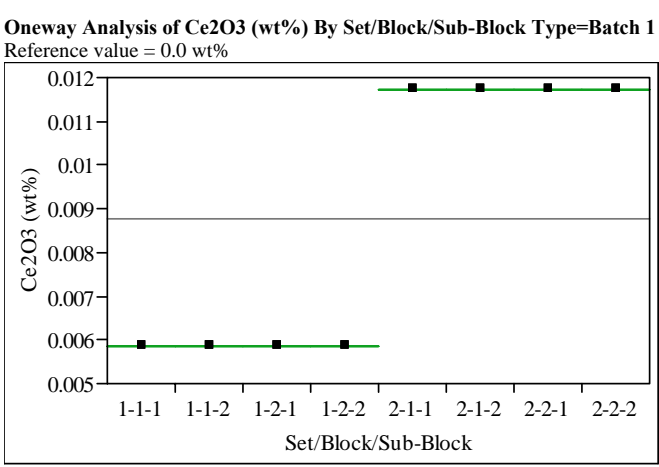

Oneway Anova
Summary of Fit

$\begin{array}{lr}\text { Rsquare } & 1 \\ \text { Adj Rsquare } & 1 \\ \text { Root Mean Square Error } & 0 \\ \text { Mean of Response } & 0.008785 \\ \text { Observations (or Sum Wgts) } & 24\end{array}$

\section{Analysis of Variance}

Source
Set/Block/Sub-Block DF Sum of Squares Mean Square F Ratio Prob $>$ F $\begin{array}{llll}\text { Set/Block/Sub-Block } & 7 & 0.00020579 & 0.000029 \\ \text { Error } & 16 & 0.00000000 & 0.000000\end{array}$

C. Total

$\begin{array}{ll}16 & 0.00000000 \\ 23 & 0.00020579\end{array}$

\begin{tabular}{lrrrrr}
$\begin{array}{l}\text { Means for Oneway Anova } \\
\text { Level }\end{array}$ & \multicolumn{1}{l}{ Number } & Mean & Std Error & Lower 95\% & Upper $\mathbf{9 5 \%}$ \\
$1-1-1$ & 3 & 0.005857 & 0 & 0.00586 & 0.00586 \\
$1-1-2$ & 3 & 0.005857 & 0 & 0.00586 & 0.00586 \\
$1-2-1$ & 3 & 0.005857 & 0 & 0.00586 & 0.00586 \\
$1-2-2$ & 3 & 0.005857 & 0 & 0.00586 & 0.00586 \\
$2-1-1$ & 3 & 0.011713 & 0 & 0.01171 & 0.01171 \\
$2-1-2$ & 3 & 0.011713 & 0 & 0.01171 & 0.01171 \\
$2-2-1$ & 3 & 0.01713 & 0 & 0.0171 & 0.01171 \\
$2-2-2$ & 3 & 0.011713 & 0 & 0.01171 & 0.01171
\end{tabular}

Std Error uses a pooled estimate of error variance

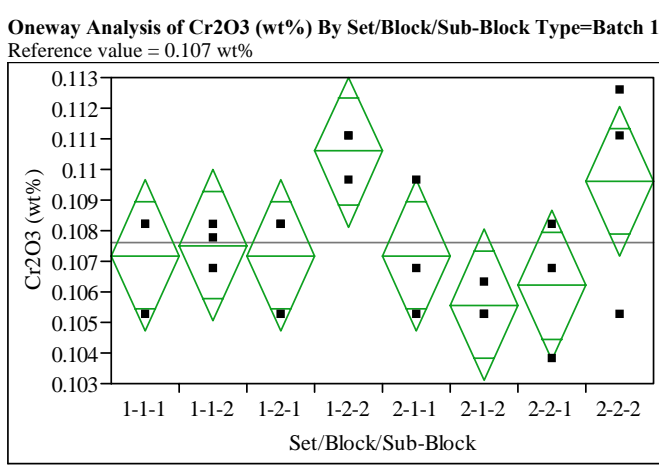

\section{Oneway Anova}

Summary of

$\begin{array}{ll}\text { Rsquare } & 0.476819 \\ \text { Adj Rsquare } & 0.247927 \\ \text { Root Mean Square Error } & 0.002008 \\ \text { Mean of Response } & 0.107635\end{array}$

$\begin{array}{lr}\text { Mean of Response } & 0.107635 \\ \text { Observations (or Sum Wgts) } & 24\end{array}$

Analysis of Variance
Source $\begin{array}{lccccc}\text { Source } & \text { DF } & \text { Sum of Squares } & \text { Mean Square } & \text { F Ratio } & \text { Prob }>\text { F } \\ \text { Set/Block/Sub-Block } & 7 & 0.00005877 & 8.3961 \mathrm{e}-6 & 2.0832 & 0.1062\end{array}$ $\begin{array}{lll}\text { Error } & 16 & 0.0000644 \\ \text { C. } & 23 & 0.0012326\end{array}$ $4.0304 \mathrm{e}-6$

\section{Means for Oneway Anova}

Level Number Mean Std Error Lower 95\% Upper 95\%

$\begin{array}{lllllll}-1-1 & 3 & 0.107184 & 0.00116 & 0.10473 & 0.10964 \\ 1-1-2 & & 3 & 0.107525 & 0.00116 & 0.10507 & 0.10998\end{array}$

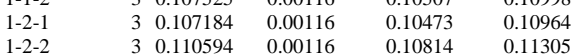

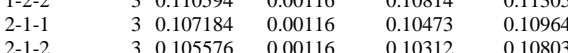

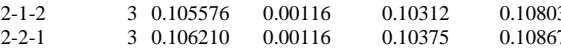

$\begin{array}{lllllll}2-2-2 & & 3 & 0.106210 & 0.00116 & 0.10375 & 0.10867 \\ 2-2-2 & & 0 & 0.109620 & 0.00116 & 0.10716 & 0.11208\end{array}$

Std Error uses a pooled estimate of error variance

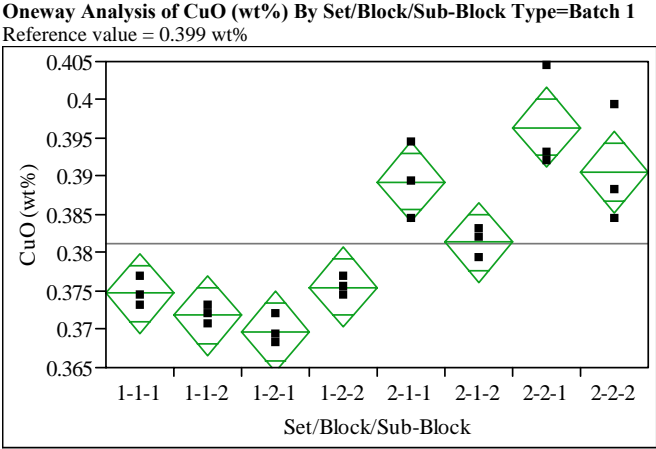

Oneway Anova

summary of Fit

$\begin{array}{ll}\text { Rsquare } & 0.873378 \\ \text { Adj Rsquare } & 0.817981 \\ \text { Root Mean Square Error } & 0.004299 \\ \text { Mean of Response } & 0.381173\end{array}$

$\begin{array}{lr}\text { Mean of Response } & 0.381173 \\ \text { Observations (or Sum Wgts) } & 24\end{array}$

Analysis of Variance
Source DF Sum of Squares Mean Square F Ratio Prob $>$ F

$\begin{array}{llllll}\text { Set/Block/Sub-Block } & 7 & 0.00203919 & 0.000291 & 15.7658 & <.0001\end{array}$

$\begin{array}{llll}\text { Error } & 16 & 0.00029564 & 0.000018 \\ \text { C. Total } & 23 & 0.00233483 & \end{array}$

Means for Oneway Anova

Level Number Mean Std Error Lower 95\% Upper 95\%

$\begin{array}{llllll}1-1-1 & 3 & 0.374705 & 0.00248 & 0.36944 & 0.3799 \\ 1-1-2 & 3 & 0.371785 & 0.00248 & 0.3652 & 0.37705\end{array}$

$\begin{array}{lllllll}1-2-1 & & 3 & 0.371785 & 0.00248 & 0.36652 & 0.37705 \\ 1-2-2 & & 3 & 0.375540 & 0.00248 & 0.36444 & 0.37496 \\ 2-1-1 & & & 0.36248 & 0.37028 & 0.38080\end{array}$

$\begin{array}{llllll}1-2-2 & 3 & 0.375940 & 0.00248 & 0.37028 & 0.38080\end{array}$

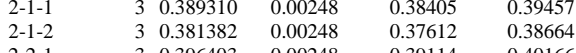

$\begin{array}{llllll}2-2-2 & 3 & 0.390562 & 0.00248 & 0.39114 & 0.40166 \\ 2 & & & & & \end{array}$

Std Error uses a pooled estimate of error variance 
SRNL-STI-2009-00465, Revision 0

Exhibit A3. LM Measurements by Analytical Set, Block, and Sub-Block for Samples of the Batch 1 and Ustd Standards by Oxide

Oneway Analysis of K2O (wt\%) By Set/Block/Sub-Block Type=Batch 1

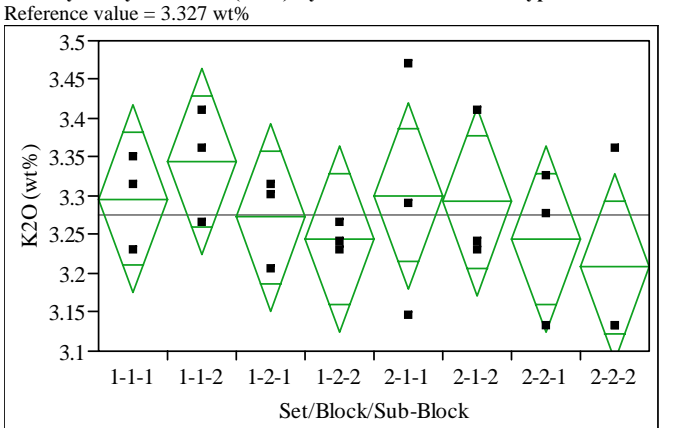

Oneway Anova
Summary of Fit

$\begin{array}{lr}\text { Rsquare } & 0.196686 \\ \text { Adj Rsquare } & -0.15476 \\ \text { Root Mean Square Error } & 0.098355\end{array}$

$\begin{array}{lr}\text { Mean of Response } & 3.275508 \\ \text { Observations (or Sum Wgts) } & 24\end{array}$

Analysis of Variance
Source

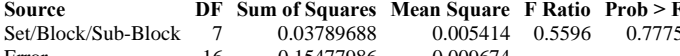

Error

$\begin{array}{ll}16 & 0.15477986 \\ 23 & 0.19267674\end{array}$

Means for Oneway Anova

Level Number Mean Std Error Lower 95\% Upper 95\%

$\begin{array}{lllllll}1-1-1 & & 3 & 3.29659 & 0.05679 & 3.1762 & 3.4170 \\ 1-1-2 & & 3 & 3.34477 & 0.05679 & 3.2244 & 3.4652\end{array}$

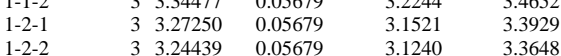

$\begin{array}{llllll}1-2-1 & 3 & 3.240060 & 0.05679 & 3.1240 & 3.3648 \\ 2-1-1 & 3 & 0.05679 & 3.1802 & 3.4210\end{array}$

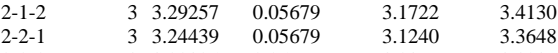

$\begin{array}{llll}3 & 3.20825 & 0.05679 & 3.0879\end{array}$
Oneway Analysis of La2O3 (wt\%) By Set/Block/Sub-Block Type=Batch 1 Reference value $=0,0 \mathrm{~W}$

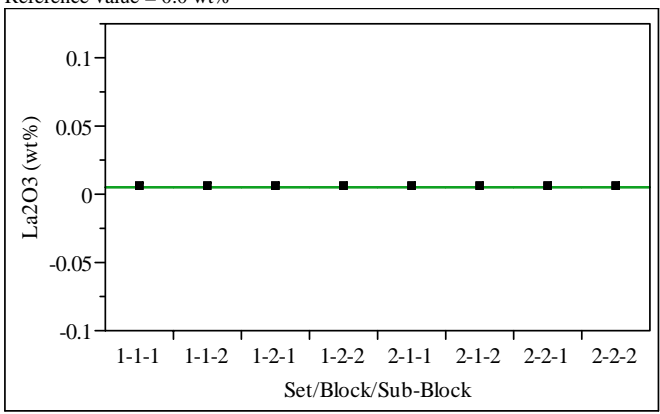

Oneway Anova

Summary of Fi

Rsquare

Root Mean Square Erro

$\begin{array}{lr}\text { Mean of Response } & 0.005278 \\ \text { Observations (or Sum Wgts) } & 24\end{array}$

Analysis of Variance

Source DF Sum of Squares Mean Square F Ratio Prob $>$ F

SelBlock/Sub-Block 7

C. Total

16
23

Means for Oneway Anova

Level Number Mean Std Error Lower 95\% Upper 95\%

$\begin{array}{llllll}1-1-1 & 3 & 0.005278 & 0 & 0.00528 & 0.00528 \\ -1-2 & 3 & 0.005278 & 0 & 0.0528 & 0.0528\end{array}$

$\begin{array}{llllll}1-1-1 & 3 & 0.005278 & 0 & 0.00528 & 0.00528 \\ 1-2-1 & 3 & 0.005278 & 0 & 0.00528 & 0.00528\end{array}$

$\begin{array}{lll}1-2-2 & 3 & 0.005278 \\ 2-1-12 & 3 & 0.005278\end{array}$

$\begin{array}{lll}2-1-2 & 3 & 0.005278 \\ 2-2-1 & 3 & 0.05278\end{array}$

$\begin{array}{lll}2-2-1 & 3 & 0.005278 \\ 2-2-2 & 3 & 0.005278\end{array}$

0.00528

$0.00528 \quad 0.00528$

Std Error uses a pooled estimate of error variance

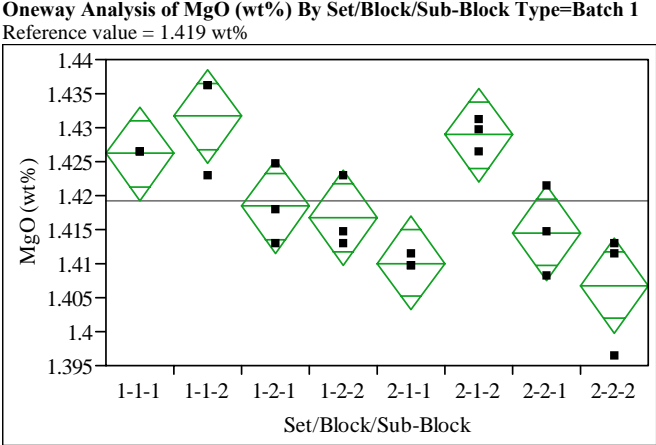

Oneway Anova

summary of Fit

$\begin{array}{ll}\text { Rsquare } & 0.767488 \\ \text { Adj Rsquare } & 0.665764\end{array}$

Root Mean Square Error $\quad 0.005654$ $\begin{array}{lr}\text { Mean of Response } & 1.419159 \\ \text { Observations (or Sum Wgts) } & 24\end{array}$

Analysis of Variance

Source DF Sum of Squares Mean Square F Ratio Prob $>$ F

Set/Block/Sub-Block $7 \quad 0.00168836$ $\begin{array}{lll}0.000241 & 7.5448 & 0.0004\end{array}$

C. Tota

$16 \quad 0.0005114$ 0.00003

Means for Oneway Anova

Level Number Mean Std Error Lower 95\% Upper 95\%

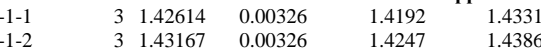

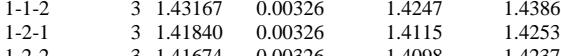

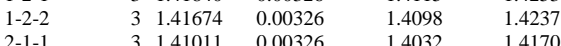

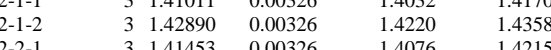

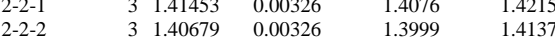

Std Error uses a pooled estimate of error variance 
Exhibit A3. LM Measurements by Analytical Set, Block, and Sub-Block for Samples of the Batch 1 and Ustd Standards by Oxide

Oneway Analysis of $\mathrm{MnO}$ (wt\%) By Set/Block/Sub-Block Type=Batch 1

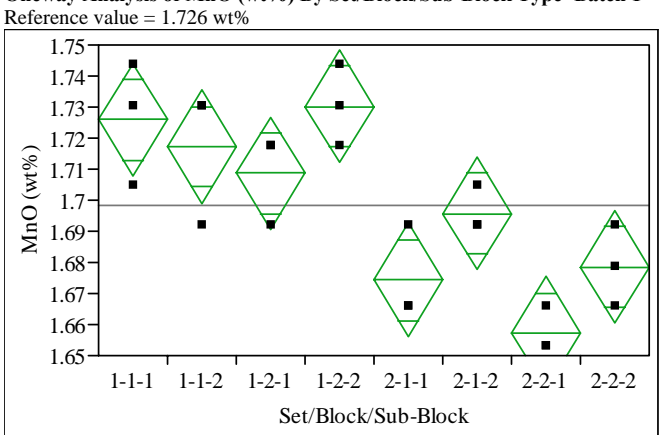

Oneway Anova
Summary of Fit

$\begin{array}{lr}\text { Rsquare } & 0.805987 \\ \text { Adj Rsquare } & 0.721106 \\ \text { Root Mean Square Error } & 0.014909 \\ \text { Mean of Response } & 1.698466 \\ \text { Observations (or Sum Wgts) } & 24\end{array}$

Observations (or Sum Wg

Analysis of Variance
Source

$\begin{array}{lrrrrr}\text { Source } & \text { DF } & \text { Sum of Squares } & \text { Mean Square } & \text { F Ratio } & \text { Prob }>\text { F } \\ \text { Set/Block/Sub-Block } & 7 & 0.01477554 & 0.002111 & 9.4955 & 0.0001\end{array}$

$\begin{array}{lrrr}\text { Set/Block/Sub-Block } & 7 & 0.01477554 & 0.002111 \\ \text { Error } & 16 & 0.00355669 & 0.000222 \\ \text { C. Total } & 23 & 0.01833223 & \end{array}$

Means for Oneway Anova

\begin{tabular}{lrrrrr}
\multicolumn{7}{c}{$\begin{array}{l}\text { Means for Oneway Anova } \\
\text { Level Number }\end{array}$} & Mean & Std Error & Lower 95\% & Upper 95\% \\
$1-1-1$ & 3 & 1.72590 & 0.00861 & 1.7077 & 1.7442 \\
$1-1-2$ & 3 & 1.71730 & 0.00861 & 1.6990 & 1.7355 \\
$1-2-1$ & 3 & 1.70869 & 0.00861 & 1.6904 & 1.7269 \\
$1-2-2$ & 3 & 1.73021 & 0.00861 & 1.7120 & 1.7485 \\
$2-1-1$ & 3 & 1.67426 & 0.00861 & 1.6560 & 1.6925 \\
$2-1-2$ & 3 & 1.69578 & 0.00861 & 1.6775 & 1.7140 \\
$2-2-1$ & 3 & 1.65704 & 0.00861 & 1.6388 & 1.6753 \\
$2-2-2$ & 3 & 1.67856 & 0.00861 & 1.6603 & 1.6968
\end{tabular}

(n)
Oneway Analysis of $\mathrm{Na2O}$ (wt\%) By Set/Block/Sub-Block Type=Batch 1 Reference value $=9.003 \mathrm{wt} \%$

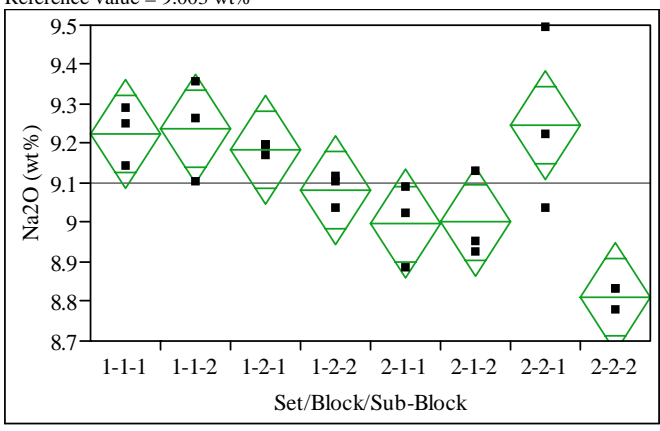

Oneway Anova

$\begin{array}{lr}\text { Rsquare } & 0.712927 \\ \text { Adj Rsquare } & 0.587332 \\ \text { Root Mean Square Error } & 0.112614 \\ \text { Mean of Response } & 9.097877 \\ \text { Observations (or Sum Wgts) } & 24\end{array}$

Analysis of Variance

Source DF Sum of Squares Mean Square F Ratio Prob > F $\begin{array}{lrllll}\text { Set/Block/Sub-Block } & 7 & 0.50391322 & 0.071988 & 5.6764 & 0.0020 \\ \text { Error } & 16 & 0.20290995 & 0.012682 & & \end{array}$ Error $\begin{array}{ll}16 & 0.20290995 \\ 23 & 0.70682317\end{array}$

Means for Oneway Anova

\begin{tabular}{|c|c|c|c|c|}
\hline & & & Lower 95\% & $\mathbf{U}_{\mathbf{I}}$ \\
\hline & 39.22481 & 0.06502 & 9.0870 & 9.3626 \\
\hline & $\begin{array}{ll}3 & 9.23829\end{array}$ & 0.06502 & 9.1005 & \\
\hline & $\begin{array}{ll}3 & 9.1843 \\
\end{array}$ & 0.06502 & 9.0465 & 9.3222 \\
\hline & $\begin{array}{ll}3 & 9.08103\end{array}$ & 0.06502 & 8.9432 & 9.2189 \\
\hline & $\begin{array}{ll}3 & 8.99565\end{array}$ & 0.06502 & 8.8578 & 9.13 \\
\hline & 39.00015 & 0.06502 & 8.8623 & 9.138 \\
\hline & 9.24728 & 0.06502 & 9.1094 & 9.38 \\
\hline & 8.81143 & 0.06502 & 8.6736 & 8.9493 \\
\hline
\end{tabular}

Std Error uses a pooled estimate of error variance
Oneway Analysis of $\mathrm{NiO}$ (wt\%) By Set/Block/Sub-Block Type=Batch 1

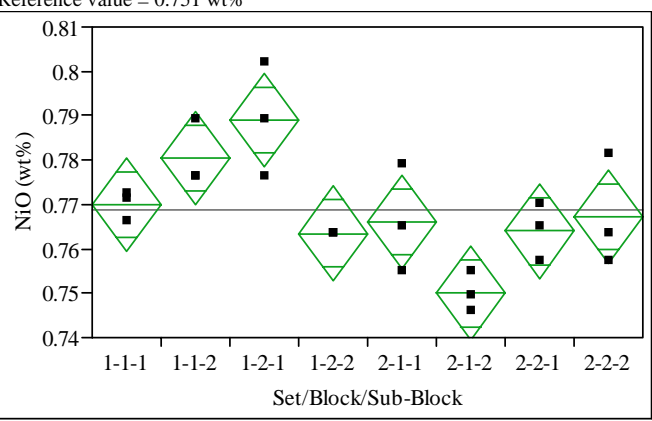

Oneway Anova

Summary of Fit

$\begin{array}{ll}\text { Rsquare } & 0.708671 \\ \text { Adj Rsquare } & 0.081215\end{array}$

Root Mean Square Error $\quad 0.008607$ $\begin{array}{lr}\text { Mean of Response } & 0.768749 \\ \text { Observations (or Sum Wgts) } & 24\end{array}$

Analysis of Variance
Source DF Sum of Squares Mean Square F Ratio Prob $>$ F $\begin{array}{lccccc}\text { Source } & \text { DF } & \text { Sum of Squares } & \text { Mean Square } & \text { F Ratio } & \text { Prob }>\text { F } \\ \text { Set/Block/Sub-Block } & 7 & 0.00288329 & 0.000412 & 5.5601 & 0.0022\end{array}$ $\begin{array}{lrrr}\text { Set/Block/Sub-Block } & 7 & 0.00288329 & 0.000412 \\ \text { Error } & 16 & 0.00118530 & 0.000074 \\ \text { C. Total } & 23 & 0.00406858 & \end{array}$

Means for Oneway Anova

Level Number Mean Std Error Lower 95\% Upper 95\%

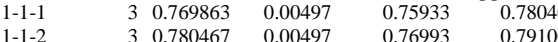

$\begin{array}{lllllll}1-2-1 & & 3 & 0.788950 & 0.00497 & 0.77842 & 0.79948 \\ 1-2-2 & & 3 & 0.763500 & 0.00497 & 0.75297 & 0.77403\end{array}$

$\begin{array}{llllll}3 & 0.766045 & 0.00497 & 0.75551 & 0.77658\end{array}$

$\begin{array}{lllllll}2-1-2 & 3 & 0.749927 & 0.00497 & 0.73939 & 0.76046\end{array}$

$\begin{array}{lllllll}2-2-1 & & 3 & 0.763924 & 0.00497 & 0.75339 & 0.77446 \\ 2-2-2 & & 3 & 0.767318 & 0.00497 & 0.75678 & 0.77785\end{array}$

Std Error uses a pooled estimate of error variance 
SRNL-STI-2009-00465, Revision 0

Exhibit A3. LM Measurements by Analytical Set, Block, and Sub-Block for Samples of the Batch 1 and Ustd Standards by Oxide

Oneway Analysis of PbO (wt\%) By Set/Block/Sub-Block Type=Batch 1

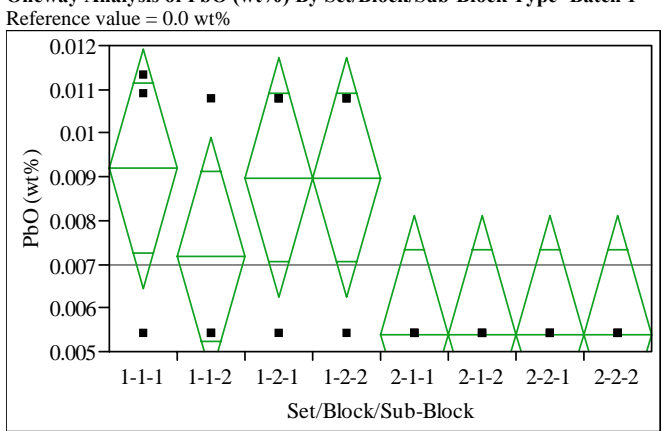

Oneway Anova
Summary of Fit

\section{$\begin{array}{ll}\text { Rsquare } & 0.464345 \\ \text { Adj Rsquare } & 0.229996\end{array}$}

Root Mean Square Error $\quad 0.002234$

$\begin{array}{lr}\text { Mean of Response } & 0.006984 \\ \text { Observations (or Sum Wgts) } & 24\end{array}$

Analysis of Variance
Source

$\begin{array}{lrrrrr}\text { Source } & & & & & \\ \text { Set/Block/Sub-Block } & 7 & 0.00006921 & 9.8874 \mathrm{e}-6 & 1.9814 & 0.1219\end{array}$

$\begin{array}{lll}\text { Error } & 16 & 0.00007984 \\ \text { C. Total } & 23 & 0.00014905\end{array}$

Means for Oneway Anova

$\begin{array}{lrrrrr}\text { Level } & \text { Number } & \text { Mean } & \text { Std Error } & \text { Lower 95\% } & \text { Upper 95\% } \\ 1-1-1 & 3 & 0.009192 & 0.00129 & 0.0064 & 0.01193\end{array}$

$\begin{array}{lllllll}1-1-2 & & 3 & 0.007181 & 0.00129 & 0.00646 & 0.01193 \\ 1-2 & & 3 & 0.00129 & 0.00445 & 0.00992\end{array}$

$\begin{array}{lllllll}1-2-1 & & 3 & 0.008977 & 0.00129 & 0.00624 & 0.01171 \\ 1-2-2 & & 3 & 0.008977 & 0.00129 & 0.00624 & 0.01171\end{array}$

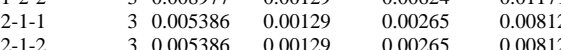

$\begin{array}{llllll}2-1-2 & 3 & 0.005386 & 0.00129 & 0.00265 & 0.00812 \\ 2-2-1 & 3 & 0.005386 & 0.00129 & 0.00265 & 0.00812\end{array}$

$\begin{array}{llllll}2-2-2 & 3 & 0.005386 & 0.00129 & 0.00265 & 0.00812\end{array}$

Std Error uses a pooled estimate of error variance
Oneway Analysis of SO4 (wt\%) By Set/Block/Sub-Block Type=Batch 1 Reference value $=0.0 \mathrm{wt} \%$

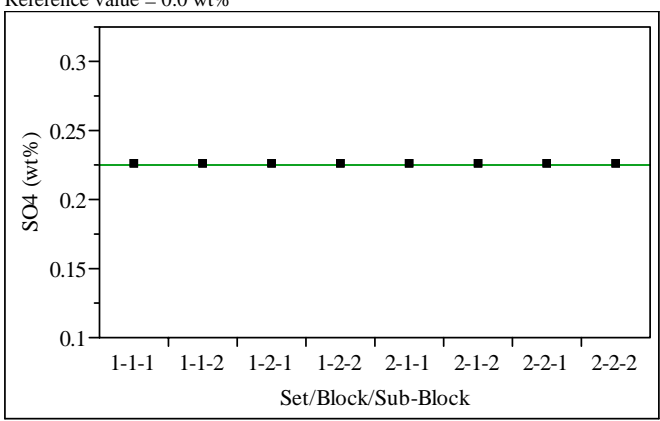

Oneway Anova

Summary of Fit

Rsquare
Adj Rsquare

Root Mean Square Error

$\begin{array}{lr}\text { Mean of Response } & 0.224693 \\ \text { Observations (or Sum Wgts) } & 24\end{array}$

Analysis of Variance

Source DF Sum of Squares Mean Square F Ratio Prob $>F$

$\begin{array}{lrrr}\text { Set/Block/Sub-Block } & 7 & 0 & 0 \\ \text { Error } & 16 & 0 & 0 \\ \text { C. Total } & 23 & 0 & \end{array}$

Means for Oneway Anova

Level Number Mean Std Error Lower 95\% Upper 95\%

$\begin{array}{lll}1-1-1 & 3 & 0.224693 \\ 1-1-2 & 3 & 0.224693 \\ 1-2-1 & 3 & 0.224693\end{array}$

$\begin{array}{llll}1-2-1 & 3 & 0.224693 \\ 1-2-2 & 3 & 0.224693\end{array}$

$\begin{array}{lll}1-2-2 & 3 & 0.224693 \\ 2-1-1 & 3 & 0.224693\end{array}$

$\begin{array}{ll}3 & 0.224693 \\ 3 & 0.224693\end{array}$

$\begin{array}{ll}0 & 0.224 \\ 0 & 0.2246 \\ 0 & 0.22469 \\ 0 & 0.22469 \\ 0 & 0.22469 \\ 0 & 0.22469 \\ 0 & 0.22469 \\ 0 & 0.2246\end{array}$

$\begin{array}{lll}2-2-1 & 3 & 0.224693 \\ 2-2-2 & 3 & 0.224693\end{array}$

Std Error uses a pooled estimate of error variance
Oneway Analysis of SiO2 (wt\%) By Set/Block/Sub-Block Type=Batch 1 Reference value $=50.22 \mathrm{wt}$

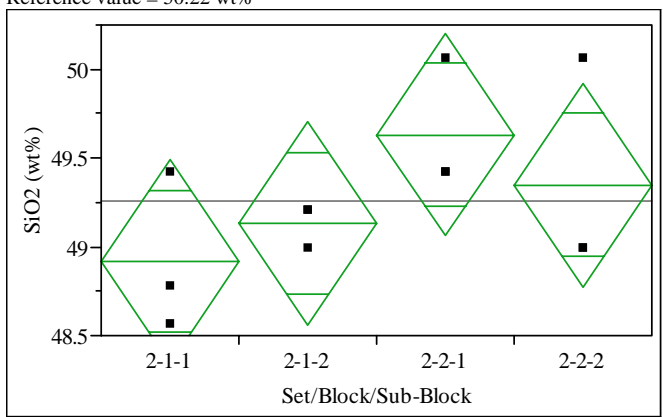

Missing Rows

Oneway Anova

Summary of Fit

$\begin{array}{ll}\text { Rsquare } & 0.363184 \\ \text { Adj Rsquare } & 0.124378\end{array}$

Root Mean Square Error $\quad \begin{array}{r}0.124378 \\ 0.42786\end{array}$

$\begin{array}{lr}\text { Mean of Response } & 49.25738 \\ \text { Observations (or Sum Wgts) } & 12\end{array}$

Analysis of Variance

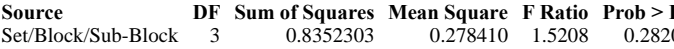
\begin{tabular}{lrrr} 
Error & 8 & 1.4645134 & 0.18306 \\
\hline
\end{tabular}

C. Tota 2.2997438

Means for Oneway Anova

Level Number Mean Std Error Lower 95\% Upper 95\% $\begin{array}{lr}\text { Level } & \text { Number } \\ 1-1-1 & 0 \\ 1-1-2 & 0\end{array}$

$\begin{array}{ll}1-1-2 & 0 \\ 1-2-1 & 0 \\ 1-2-2 & 0 \\ 2-1-1 & 3\end{array}$

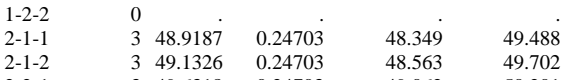

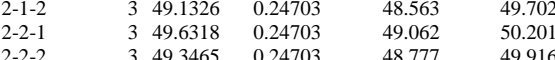

Std Error uses a pooled estimate of error variance 
SRNL-STI-2009-00465, Revision 0

Exhibit A3. LM Measurements by Analytical Set, Block, and Sub-Block for Samples of the Batch 1 and Ustd Standards by Oxide

Oneway Analysis of TiO2 (wt\%) By Set/Block/Sub-Block Type=Batch 1

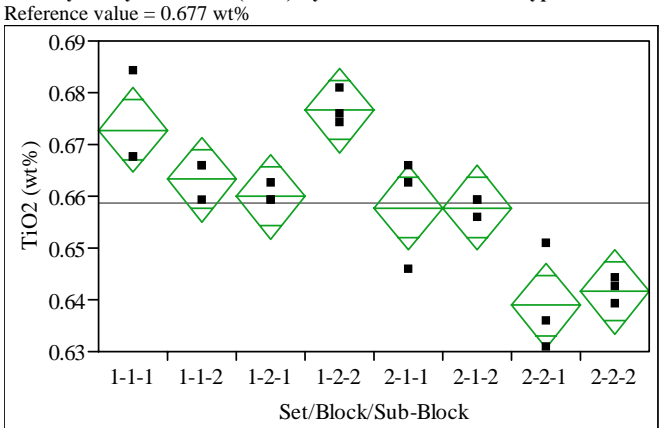

Oneway Anova
Summary of Fit

$\begin{array}{ll}\text { Rsquare } & 0.837895 \\ \text { Adj Rsquare } & 0.766974 \\ \text { Root Mean Square Error } & 0.006681 \\ \text { Mean of Response } & 0.658582\end{array}$

$\begin{array}{lr}\text { Mean of Response } & 0.058582 \\ \text { Observations (or Sum Wgts) } & 24\end{array}$

Analysis of Variance
Source

$\begin{array}{lrrrrr}\text { Source } & \text { DF } & \text { Sum of Squares } & & & \\ \text { Set/Block/Sub-Block } & 7 & 0.00369108 & 0.000527 & 11.8145 & <.0001\end{array}$

Error
C. Total

$\begin{array}{ll}16 \\ 23 & 0.00041410519\end{array}$

Means for Oneway Anova

Level Number Mean Std Error Lower 95\% Upper 95\%

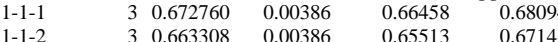

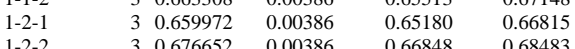

$\begin{array}{lllllll}1-2-2 & & 3 & 0.676652 & 0.00386 & 0.66848 & 0.68483 \\ 2-1-1 & & 3 & 0.657748 & 0.00386 & 0.64957 & 0.66592\end{array}$

$\begin{array}{lllllll}2-1-1 & & 3 & 0.657748 & 0.00386 & 0.64957 & 0.66592 \\ 2-1-2 & & 3 & 0.657748 & 0.00386 & 0.64957 & 0.66592\end{array}$

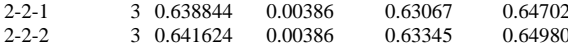

Std Error uses a pooled estimate of error variance
Oneway Analysis of U3O8 (wt\%) By Set/Block/Sub-Block Type=Batch 1

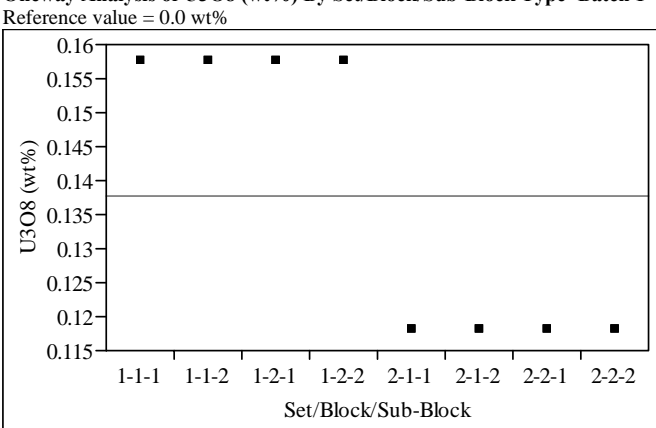

Oneway Anova

ary of Fit

Rsquare

Root Mean Square Erro

$\begin{array}{lr}\text { Mean of Response } & 0.137672 \\ \text { Observations (or Sum Wgts) } & 24\end{array}$

Analysis of Variance

Source De 1 Dlock/Sub-Block of Squares Mean Square F Ratio Prob $>$ F $\begin{array}{lrrrrr} & 0.0093630 & 0.00134 & -1.2 \mathrm{e}+16 & 0.0000 \\ \text { Set/Block/Sub-Block } & 7 & -1.735 \mathrm{e}-18 & -1.1 \mathrm{e}-19 & & \end{array}$

C. Total $-1.1 \mathrm{e}-19$

Means for Oneway Anova

Level Number Mean Std Error Lower 95\% Upper 95\%

$\begin{array}{rrr}1-1-1 & 3 & 0.157423 \\ 1-1-2 & 3 & 0.17423\end{array}$

$\begin{array}{lll}1-2-1 & 3 & 0.157423 \\ 1-2-2 & 3 & 0.15723\end{array}$

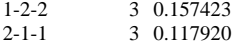

$\begin{array}{lll}2-1-2 & 3 & 0.117920 \\ 2-2-1 & 3 & 0.117920 \\ 2 & 3 & 0.11720\end{array}$

$2-2-2 \quad 3 \quad 0.11792$

Std Error uses a pooled estimate of error variance
Oneway Analysis of $\mathrm{ZnO}$ (wt\%) By Set/Block/Sub-Block Type=Batch 1 Reference vales

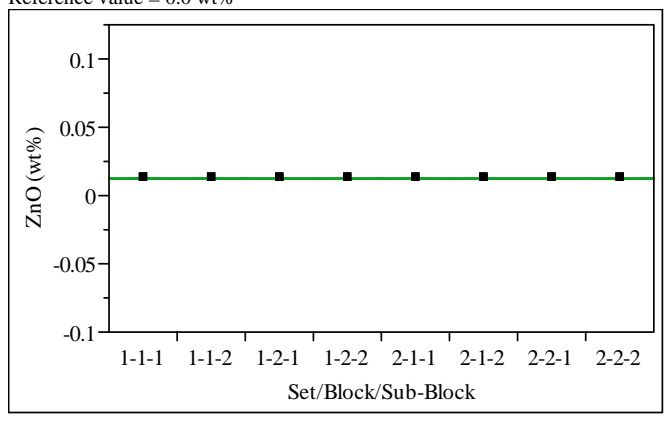

Oneway Anova

ary of Fit

Rsquare

$\begin{array}{lr}\text { Root Mean Square Error } & 0 \\ \text { Mean of Response } & 0.012448 \\ \text { Observations (or Sum Wgts) } & 24\end{array}$

Analysis of Variance

Source DF Sum of Squares Mean Square F Ratio Prob $>$ F

$\begin{array}{lrrr}\text { Set/Block/Sub-Block } & 7 & 0 & 0 \\ \text { Error } & 16 & 0 & 0 \\ \text { C. Total } & 23 & 0 & \end{array}$

Means for Oneway Anova

Level Number Mean Std Error Lower 95\% Upper 95\%

$\begin{array}{llllll} & 3 & 0.012448 & 0 & 0.01245 & 0.01245 \\ 1-1-1 & & 3 & 0.01248 \\ 1-1-2 & & 0 & 0.0245 & 0.0245\end{array}$

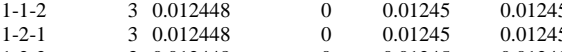

$\begin{array}{lll}1-2-2 & 3 & 0.012448\end{array}$

(1)

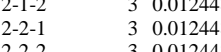

$\begin{array}{ll}0.01245 & 0.01245 \\ 0.01245 & 0.01245\end{array}$

$0.01245 \quad 0.0124$

Std Error uses a pooled estimate of error variance 
Exhibit A3. LM Measurements by Analytical Set, Block, and Sub-Block for Samples of the Batch 1 and Ustd Standards by Oxide

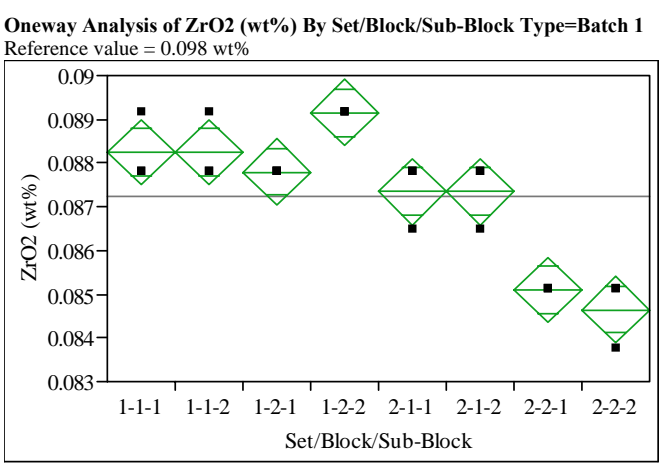

Oneway Anova
Summary of Fit

$\begin{array}{ll}\text { Rsquare } & 0.895288 \\ \text { Adj Rsquare } & 0.849476 \\ \text { Root Mean Square Error } & 0.000617 \\ \text { Mean of Response } & 0.087239\end{array}$

Root Mean Square Error $\quad 0.000617$

$\begin{array}{lr}\text { Mean of Response } & 0.087239 \\ \text { Observations (or Sum Wgts) } & 24\end{array}$

\begin{tabular}{lrrrrr}
$\begin{array}{l}\text { Analysis of Variance } \\
\text { Source }\end{array}$ & & & & \\
DFet/Block/Sub-Block & 7 & Sum of Squares & Mean Square & F Ratio & Prob $>$ F \\
Se.00005200 & $7.429 \mathrm{e}-6$ & 19.5429 & $<.0001$ \\
\hline & 16 & 0.00000608 & $3.8014 \mathrm{e}-7$ & &
\end{tabular}

$\begin{array}{lrrr}\text { Set/Block/Sub-Block } & 7 & 0.00005200 & 7.429 \mathrm{e}-6 \\ \text { Error } & 16 & 0.00000608 & 3.8014 \mathrm{e}-7 \\ \text { C. Total } & 23 & 0.00005809 & \end{array}$

Means for Oneway Anova

$\begin{array}{lrrrrr}\text { Level } & \text { Number } & \text { Mean } & \text { Std Error } & \text { Lower 95\% } & \text { Upper 95\% } \\ 1-1-1 & 3 & 0.088252 & 0.00036 & 0.08750 & 0.08901 \\ 1-1-2 & 3 & 0.088252 & 0.00036 & 0.08750 & 0.08901 \\ 1-2-1 & 3 & 0.087502 & 0.00036 & 0.08705 & 0.08856 \\ 1-2-2 & 3 & 0.089153 & 0.00036 & 0.08840 & 0.08991 \\ 2-1-1 & 3 & 0.087352 & 0.00036 & 0.08660 & 0.08811 \\ 2-1-2 & 3 & 0.087352 & 0.00036 & 0.08660 & 0.08811 \\ 2-2-1 & 3 & 0.085100 & 0.00036 & 0.08435 & 0.08586 \\ 2-2-2 & 3 & 0.084650 & 0.00036 & 0.08390 & 0.08540\end{array}$

Std Error uses a pooled estimate of error variance

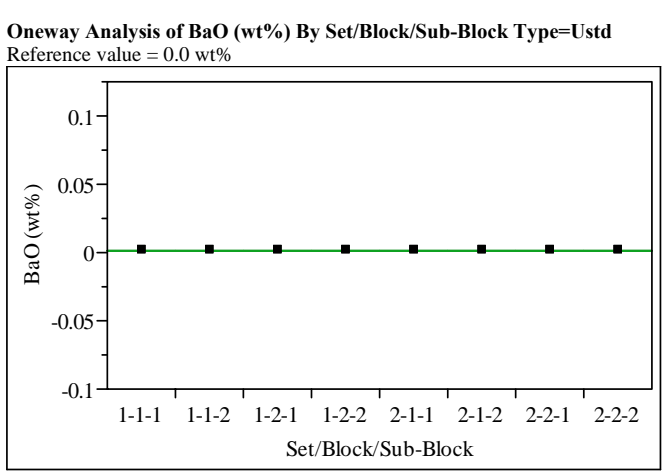

Oneway Anova

Summary of Fit

Rsquare
Adj Rsquare

Root Mean Square Erro

Mean of Response

Observations (or Sum Wgts) $\quad 24$

Analysis of Variance

Source DF Sum of Squares Mean Square F Ratio Prob $>$ F

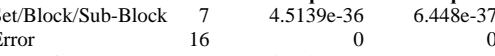

C. Total

$\begin{array}{rr}16 & 0 \\ 23 & 4.5139 \mathrm{e}-36\end{array}$

Means for Oneway Anova

Level Number Mean Std Error Lower 95\% Upper 95\%

$\begin{array}{llllll}1-1-1 & 3 & 0.001117 & 0 & 0.00112 & 0.00112 \\ 1-1-2 & 3 & 0.001117 & 0 & 0.00112 & 0.00112\end{array}$

$\begin{array}{lll}1-2-1 & 3 & 0.001117 \\ 1-2-2 & 3 & 0.00117\end{array}$

$\begin{array}{lll}1-2-2 & 3 & 0.00111 \\ 2-1-1 & 3 & 0.00111\end{array}$

$\begin{array}{lll}2-1-2 & 3 & 0.001117 \\ 2-2-1 & 3 & 0.001117\end{array}$

$\begin{array}{ll}3 & 0.001117 \\ 3 & 0.001117\end{array}$

$\begin{array}{ll}0.00112 & 0.00112 \\ 0.00112 & 0.00112 \\ 0.00112 & 0.00112 \\ 0.00112 & 0.00112 \\ 0.00112 & 0.00112 \\ 0.00112 & 0.00112\end{array}$

Std Error uses a pooled estimate of error variance
Oneway Analysis of $\mathrm{CaO}(\mathrm{wt} \%) \mathrm{By}$ Set/Block/Sub-Block Type=Ustd

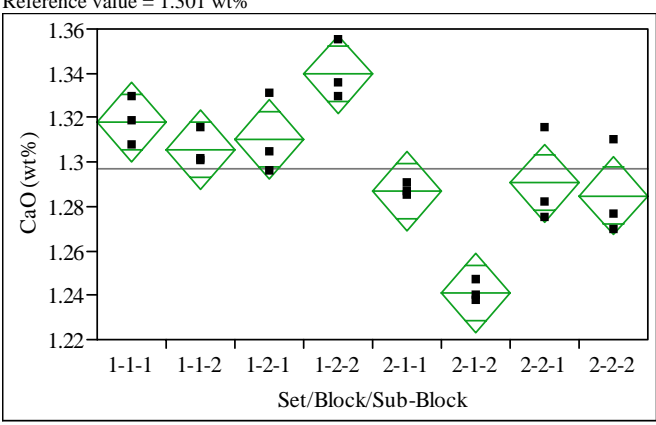

Oneway Anova

Summary of Fit

$\begin{array}{lr}\text { Rsquare } & 0.840604 \\ \text { Adj Rsquare } & 0.770868 \\ \text { Root Mean Square Error } & 0.014496 \\ \text { Mean of Response } & 1.297058 \\ \text { Observations (or Sum Wgts) } & 24\end{array}$

Analysis of Variance
Source DF Sum of Squares Mean Square F Ratio Prob $>$ F

$\begin{array}{lrrrrr}\text { Source } & \text { DF } & \text { Sum of Squares } & \text { Mean Square } & \text { F Ratio } & \text { Prob }>\text { F } \\ \text { Set/Block/Sub-Block } & 7 & 0.01773079 & 0.002533 & 12.0541 & <.0001\end{array}$ $\begin{array}{lrrr}\text { Set/Block/Sub-Block } & 7 & 0.01773079 & 0.002533 \\ \text { Error } & 16 & 0.00336213 & 0.000210 \\ \text { C. Total } & 23 & 0.02109291 & \end{array}$

Means for Oneway Anova

Level Number Mean Std Error Lower 95\% Upper 95\% $\begin{array}{lllllll}1-1-1 & & 3 & 1.31805 & 0.00837 & 1.3003 & 1.3358 \\ 1-1-2 & & 3 & 1.30545 & 0.00837 & 1.2877 & 1.3232\end{array}$

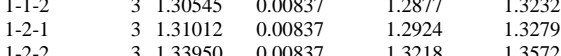

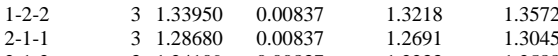

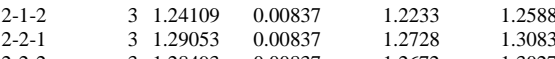

$\begin{array}{llllll}2-2-2 & 3 & 1.28493 & 0.00837 & 1.2672 & 1.3027\end{array}$ 
SRNL-STI-2009-00465, Revision 0

Exhibit A3. LM Measurements by Analytical Set, Block, and Sub-Block for Samples of the Batch 1 and Ustd Standards by Oxide

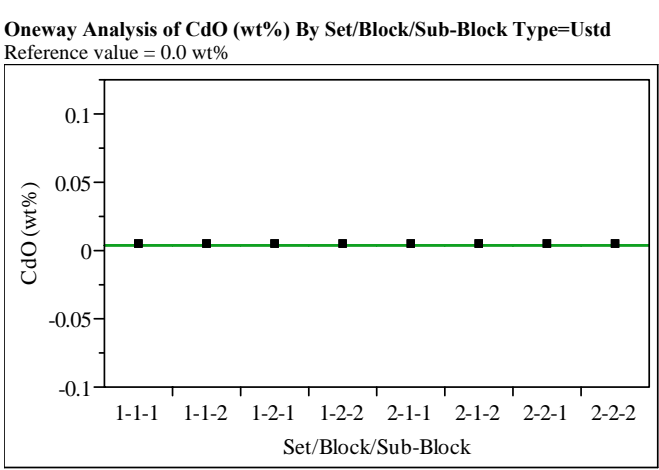

Oneway Anova
Summary of Fit

Rsquare

Root Mean Square Error

$\begin{array}{lr}\text { Mean of Response } & 0.003998 \\ \text { Observations (or Sum Wgts) } & 24\end{array}$

Analysis of Variance

Source
Set/Block/Sub-Block

Error 16

C. Total

23

Means for Oneway Anova

Level Number Mean Std Error Lower 95\% Upper 95\%

$\begin{array}{llllll}1-1-2 & 3 & 0.003998 & 0 & 0.00400 & 0.00400 \\ 1-12 & & 0.00398 & 0 & 0.00400 & 0.00400\end{array}$

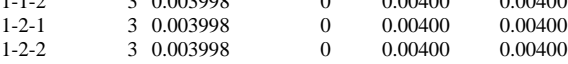

3 0.003998

$\begin{array}{lll}2-2-1 & 3 & 0.003998 \\ 2-2-2 & 3 & 0.003998\end{array}$

0.0400

$0.00400 \quad 0.00400$

Std Error uses a pooled estimate of error variance

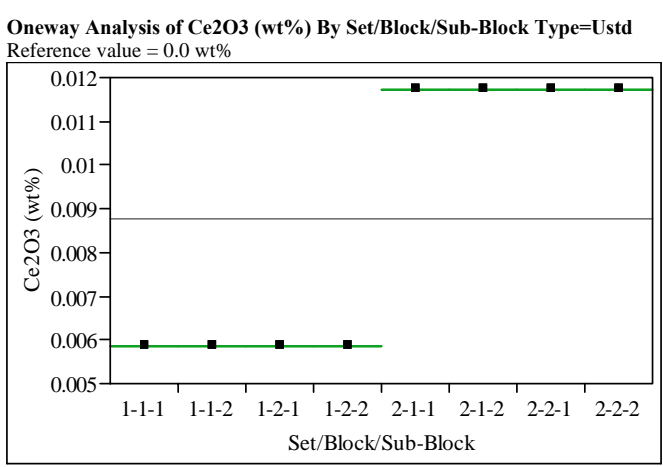

\section{Oneway Anova}

ummary of Fit

Rsquare

Root Mean Square Erro

$\begin{array}{lr}\text { Mean of Response } & 0.008785 \\ \text { Observations (or Sum Wgts) } & 24\end{array}$

Analysis of Variance

Source $\quad$ DF Sum of Squares Mean Square F Ratio Prob $>$ F $\begin{array}{lrrr} & 0.00020579 & 0.000029 \\ \text { Error } & 7 & & \\ \text { Erock/Sub-Block } & 76 & 0.0000000 & 0.000000\end{array}$

C. Total

$\begin{array}{ll}16 & 0.00000000 \\ 23 & 0.00020579\end{array}$

Means for Oneway Anova

$\begin{array}{lrrrrr}\text { Level Number } & \text { Mean } & \text { Std Error } & \text { Lower 95\% } & \text { Upper 95\% } \\ 1-1-1 & 3 & 0.005857 & 0 & 0.00586 & 0.00586 \\ 1-1-2 & 3 & 0.005857 & 0 & 0.00586 & 0.00586 \\ 1-2-1 & 3 & 0.005857 & 0 & 0.00586 & 0.00586 \\ 1-2-2 & 3 & 0.005857 & 0 & 0.00586 & 0.00586 \\ 2-1-1 & 3 & 0.011713 & 0 & 0.01171 & 0.01171 \\ 2-1-2 & 3 & 0.011713 & 0 & 0.01171 & 0.01171 \\ 2-2-1 & 3 & 0.011713 & 0 & 0.01171 & 0.01171 \\ 2-2-2 & 3 & 0.011713 & 0 & 0.01171 & 0.01171\end{array}$

Std Error uses a pooled estimate of error variance
Oneway Analysis of Cr2O3 (wt\%) By Set/Block/Sub-Block Type=Ustd

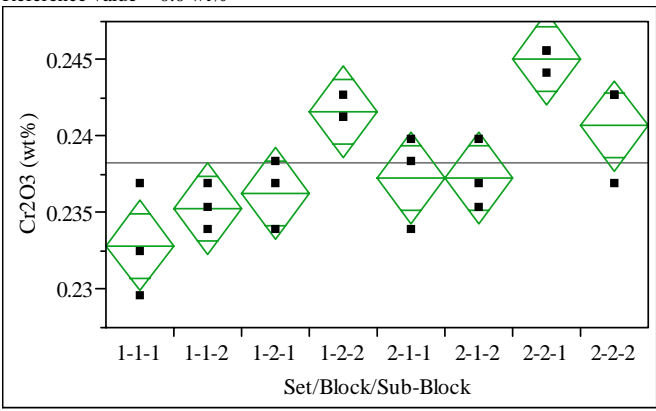

Oneway Anova

ummary of Fit

$\begin{array}{lr}\text { Rsquare } & 0.770891 \\ \text { Adj Rsquare } & 0.670656 \\ \text { Root Mean Square Error } & 0.002442 \\ \text { Mean of Response } & 0.238302 \\ \text { Observations (or Sum Wgts) } & 24\end{array}$

Analysis of Variance

Source DF Sum of Squares Mean Square F Ratio Prob $>$ F

Set/Block/Sub-Block $7 \quad 0.0003210$

$\begin{array}{lll}\text { Error } & 16 & 0.00009542 \\ \text { C. Total } & 23 & 0.00041648\end{array}$

$5.964 \mathrm{e}-6$

Means for Oneway Anova

Level Number Mean Std Error Lower 95\% Upper 95\%

$\begin{array}{lllll}3 & 0.232882 & 0.00141 & 0.22989 & 0.2358\end{array}$

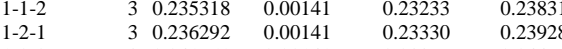

$\begin{array}{llllll}1-2-2 & 3 & 0.241651 & 0.00141 & 0.23866 & 0.24464\end{array}$

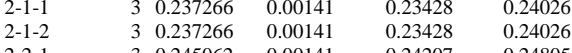

$\begin{array}{lllllll}2-2-2 & 3 & 0.240677 & 0.00141 & 0.24207 & 0.2480 \\ 2 & & 0.23769 & 0.24367\end{array}$

Std Error uses a pooled estimate of error variance 
SRNL-STI-2009-00465, Revision 0

Exhibit A3. LM Measurements by Analytical Set, Block, and Sub-Block for Samples of the Batch 1 and Ustd Standards by Oxide

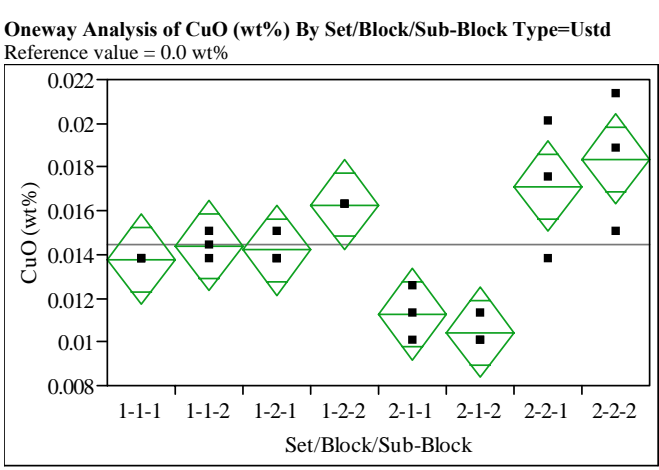

Oneway Anova
Summary of Fit

$\begin{array}{lr}\text { Rsquare } & 0.775046 \\ \text { Adj Rsquare } & 0.676629 \\ \text { Root Mean Square Error } & 0.00169 \\ \text { Mean of Response } & 0.014474 \\ \text { Observations (or Sum Wgts) } & 24\end{array}$

Analysis of Variance
Source

$\begin{array}{llllll}\text { Set/Block/Sub-Block } & 7 & 0.00015747 & 0.000022 & 7.8751 & 0.0003\end{array}$

$\begin{array}{lll}\text { Error } & 16 & 0.00004570 \\ \text { C. Total } & 23 & 0.00020317\end{array}$

Means for Oneway Anova

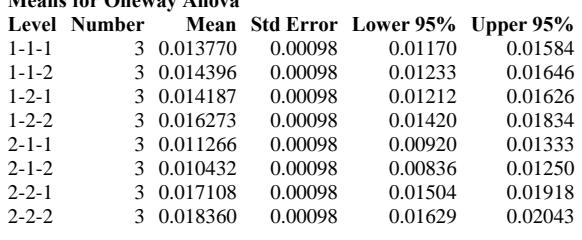

Std Error uses a pooled estimate of error variance
Oneway Analysis of K2O (wt\%) By Set/Block/Sub-Block Type=Ustd

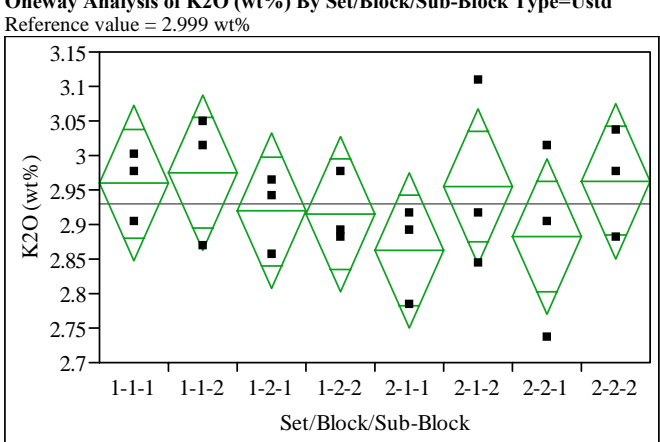

Oneway Anova

Summary of Fit

Rsquare

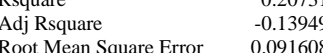
Mean of Response $\quad 2.929186$ Observations (or Sum Wgts) $\quad 24$

Analysis of Variance

Source DF Sum of Squares Mean Square F Ratio Prob $>$ F $\begin{array}{llllll}\text { Set/Block/Sub-Block } & 7 & 0.03511568 & 0.005017 & 0.5978 & 0.7490\end{array}$ C. Total

$\begin{array}{ll}16 & 0.13427153 \\ 23 & 0.16938721\end{array}$ 0.008392

Means for Oneway Anova

Level Number Mean Std Error Lower 95\% Upper 95\%

$\begin{array}{lll}1-1-1 & 3 & 2.95930 \\ 1-1-2 & 3 & 2.97536\end{array}$

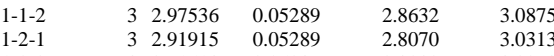

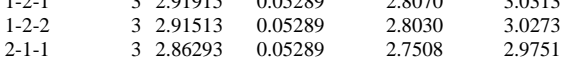

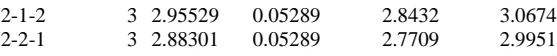

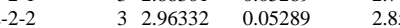

Std Error uses a pooled estimate of error variance
Oneway Analysis of La2O3 (wt\%) By Set/Block/Sub-Block Type=Ustd Reference v

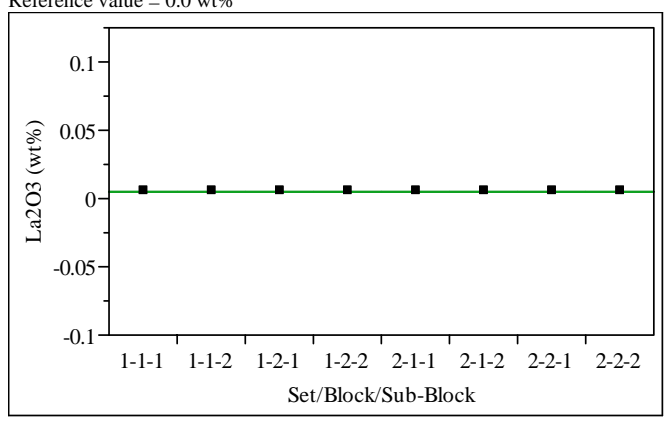

Oneway Anova

Summary of Fit

Rsquare
Adj Rsquare

$\begin{array}{lr}\text { Root Mean Square Error } & 0 \\ \text { Mean of Response } & 0.005278\end{array}$

Observations (or Sum Wgts)

Analysis of Variance

Source DF Sum of Squares Mean Square F Ratio Prob $>$ F

$\begin{array}{lrll} & & 0 & 0 \\ \text { Error } & 16 & 0 & 0 \\ \text { C. Total } & 23 & 0 & \end{array}$

Means for Oneway Anova

Level Number Mean Std Error Lower 95\% Upper 95\%

$\begin{array}{llllll}1-1-1 & 3 & 0.005278 & 0 & 0.00528 & 0.00528 \\ 1-1-2 & 3 & 0.00578 & 0 & 0.0528 & 0.0528\end{array}$

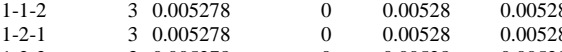

$\begin{array}{lllllll}1-2-2 & 3 & 0.005278 & 0 & 0.00528 & 0.00528\end{array}$

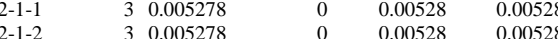

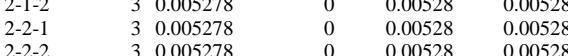

Std Error uses a pooled estimate of error variance 
SRNL-STI-2009-00465, Revision 0

Exhibit A3. LM Measurements by Analytical Set, Block, and Sub-Block for Samples of the Batch 1 and Ustd Standards by Oxide

Oneway Analysis of MgO (wt\%) By Set/Block/Sub-Block Type=Ustd

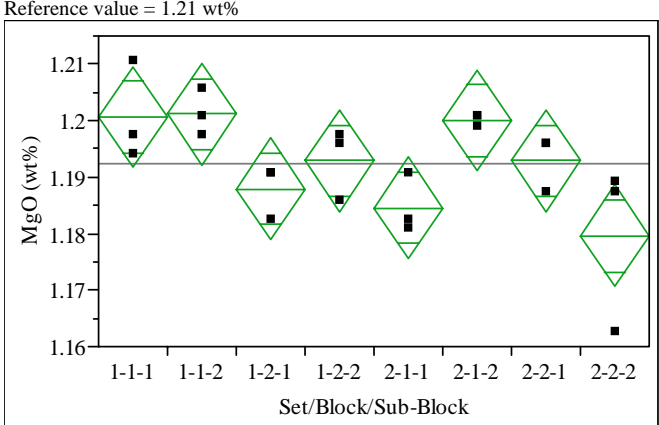

Oneway Anova
Summary of Fit

$\begin{array}{lr}\text { Rsquare } & 0.61006 \\ \text { Adj Rsquare } & 0.439462 \\ \text { Root Mean Square Error } & 0.007331 \\ \text { Mean of Response } & 1.192456 \\ \text { Observations (or Sum Wgts) } & 24\end{array}$

Observations (or Sum Wgs)

Analysis of Variance
Source

$\begin{array}{lrrrrr}\text { Source } & \text { DF } & \text { Sum of Squares } & \text { Mean Square } & \text { F Ratio } & \text { Prob }>\text { F } \\ \text { Set/Block/Sub-Block } & 7 & 0.00134519 & 0.000192 & 3.5760 & 0.0165\end{array}$

$\begin{array}{lll}\text { Error } & 16 & 0.00085982 \\ \text { C. Total } & 23 & 0.00220501\end{array}$

Means for Oneway Anova

\begin{tabular}{lrrrrr}
\multicolumn{7}{c}{$\begin{array}{l}\text { Means for Oneway Anova } \\
\text { Level Number }\end{array}$} & \multicolumn{1}{c}{ Mean } & Std Error & Lower 95\% & Upper 95\% \\
$1-1-1$ & 3 & 1.20061 & 0.00423 & 1.1916 & 1.2096 \\
$1-1-2$ & 3 & 1.20116 & 0.00423 & 1.1922 & 1.2101 \\
$1-2-1$ & 3 & 1.18790 & 0.00423 & 1.1789 & 1.1969 \\
$1-2-2$ & 3 & 1.19287 & 0.00423 & 1.1839 & 1.2018 \\
$2-1-1$ & 3 & 1.18458 & 0.00423 & 1.1756 & 1.1936 \\
$2-1-2$ & 3 & 1.20006 & 0.00423 & 1.1911 & 1.2090 \\
$2-2-1$ & 3 & 1.19287 & 0.0423 & 1.1839 & 1.2018 \\
$2-2-2$ & 3 & 1.17960 & 0.00423 & 1.1706 & 1.1886
\end{tabular}

Std Error uses a pooled estimate of error variance

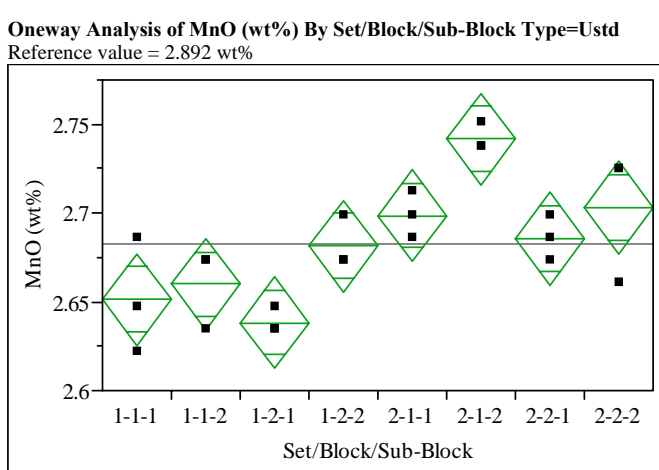

Oneway Anova

Summary of Fit

Rsquare

$\begin{array}{ll}\text { Adj Rsquare } & 0.654894 \\ \text { Root Mean Square Error } \quad 0.021249\end{array}$ Mean of Response $\quad 2.682468$ Observations (or Sum Wgts) 24

Analysis of Variance

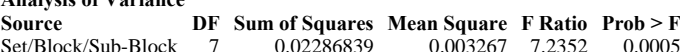
$\begin{array}{lrrrrr} & & & \\ \text { Set/Block/Sub-Block } & 7 & 0.02286839 & 0.003267 & 7.2352 & 0.0005 \\ \text { Error } & 16 & 0.00722452 & 0.000452 & & \end{array}$

C. Total

0.00722452
0.03009291

Means for Oneway Anova

Level Number Mean Std Error Lower 95\% Upper 95\%

$\begin{array}{lllllll}1-1-1 & & 3 & 2.65126 & 0.01227 & 2.6253 & 2.6773 \\ 1-1-2 & & 3 & 2.65987 & 0.01227 & 2.6339 & 2.6859 \\ 1-2-1 & & & 3 & 2.6385 & 0.0227 & \end{array}$

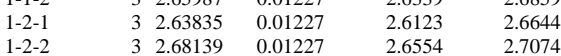

$\begin{array}{llll}2-1-1 & 3 & 2.69861 & 0 \\ 2 & 3 & 2.7465 & 0\end{array}$

$2.7156 \quad 2.7677$

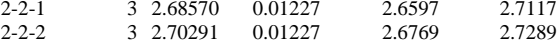

Std Error uses a pooled estimate of error variance
Oneway Analysis of $\mathrm{Na2O}$ (wt\%) By Set/Block/Sub-Block Type=Ustd Reference value $=11.79$

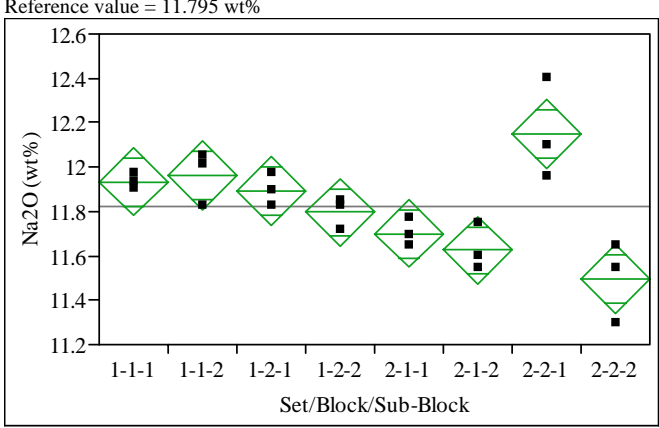

Oneway Anova

Summary of Fit

$\begin{array}{lr}\text { Rsquare } & 0.785425 \\ \text { Adj Rsquare } & 0.691548 \\ \text { Root Mean Square Error } & 0.125371 \\ \text { Mean of Response } & 11.81915 \\ \text { Observations (or Sum Wgts) } & 24\end{array}$

Analysis of Variance
Source DF Sum of Squares Mean Square F Ratio Prob $>$ F

$\begin{array}{llrrrr}\text { Source } & \text { DF } & \text { Sum of Squares } & \text { Mean Square } & \text { F Ratio } & \text { Prob }>\text { F } \\ \text { Set/Block/Sub-Block } & 7 & 0.9205373 & 0.131505 & 8.3666 & 0.0002\end{array}$ $\begin{array}{lrrr}\text { Error } & 16 & 0.92513472 & 0.015718 \\ \text { C. Total } & 23 & 1.1720245 & \end{array}$

Means for Oneway Anova

Level Number Mean Std Error Lower 95\% Upper 95\%

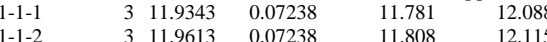

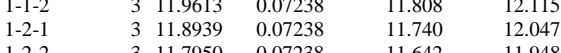

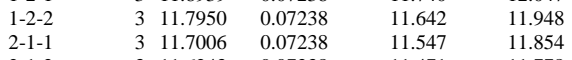

$\begin{array}{llllll}2-1-2 & 3 & 11.6243 & 0.07238 & 11.471 & 11.778 \\ 2-2-1 & 3 & 1.1500 & 0.07238 & 11.997 & 1238\end{array}$

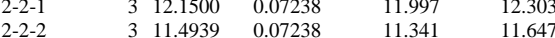

Std Error uses a pooled estimate of error variance 
SRNL-STI-2009-00465, Revision 0

Exhibit A3. LM Measurements by Analytical Set, Block, and Sub-Block for Samples of the Batch 1 and Ustd Standards by Oxide

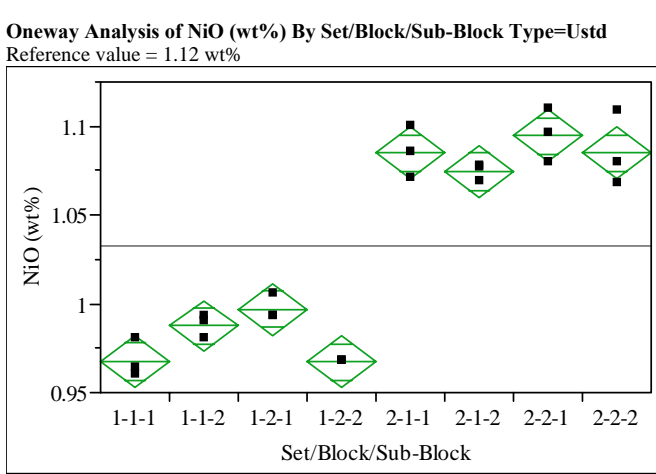

Oneway Anova
Summary of Fit

$\begin{array}{lr}\text { Rsquare } & 0.96826 \\ \text { Adj Rsquare } & 0.954374 \\ \text { Root Mean Square Error } & 0.011878 \\ \text { Mean of Response } & 1.032263 \\ \text { Observations (or Sum Wgts) } & 24\end{array}$

Analysis of Variance
Source

$\begin{array}{lrrrrr}\text { Source } & \text { DF } & & & \\ \text { Set/Block/Sub-Block } & 7 & 0.06886042 & 0.009837 & 69.7290 & <.000\end{array}$

Error
C. Total

$23-0.07111767$

Means for Oneway Anova

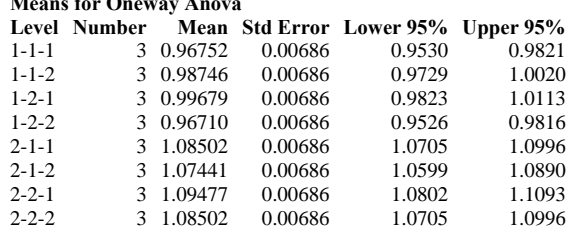

Std Error uses a pooled estimate of error variance

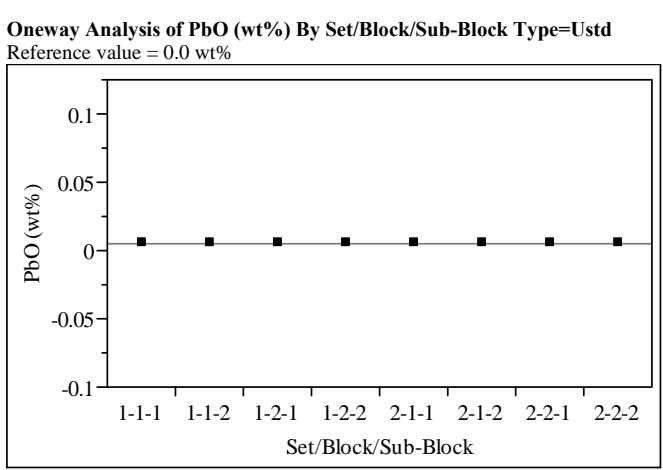

Oneway Anova

Summary of Fit

Rsquare
Adj Rsquare

Root Mean Square Error

$\begin{array}{lr}\text { Mean of Response } & 0.005386 \\ \text { Observations (or Sum Wgts) } & 24\end{array}$

Analysis of Variance

Source DF Sum of Squares Mean Square F Ratio Prob $>$ F

$\begin{array}{llllll}\text { Se/Block/Sub-Block } & 7 & 7.2222 \mathrm{e}-35 & 1.032 \mathrm{e}-35 & -3.0476 & 0.0000\end{array}$

C. Total

$\begin{array}{lr}16 & -5.417 \mathrm{e}-35 \\ 23 & 1.8056 \mathrm{e}-35\end{array}$

$-3.39 \mathrm{e}-36$

Means for Oneway Anova

Level Number Mean Std Error Lower 95\% Upper 95\%

$\begin{array}{lll}1-1-1 & 3 & 0.005386 \\ 1-1-2 & 3 & 0.005386\end{array}$

$\begin{array}{lll}1-2-1 & 3 & 0.005386\end{array}$

$\begin{array}{lll}1-2-2 & 3 & 0.005386 \\ 2-1-1 & 3 & 0.005386\end{array}$

$\begin{array}{lll}2-1-2 & 3 & 0.005386 \\ 2-2-1 & 3 & 0.005386\end{array}$

$2-2-2 \quad 3 \quad 0.00538$

Std Error uses a pooled estimate of error variance
Oneway Analysis of SO4 (wt\%) By Set/Block/Sub-Block Type=Ustd Reference value $=0.0 \mathrm{wt} \%$

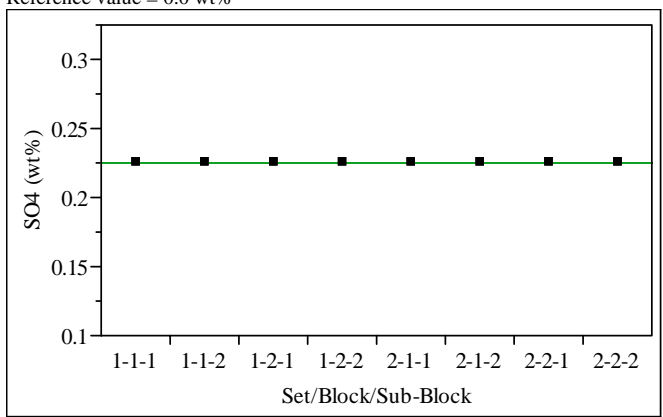

Oneway Anova

Summary of Fit

Rsquare
Adj Rsquare

Adj Rsquare

$\begin{array}{lr}\text { Mean of Response } & 0.224693 \\ \text { Observations (or Sum Wgts) } & 24\end{array}$

Analysis of Variance
Source DF Sum of Squares Mean Square F Ratio Prob $>F$

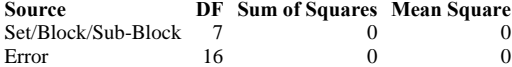

Error

23

Means for Oneway Anova

Level Number Mean Std Error Lower 95\% Upper 95\%

$\begin{array}{llllll}1-1-1 & 3 & 0.224693 & 0 & 0.22469 & 0.22469 \\ 1-1-2 & 3 & 0.224693 & 0 & 0.22469 & 0.2469\end{array}$

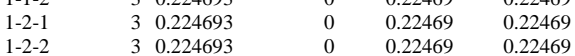

$\begin{array}{llllll}2-1-1 & 3 & 0.224693 & 0 & 0.22469 & 0.22469\end{array}$

$\begin{array}{lllllll}2-1-2 & 3 & 0.224693 & & 0 & 0.22469 & 0.22469 \\ 2-2-1 & 3 & 0.224693 & 0 & 0.22469 & 0.22469\end{array}$

Std Error uses a pooled estimate of error variance 
Exhibit A3. LM Measurements by Analytical Set, Block, and Sub-Block for Samples of the Batch 1 and Ustd Standards by Oxide

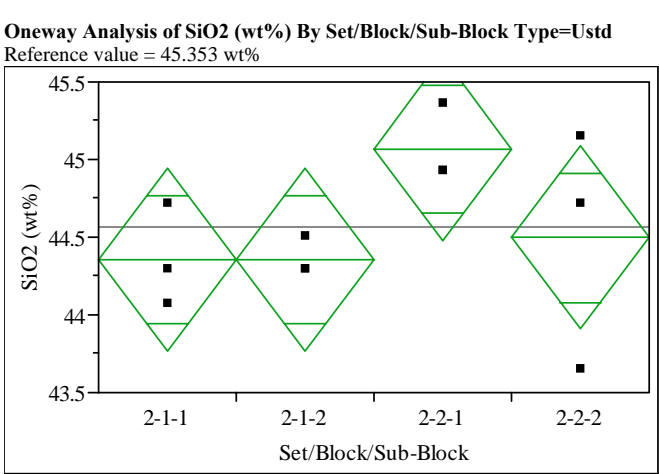

Missing Rows

12

Oneway Anova
Summary of Fit

$\begin{array}{lr}\text { Rsquare } & 0.4 \\ \text { Adj Rsquare } & 0.175 \\ \text { Root Mean Square Error } & 0.441028 \\ \text { Mean of Response } & 44.56875 \\ \text { Observations (or Sum Wgts) } & 12\end{array}$

Observations (or Sum W

$\begin{array}{lll}\text { Analysis of Variance } & & \\ \text { Source } & \text { DF Sum of Squares Mean Square F Ratio Prob }>\text { F }\end{array}$

$\begin{array}{lrrrrr}\text { Source } & \text { DF } & \text { Sum of Squares } & \text { Mean Square } & \text { F Ratio } & \text { Prob }>\text { F } \\ \text { Set/Block/Sub-Block } & 3 & 1.0373637 & 0.345788 & 1.7778 & 0.2290\end{array}$

$\begin{array}{lrr}\text { Error } & 8 & 1.5560455 \\ \text { C. Total } & 11 & 2.5934092\end{array}$

Means for Oneway Anova

Level Number Mean Std Error Lower 95\% Upper 95\% $1-1-1$

$\begin{array}{ll}1-1-2 & 0 \\ 1-2-1 & 0 \\ 1-2-2 & 0\end{array}$

$\begin{array}{llllll}2-1-1 & 3 & 44.3548 & 0.25463 & 43.768 & 44.942\end{array}$

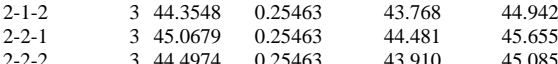

Std Error uses a pooled estimate of error variance

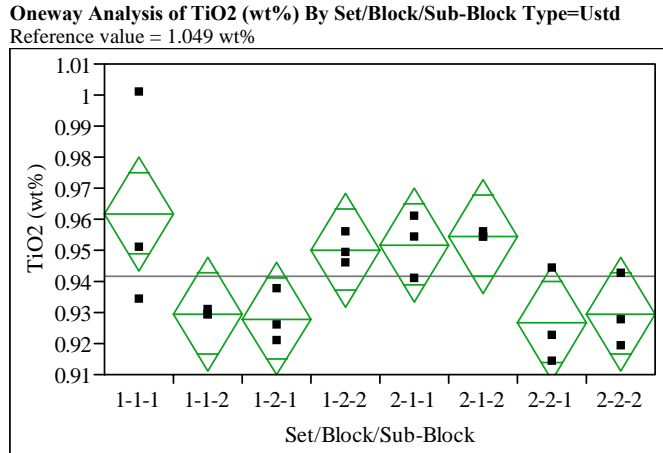

Oneway Anova

Summary of Fit

Rsquare

Root Mean Square Error $\quad 0.014962$ $\begin{array}{lr}\text { Mean of Response } & 0.941586 \\ \text { Observations (or Sum Wgts) } & 24\end{array}$

Analysis of Variance

Source DF Sum of Squares Mean Square F Ratio Prob $>$ F $\begin{array}{lrrrrr}\text { Set/Block/Sub-Block } & 7 & 0.00435325 & 0.000622 & 2.7781 & 0.0429\end{array}$ C. Total

$\begin{array}{ll}16 & 0.00435325 \\ 23 & 0.00358165 \\ & \end{array}$

Means for Oneway Anova

Level Number Mean Std Error Lower 95\% Upper 95\%

$\begin{array}{lllllll}1-1-1 & 3 & 0.961880 & 0.00864 & 0.94357 & 0.98019 \\ 1-1-2 & & 3 & 0.929632 & 0.00864 & 0.91132 & 0.94794\end{array}$

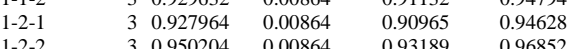

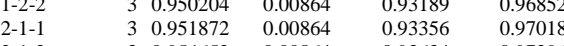

$\begin{array}{lllllll}2-1-2 & 3 & 0.954652 & 0.00864 & 0.93634 & 0.97296\end{array}$

$\begin{array}{lllllll}2-2-1 & & 3 & 0.926852 & 0.00864 & 0.90854 & 0.94516 \\ 2-2-2 & & 3 & 0.929632 & 0.00864 & 0.91132 & 0.94794\end{array}$

Std Error uses a pooled estimate of error variance
Oneway Analysis of U3O8 (wt\%) By Set/Block/Sub-Block Type=Ustd

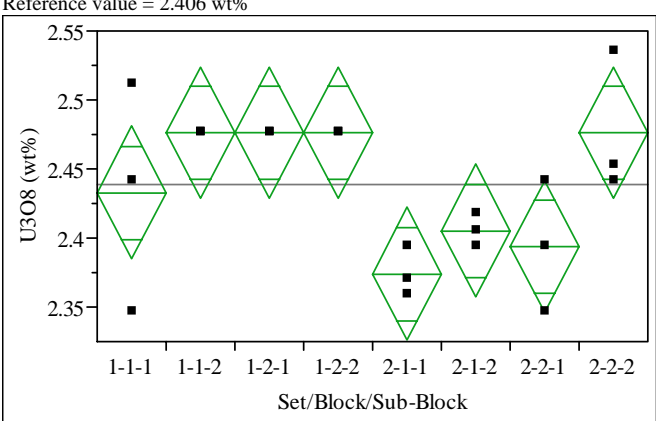

Oneway Anova

Summary of Fit

Rsquare

$\begin{array}{ll} & 0.446468 \\ \text { Root Mean Square Error } & 0.039035\end{array}$ $\begin{array}{lr}\text { Mean of Response } & 2.438979 \\ \text { Observations (or Sum Wgts) } & 24\end{array}$

Analysis of Variance
Source DF Sum of Squares Mean Square F Ratio Prob $>$ F $\begin{array}{lccccc}\text { Source } & \text { DF } & \text { Sum of Squares } & \text { Mean Square } & \text { F Ratio } & \text { Prob }>\text { F } \\ \text { Set/Block/Sub-Block } & 7 & 0.03893435 & 0.005562 & 3.6502 & 0.0152\end{array}$ $\begin{array}{lrrr}\text { Error } & 16 & 0.03893435 & 0.005562 \\ \text { C. Total } & 23 & 0.0633032 & 0.001524 \\ & & \end{array}$

Means for Oneway Anova

Level Number Mean Std Error Lower 95\% Upper 95\% $\begin{array}{lllllll}1-1-1 & & 3 & 2.43308 & 0.02254 & 2.3853 & 2.4809 \\ 1-1-2 & & 3 & 2.47632 & 0.02254 & 2.4285 & 2.5241 \\ 1-2-1 & & 3 & 2.4762 & 0.0254 & 2.425 & 2.5241\end{array}$

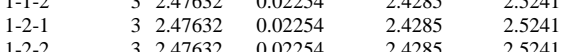

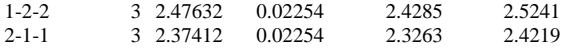
$\begin{array}{llllll}2-1-2 & 3 & 2.40557 & 0.02254 & 2.3578 & 2.4533\end{array}$

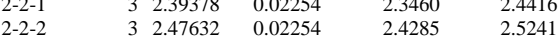

Std Error uses a pooled estimate of error variance 
Exhibit A3. LM Measurements by Analytical Set, Block, and Sub-Block for Samples of the Batch 1 and Ustd Standards by Oxide

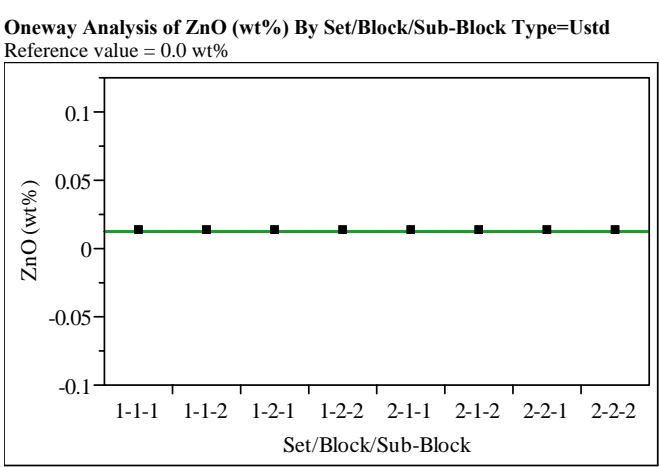

Oneway Anova
Summary of Fit

\section{Rsquare}

Root Mean Square Erro

Mean of Response

0.012448
Observations (or Sum Wgts)

Analysis of Variance

Source $\quad$ DF Sum of Squares Mean Square F Ratio Prob $>$ F

Error

C. Total

16
23

Means for Oneway Anova

Level Number Mean Std Error Lower 95\% Upper 95\%

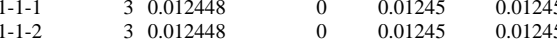

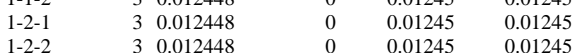

$1-2-2$

$2-1-2$

$\begin{array}{llll}3 & 0.012448 \\ 2-2-1 & 3 & 0.01248\end{array}$

$0.01245 \quad 0.01245$

$\begin{array}{ll}0.01245 & 0.01245 \\ 0.01245 & 0.01245 \\ 0.01245 & 0.01245\end{array}$

Std Error uses a pooled estimate of error variance

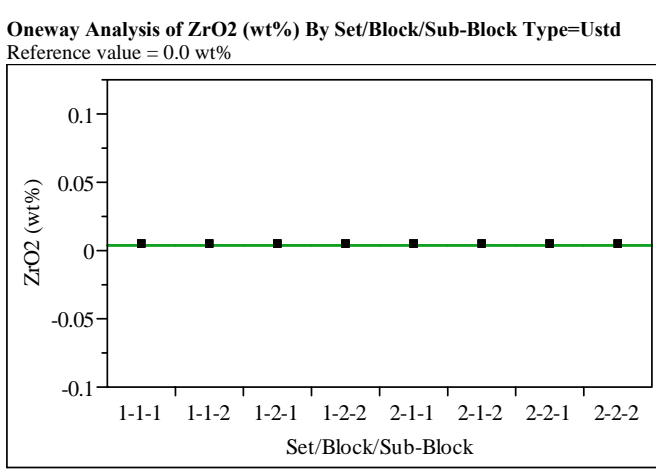

Oneway Anova

ary of $F$

Rsquare

Root Mean Square Error

$\begin{array}{lr}\text { Mean of Response } & 0.003377 \\ \text { Observations (or Sum Wgts) } & 24\end{array}$

Analysis of Variance

Source DF Sum of Squares Mean Square F Ratio Prob $>$ F

Set/Block/Sub-Block 7

C. Total

16
23

Means for Oneway Anova

Level Number Mean Std Error Lower 95\% Upper 95\%

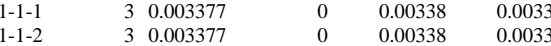

$\begin{array}{llllll}1-2-1 & 3 & 0.003377 & 0 & 0.00338 & 0.00338\end{array}$

$\begin{array}{lllllll}1-2-2 & 3 & 0.003377 & 0 & 0.00338 & 0.00338 \\ 2-1-1 & & 3 & 0.003377 & 0 & 0.00338 & 0.00338\end{array}$

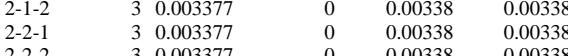

Std Error uses a pooled estimate of error variance 
SRNL-STI-2009-00465, Revision 0

Exhibit A4. PF Measurements by Analytical Set, Block, and Sub-Block for Samples of the Batch 1 and Ustd Standards by Oxide

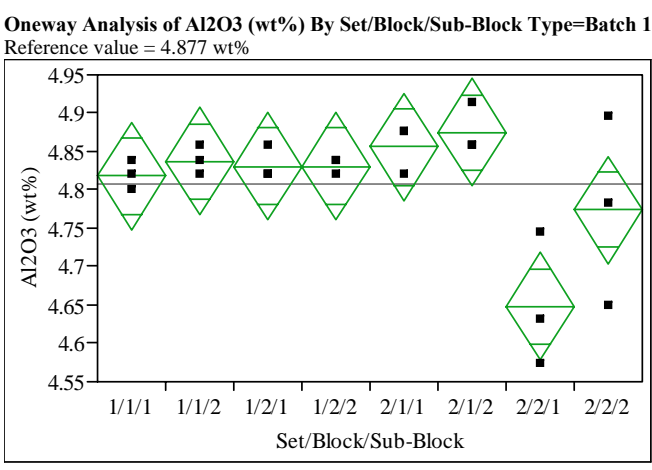

Oneway Anova

Rsquare

$\begin{array}{ll}\text { Rsquare } & 0.671171 \\ \text { Adj Rsquare } & 0.527309 \\ \text { Root Mean Square Error } & 0.057077\end{array}$

Mean of

Observations (or Sum Wgts)

Analysis of Variance

$\begin{array}{lrrrrr}\text { Source } & \text { DF } & \text { Sum of Squares } & \text { Mean Square } & \text { F Ratio } & \text { Prob }>\text { F } \\ \text { Set/Block/Sub-Block } & 7 & 0.10639227 & 0.015199 & 4.6654 & 0.0051 \\ \text { Error } & 16 & 0.05212507 & 0.003258 & & \end{array}$

Means for Oneway Anova

Level Number Mean Std Error Lower 95\% Upper 95\%

$\begin{array}{lrrrrr}\text { Level } & \text { Number } & \text { Mean } & \text { Std Error } & \text { Lower 95\% } & \text { Upper 95\% } \\ 1 / 1 / 1 & 3 & 4.81822 & 0.03295 & 4.7484 & 4.8881 \\ 11 / 1 / 2 & 3 & 4.83712 & 0.03295 & 4.7673 & 4.9070 \\ 1 / 2 / 1 & 3 & 4.83082 & 0.03295 & 4.7610 & 4.9007 \\ 1 / 2 / 2 & 3 & 4.83082 & 0.03225 & 4.7610 & 4.9007 \\ 2 / 1 / 1 & 3 & 4.85002 & 0.03295 & 4.7862 & 4.90259 \\ 2 / 1 / 2 & 3 & 4.87491 & 0.03295 & 4.8051 & 4.9448 \\ 2 / 2 / 1 & 3 & 4.64817 & 0.03295 & 4.5783 & 4.7180 \\ 2 / 2 / 2 & 3 & 4.77414 & 0.03295 & 4.7043 & 4.8440\end{array}$

Std Error uses a pooled estimate of error variance

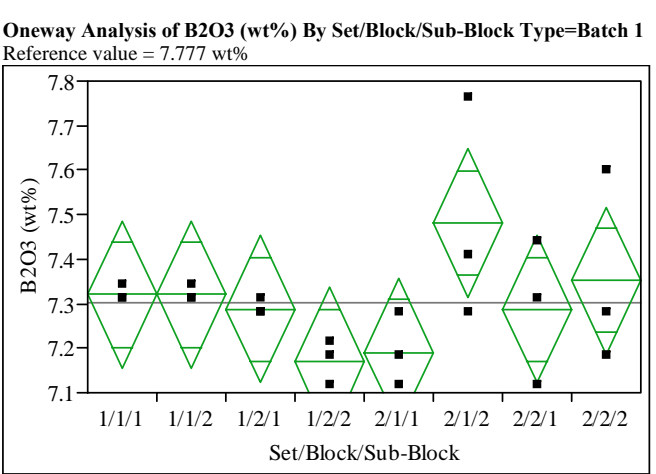

Oneway Anova
Summary of Fit

$\begin{array}{ll}\text { Rsquare } & 0.400353 \\ \text { Adj Rsquare } & 0.138007 \\ \text { Root Mean Square Frror } & 0.135497\end{array}$

$\begin{array}{ll}\text { Root Mean Square Error } & 0.1350497 \\ \text { Mean of Response } & 7.301123\end{array}$

$\begin{array}{lr}\text { Mean of Response } & 7.301123 \\ \text { Observations (or Sum Wgts) } & 24\end{array}$

Analysis of Variance

$\begin{array}{lllrrr}\begin{array}{l}\text { Analysis of Variance } \\ \text { Source }\end{array} & \text { DF } & \text { Sum of Squares } & \text { Mean Square } & \text { F Ratio } & \text { Prob }>\text { F } \\ \text { Set/Block/Sub-Block } & 7 & 0.19612338 & 0.028018 & 1.5261 & 0.2282\end{array}$

Error

$\begin{array}{ll}16 & 0.29375309 \\ 23 & 0.48987647\end{array}$

0.01836

Means for Oneway Anova

\begin{tabular}{|c|c|c|c|c|c|}
\hline Level & Number & Mean & Std Error & Lower 95\% & Upper $95 \%$ \\
\hline $1 / 1 / 1$ & & 7.31991 & 0.07823 & 7.1541 & 7.4857 \\
\hline 1/1/2 & 3 & 7.31991 & 0.07823 & 7.1541 & 7.4857 \\
\hline $1 / 2 / 1$ & 3 & 7.28771 & 0.07823 & 7.1219 & 7.4535 \\
\hline $1 / 2 / 2$ & 3 & 7.16964 & 0.07823 & 7.0038 & 7.3355 \\
\hline $2 / 1 / 1$ & 3 & 7.19111 & 0.07823 & 7.0253 & 7.3569 \\
\hline $2 / 1 / 2$ & 3 & 7.48090 & 0.07823 & 7.3151 & $\begin{array}{l}7.3509 \\
7.6467\end{array}$ \\
\hline 2/2/1 & 3 & $\begin{array}{l}7.480771 \\
7.2871\end{array}$ & 0.07823 & $\begin{array}{l}7.31519 \\
7.1219\end{array}$ & $\begin{array}{l}7.6467 \\
7.4535\end{array}$ \\
\hline $2 / 2 / 2$ & 3 & 7.35211 & 0.07823 & 7.1863 & 7.5179 \\
\hline
\end{tabular}

Std Error uses a pooled estimate of error variance
Oneway Analysis of Fe2O3 (wt\%) By Set/Block/Sub-Block Type=Batch

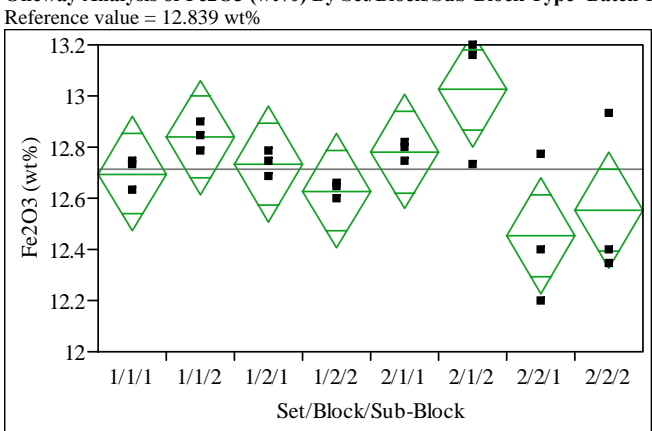

Oneway Anova

$\begin{array}{lr}\text { Rsquare } & 0.54981 \\ \text { Adj Rsquare } & 0.352852 \\ \text { Megrat } & 0.1232761\end{array}$

Root Mean Square Error $\quad 0.183277$

Mean of Response 12.71361

Analysis of Variance

Source DF Sum of Squares Mean Square F Ratio Prob $>$ F $\begin{array}{lrllll}\text { Set/Block/Sub-Block } & 7 & 0.6563760 & 0.093768 & 2.7915 & 0.0422\end{array}$ Error 0.033590

Means for Oneway Anova

Level Number Mean Std Error Lower 95\% Upper 95\%

$\begin{array}{llllll}1 / 1 / 1 & 3 & 12.6957 & 0.10581 & 12.471 & 12.920\end{array}$

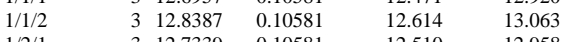

$1 / 2 / 2$

$\begin{array}{llllll}2 / 2 / 1 & 3 & 12.4527 & 0.10581 & 12.228 & 12.677 \\ 2 / 2 / 2 & 3 & 12.5528 & 0.10581 & 12.328 & 12.777\end{array}$

Std Error uses a pooled estimate of error variance 
SRNL-STI-2009-00465, Revision 0

Exhibit A4. PF Measurements by Analytical Set, Block, and Sub-Block for Samples of the Batch 1 and Ustd Standards by Oxide

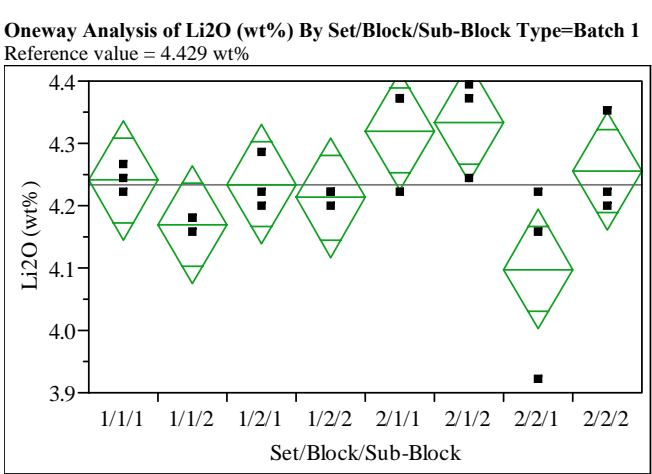

Oneway Anova
Summary of Fit

$\begin{array}{lr}\text { Rsquare } & 0.558929 \\ \text { Adj Rsquare } & 0.36596 \\ \text { Root Mean Square Error } & 0.07812 \\ \text { Mean of Response } & 4.23314 \\ \text { Observations (or Sum Wgts) } & 24\end{array}$

Analysis of Variance
Source

$\begin{array}{lrrrrr}\text { Set/Block/Sub-Block } & 7 & 0.12373461 & 0.017676 & 2.8965 & 0.0370\end{array}$

$\begin{array}{lll}\text { Error } & 16 & 0.09764355 \\ \text { C. Total } & 23 & 0.22137816\end{array}$

Means for Oneway Anova

Level Number Mean Std Error Lower 95\% Upper 95\%

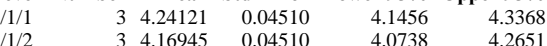

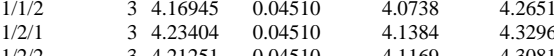

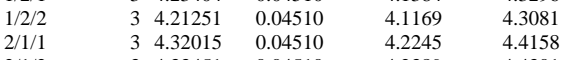

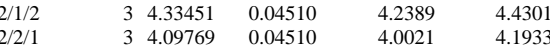

$\begin{array}{llllll}2 / 2 / 2 & 3 & 4.25557 & 0.04510 & 4.1600 & 4.3512\end{array}$

Std Error uses a pooled estimate of error variance

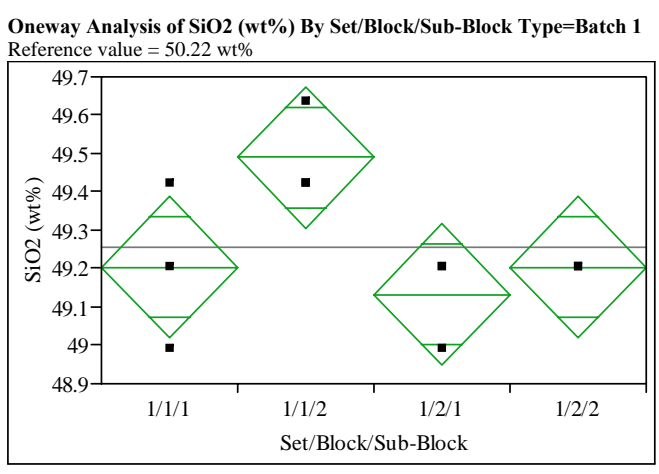

Missing Rows

Oneway Anova
Summary of Fit

$\begin{array}{lr}\text { Rsquare } & 0.59596 \\ \text { Adj Rsquare } & 0.444444\end{array}$

Root Mean Square Error $\quad 0.13809$

Observations (or Sum Wats) 49.25738

Analysis of Variance

DF Sum of Squares Mean Square F Ratio Prob $>$ F $\begin{array}{lrrrrr}\text { Set/Block/Sub-Block } & 3 & 0.22501639 & 0.075005 & 3.9333 & 0.0539\end{array}$ C. Total 0.37756987

Means for Oneway Anova

Level Number Mean Std Error Lower 95\% Upper 95\%

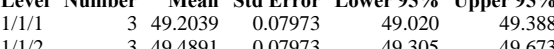

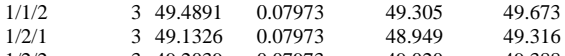

$1 / 2 / 2$

$2 / 1 / 2$

$2 / 2 / 1$

Std Error uses a pooled estimate of error variance
Oneway Analysis of Al2O3 (wt\%) By Set/Block/Sub-Block Type=Usto

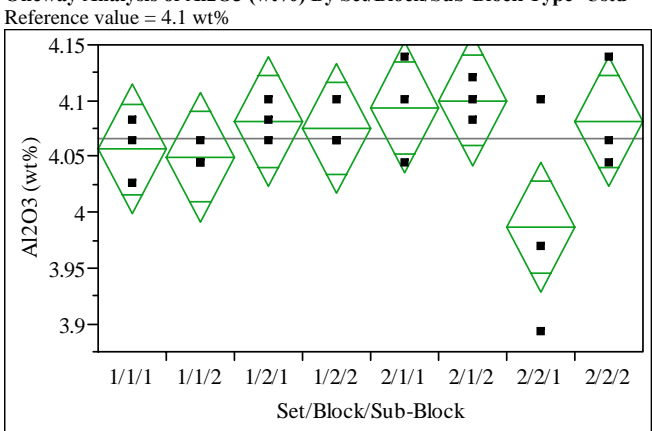

Oneway Anova

$\begin{array}{ll} & 0.432331 \\ \text { Adj Rsquare } & 0.183976\end{array}$

Root Mean Square Error $\quad 0.047395$

Mean of Response 4.065574

Analysis of Variance

Dource Sum of Squares Mean Square F Ratio Prob $>$ F $\begin{array}{llllll}\text { Set/Block/Sub-Block } & 7 & 0.02737161 & 0.003910 & 1.7408 & 0.1695\end{array}$ Error $\quad-16-0.03594012$ 0.002246

Means for Oneway Anova

Level Number Mean Std Error Lower 95\% Upper 95\%

$\begin{array}{llllll}1 / 1 / 1 & 3 & 4.05613 & 0.02736 & 3.9981 & 4.1141\end{array}$

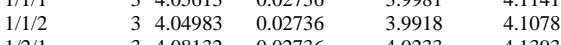

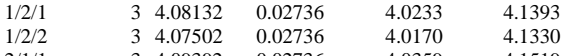

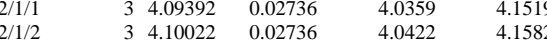

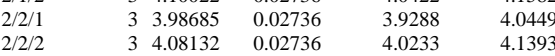

Std Error uses a pooled estimate of error variance 
SRNL-STI-2009-00465, Revision 0

Exhibit A4. PF Measurements by Analytical Set, Block, and Sub-Block for Samples of the Batch 1 and Ustd Standards by Oxide

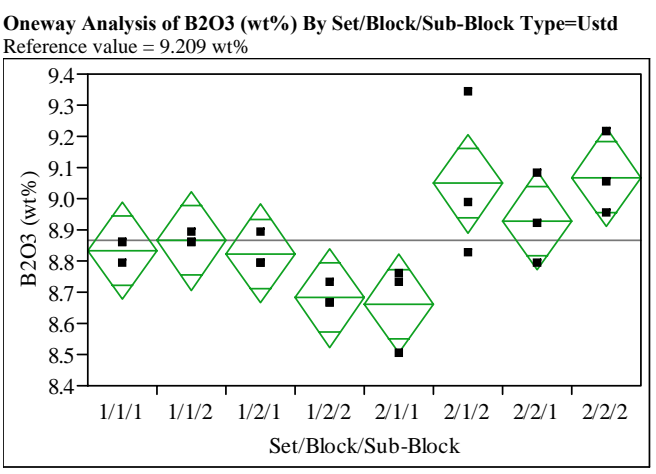

Oneway Anova

nary of Fit

$\begin{array}{lr}\text { Rsquare } & 0.638047 \\ \text { Adj Rsquare } & 0.479693 \\ \text { Root Mean Square Error } & 0.129131 \\ \text { Mean of Response } & 8.864116 \\ \text { Observations (or Sum Wgts) } & 24\end{array}$

Analysis of Variance

$\begin{array}{lrrrrr}\text { Source } & \text { DF } & \text { Sum of Squares } & & & \\ \text { Set/Block/Sub-Block Square } & 7 & 0.47030733 & 0.067187 & 4.0292 & 0.0100\end{array}$

$\begin{array}{lll}\text { Error } & 16 & 0.26679692 \\ \text { C. Total } & 23 & 0.73710425\end{array}$

Means for Oneway Anova

Level Number Mean Std Error Lower 95\% Upper 95\%

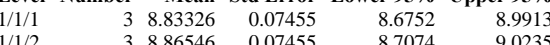

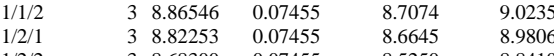

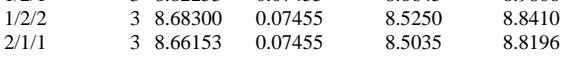

$\begin{array}{llllll}2 / 1 / 2 & 3 & 9.04792 & 0.07455 & 8.8899 & 9.2060 \\ 2 / 2 / 1 & 3 & 3.92986 & 0.07455 & 8.7718 & 9.0879\end{array}$

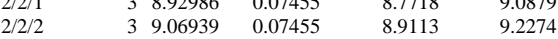

Std Error uses a pooled estimate of error variance

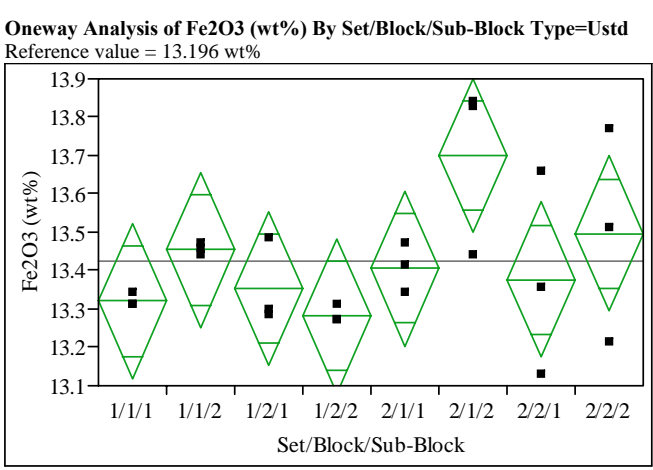

Oneway Anova

$\begin{array}{lr}\text { Rsquare } & 0.455839 \\ \text { Adj Rsquare } & 0.217769 \\ \text { Root Mean Square Error } & 0.164907 \\ \text { Mean of Response } & 13.42369 \\ \text { Observations (or Sum Wgts) } & 24\end{array}$

Analysis of Variance

Source $/$ Block/Sub Sum of Squares Mean Square F Ratio Prob $>$ $\begin{array}{lrrrrr}\text { Set/Block/Sub-Block } & 7 & 0.36448677 & 0.052070 & 1.9147 & 0.1335 \\ \text { Error } & 16 & 0.43510843 & 0.027194 & & \end{array}$ C. Tota 0.027194

$\begin{array}{lrrrrr}\text { Level } & \text { Number } & \text { Mean } & \text { Std Error } & \text { Lower 95\% } & \text { Upper 95\% } \\ 1 / 1 / 1 & 3 & 13.3200 & 0.09521 & 13.118 & 13.522 \\ 1 / 1 / 2 & 3 & 13.4535 & 0.09521 & 13.252 & 13.655 \\ 1 / 2 / 1 & 3 & 13.3534 & 0.09521 & 13.152 & 13.555 \\ 1 / 2 / 2 & 3 & 13.2819 & 0.09521 & 13.080 & 13.484 \\ 2 / 1 / 1 & 3 & 13.4058 & 0.09521 & 13.204 & 13.608 \\ 2 / 1 / 2 & 3 & 13.7013 & 0.09521 & 13.499 & 13.903 \\ 2 / 2 / 1 & 3 & 13.3772 & 0.09521 & 13.175 & 13.579 \\ 2 / 2 / 2 & 3 & 13.4964 & 0.09521 & 13.295 & 13.698\end{array}$

Std Error uses a pooled estimate of error variance
Oneway Analysis of Li2O (wt\%) By Set/Block/Sub-Block Type=Ustd

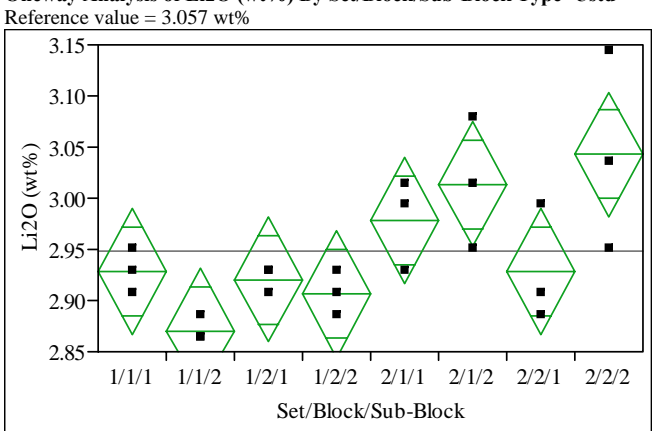

Oneway Anova

$\begin{array}{lr}0.637315 \\ \text { Adj Rsquare } & 0.47864\end{array}$

Root Mean Square Error $\quad 0.050106$

Mean of Response 2.948576

Analysis of Variance

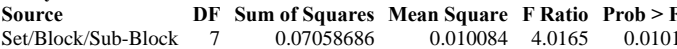
Error

$\begin{array}{lll}\text { C. Total } & 23 & 0.11075667\end{array}$ 0.00251

Means for Oneway Anova

Level Number Mean Std Error Lower 95\% Upper 95\%

$\begin{array}{llllll}1 / 1 / 1 & 3 & 2.92794 & 0.02893 & 2.8666 & 2.9893\end{array}$

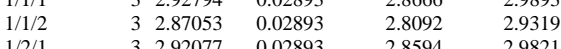

$1 / 2 / 2$
$2 / 1 / 1$

$\begin{array}{llllll}2 / 2 / 2 & 3 & 2.92794 & 0.02893 & 2.8666 & 2.9893 \\ 2 / 2 / 2 & 3 & 3.04277 & 0.02893 & 2.9814 & 3.1041\end{array}$

Std Error uses a pooled estimate of error variance 
Exhibit A4. PF Measurements by Analytical Set, Block, and Sub-Block for Samples of the Batch 1 and Ustd Standards by Oxide

Oneway Analysis of SiO2 (wt\%) By Set/Block/Sub-Block Type=Ustd

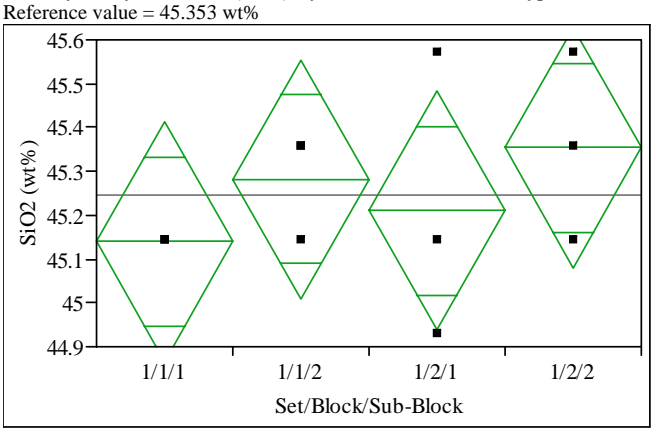

Missing Rows

Oneway Anova
Summary of Fit

$\begin{array}{lr}\text { Rsquare } & 0.185185 \\ \text { Adj Rsquare } & -0.12037 \\ \text { Root Mean Square Error } & 0.204822 \\ \text { Mean of Response } & 45.2462\end{array}$

Analysis of Variance

Source DF Sum of Squares Mean Square F Ratio Prob $>\mathbf{F}$

$\begin{array}{llllll}\text { Set/Block/Sub-Block } & 3 & 0.07627674 & 0.025426 & 0.6061 & 0.6294 \\ \text { Error } & 8 & 0.3561766 & 0.041952 & & \end{array}$

$\begin{array}{lrr}\text { Error } & 8 & 0.33561766 \\ \text { C. Total } & 11 & 0.41189440\end{array}$

Means for Oneway Anova

Level Number Mean Std Error Lower 95\% Upper 95\%

$\begin{array}{llrrrr}1 / 1 / 1 & 3 & 45.1392 & 0.11825 & 44.867 & 45.412 \\ 1 / 1 / 2 & 3 & 45.2819 & 0.11825 & 45.009 & 45.555\end{array}$

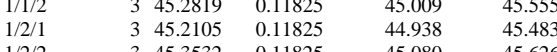

$2 / 1 / 2$

$\begin{array}{ll}2 / 2 / 1 & 0 \\ 2 / 2 / 2 & 0\end{array}$

Std Error uses a pooled estimate of error variance 
SRNL-STI-2009-00465, Revision 0

Exhibit A5. Oxide Measurements by Lab ID within Glass ID Sorted by Targeted Concentrations

Set $=1$, Analyte $=\mathrm{Al2O} 3(\mathrm{wt} \%)$ Variability Chart for Measured

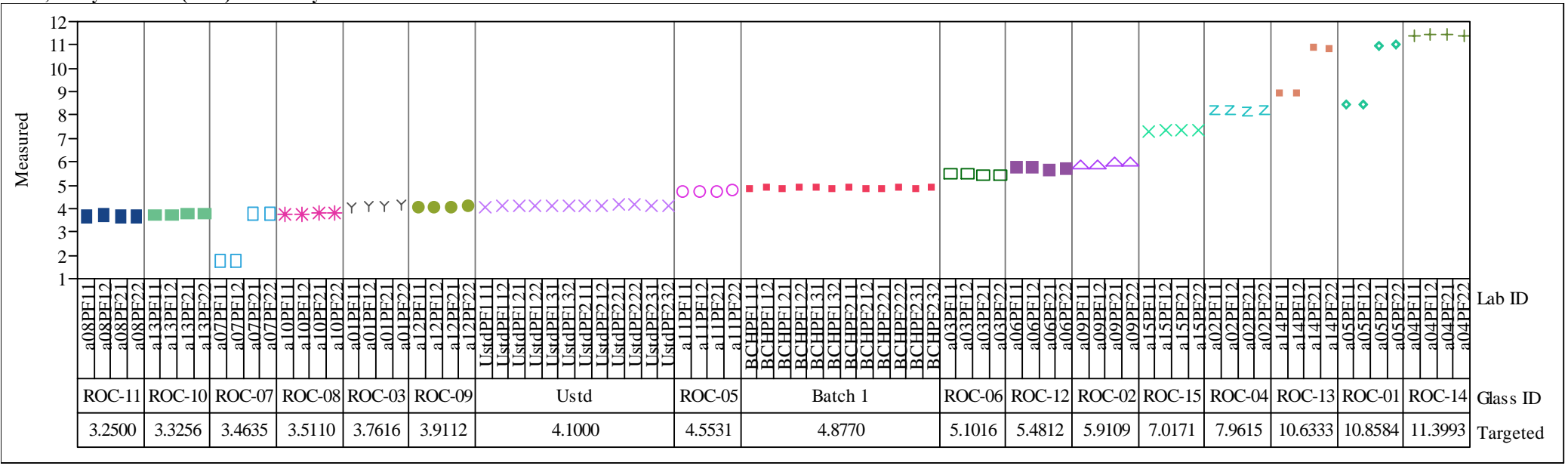

Set=1, Analyte=Al2O3 (wt\%) Variability Chart for Measured bc

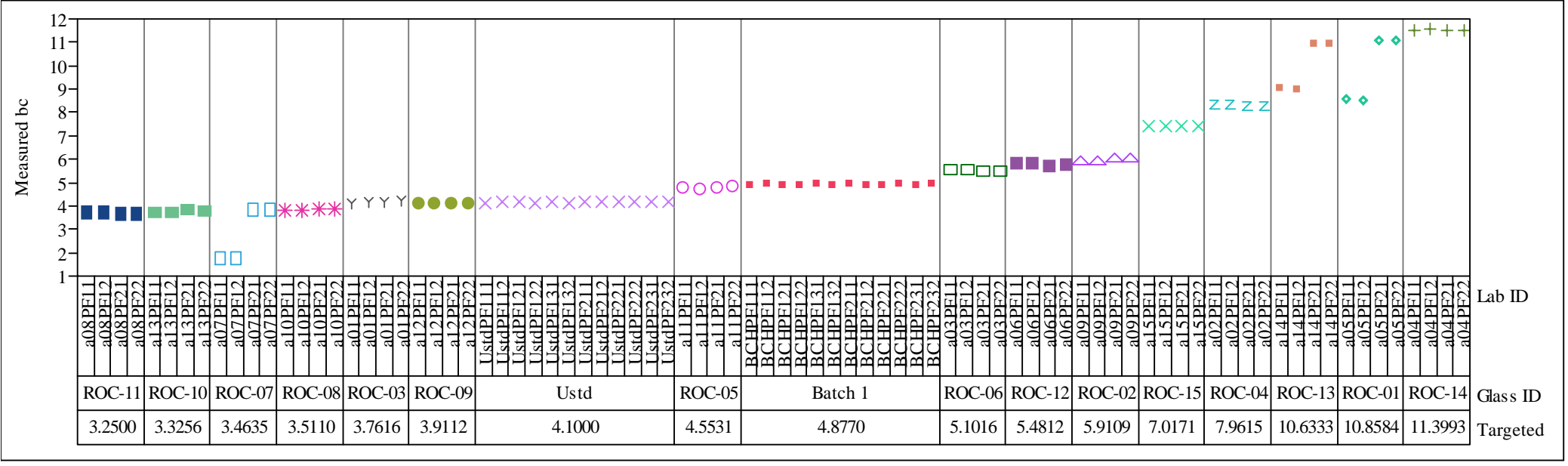


SRNL-STI-2009-00465, Revision 0

Exhibit A5. Oxide Measurements by Lab ID within Glass ID Sorted by Targeted Concentrations

Set=1, Analyte $=\mathbf{B 2 O} 3($ wt $\%)$ Variability Chart for Measured

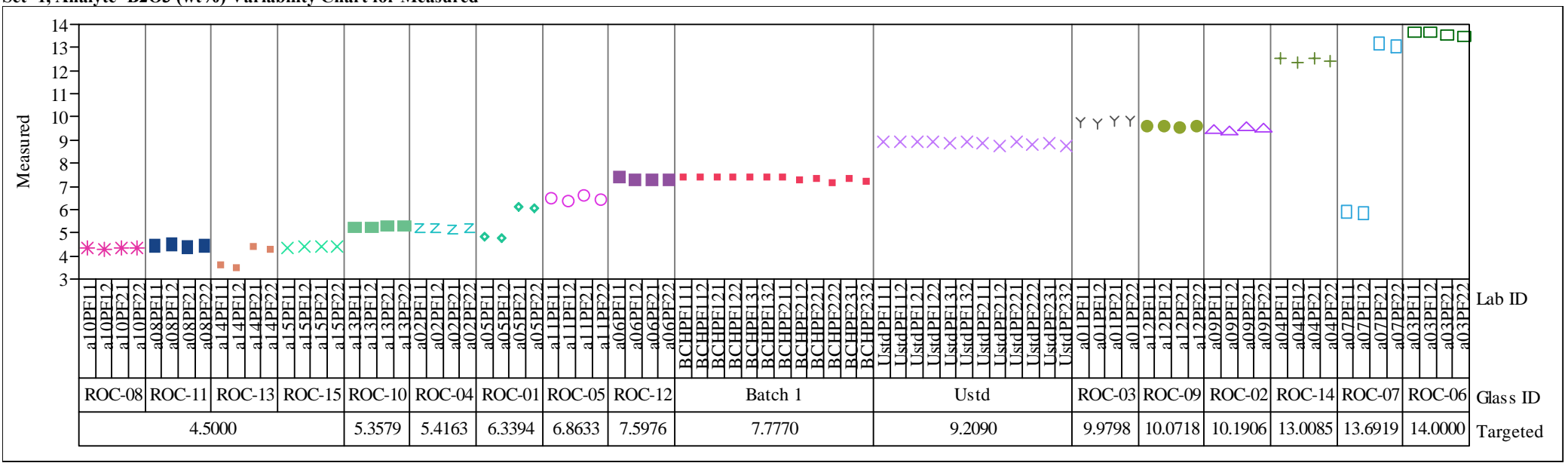

Set=1, Analyte=B2O3 (wt\%) Variability Chart for Measured bc

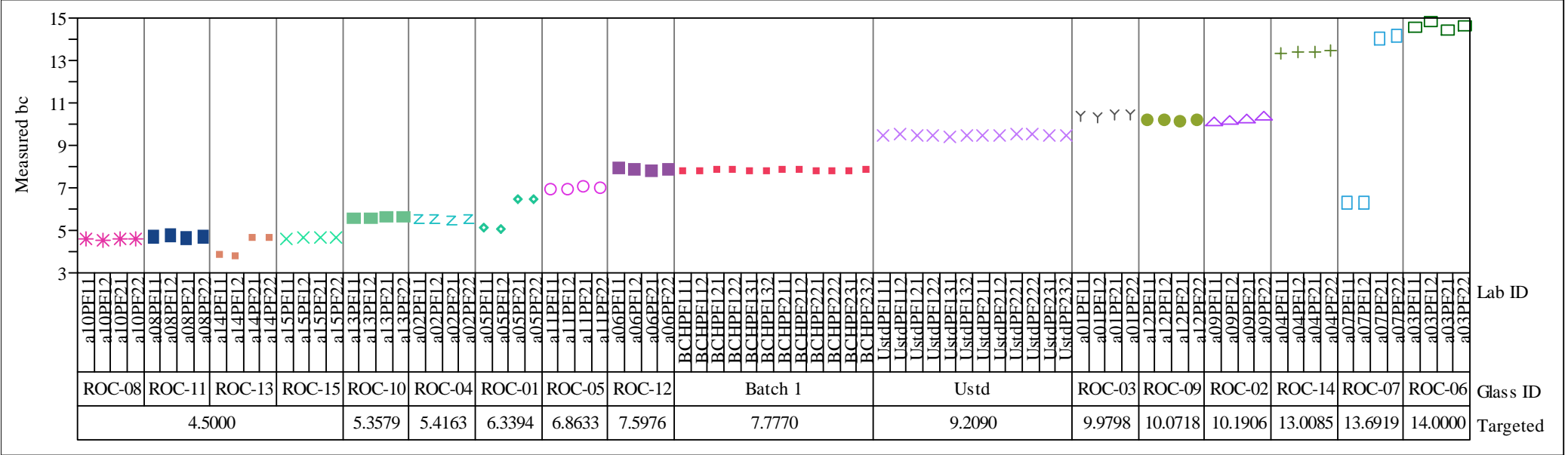


SRNL-STI-2009-00465, Revision 0

Exhibit A5. Oxide Measurements by Lab ID within Glass ID Sorted by Targeted Concentrations

Set $=1$, Analyte $=\mathrm{BaO}($ wt $\%)$ Variability Chart for Measured

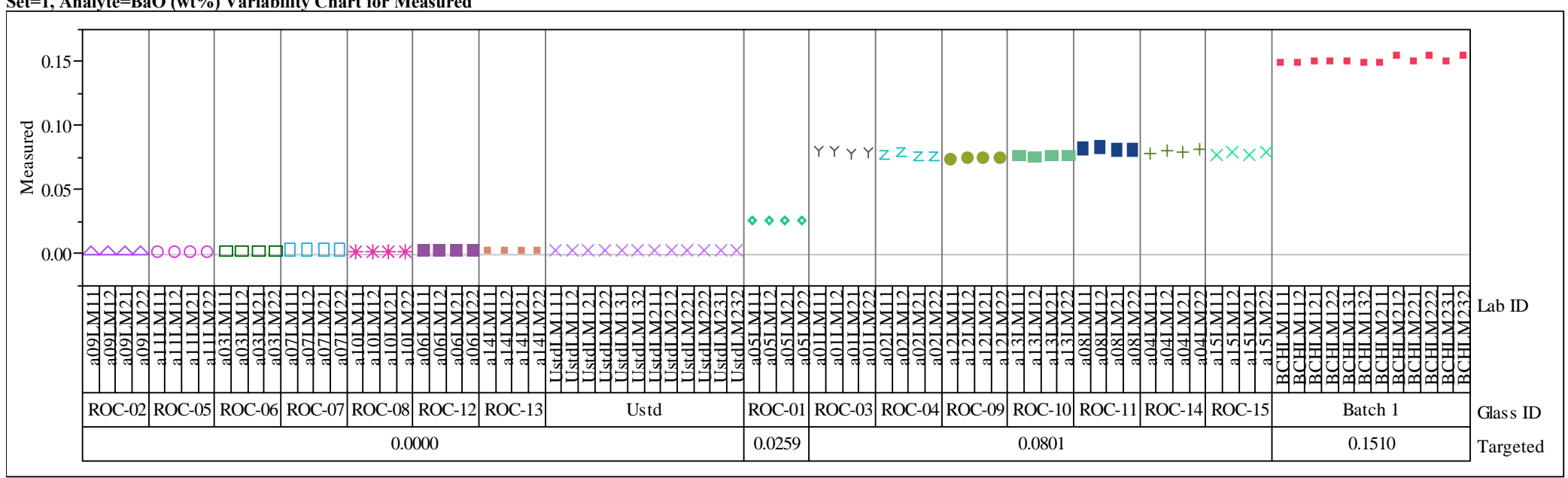

Set $=1$, Analyte $=\mathrm{BaO}(\mathrm{wt} \%)$ Variability Chart for Measured bc

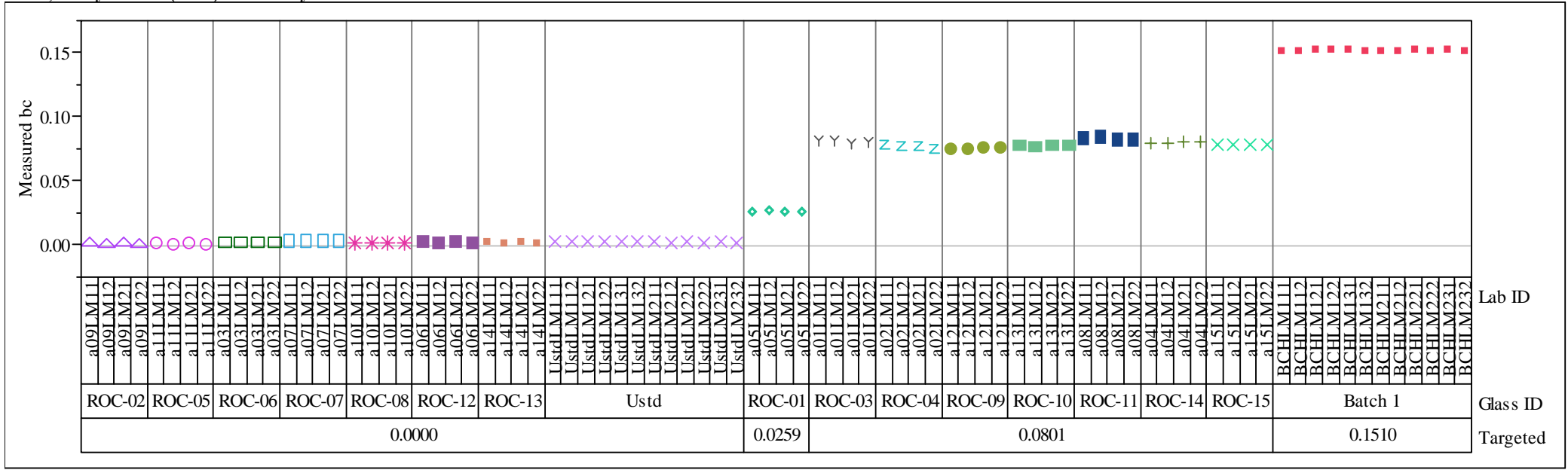


SRNL-STI-2009-00465, Revision 0

Exhibit A5. Oxide Measurements by Lab ID within Glass ID Sorted by Targeted Concentrations

Set $=1$, Analyte $=\mathrm{CaO}(\mathrm{wt} \%)$ Variability Chart for Measured

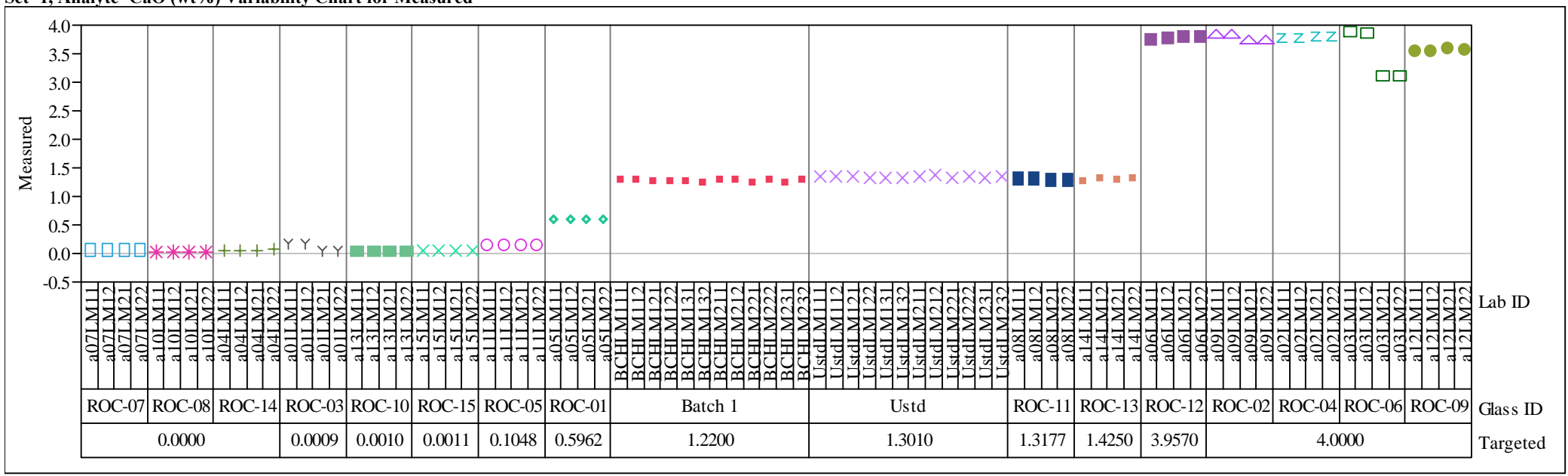

Set $=1$, Analyte $=\mathrm{CaO}(\mathrm{wt} \%)$ Variability Chart for Measured bc

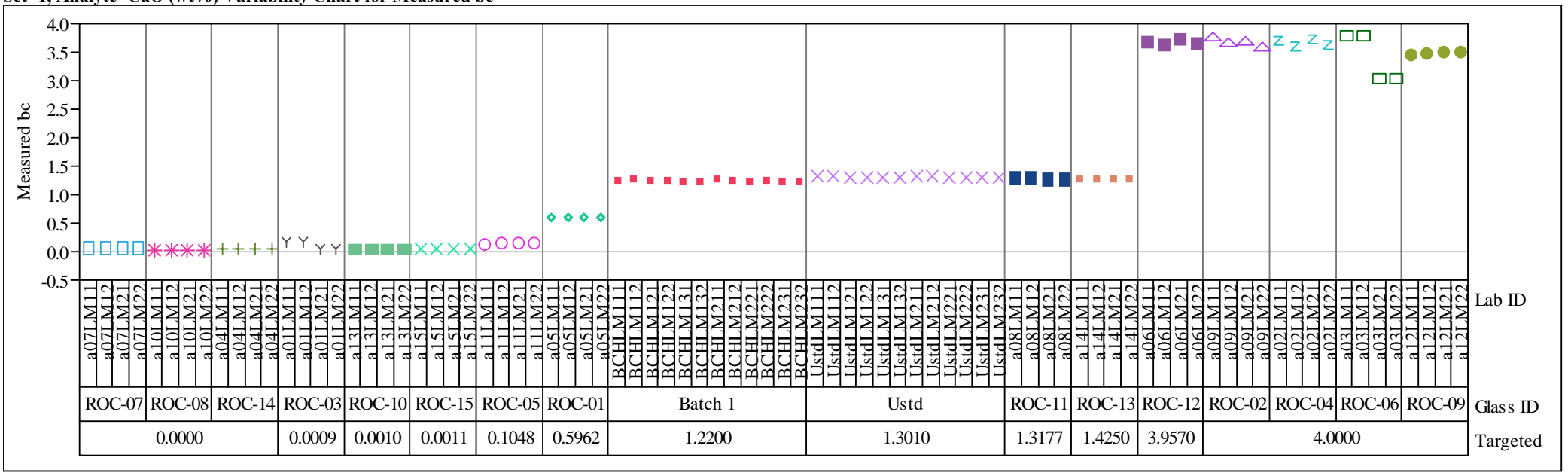


SRNL-STI-2009-00465, Revision 0

Exhibit A5. Oxide Measurements by Lab ID within Glass ID Sorted by Targeted Concentrations

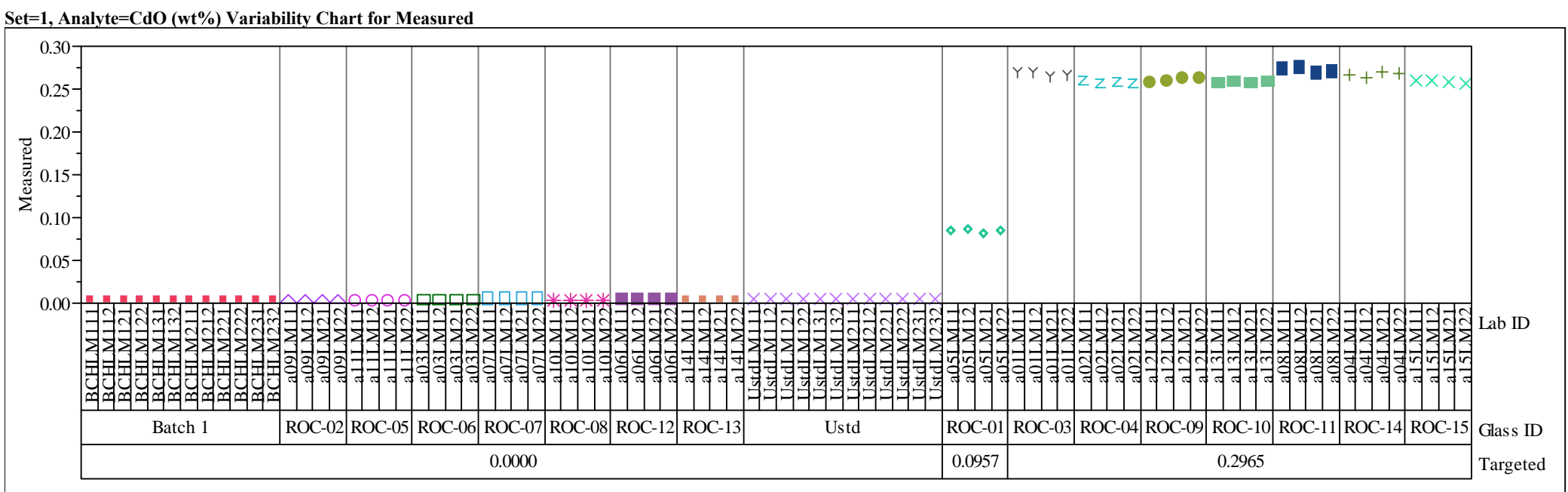

Set $=1$, Analyte $=\mathbf{C d O}($ wt $\%)$ Variability Chart for Measured bc

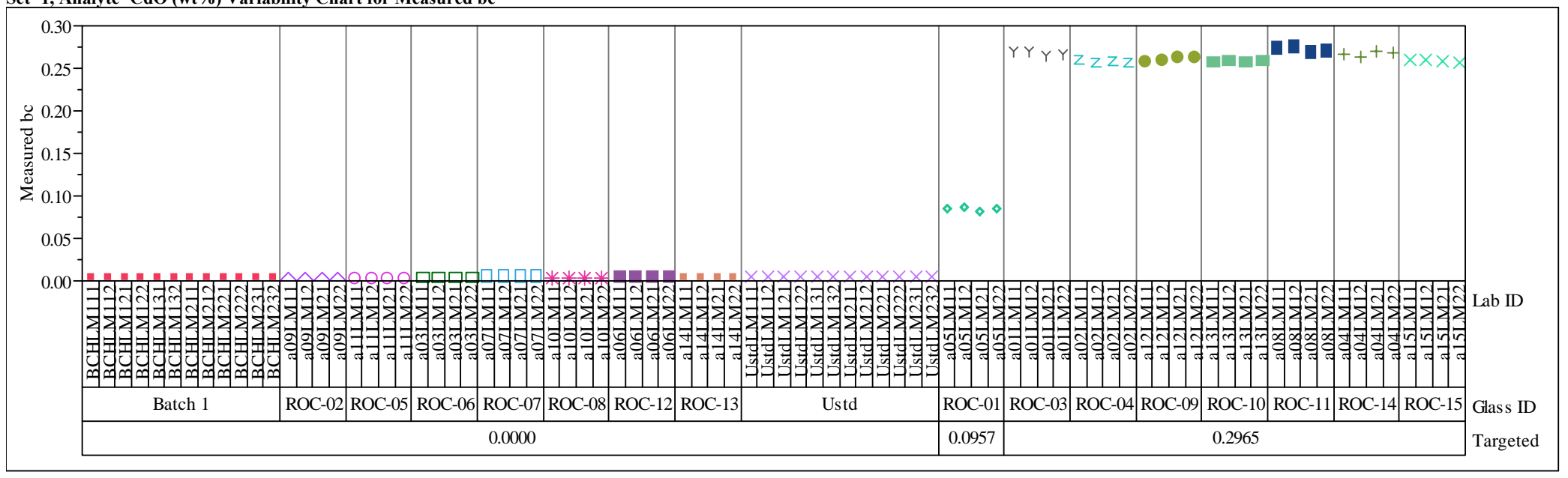


SRNL-STI-2009-00465, Revision 0

Exhibit A5. Oxide Measurements by Lab ID within Glass ID Sorted by Targeted Concentrations

Set $=1$, Analyte $=\mathrm{Ce} 2 \mathrm{O} 3(\mathrm{wt} \%)$ Variability Chart for Measured

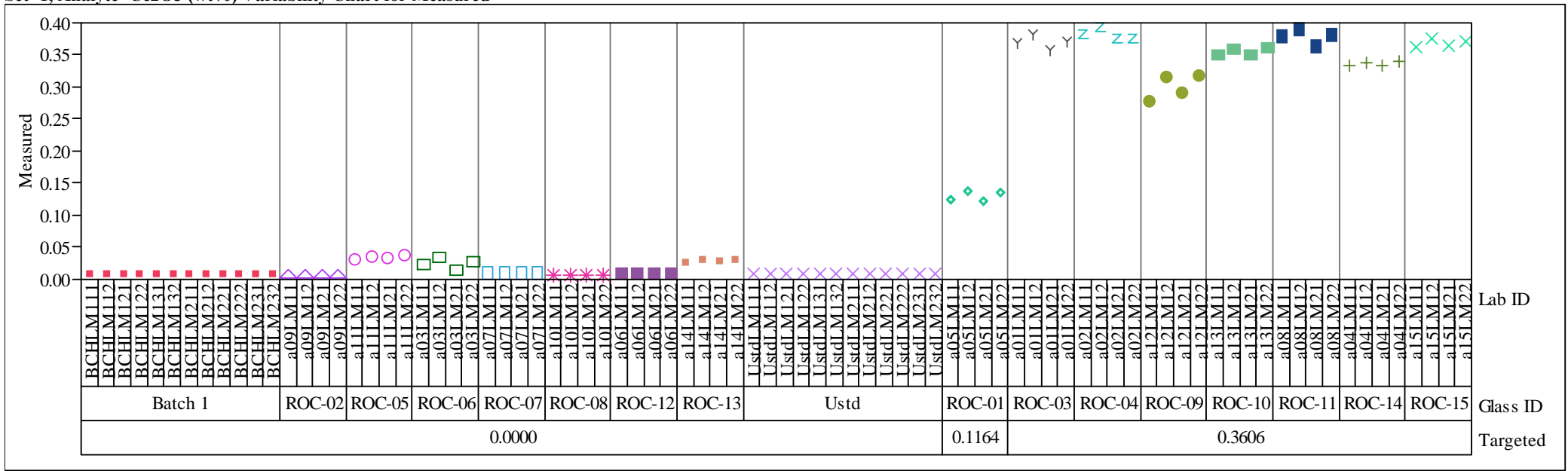

Set=1, Analyte=Ce2O3 (wt\%) Variability Chart for Measured bc

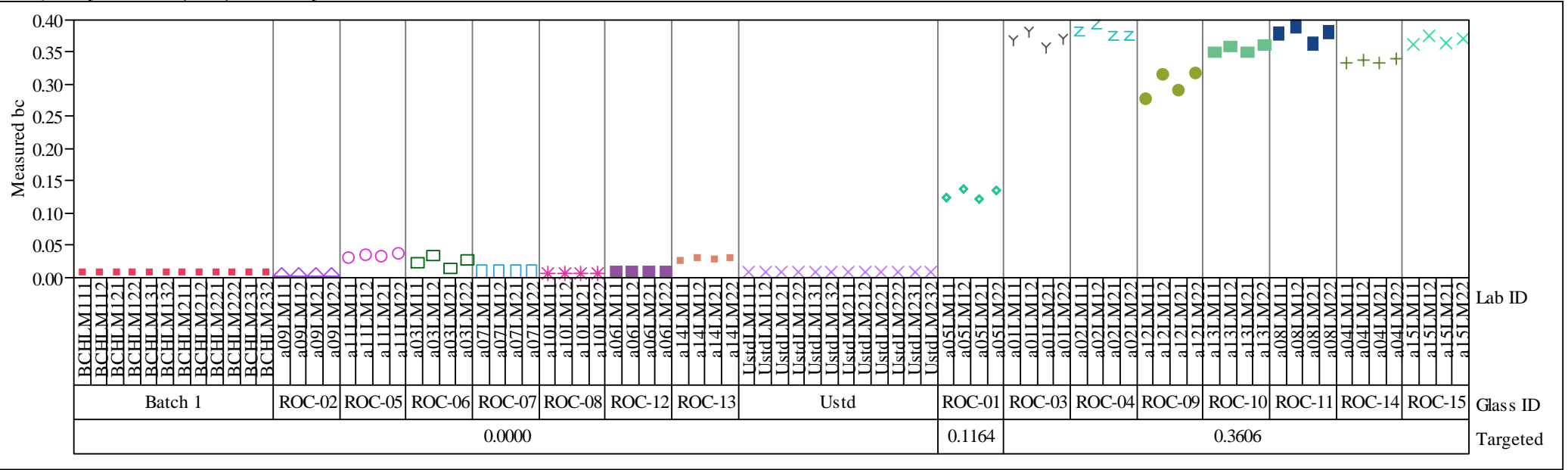


SRNL-STI-2009-00465, Revision 0

Exhibit A5. Oxide Measurements by Lab ID within Glass ID Sorted by Targeted Concentrations

Set $=1$, Analyte $=\mathbf{C r} 203($ wt $\%)$ Variability Chart for Measured

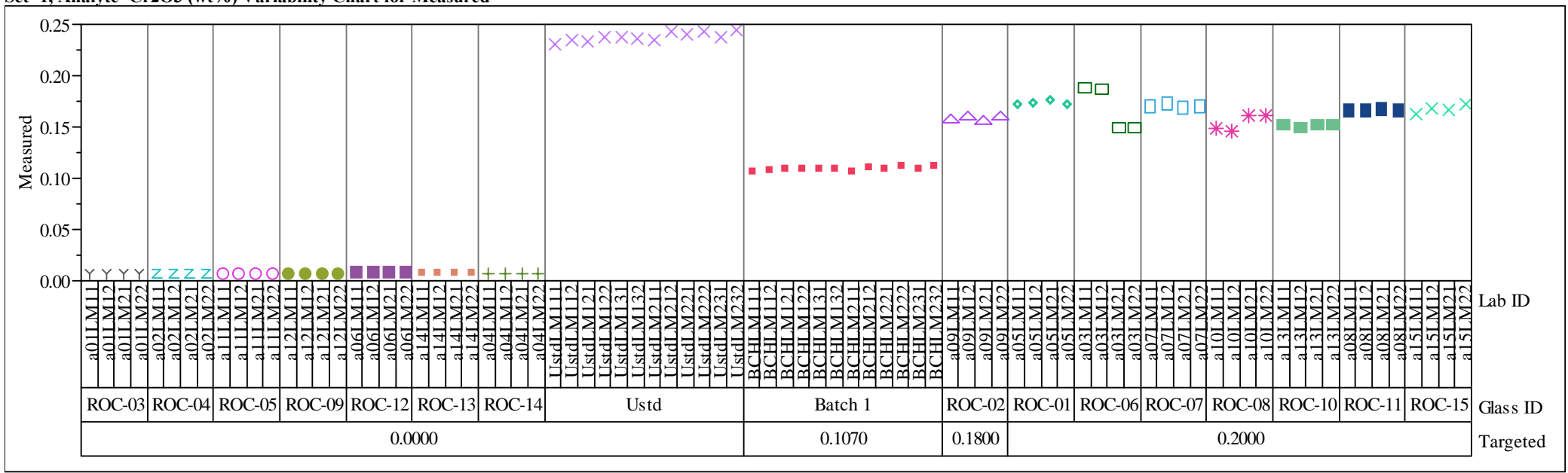

Set=1, Analyte= $\mathrm{Cr} 2 \mathrm{O} 3(\mathrm{wt} \%)$ Variability Chart for Measured bc

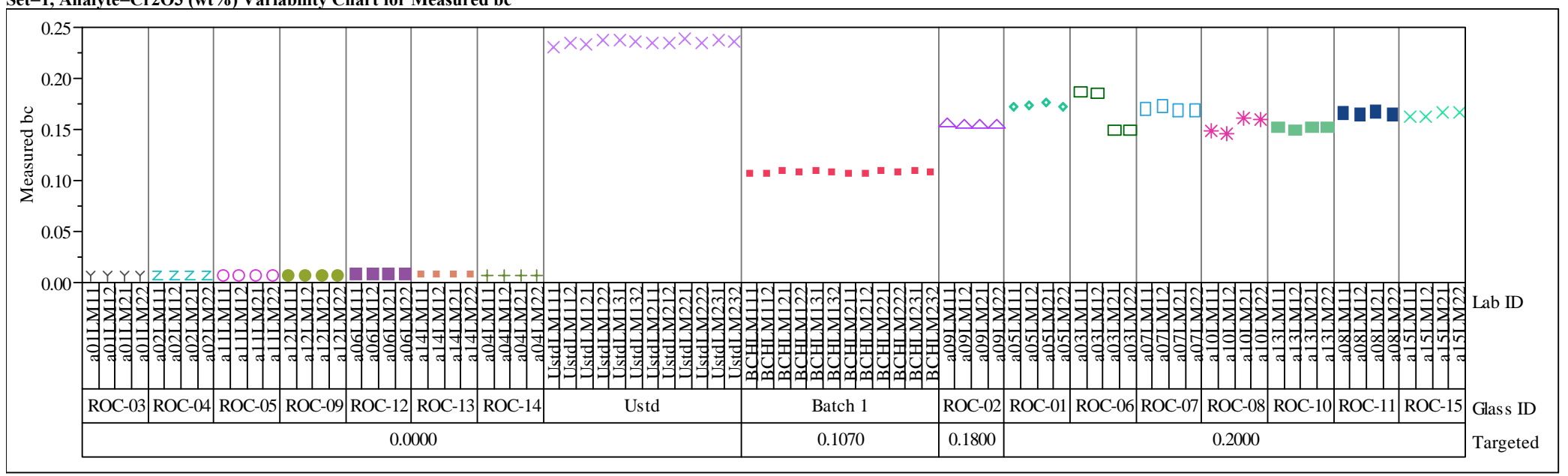


SRNL-STI-2009-00465, Revision 0

Exhibit A5. Oxide Measurements by Lab ID within Glass ID Sorted by Targeted Concentrations

Set $=1$, Analyte $=\mathrm{CuO}(\mathrm{wt} \%)$ Variability Chart for Measured

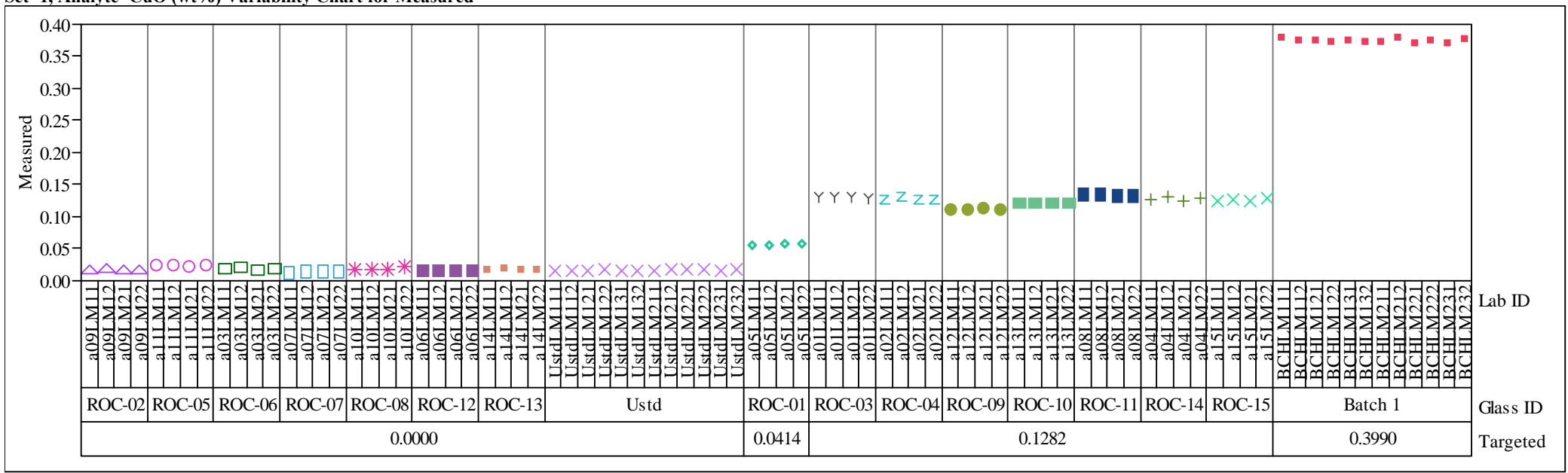

Set $=1$, Analyte $=\mathrm{CuO}($ wt $\%)$ Variability Chart for Measured bc

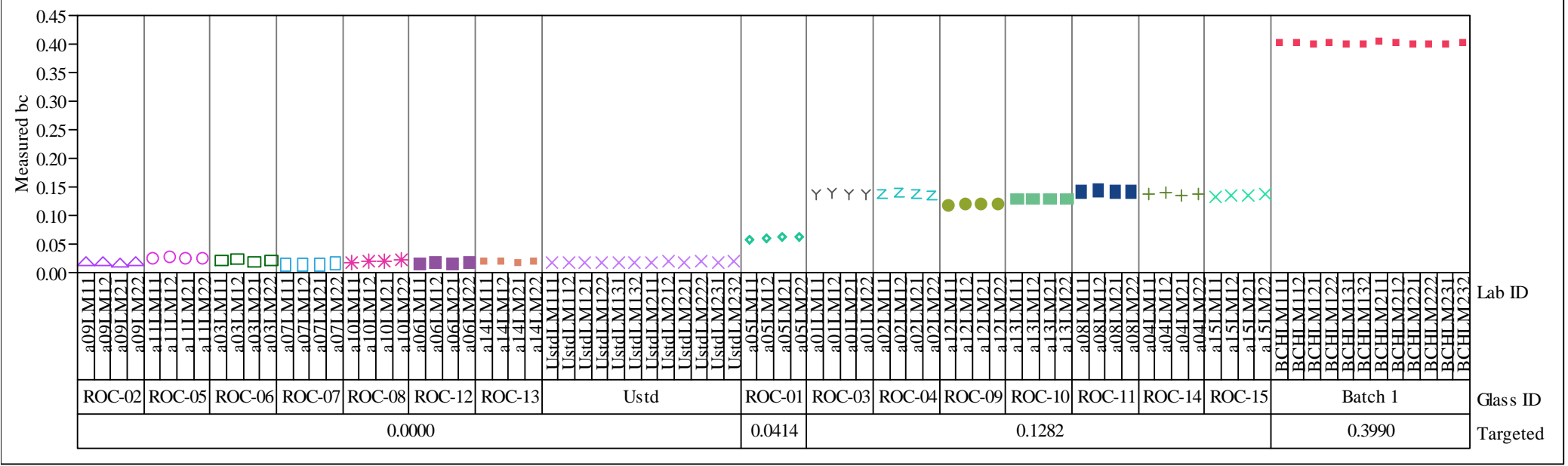


SRNL-STI-2009-00465, Revision 0

Exhibit A5. Oxide Measurements by Lab ID within Glass ID Sorted by Targeted Concentrations

Set=1, Analyte=Fe2O3 (wt \%) Variability Chart for Measured

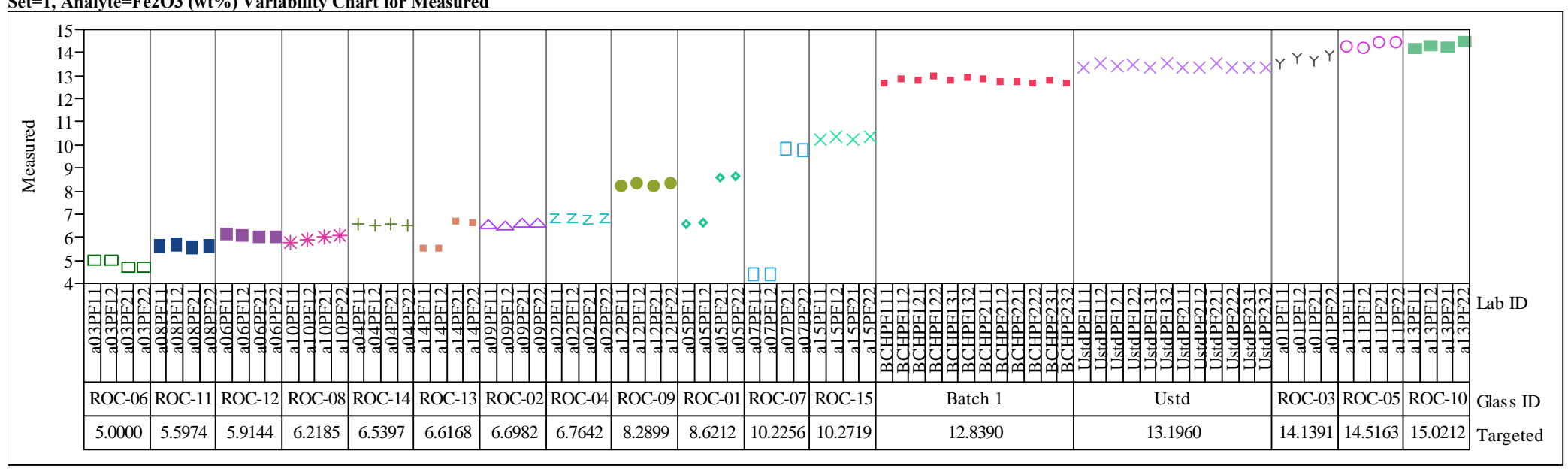

Set=1, Analyte=Fe2O3 (wt\%) Variability Chart for Measured bc

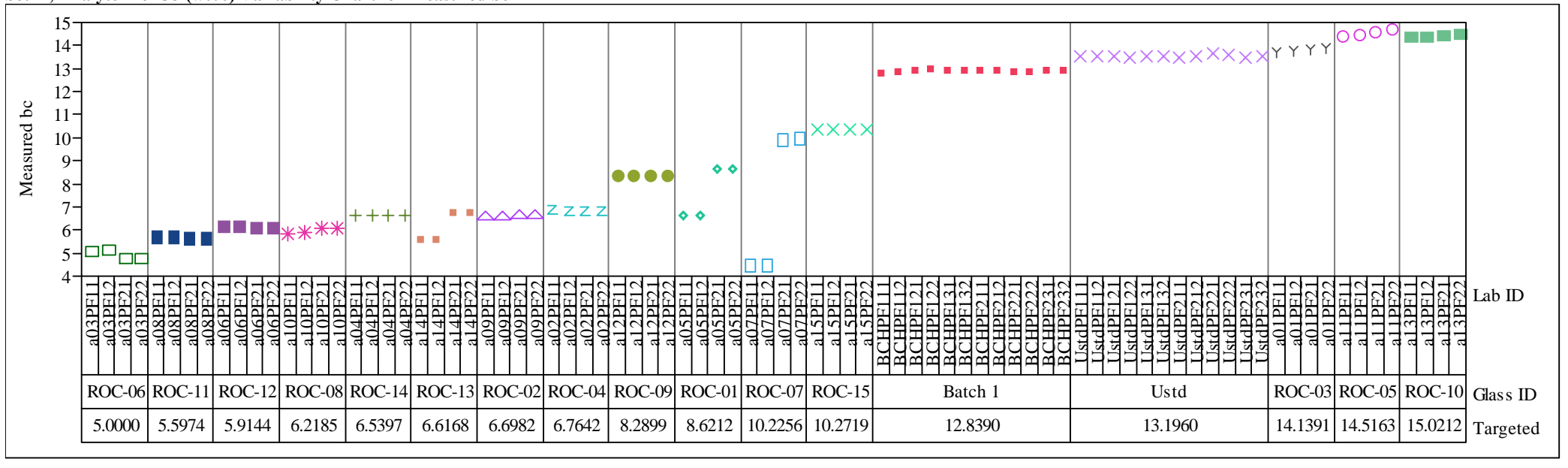


SRNL-STI-2009-00465, Revision 0

Exhibit A5. Oxide Measurements by Lab ID within Glass ID Sorted by Targeted Concentrations

Set $=1$, Analyte $=K 2 O(w t \%)$ Variability Chart for Measured

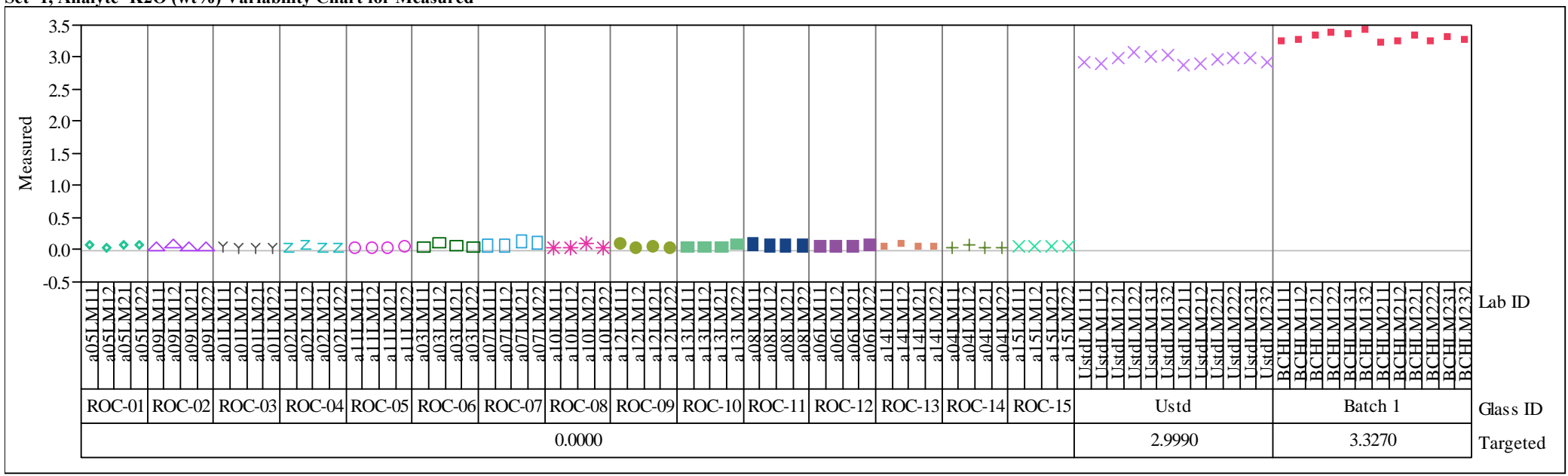

Set $=1$, Analyte $=K 2 O(w t \%)$ Variability Chart for Measured bc

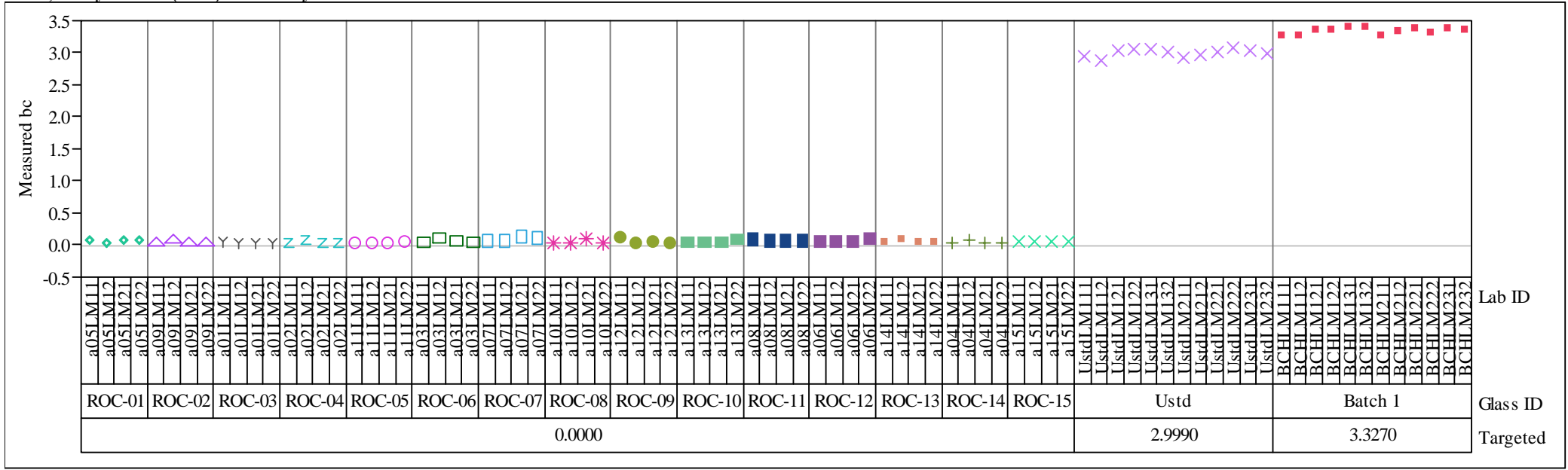


SRNL-STI-2009-00465, Revision 0

Exhibit A5. Oxide Measurements by Lab ID within Glass ID Sorted by Targeted Concentrations

Set=1, Analyte=La2O3 (wt\%) Variability Chart for Measured

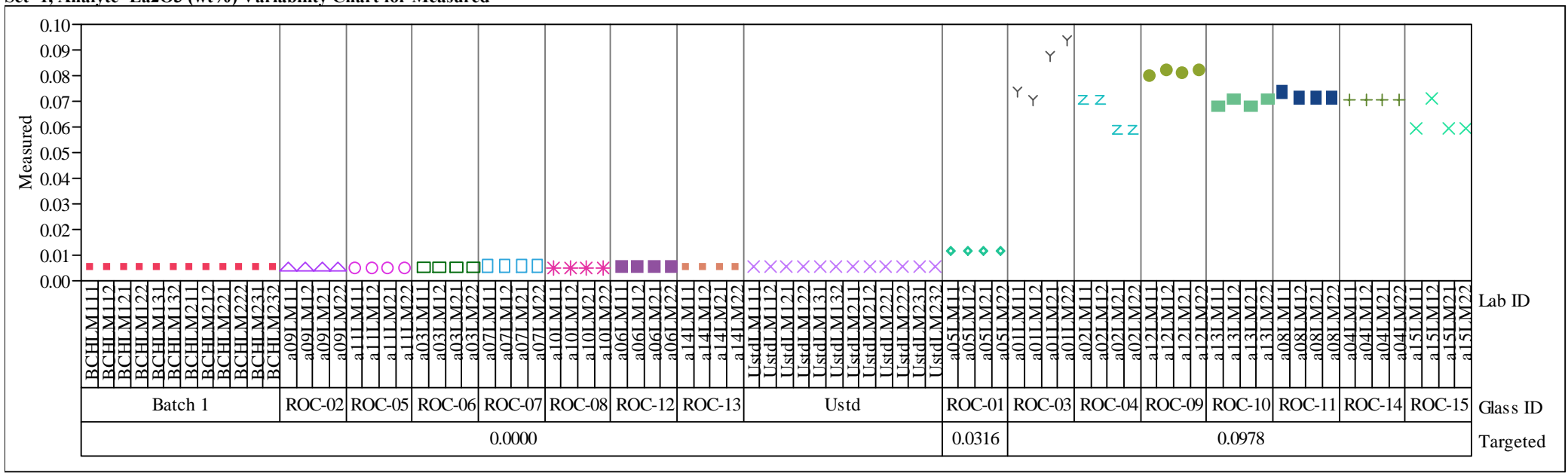

Set=1, Analyte=La2O3 (wt\%) Variability Chart for Measured bc

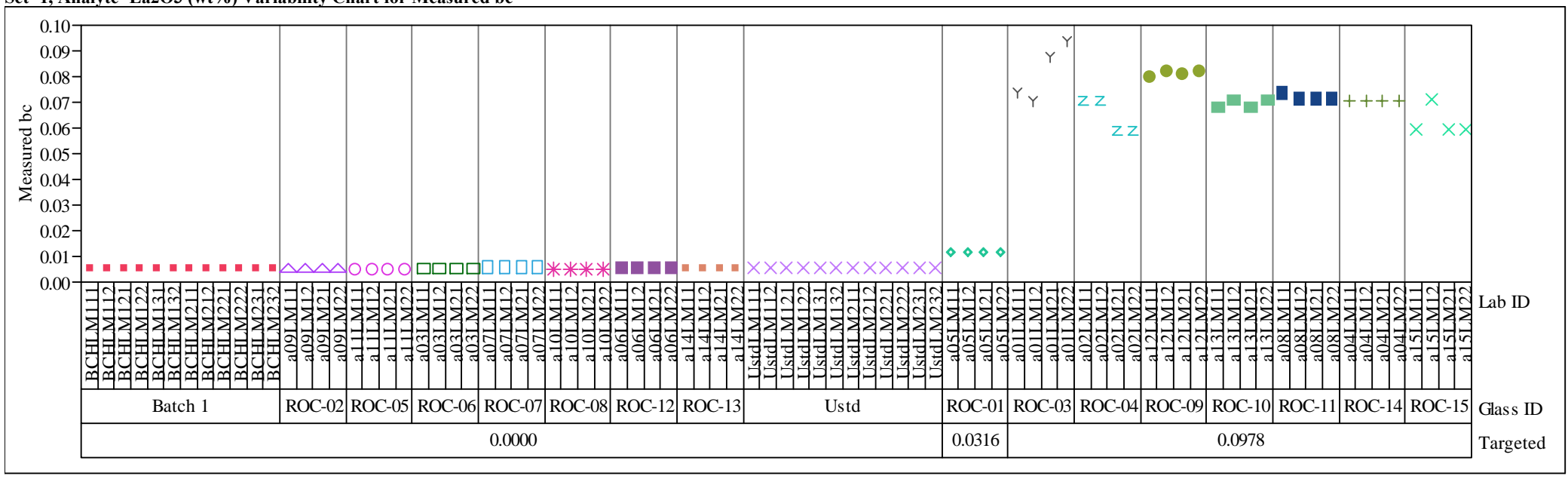


SRNL-STI-2009-00465, Revision 0

Exhibit A5. Oxide Measurements by Lab ID within Glass ID Sorted by Targeted Concentrations

Set=1, Analyte $=$ Li2O $(w t \%)$ Variability Chart for Measured

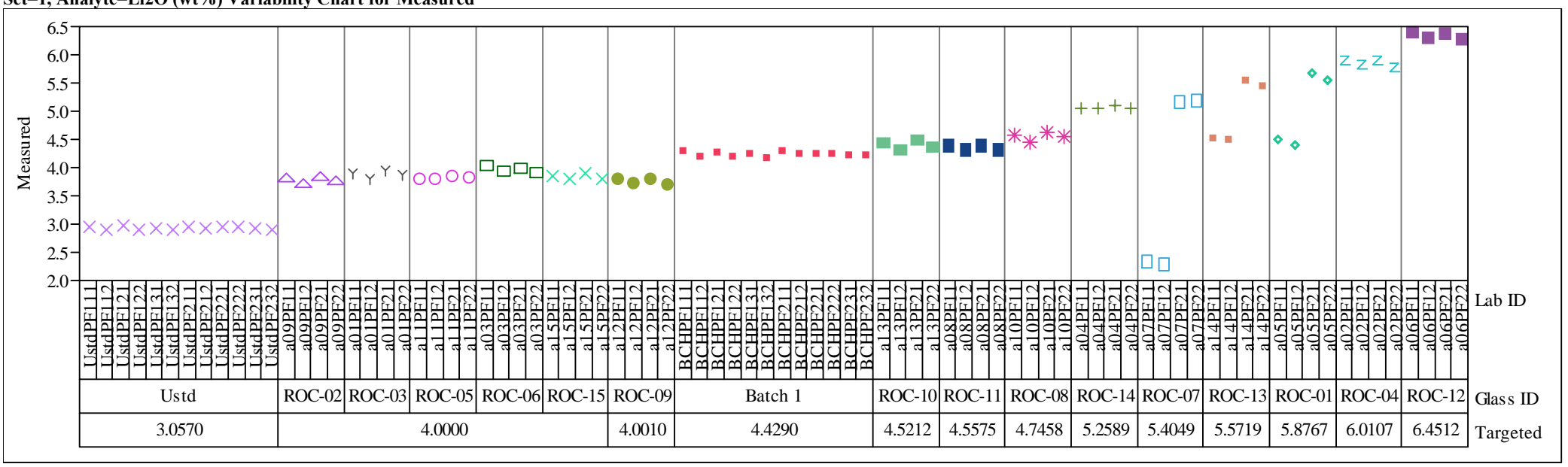

Set=1, Analyte $=$ Li2O $($ wt $\%)$ Variability Chart for Measured bc

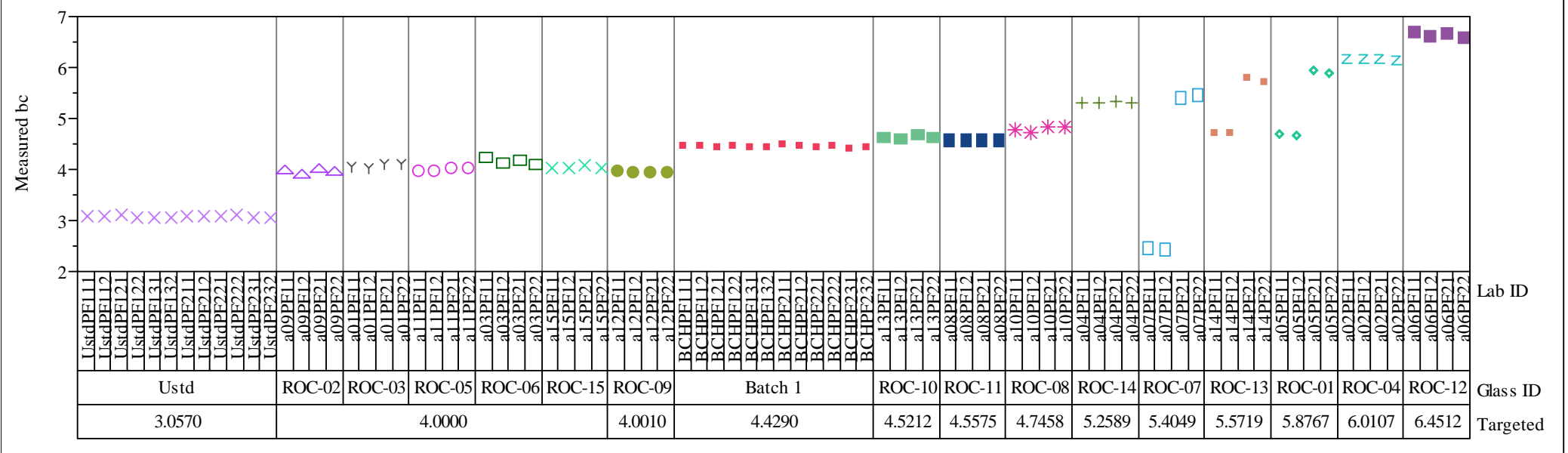


SRNL-STI-2009-00465, Revision 0

Exhibit A5. Oxide Measurements by Lab ID within Glass ID Sorted by Targeted Concentrations

Set $=1$, Analyte $=\mathrm{MgO}($ wt $\%)$ Variability Chart for Measured

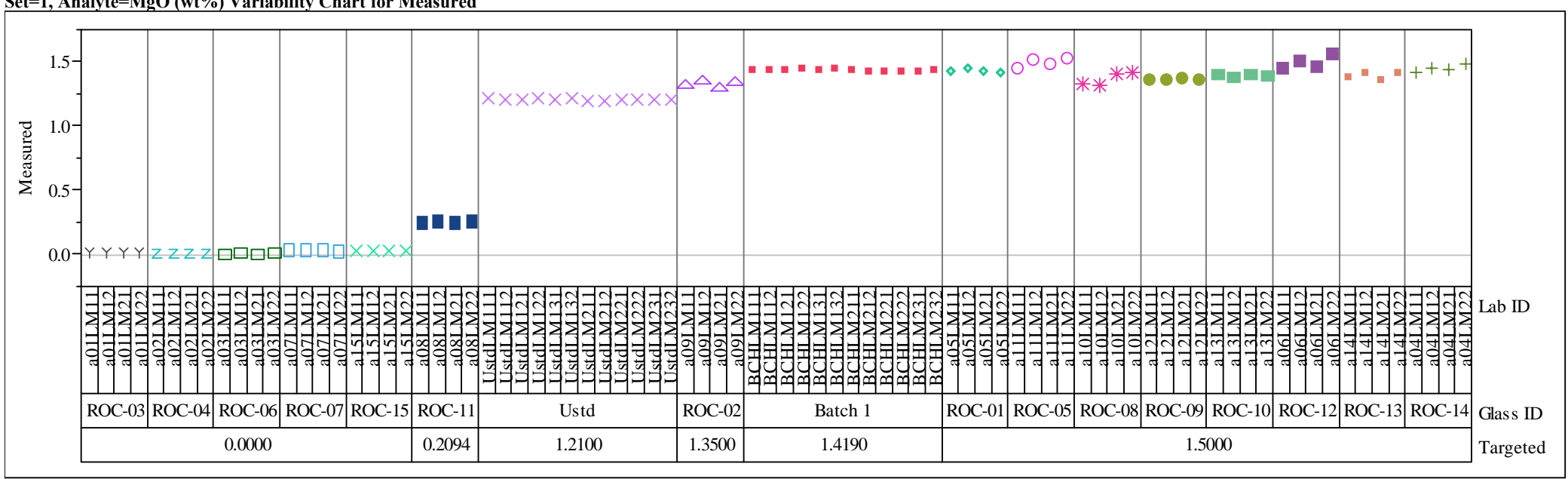

Set=1, Analyte $=\mathrm{MgO}(\mathrm{wt} \%)$ Variability Chart for Measured be

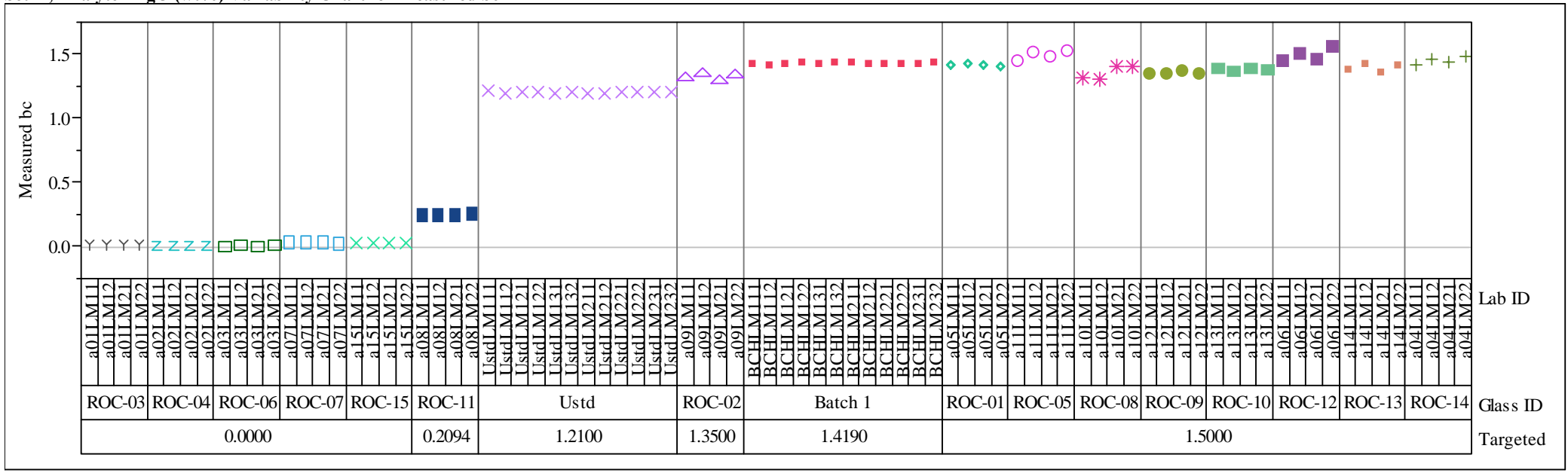


SRNL-STI-2009-00465, Revision 0

Exhibit A5. Oxide Measurements by Lab ID within Glass ID Sorted by Targeted Concentrations

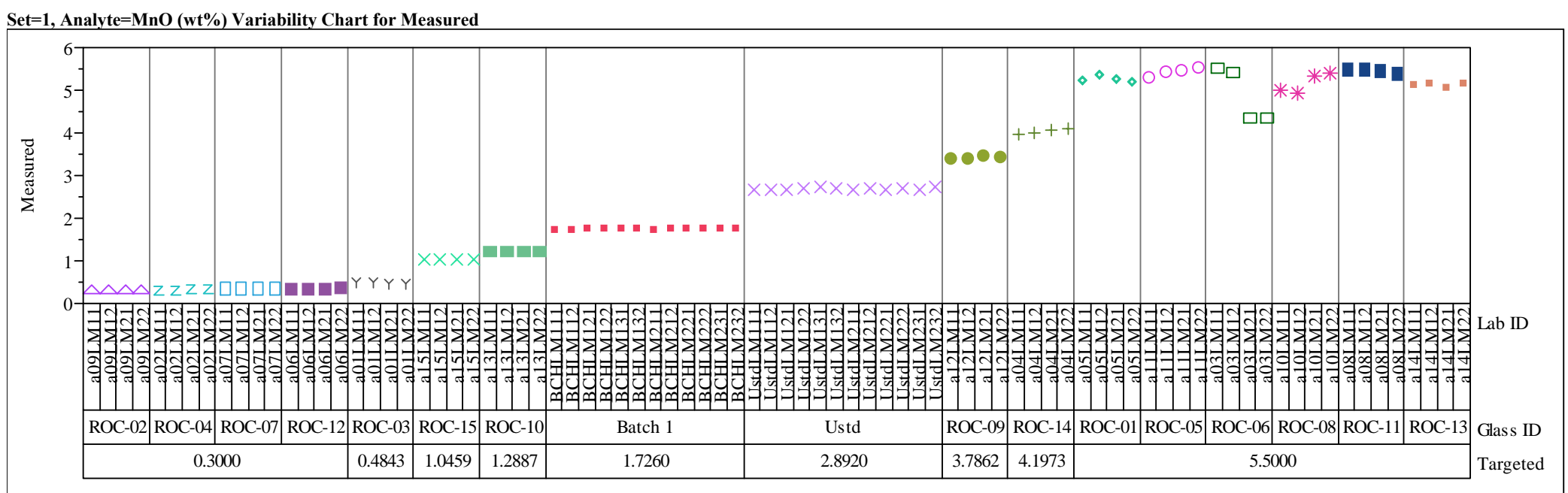

Set $=1$, Analyte $=\mathrm{MnO}(\mathrm{wt} \%)$ Variability Chart for Measured bc

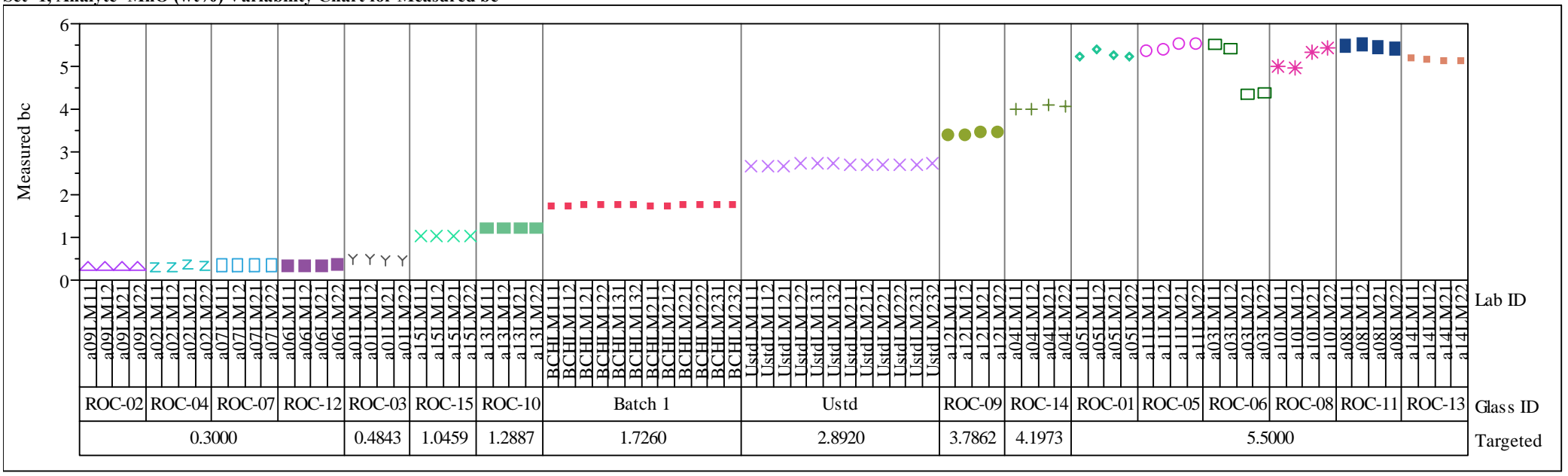


SRNL-STI-2009-00465, Revision 0

Exhibit A5. Oxide Measurements by Lab ID within Glass ID Sorted by Targeted Concentrations

Set $=1$, Analyte $=\mathrm{Na} 2 \mathrm{O}(\mathrm{wt} \%)$ Variability Chart for Measured

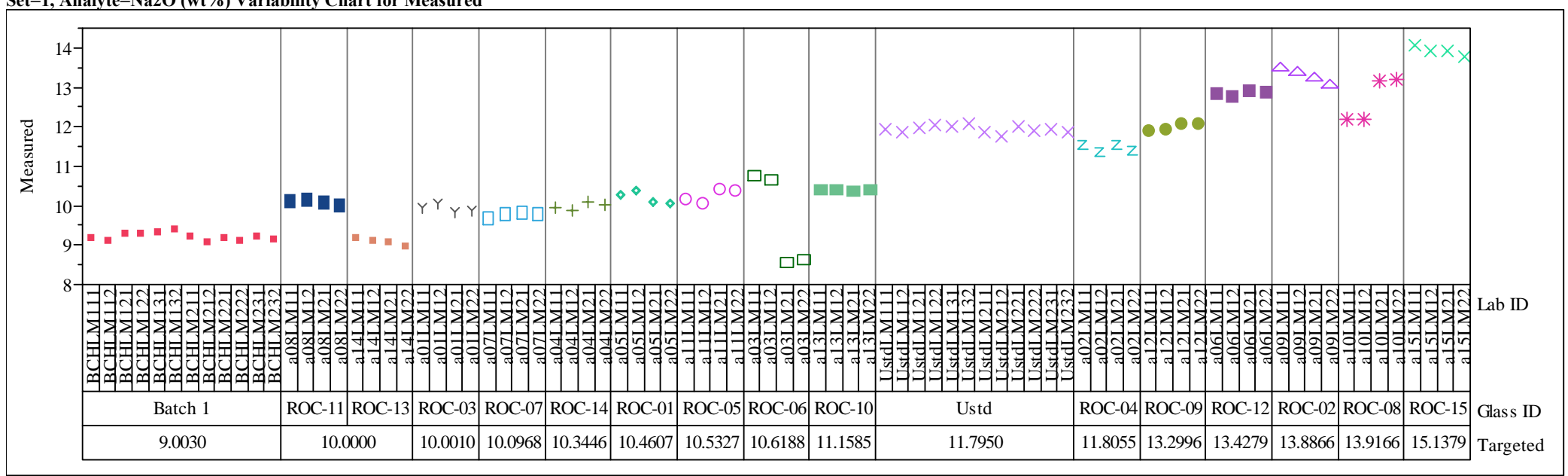

Set=1, Analyte=Na2O (wt\%) Variability Chart for Measured bc

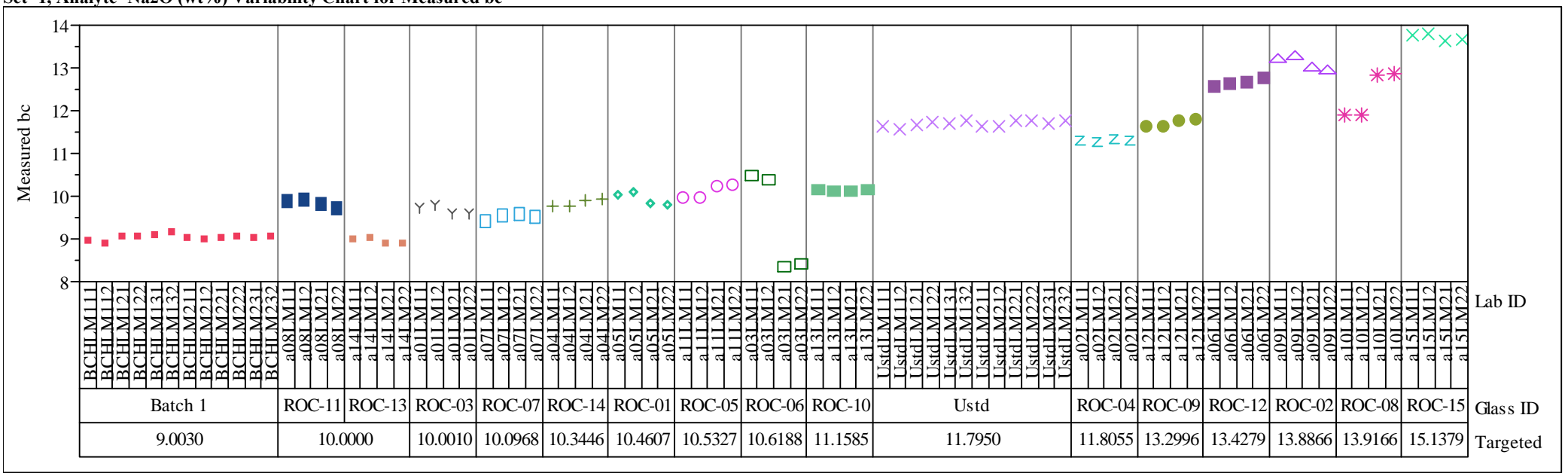


SRNL-STI-2009-00465, Revision 0

Exhibit A5. Oxide Measurements by Lab ID within Glass ID Sorted by Targeted Concentrations

Set=1, Analyte=NiO (wt\%) Variability Chart for Measured

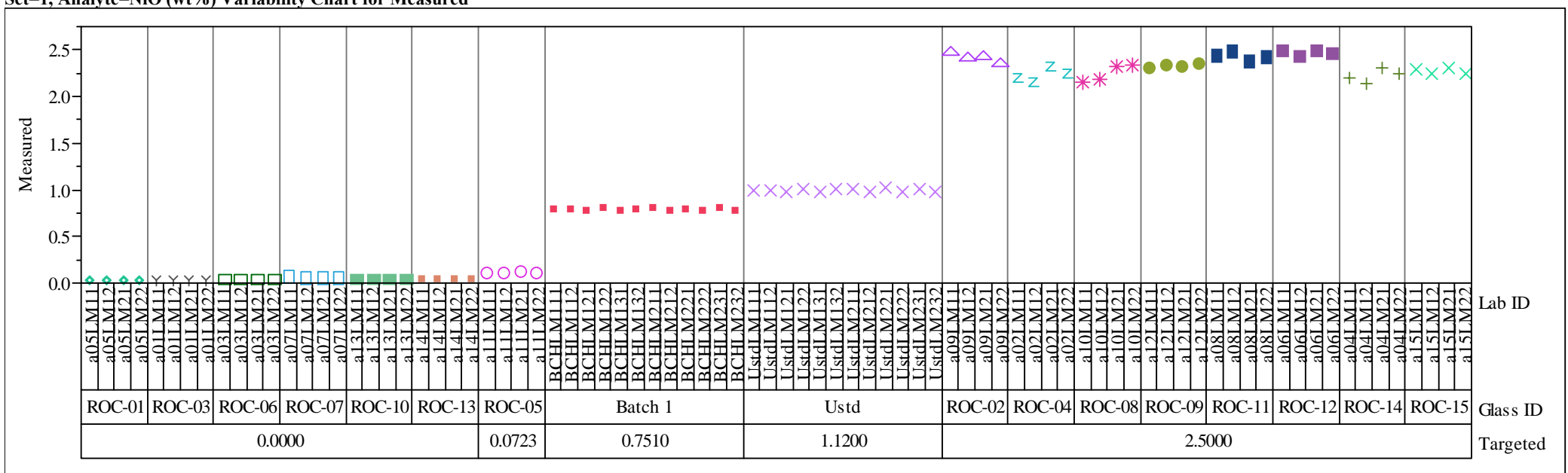

Set $=1$, Analyte=NiO (wt\%) Variability Chart for Measured bc

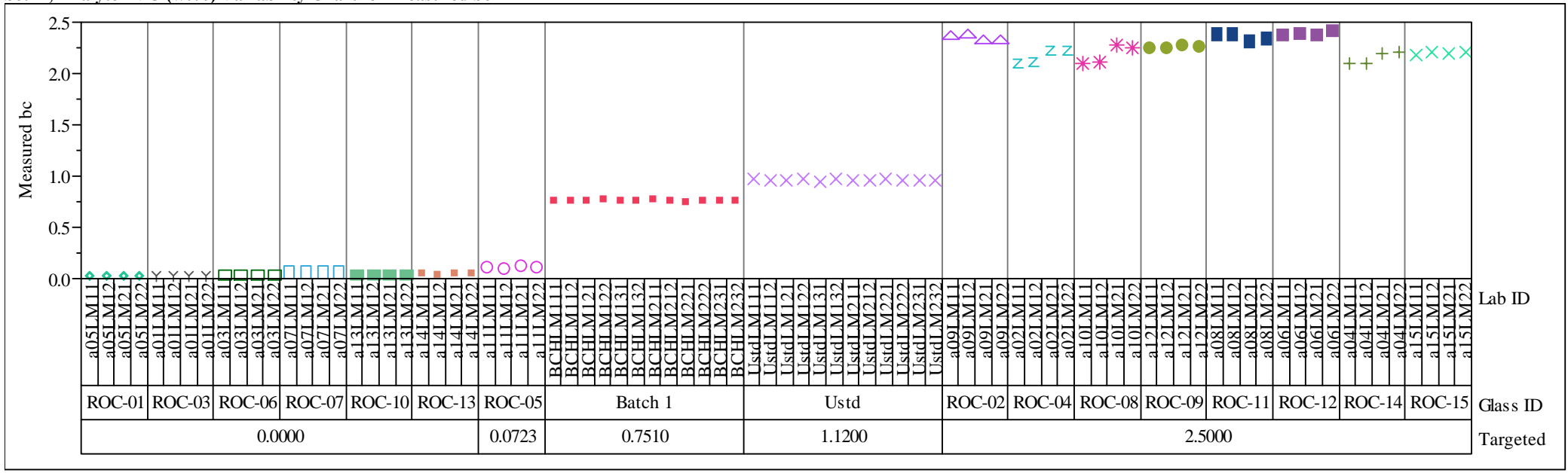




\section{Exhibit A5. Oxide Measurements by Lab ID within Glass ID Sorted by Targeted Concentrations}

Set=1, Analyte=PbO (wt\%) Variability Chart for Measured

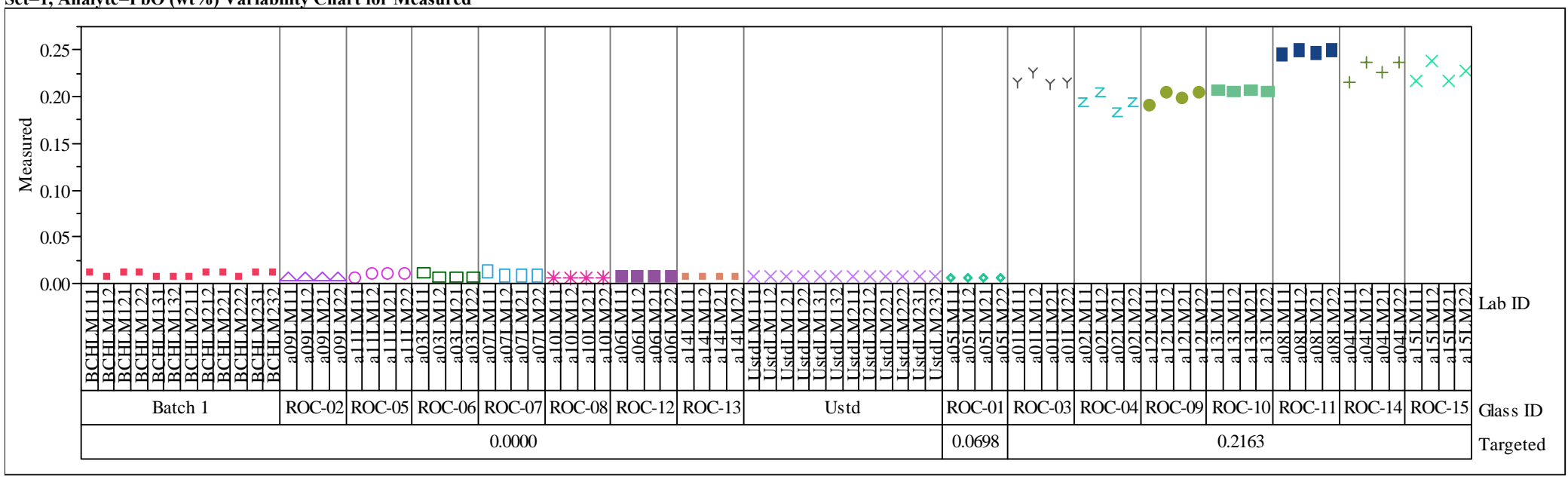

Set=1, Analyte $=\mathbf{P b O}($ wt $\%)$ Variability Chart for Measured bc

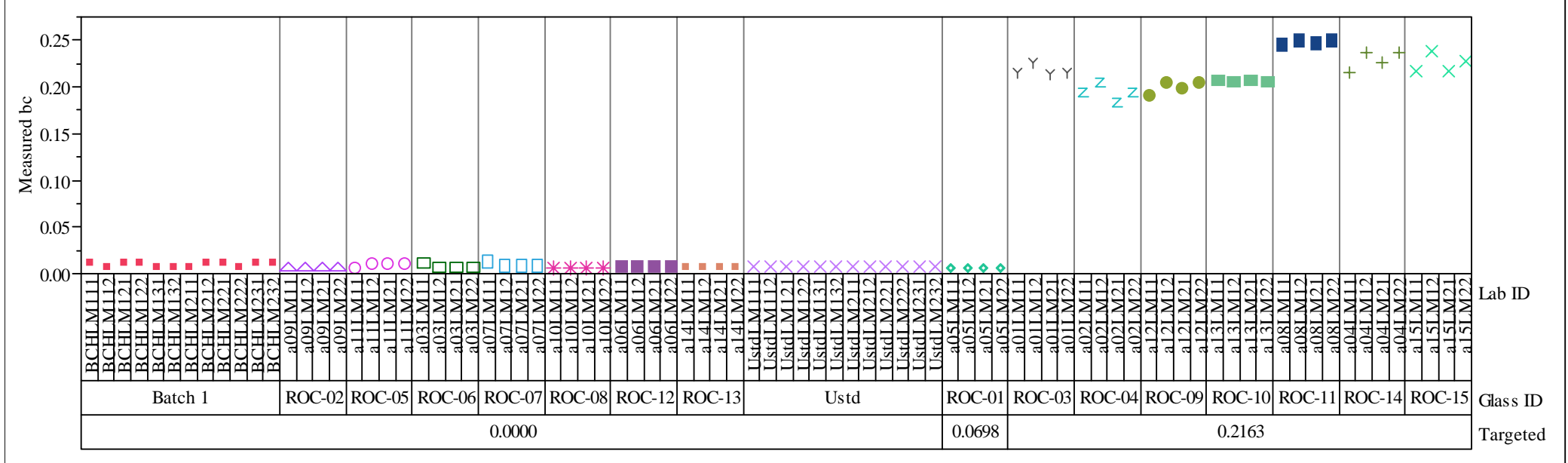


SRNL-STI-2009-00465, Revision 0

Exhibit A5. Oxide Measurements by Lab ID within Glass ID Sorted by Targeted Concentrations

Set $=1$, Analyte $=\mathrm{SiO} 2(\mathrm{wt} \%)$ Variability Chart for Measured

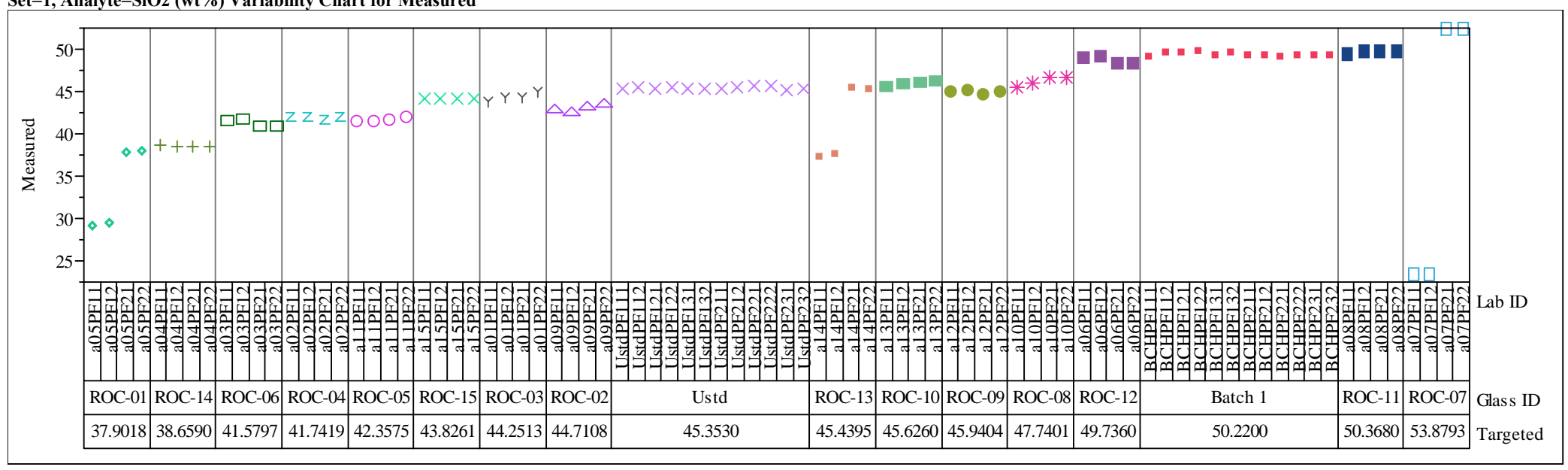

Set=1, Analyte=SiO2 (wt\%) Variability Chart for Measured bc

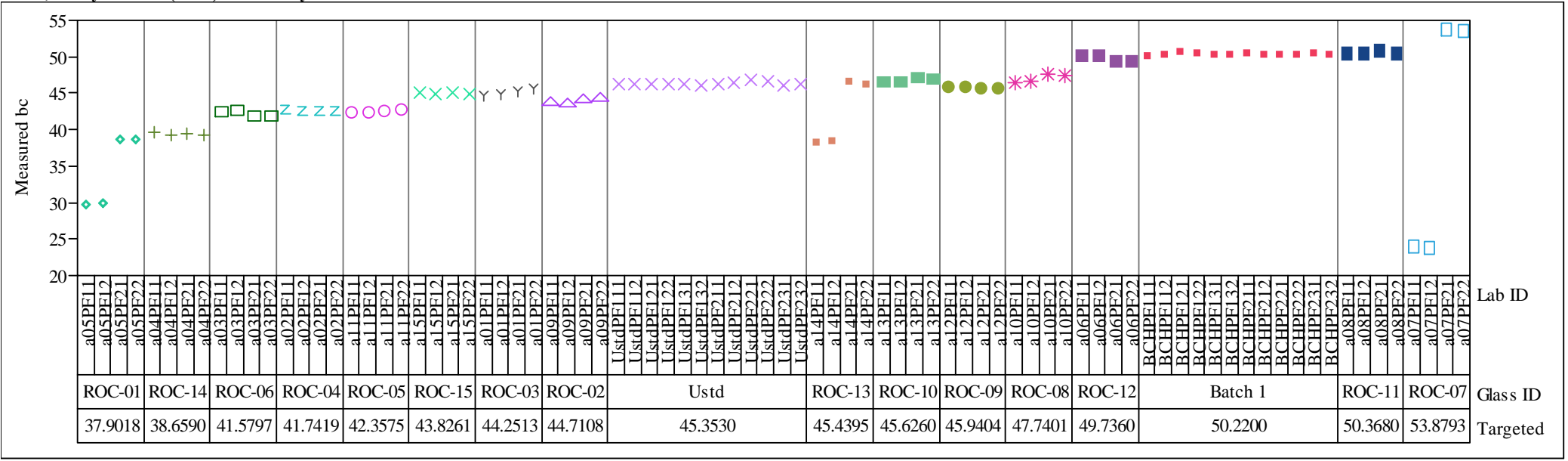


SRNL-STI-2009-00465, Revision 0

Exhibit A5. Oxide Measurements by Lab ID within Glass ID Sorted by Targeted Concentrations

Set $=1$, Analyte $=\mathrm{SO} 4(\mathrm{wt} \%)$ Variability Chart for Measured

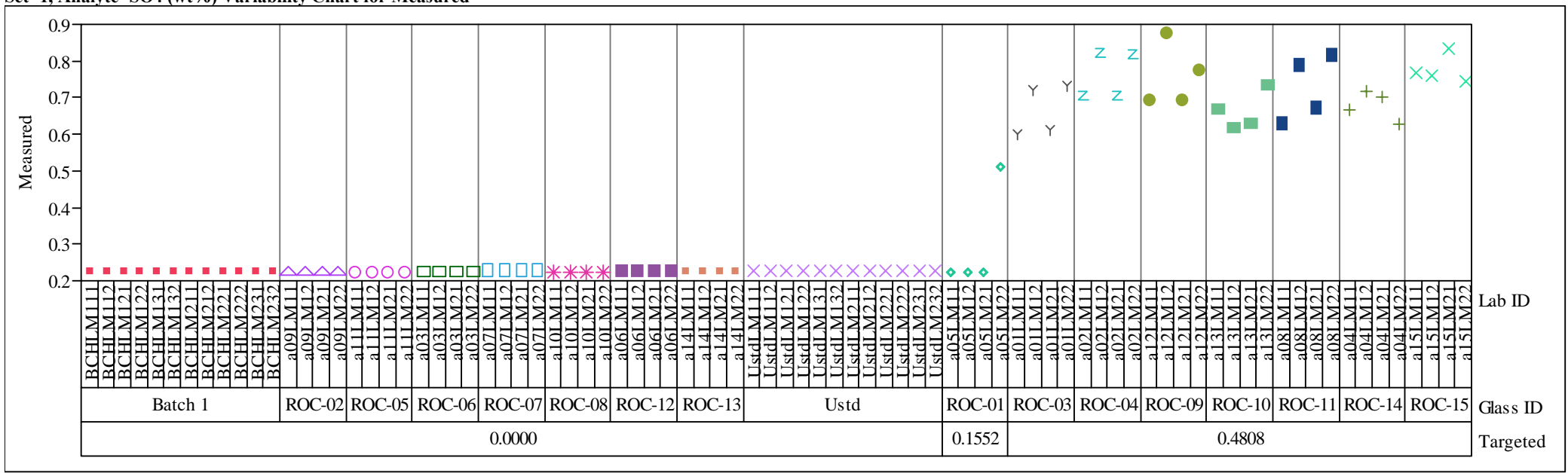

Set $=1$, Analyte $=\mathrm{SO} 4(\mathrm{wt} \%)$ Variability Chart for Measured bc

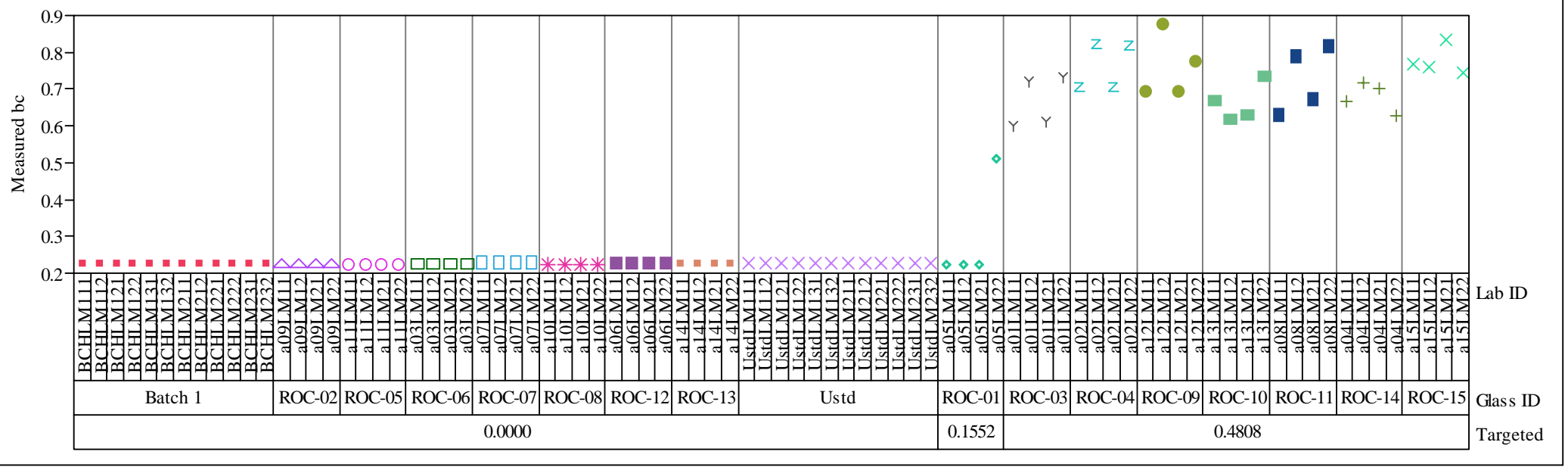




\section{Exhibit A5. Oxide Measurements by Lab ID within Glass ID Sorted by Targeted Concentrations}

Set=1, Analyte=TiO2 (wt\%) Variability Chart for Measured

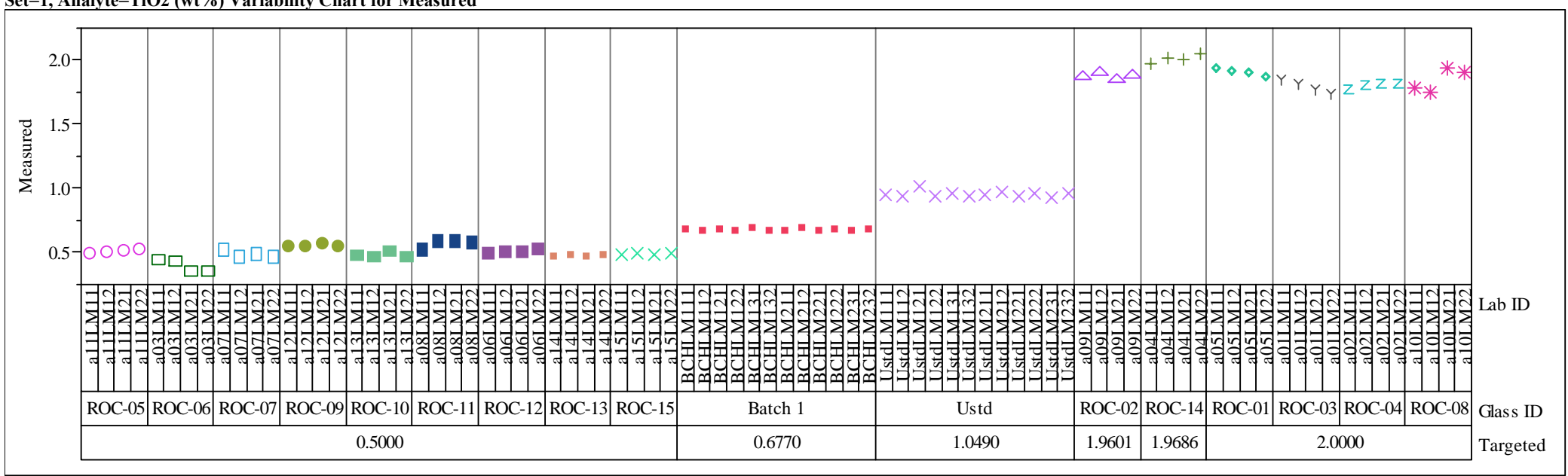

Set=1, Analyte=TiO2 (wt \%) Variability Chart for Measured bc

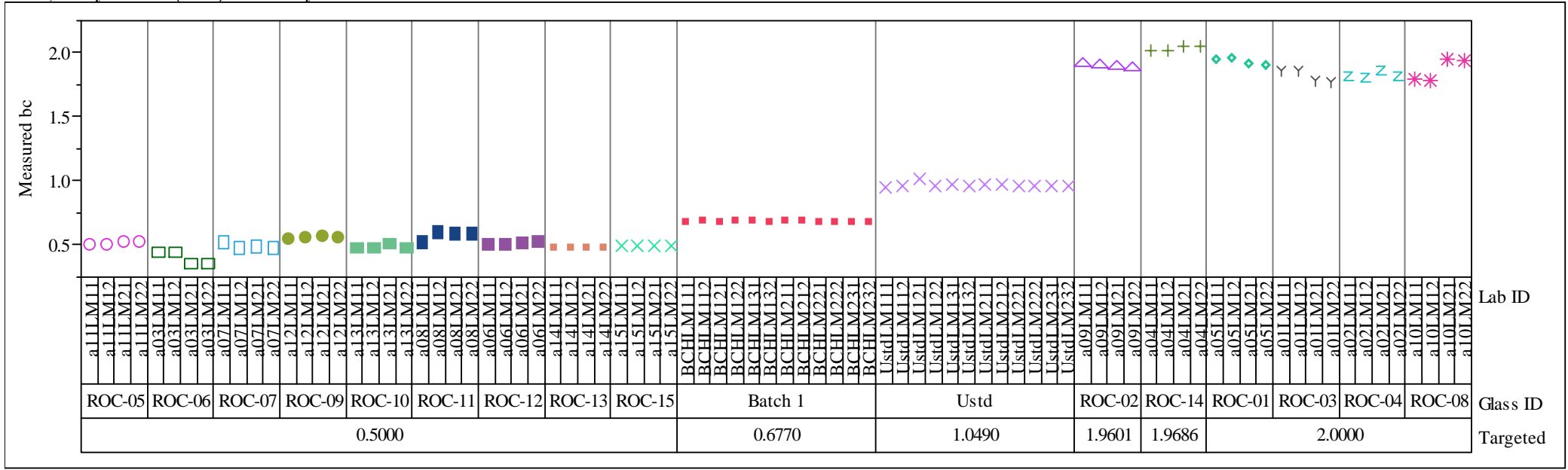


SRNL-STI-2009-00465, Revision 0

Exhibit A5. Oxide Measurements by Lab ID within Glass ID Sorted by Targeted Concentrations

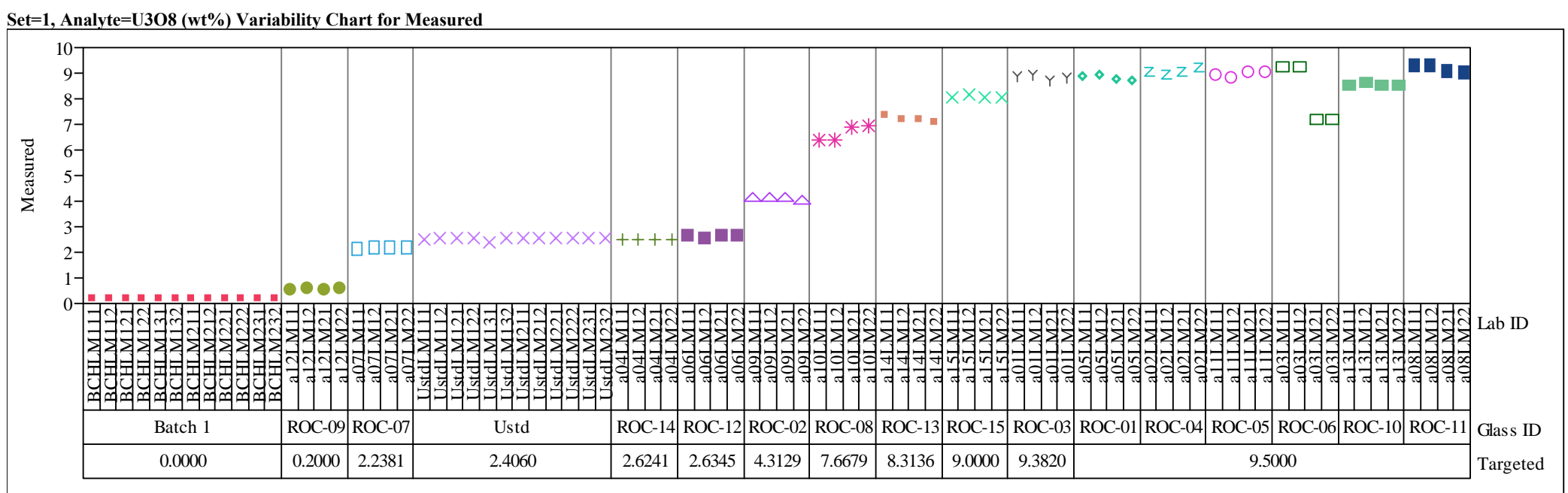

Set=1, Analyte=U308 (wt\%) Variability Chart for Measured bc

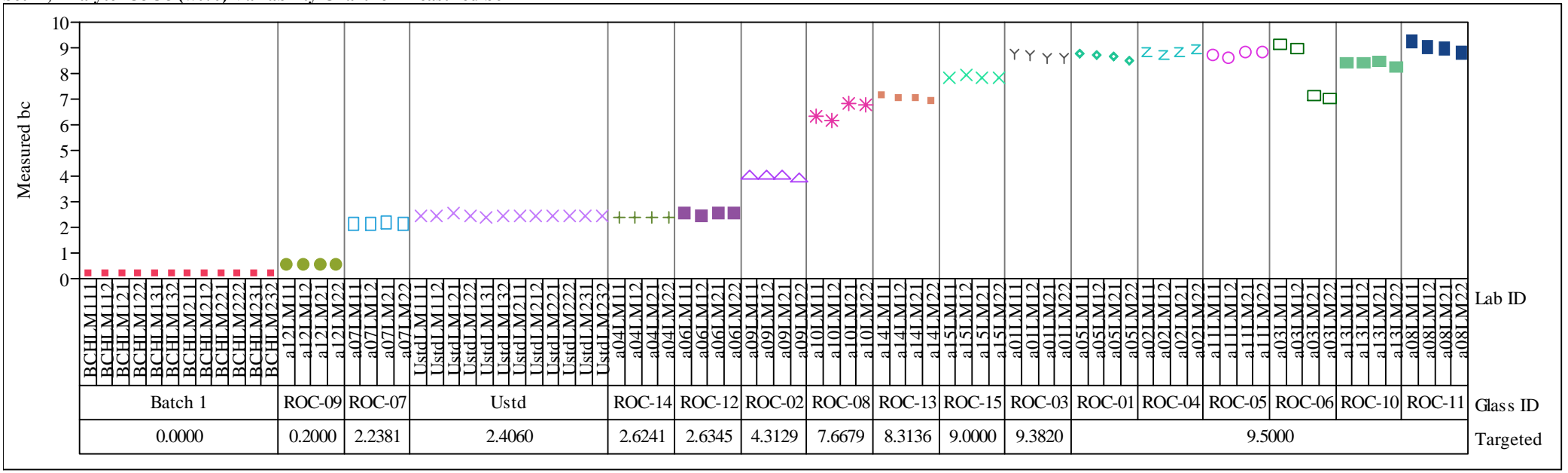


SRNL-STI-2009-00465, Revision 0

Exhibit A5. Oxide Measurements by Lab ID within Glass ID Sorted by Targeted Concentrations

Set $=1$, Analyte $=\mathrm{ZnO}(\mathrm{wt} \%)$ Variability Chart for Measured

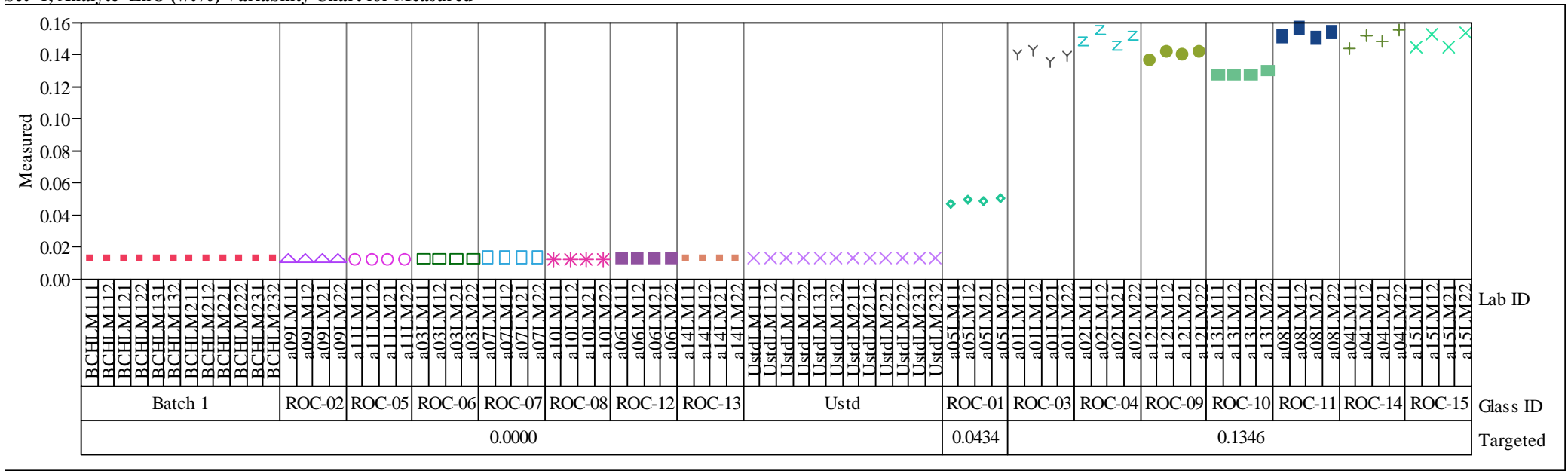

Set $=1$, Analyte $=\mathbf{Z n O}($ wt $\%)$ Variability Chart for Measured bc

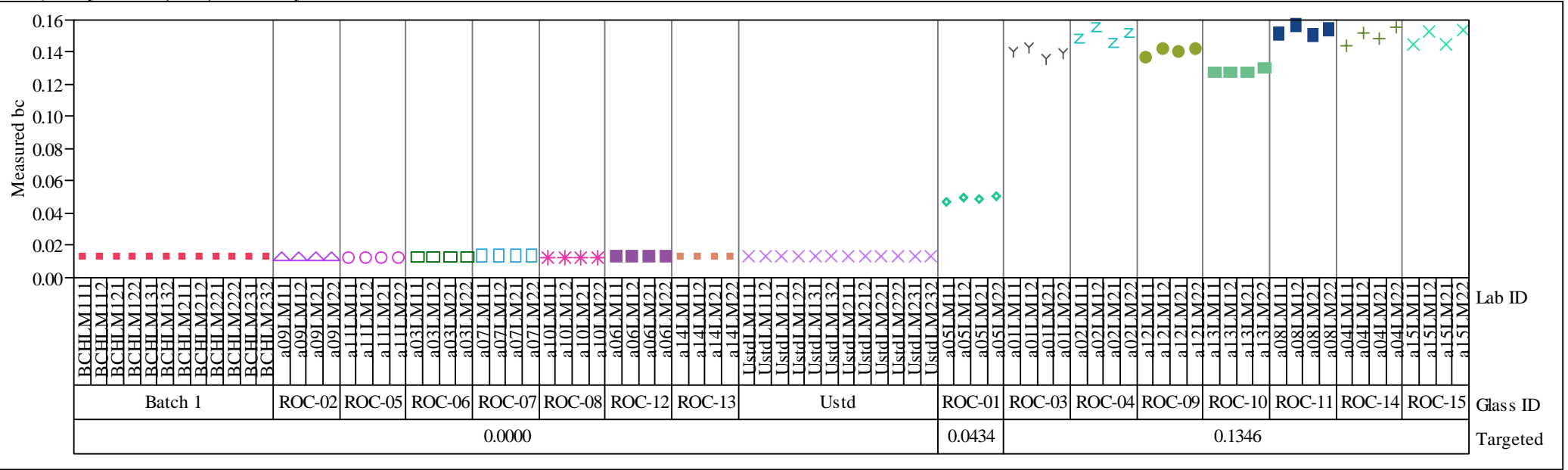


SRNL-STI-2009-00465, Revision 0

Exhibit A5. Oxide Measurements by Lab ID within Glass ID Sorted by Targeted Concentrations

Set $=1$, Analyte $=\mathrm{ZrO} 2(w t \%)$ Variability Chart for Measured

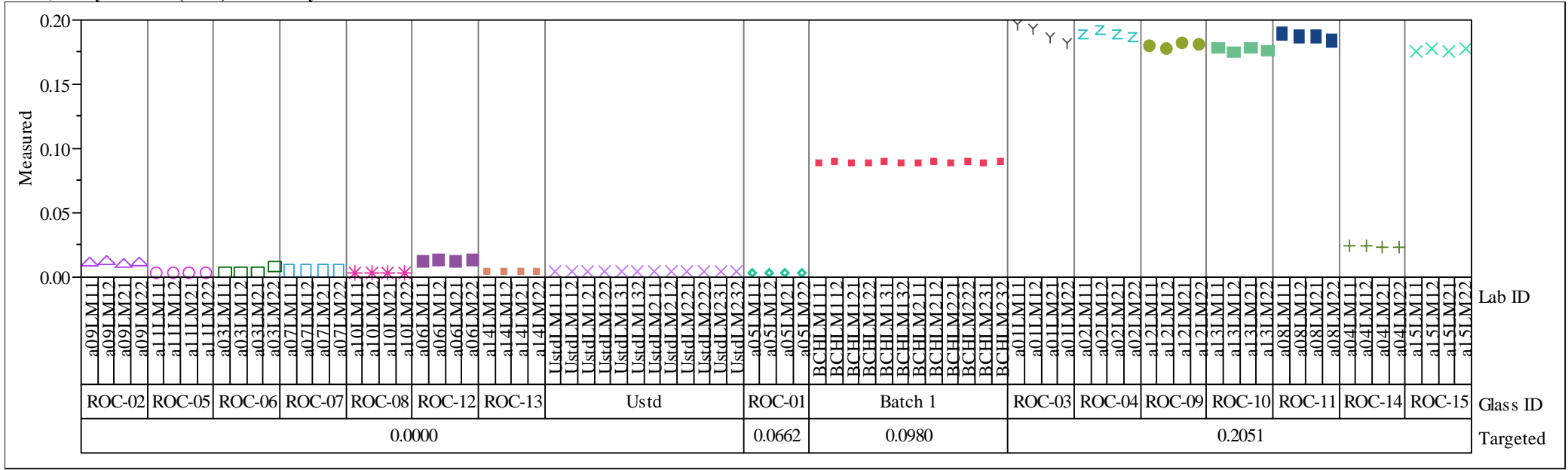

Set=1, Analyte=ZrO2 (wt\%) Variability Chart for Measured bc

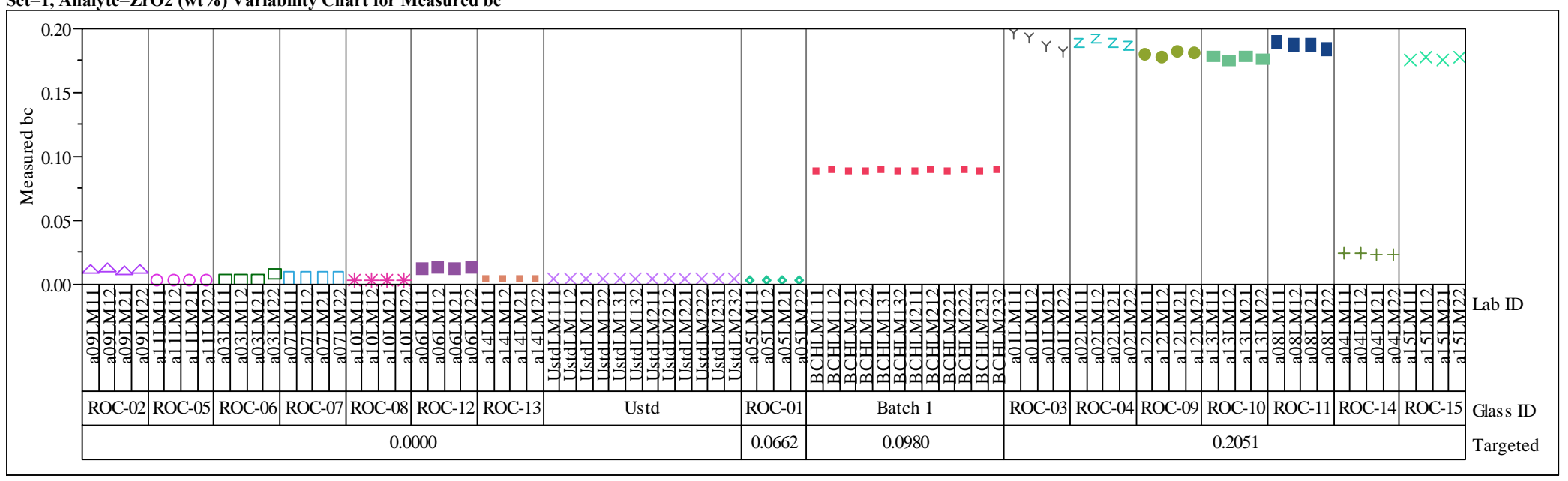


SRNL-STI-2009-00465, Revision 0

\section{Exhibit A5. Oxide Measurements by Lab ID within Glass ID Sorted by Targeted Concentrations}

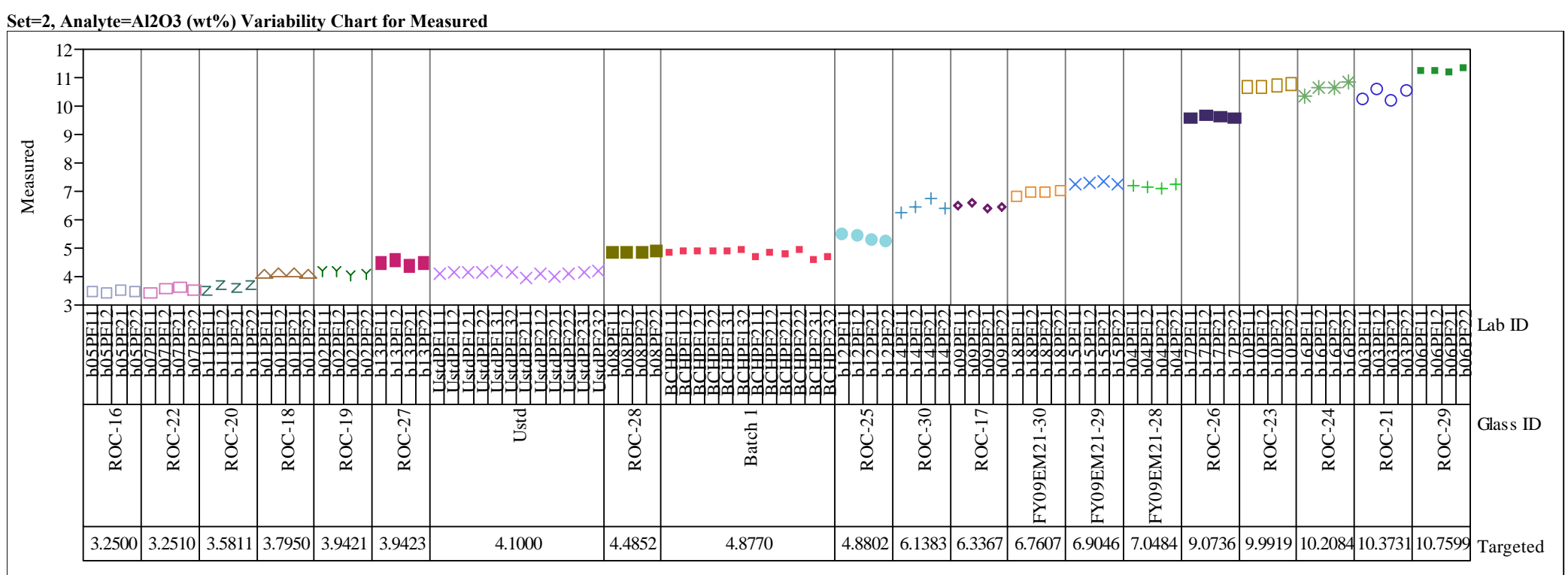

Set $=2$, Analyte $=\mathrm{Al2O}$ (wt $\%$ ) Variability Chart for Measured bc

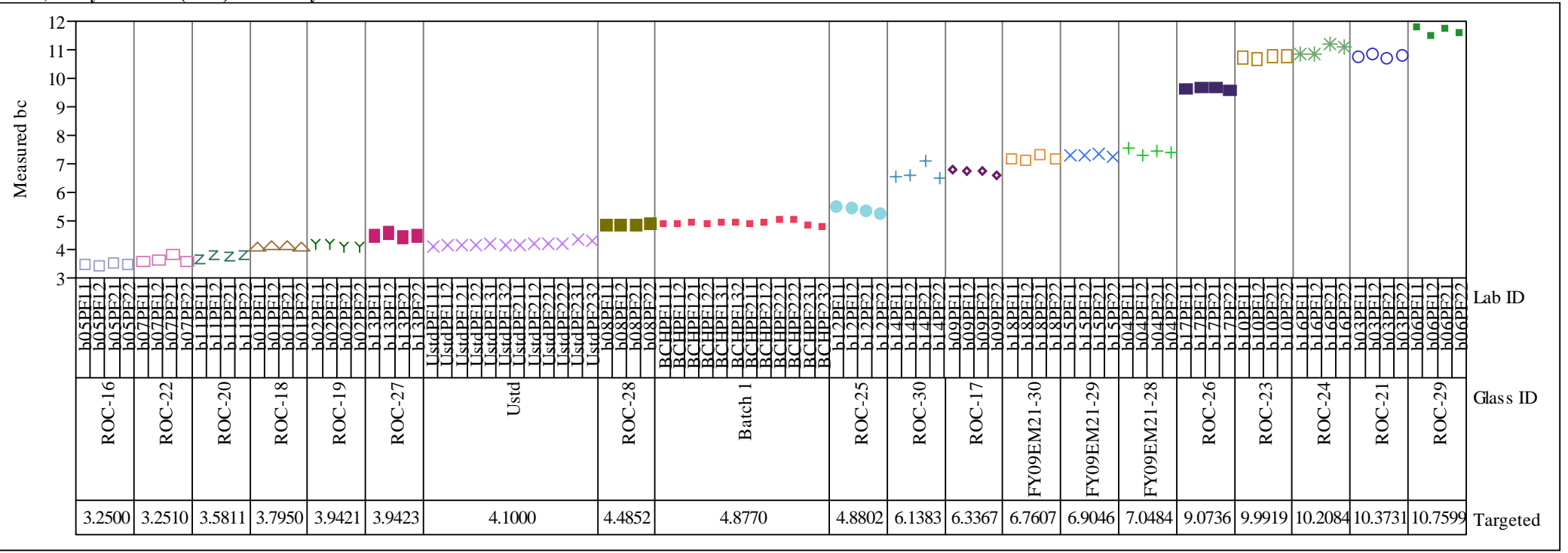


SRNL-STI-2009-00465, Revision 0

Exhibit A5. Oxide Measurements by Lab ID within Glass ID Sorted by Targeted Concentrations

Set $=2$, Analyte $=\mathbf{B 2 O} 3(\mathrm{wt} \%)$ Variability Chart for Measured

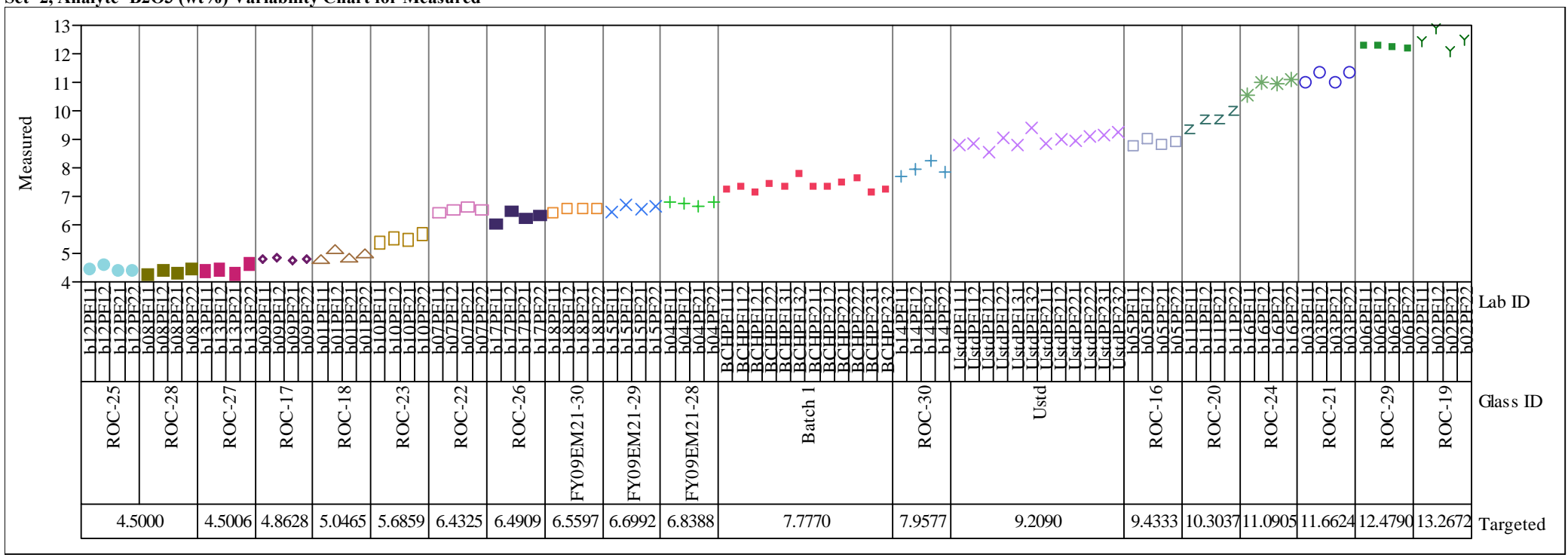

Set=2, Analyte $=\mathbf{B 2 O 3}(\mathrm{wt} \%)$ Variability Chart for Measured bc

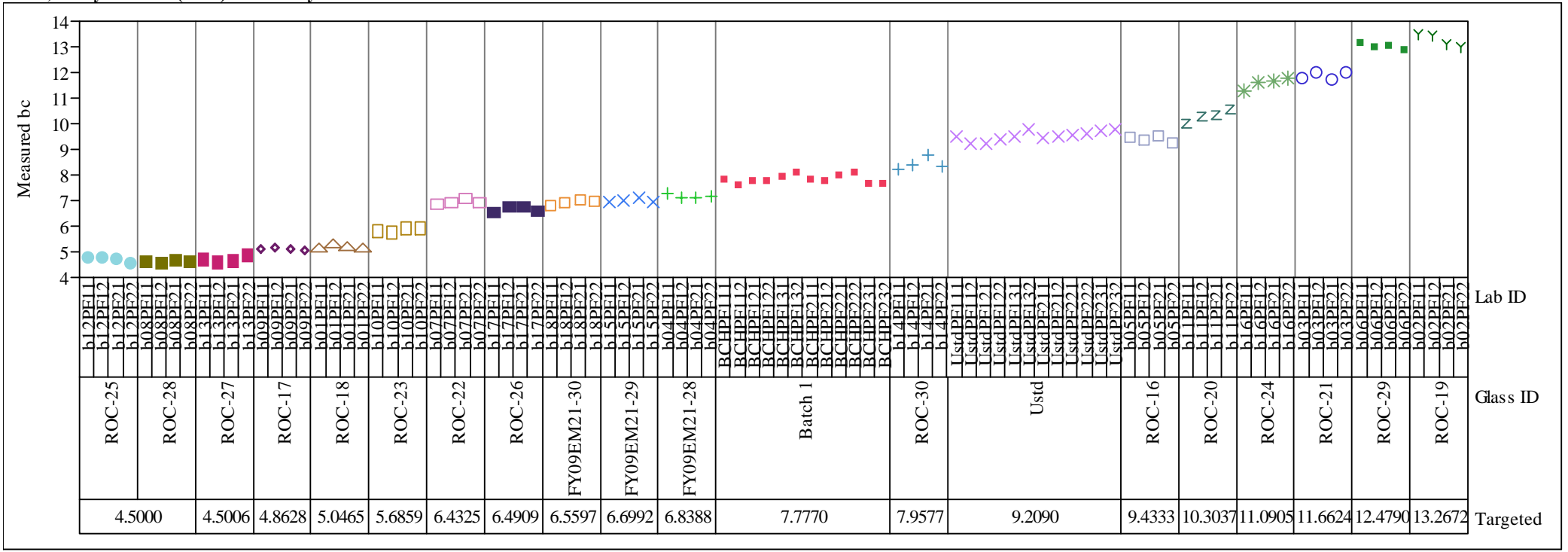


SRNL-STI-2009-00465, Revision 0

Exhibit A5. Oxide Measurements by Lab ID within Glass ID Sorted by Targeted Concentrations

Set $=2$, Analyte $=\mathrm{BaO}(\mathrm{wt} \%)$ Variability Chart for Measured

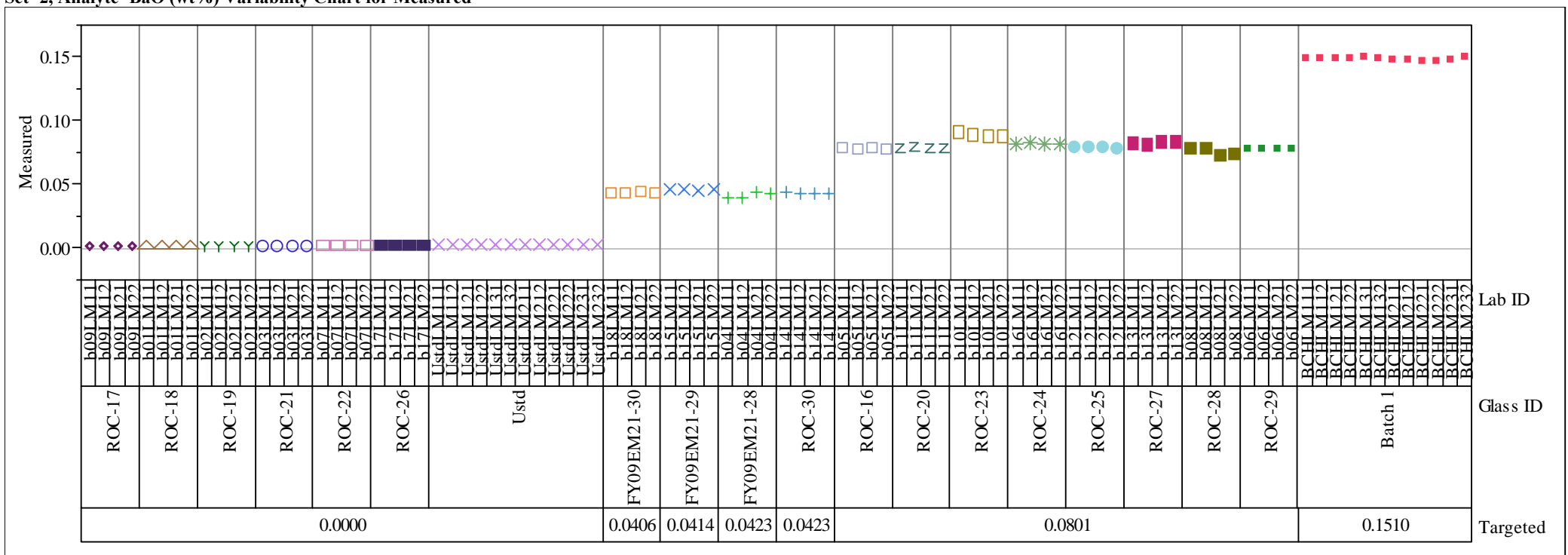

Set $=2$, Analyte $=\mathrm{BaO}(\mathrm{wt} \%)$ Variability Chart for Measured bc

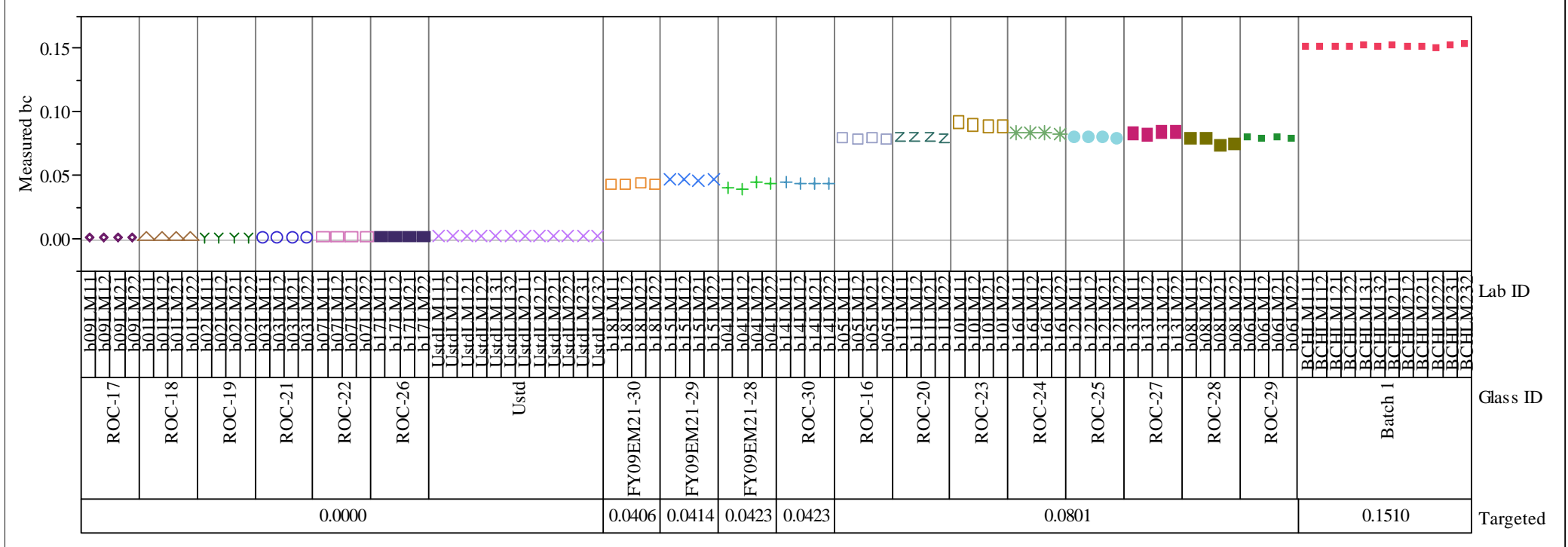


SRNL-STI-2009-00465, Revision 0

\section{Exhibit A5. Oxide Measurements by Lab ID within Glass ID Sorted by Targeted Concentrations}

Set $=2$, Analyte $=\mathrm{CaO}(w \mathrm{t} \%)$ Variability Chart for Measured

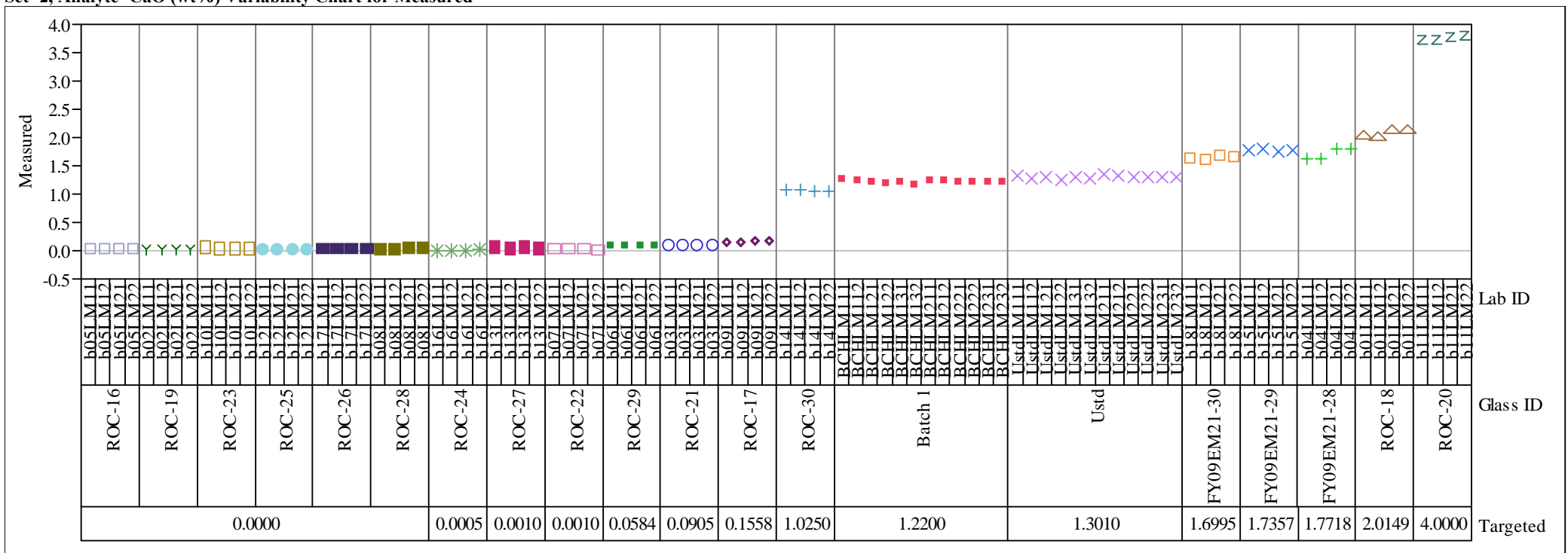

Set $=2$, Analyte $=\mathrm{CaO}(\mathrm{wt} \%)$ Variability Chart for Measured bc

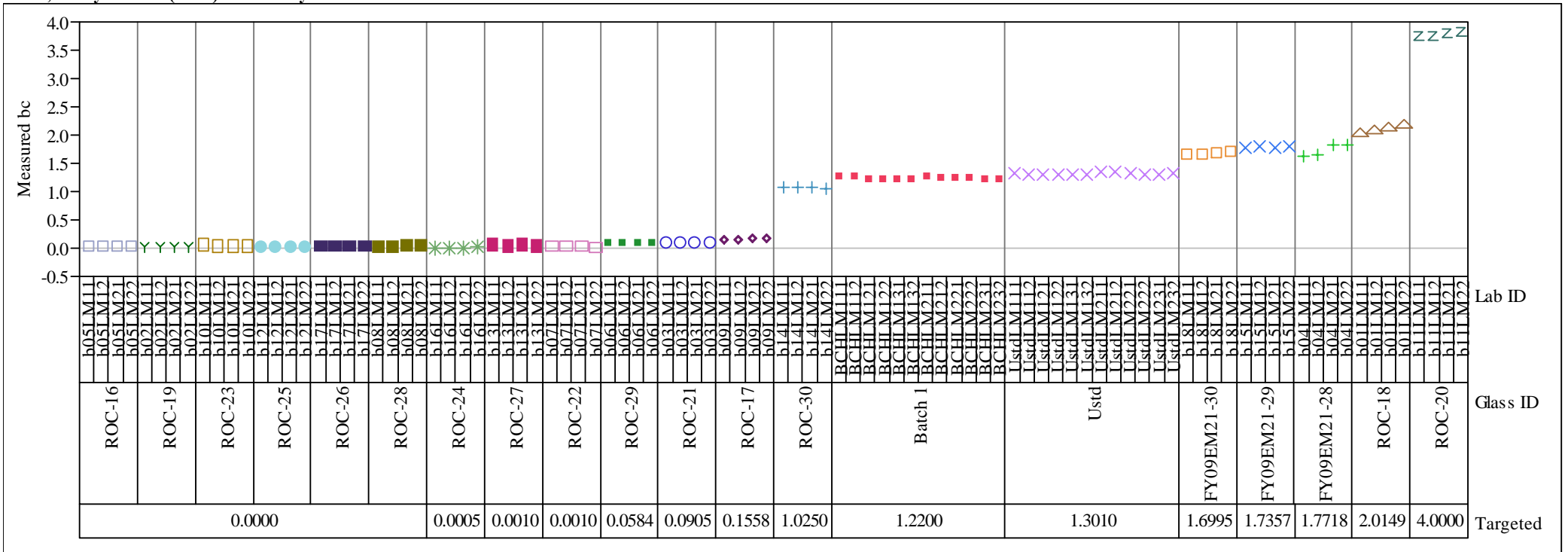


SRNL-STI-2009-00465, Revision 0

Exhibit A5. Oxide Measurements by Lab ID within Glass ID Sorted by Targeted Concentrations

Set $=2$, Analyte $=\mathrm{CdO}(\mathrm{wt} \%)$ Variability Chart for Measured

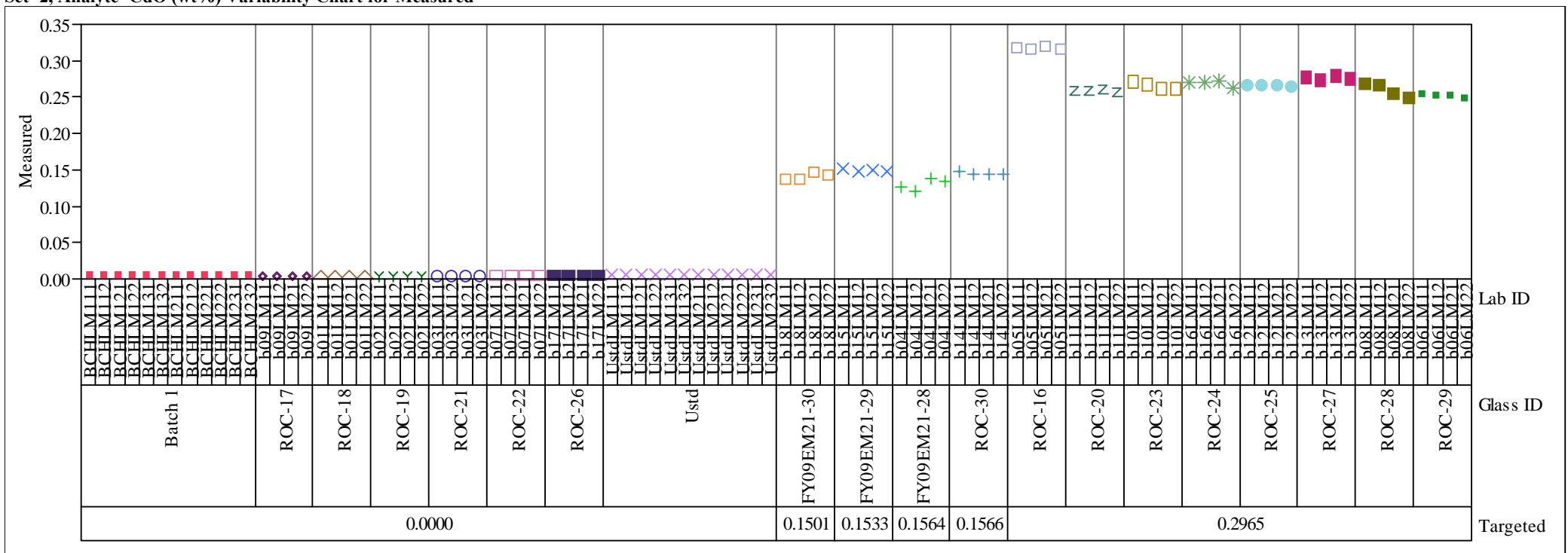

Set $=2$, Analyte $=\mathbf{C d O}($ wt $\%)$ Variability Chart for Measured bc

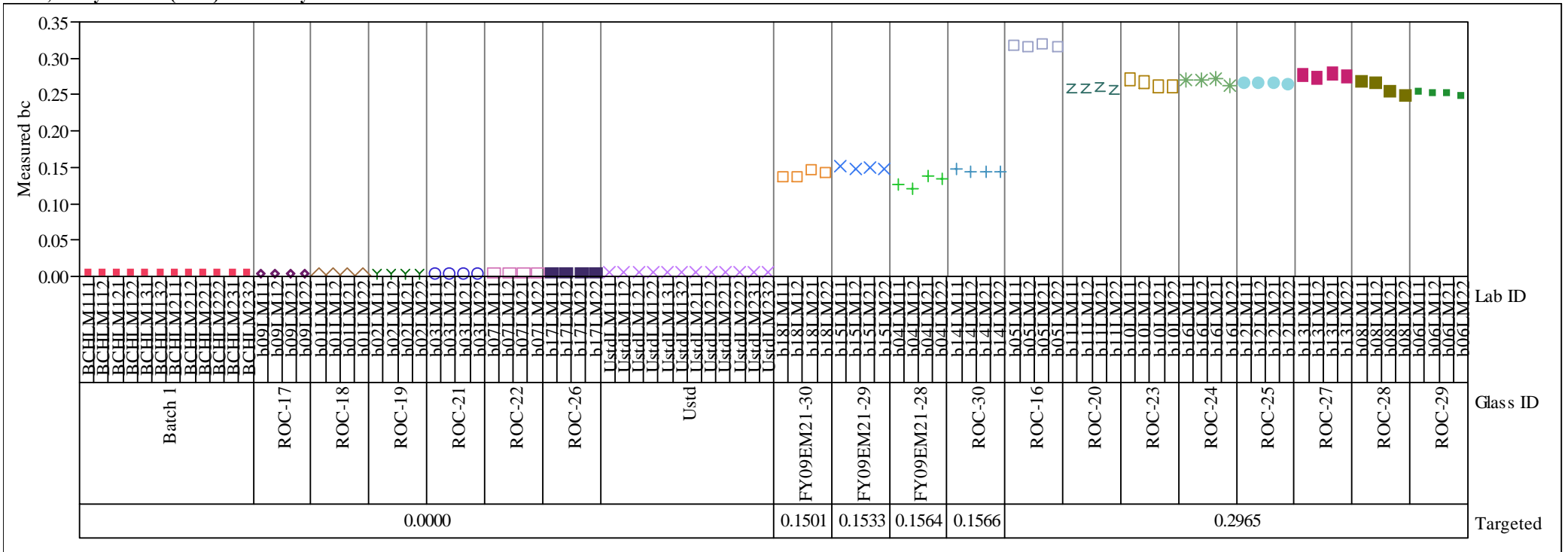

- 125 - 
SRNL-STI-2009-00465, Revision 0

Exhibit A5. Oxide Measurements by Lab ID within Glass ID Sorted by Targeted Concentrations

Set $=2$, Analyte $=\mathrm{Ce} 2 \mathrm{O} 3(\mathrm{wt} \%)$ Variability Chart for Measured

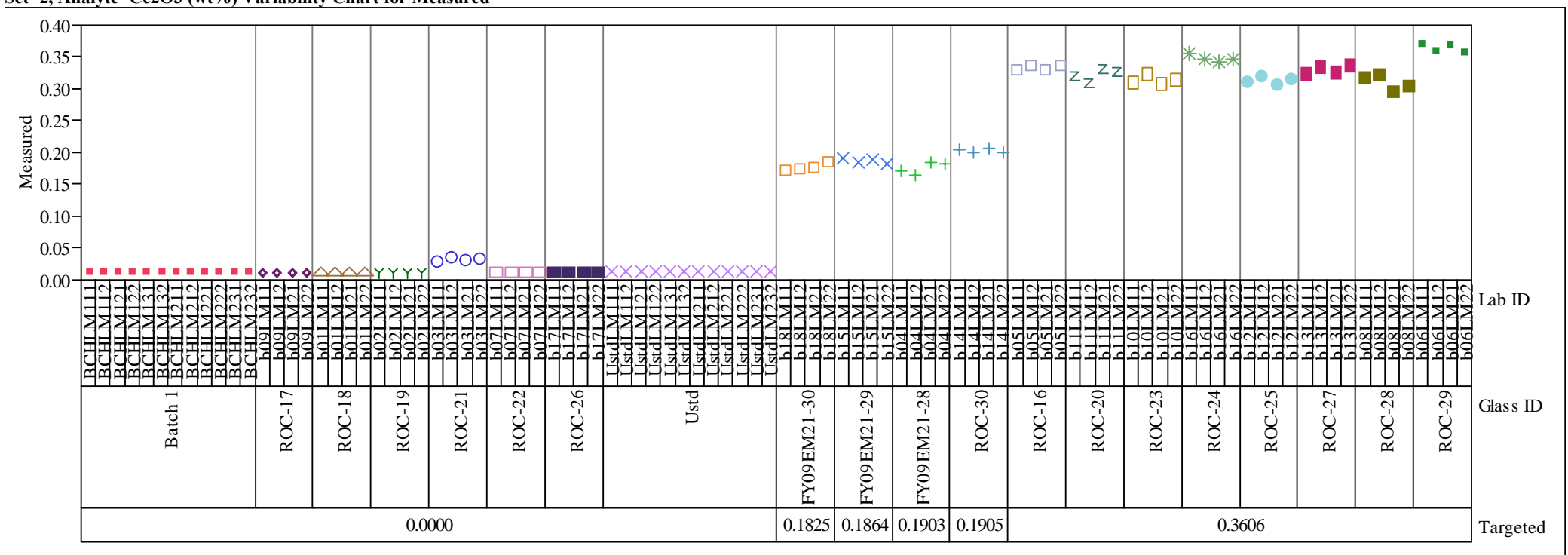

Set $=2$, Analyte $=\mathrm{Ce} 2 \mathrm{O} 3(\mathrm{wt} \%)$ Variability Chart for Measured bc

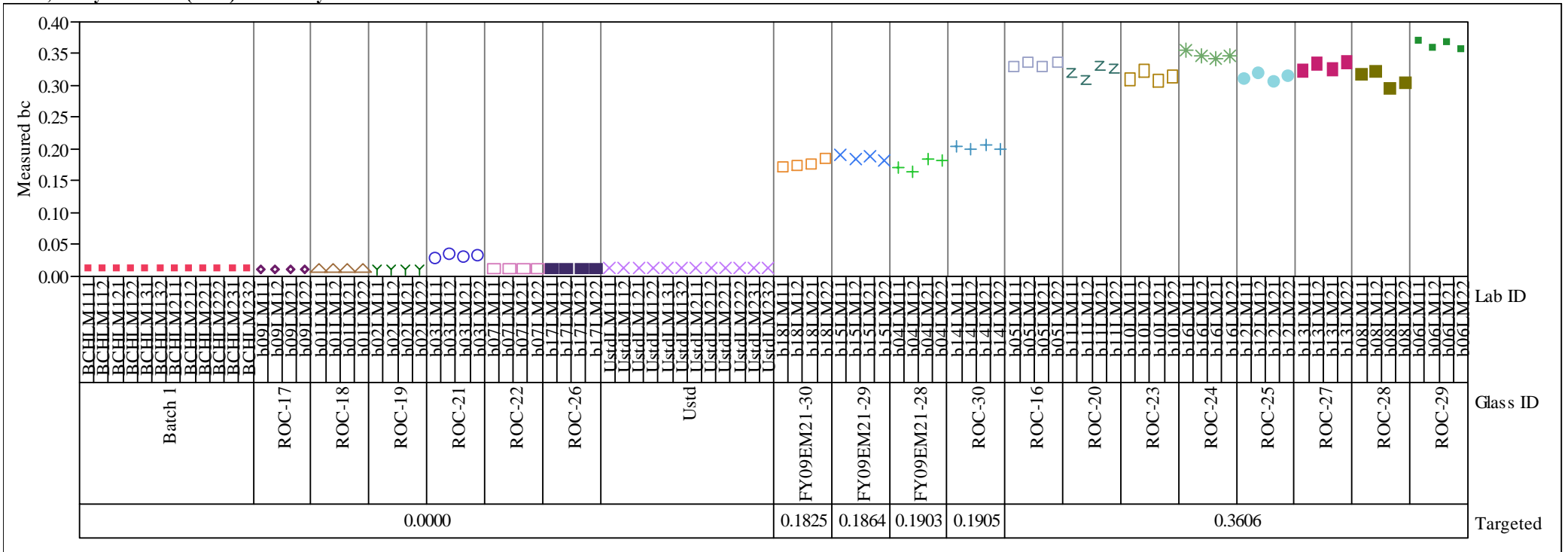


SRNL-STI-2009-00465, Revision 0

Exhibit A5. Oxide Measurements by Lab ID within Glass ID Sorted by Targeted Concentrations

Set $=2$, Analyte $=\mathbf{C r} 203(w t \%)$ Variability Chart for Measured

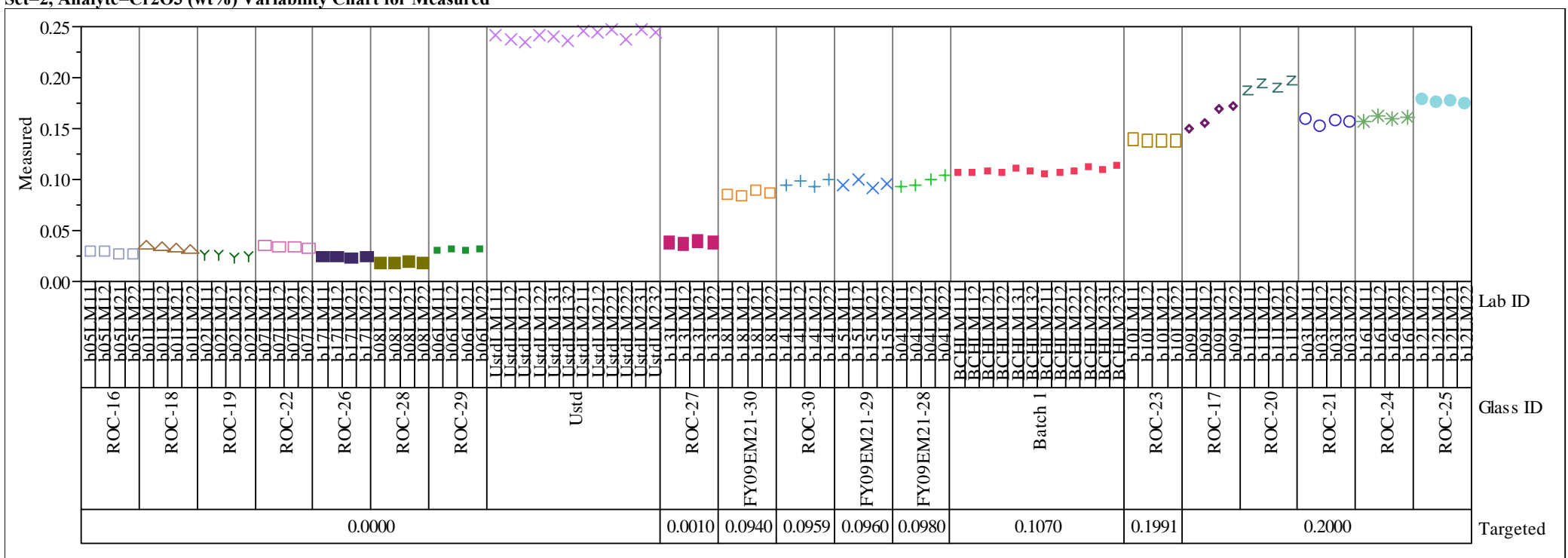

Set $=2$, Analyte $=\mathrm{Cr} 2 \mathrm{O} 3(\mathrm{wt} \%)$ Variability Chart for Measured bc

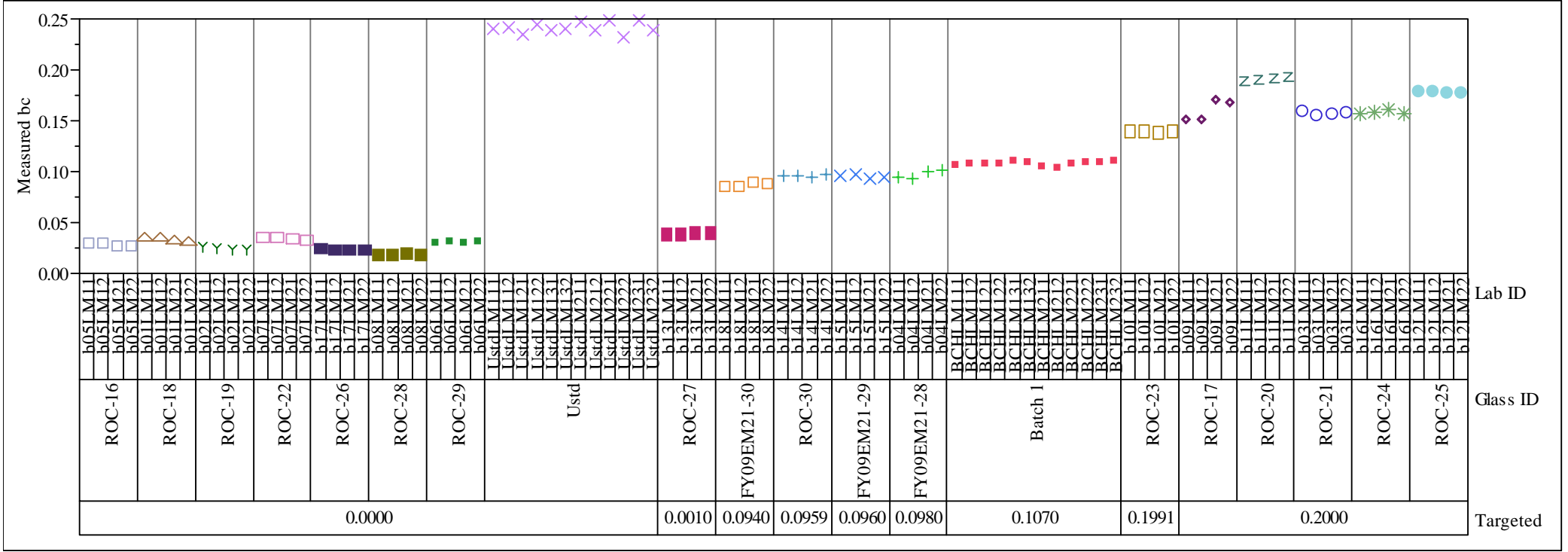


SRNL-STI-2009-00465, Revision 0

Exhibit A5. Oxide Measurements by Lab ID within Glass ID Sorted by Targeted Concentrations

Set $=2$, Analyte $=\mathrm{CuO}(\mathrm{wt} \%)$ Variability Chart for Measured

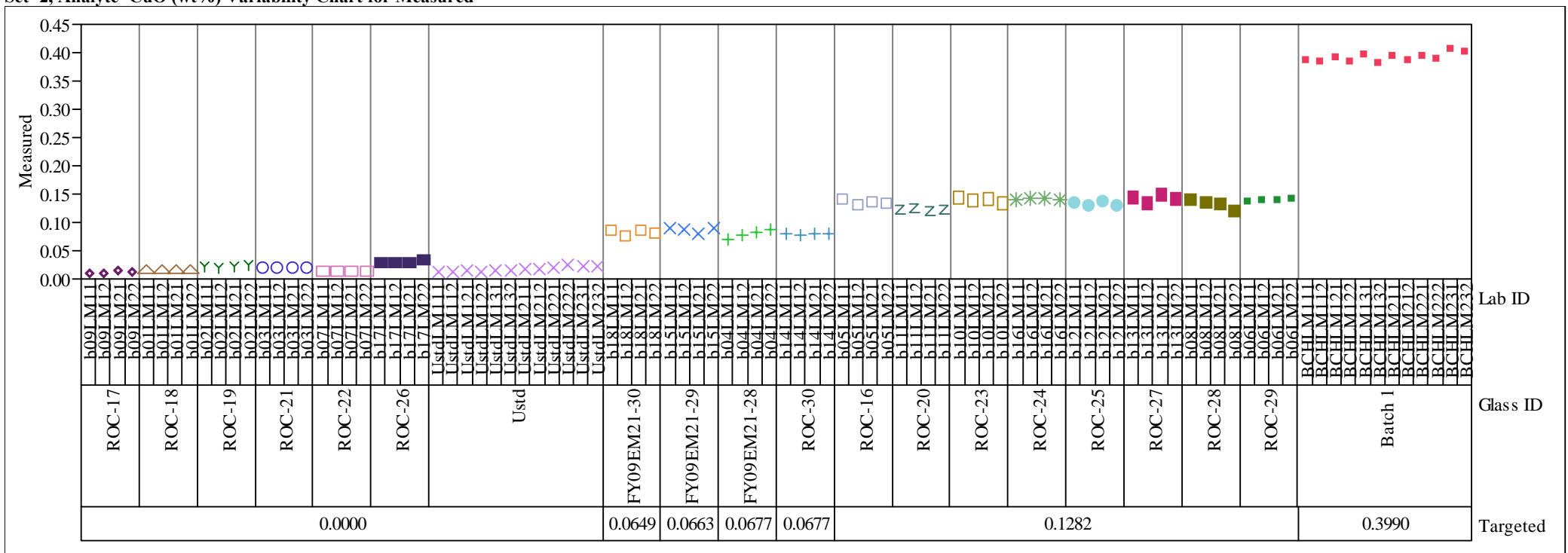

Set $=2$, Analyte $=\mathrm{CuO}(\mathrm{wt} \%)$ Variability Chart for Measured bc

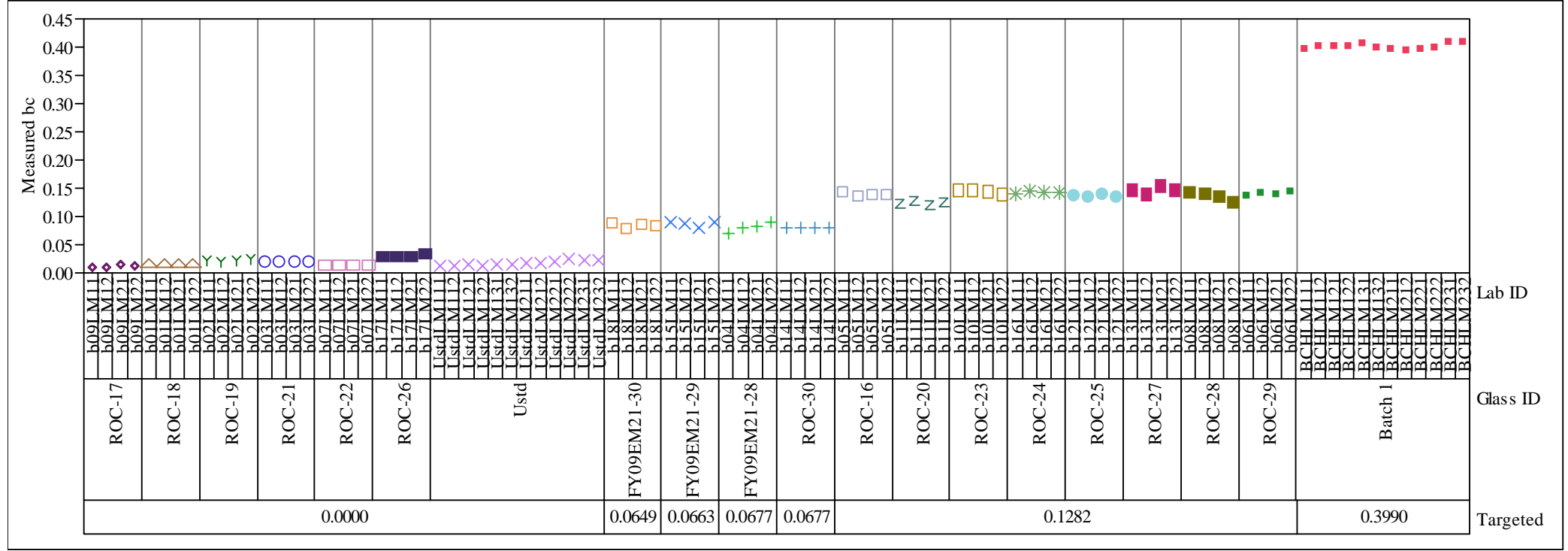


SRNL-STI-2009-00465, Revision 0

\section{Exhibit A5. Oxide Measurements by Lab ID within Glass ID Sorted by Targeted Concentrations}

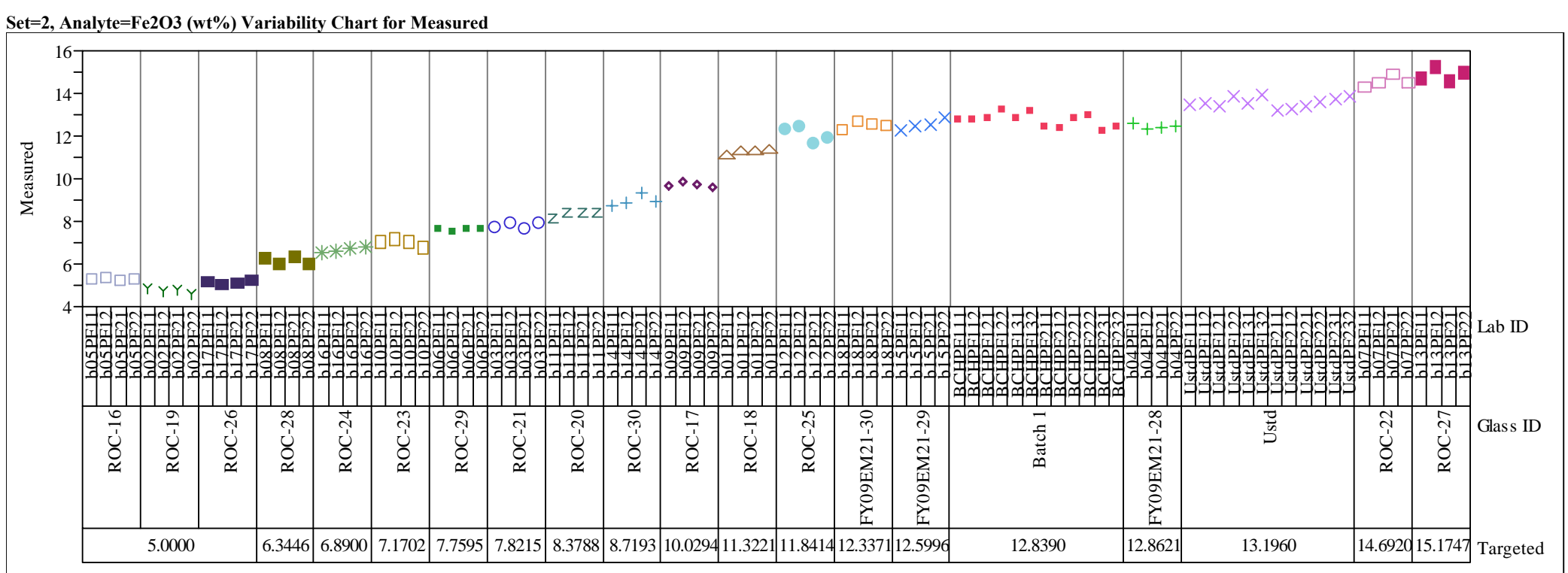

Set=2, Analyte=Fe2O3 (wt\%) Variability Chart for Measured bc

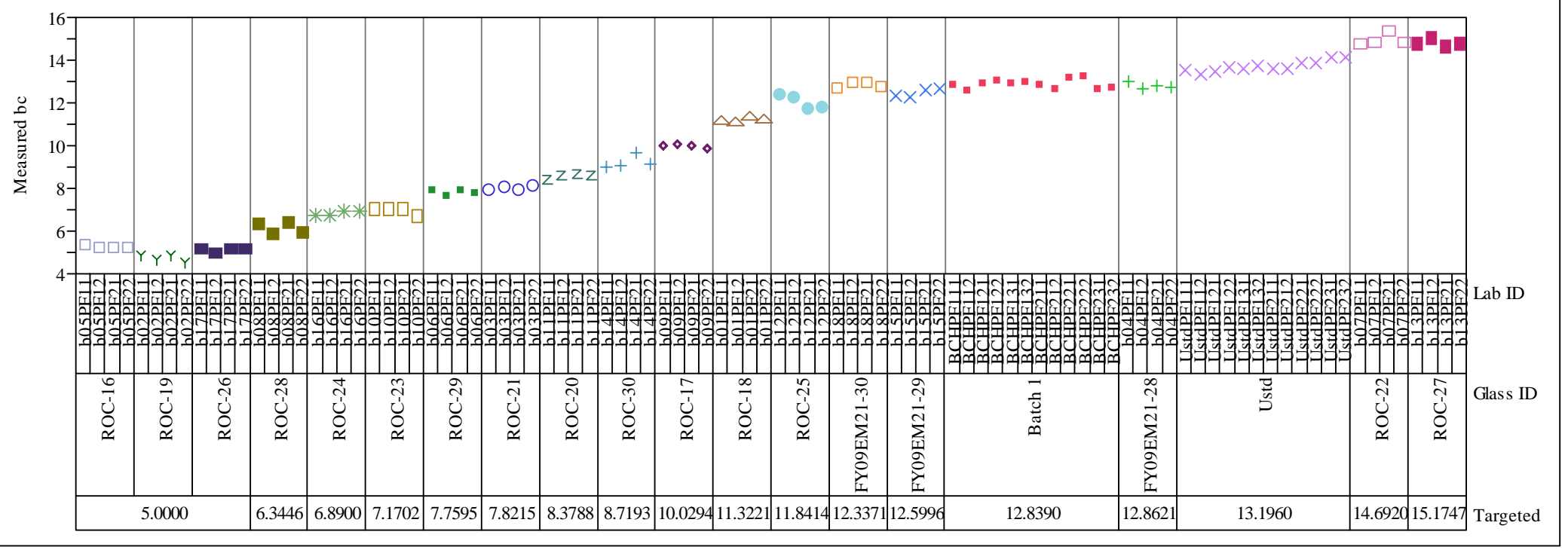




\section{Exhibit A5. Oxide Measurements by Lab ID within Glass ID Sorted by Targeted Concentrations}

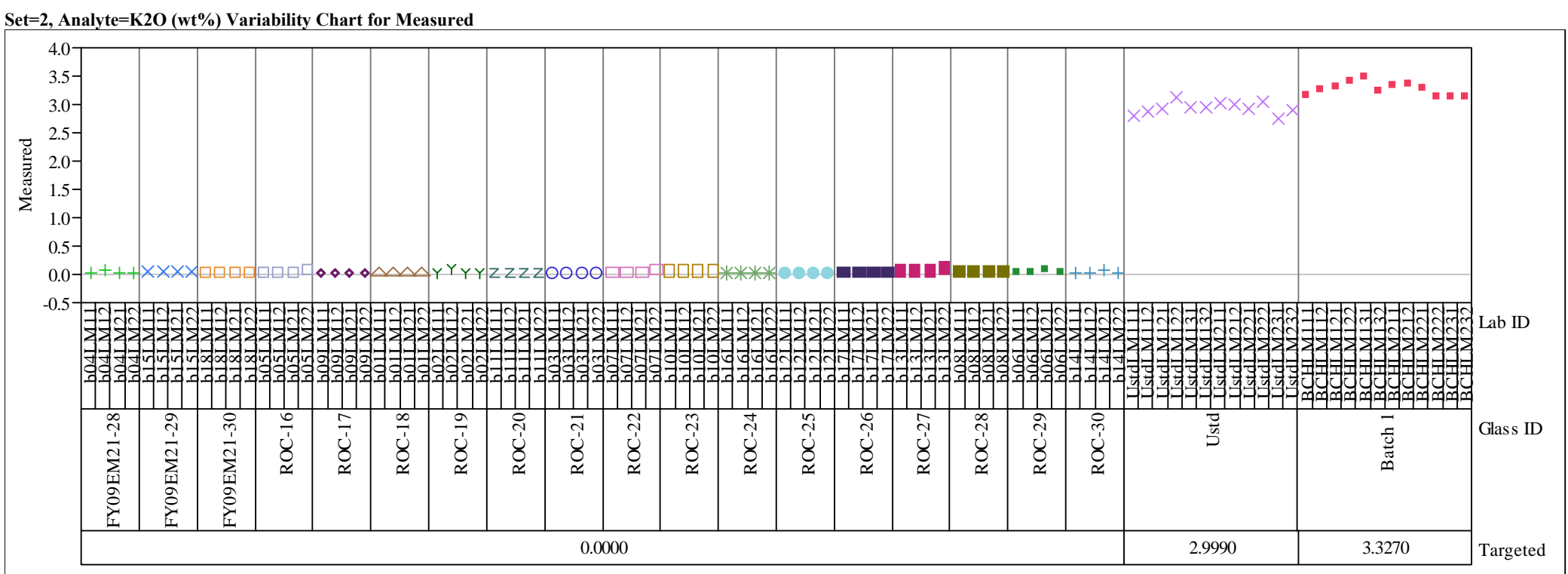

Set $=2$, Analyte $=$ K2O (wt\%) Variability Chart for Measured bc

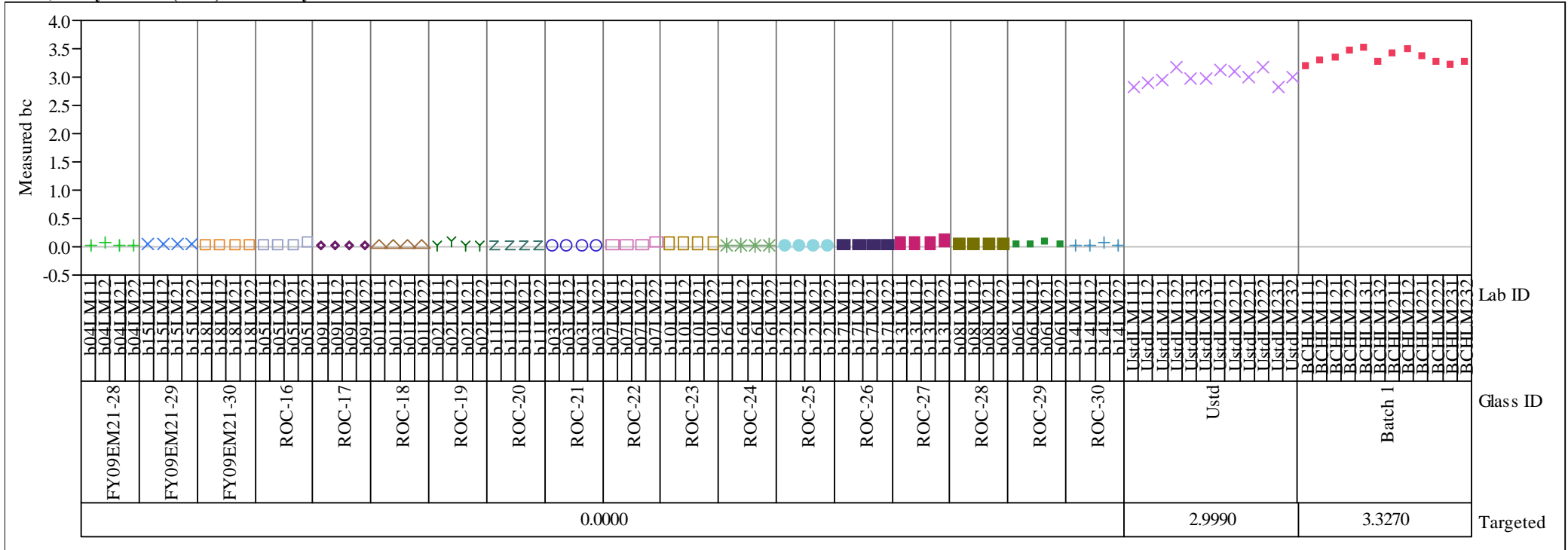


SRNL-STI-2009-00465, Revision 0

Exhibit A5. Oxide Measurements by Lab ID within Glass ID Sorted by Targeted Concentrations

Set $=2$, Analyte $=\mathrm{La2O3}(\mathrm{wt} \%)$ Variability Chart for Measured

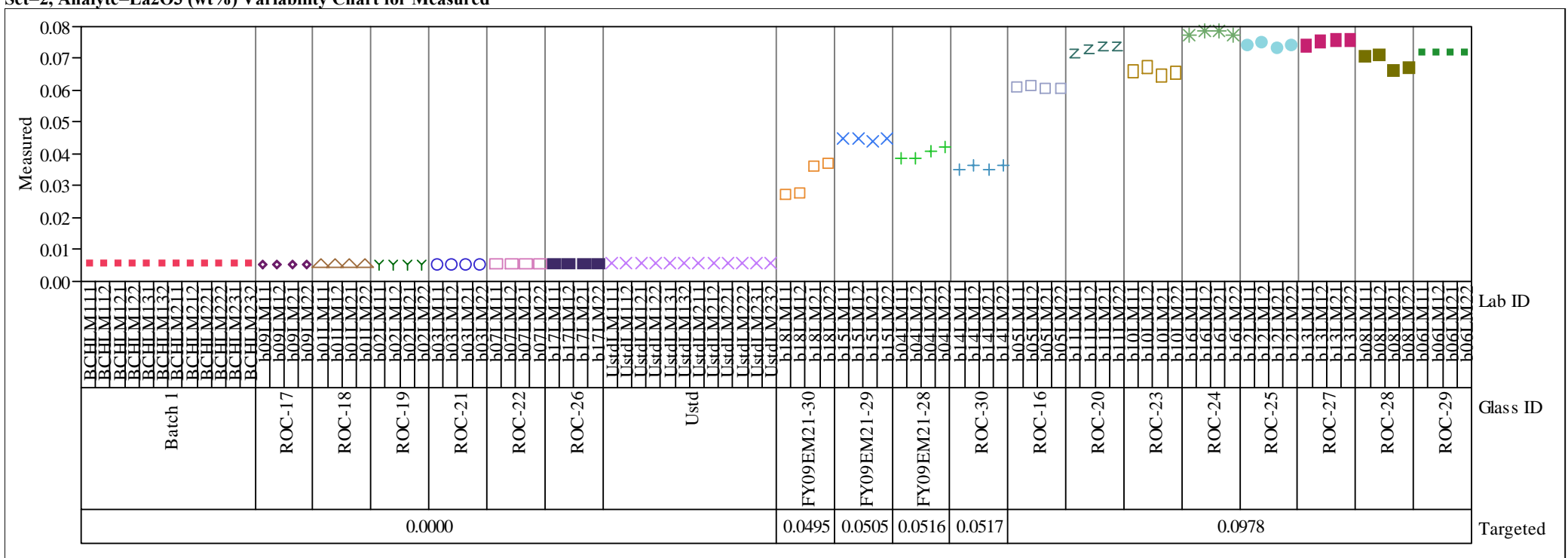

Set $=2$, Analyte $=$ La2O3 (wt $\%$ ) Variability Chart for Measured bc

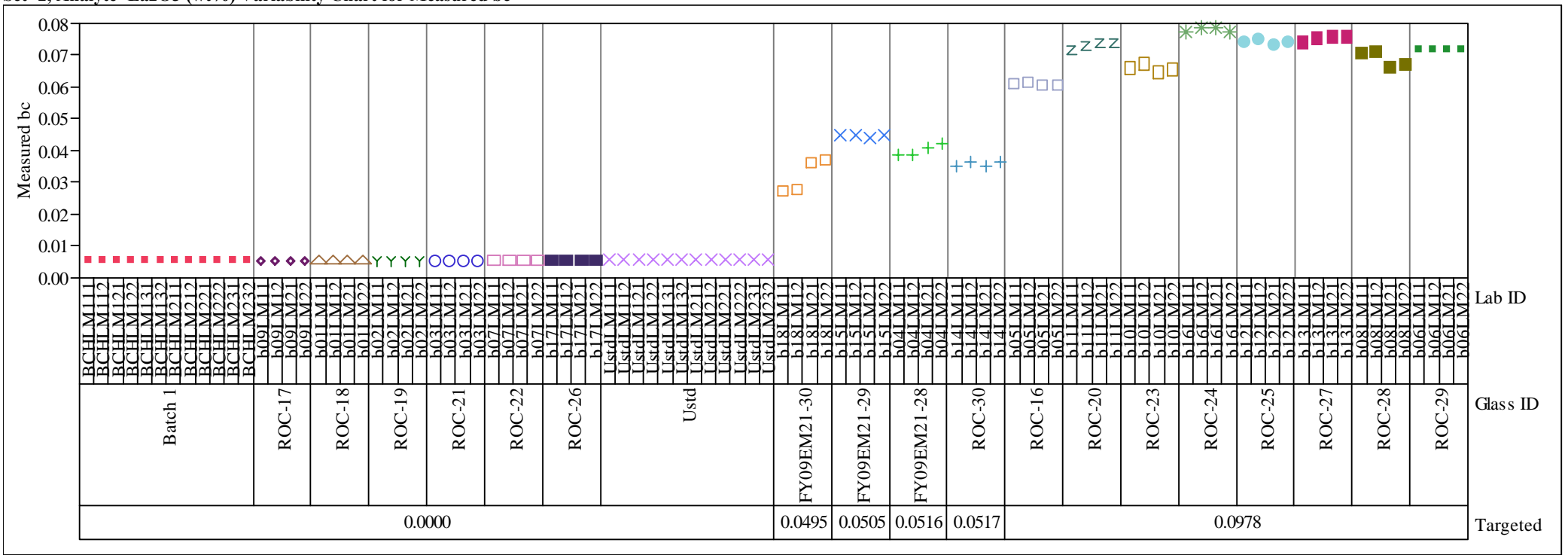


SRNL-STI-2009-00465, Revision 0

Exhibit A5. Oxide Measurements by Lab ID within Glass ID Sorted by Targeted Concentrations

Set $=2$, Analyte $=$ Li2O $($ wt $\%)$ Variability Chart for Measured

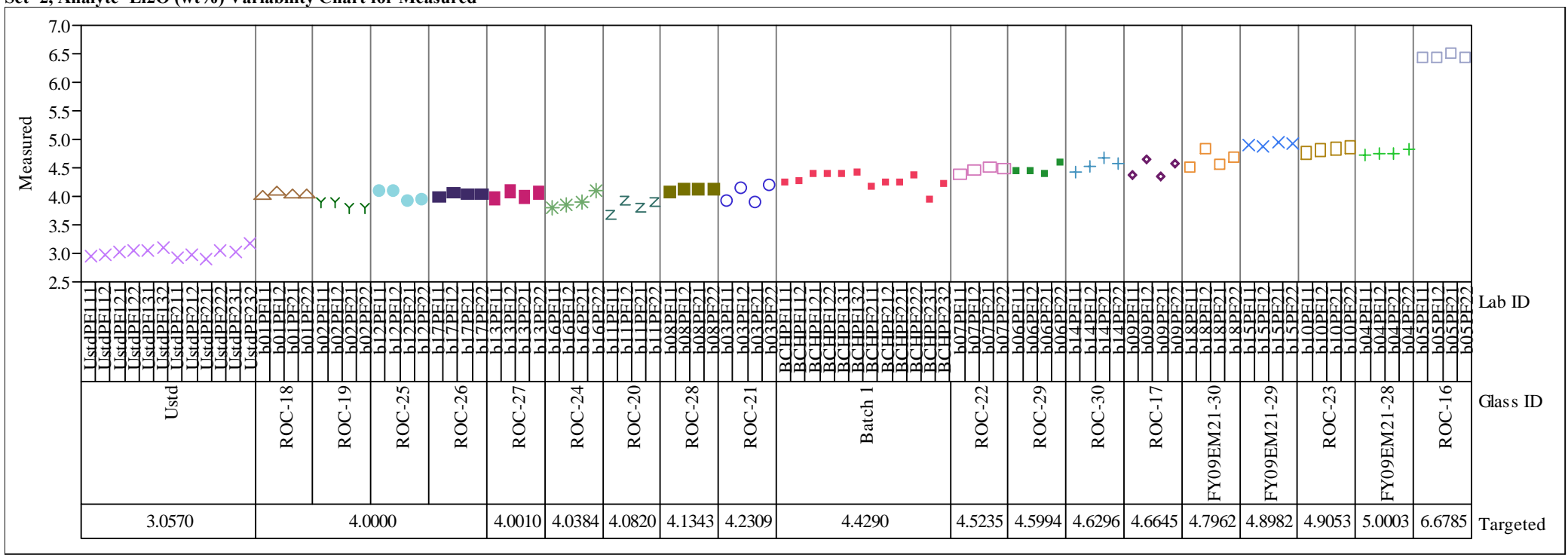

Set=2, Analyte=Li2O (wt\%) Variability Chart for Measured bc

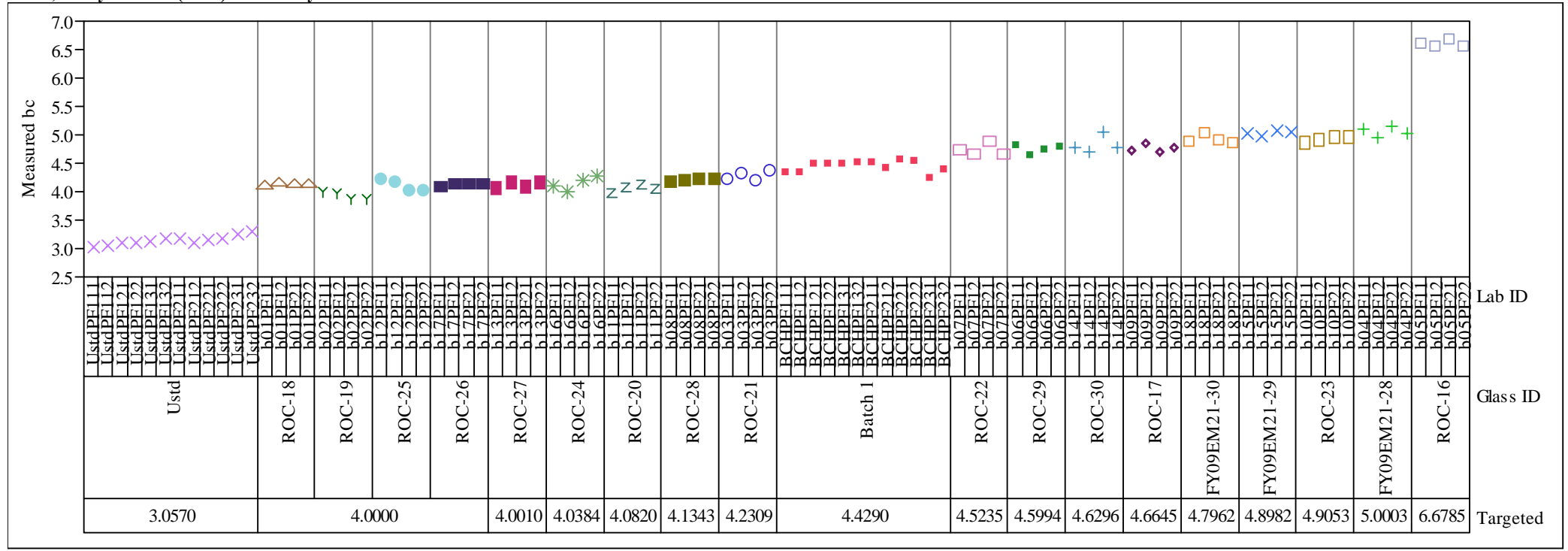


SRNL-STI-2009-00465, Revision 0

Exhibit A5. Oxide Measurements by Lab ID within Glass ID Sorted by Targeted Concentrations

Set $=2$, Analyte $=\mathrm{MgO}(\mathrm{wt} \%)$ Variability Chart for Measured

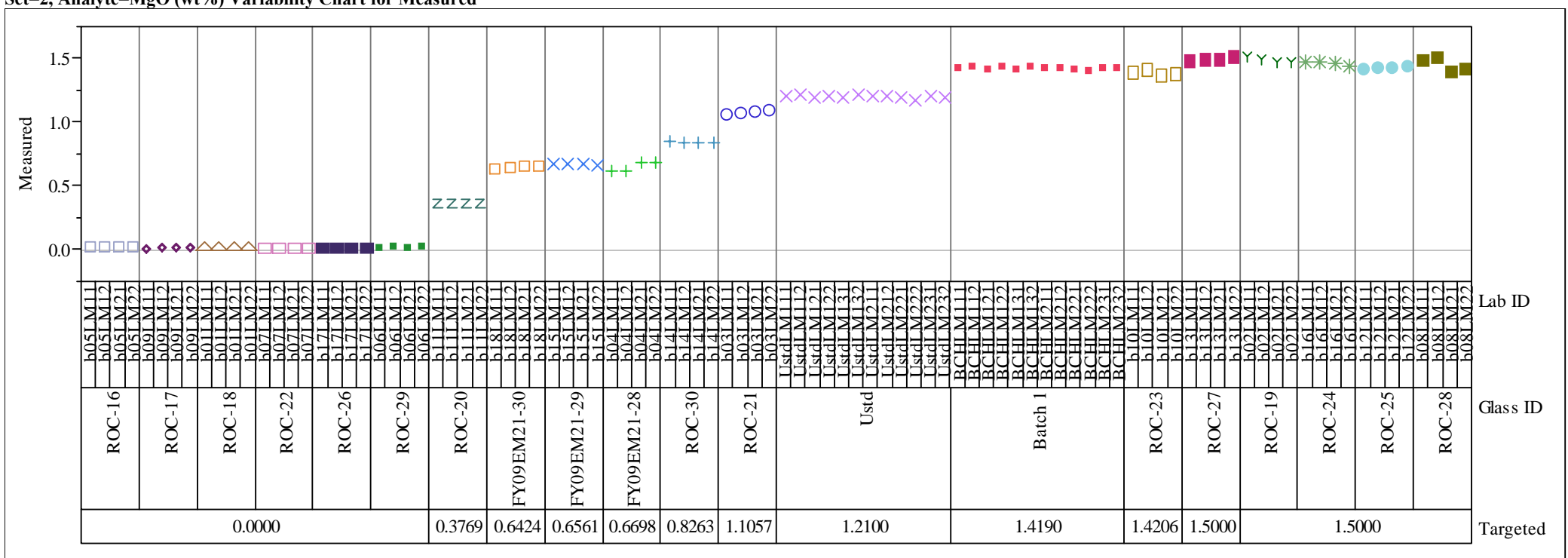

Set $=2$, Analyte $=\mathbf{M g O}(\mathrm{wt} \%)$ Variability Chart for Measured bc

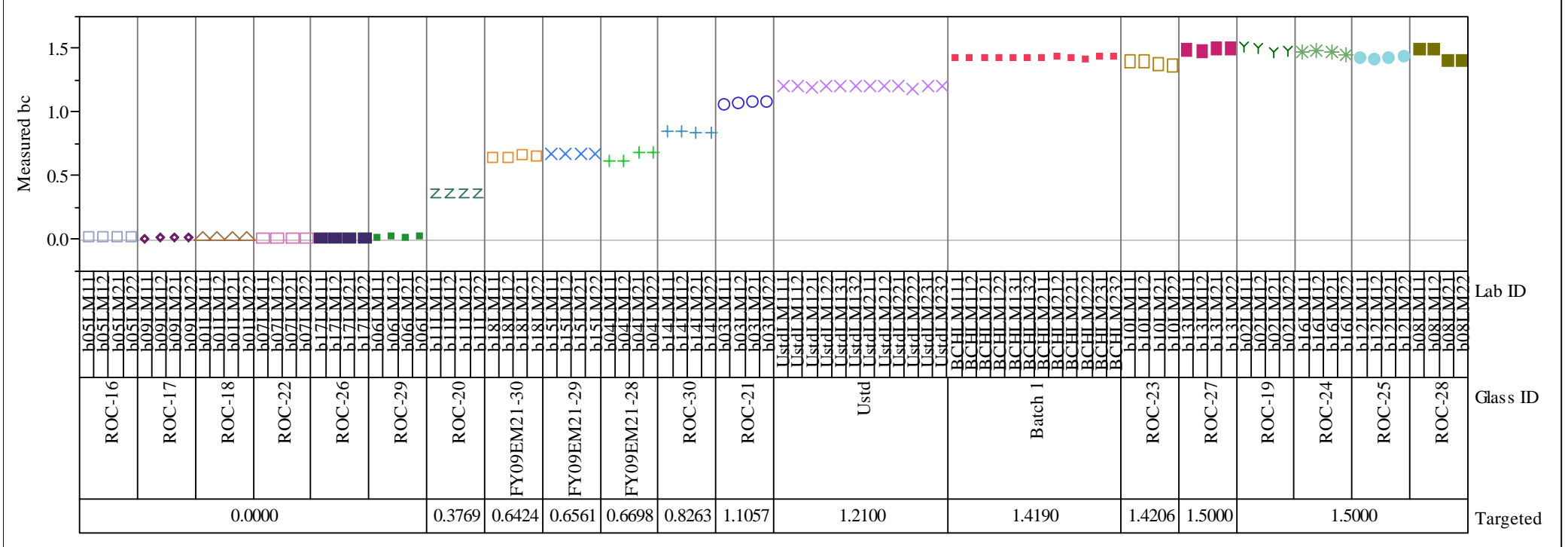




\section{Exhibit A5. Oxide Measurements by Lab ID within Glass ID Sorted by Targeted Concentrations}

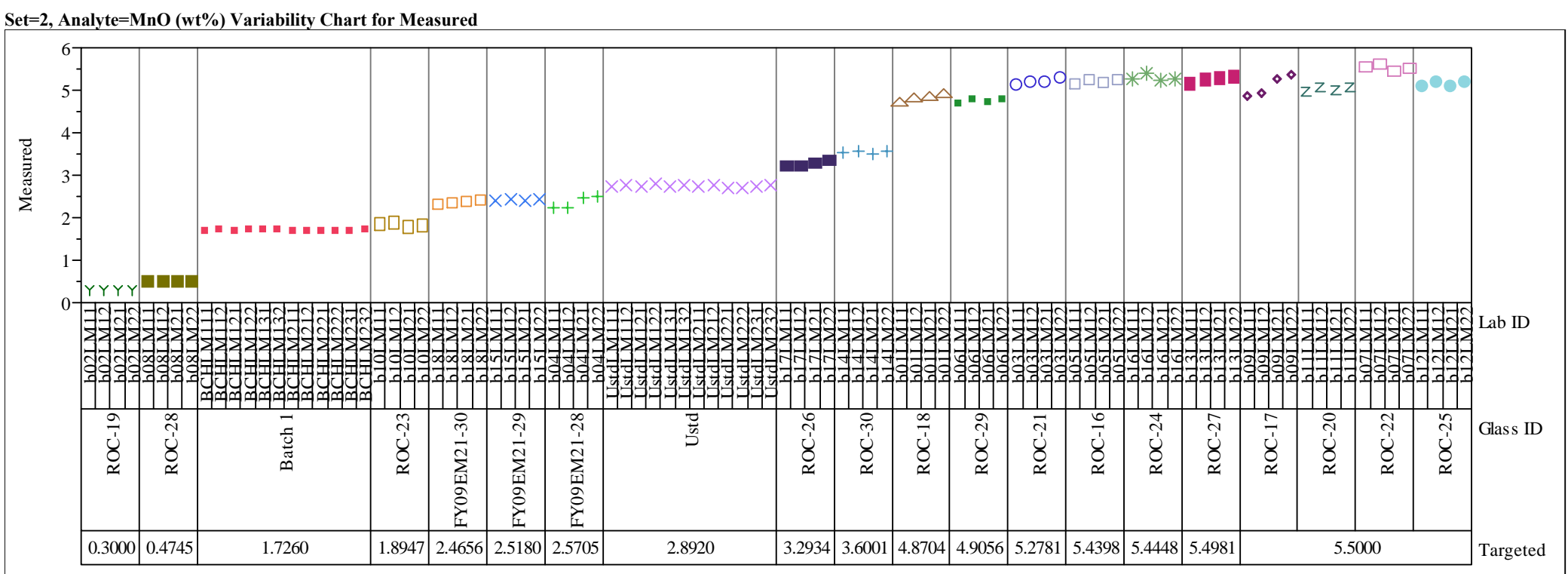

Set $=2$, Analyte $=\mathrm{MnO}(\mathrm{wt} \%)$ Variability Chart for Measured bc

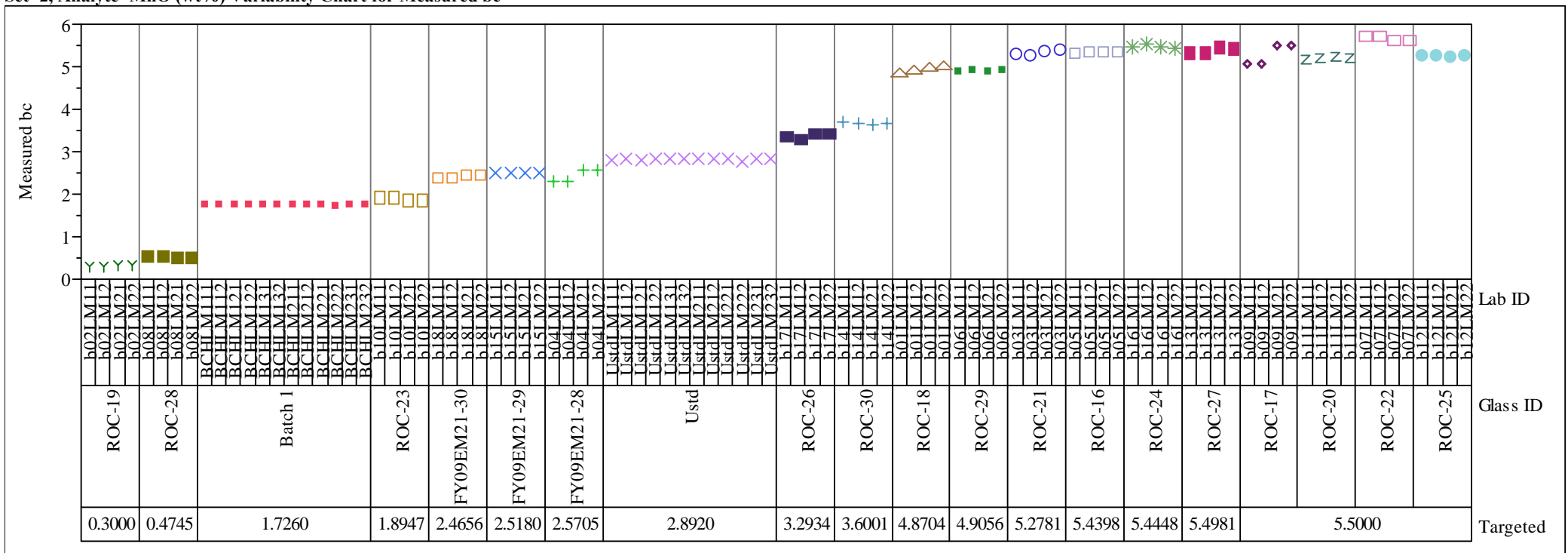


SRNL-STI-2009-00465, Revision 0

Exhibit A5. Oxide Measurements by Lab ID within Glass ID Sorted by Targeted Concentrations

Set $=2$, Analyte $=\mathrm{Na2O}(\mathrm{wt} \%)$ Variability Chart for Measured

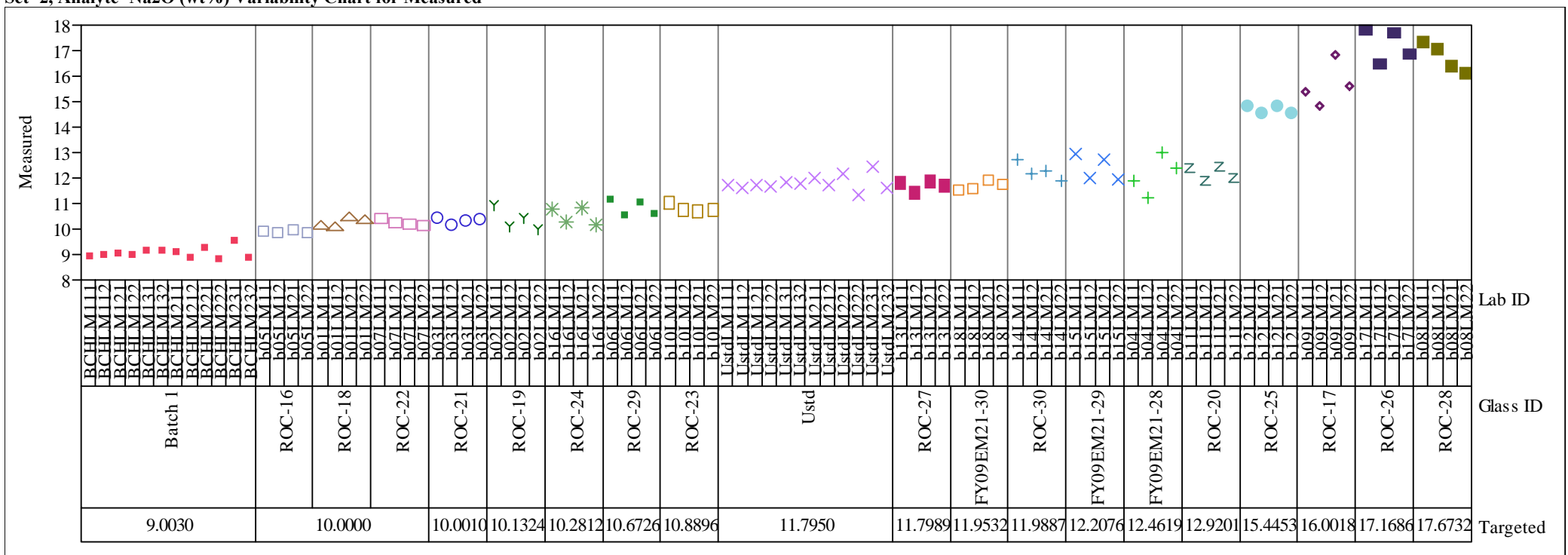

Set $=2$, Analyte $=\mathrm{Na} 2 \mathrm{O}(\mathrm{wt} \%)$ Variability Chart for Measured bc

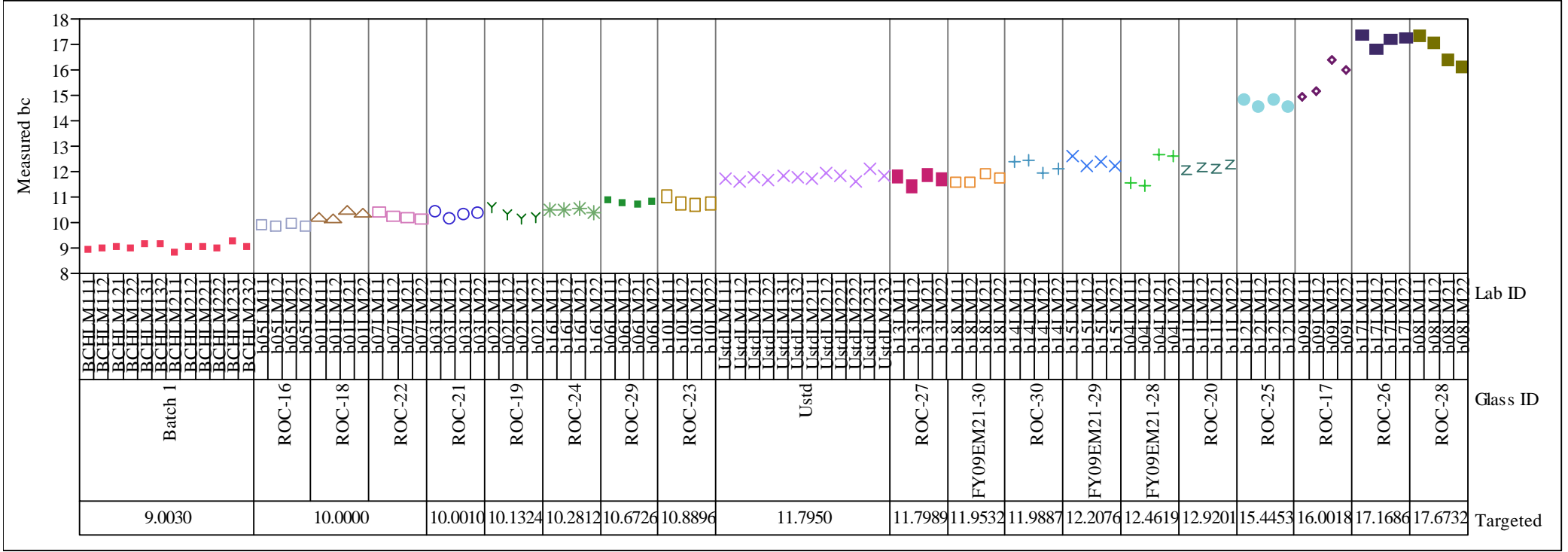

- 135 - 


\section{Exhibit A5. Oxide Measurements by Lab ID within Glass ID Sorted by Targeted Concentrations}

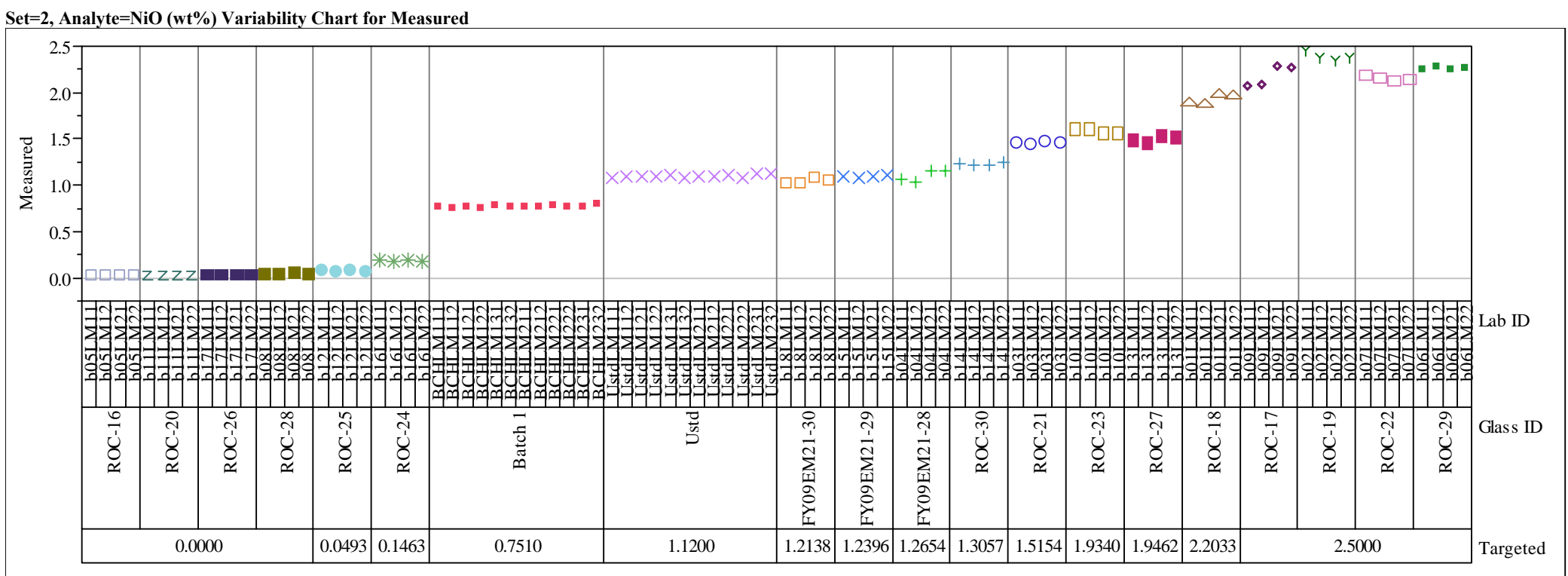

Set=2, Analyte=NiO (wt\%) Variability Chart for Measured bc

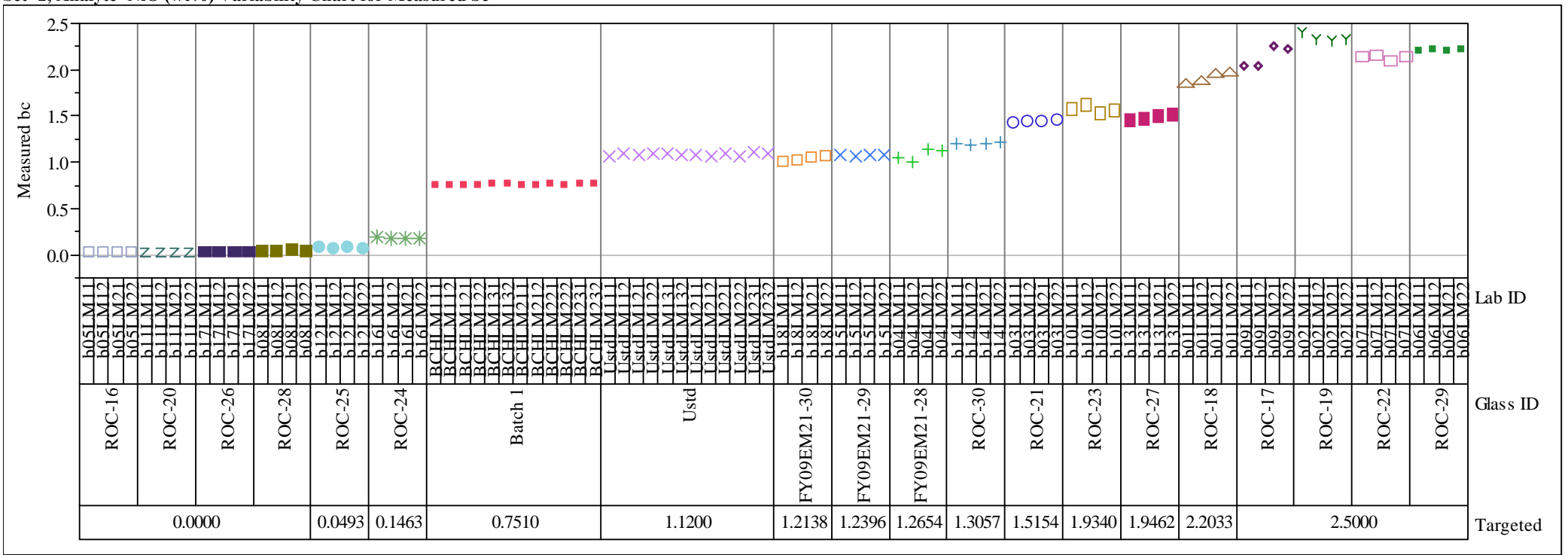




\section{Exhibit A5. Oxide Measurements by Lab ID within Glass ID Sorted by Targeted Concentrations}

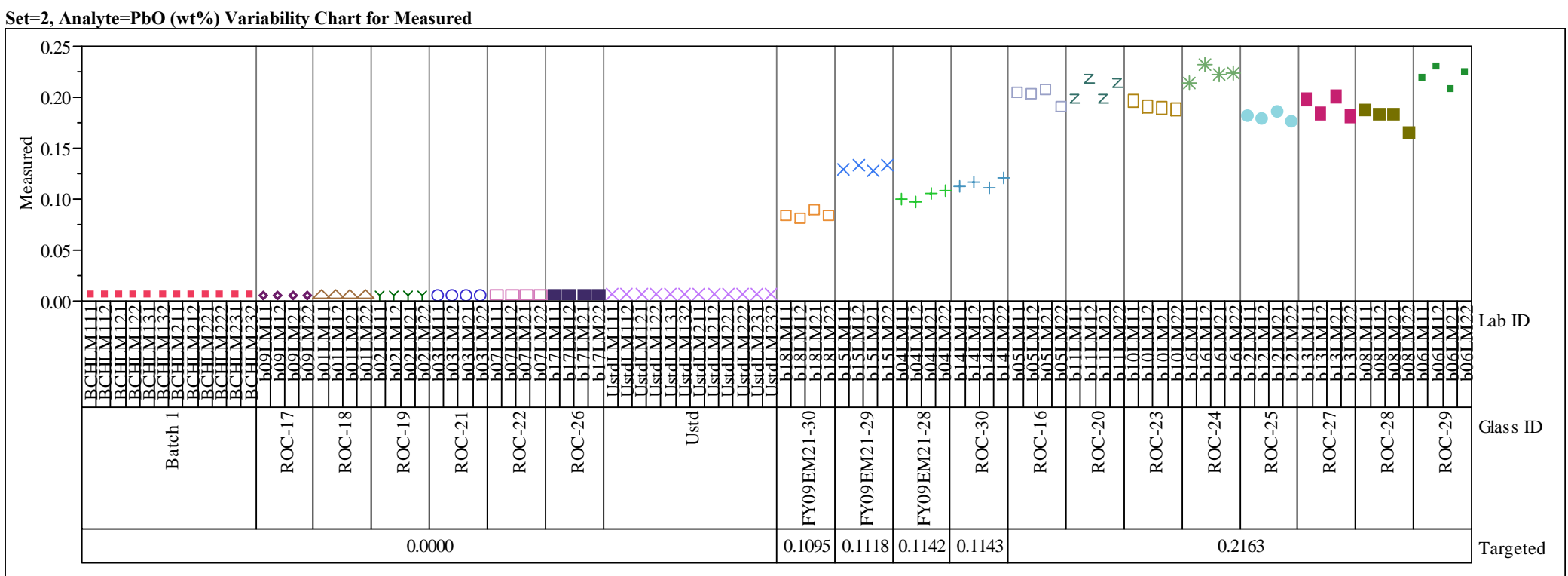

Set $=2$, Analyte $=\mathbf{P b O}($ wt $\%)$ Variability Chart for Measured bc

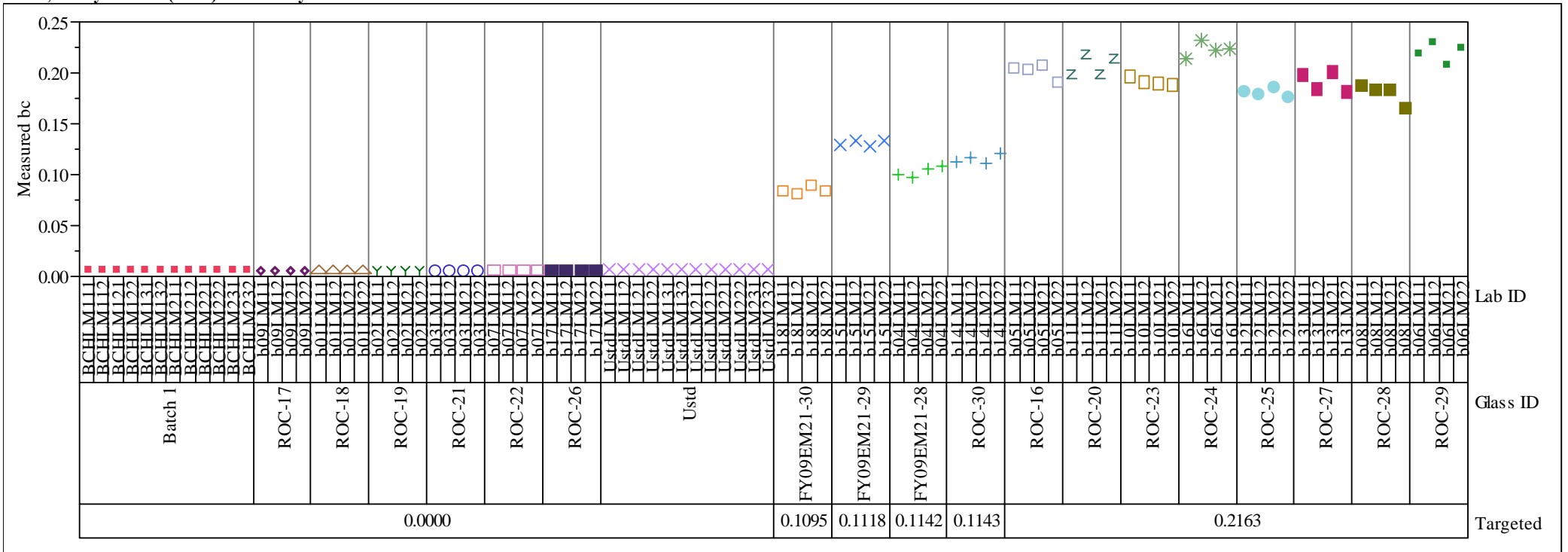


SRNL-STI-2009-00465, Revision 0

\section{Exhibit A5. Oxide Measurements by Lab ID within Glass ID Sorted by Targeted Concentrations}

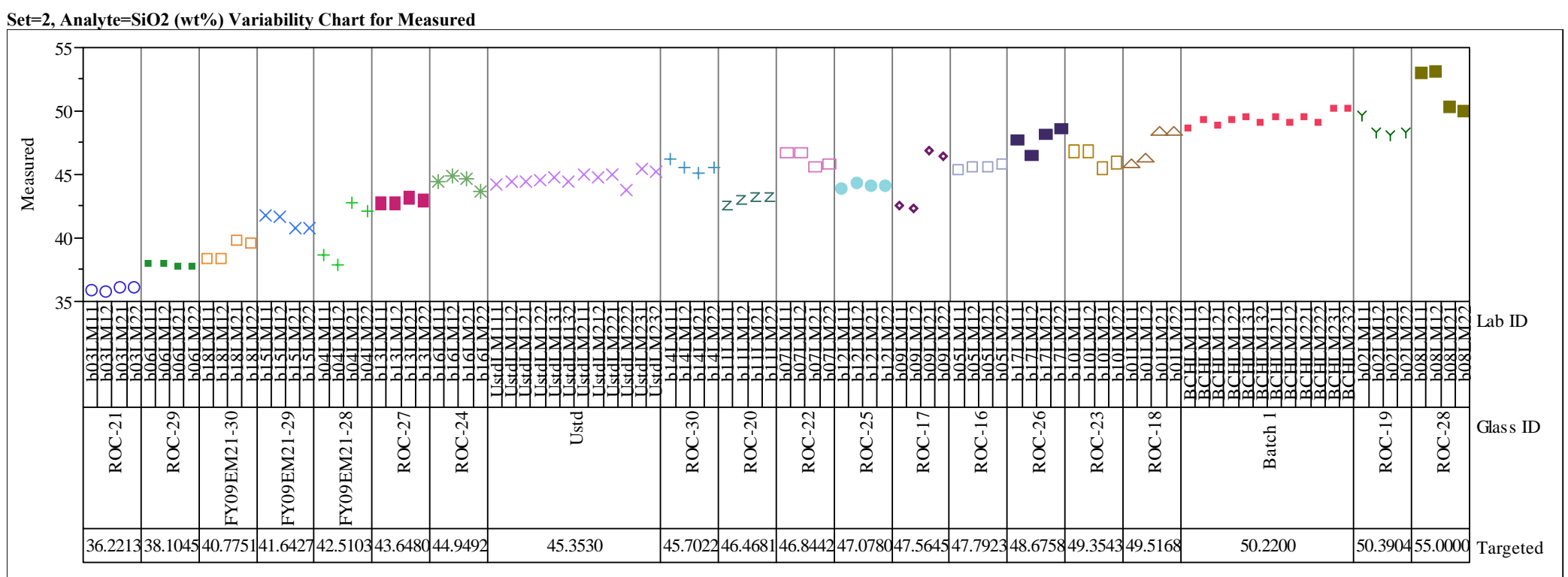

Set=2, Analyte=SiO2 (wt\%) Variability Chart for Measured bc

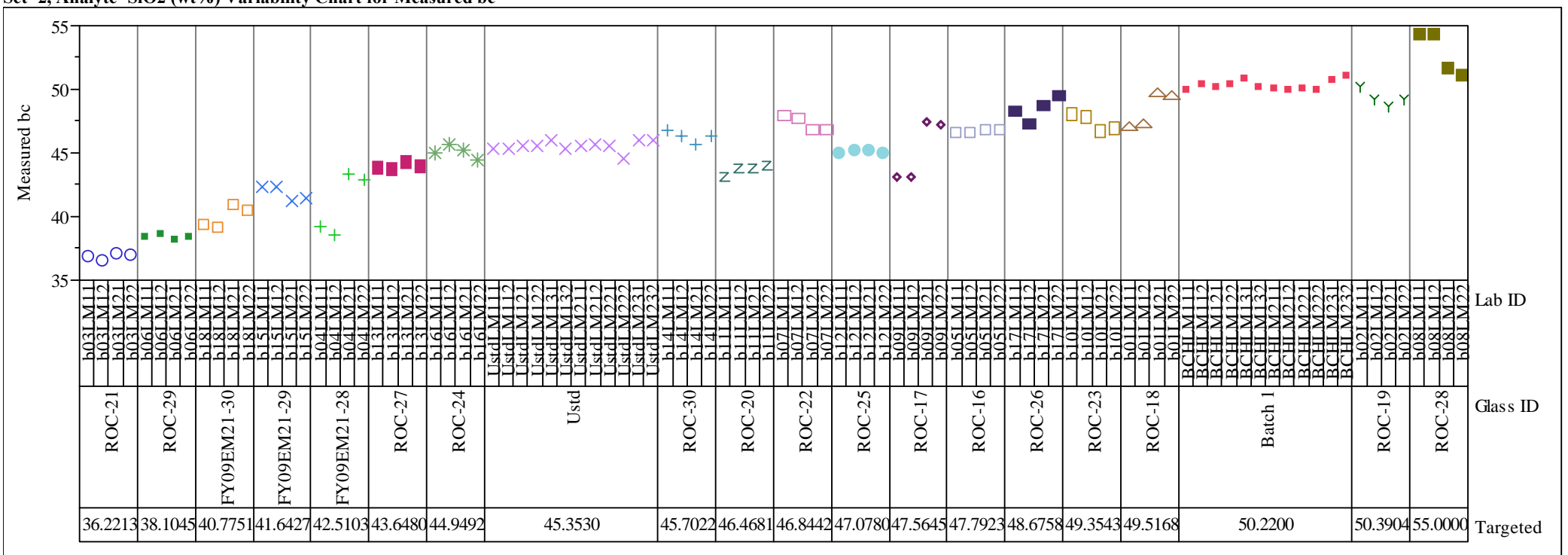


SRNL-STI-2009-00465, Revision 0

Exhibit A5. Oxide Measurements by Lab ID within Glass ID Sorted by Targeted Concentrations

Set $=2$, Analyte $=\mathrm{SO} 4(\mathrm{wt} \%)$ Variability Chart for Measured

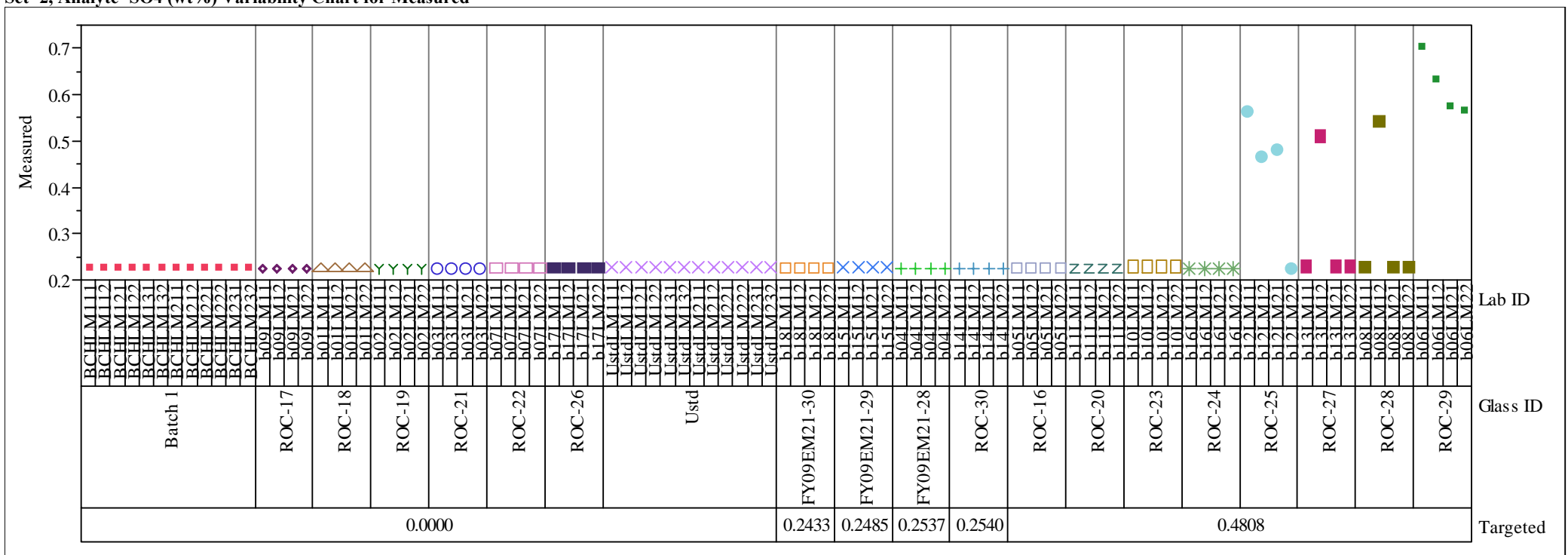

Set $=2$, Analyte $=\mathrm{SO} 4(\mathrm{wt} \%)$ Variability Chart for Measured bc

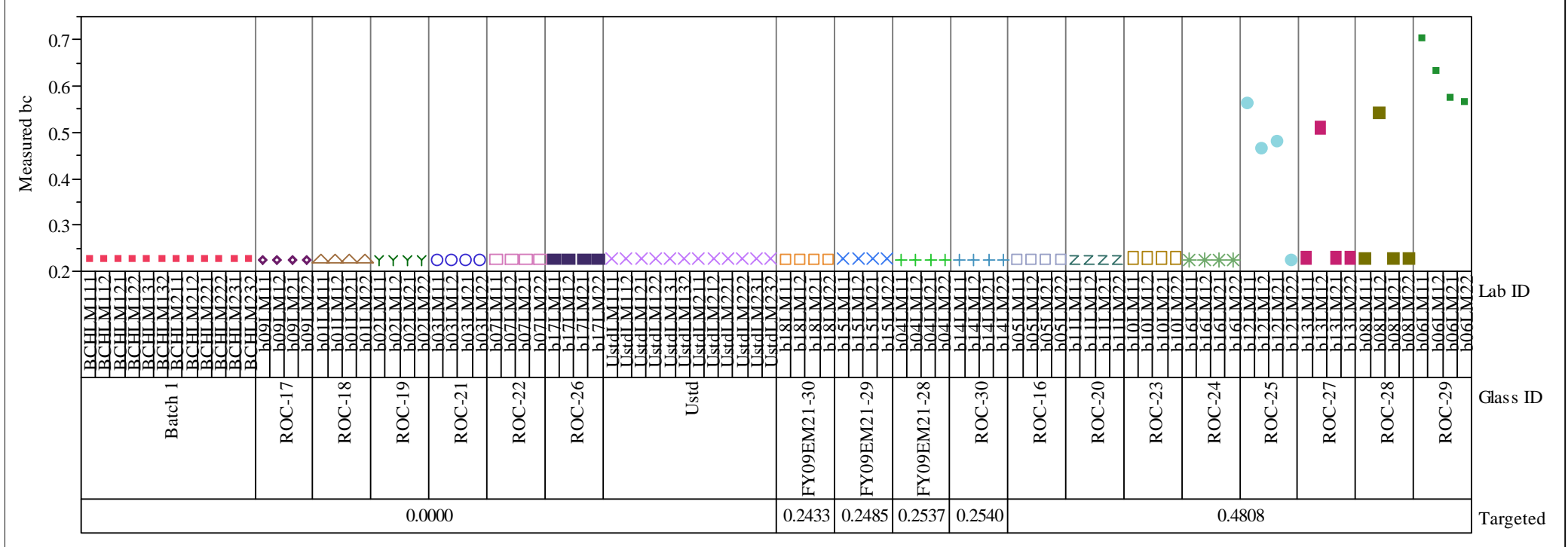


SRNL-STI-2009-00465, Revision 0

Exhibit A5. Oxide Measurements by Lab ID within Glass ID Sorted by Targeted Concentrations

Set $=2$, Analyte $=\mathrm{TiO} 2(\mathrm{wt} \%)$ Variability Chart for Measured

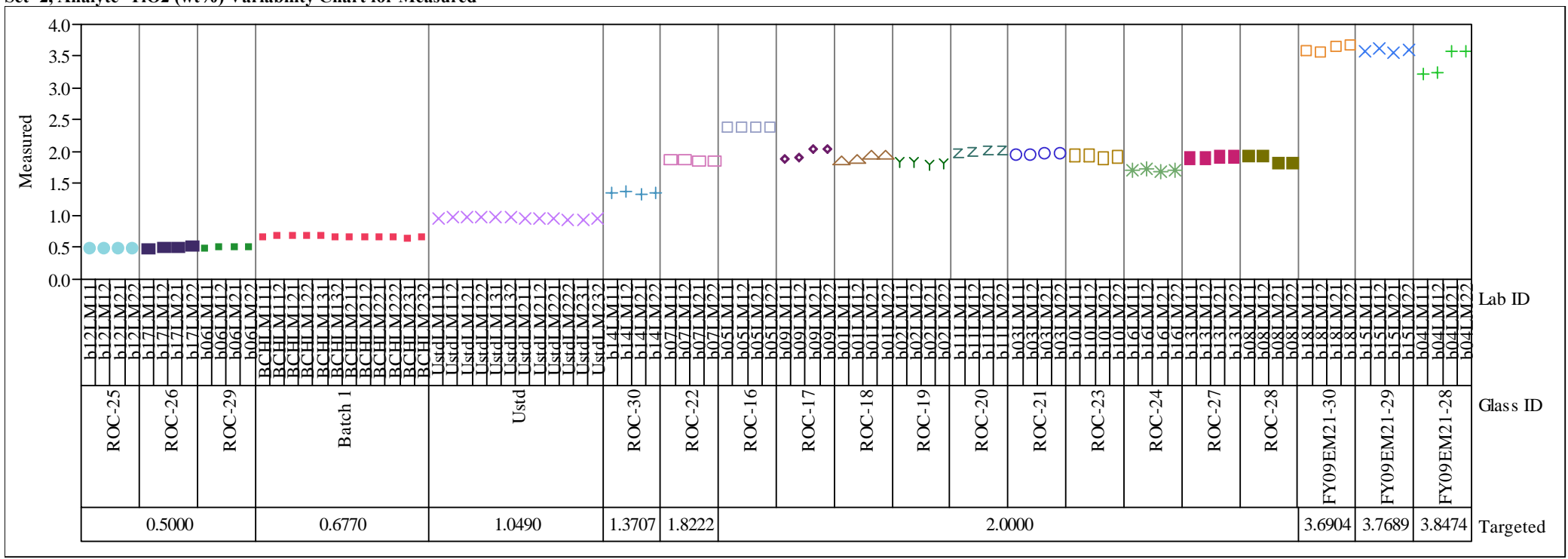

Set=2, Analyte $=\mathrm{TiO2}(\mathrm{wt} \%)$ Variability Chart for Measured bc

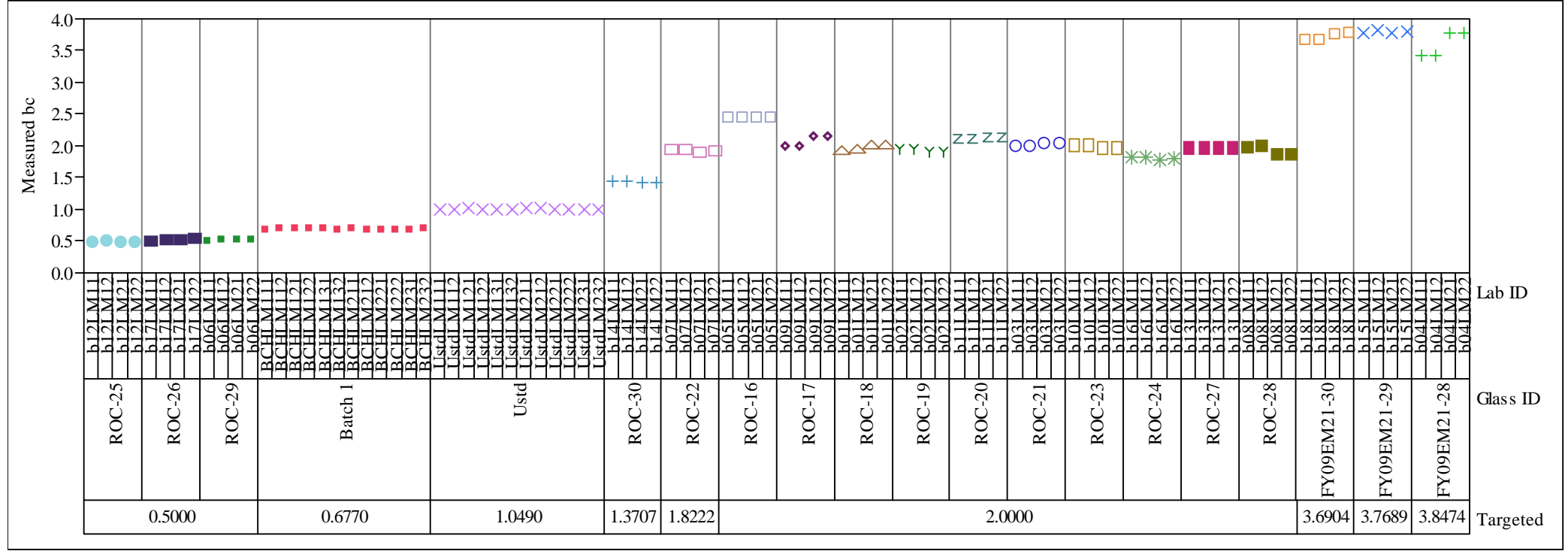

$-140-$ 
SRNL-STI-2009-00465, Revision 0

Exhibit A5. Oxide Measurements by Lab ID within Glass ID Sorted by Targeted Concentrations

Set $=2$, Analyte $=\mathbf{U} 308$ (wt $\%)$ Variability Chart for Measured

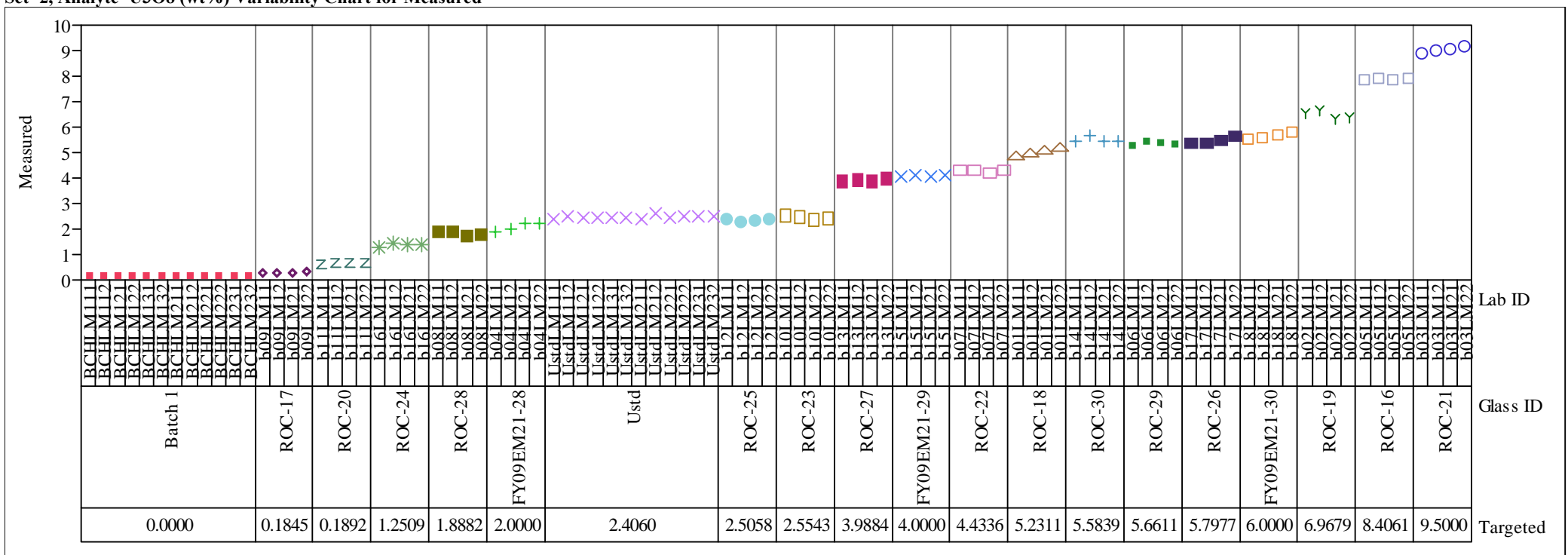

Set=2, Analyte=U3O8 (wt\%) Variability Chart for Measured bc

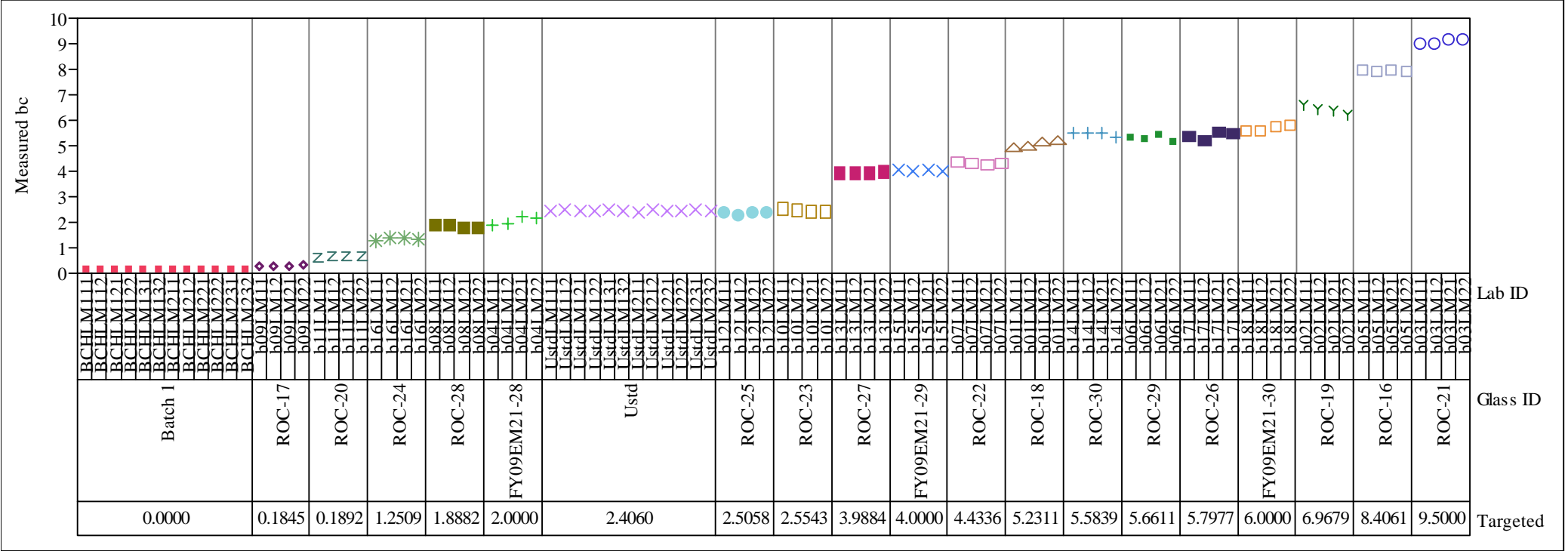


SRNL-STI-2009-00465, Revision 0

Exhibit A5. Oxide Measurements by Lab ID within Glass ID Sorted by Targeted Concentrations

Set $=2$, Analyte $=\mathrm{ZnO}(\mathrm{wt} \%)$ Variability Chart for Measured

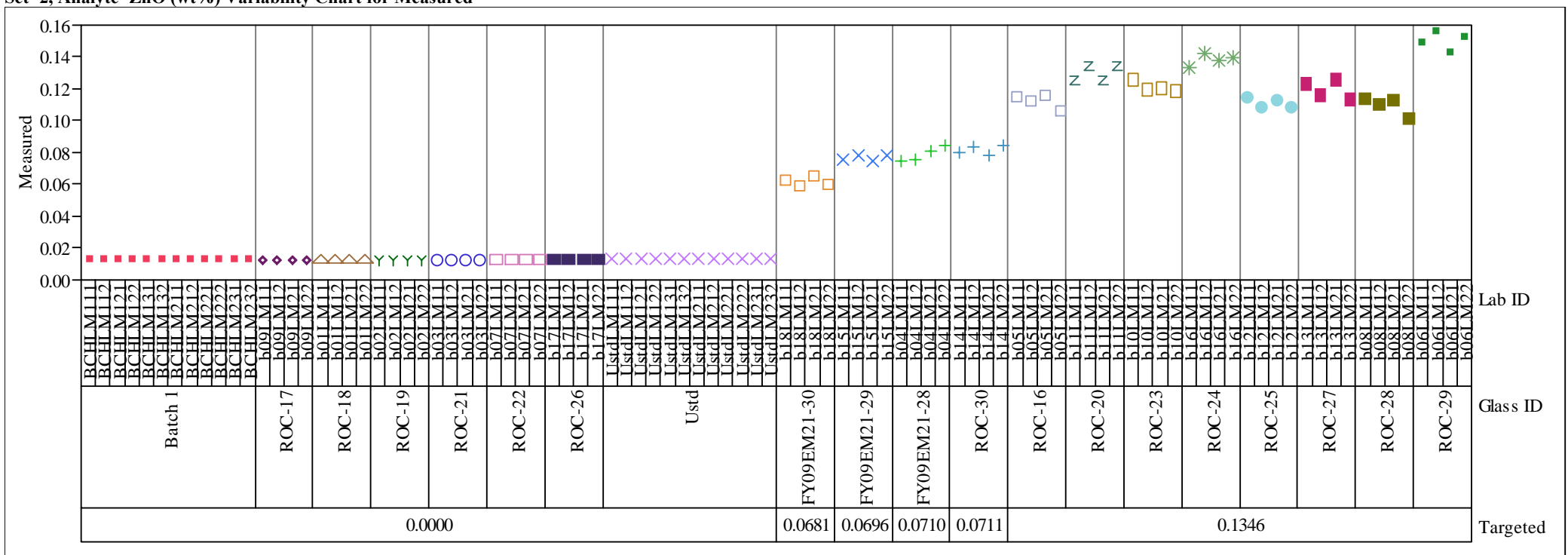

Set $=2$, Analyte $=\mathrm{ZnO}(\mathrm{wt} \%)$ Variability Chart for Measured bc

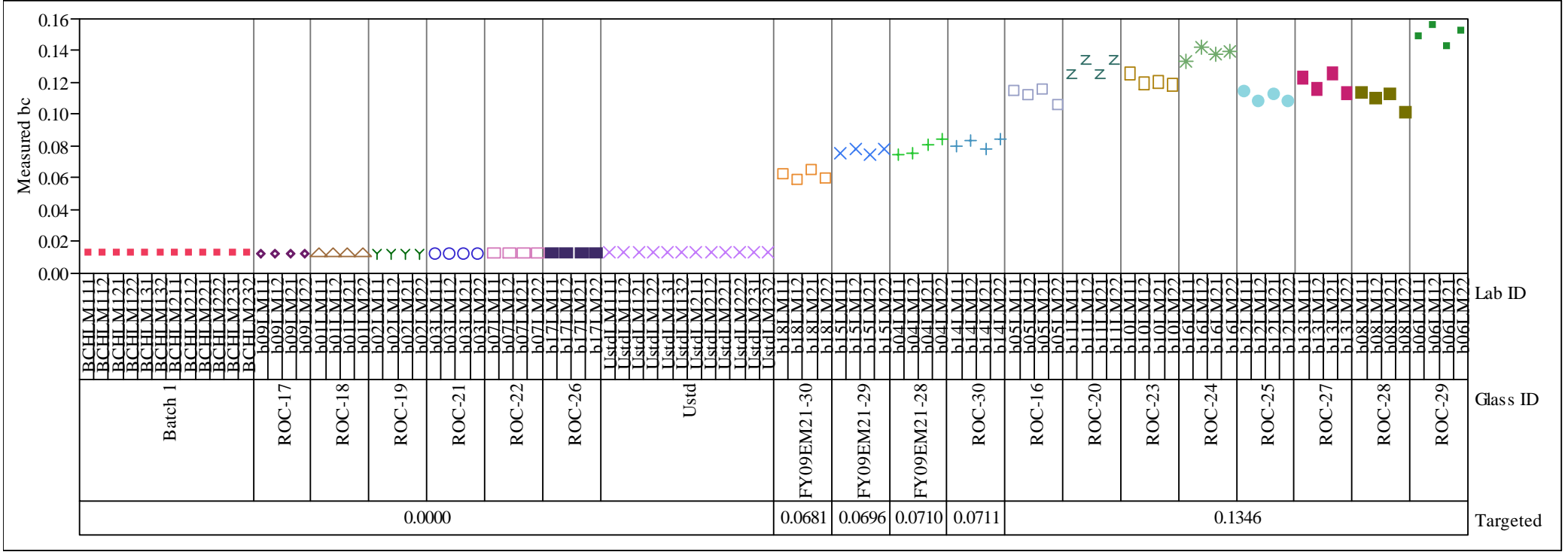


SRNL-STI-2009-00465, Revision 0

Exhibit A5. Oxide Measurements by Lab ID within Glass ID Sorted by Targeted Concentrations

Set $=2$, Analyte $=\mathrm{ZrO} 2(w t \%)$ Variability Chart for Measured

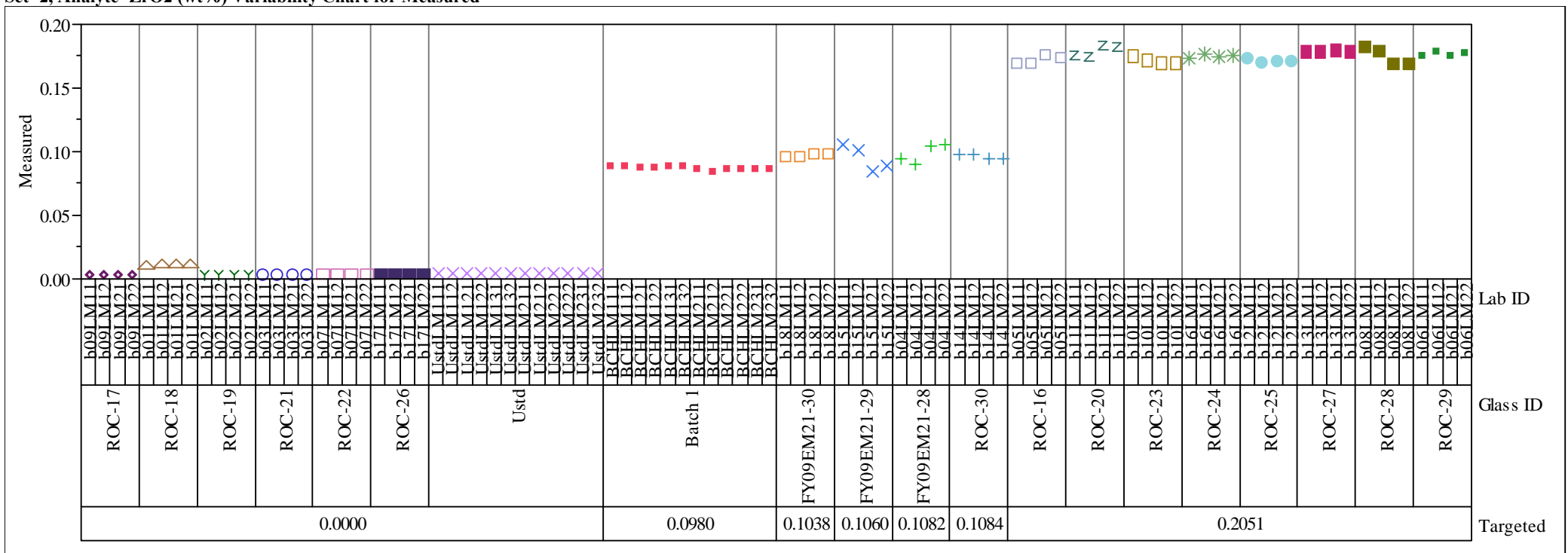

Set $=2$, Analyte $=\mathrm{ZrO2}(\mathrm{wt} \%)$ Variability Chart for Measured bc

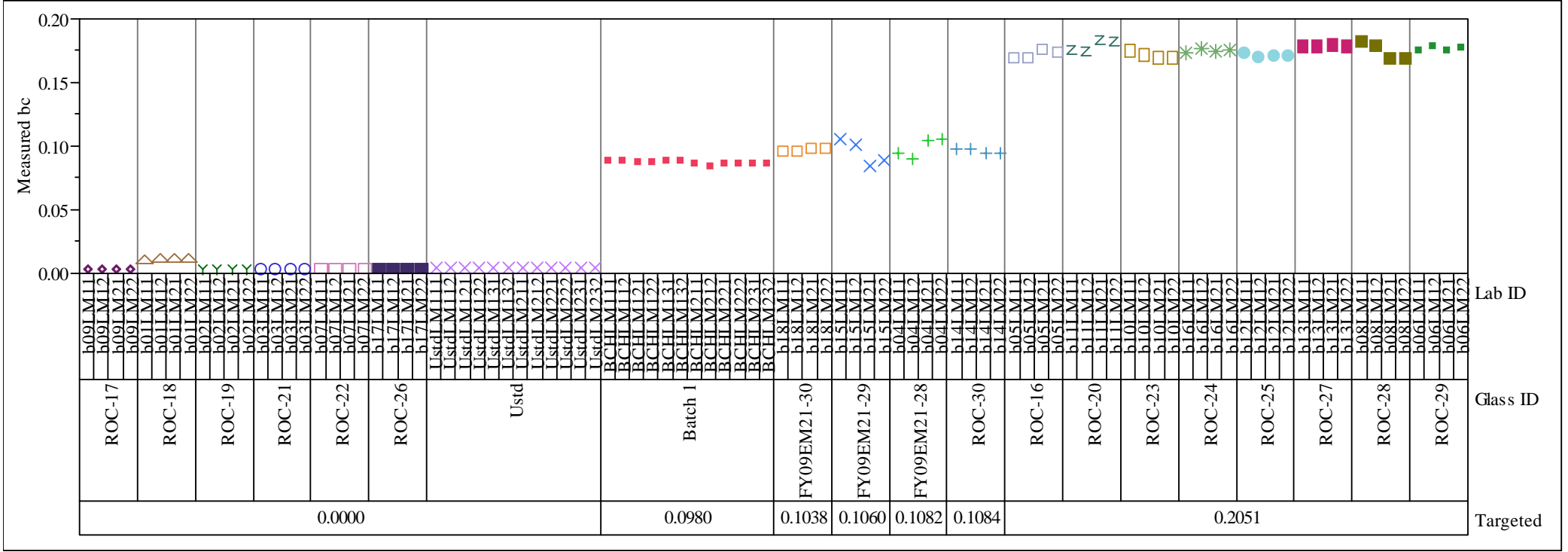


Exhibit A5. Oxide Measurements by Lab ID within Glass ID Sorted by Targeted Concentrations 
Exhibit A6. Average Measured and Bias-Corrected (bc) Versus Targeted Compositions by Glass ID by Oxide
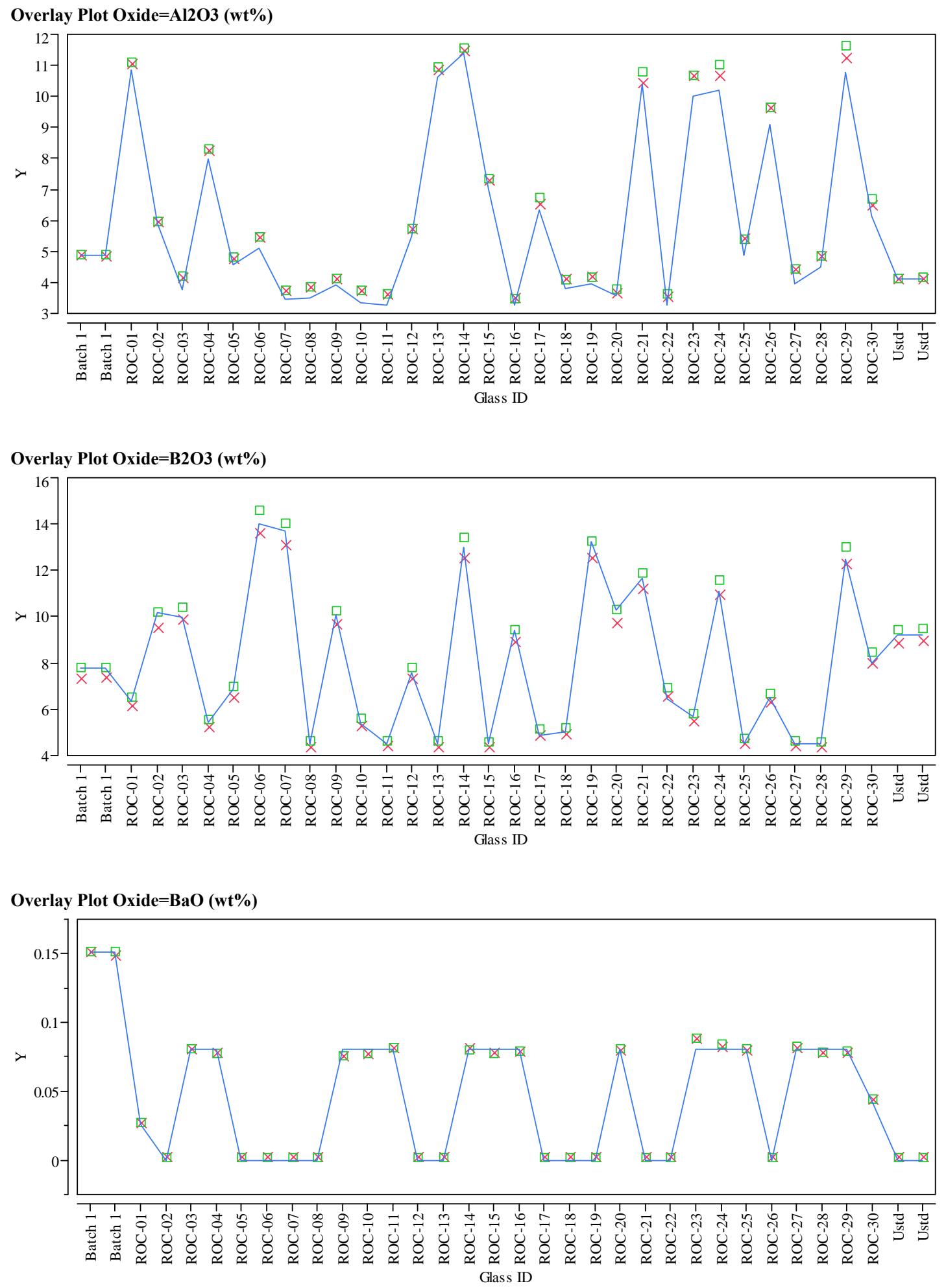

Y $\times$ Measured $\square$ Measured bc —Targeted 
Exhibit A6. Average Measured and Bias-Corrected (bc) Versus Targeted Compositions by Glass ID by Oxide
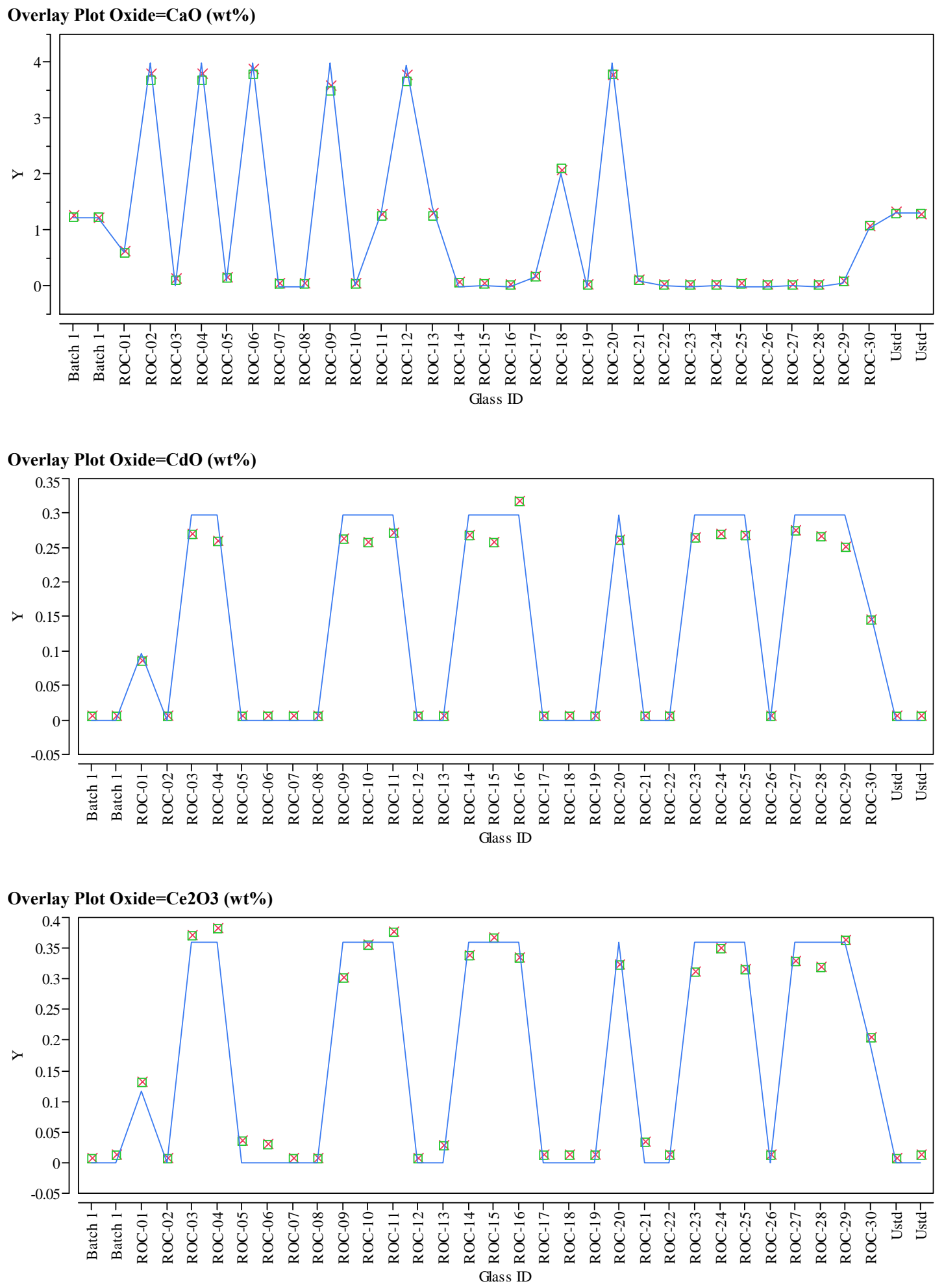

$\mathrm{Y} \times$ Measured $₫$ Measured bc 一 Targeted 
Exhibit A6. Average Measured and Bias-Corrected (bc) Versus Targeted Compositions by Glass ID by Oxide
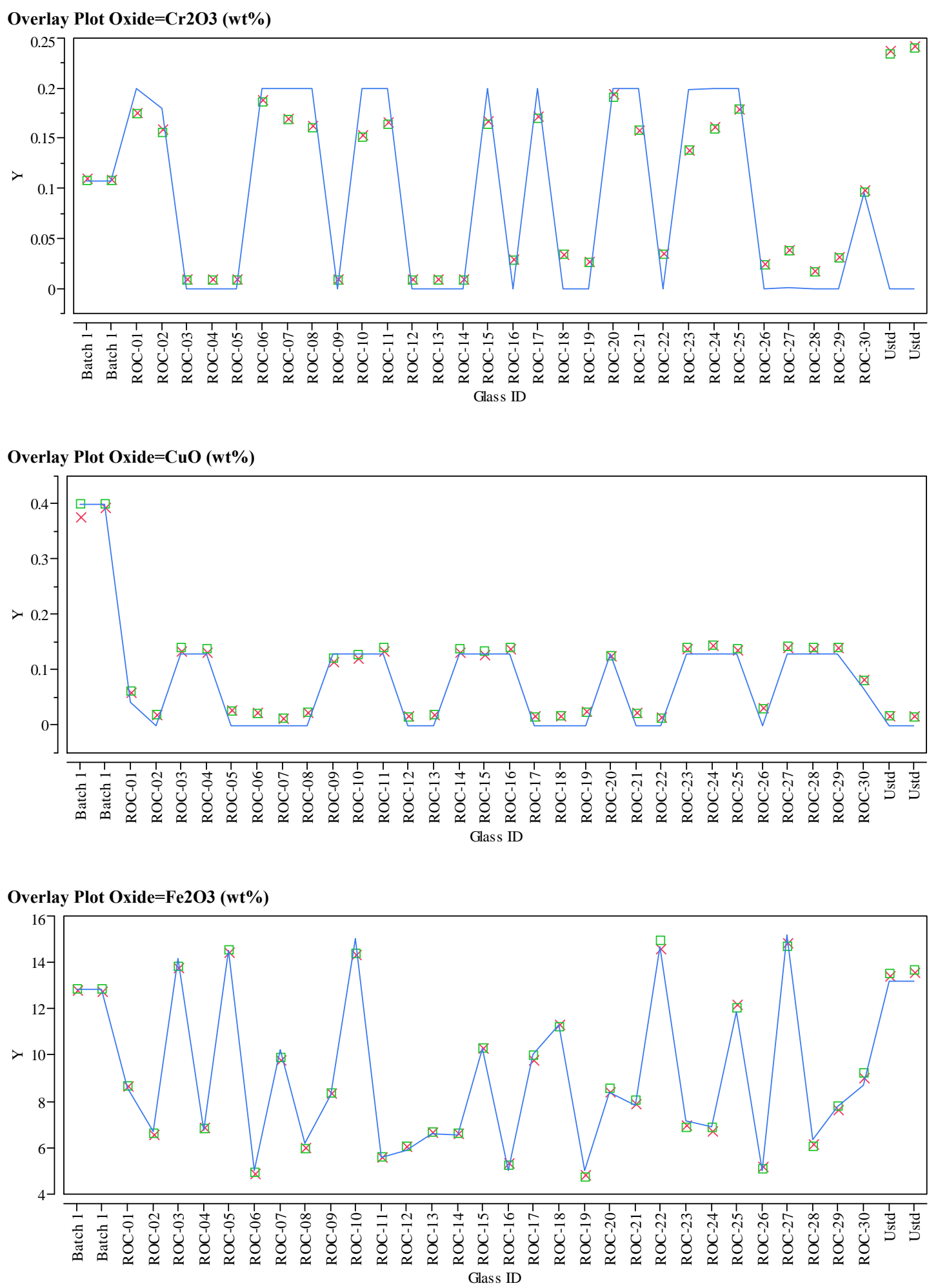

Y x Measured $\square$ Measured bc — Targeted 


\section{Exhibit A6. Average Measured and Bias-Corrected (bc) Versus Targeted Compositions by Glass ID by Oxide}
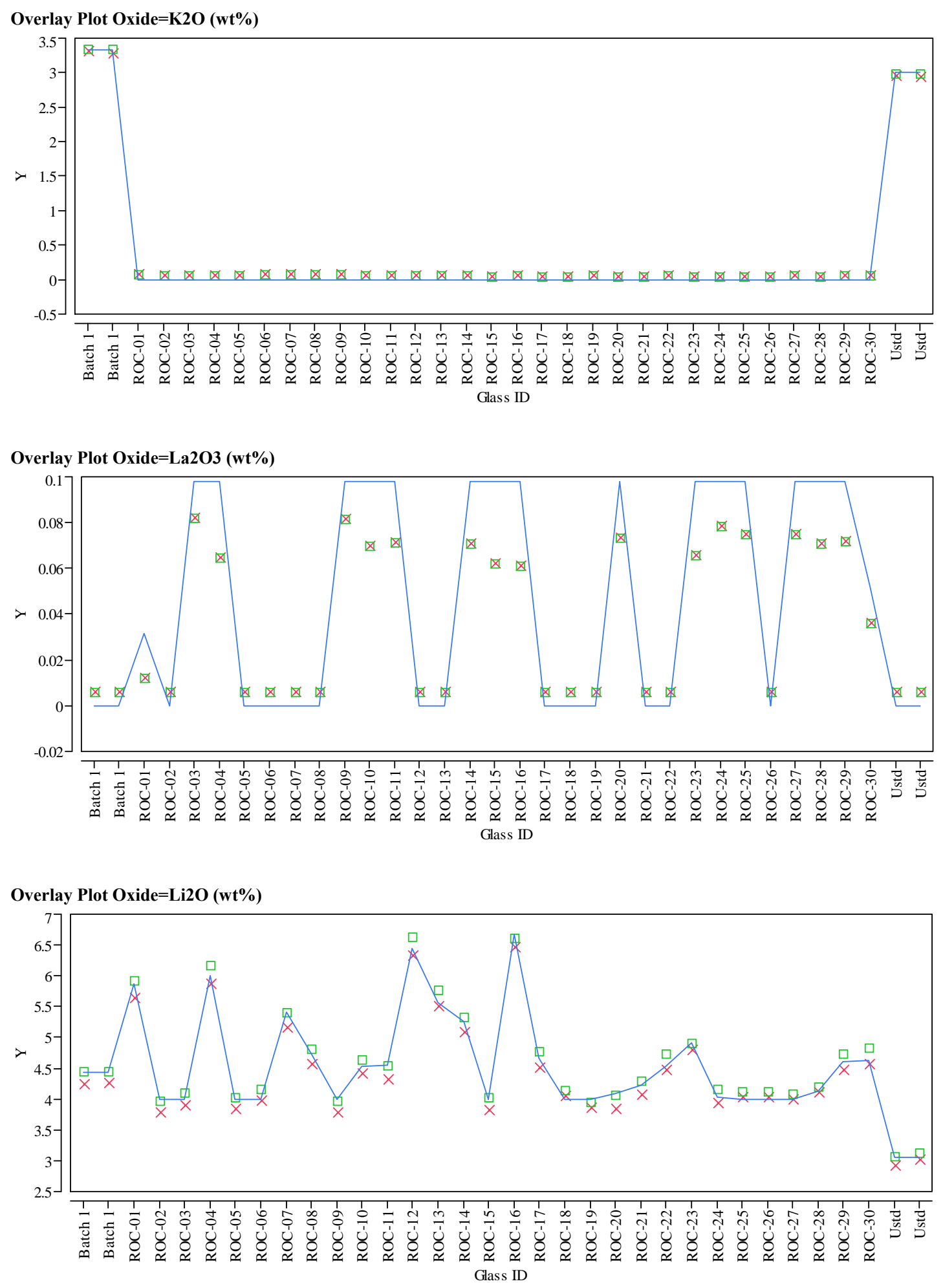

Y x Measured @ Measured bc — Targeted 
Exhibit A6. Average Measured and Bias-Corrected (bc) Versus Targeted Compositions by Glass ID by Oxide

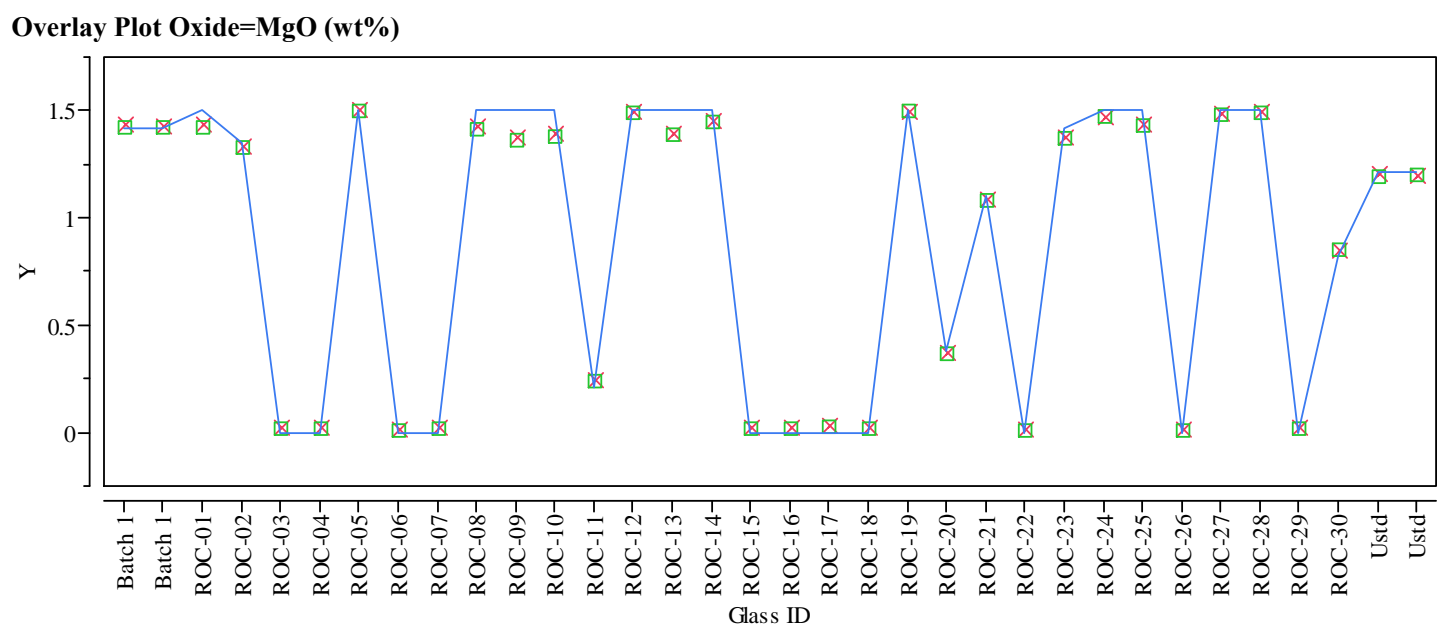

Overlay Plot Oxide $=\mathrm{MnO}(\mathrm{wt} \%)$
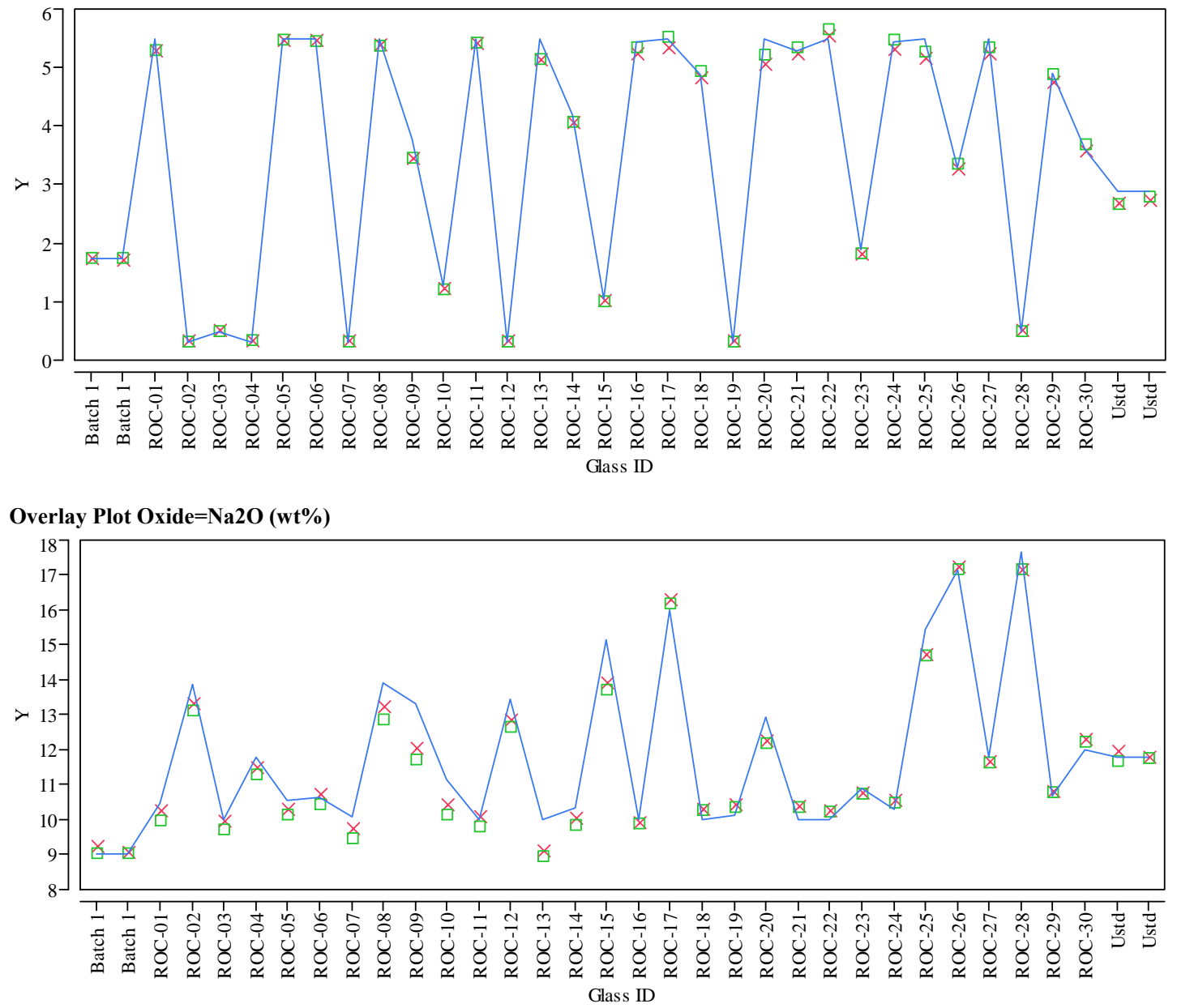

$\mathrm{Y} \times$ Measured $\square$ Measured bc — Targeted 


\section{Exhibit A6. Average Measured and Bias-Corrected (bc) Versus Targeted Compositions by Glass ID by Oxide}
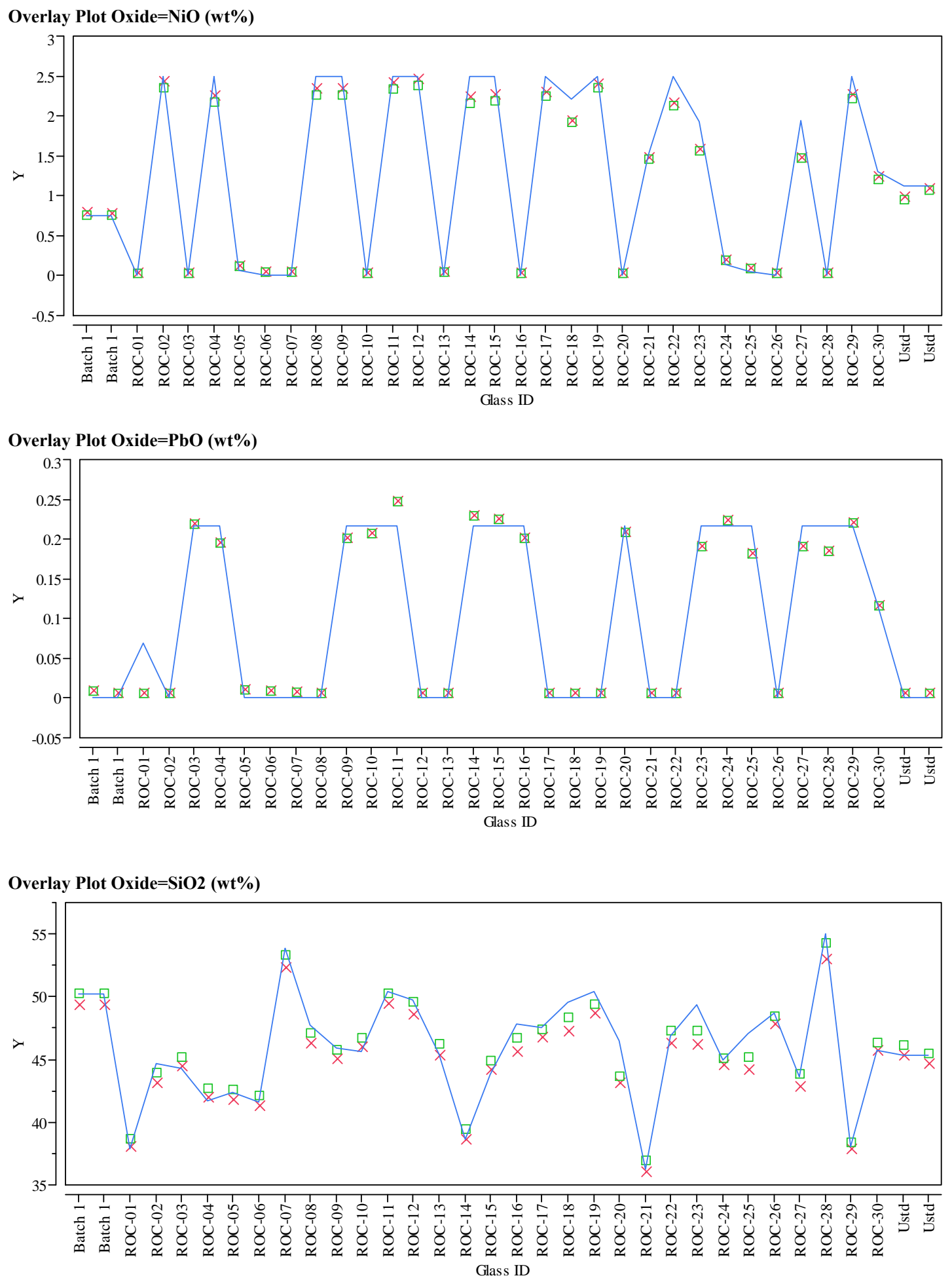

Y x Measured $\square$ Measured bc — Targeted 
Exhibit A6. Average Measured and Bias-Corrected (bc) Versus Targeted Compositions by Glass ID by Oxide
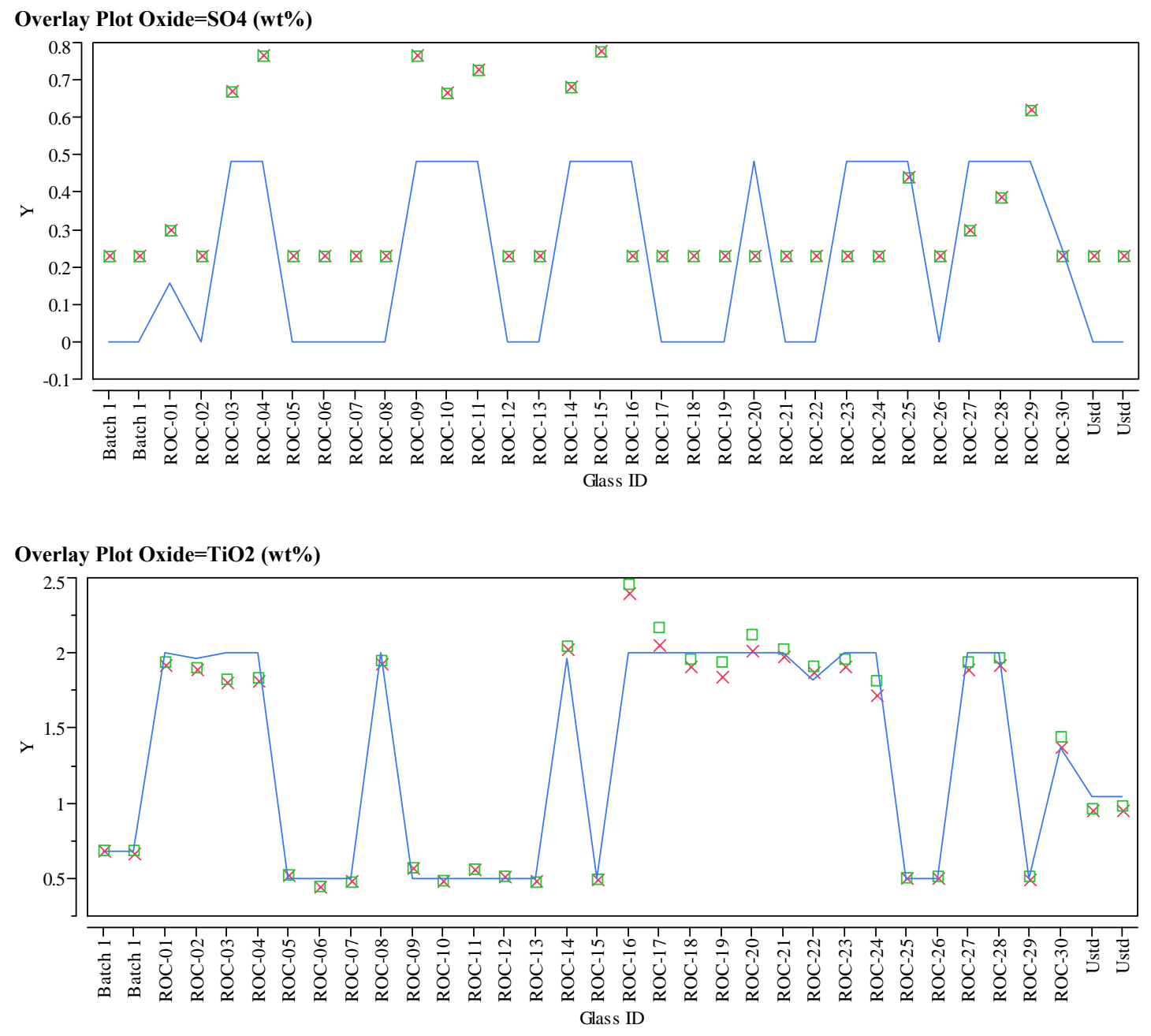

Overlay Plot Oxide $=$ U3O8 (wt\%)

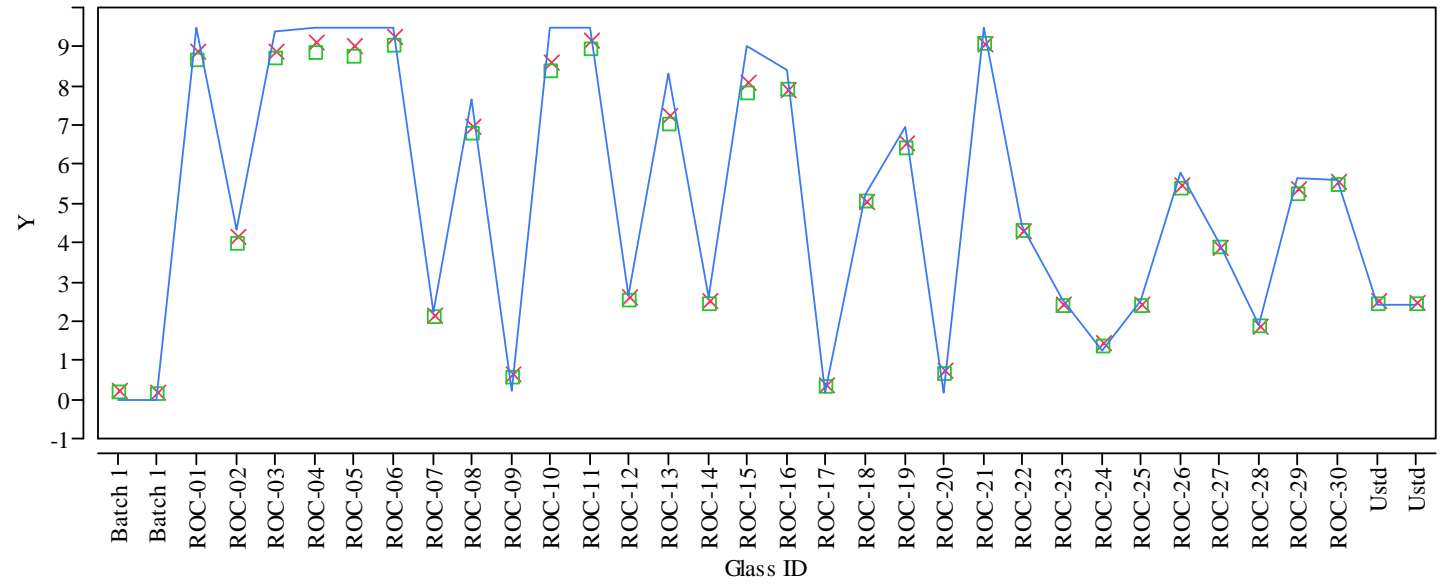

$\mathrm{Y} \times$ Measured $\square$ Measured bc — Targeted 


\section{Exhibit A6. Average Measured and Bias-Corrected (bc) Versus Targeted Compositions by Glass ID by Oxide}
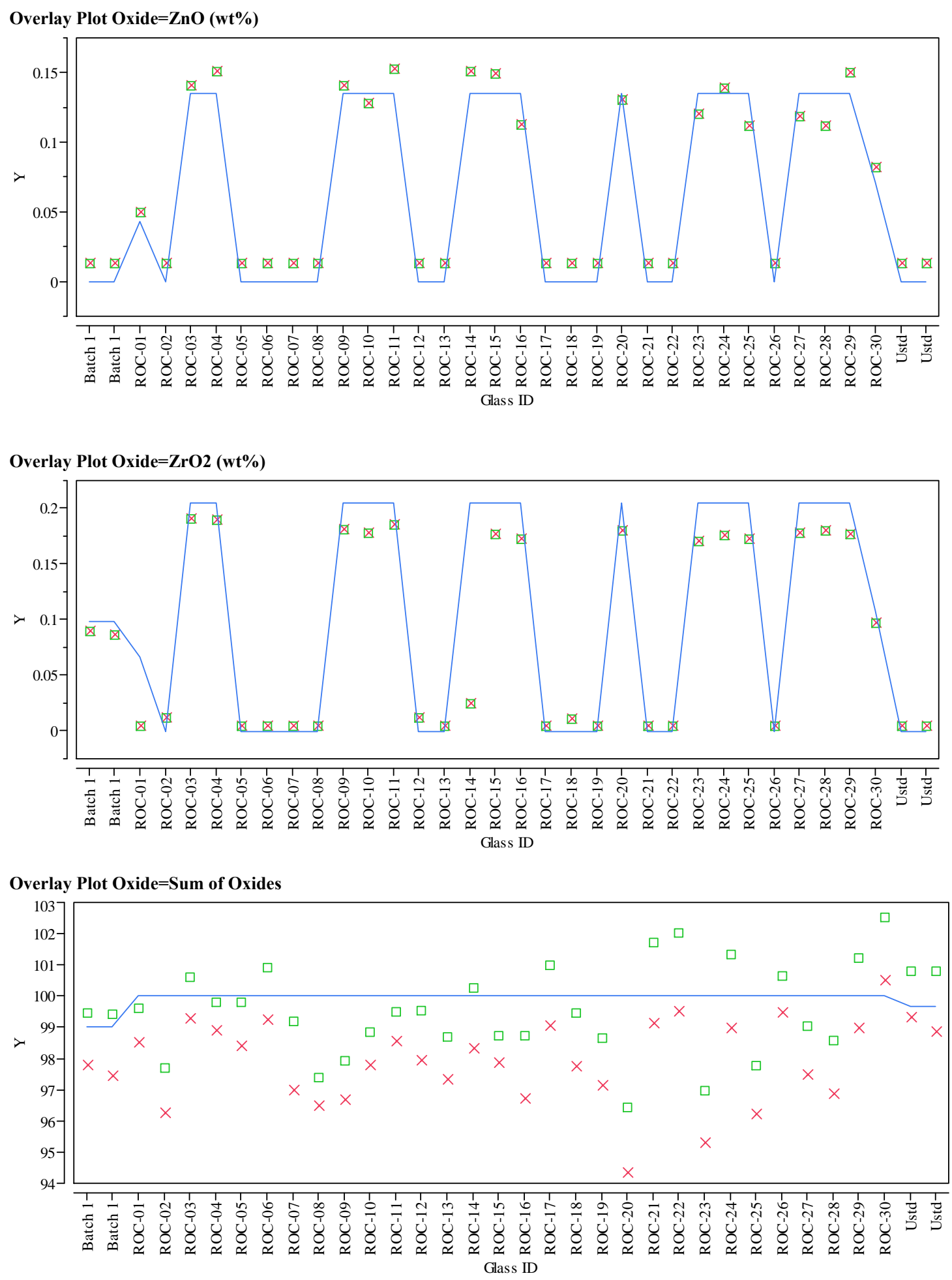

$\mathrm{Y} \times$ Measured $\square$ Measured bc — Targeted 


\section{Appendix B:}

\section{Tables and Exhibits Supporting the Analysis of the PCT Results for the Study Glasses}


SRNL-STI-2009-00465, Revision 0

This page intentionally left blank.

$-154-$ 
SRNL-STI-2009-00465, Revision 0

Table B1. Analytical Development's Measurements of the PCT Solutions AsReceived (ar) and After Appropriate Adjustments (in ppm)

\begin{tabular}{|c|c|c|c|c|c|c|c|c|c|c|c|c|c|c|}
\hline Set & Glass ID & \begin{tabular}{|c|} 
Heat \\
Treatment
\end{tabular} & Block & Seq & $\begin{array}{c}\text { Analytical } \\
\text { Sequence } \\
\end{array}$ & $\begin{array}{c}\text { Lab } \\
\text { ID }\end{array}$ & $\begin{array}{c}\text { B } \\
\text { ar }\end{array}$ & $\begin{array}{l}\mathrm{Li} \\
\mathrm{ar}\end{array}$ & $\begin{array}{l}\mathrm{Na} \\
\mathrm{ar}\end{array}$ & $\begin{array}{l}\mathrm{Si} \\
\text { ar }\end{array}$ & $\begin{array}{c}\text { B } \\
(\mathbf{p p m})\end{array}$ & $\begin{array}{c}\mathbf{L i} \\
(\mathbf{p p m})\end{array}$ & $\begin{array}{c}\mathrm{Na} \\
(\mathrm{ppm})\end{array}$ & $\begin{array}{c}\text { Si ( } \\
\text { ppm) }\end{array}$ \\
\hline 1 & Soln Std & \begin{tabular}{|l|} 
ref \\
\end{tabular} & 1 & 1 & 1 & std-11 & 19.8 & 10.1 & 82.4 & 50.4 & 19.800 & 10.100 & 82.400 & 50.400 \\
\hline 1 & ROC-10 & quenched & 1 & 2 & 2 & $\mathrm{c} 17$ & 12.8 & 15.7 & 52.6 & 91.1 & 21.334 & 26.167 & 87.668 & 151.836 \\
\hline 1 & ROC-04 & quenched & 1 & 3 & 3 & c51 & 7.29 & 14.0 & 46.7 & 50.9 & 12.150 & 23.334 & 77.835 & 84.835 \\
\hline 1 & ROC-10 & CCC & 1 & 4 & 4 & c20 & 12.7 & 15.9 & 52.4 & 93.8 & 21.167 & 26.501 & 87.335 & 156.336 \\
\hline 1 & ROC-02 & CCC & 1 & 5 & 5 & c23 & 14.4 & 8.62 & 51.4 & 50.4 & 24.000 & 14.367 & 85.668 & 84.002 \\
\hline 1 & ROC-06 & $\mathrm{CCC}$ & 1 & 6 & 6 & c36 & 52.4 & 21.8 & 75.8 & 59.1 & 87.335 & 36.334 & 126.336 & 98.502 \\
\hline 1 & ROC-05 & CCC & 1 & 7 & 7 & c27 & 16.4 & 17.6 & 48.9 & 86.1 & 27.334 & 29.334 & 81.502 & 143.503 \\
\hline 1 & ROC-06 & quenched & 1 & 8 & 8 & c56 & 58.3 & 23.9 & 82.2 & 57.8 & 97.169 & 39.834 & 137.003 & 96.335 \\
\hline 1 & ROC-03 & CCC & 1 & 9 & 9 & c32 & 15.1 & 10.2 & 25.6 & 56.3 & 25.167 & 17.000 & 42.668 & 93.835 \\
\hline 1 & ROC-01 & quenched & 1 & 10 & 10 & c09 & 12.4 & 17.0 & 42.3 & 62.3 & 20.667 & 28.334 & 70.501 & 103.835 \\
\hline 1 & blank & ref & 1 & 11 & 11 & c06 & $<0.460$ & $<0.102$ & $<0.332$ & $<0.301$ & 0.383 & 0.085 & 0.277 & \begin{tabular}{|l|}
0.251 \\
\end{tabular} \\
\hline 1 & ROC-09 & CCC & 1 & 12 & 12 & c65 & 72.1 & 35.4 & 194 & 178 & 120.169 & 59.001 & 323.340 & 296.673 \\
\hline 1 & Soln Std & ref & 1 & 13 & 13 & std-12 & 19.9 & 10.1 & 81.8 & 50.2 & 19.900 & 10.100 & 81.800 & 50.200 \\
\hline 1 & ROC-03 & quenched & 1 & 14 & 14 & c41 & 14.50 & 10.2 & 24.1 & 54.2 & 24.167 & 17.000 & 40.167 & 90.335 \\
\hline 1 & EA & ref & 1 & 15 & 15 & $\mathrm{c46}$ & 33.9 & 11.0 & 94.7 & 53.5 & 565.001 & 183.334 & 1578.336 & 891.668 \\
\hline 1 & ROC-07 & CCC & 1 & 16 & 16 & $\mathrm{c08}$ & 44.6 & 25.4 & 53.2 & 106 & 74.335 & 42.334 & 88.668 & 176.670 \\
\hline 1 & ROC-05 & quenched & 1 & 17 & 17 & c50 & 16.2 & 13.5 & 47.2 & 73.1 & 27.001 & 22.500 & 78.668 & 121.836 \\
\hline 1 & ROC-08 & quenched & 1 & 18 & 18 & c44 & 27.4 & 35.5 & 167 & 214 & 45.668 & 59.168 & 278.339 & 356.674 \\
\hline 1 & ROC-01 & $\mathrm{CCC}$ & 1 & 19 & 19 & c52 & 13.1 & 21.5 & 44.1 & 75.7 & 21.834 & 35.834 & 73.501 & 126.169 \\
\hline 1 & ROC-08 & $\mathrm{CCC}$ & 1 & 20 & 20 & c12 & 26.2 & 37.6 & 161 & 222 & 43.668 & 62.668 & 268.339 & 370.007 \\
\hline 1 & ROC-02 & quenched & 1 & 21 & 21 & c57 & 17.6 & 10.4 & 65.1 & 60.6 & 29.334 & 17.334 & 108.502 & 101.002 \\
\hline 1 & ROC-07 & quenched & 1 & 22 & 22 & c64 & 50.3 & 28.1 & 56.8 & 101 & 83.835 & 46.834 & 94.669 & 168.337 \\
\hline 1 & ARM-1 & ref & 1 & 23 & 23 & c26 & 10.5 & 8.32 & 22.0 & 36.7 & 17.500 & 13.867 & 36.667 & \begin{tabular}{|l|}
61.168 \\
\end{tabular} \\
\hline 1 & ROC-09 & quenched & 1 & 24 & 24 & c48 & 185 & 82.1 & 469 & 289 & 308.340 & 136.836 & 781.682 & 481.676 \\
\hline 1 & ROC-04 & $\mathrm{CCC}$ & 1 & 25 & 25 & c68 & 5.85 & 18.6 & 43.2 & 55.0 & 9.750 & 31.001 & 72.001 & \begin{tabular}{|l|}
91.669 \\
\end{tabular} \\
\hline 1 & Soln Std & ref & 1 & 26 & 26 & std-13 & 20.1 & 10.0 & 82.9 & 50.7 & 20.100 & 10.000 & 82.900 & 50.700 \\
\hline 1 & Soln Std & ref & 2 & 1 & 27 & std-21 & 19.6 & 9.96 & 81.1 & 49.8 & 19.600 & 9.960 & 81.100 & 49.800 \\
\hline 1 & ARM-1 & ref & 2 & 2 & 28 & c60 & 9.98 & 8.11 & 21.3 & 35.8 & 16.634 & 13.517 & 35.501 & 59.668 \\
\hline 1 & ROC-02 & CCC & 2 & 3 & 29 & c21 & 14.4 & 8.74 & 51.8 & 50.8 & 24.000 & 14.567 & 86.335 & 84.668 \\
\hline 1 & ROC-06 & quenched & 2 & 4 & 30 & c11 & 58.5 & 24.1 & 83.3 & 58.3 & 97.502 & 40.167 & 138.836 & 97.169 \\
\hline 1 & ROC-10 & quenched & 2 & 5 & 31 & c15 & 12.7 & 15.6 & 52.8 & 91.9 & 21.167 & 26.001 & 88.002 & 153.170 \\
\hline 1 & ROC-05 & quenched & 2 & 6 & 32 & $\mathrm{c} 01$ & 15.9 & 13.3 & 46.5 & 70.1 & 26.501 & 22.167 & 77.502 & 116.836 \\
\hline 1 & ROC-02 & quenched & 2 & 7 & 33 & $\mathrm{c05}$ & 17.3 & 10.4 & 64.6 & 59.6 & 28.834 & 17.334 & 107.669 & 99.335 \\
\hline 1 & ROC-01 & $\mathrm{CCC}$ & 2 & 8 & 34 & c35 & 12.9 & 20.9 & 43.0 & 74.2 & 21.500 & 34.834 & 71.668 & 123.669 \\
\hline 1 & ROC-08 & quenched & 2 & 9 & 35 & c29 & 28.5 & 36.9 & 173 & 223 & 47.501 & 61.501 & 288.339 & 371.674 \\
\hline 1 & ROC-06 & CCC & 2 & 10 & 36 & c19 & 55.2 & 23.1 & 80.6 & 62.7 & 92.002 & 38.501 & 134.336 & 104.502 \\
\hline 1 & ROC-05 & CCC & 2 & 11 & 37 & c13 & 15.7 & 16.8 & 47.1 & 82.2 & 26.167 & 28.001 & 78.502 & 137.003 \\
\hline 1 & ROC-10 & $\mathrm{CCC}$ & 2 & 12 & 38 & c58 & 12.9 & 16.2 & 52.9 & 93.1 & 21.500 & 27.001 & 88.168 & 155.170 \\
\hline 1 & Soln Std & ref & 2 & 13 & 39 & std-22 & 19.8 & 10.0 & 81.9 & 50.1 & 19.800 & 10.000 & 81.900 & 50.100 \\
\hline 1 & ROC-09 & quenched & 2 & 14 & 40 & $\mathrm{c} 10$ & 178 & 79.8 & 459 & 282 & 296.673 & 133.003 & 765.015 & 470.009 \\
\hline 1 & ROC-03 & $\mathrm{CCC}$ & 2 & 15 & 41 & c63 & 15.2 & 10.2 & 25.8 & 56.9 & 25.334 & 17.000 & 43.001 & 94.835 \\
\hline 1 & ROC-07 & $\mathrm{CCC}$ & 2 & 16 & 42 & $\mathrm{c03}$ & 43.0 & 24.5 & 51.7 & 103 & 71.668 & 40.834 & 86.168 & 171.670 \\
\hline 1 & ROC-08 & CCC & 2 & 17 & 43 & c43 & 25.2 & 37.0 & 159 & 229 & 42.001 & 61.668 & 265.005 & 381.674 \\
\hline 1 & ROC-04 & CCC & 2 & 18 & 44 & c67 & 5.79 & 18.9 & 44.1 & 56.0 & 9.650 & 31.501 & 73.501 & 93.335 \\
\hline 1 & ROC-09 & CCC & 2 & 19 & 45 & c31 & 66.1 & 31.4 & 174 & 132 & 110.169 & 52.334 & 290.006 & 220.004 \\
\hline 1 & ROC-04 & quenched & 2 & 20 & 46 & c25 & 7.25 & 13.7 & 46.0 & 50.6 & 12.084 & 22.834 & 76.668 & 84.335 \\
\hline 1 & ROC-07 & quenched & 2 & 21 & 47 & c53 & 53.5 & 29.9 & 61.2 & 109 & 89.168 & 49.834 & 102.002 & 181.670 \\
\hline 1 & ROC-03 & quenched & 2 & 22 & 48 & c54 & 14.4 & 10.0 & 23.9 & 53.8 & 24.000 & 16.667 & 39.834 & \begin{tabular}{|l|}
89.668 \\
\end{tabular} \\
\hline 1 & ROC-01 & quenched & 2 & 23 & 49 & $\mathrm{c04}$ & 12.9 & 17.4 & 44.1 & 64.3 & 21.500 & 29.001 & 73.501 & 107.169 \\
\hline 1 & EA & ref & 2 & 24 & 50 & $\mathrm{c} 45$ & 35.8 & 11.5 & 99.6 & 56.0 & 596.668 & 191.667 & 1660.003 & 933.335 \\
\hline 1 & Soln Std & ref & 2 & 25 & 51 & std-23 & 19.9 & 9.96 & 82.2 & 50.2 & 19.900 & 9.960 & 82.200 & 50.200 \\
\hline 1 & Soln Std & ref & 3 & 1 & 52 & std-31 & 19.8 & 10.0 & 81.6 & 50.2 & 19.800 & 10.000 & 81.600 & 50.200 \\
\hline 1 & ROC-04 & CCC & 3 & 2 & 53 & c38 & 5.75 & 18.7 & 43.2 & 55.2 & 9.584 & 31.167 & 72.001 & 92.002 \\
\hline 1 & ROC-04 & quenched & 3 & 3 & 54 & c34 & 7.56 & 14.5 & 48.1 & 53.0 & 12.600 & 24.167 & 80.168 & 88.335 \\
\hline 1 & ROC-06 & CCC & 3 & 4 & 55 & c14 & 50.7 & 21.1 & 73.1 & 57.1 & 84.502 & 35.167 & 121.836 & \begin{tabular}{|l|}
95.169 \\
\end{tabular} \\
\hline 1 & ROC-07 & CCC & 3 & 5 & 56 & c49 & 44.8 & 25.5 & 53.3 & 107 & 74.668 & 42.501 & 88.835 & 178.337 \\
\hline 1 & ROC-08 & quenched & 3 & 6 & 57 & c62 & 28.0 & 36.1 & 169 & 218 & 46.668 & 60.168 & 281.672 & 363.341 \\
\hline 1 & ROC-01 & $\mathrm{CCC}$ & 3 & 7 & 58 & $\mathrm{c} 40$ & 13.4 & 21.7 & 44.3 & 77.4 & 22.334 & 36.167 & 73.835 & 129.003 \\
\hline 1 & ROC-03 & CCC & 3 & 8 & 59 & c18 & 15.0 & 10.1 & 25.3 & 56.3 & 25.001 & 16.834 & 42.168 & 93.835 \\
\hline 1 & ROC-07 & quenched & 3 & 9 & 60 & c42 & 54.6 & 30.4 & 61.6 & 109 & 91.002 & 50.668 & 102.669 & 181.670 \\
\hline 1 & ROC-01 & quenched & 3 & 10 & 61 & c24 & 12.1 & 16.7 & 40.8 & 60.2 & 20.167 & 27.834 & 68.001 & 100.335 \\
\hline 1 & blank & ref & 3 & 11 & 62 & c47 & $<0.460$ & $<0.102$ & $<0.332$ & $<0.301$ & 0.383 & 0.085 & 0.277 & $\begin{array}{l}0.251 \\
\end{array}$ \\
\hline 1 & ROC-06 & quenched & 3 & 12 & 63 & c33 & 57.5 & 23.4 & 80.3 & 57.2 & 95.835 & 39.001 & 133.836 & 95.335 \\
\hline 1 & Soln Std & ref & 3 & 13 & 64 & std-32 & 19.9 & 9.98 & 81.2 & 50.3 & 19.900 & 9.980 & 81.200 & 50.300 \\
\hline 1 & ROC-09 & quenched & 3 & 14 & 65 & c22 & 182 & 80.5 & 463 & 284 & 303.339 & 134.169 & 771.682 & 473.343 \\
\hline 1 & ROC-03 & quenched & 3 & 15 & 66 & c66 & 15.5 & 10.8 & 25.5 & 57.9 & 25.834 & 18.000 & 42.501 & \begin{tabular}{|l|}
96.502 \\
\end{tabular} \\
\hline 1 & ROC-02 & quenched & 3 & 16 & 67 & $\mathrm{c07}$ & 17.4 & 10.2 & 63.7 & 59.6 & 29.001 & 17.000 & 106.169 & 99.335 \\
\hline 1 & ROC-09 & CCC & 3 & 17 & 68 & $\mathrm{c02}$ & 63.0 & 30.0 & 163 & 129 & 105.002 & 50.001 & 271.672 & 215.004 \\
\hline 1 & ARM-1 & ref & 3 & 18 & 69 & c37 & 10.8 & 8.47 & 22.6 & 38.0 & 18.000 & 14.117 & 37.667 & 63.335 \\
\hline 1 & ROC-02 & $\mathrm{CCC}$ & 3 & 19 & 70 & c55 & 15.3 & 9.07 & 54.1 & 53.4 & 25.501 & 15.117 & 90.168 & 89.002 \\
\hline
\end{tabular}


SRNL-STI-2009-00465, Revision 0

Table B1. Analytical Development's Measurements of the PCT Solutions AsReceived (ar) and After Appropriate Adjustments (in ppm)

\begin{tabular}{|c|c|c|c|c|c|c|c|c|c|c|c|c|c|c|}
\hline Set & Glass ID & $\begin{array}{c}\text { Heat } \\
\text { Treatment } \\
\end{array}$ & Block & Seq & $\begin{array}{c}\text { Analytical } \\
\text { Sequence } \\
\end{array}$ & $\begin{array}{c}\text { Lab } \\
\text { ID }\end{array}$ & $\begin{array}{c}\text { B } \\
\text { ar }\end{array}$ & $\begin{array}{l}\mathrm{Li} \\
\mathrm{ar}\end{array}$ & $\begin{array}{l}\mathrm{Na} \\
\mathrm{ar}\end{array}$ & $\begin{array}{l}\text { Si } \\
\text { ar }\end{array}$ & $\begin{array}{c}\text { B } \\
(\mathbf{p p m})\end{array}$ & $\begin{array}{c}\mathbf{L i} \\
(\mathbf{p p m})\end{array}$ & $\begin{array}{c}\mathrm{Na} \\
(\mathrm{ppm})\end{array}$ & $\begin{array}{c}\text { Si ( } \\
\text { ppm) }\end{array}$ \\
\hline 1 & ROC-05 & \begin{tabular}{|c|} 
CCC \\
\end{tabular} & 3 & 20 & \begin{tabular}{|l|}
71 \\
\end{tabular} & c59 & 15.5 & 16.0 & 45.3 & 80.3 & 25.834 & 26.667 & 75.502 & 133.836 \\
\hline 1 & ROC-08 & CCC & 3 & 21 & 72 & c28 & 26.8 & 37.2 & 160 & 221 & 44.668 & 62.001 & 266.672 & 368.341 \\
\hline 1 & ROC-10 & $\mathrm{CCC}$ & 3 & 22 & 73 & c39 & 9.86 & 11.9 & 39.8 & 71.6 & 16.434 & 19.834 & 66.335 & 119.336 \\
\hline 1 & EA & ref & 3 & 23 & 74 & c16 & 37.8 & 11.4 & 103 & 55.5 & 630.001 & 190.000 & 1716.670 & 925.002 \\
\hline 1 & ROC-05 & quenched & 3 & 24 & 75 & c61 & 15.60 & 12.6 & 44.3 & 68.9 & 26.001 & 21.000 & 73.835 & 114.836 \\
\hline 1 & ROC-10 & quenched & 3 & 25 & 76 & c30 & 13.0 & 15.4 & 52.5 & 93.5 & 21.667 & 25.667 & 87.502 & 155.836 \\
\hline 1 & Soln Std & ref & 3 & 26 & 77 & std-33 & 20.5 & 10.0 & 81.8 & 51.0 & 20.500 & 10.000 & 81.800 & 51.000 \\
\hline 2 & Soln Std & ref & 1 & 1 & 78 & std-11 & 20.1 & 9.93 & 81.5 & 49.9 & 20.100 & 9.930 & 81.500 & 49.900 \\
\hline 2 & ROC-20 & quenched & 1 & 2 & 79 & $\mathrm{~d} 17$ & 159 & 71.7 & 368 & 245 & 265.005 & 119.502 & 613.346 & 408.342 \\
\hline 2 & ROC-14 & quenched & 1 & 3 & 80 & d51 & 25.7 & 14.3 & 35.0 & 45.1 & 42.834 & 23.834 & 58.335 & 75.168 \\
\hline 2 & ROC-20 & CCC & 1 & 4 & 81 & d20 & 122 & 58.3 & 288 & 221 & 203.337 & 97.169 & 480.010 & 368.341 \\
\hline 2 & ROC-12 & CCC & 1 & 5 & 82 & d23 & 12.0 & 19.3 & 63.5 & 72.5 & 20.000 & 32.167 & 105.835 & 120.836 \\
\hline 2 & ROC-16 & $\mathrm{CCC}$ & 1 & 6 & 83 & d36 & 84.6 & 77.9 & 138 & 250 & 141.003 & 129.836 & 230.005 & 416.675 \\
\hline 2 & ROC-15 & CCC & 1 & 7 & 84 & d27 & 6.79 & 10.5 & 73.4 & 80.9 & 11.317 & 17.500 & 122.336 & 134.836 \\
\hline 2 & ROC-16 & quenched & 1 & 8 & 85 & d56 & 85.0 & 77.2 & 140 & 247 & 141.670 & 128.669 & 233.338 & 411.675 \\
\hline 2 & ROC-13 & CCC & 1 & 9 & 86 & $\mathrm{~d} 32$ & 6.59 & 13.6 & 30.5 & 63.9 & 10.984 & 22.667 & 50.834 & 106.502 \\
\hline 2 & ROC-11 & quenched & 1 & 10 & 87 & d09 & 9.38 & 13.5 & 43.6 & 91.6 & 15.634 & 22.500 & 72.668 & 152.670 \\
\hline 2 & blank & ref & 1 & 11 & 88 & d06 & $<0.460$ & $<0.102$ & $<0.332$ & $<0.301$ & 0.383 & 0.085 & 0.277 & 0.251 \\
\hline 2 & ROC-19 & $\mathrm{CCC}$ & 1 & 12 & 89 & $\mathrm{~d} 65$ & 50.0 & 20.9 & 63.2 & 103 & 83.335 & 34.834 & 105.335 & 171.670 \\
\hline 2 & Soln Std & ref & 1 & 13 & 90 & std-12 & 20.1 & 9.92 & 81.2 & 50.1 & 20.100 & 9.920 & 81.200 & 50.100 \\
\hline 2 & ROC-13 & quenched & 1 & 14 & 91 & \begin{tabular}{|l|}
$\mathrm{d} 41$ \\
\end{tabular} & 6.32 & 12.7 & 30.4 & 61.5 & 10.534 & 21.167 & 50.668 & 102.502 \\
\hline 2 & EA & ref & 1 & 15 & 92 & $\mathrm{~d} 46$ & 37.6 & 11.9 & 104 & 58.3 & 626.668 & 198.334 & 1733.337 & 971.669 \\
\hline 2 & ROC-17 & CCC & 1 & 16 & 93 & d08 & 9.19 & 16.5 & 96.3 & 102 & 15.317 & 27.501 & 160.503 & 170.003 \\
\hline 2 & ROC-15 & quenched & 1 & 17 & 94 & $\mathrm{~d} 50$ & 6.92 & 8.60 & 68.2 & 71.7 & 11.534 & 14.334 & 113.669 & 119.502 \\
\hline 2 & ROC-18 & quenched & 1 & 18 & 95 & $\mathrm{~d} 44$ & 9.42 & 11.7 & 40.9 & 80.3 & 15.700 & 19.500 & 68.168 & 133.836 \\
\hline 2 & ROC-11 & CCC & 1 & 19 & 96 & $\mathrm{~d} 52$ & 8.26 & 13.2 & 38.2 & 86.8 & 13.767 & 22.000 & 63.668 & 144.670 \\
\hline 2 & ROC-18 & CCC & 1 & 20 & 97 & $\mathrm{~d} 12$ & 7.52 & 10.7 & 34.1 & 73.4 & 12.534 & 17.834 & 56.834 & 122.336 \\
\hline 2 & ROC-12 & quenched & 1 & 21 & 98 & $\mathrm{~d} 57$ & 12.6 & 19.8 & 69.9 & 78.0 & 21.000 & 33.001 & 116.502 & 130.003 \\
\hline 2 & ROC-17 & quenched & 1 & 22 & 99 & d64 & 10.6 & 14.3 & 96.3 & 97.6 & 17.667 & 23.834 & 160.503 & 162.670 \\
\hline 2 & ARM-1 & ref & 1 & 23 & 100 & d26 & 12.7 & 9.16 & 24.1 & 39.2 & 21.167 & 15.267 & 40.167 & 65.335 \\
\hline 2 & ROC-19 & quenched & 1 & 24 & 101 & $\mathrm{~d} 48$ & 50.4 & 21.0 & 62.6 & 101 & 84.002 & 35.001 & 104.335 & 168.337 \\
\hline 2 & ROC-14 & $\mathrm{CCC}$ & 1 & 25 & 102 & $\mathrm{~d} 68$ & 35.8 & 19.3 & 42.6 & 45.9 & 59.668 & 32.167 & 71.001 & \begin{tabular}{|l|}
76.502 \\
\end{tabular} \\
\hline 2 & Soln Std & ref & 1 & 26 & 103 & std-13 & 20.1 & 9.84 & 81.6 & 50.1 & 20.100 & 9.840 & 81.600 & 50.100 \\
\hline 2 & Soln Std & ref & 2 & 1 & 104 & std-21 & 20.1 & 9.99 & 82.3 & 50.2 & 20.100 & 9.990 & 82.300 & 50.200 \\
\hline 2 & ARM-1 & ref & 2 & 2 & 105 & d60 & 10.8 & 8.27 & 21.5 & 36.1 & 18.000 & 13.784 & 35.834 & 60.168 \\
\hline 2 & ROC-12 & CCC & 2 & 3 & 106 & d21 & 12.1 & 19.7 & 64.6 & 73.6 & 20.167 & 32.834 & 107.669 & 122.669 \\
\hline 2 & ROC-16 & quenched & 2 & 4 & 107 & d11 & 90.2 & 81.8 & 150 & 262 & 150.336 & 136.336 & 250.005 & 436.675 \\
\hline 2 & ROC-20 & quenched & 2 & 5 & 108 & $\mathrm{~d} 15$ & 154 & 69.0 & 359 & 238 & 256.672 & 115.002 & 598.345 & 396.675 \\
\hline 2 & ROC-15 & quenched & 2 & 6 & 109 & $\mathrm{~d} 01$ & 6.52 & 8.12 & 64.1 & 66.7 & 10.867 & 13.534 & 106.835 & 111.169 \\
\hline 2 & ROC-12 & quenched & 2 & 7 & 110 & d05 & 13.0 & 20.4 & 73.7 & 79.5 & 21.667 & 34.001 & 122.836 & 132.503 \\
\hline 2 & ROC-11 & CCC & 2 & 8 & 111 & $\mathrm{~d} 35$ & 8.15 & 13.2 & 38.8 & 87.0 & 13.584 & 22.000 & 64.668 & 145.003 \\
\hline 2 & ROC-18 & quenched & 2 & 9 & 112 & d29 & 9.32 & 11.7 & 40.7 & 79.5 & 15.534 & 19.500 & 67.835 & 132.503 \\
\hline 2 & ROC-16 & CCC & 2 & 10 & 113 & d19 & 84.1 & 78.3 & 139 & 251 & 140.169 & 130.503 & 231.671 & 418.342 \\
\hline 2 & ROC-15 & CCC & 2 & 11 & 114 & $\mathrm{~d} 13$ & 6.59 & 10.4 & 72.8 & 79.6 & 10.984 & 17.334 & 121.336 & 132.669 \\
\hline 2 & ROC-20 & $\mathrm{CCC}$ & 2 & 12 & 115 & \begin{tabular}{|l}
$\mathrm{d} 58$ \\
\end{tabular} & 123 & 59.3 & 293 & 224 & 205.004 & 98.835 & 488.343 & 373.341 \\
\hline 2 & Soln Std & ref & 2 & 13 & 116 & std-22 & 20.1 & 10.0 & 81.8 & 50.1 & 20.100 & 10.000 & 81.800 & 50.100 \\
\hline 2 & ROC-19 & quenched & 2 & 14 & 117 & $\mathrm{~d} 10$ & 51.4 & 22.0 & 64.8 & 103 & 85.668 & 36.667 & 108.002 & 171.670 \\
\hline 2 & ROC-13 & $\mathrm{CCC}$ & 2 & 15 & 118 & d63 & 6.52 & 13.7 & 30.5 & 63.2 & 10.867 & 22.834 & 50.834 & 105.335 \\
\hline 2 & ROC-17 & CCC & 2 & 16 & 119 & d03 & 9.24 & 16.8 & 97.9 & 102 & 15.400 & 28.001 & 163.170 & 170.003 \\
\hline 2 & ROC-18 & $\mathrm{CCC}$ & 2 & 17 & 120 & $\mathrm{~d} 43$ & 7.19 & 10.4 & 32.8 & 71.1 & 11.984 & 17.334 & 54.668 & 118.502 \\
\hline 2 & ROC-14 & CCC & 2 & 18 & 121 & $\mathrm{~d} 67$ & 37.6 & 20.9 & 45.9 & 47.9 & 62.668 & 34.834 & 76.502 & 79.835 \\
\hline 2 & ROC-19 & $\mathrm{CCC}$ & 2 & 19 & 122 & d31 & 50.4 & 21.5 & 64.3 & 105 & 84.002 & 35.834 & 107.169 & 175.004 \\
\hline 2 & ROC-14 & quenched & 2 & 20 & 123 & d25 & 23.4 & 13.3 & 32.5 & 42.3 & 39.001 & 22.167 & 54.168 & 70.501 \\
\hline 2 & ROC-17 & quenched & 2 & 21 & 124 & $\mathrm{~d} 53$ & 10.6 & 14.6 & 97.3 & 99.6 & 17.667 & 24.334 & 162.170 & 166.003 \\
\hline 2 & ROC-13 & quenched & 2 & 22 & 125 & $\mathrm{~d} 54$ & 6.60 & 13.4 & 32.6 & 64.1 & 11.000 & 22.334 & 54.334 & 106.835 \\
\hline 2 & ROC-11 & quenched & 2 & 23 & 126 & $\mathrm{~d} 04$ & 8.96 & 13.2 & 42.1 & 87.6 & 14.934 & 22.000 & 70.168 & 146.003 \\
\hline 2 & EA & ref & 2 & 24 & 127 & $\mathrm{~d} 45$ & 38.7 & 11.9 & 107 & 56.6 & 645.001 & 198.334 & 1783.337 & 943.335 \\
\hline 2 & Soln Std & ref & 2 & 25 & 128 & std-23 & 20.0 & 9.93 & 81.9 & 50.0 & 20.000 & 9.930 & 81.900 & 50.000 \\
\hline 2 & Soln Std & ref & 3 & 1 & 129 & std-31 & 20.3 & 10.0 & 81.6 & 50.0 & 20.300 & 10.000 & 81.600 & 50.000 \\
\hline 2 & ROC-14 & CCC & 3 & 2 & 130 & $\mathrm{~d} 38$ & 37.2 & 20.6 & 44.2 & 45.5 & 62.001 & 34.334 & 73.668 & 75.835 \\
\hline 2 & ROC-14 & quenched & 3 & 3 & 131 & d34 & 24.5 & 14.1 & 33.9 & 43.9 & 40.834 & 23.500 & 56.501 & 73.168 \\
\hline 2 & ROC-16 & CCC & 3 & 4 & 132 & $\mathrm{~d} 14$ & 84.2 & 80.2 & 138 & 252 & 140.336 & 133.669 & 230.005 & 420.008 \\
\hline 2 & ROC-17 & CCC & 3 & 5 & 133 & $\mathrm{~d} 49$ & 9.08 & 16.7 & 96.1 & 102 & 15.134 & 27.834 & 160.170 & 170.003 \\
\hline 2 & ROC-18 & quenched & 3 & 6 & 134 & $\mathrm{~d} 62$ & 9.30 & 11.7 & 40.5 & 77.8 & 15.500 & 19.500 & 67.501 & 129.669 \\
\hline 2 & ROC-11 & CCC & 3 & 7 & 135 & $\mathrm{~d} 40$ & 8.28 & 13.5 & 38.5 & 87.0 & 13.800 & 22.500 & 64.168 & 145.003 \\
\hline 2 & ROC-13 & CCC & 3 & 8 & 136 & d18 & 6.76 & 14.4 & 31.7 & 65.0 & 11.267 & 24.000 & 52.834 & 108.336 \\
\hline 2 & ROC-17 & quenched & 3 & 9 & 137 & $\mathrm{~d} 42$ & 10.8 & 15.0 & 99.1 & 103 & 18.000 & 25.001 & 165.170 & 171.670 \\
\hline 2 & ROC-11 & quenched & 3 & 10 & 138 & d24 & 9.63 & 14.1 & 44.2 & 93.2 & 16.050 & 23.500 & 73.668 & 155.336 \\
\hline 2 & blank & ref & 3 & 11 & 139 & $\mathrm{~d} 47$ & $<0.460$ & $<0.102$ & $<0.332$ & $<0.301$ & 0.383 & 0.085 & 0.277 & 0.251 \\
\hline 2 & ROC-16 & quenched & 3 & 12 & 140 & d33 & 86.5 & 80.7 & 142 & 251 & 144.170 & 134.503 & 236.671 & 418.342 \\
\hline
\end{tabular}


SRNL-STI-2009-00465, Revision 0

Table B1. Analytical Development's Measurements of the PCT Solutions AsReceived (ar) and After Appropriate Adjustments (in ppm)

\begin{tabular}{|c|c|c|c|c|c|c|c|c|c|c|c|c|c|c|}
\hline Set & Glass ID & $\begin{array}{c}\text { Heat } \\
\text { Treatment }\end{array}$ & Block & Seq & $\begin{array}{l}\text { Analytical } \\
\text { Sequence }\end{array}$ & $\begin{array}{c}\text { Lab } \\
\text { ID }\end{array}$ & $\begin{array}{c}\text { B } \\
\text { ar }\end{array}$ & $\begin{array}{l}\mathrm{Li} \\
\text { ar }\end{array}$ & $\begin{array}{l}\mathrm{Na} \\
\mathrm{ar}\end{array}$ & $\begin{array}{l}\text { Si } \\
\text { ar }\end{array}$ & $\begin{array}{c}\mathbf{B} \\
(\mathbf{p p m})\end{array}$ & $\begin{array}{c}\mathbf{L i} \\
(\mathbf{p p m})\end{array}$ & $\begin{array}{c}\mathrm{Na} \\
(\mathrm{ppm})\end{array}$ & $\begin{array}{c}\mathrm{Si}( \\
\text { ppm) }\end{array}$ \\
\hline 2 & Soln Std & ref & 3 & 13 & 141 & std-32 & 20.1 & 10.1 & 82.0 & 49.7 & 20.100 & 10.100 & 82.000 & 49.700 \\
\hline 2 & ROC-19 & quenched & 3 & 14 & 142 & $\mathrm{~d} 22$ & 51.0 & 22.0 & 63.9 & 102 & 85.002 & 36.667 & 106.502 & 170.003 \\
\hline 2 & ROC-13 & quenched & 3 & 15 & 143 & d66 & 6.65 & 13.6 & 32.2 & 64.3 & 11.084 & 22.667 & 53.668 & \begin{tabular}{|l}
107.169 \\
\end{tabular} \\
\hline 2 & ROC-12 & quenched & 3 & 16 & 144 & d07 & 12.7 & 20.4 & 71.4 & 78.0 & 21.167 & 34.001 & 119.002 & 130.003 \\
\hline 2 & ROC-19 & $\mathrm{CCC}$ & 3 & 17 & 145 & d02 & 49.7 & 21.2 & 63.2 & 103 & 82.835 & 35.334 & 105.335 & 171.670 \\
\hline 2 & ARM-1 & ref & 3 & 18 & 146 & d37 & 11.0 & 8.60 & 22.3 & 37.0 & 18.334 & 14.334 & 37.167 & 61.668 \\
\hline 2 & ROC-12 & CCC & 3 & 19 & 147 & d55 & 12.2 & 20.2 & 64.7 & 74.6 & 20.334 & 33.667 & 107.835 & 124.336 \\
\hline 2 & ROC-15 & CCC & 3 & 20 & 148 & d59 & 6.67 & 10.6 & 72.8 & 79.2 & 11.117 & 17.667 & 121.336 & \begin{tabular}{|l|}
132.003 \\
\end{tabular} \\
\hline 2 & ROC-18 & CCC & 3 & 21 & 149 & d28 & 7.45 & 10.9 & 33.9 & 71.8 & 12.417 & 18.167 & 56.501 & 119.669 \\
\hline 2 & ROC-20 & CCC & 3 & 22 & 150 & d39 & 120 & 59.2 & 285 & 218 & 200.004 & 98.669 & 475.010 & 363.341 \\
\hline 2 & EA & ref & 3 & 23 & 151 & $\mathrm{~d} 16$ & 34.0 & 10.6 & 93.7 & 49.2 & 566.668 & 176.667 & 1561.670 & 820.002 \\
\hline 2 & ROC-15 & quenched & 3 & 24 & 152 & $\mathrm{~d} 61$ & 7.34 & 9.07 & 71.5 & 74.1 & 12.234 & 15.117 & 119.169 & \begin{tabular}{|l}
123.502 \\
\end{tabular} \\
\hline 2 & ROC-20 & quenched & 3 & 25 & 153 & $\mathrm{~d} 30$ & 161 & 73.6 & 376 & 247 & 268.339 & 122.669 & 626.679 & 411.675 \\
\hline 2 & Soln Std & ref & 3 & 26 & 154 & std-33 & 20.2 & 10.1 & 82.5 & 50.1 & 20.200 & 10.100 & 82.500 & \begin{tabular}{|l|}
50.100 \\
\end{tabular} \\
\hline 3 & Soln Std & ref & 1 & 1 & 155 & std-11 & 20.1 & 10.0 & 81.0 & 50.2 & 20.100 & 10.000 & 81.000 & 50.200 \\
\hline 3 & ROC-22 & CCC & 1 & 2 & 156 & $\mathrm{e} 23$ & 13.1 & 15.2 & 41.1 & 86.7 & 21.834 & 25.334 & 68.501 & 144.503 \\
\hline 3 & $\begin{array}{c}\text { FY09EM21- } \\
29\end{array}$ & CCC & 1 & 3 & 157 & e78 & 9.91 & 12.4 & 49.1 & 66.5 & 16.517 & 20.667 & 81.835 & 110.836 \\
\hline 3 & ROC-28 & quenched & 1 & 4 & 158 & e44 & 21.8 & 24.2 & 197 & 229 & 36.334 & 40.334 & 328.340 & 381.674 \\
\hline 3 & ROC-21 & quenched & 1 & 5 & 159 & e09 & 21.0 & 11.4 & 33.6 & 48.5 & 35.001 & 19.000 & 56.001 & 80.835 \\
\hline 3 & ROC-29 & $\mathrm{CCC}$ & 1 & 6 & 160 & e65 & 38.7 & 19.7 & 47.8 & 50.6 & 64.501 & 32.834 & 79.668 & 84.335 \\
\hline 3 & ROC-24 & CCC & 1 & 7 & 161 & e68 & 12.0 & 7.38 & 22.3 & 47.3 & 20.000 & 12.300 & 37.167 & 78.835 \\
\hline 3 & ROC-24 & quenched & 1 & 8 & 162 & e51 & 12.4 & 7.95 & 22.6 & 46.8 & 20.667 & 13.250 & 37.667 & 78.002 \\
\hline 3 & ROC-28 & CCC & 1 & 9 & 163 & e12 & 16.7 & 20.0 & 157 & 175 & 27.834 & 33.334 & 261.672 & 291.673 \\
\hline 3 & ROC-09 & CCC & $\frac{1}{1}$ & 10 & 164 & e92 & 68.9 & 34.2 & 188 & 148 & 114.836 & 57.001 & 313.340 & \begin{tabular}{|l}
246.672 \\
\end{tabular} \\
\hline 3 & ROC-25 & $\mathrm{CCC}$ & 1 & 11 & 165 & $\mathrm{e} 27$ & 10.4 & 13.1 & 86.1 & 95.1 & 17.334 & 21.834 & 143.503 & 158.503 \\
\hline 3 & ROC-23 & CCC & 1 & 12 & 166 & e32 & 5.95 & 9.56 & 26.6 & 60.8 & 9.917 & 15.934 & 44.334 & 101.335 \\
\hline 3 & ROC-21 & CCC & 1 & 13 & 167 & e52 & 19.8 & 11.0 & 31.9 & 49.6 & 33.001 & 18.334 & 53.168 & 82.668 \\
\hline 3 & $\begin{array}{c}\text { FY09EM21- } \\
30\end{array}$ & CCC & 1 & 14 & 168 & e70 & 9.98 & 12.7 & 47.1 & 67.1 & 16.634 & 21.167 & 78.502 & 111.836 \\
\hline 3 & ROC-22 & quenched & 1 & 15 & 169 & e57 & 17.9 & 18.5 & 54.6 & 101 & 29.834 & 30.834 & 91.002 & 168.337 \\
\hline 3 & ROC-26 & quenched & 1 & 16 & 170 & e56 & 10.0 & 9.34 & 86.8 & 77.7 & 16.667 & 15.567 & 144.670 & 129.503 \\
\hline 3 & Soln Std & ref & 1 & 17 & 171 & std-12 & 20.0 & 9.99 & 81.9 & 50.0 & 20.000 & 9.990 & 81.900 & 50.000 \\
\hline 3 & $\begin{array}{c}\text { FY09EM21- } \\
29\end{array}$ & quenched & 1 & 18 & 172 & $\mathrm{e} 73$ & 14.6 & 16.1 & 61.4 & 76.6 & 24.334 & 26.834 & 102.335 & \begin{tabular}{|l|}
127.669 \\
\end{tabular} \\
\hline 3 & ROC-26 & $\mathrm{CCC}$ & 1 & 19 & 173 & e36 & 8.59 & 8.84 & 71.9 & 72.6 & 14.317 & 14.734 & 119.836 & 121.002 \\
\hline 3 & EA & ref & 1 & 20 & 174 & e46 & 36.5 & 11.6 & 101 & 55.6 & 608.335 & 193.334 & 1683.337 & 926.669 \\
\hline 3 & ARM-1 & ref & 1 & 21 & 175 & e26 & 10.5 & 8.31 & 21.1 & 36.9 & 17.500 & 13.850 & 35.167 & \begin{tabular}{|l|}
61.501 \\
\end{tabular} \\
\hline 3 & ROC-27 & CCC & 1 & 22 & 176 & $\mathrm{e} 08$ & 10.2 & 14.8 & 61.3 & 93.4 & 17.000 & 24.667 & 102.169 & 155.670 \\
\hline 3 & $\begin{array}{c}\text { FY09EM21- } \\
28\end{array}$ & quenched & 1 & 23 & 177 & e74 & 13.4 & 14.6 & 57.4 & 68.9 & 22.334 & 24.334 & 95.669 & 114.836 \\
\hline 3 & ROC-25 & quenched & 1 & 24 & 178 & e50 & 11.4 & 13.4 & 99.4 & 105 & 19.000 & 22.334 & 165.670 & 175.004 \\
\hline 3 & ROC-30 & quenched & $\frac{1}{1}$ & 25 & 179 & $\mathrm{e} 17$ & 12.8 & 11.3 & 45.9 & 67.5 & 21.334 & 18.834 & 76.502 & \begin{tabular}{|l}
112.502 \\
17.5
\end{tabular} \\
\hline 3 & blank & ref & 1 & 26 & 180 & e06 & $<0.460$ & $<0.102$ & $<0.332$ & $<0.301$ & 0.383 & 0.085 & 0.277 & 0.251 \\
\hline 3 & $\begin{array}{c}\text { FY09EM21- } \\
30\end{array}$ & quenched & 1 & 27 & 181 & e69 & 13.3 & 14.6 & 54.6 & 68.8 & 22.167 & 24.334 & 91.002 & 114.669 \\
\hline 3 & ROC-29 & quenched & 1 & 28 & 182 & e48 & 27.6 & 14.7 & 40.8 & 49.4 & 46.001 & 24.500 & 68.001 & 82.335 \\
\hline 3 & ROC-30 & CCC & 1 & 29 & 183 & $\mathrm{e} 20$ & 13.5 & 12.3 & 46.2 & 70.4 & 22.500 & 20.500 & 77.002 & 117.336 \\
\hline 3 & ROC-27 & quenched & 1 & 30 & 184 & e64 & 12.5 & 15.2 & 69.6 & 94.8 & 20.834 & 25.334 & 116.002 & 158.003 \\
\hline 3 & ROC-23 & quenched & 1 & 31 & 185 & e41 & 7.26 & 10.5 & 29.1 & 62.8 & 12.100 & 17.500 & 48.501 & \begin{tabular}{|l}
104.669 \\
\end{tabular} \\
\hline 3 & ROC-09 & quenched & 1 & 32 & 186 & $\mathrm{e} 87$ & 184 & 83.1 & 465 & 284 & 306.673 & 138.503 & 775.016 & 473.343 \\
\hline 3 & $\begin{array}{c}\text { FY09EM21- } \\
28\end{array}$ & CCC & 1 & 33 & 187 & e72 & 10.5 & 13.2 & 51.4 & 68.7 & 17.500 & 22.000 & 85.668 & 114.502 \\
\hline 3 & Soln Std & ref & 1 & 34 & 188 & std-13 & 20.2 & 10.0 & 82.0 & 50.0 & 20.200 & 10.000 & 82.000 & 50.000 \\
\hline 3 & Soln Std & ref & 2 & 1 & 189 & std-21 & 20.6 & 10.1 & 81.0 & 51.0 & 20.600 & 10.100 & 81.000 & 51.000 \\
\hline 3 & $\begin{array}{c}\text { FY09EM21- } \\
28\end{array}$ & quenched & 2 & 2 & 190 & e79 & 13.9 & 14.9 & 58.8 & 71.6 & 23.167 & 24.834 & 98.002 & 119.336 \\
\hline 3 & ROC-22 & quenched & 2 & 3 & 191 & $\mathrm{e} 05$ & 20.5 & 20.4 & 58.4 & 105 & 34.167 & 34.001 & 97.335 & 175.004 \\
\hline 3 & ROC-26 & CCC & 2 & 4 & 192 & e19 & 9.21 & 9.48 & 77.5 & 79.6 & 15.350 & 15.800 & 129.169 & \begin{tabular}{|l}
132.669 \\
132.69 \\
\end{tabular} \\
\hline 3 & ROC-21 & CCC & 2 & 5 & 193 & e35 & 20.5 & 11.2 & 32.8 & 51.4 & 34.167 & 18.667 & 54.668 & 85.668 \\
\hline 3 & ROC-24 & $\mathrm{CCC}$ & 2 & 6 & 194 & $\mathrm{e} 67$ & 12.5 & $\begin{array}{l}1 . .2 \\
7.56\end{array}$ & 24.7 & 49.3 & 20.834 & 12.600 & 41.167 & 82.168 \\
\hline 3 & ROC-29 & quenched & 2 & 7 & 195 & e10 & 27.5 & 14.4 & 41.3 & 49.0 & 45.834 & 24.000 & 68.835 & 81.668 \\
\hline 3 & $\begin{array}{c}\text { FY09EM21- } \\
30\end{array}$ & quenched & 2 & 8 & 196 & e71 & 13.5 & 14.7 & 55.7 & 69.2 & 22.500 & 24.500 & 92.835 & 115.336 \\
\hline 3 & ROC-21 & quenched & 2 & 9 & 197 & $\mathrm{e} 04$ & 22.0 & 11.9 & 35.2 & 49.9 & 36.667 & 19.834 & 58.668 & 83.168 \\
\hline 3 & $\begin{array}{c}\text { FY09EM21- } \\
28\end{array}$ & CCC & 2 & 10 & 198 & e76 & 9.84 & 12.5 & 48.5 & 66.7 & 16.400 & 20.834 & 80.835 & 111.169 \\
\hline 3 & $\begin{array}{c}\text { FY09EM21- } \\
29\end{array}$ & quenched & 2 & 11 & 199 & e77 & 14.3 & 15.6 & 59.5 & 74.5 & 23.834 & 26.001 & 99.169 & 124.169 \\
\hline 3 & EA & ref & 2 & 12 & 200 & e45 & 36.7 & 11.6 & 98.8 & 55.2 & 611.668 & 193.334 & 1646.670 & 920.002 \\
\hline 3 & ROC-25 & quenched & 2 & 13 & 201 & $\mathrm{e} 01$ & 11.4 & 13.3 & 98.1 & 100 & 19.000 & 22.167 & 163.503 & 166.670 \\
\hline
\end{tabular}


SRNL-STI-2009-00465, Revision 0

Table B1. Analytical Development's Measurements of the PCT Solutions AsReceived (ar) and After Appropriate Adjustments (in ppm)

\begin{tabular}{|c|c|c|c|c|c|c|c|c|c|c|c|c|c|c|}
\hline Set & Glass ID & $\begin{array}{c}\text { Heat } \\
\text { Treatment }\end{array}$ & Block & Seq & $\begin{array}{l}\text { Analytical } \\
\text { Sequence }\end{array}$ & $\begin{array}{c}\text { Lab } \\
\text { ID }\end{array}$ & $\begin{array}{l}\text { B } \\
\text { ar }\end{array}$ & $\begin{array}{l}\mathbf{L i} \\
\text { ar }\end{array}$ & $\begin{array}{c}\mathrm{Na} \\
\mathrm{ar}\end{array}$ & $\begin{array}{l}\text { Si } \\
\text { ar }\end{array}$ & $\begin{array}{c}\text { B } \\
(\mathbf{p p m})\end{array}$ & $\begin{array}{c}\mathbf{L i} \\
(\mathbf{p p m})\end{array}$ & $\begin{array}{c}\mathrm{Na} \\
(\mathrm{ppm})\end{array}$ & $\begin{array}{c}\text { Si ( } \\
\text { ppm) }\end{array}$ \\
\hline 3 & ROC-29 & CCC & 2 & 14 & 202 & e31 & 36.4 & 18.5 & 46.6 & 50.6 & 60.668 & 30.834 & 77.668 & 84.335 \\
\hline 3 & ROC-22 & $\mathrm{CCC}$ & 2 & 15 & 203 & e21 & 13.6 & 15.7 & 41.4 & 89.9 & 22.667 & 26.167 & 69.001 & 149.836 \\
\hline 3 & ROC-28 & $\mathrm{CCC}$ & 2 & 16 & 204 & e43 & 16.0 & 18.7 & 147 & 160 & 26.667 & 31.167 & 245.005 & 266.672 \\
\hline 3 & Soln Std & ref & 2 & 17 & 205 & std-22 & 20.3 & 10.0 & 79.9 & 50.3 & 20.300 & 10.000 & 79.900 & 50.300 \\
\hline 3 & ROC-23 & quenched & 2 & 18 & 206 & e54 & 7.42 & 10.5 & 28.0 & 63.9 & 12.367 & 17.500 & 46.668 & 106.502 \\
\hline 3 & ROC-30 & CCC & 2 & 19 & 207 & e58 & 14.0 & 12.7 & 46.7 & 73.4 & 23.334 & 21.167 & 77.835 & 122.336 \\
\hline 3 & ROC-09 & $\mathrm{CCC}$ & 2 & 20 & 208 & $\mathrm{e} 89$ & 69.5 & 34.1 & 183 & 147 & 115.836 & 56.834 & 305.006 & 245.005 \\
\hline 3 & ROC-23 & $\mathrm{CCC}$ & 2 & 21 & 209 & e63 & 5.93 & 9.39 & 24.7 & 60.0 & 9.884 & 15.650 & 41.167 & 100.002 \\
\hline 3 & ROC-26 & quenched & 2 & 22 & 210 & e11 & 10.3 & 9.43 & 85.6 & 79.1 & 17.167 & 15.717 & 142.670 & 131.836 \\
\hline 3 & ARM-1 & ref & 2 & 23 & 211 & e60 & 11.4 & 8.8 & 22.8 & 38.8 & 19.000 & 14.667 & 38.001 & 64.668 \\
\hline 3 & ROC-24 & quenched & 2 & 24 & 212 & e25 & 12.7 & 7.99 & 23.6 & 48.2 & 21.167 & 13.317 & 39.334 & 80.335 \\
\hline 3 & ROC-28 & quenched & 2 & 25 & 213 & e29 & 20.8 & 22.8 & 183 & 180 & 34.667 & 38.001 & 305.006 & 300.006 \\
\hline 3 & ROC-27 & quenched & 2 & 26 & 214 & e53 & 12.8 & 15.2 & 69.5 & 95.6 & 21.334 & 25.334 & 115.836 & 159.337 \\
\hline 3 & ROC-30 & quenched & 2 & 27 & 215 & e15 & 13.8 & 12.1 & 48.5 & 71.6 & 23.000 & 20.167 & 80.835 & 119.336 \\
\hline 3 & ROC-25 & CCC & 2 & 28 & 216 & $\mathrm{e} 13$ & 10.4 & 12.9 & 83.6 & 96.6 & 17.334 & 21.500 & 139.336 & 161.003 \\
\hline 3 & ROC-09 & quenched & 2 & 29 & 217 & e91 & 201 & 89 & 497 & 307 & 335.007 & 148.336 & 828.350 & 511.677 \\
\hline 3 & $\begin{array}{c}\text { FY09EM21- } \\
30 \\
\end{array}$ & $\mathrm{CCC}$ & 2 & 30 & 218 & e75 & 10.3 & 12.9 & 46.8 & 69.2 & 17.167 & 21.500 & 78.002 & 115.336 \\
\hline 3 & ROC-27 & $\mathrm{CCC}$ & 2 & 31 & 219 & $\mathrm{e} 03$ & 10.5 & 15.1 & 60.4 & 95.6 & 17.500 & 25.167 & 100.669 & 159.337 \\
\hline 3 & $\begin{array}{c}\text { FY09EM21- } \\
29 \\
\end{array}$ & $\mathrm{CCC}$ & 2 & 32 & 220 & e81 & 10.8 & 13.1 & 50.5 & 70.9 & 18.000 & 21.834 & 84.168 & 118.169 \\
\hline 3 & Soln Std & ref & 2 & 33 & 221 & std-23 & 20.5 & 10.1 & 80.7 & 51.0 & 20.500 & 10.100 & 80.700 & 51.000 \\
\hline 3 & Soln Std & ref & 3 & 1 & 222 & std-31 & 20.4 & 10.1 & 81.6 & 51.0 & 20.400 & 10.100 & 81.600 & 51.000 \\
\hline 3 & ROC-21 & quenched & 3 & 2 & 223 & $\mathrm{e} 24$ & 22.2 & 11.9 & 34.0 & 49.0 & 37.001 & 19.834 & 56.668 & 81.668 \\
\hline 3 & ROC-25 & quenched & 3 & 3 & 224 & e61 & 11.6 & 13.4 & 99.1 & 102 & 19.334 & 22.334 & 165.170 & 170.003 \\
\hline 3 & ROC-24 & quenched & 3 & 4 & 225 & e34 & 12.5 & 7.94 & 23.3 & 47.5 & 20.834 & 13.234 & 38.834 & 79.168 \\
\hline 3 & ROC-27 & quenched & 3 & 5 & 226 & e42 & 12.8 & 15.4 & 70.5 & 102 & 21.334 & 25.667 & 117.502 & 170.003 \\
\hline 3 & ARM-1 & ref & 3 & 6 & 227 & e37 & 11.1 & 8.57 & 22.7 & 37.6 & 18.500 & 14.284 & 37.834 & 62.668 \\
\hline 3 & ROC-29 & CCC & 3 & 7 & 228 & $\mathrm{e} 02$ & 38.4 & 19.3 & 47.1 & 50.1 & 64.001 & 32.167 & 78.502 & 83.502 \\
\hline 3 & $\begin{array}{c}\text { FY09EM21- } \\
29 \\
\end{array}$ & quenched & 3 & 8 & 229 & e83 & 13.8 & 15.1 & 58.2 & 73.0 & 23.000 & 25.167 & 97.002 & 121.669 \\
\hline 3 & EA & ref & 3 & 9 & 230 & e16 & 37.7 & 11.6 & 101 & 55.0 & 628.335 & 193.334 & 1683.337 & 916.669 \\
\hline 3 & ROC-30 & quenched & 3 & 10 & 231 & e30 & 14.0 & 12.5 & 49.0 & 73.7 & 23.334 & 20.834 & 81.668 & 122.836 \\
\hline 3 & ROC-22 & CCC & 3 & 11 & 232 & e55 & 14.3 & 16.4 & 44.0 & 96.3 & 23.834 & 27.334 & 73.335 & 160.503 \\
\hline 3 & $\begin{array}{c}\text { FY09EM21- } \\
30 \\
\end{array}$ & quenched & 3 & 12 & 233 & e86 & 14.0 & 15.3 & 57.7 & 72.7 & 23.334 & 25.501 & 96.169 & 121.169 \\
\hline 3 & blank & ref & 3 & 13 & 234 & e47 & $<0.460$ & $<0.102$ & $<0.332$ & $<0.301$ & 0.383 & 0.085 & 0.277 & 0.251 \\
\hline 3 & ROC-30 & $\mathrm{CCC}$ & 3 & 14 & 235 & e39 & 13.8 & 12.7 & 48.3 & 73.1 & 23.000 & 21.167 & 80.502 & 121.836 \\
\hline 3 & ROC-22 & quenched & 3 & 15 & 236 & $\mathrm{e} 07$ & 18.0 & 18.6 & 54.6 & 101 & 30.001 & 31.001 & 91.002 & 168.337 \\
\hline 3 & ROC-21 & CCC & 3 & 16 & 237 & $\mathrm{e} 40$ & 21.3 & 11.8 & 33.7 & 53.3 & 35.501 & 19.667 & 56.168 & 88.835 \\
\hline 3 & Soln Std & ref & 3 & 17 & 238 & std-32 & 20.1 & 10.0 & 81.9 & 50.4 & 20.100 & 10.000 & 81.900 & 50.400 \\
\hline 3 & ROC-28 & quenched & 3 & 18 & 239 & $\mathrm{e} 62$ & 21.8 & 24.4 & 195 & 211 & 36.334 & 40.667 & 325.007 & 351.674 \\
\hline 3 & $\begin{array}{c}\text { FY09EM21- } \\
28 \\
\end{array}$ & quenched & 3 & 19 & 240 & e85 & 13.8 & 15.2 & 57.9 & 72.4 & 23.000 & 25.334 & 96.502 & 120.669 \\
\hline 3 & ROC-29 & quenched & 3 & 20 & 241 & e22 & 27.7 & 14.7 & 40.8 & 49.9 & 46.168 & 24.500 & 68.001 & 83.168 \\
\hline 3 & ROC-23 & CCC & 3 & 21 & 242 & e18 & 5.73 & 9.41 & 25.4 & 60.1 & 9.550 & 15.684 & 42.334 & 100.169 \\
\hline 3 & ROC-26 & CCC & 3 & 22 & 243 & e14 & 8.41 & 8.84 & 70.6 & 72.7 & 14.017 & 14.734 & 117.669 & 121.169 \\
\hline 3 & ROC-26 & quenched & 3 & 23 & 244 & e33 & 9.79 & 9.17 & 85.3 & 77.4 & 16.317 & 15.284 & 142.170 & 129.003 \\
\hline 3 & ROC-09 & $\mathrm{CCC}$ & 3 & 24 & 245 & e88 & 69.0 & 33.7 & 185 & 148 & 115.002 & 56.168 & 308.340 & 246.672 \\
\hline 3 & ROC-23 & quenched & 3 & 25 & 246 & e66 & 6.89 & 10.1 & 26.8 & 61.7 & 11.484 & 16.834 & 44.668 & 102.835 \\
\hline 3 & ROC-25 & CCC & 3 & 26 & 247 & e59 & 10.2 & 13.2 & 83.1 & 103 & 17.000 & 22.000 & 138.503 & 171.670 \\
\hline 3 & $\begin{array}{c}\text { FY09EM21- } \\
29 \\
\end{array}$ & $\mathrm{CCC}$ & 3 & 27 & 248 & e84 & 9.50 & 11.9 & 46.9 & 65.4 & 15.834 & 19.834 & 78.168 & 109.002 \\
\hline 3 & ROC-24 & $\mathrm{CCC}$ & 3 & 28 & 249 & e38 & 12.3 & 7.59 & 23.9 & 48.9 & 20.500 & 12.650 & 39.834 & 81.502 \\
\hline 3 & ROC-28 & $\mathrm{CCC}$ & 3 & 29 & 250 & e28 & 15.9 & 19.0 & 148 & 201 & 26.501 & 31.667 & 246.672 & 335.007 \\
\hline 3 & $\begin{array}{c}\text { FY09EM21- } \\
28 \\
\end{array}$ & CCC & 3 & 30 & 251 & e80 & 9.68 & 12.4 & 47.0 & 66.5 & 16.134 & 20.667 & 78.335 & 110.836 \\
\hline 3 & $\begin{array}{c}\text { FY09EM21- } \\
30 \\
\end{array}$ & CCC & 3 & 31 & 252 & $\mathrm{e} 82$ & 9.98 & 13.0 & 47.3 & 68.6 & 16.634 & 21.667 & 78.835 & 114.336 \\
\hline 3 & ROC-09 & quenched & 3 & 32 & 253 & e90 & 188 & 84.1 & 461 & 287 & 313.340 & 140.169 & 768.349 & 478.343 \\
\hline 3 & ROC-27 & $\mathrm{CCC}$ & 3 & 33 & 254 & e49 & 9.92 & 14.6 & 58.3 & 94.1 & 16.534 & 24.334 & 97.169 & 156.836 \\
\hline 3 & Soln Std & ref & 3 & 34 & 255 & std-33 & 20.1 & 10.0 & 79.5 & 50.5 & 20.100 & 10.000 & 79.500 & 50.500 \\
\hline
\end{tabular}


SRNL-STI-2009-00465, Revision 0

Exhibit B1. PCT Measurements in Analytical Sequence over All of the Analytical Plans
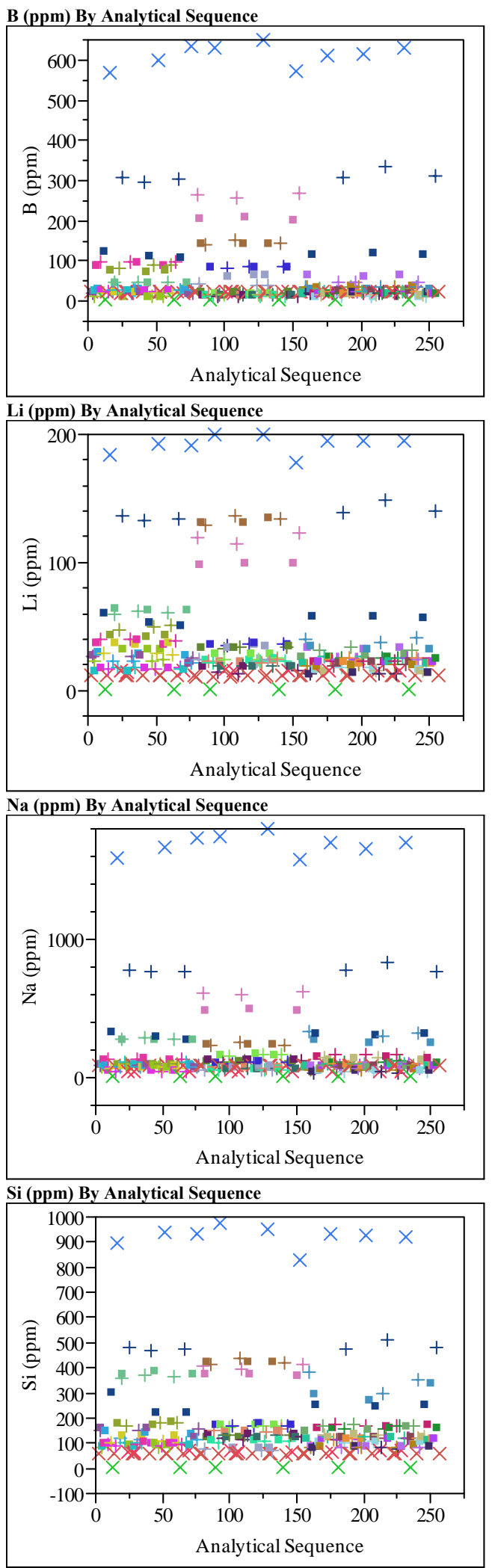

$\log [$ B ppm] By Analytical Sequence
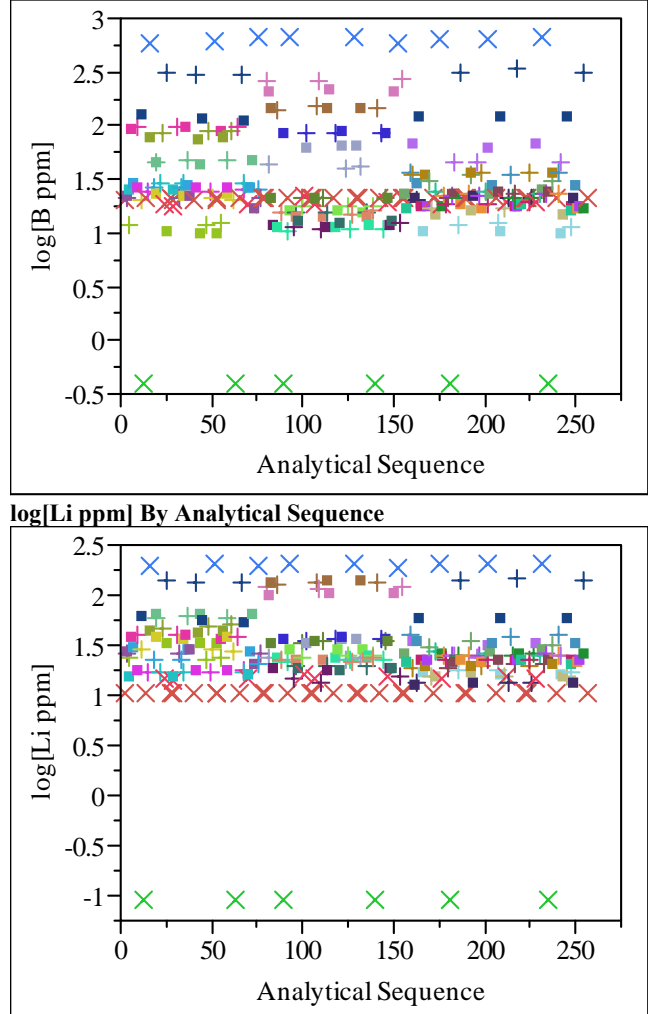

log[Na ppm] By Analytical Sequence

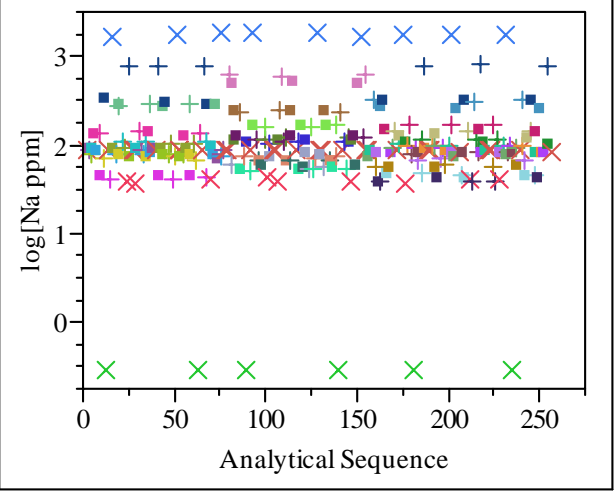

$\log [\mathrm{Si}$ ppm] By Analytical Sequence

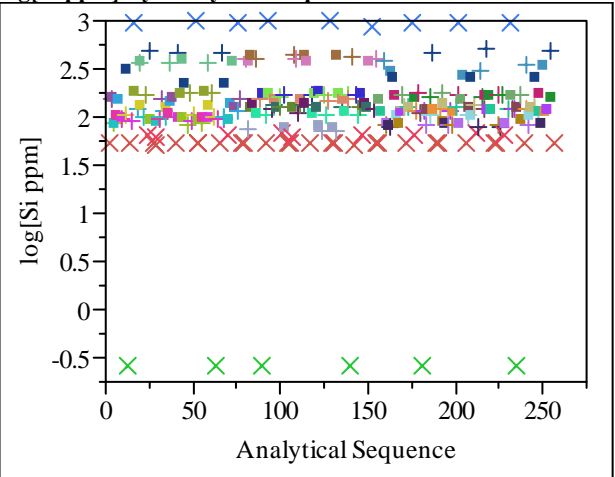


SRNL-STI-2009-00465, Revision 0

\section{Exhibit B2. Measurements of the Multi-Element Solution Standard by ICP Block}

Oneway Analysis of B (ppm) By Set/Block

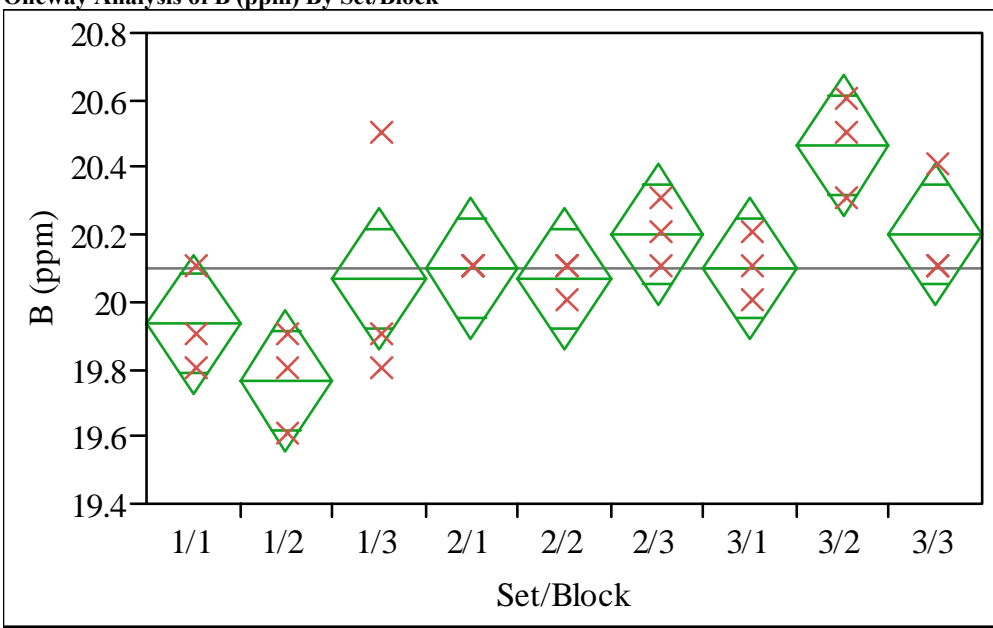

Oneway Anova

Summary of Fit

Rsquare

Adj Rsquare $\quad 0.45748$

0.172133

$\begin{array}{lr}\text { Mean of Response } & 20.1 \\ \text { Observations (or Sum Wgts) } & 27\end{array}$

Analysis of Variance

Source DF Sum of Squares Mean Square F Ratio Prob > F

$\begin{array}{lrrrrr}\text { Set/Block } & 8 & 0.8866667 & 0.110833 & 3.7406 & 0.0096\end{array}$

$\begin{array}{lrrr}\text { Error } & 18 & 0.58333333 & 0.029630 \\ \text { C. Total } & 26 & 1.4200000 & \end{array}$

Means for Oneway Anova

Level Number Mean Std Error Lower 95\% Upper 95\%

$1 / 1$

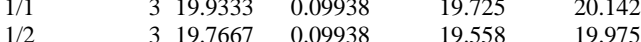

$\begin{array}{llllll}1 / 3 & 3 & 20.0667 & 0.09938 & 19.858 & 20.275\end{array}$

$\begin{array}{llllll}2 / 1 & 3 & 20.1000 & 0.09938 & 19.891 & 20.309 \\ 2 / 2 & 3 & 20.0667 & 0.09938 & 19.858 & 20.275\end{array}$

$\begin{array}{llllll}2 / 2 & 3 & 20.0667 & 0.09938 & 19.858 & 20.275 \\ 2 / 3 & 3 & 20.2000 & 0.09938 & 19.991 & 20.409\end{array}$

$\begin{array}{llllll}2 / 3 & 3 & 20.2000 & 0.09938 & 19.991 & 20.409 \\ 3 / 1 & 3 & 20.1000 & 0.09938 & 19.891 & 20.309\end{array}$

$\begin{array}{llllll}3 / 2 & 3 & 20.4667 & 0.09938 & 20.258 & 20.675\end{array}$

Std Error uses a pooled estimate of error variance
Oneway Analysis of Li (ppm) By Set/Block

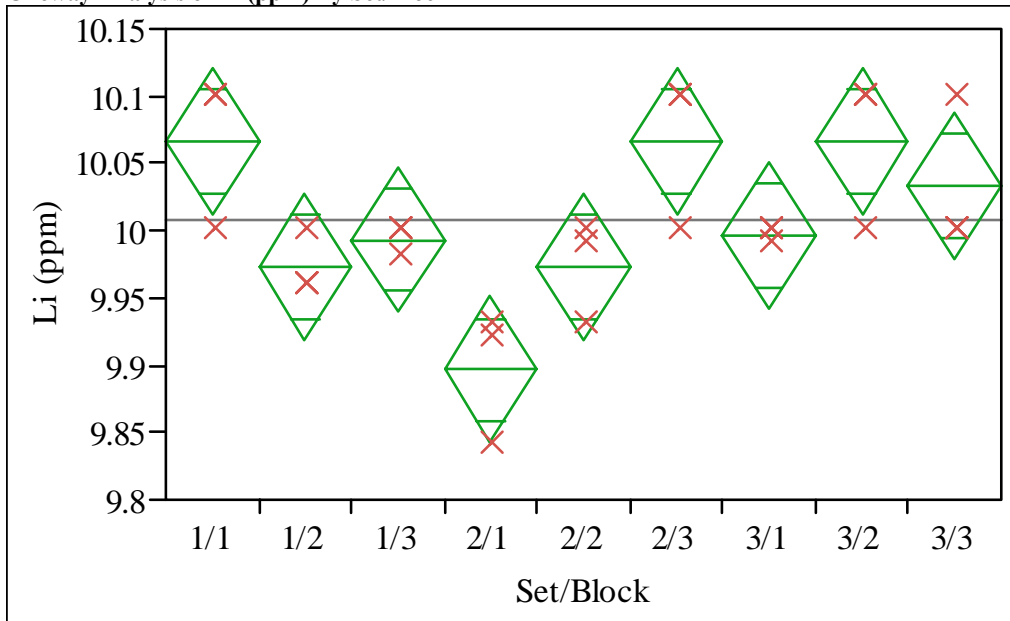

Oneway Anova

Summary of Fit

Rsquare

Adj Rsquare $\quad 0.546865$

Root Mean Square Error $\quad 0.044597$

Mean of Response 10.0074

Analysis of Variance

Source DF Sum of Squares Mean Square F Ratio Prob $>$ F

$\begin{array}{lllrrr}\text { Set/Block } & 8 & 0.07831852 & 0.009790 & 4.9223 & 0.0024\end{array}$

Error 18

0.001989

Means for Oneway Anova

Level Number Mean Std Error Lower 95\% Upper 95\%

$\begin{array}{rrrrrr}1 / 1 & 3 & 10.0667 & 0.02575 & 10.013 & 10.121\end{array}$

$\begin{array}{llllll}1 / 2 & 3 & 9.9733 & 0.02575 & 9.919 & 10.027 \\ 1 / 3 & 3 & 9.9933 & 0.02575 & 9.939 & 10.047\end{array}$

$\begin{array}{rrrrrr}1 / 3 & 3 & 9.9933 & 0.02575 & 9.939 & 10.047 \\ 2 / 1 & 3 & 9.8967 & 0.02575 & 9.843 & 9.951\end{array}$

$\begin{array}{rrrrrr}2 / 2 & 3 & 9.9733 & 0.02575 & 9.919 & 10.027\end{array}$

$\begin{array}{rrrrrr}2 / 3 & 3 & 10.0667 & 0.02575 & 10.013 & 10.121 \\ 3 / 1 & 3 & 9.9967 & 0.02575 & 9.943 & 10.051\end{array}$

$\begin{array}{rrrrrr}3 / 2 & 3 & 10.0667 & 0.02575 & 10.013 & 10.121\end{array}$

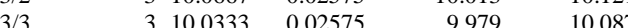

Std Error uses a pooled estimate of error variance 
SRNL-STI-2009-00465, Revision 0

\section{Exhibit B2. Measurements of the Multi-Element Solution Standard by ICP Block}

Oneway Analysis of Na (ppm) By Set/Block

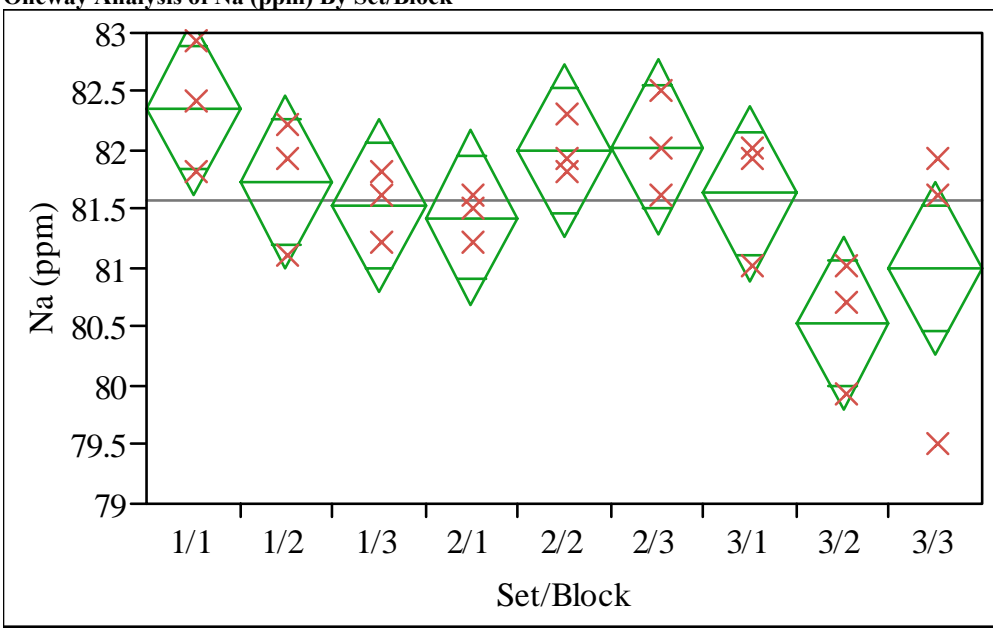

Oneway Anova

Summary of Fit

Rsquare

Adj Rsquare $\quad 0.524684$

$\begin{array}{ll} & 0.313433 \\ \text { Root Mean Square Error } \quad 0.612221\end{array}$

Mean of Response $\quad 81.58519$

Observations (or Sum Wgts) $\quad 27$

Analysis of Variance

Source DF Sum of Squares Mean Square F Ratio Prob $>$ F

$\begin{array}{lrrrrr}\text { Set/Block } & 8 & 7.447407 & 0.930926 & 2.4837 & 0.0519\end{array}$

$\begin{array}{lrrr}\text { Error } & 18 & 6.746667 & 0.374815\end{array}$

Means for Oneway Anova

Level Number Mean Std Error Lower 95\% Upper 95\%

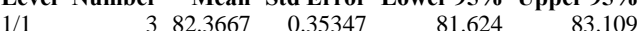

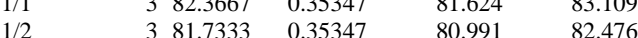

$\begin{array}{llllll}1 / 2 & 3 & 81.5333 & 0.35347 & 80.791 & 82.276\end{array}$

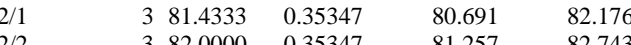

$\begin{array}{llllll}2 / 2 & 3 & 82.0000 & 0.35347 & 81.257 & 82.743 \\ 2 / 3 & 3 & 82.0333 & 0.35347 & 81.291 & 82.776\end{array}$

$\begin{array}{llllll}2 / 3 & 3 & 82.0333 & 0.35347 & 81.291 & 82.776 \\ 3 / 1 & 3 & 81.6333 & 0.35347 & 80.891 & 82.376\end{array}$

$\begin{array}{llllll}3 / 2 & 3 & 80.5333 & 0.35347 & 79.791 & 81.276\end{array}$

Std Error uses a pooled estimate of error variance
Oneway Analysis of Si (ppm) By Set/Block

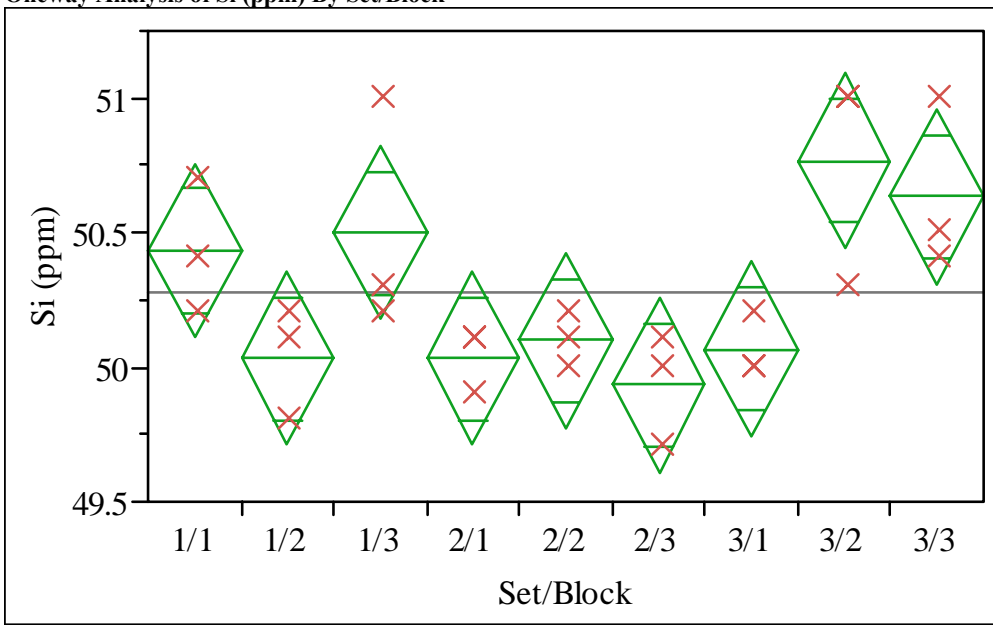

Oneway Anova

Summary of Fit

Rsquare

0.637218

Adj Rsquare $\quad 0.475982$

Root Mean Square Error $\quad 0.26736$

Mean of Response

50.27778

Analysis of Variance

Source DF Sum of Squares Mean Square F Ratio Prob $>$ F

$\begin{array}{llrrr} & 2.2600000 & 0.282500 & 3.9521 & 0.0074\end{array}$

$\begin{array}{lll}\text { Error } & 18 & 1.2866667\end{array}$

0.071481

Means for Oneway Anova

Level Number Mean Std Error Lower 95\% Upper 95\%

$\begin{array}{lrrrrr}1 / 1 & 3 & 50.4333 & 0.15436 & 50.109 & 50.758\end{array}$

$\begin{array}{llllll}1 / 2 & 3 & 50.0333 & 0.15436 & 49.709 & 50.358 \\ 1 / 3 & 3 & 50.5000 & 0.15436 & 50.176 & 50.824\end{array}$

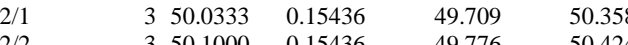

$\begin{array}{llllll}2 / 2 & 3 & 50.1000 & 0.15436 & 49.776 & 50.424 \\ 2 / 3 & 3 & 49.9333 & 0.15436 & 49.609 & 50.258\end{array}$

$\begin{array}{llllll}2 / 3 & 3 & 49.9333 & 0.15436 & 49.609 & 50.258 \\ 3 / 1 & 3 & 50.0667 & 0.15436 & 49.742 & 50.391\end{array}$

Std Error uses a pooled estimate of error variance 


\section{Exhibit B3. Laboratory PCT Measurements by Glass Identifier for Study Glasses and Standards}

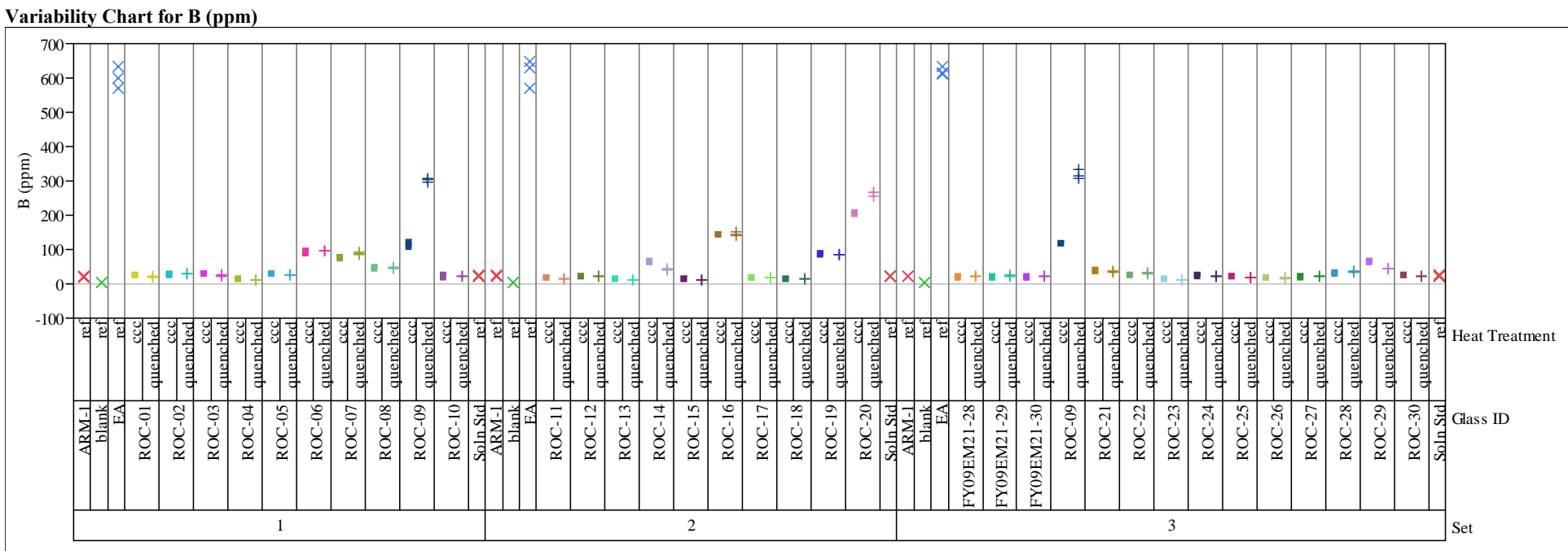

Variability Chart for Li (ppm)
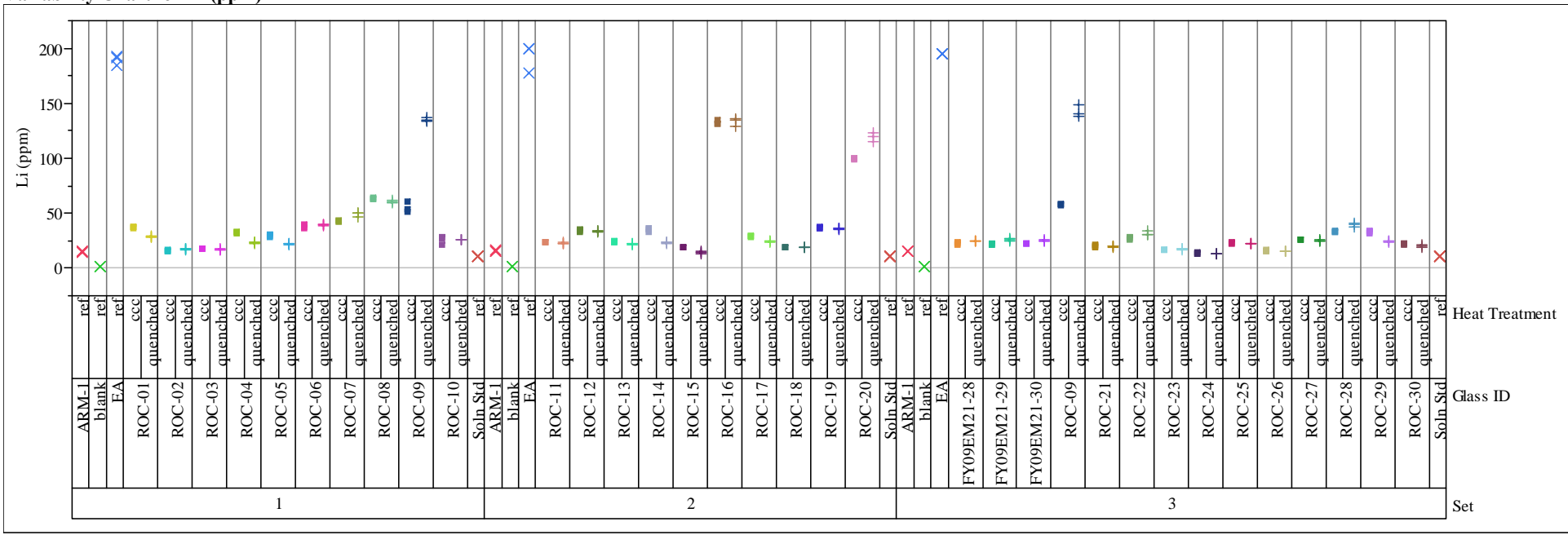


\section{Exhibit B3. Laboratory PCT Measurements by Glass Identifier for Study Glasses and Standards}
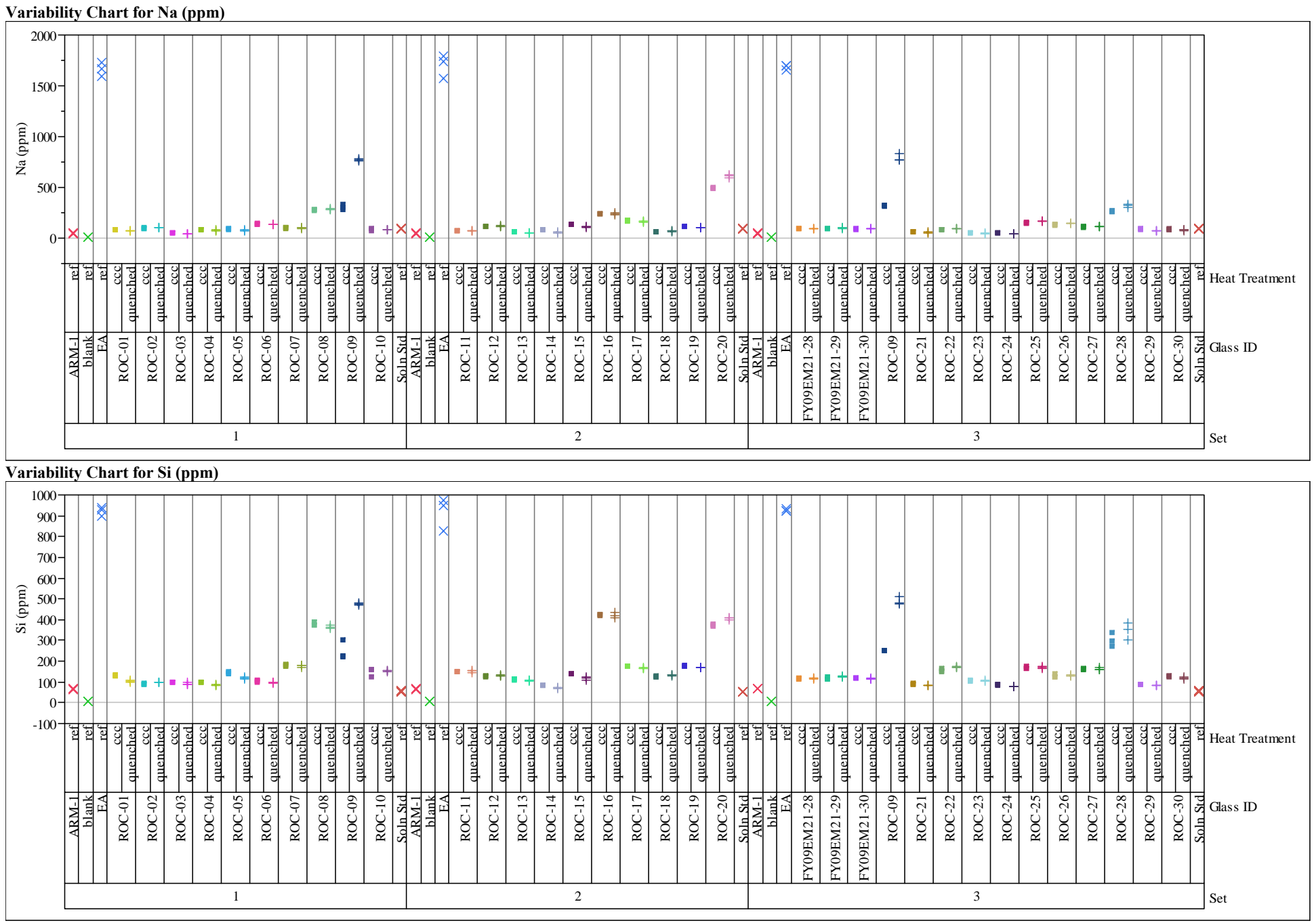


\section{Exhibit B3. Laboratory PCT Measurements by Glass Identifier for Study Glasses and Standards}

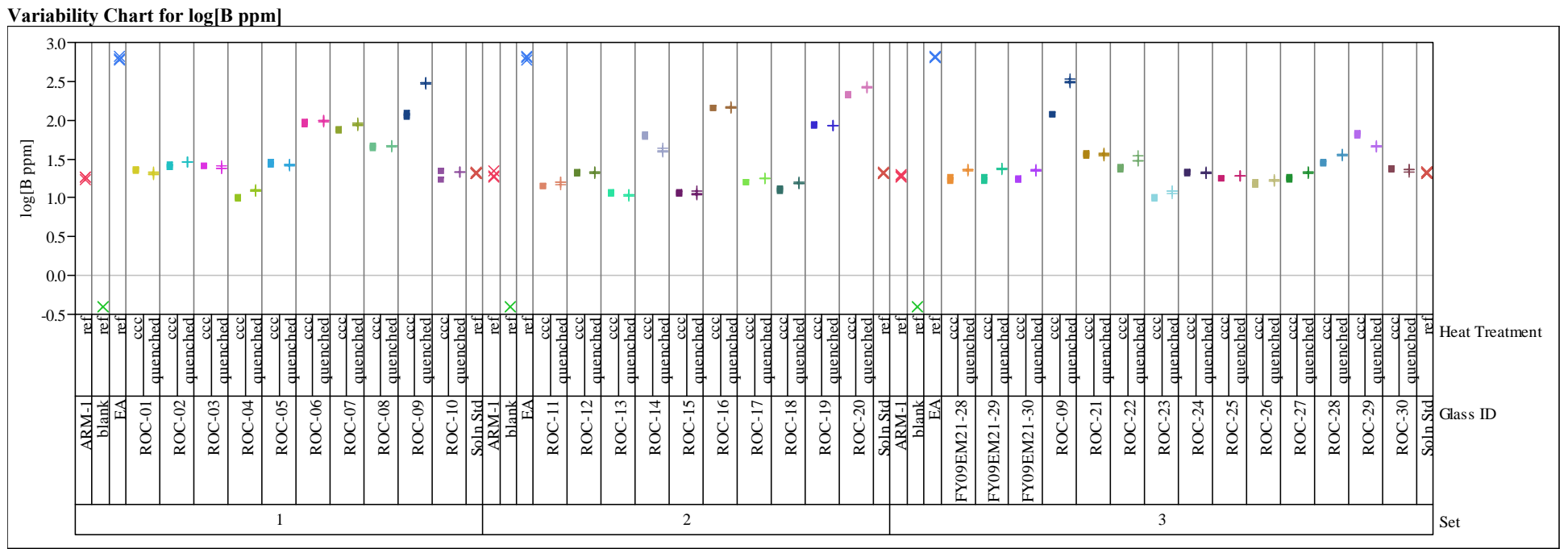

Variability Chart for log[Li ppm]

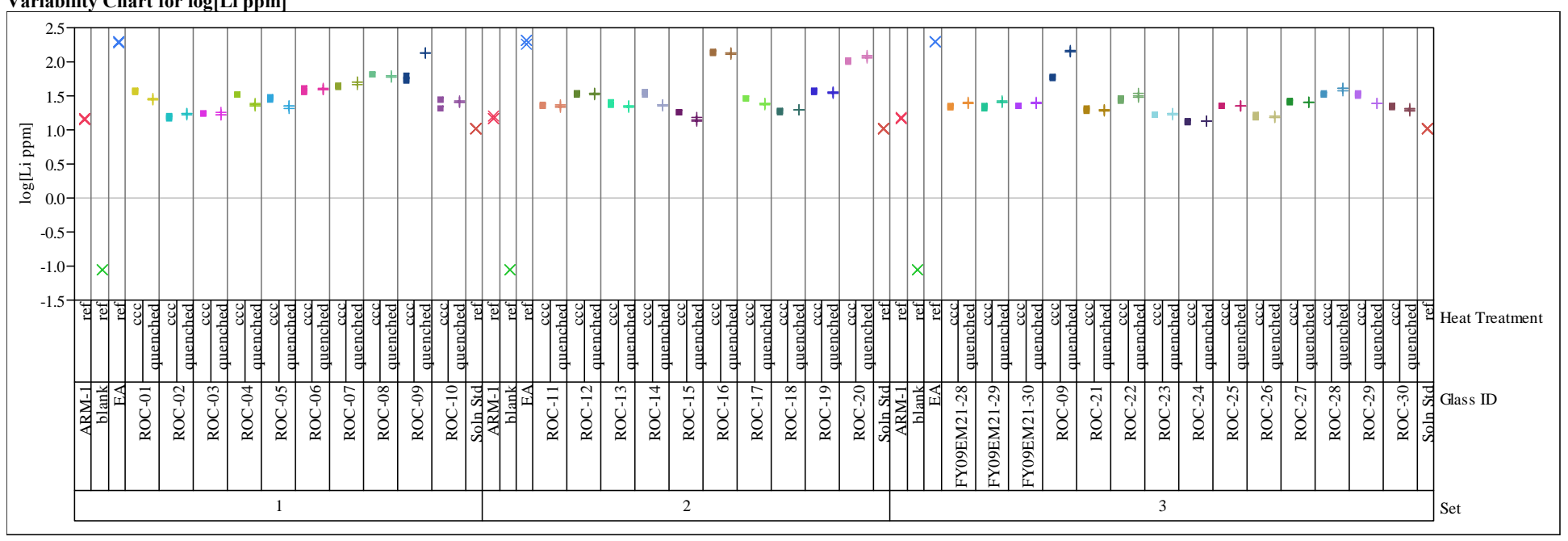


SRNL-STI-2009-00465, Revision 0

Exhibit B3. Laboratory PCT Measurements by Glass Identifier for Study Glasses and Standards

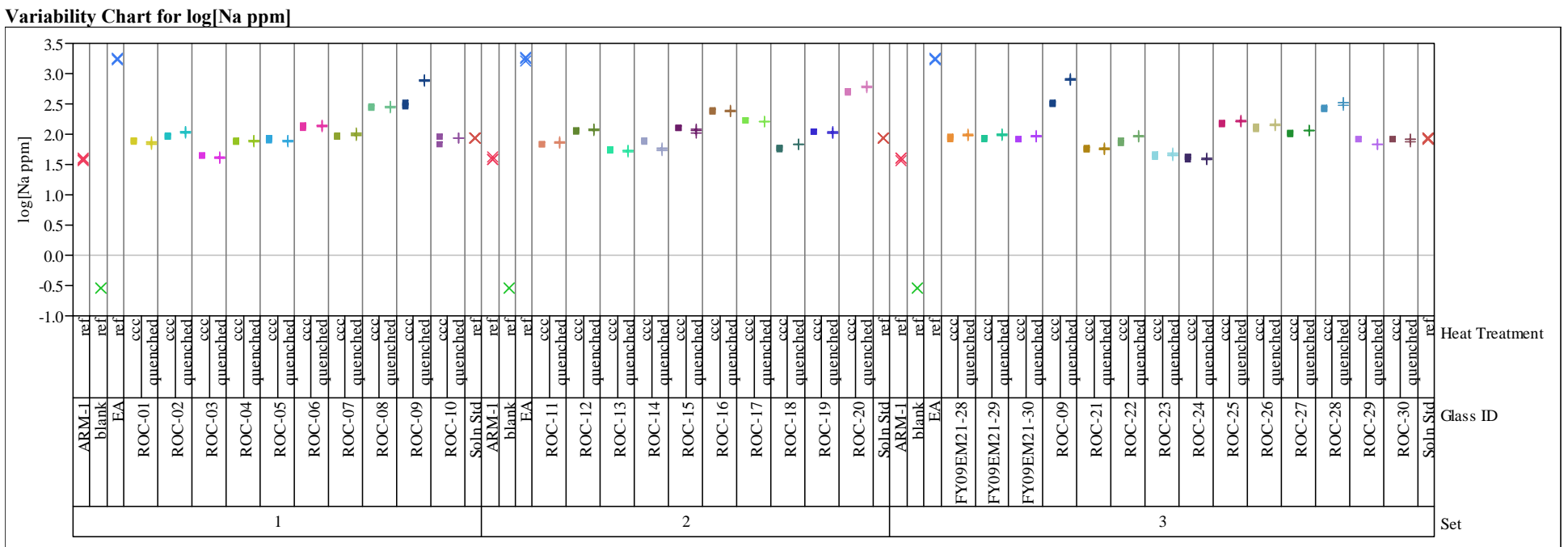

Variability Chart for log[Si ppm]

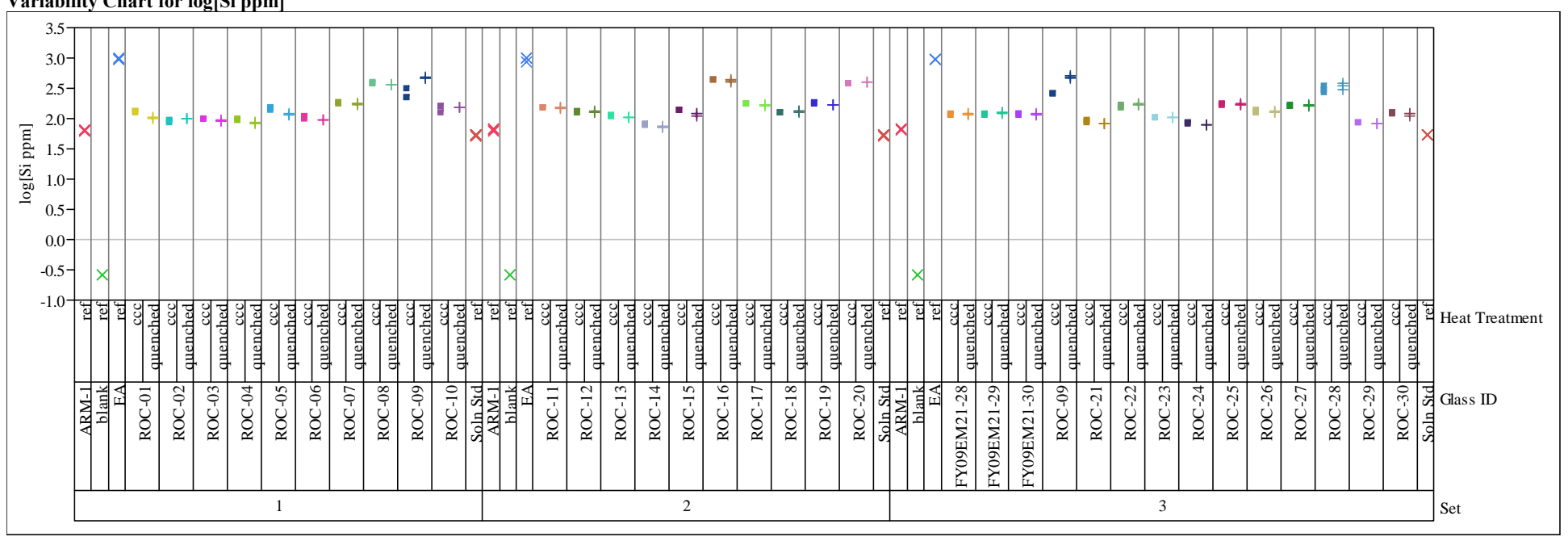




\section{Exhibit B4. Correlations and Scatter Plots of Normalized PCTs} Over All Compositional Views and Heat Treatments

Multivariate

Correlations

$\begin{array}{lrrrr} & \log \mathbf{N L}[\mathbf{B}(\mathbf{g} / \mathbf{L})] & \log \mathbf{N L}[\mathbf{L i}(\mathbf{g} / \mathbf{L})] & \log \mathbf{N L}[\mathbf{N a}(\mathbf{g} / \mathbf{L})] & \log \mathbf{N L}[\mathbf{S i}(\mathbf{g} / \mathbf{L})] \\ \log \mathrm{NL}[\mathrm{B}(\mathrm{g} / \mathrm{L})] & 1.0000 & 0.9882 & 0.9608 & 0.9170 \\ \log \mathrm{NL}[\mathrm{Li}(\mathrm{g} / \mathrm{L})] & 0.9882 & 1.0000 & 0.9576 & 0.9152 \\ \log \mathrm{NL}[\mathrm{Na}(\mathrm{g} / \mathrm{L})] & 0.9608 & 0.9576 & 1.0000 & 0.9371 \\ \log \mathrm{NL}[\mathrm{Si}(\mathrm{g} / \mathrm{L})] & 0.9170 & 0.9152 & 0.9371 & 1.0000\end{array}$

\section{Scatterplot Matrix}

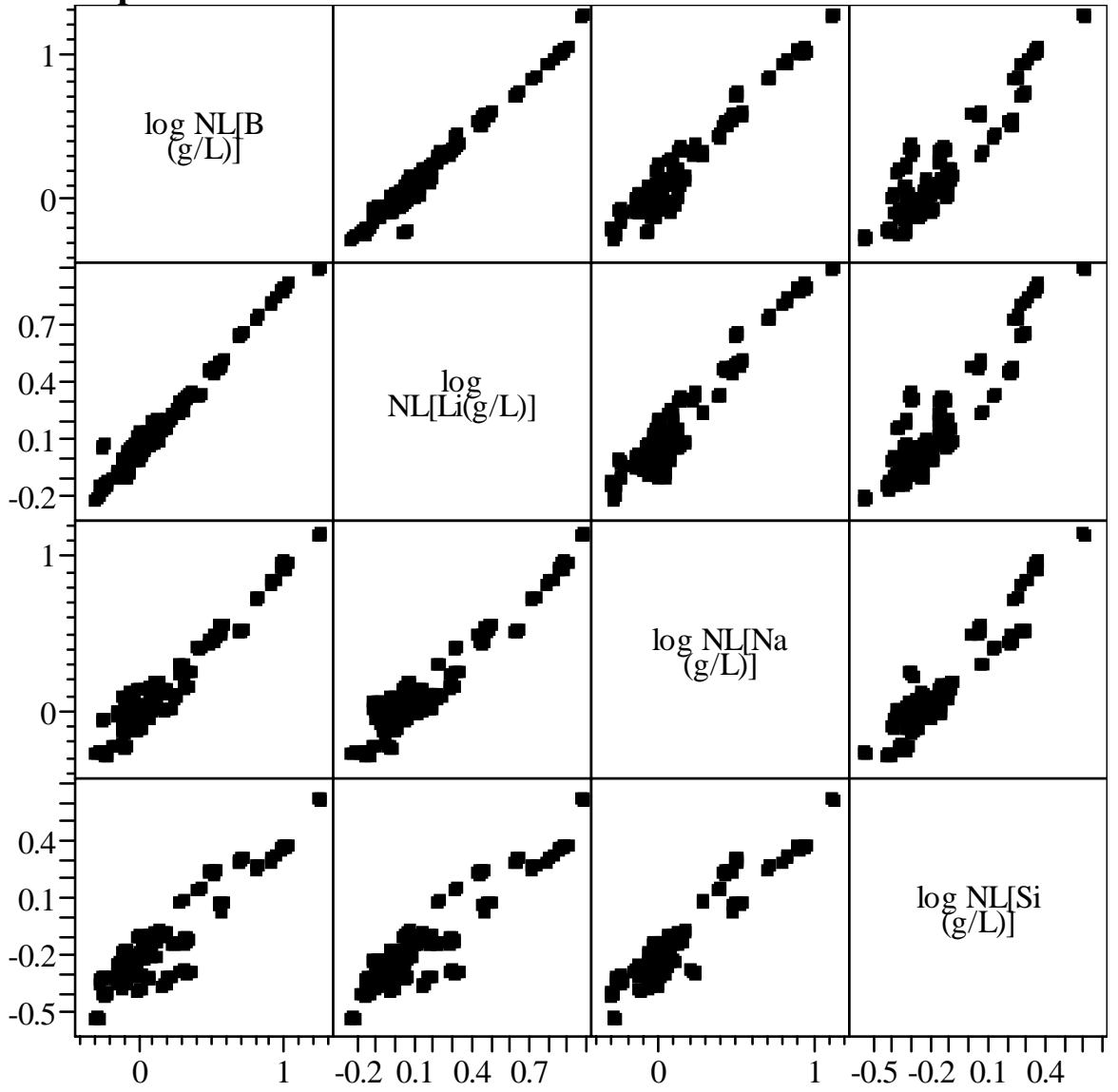




\section{Exhibit B5. Effects of Heat Treatment for Study Glasses by Compositional View}

Comp View=measured

Variability Chart for $\log$ NL[B (g/L)]

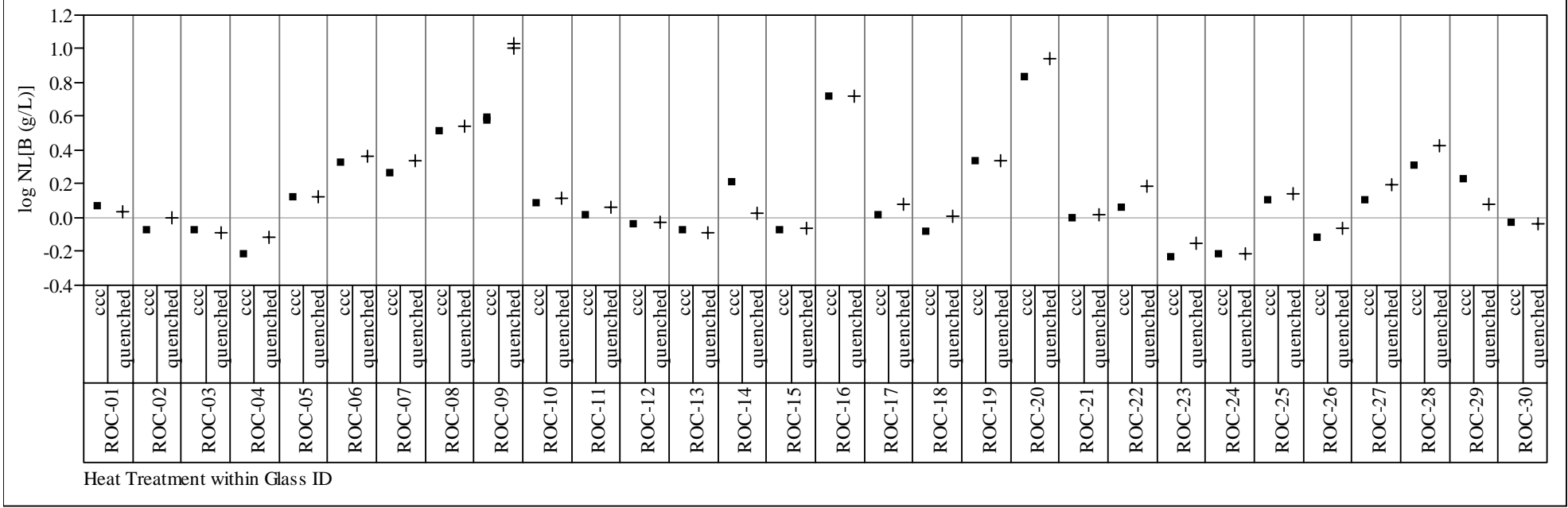

Comp View=measured

Variability Chart for $\log \mathrm{NL}[\mathrm{Li}(\mathrm{g} / \mathrm{L})$

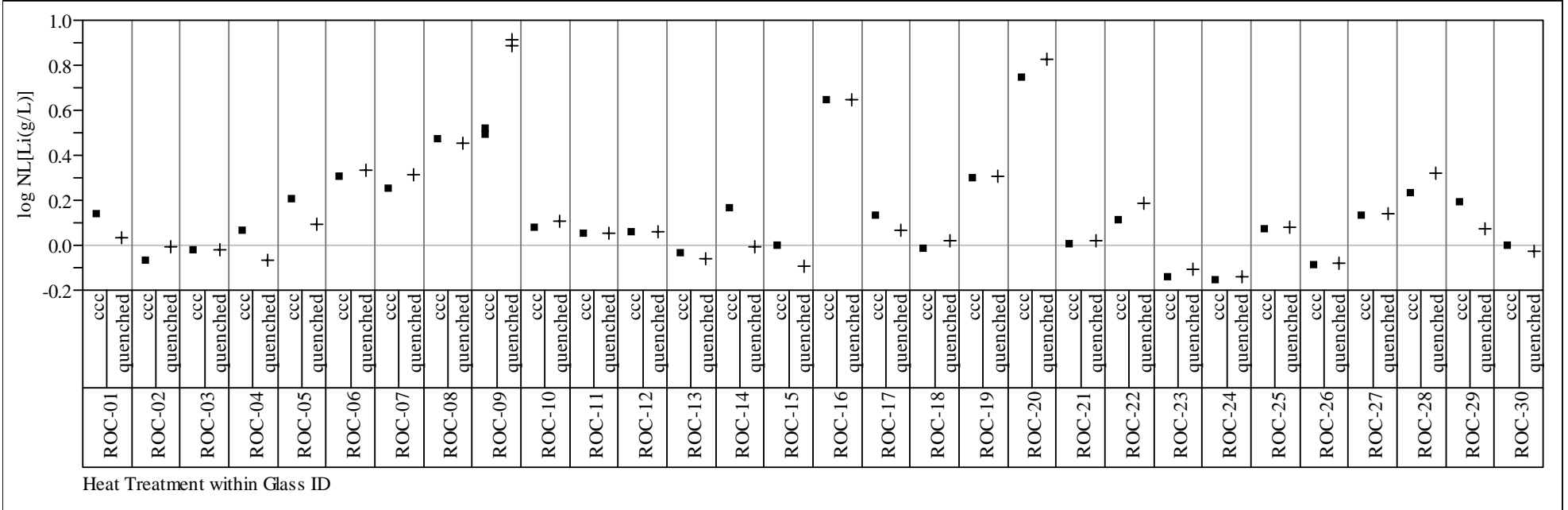




\section{Exhibit B5. Effects of Heat Treatment for Study Glasses by Compositional View}

Comp View=measured

Variability Chart for log NL[Na (g/L)]

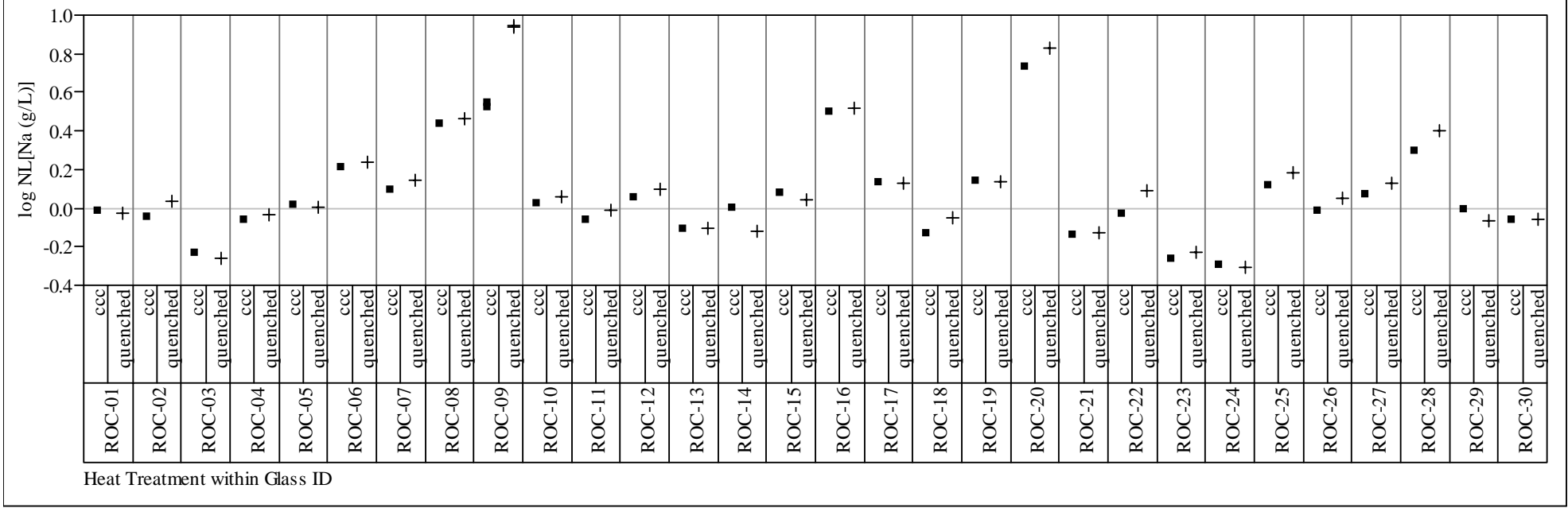

Comp View=measured

Variability Chart for $\log \mathrm{NL}[\mathrm{Si}(\mathrm{g} / \mathrm{L})$

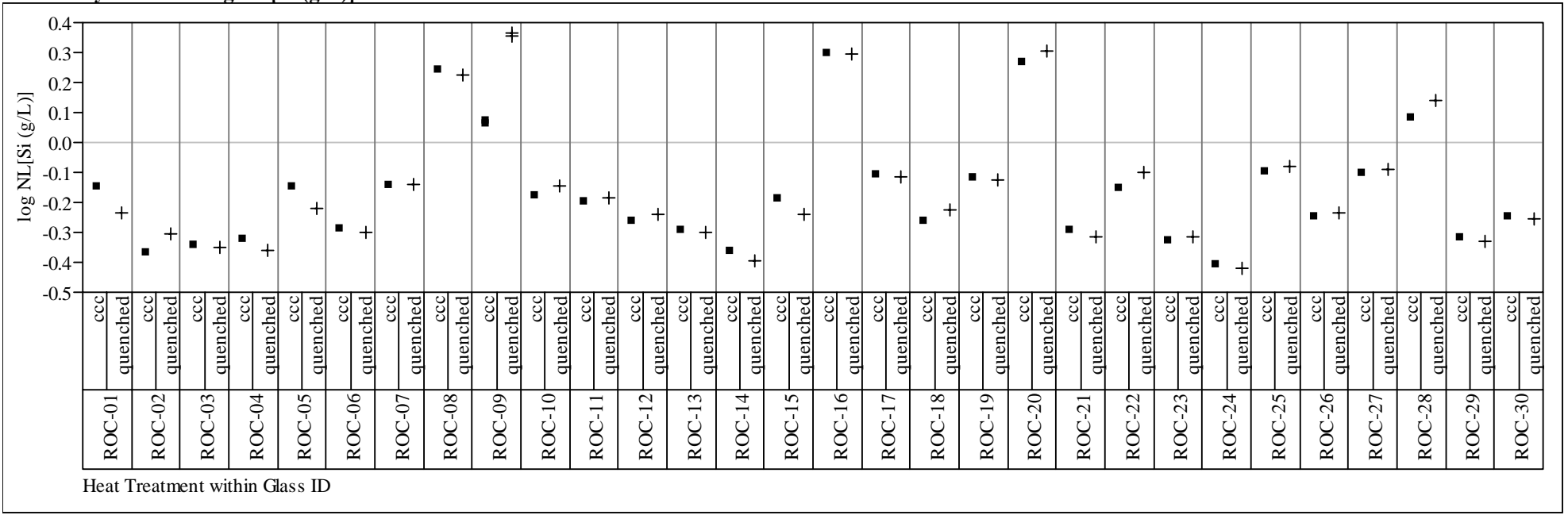




\section{Exhibit B5. Effects of Heat Treatment for Study Glasses by Compositional View}

Comp View=measured bc

Variability Chart for log NL[B (g/L)]

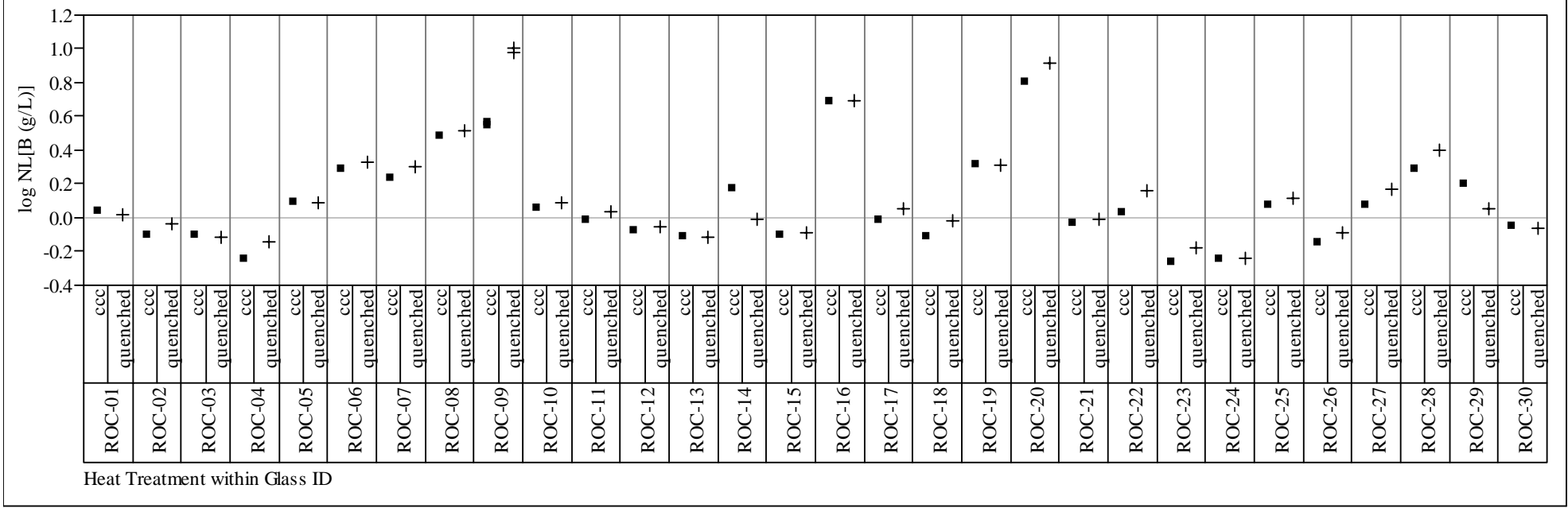

Comp View=measured bc

Variability Chart for $\log \mathrm{NL} / \mathrm{Li}(\mathrm{g} / \mathrm{L})$

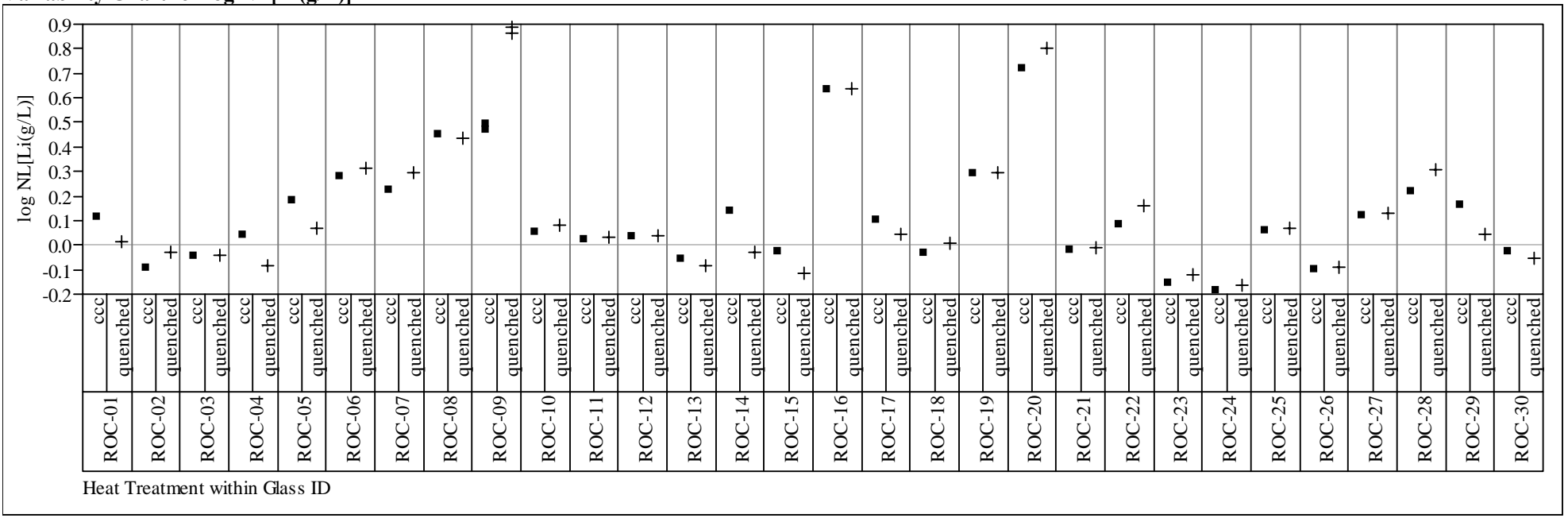




\section{Exhibit B5. Effects of Heat Treatment for Study Glasses by Compositional View}

Comp View=measured bc

Variability Chart for log NL[Na (g/L)]

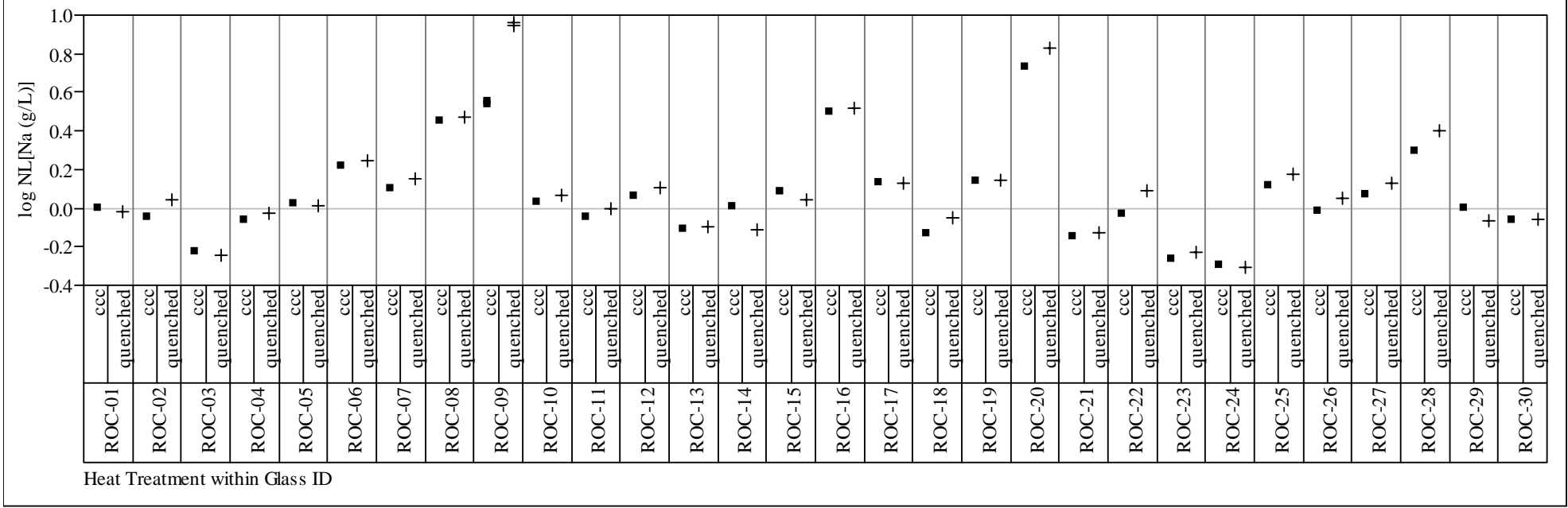

Comp View=measured bc

Variability Chart for $\log \mathrm{NL}[\mathrm{Si}(\mathrm{g} / \mathrm{L})$

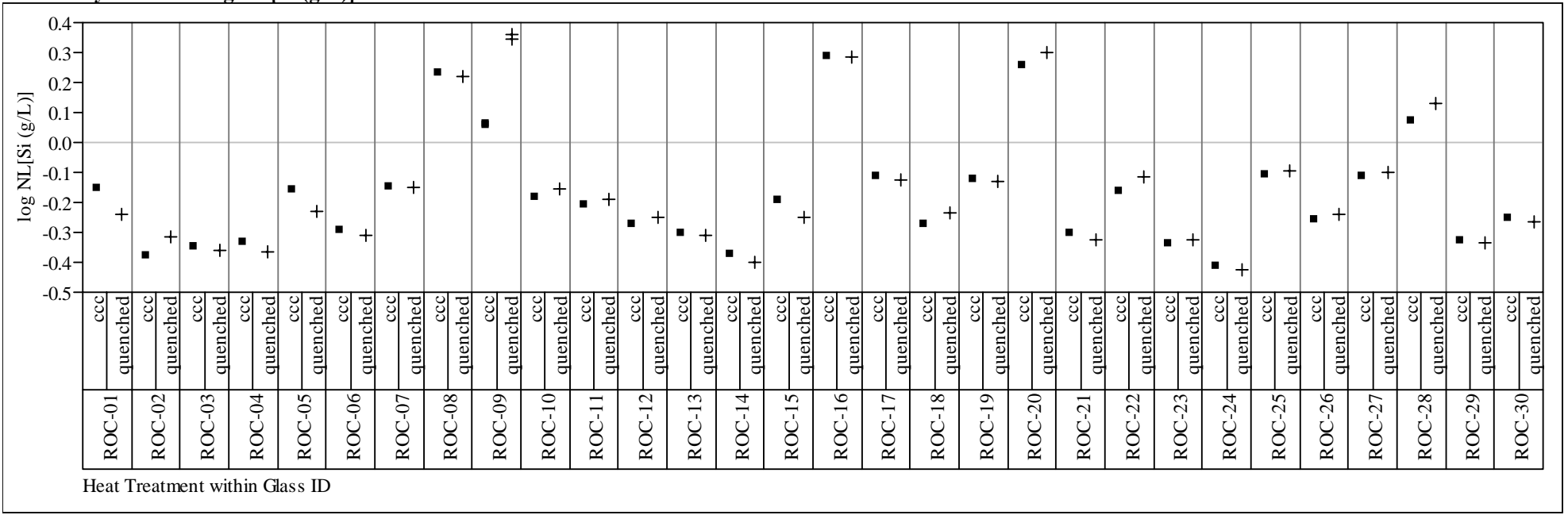




\section{Exhibit B5. Effects of Heat Treatment for Study Glasses by Compositional View}

Comp View=targeted

Variability Chart for $\log \mathrm{NL}[\mathrm{B}(\mathrm{g} / \mathrm{L})]$

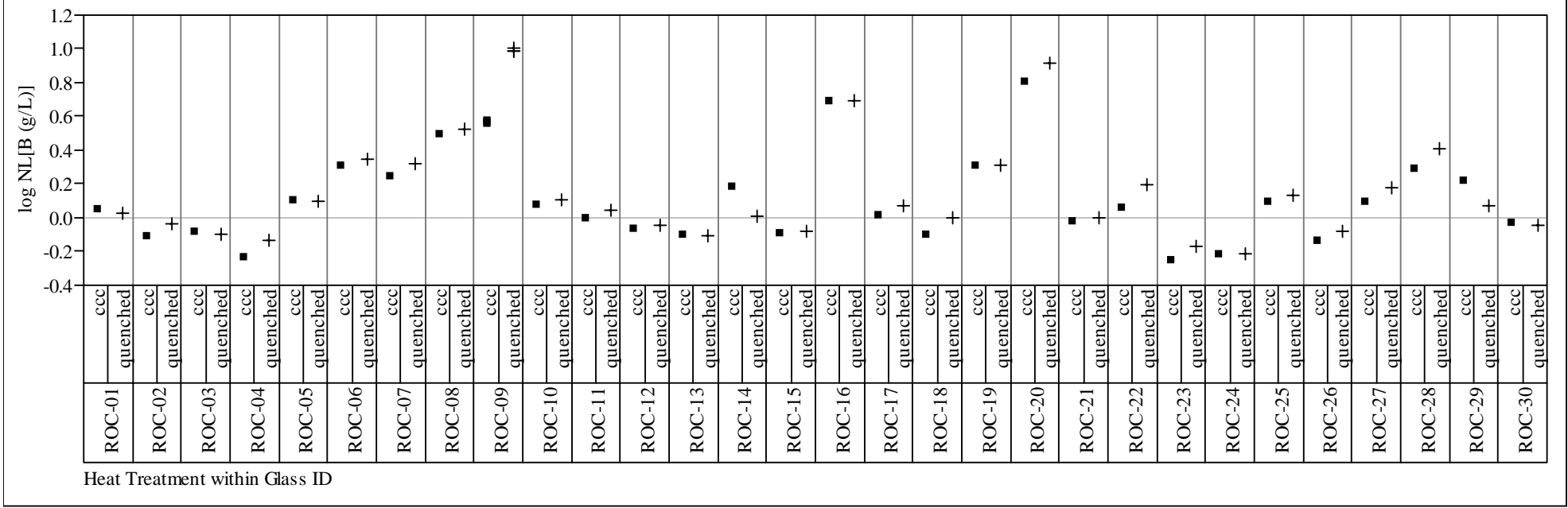

Comp View=targeted

Variability Chart for $\log \mathrm{NL}[\mathrm{Li}(\mathrm{g} / \mathrm{L})]$

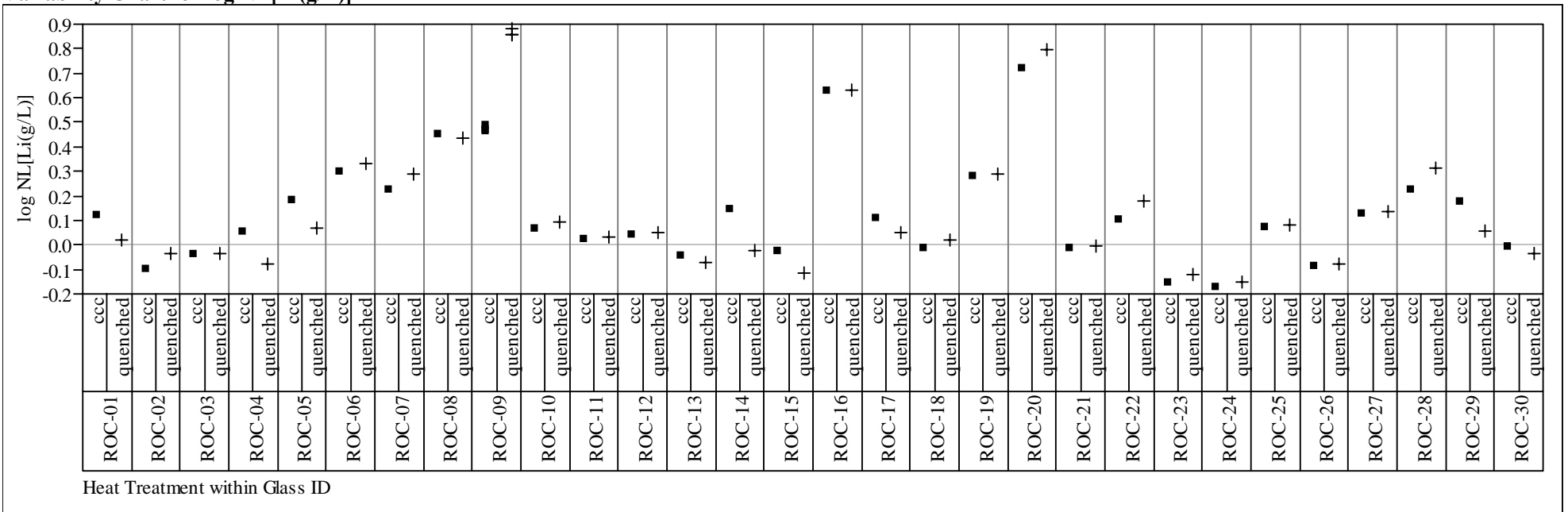




\section{Exhibit B5. Effects of Heat Treatment for Study Glasses by Compositional View}

Comp View=targeted

Variability Chart for log NL[Na (g/L)]

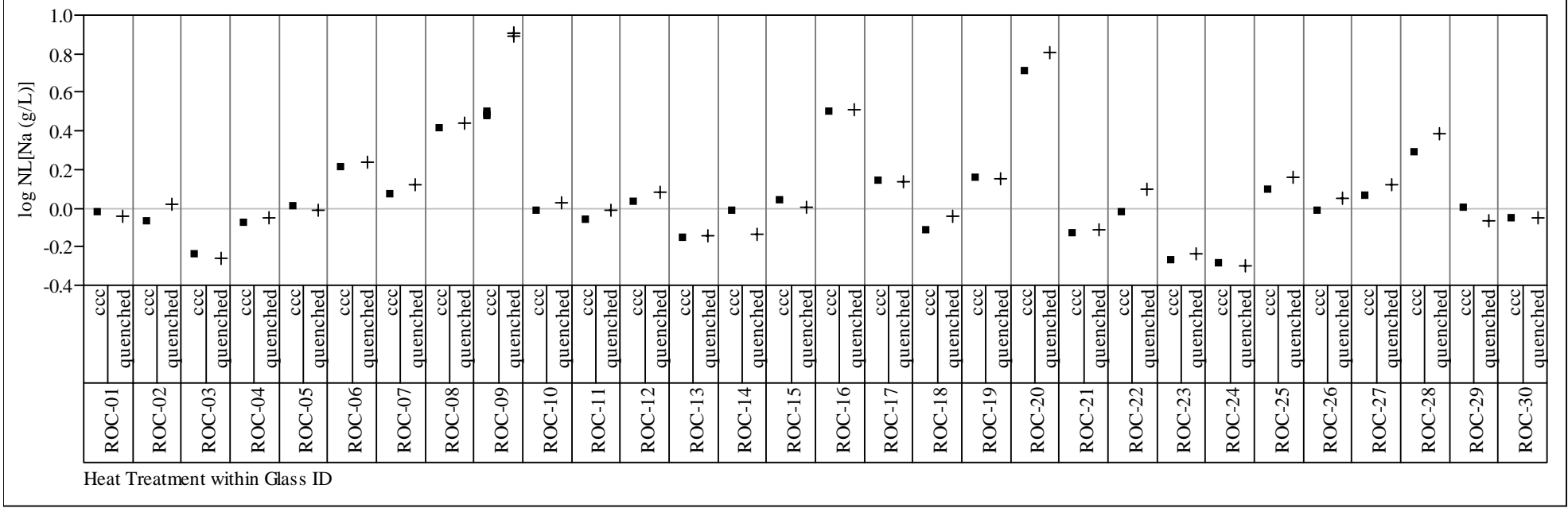

Comp View=targeted

Variability Chart for $\log \mathbf{N L}[\mathrm{Si}(\mathrm{g} / \mathrm{L})$

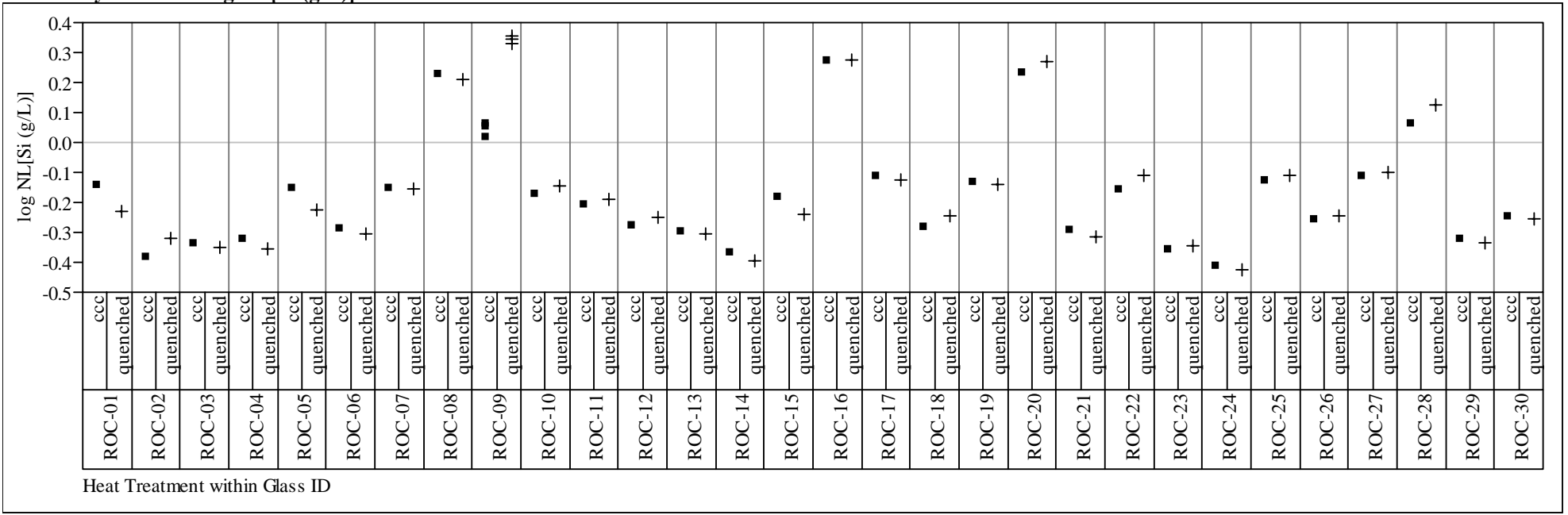


Exhibit B6. del Gp $\left(\Delta G_{p}\right)$ Predictions versus Common Logarithm Normalized Leachate $(\log$ NL[.]) for B over All Compositional Views and Heat Treatments

Legend

\begin{tabular}{|c|c|}
\hline Symbol & $\begin{array}{c}\text { Standard/ } \\
\text { Comp View-Heat Treatment }\end{array}$ \\
\hline $\mathrm{z}$ & EA \\
\hline$\diamond$ & ARM \\
\hline$\square$ & Measured-ccc \\
\hline$\square$ & Measured bc -ccc \\
\hline$\square$ & Targeted-ccc \\
\hline$\bullet$ & Measured-quenched \\
\hline$\bullet$ & Measured bc - quenched \\
\hline$\bullet$ & Targeted- quenched \\
\hline
\end{tabular}

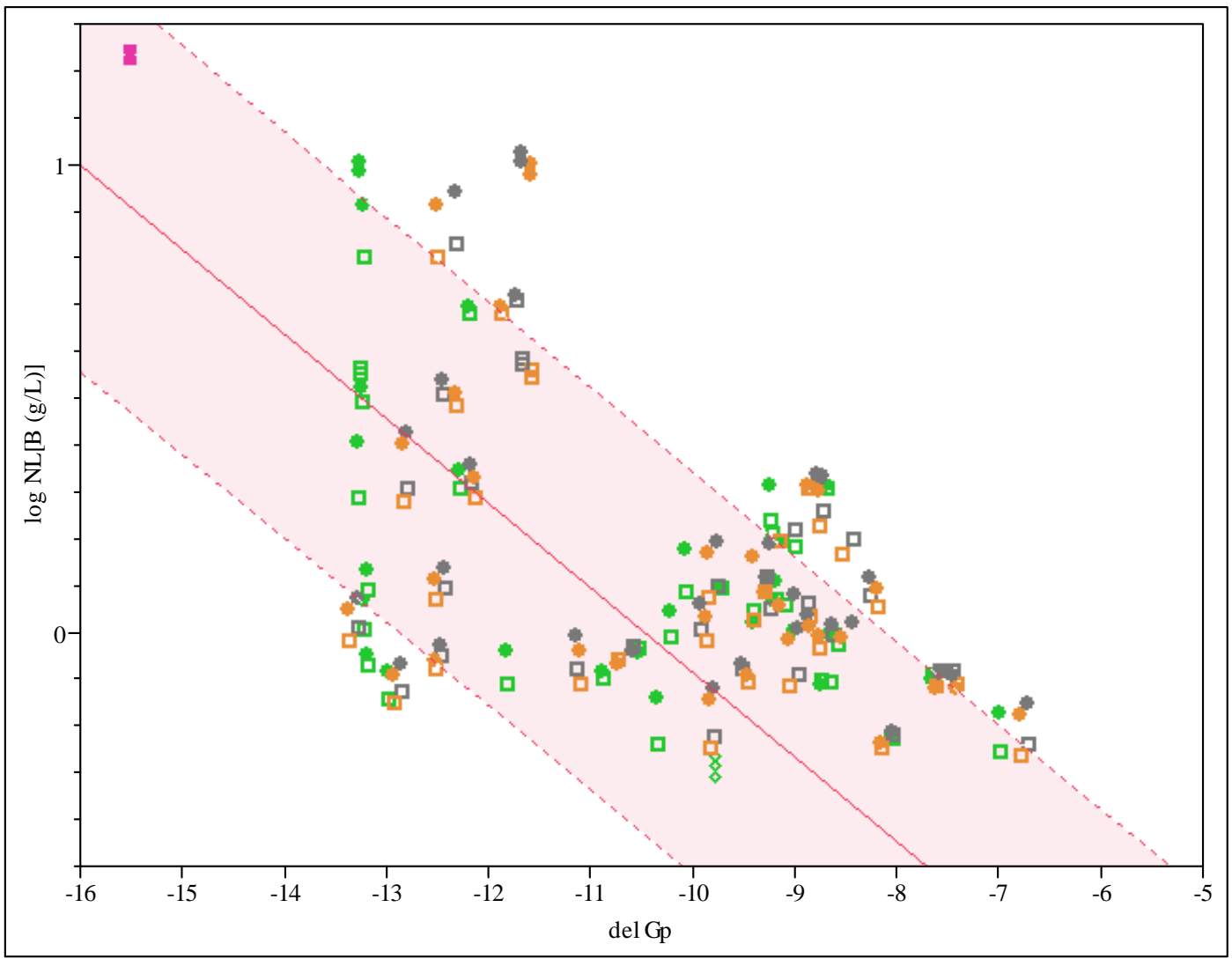


Exhibit B7. del Gp $\left(\Delta G_{p}\right)$ Predictions versus Common Logarithm Normalized Leachate $(\log N L[]$.$) for Li over All Compositional Views and Heat Treatments$

Legend

\begin{tabular}{|c|c|}
\hline Symbol & $\begin{array}{c}\text { Standard/ } \\
\text { Comp View-Heat Treatment }\end{array}$ \\
\hline $\mathrm{z}$ & EA \\
\hline$\triangleright$ & ARM \\
\hline$\square$ & Measured-ccc \\
\hline$\square$ & Measured bc -ccc \\
\hline$\square$ & Targeted-ccc \\
\hline$\bullet$ & Measured-quenched \\
\hline$\bullet$ & Measured bc - quenched \\
\hline$\bullet$ & Targeted- quenched \\
\hline
\end{tabular}

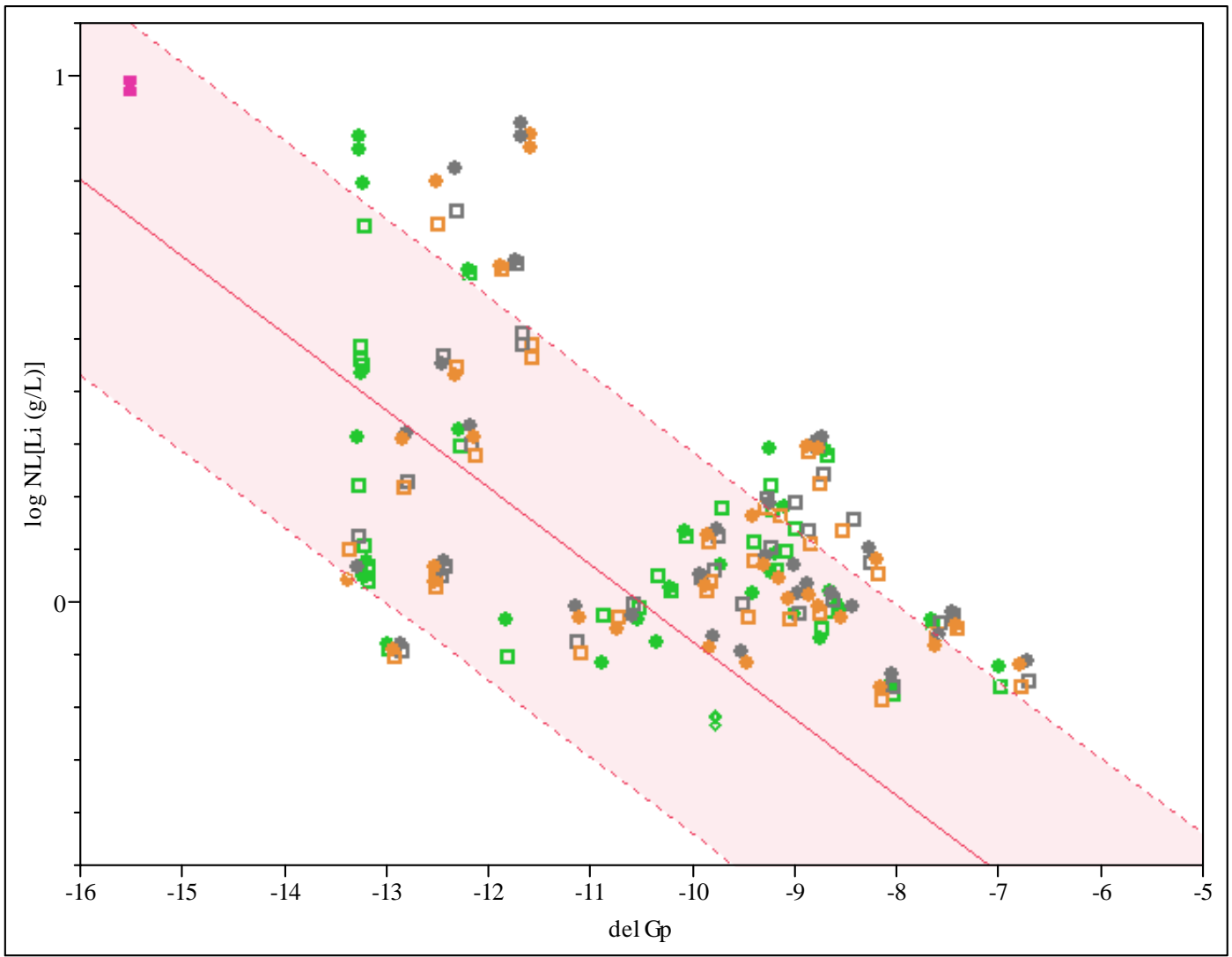


Exhibit B8. del $G p\left(\Delta G_{p}\right)$ Predictions versus Common Logarithm Normalized Leachate $(\log N L[]$.$) for Na over All Compositional Views and Heat Treatments$

Legend

\begin{tabular}{|c|c|}
\hline Symbol & $\begin{array}{c}\text { Standard/ } \\
\text { Comp View-Heat Treatment }\end{array}$ \\
\hline $\mathrm{Z}$ & EA \\
\hline$\triangleright$ & ARM \\
\hline$\square$ & Measured-ccc \\
\hline$\square$ & Measured bc -ccc \\
\hline$\square$ & Targeted-ccc \\
\hline$\bullet$ & Measured-quenched \\
\hline$\bullet$ & Measured bc - quenched \\
\hline$\bullet$ & Targeted- quenched \\
\hline
\end{tabular}

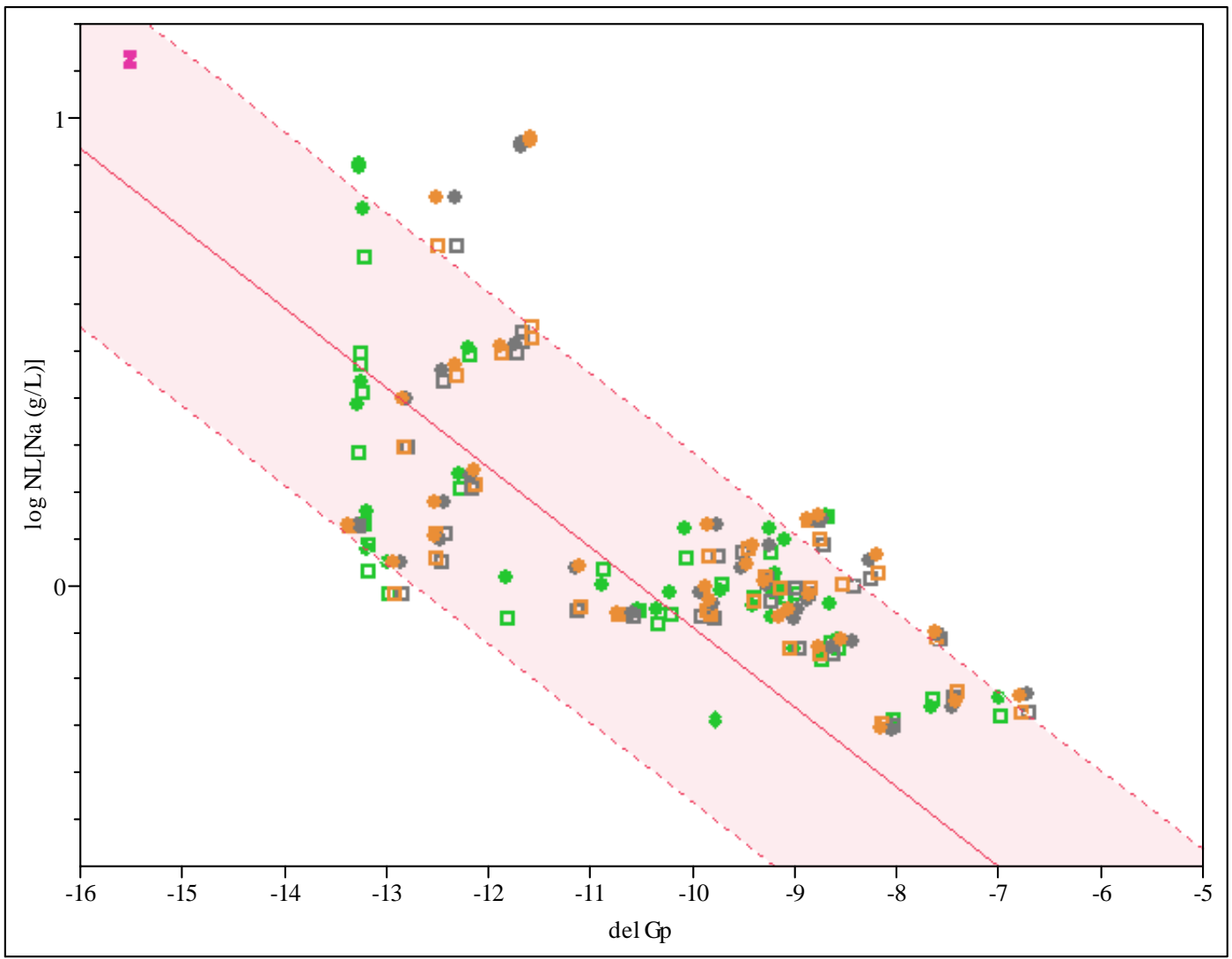


Exhibit B9. del $G p\left(\Delta G_{p}\right)$ Predictions versus Common Logarithm Normalized Leachate $(\log \mathrm{NL}[]$.$) for Si over All Compositional Views and Heat Treatments$

Legend

\begin{tabular}{|c|c|}
\hline Symbol & $\begin{array}{c}\text { Standard/ } \\
\text { Comp View-Heat Treatment }\end{array}$ \\
\hline $\mathrm{z}$ & EA \\
\hline$\diamond$ & ARM \\
\hline$\square$ & Measured-ccc \\
\hline$\square$ & Measured bc -ccc \\
\hline$\square$ & Targeted-ccc \\
\hline$\bullet$ & Measured-quenched \\
\hline$\bullet$ & Measured bc - quenched \\
\hline$\bullet$ & Targeted- quenched \\
\hline
\end{tabular}

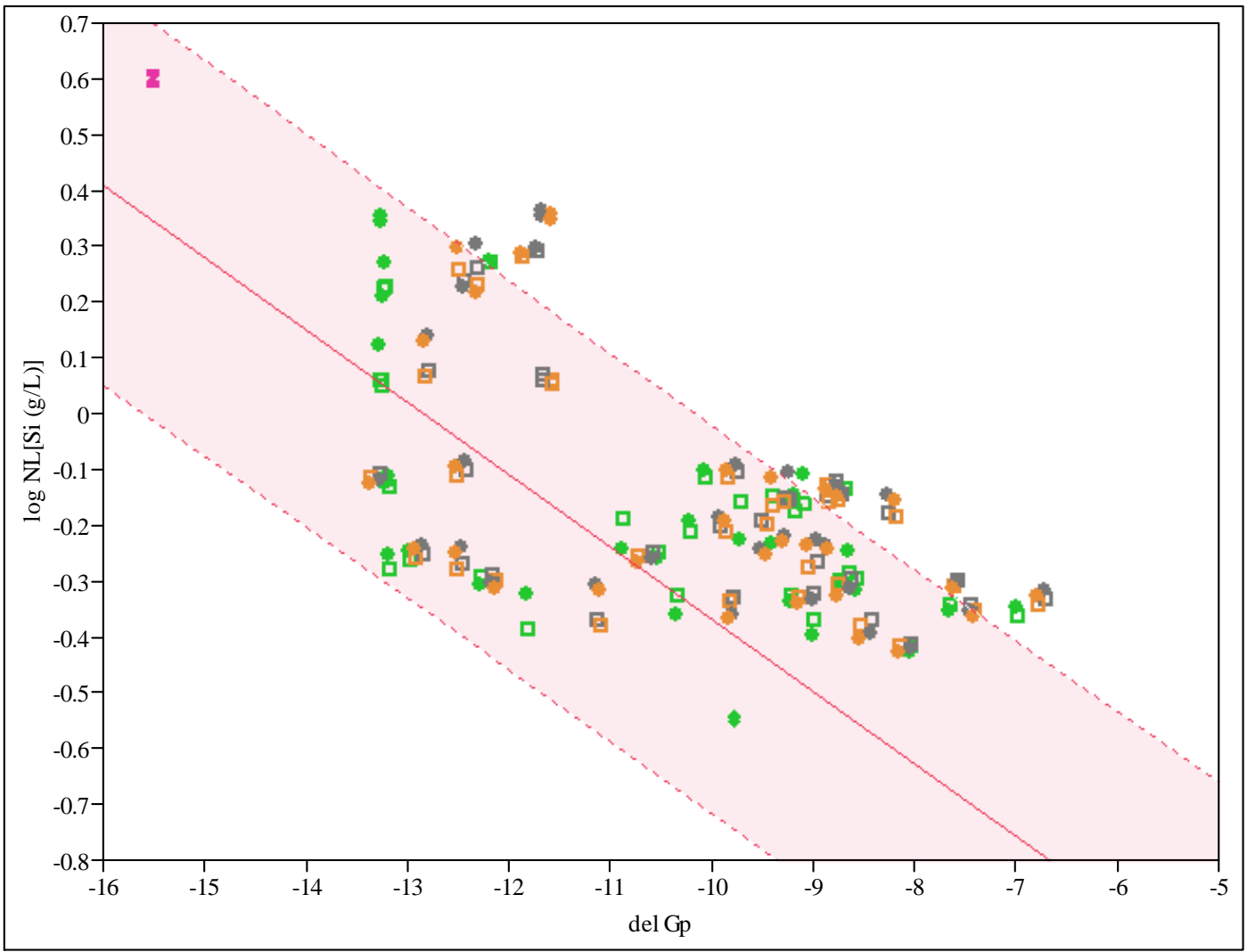




\section{Distribution:}

C.J. Bannochie, 773-42A

A.B. Barnes, 999-W

A.L. Billings, 999-W

J.M. Bricker, 704-27S

M.A. Broome, 704-29S

D.A. Crowley, 773-43A

T.B. Edwards, 999-W

T.L. Fellinger, 704-26S

S.D. Fink, 773-A

K.M. Fox, 999-W

B.J. Giddings, 786-5A

J.M. Gillam, 766-H

B.A. Hamm, 766-H

C.C. Herman, 999-W

R.N. Hinds, 704-S

E.W. Holtzscheiter, 704-15S

J.F. Iaukea, 704-30S

C.M. Jantzen, 773-A

D.D. Larsen, 766-H

S.L. Marra, 773-A

R.T. McNew, 704-27S

J.E. Occhipinti, 704-S

D.K. Peeler, 999-W

F.M. Pennebaker, 773-42A

H.M. Pittman, 704-27S

F.C. Raszewski, 999-W

J.W. Ray, 704-S

J.H. Scogin, 773-A

H.B. Shah, 766-H

D.C. Sherburne, 704-S

M.E. Stone, 999-W

J.P. Vaughan, 773-41A 